\title{
A Preliminary Evaluation of Alternatives for Disposal of INEL Low-Level Waste and Low-Level Mixed Waste
}

\author{
T. H. Smith \\ W. S. Roesener \\ M. J. Jorgenson-Waters \\ Published July 1993 \\ Idaho National Engineering Laboratory \\ EG\&G Idaho, Inc. \\ Idaho Falls, Idaho 83415
}




\section{ABSTRACT}

The Mixed and Low-Level Waste Disposal Facility (MLLWDF) project was established in 1992 by the U.S. Department of Energy Idaho Operations Office to provide enhanced disposal capabilities for Idaho National Engineering Laboratory (INEL) low-level mixed waste and low-level waste. This Preliminary Evaluation of Alternatives for Disposal of INEL Low-Level Waste and Low-Level Mixed Waste identifies and evaluates-on a preliminary, overview basis-the alternatives for disposal of that waste. Five disposal alternatives, ranging from "no-action" to constructing and operating the MLLWDF, are identified and evaluated. Several subalternatives are formulated within the MLLWDF alternative. The subalternatives involve various disposal technologies as well as various scenarios related to the waste volumes and waste forms to be received for disposal. The evaluations include qualitative comparisons of the projected isolation performance for each alternative, and facility, health and safety, environmental, institutional, schedule, and rough order-of-magnitude life-cycle cost comparisons. The performance of each alternative is evaluated against lists of "musts" and "wants." Also included is a discussion of other key considerations for decisionmaking. The analysis of results indicated further study is necessary to obtain the best estimate of long-term future waste volume and characteristics from the INEL Environmental Restoration activities and the expanded INEL Decontamination and Decommissioning Program. Several performanceverification support studies are also needed. It is recommended that activities proceed on the offsite alternative and/or the onsite shallow land burial alternatives while reevaluating the waste volume projections. This approach will allow the INEL to develop the necessary disposal capacity for these wastes as soon as feasible. It is also recommended that a preliminary performance assessment be conducted in FY 1995 and FY 1996 when conceptual design is underway, and a detailed performance assessment be performed after Title II design in FY 1999 and FY 2000. 


\section{CONTENTS}

ABSTRACT $\ldots \ldots \ldots \ldots \ldots \ldots \ldots \ldots \ldots \ldots \ldots \ldots \ldots \ldots \ldots \ldots \ldots \ldots \ldots$

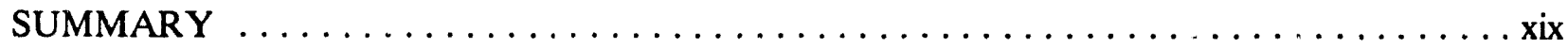

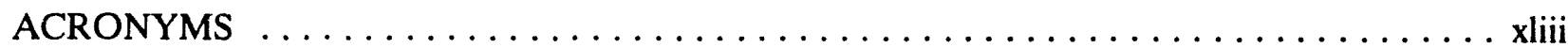

REGULATORY SOURCES CITED $\ldots \ldots \ldots \ldots \ldots \ldots \ldots \ldots \ldots \ldots \ldots \ldots \ldots \ldots$

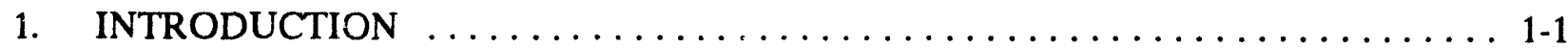

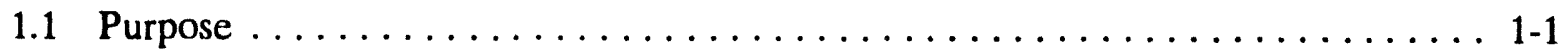

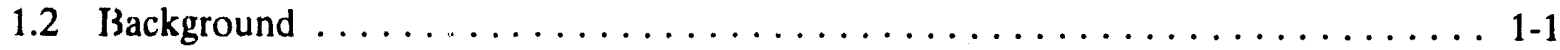

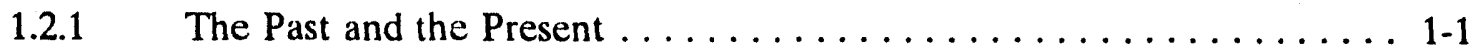

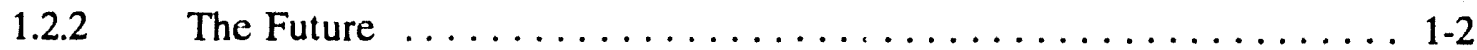

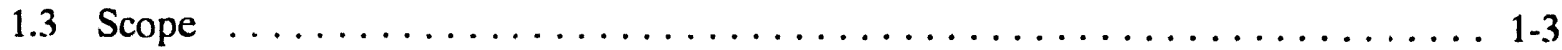

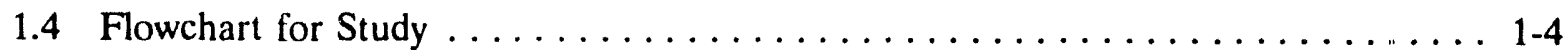

2. DESCRIPTION OF WASTE STREAMS AND REQUIREMENTS AFFECTING

DISPOSAL

2.1 Sources and Types of Waste to be Disposed of $\ldots \ldots \ldots \ldots \ldots \ldots \ldots \ldots$

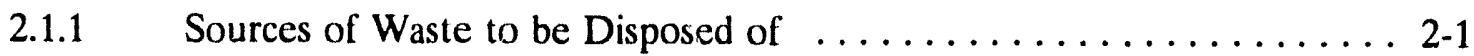

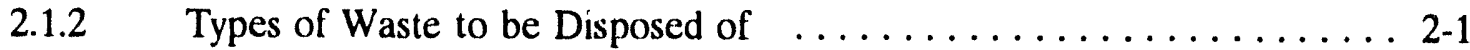

2.2 Volumes and Characteristics of Waste To Be Disposed of $\ldots \ldots \ldots \ldots \ldots \ldots$

2.2.1 Waste from Ongoing Operations $\ldots \ldots \ldots \ldots \ldots \ldots \ldots \ldots \ldots \ldots \ldots . \ldots \ldots$

2.2.2 Waste from Large D\&D Scenario . . . . . . . . . . . . . . 2-7

2.2.3 Waste from Large ER Scenario . . . . . . . . . . . . . . . 2-10

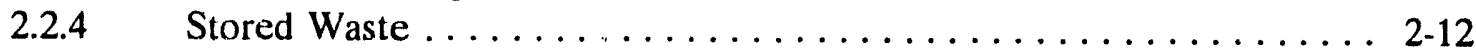

2.2.5 Total Waste .. . . . . . . . . . . . . . . . . 2-15

2.3 Regulatory Requirements Assumed to Govern Waste Disposal . . . . . . . . 2-17

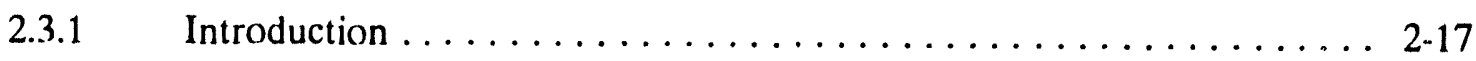

2.3.2 Requirements in DOE Order $5820.2 \mathrm{~A} \ldots \ldots \ldots \ldots \ldots \ldots \ldots . \ldots . \ldots . . \ldots 21$

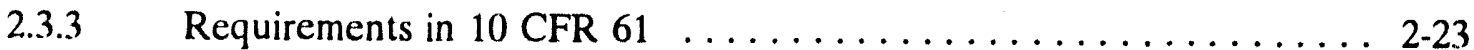

2.3.4 Requirements in Draft 40 CFR $193 \ldots \ldots \ldots \ldots \ldots \ldots \ldots . \ldots \ldots 2-24$

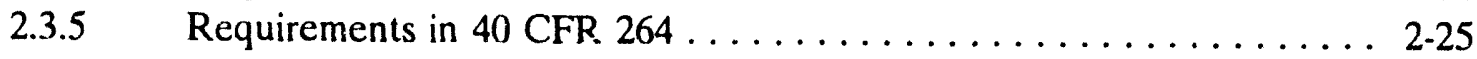

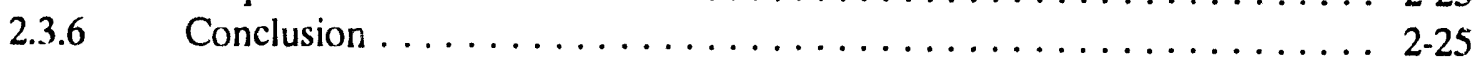




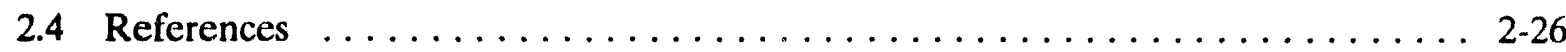

3. ASSUMPTIONS FOR STUDY OF ALTERNATIVES $\ldots \ldots \ldots \ldots \ldots \ldots \ldots \ldots$

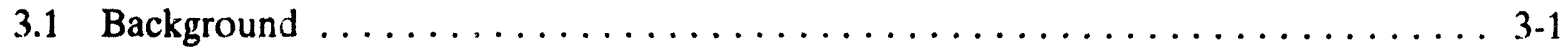

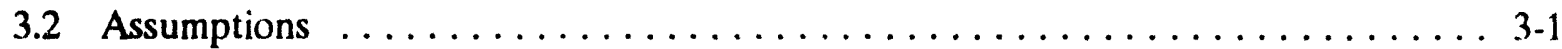

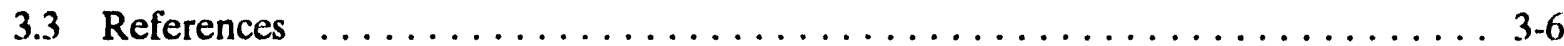

4. OVERVIEW OF ALTERNATIVES $\ldots \ldots \ldots \ldots \ldots \ldots \ldots \ldots \ldots \ldots \ldots \ldots$

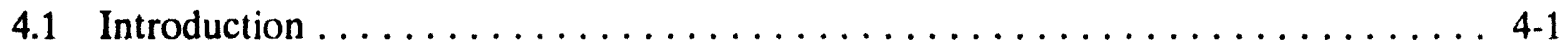

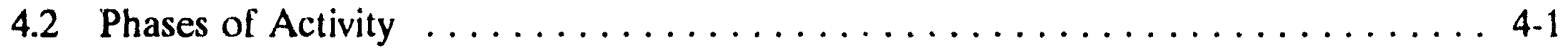

4.3 Overview Descriptions of Alternatives $\ldots \ldots \ldots \ldots \ldots \ldots \ldots \ldots \ldots \ldots \ldots$

4.3.1 Alternative 1: Construct and Operate MLLWDF ......... 4-3

4.3.2 Alternative 2: Continue Disposal at RWMC ............. 4-15

4.3.3 Alternative 3: Ship Waste Offsite for Disposal ............ 4-16

4.3.4 Alternative 4: Combinations of Alternatives ............

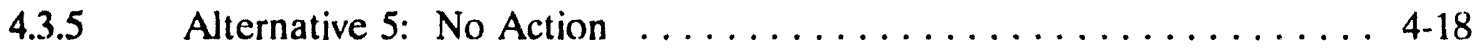

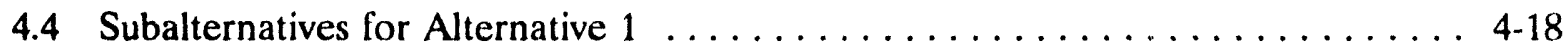

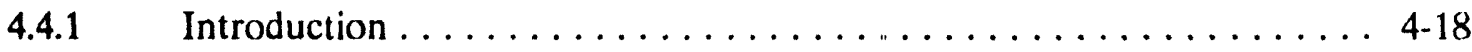

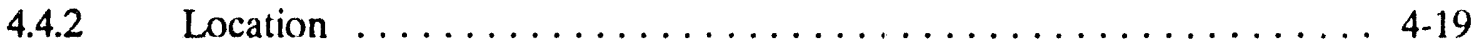

4.4.3 Disposal Technology . . . . . . . . . . . . . . . . . . 4-19

4.4.4 Combinations of Technologies . . . . . . . . . . . . . . . 4-20

4.4.5 Volume of Waste and Extent of Waste Treatment .......... 4.23

4.4.6 Complete Set of Subalternatives ................ 4-24

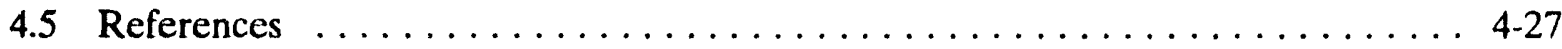

5. DESCRIPTION OF ALTERNATIVES $\ldots \ldots \ldots \ldots \ldots \ldots \ldots \ldots \ldots \ldots \ldots$

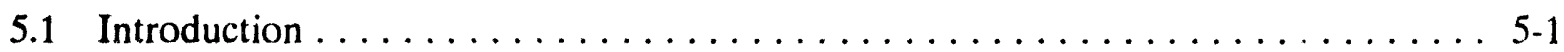

5.2 Basis for Disposal Design Concepts Evaluated $\ldots \ldots \ldots \ldots \ldots \ldots \ldots \ldots \ldots$

5.3 Alternative 1: Construct and Operate MLLWDF $\ldots \ldots \ldots \ldots \ldots \ldots \ldots \ldots$ 5-12

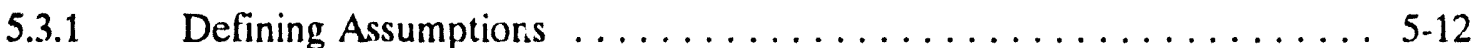

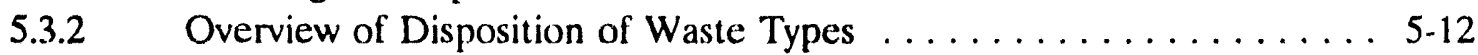

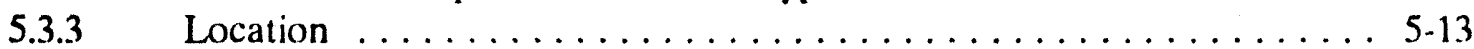

5.3.4 Subalternative 1A: Abovegrade Engineered Structures . . . . . . 5-16 
5.3.5 Subalternative 1B: Belowgrade Engineered Structures . . . . . . . . 5 5-42

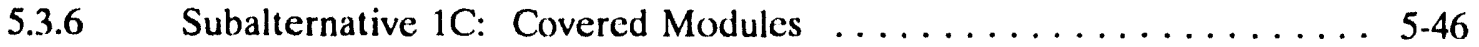

5.3.7 Subalternative 1D: Greater-depth Burial/Borcholes ........... $5-54$

5.3.8 Subalternative 1E: Shallow Land Burial/Boreholes . . . . . . . . . 5-62

5.3.9 Subalternative 1F: Shallow Land Burial/Abovegrade Engineered Structures ......................... 5-67

5.3.10 Subalternative 1G: Effect of Waste Volume . . . . . . . . . . . 5-70

5.3.11 Subalternative $1 \mathrm{H}$ : Effect of Waste Treatment .......... 5-70

5.4 Alternative 2: Continue Disposal at RWMC $\ldots \ldots \ldots \ldots \ldots \ldots \ldots \ldots$ 5-74

5.4.1 Defining Assumptions $\ldots \ldots \ldots \ldots \ldots \ldots \ldots \ldots \ldots \ldots \ldots \ldots \ldots \ldots$

5.4 .2 Overview of Disposition of Waste Types $\ldots \ldots \ldots \ldots \ldots \ldots \ldots$. $\ldots \ldots 5$

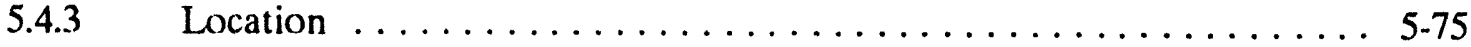

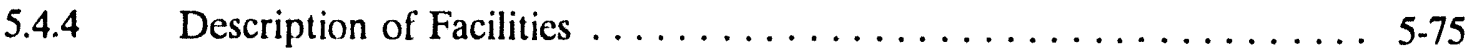

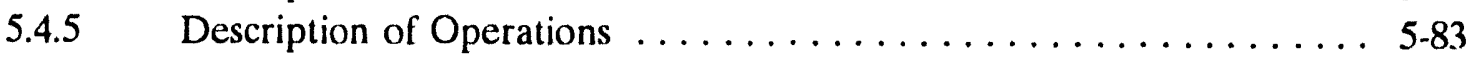

5.4.6 Closure and Postclosure Activities ................. 5-84

5.5 Alternative 3: Ship Waste Offsite for Disposal $\ldots \ldots \ldots \ldots \ldots \ldots \ldots$. . . . . .

$5.5 .1 \quad$ Defining Assumptions $\ldots \ldots \ldots \ldots \ldots \ldots \ldots \ldots \ldots \ldots . \ldots \ldots 5$

5.5 .2 Overview of Disposition of Waste Types $\ldots \ldots \ldots \ldots \ldots \ldots \ldots . . \ldots 6$

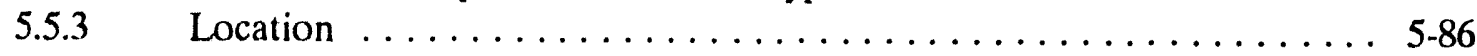

5.5 .4 Description of Facilities $\ldots \ldots \ldots \ldots \ldots \ldots \ldots \ldots \ldots \ldots \ldots \ldots \ldots$

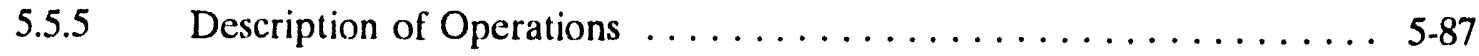

5.5.6 Closure and Postclosure Activities ................ 5-87

5.6 Alternative 4 : Combinations of Alternatives $\ldots \ldots \ldots \ldots \ldots \ldots \ldots$. $\ldots \ldots 7$

$5.6 .1 \quad$ Defining Assumptions $\ldots \ldots \ldots \ldots \ldots \ldots \ldots \ldots \ldots$ 5-87

5.6.2 Overview of Disposition of Waste Types $\ldots \ldots \ldots \ldots \ldots \ldots \ldots$. $\ldots \ldots 8$

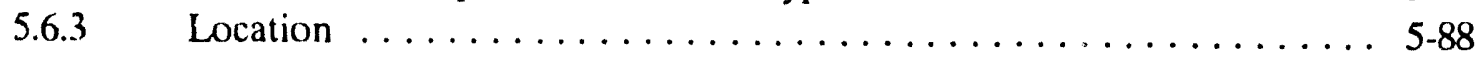

5.6 .4 Description of Facilities $\ldots \ldots \ldots \ldots \ldots \ldots \ldots \ldots \ldots \ldots \ldots \ldots . . \ldots \ldots$

5.6 .5 Description of Operations $\ldots \ldots \ldots \ldots \ldots \ldots \ldots \ldots \ldots \ldots \ldots . \ldots \ldots$

5.6.6 Closure and Postclosure Activities . . . . . . . . . . . . 5-90

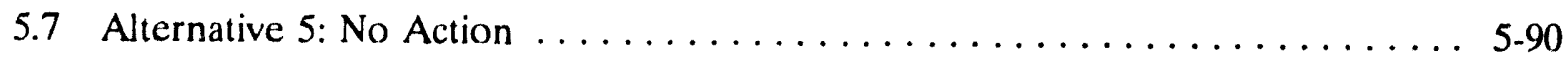

$5.7 .1 \quad$ Defining Assumptions $\ldots \ldots \ldots \ldots \ldots \ldots \ldots \ldots \ldots \ldots \ldots . \ldots \ldots$

5.7 .2 Overview of Disposition of Waste Types $\ldots \ldots \ldots \ldots \ldots \ldots \ldots$. . . . . .

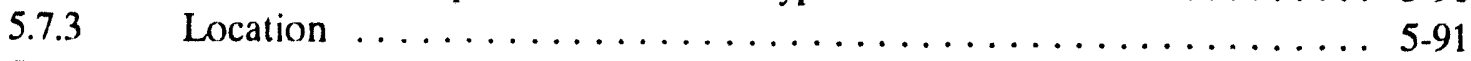

5.7 .4 Description of Facilities $\ldots \ldots \ldots \ldots \ldots \ldots \ldots \ldots \ldots \ldots \ldots . \ldots \ldots$. $5.9 \ldots$

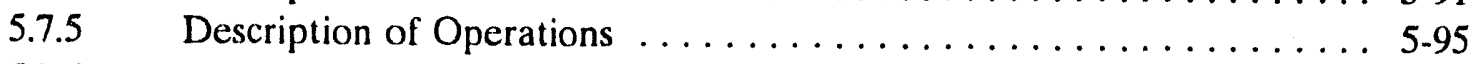

5.7.6 Closure and Postclosure Activities . . . . . . . . . . . . 5.96

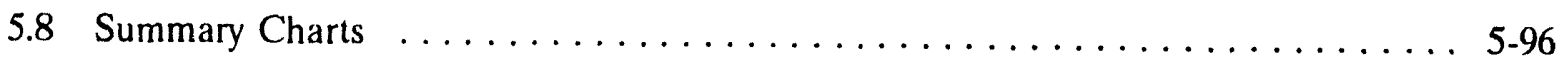


5.9 References

6. OVERVIEW OF EVALUATIONS $\ldots \ldots \ldots \ldots \ldots \ldots \ldots \ldots \ldots \ldots \ldots \ldots \ldots$

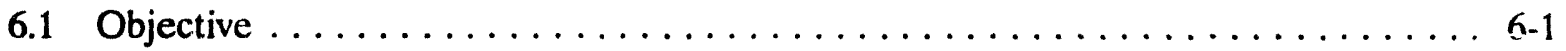

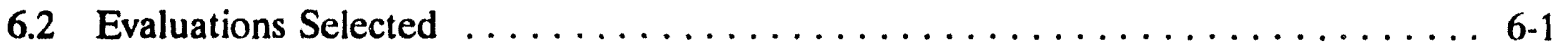

6.2.1 Operability and Isolation Performance Evaluations . . . . . . . . . . 6-1

6.2.2 Evaluation of Relationship to Other INEL Waste Management

Facilities ....................... 6-1

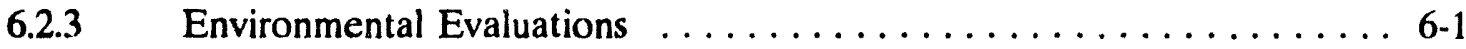

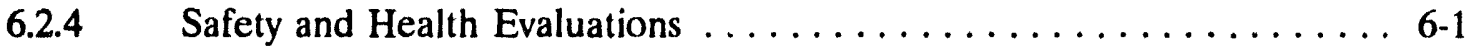

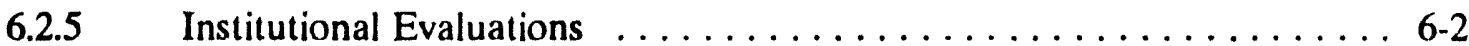

6.2.6 Schedule Evaluations . . . . . . . . . . . . . . . . . . . . . 6-2

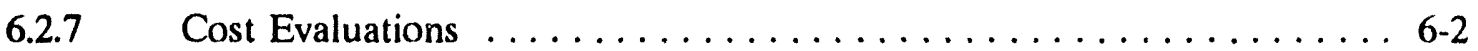

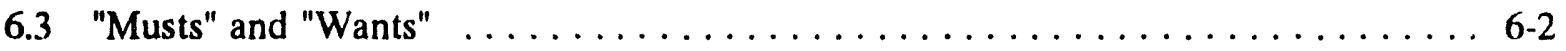

7. FACILITY AND PERFORMANCE EVALUATIONS $\ldots \ldots \ldots \ldots \ldots \ldots \ldots \ldots \ldots$.1

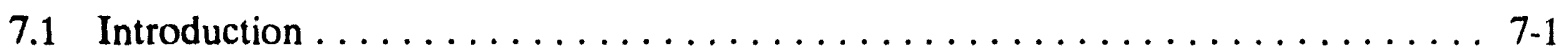

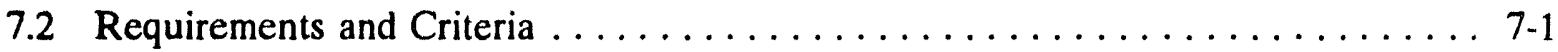

7.2.1 Disposal Standards ........................ 7-1

7.2.2 Development Risk . . . . . . . . . . . . . . .

7.2.3 Constructability, Operability, Reliability, and Maintainability ..... . 7-2

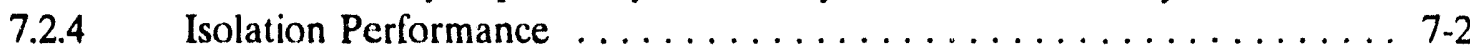

7.3 Evaluations of Alternatives Against Requirements/Criteria $\ldots \ldots \ldots \ldots \ldots \ldots$ 7-3

7.3.1 Disposal Standards and the Ability to Dispose of All Waste . . . . . . 7-3

7.3.2 Developmental Risk ..................... 7-3

7.3.3 Relative Constructability, Operability, Reliability, and Maintainability .. 7-5

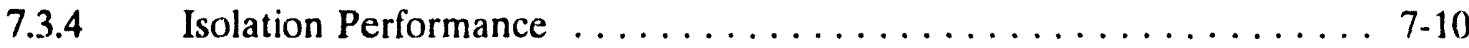

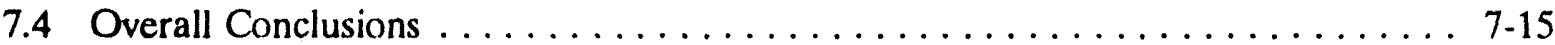

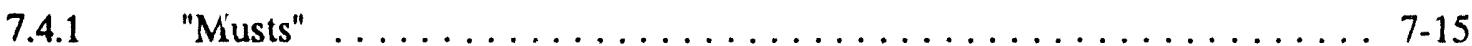

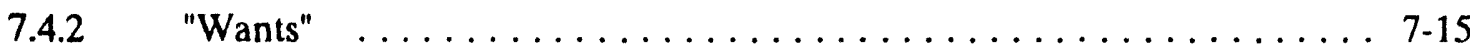

8. EVALUATION OF INTERFACES WITH OTHER WASTE MANAGEMENT

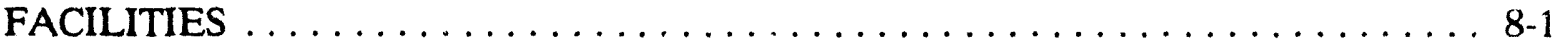

8.1 Introduction $\ldots \ldots \ldots \ldots \ldots \ldots \ldots \ldots \ldots \ldots \ldots \ldots \ldots \ldots \ldots \ldots \ldots \ldots$ 


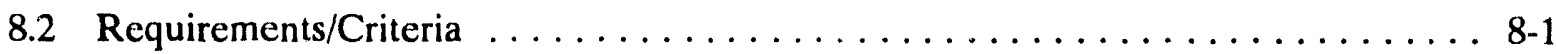

8.3 Interface of Disposal Alternatives with RWMC $\ldots \ldots \ldots \ldots \ldots \ldots \ldots .2$

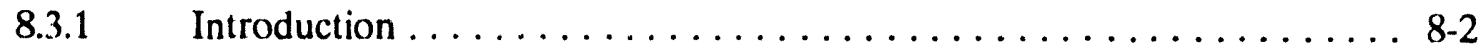

8.3.2 Evaluation of Interfaces $\ldots \ldots \ldots \ldots \ldots \ldots \ldots \ldots \ldots \ldots, 8 \ldots \ldots \ldots \ldots, 2$

8.4 Interface of Disposal Alternatives with MLLWTF and IWPF $\ldots \ldots \ldots \ldots \ldots 8-3$

8.4.1 Introduction $\ldots \ldots \ldots \ldots \ldots \ldots \ldots \ldots \ldots \ldots \ldots \ldots, 8,3$

8.4.2 Evaluation of Interfaces $\ldots \ldots \ldots \ldots \ldots \ldots \ldots \ldots \ldots \ldots, 8,3$

8.5 Interface of Disposal Alternatives with Offsite Treatment Facility $\ldots \ldots \ldots \ldots 8-4$

8.5.1 Introduction $\ldots \ldots \ldots \ldots \ldots \ldots \ldots \ldots \ldots \ldots \ldots \ldots, \ldots \ldots \ldots$

8.5.2 Evaluation of Interfaces $\ldots \ldots \ldots \ldots \ldots \ldots \ldots \ldots \ldots \ldots, 8.4$

8.6 Overall Conclusions $\ldots \ldots \ldots \ldots \ldots \ldots \ldots \ldots \ldots \ldots \ldots \ldots \ldots \ldots \ldots \ldots$

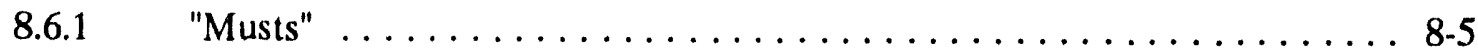

8.6.2 "Wants" ................................ 8-5

9. ENVIRONMENTAL EVALUATIONS $\ldots \ldots \ldots \ldots \ldots \ldots \ldots \ldots \ldots \ldots . \ldots . \ldots \ldots$

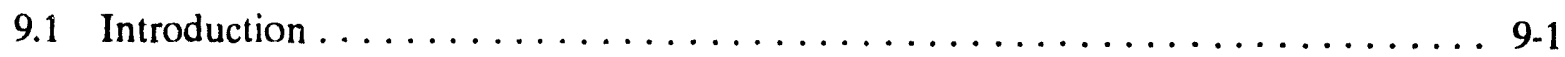

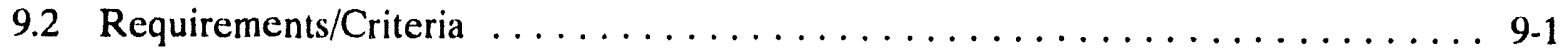

9.2.1 Overview of Requirements/Criteria . . . . . . . . . . . . . . . 9-1

9.2.2 National Environmental Policy Act Requirements ............ 9-2

9.2.3 Siting Requirements/Criteria $\ldots \ldots \ldots \ldots \ldots \ldots \ldots \ldots \ldots . \ldots . \ldots .2$

9.2.4 Permitting Requirements ......................... 9-3

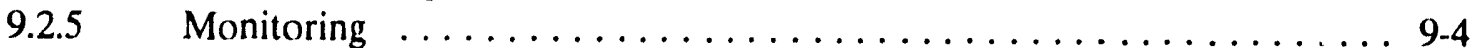

9.3 Evaluations of Alternatives Against Requircments ................. 9.5

9.3.1 Construct and Operate MLLWDF . . . . . . . . . . . . . . . .

9.3.2 Continue Disposal at RWMC $\ldots \ldots \ldots \ldots \ldots \ldots \ldots \ldots \ldots . \ldots . \ldots \ldots$

9.3.3 Offsite Treatment ......................... 9-11

9.3.4 Combinations of Alternatives ...................... 9-11

9.3.5 No Action ............................... 9-12

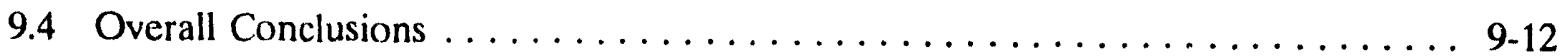

9.4.1 "Musts" ............................... 9-12

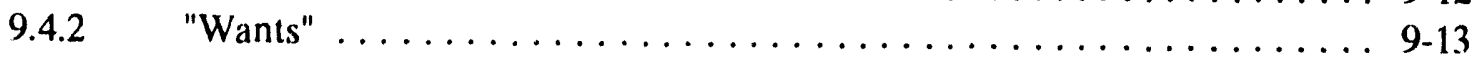


10. SAFETY AND HEALTH EVALUATIONS $\ldots \ldots \ldots \ldots \ldots \ldots \ldots \ldots \ldots \ldots \ldots$

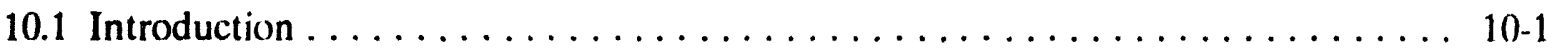

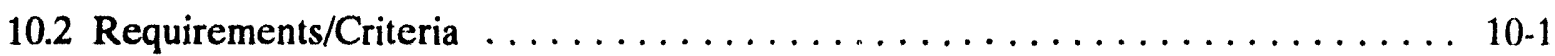

10.3 Hazards Associated with Storing, Shipping, and Disposing of INEL Mixed Waste

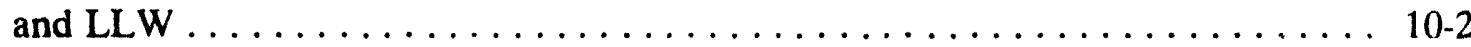

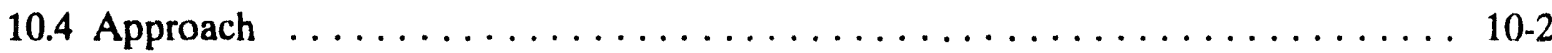

10.5 Evaluations of Alternatives Against Requirements/Criteria $\ldots \ldots \ldots \ldots \ldots \ldots$ 10-4

10.5.1 Safety and Health During Construction $\ldots \ldots \ldots \ldots \ldots \ldots \ldots$. . . . . . . .

10.5.2 Safety and Health During Operations and Closure . . . . . . . . . 10.7

10.5.3 Safety and Health During Offsite Shipment ............ 10-11

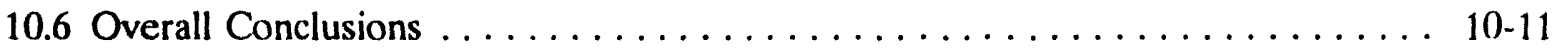

10.6.1 "Musts" . . . . . . . . . . . . . . . . . . . 10-11

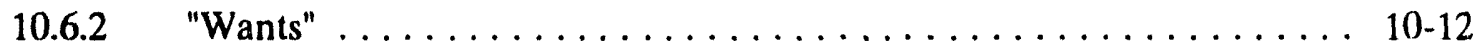

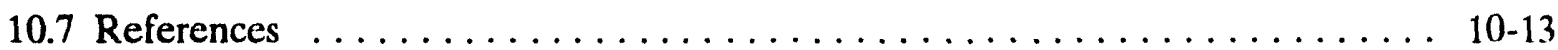

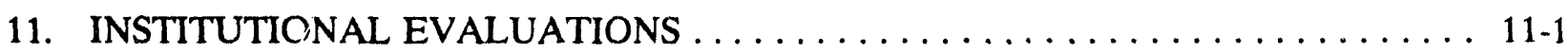

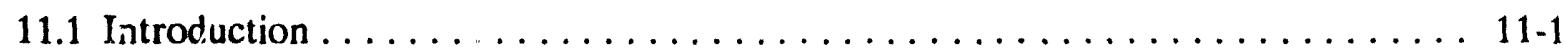

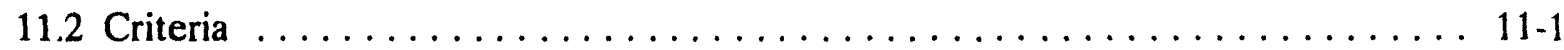

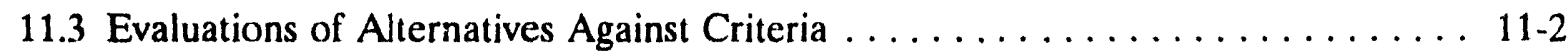

11.3.1 Construct and Operate MLLWDF $\ldots \ldots \ldots \ldots \ldots \ldots \ldots \ldots \ldots . \ldots \ldots 11-2$

11.3.2 Continue Disposal at RWMC .................. 11-4

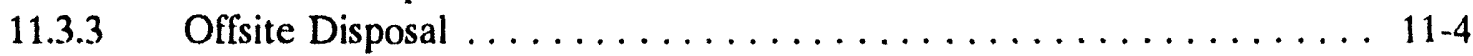

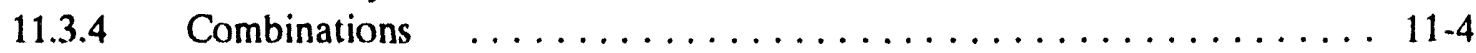

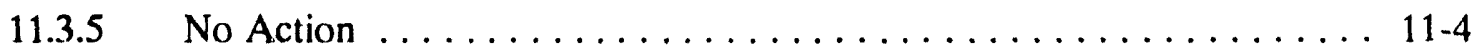

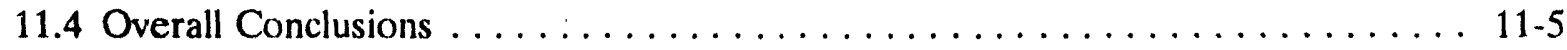

11.4.1 "Musts" ... . . . . . . . . . . . . . . . . . . . 11-5

11.4.2 "Wants" ............................. 11.5

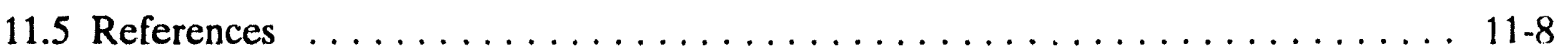




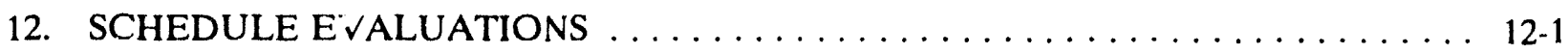

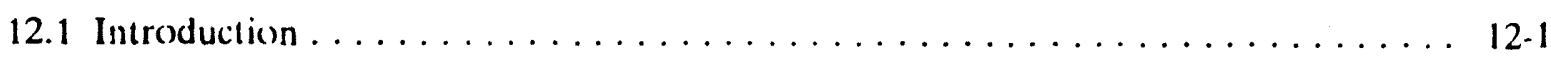

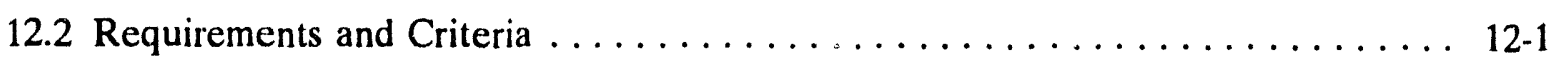

12.3 Evaluations of Alternatives Against Requirements/Criteria . . . . . . . . . 12-2

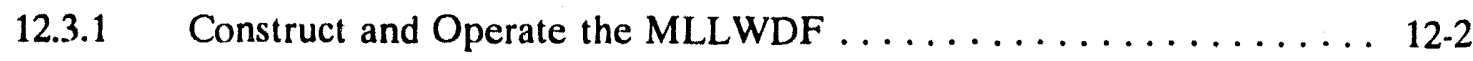

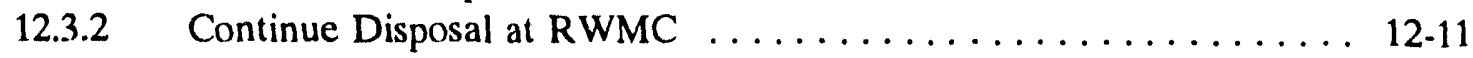

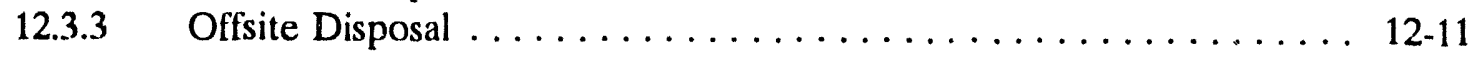

12.3.4 Combinations of Alternatives .................. 12-12

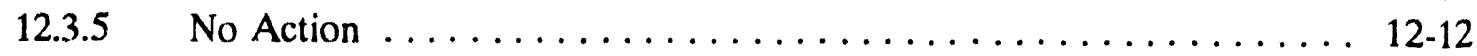

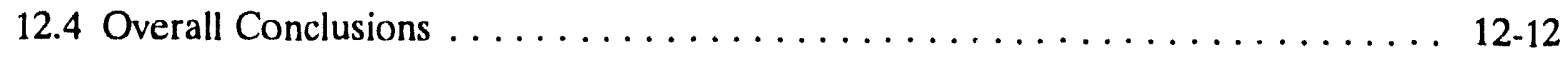

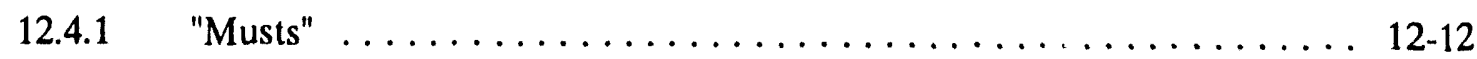

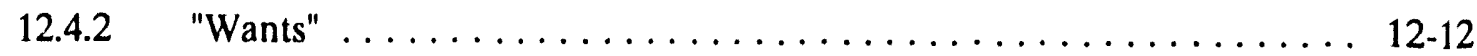

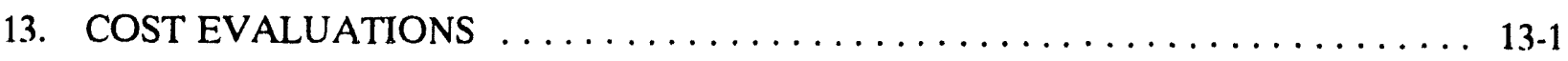

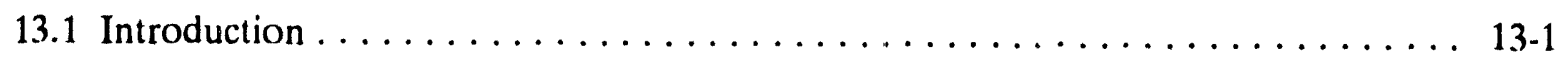

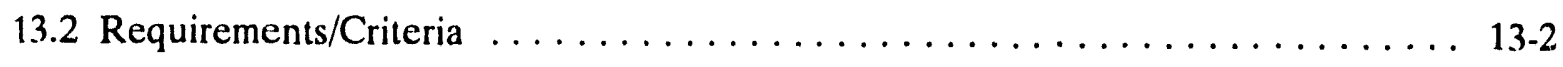

13.3 Evaluations of Alternatives Against the Requirements/Criteria . . . . . . . 13-2

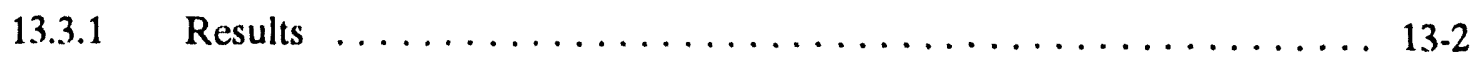

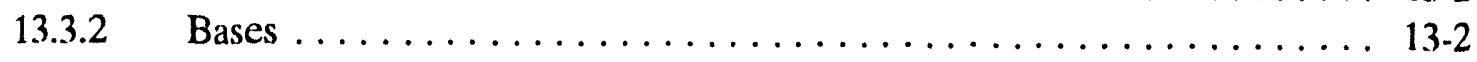

13.3.3 Discussion of Results $\ldots \ldots \ldots \ldots \ldots \ldots \ldots \ldots \ldots \ldots \ldots$ 13-6

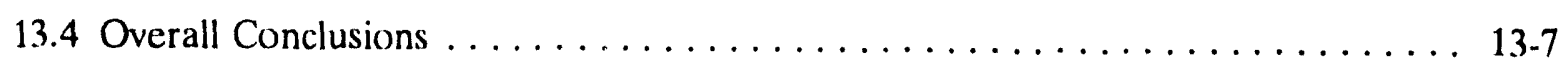

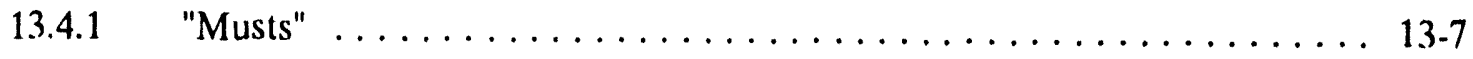

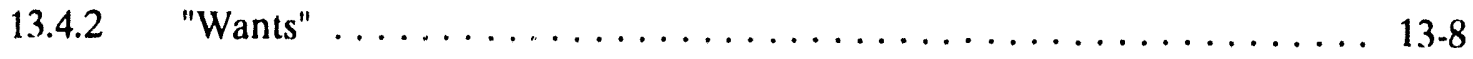

13.4.3 Escalation and Discount Factors $\ldots \ldots \ldots \ldots \ldots \ldots \ldots \ldots \ldots$ 13-8

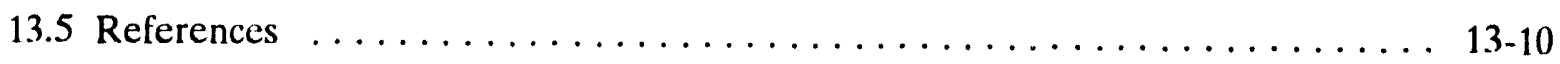

14. KEY UNCEPTAINTIES $\ldots \ldots \ldots \ldots \ldots \ldots \ldots \ldots \ldots \ldots \ldots \ldots \ldots \ldots \ldots$ 14-1

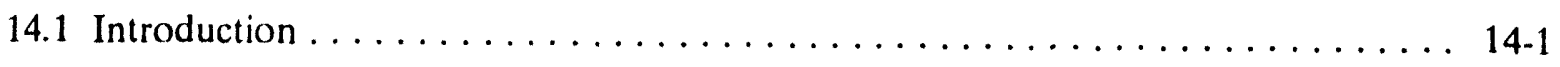

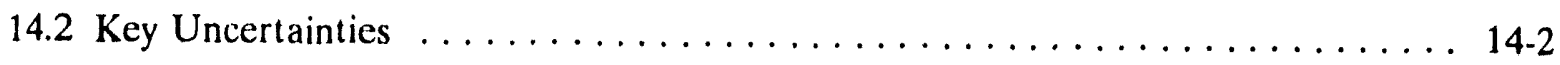

14.2.1 Shifting Regulations . . . . . . . . . . . . . . . . . .

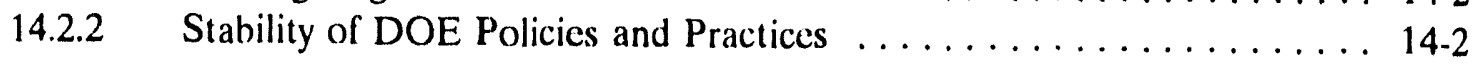


14.2.3 Volumes and Characteristics of Waste that Will Be Generated . . . . . . 14-2

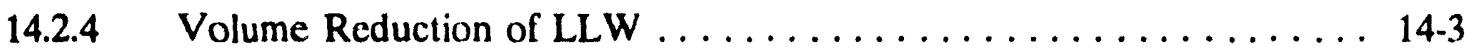

14.2.5 Outcome of Privatization Initiatives ................ 14-3

14.2.6 Assumptions Concerning the Future of Other INEL Waste Management Facilities ........................ 14-3

14.2.7 Uncertainties of Disposal at Another DOE Site . . . . . . . . . . . 14-3

14.2.8 Impacts of the Performance Assessment of the MLLWDF . . . . . . . 14-4

14.2.9 Use of Technologies that Are Not Totally Proven . . . . . . . . . . . 14-4

14.2.10 Lack of Preconceptual Designs . . . . . . . . . . . . . . . . 14-4

14.2.11 Schedule Uncertainties . . . . . . . . . . . . . . . . . . . . . 14-5

14.2.12 Cost Uncertainties . . . . . . . . . . . . . . . . . . 14-5

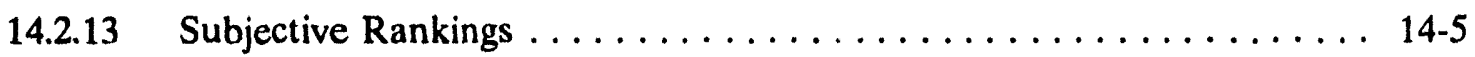

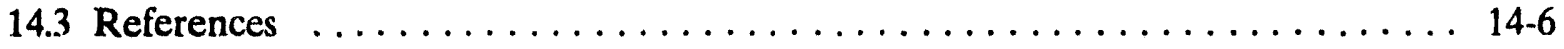

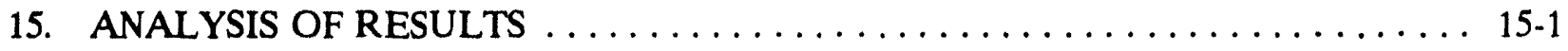

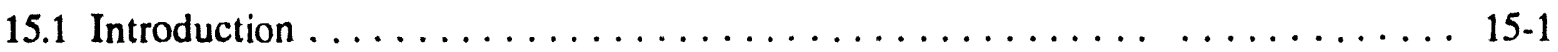

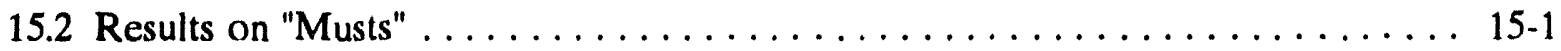

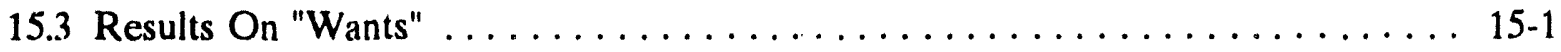

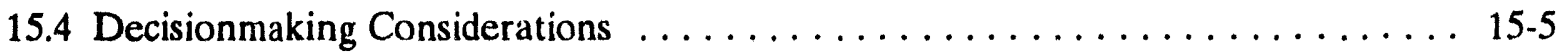

15.4.1 Should a Decision on Enhanced Disposal Capability Be Pursued Now or Delayed ........................ 15-5

15.4.2 The Effect of Waste Treatment on Disposal Requirements. . . . . . . 15-6

15.4.3 Waste Volume Projections . . . . . . . . . . . . . . . . . . .

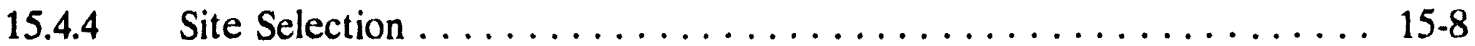

15.4.5 Technology Risk . . . . . . . . . . . . . . . . . . . 15-9

15.4.6 Will Other Facilities Be Available? . . . . . . . . . . . . . . . 15-9

15.4.7 Public Perception . . . . . . . . . . . . . . . . . . . 15-9

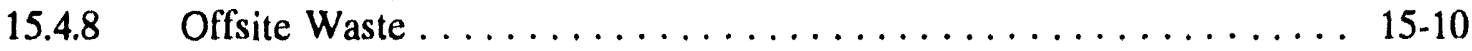

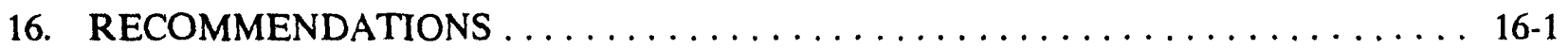

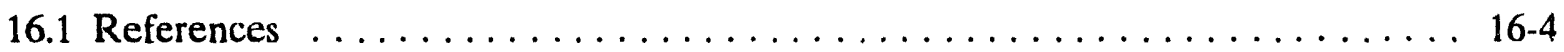

Appendix A-Details and Bases for Cost Estimates $\ldots \ldots \ldots \ldots \ldots \ldots \ldots \ldots \ldots \ldots \ldots \ldots$ 


\section{FIGURES}

1-1. Logic flowchart for current study $\ldots \ldots \ldots \ldots \ldots \ldots \ldots \ldots \ldots \ldots \ldots$

2-1. The four potential sources of mixed waste and LLW at the INEL $\ldots \ldots \ldots \ldots 2-2$

2-2. Pretreatment and posttreatment types and volumes of waste for ongoing operations

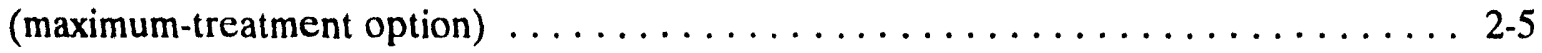

2-3. Ongoing operations waste types and volumes for the minimum-treatment option . . . 2-8

2-4. D\&D waste types and volumes for the maximum-treatment option . . . . . . . . 2-9

2-5. D\&D waste types and volumes for the minimum-treatment option, Large D\&D

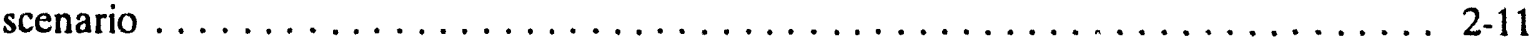

2-6. Types and volumes of waste in storage for the maximum- and minimum-treatment

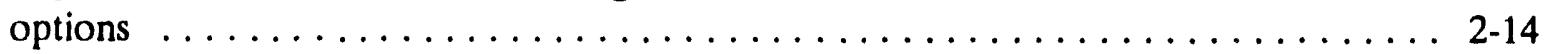

2-7. Estimated campaign length for each source of waste $\ldots \ldots \ldots \ldots \ldots \ldots \ldots$

4-1. Phases in the life of a waste-disposal facility $\ldots \ldots \ldots \ldots \ldots \ldots \ldots \ldots \ldots$

4-2. Highly simplified flow diagrams for the disposal alternatives and subalternatives

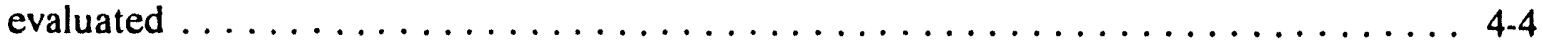

5-1. Map of candidate sites of primary interest for the MLLWDF. . . . . . . . . 5-14

5-2. General disposal site layout for Subalternative $1 \mathrm{~A}$ (not to scale) $\ldots \ldots \ldots \ldots . . \ldots 5$

5-3. Design concept for disposal structures for contact-handled waste in

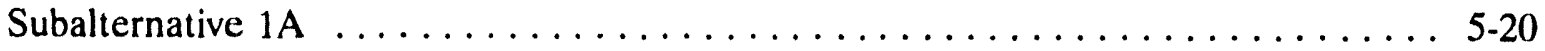

5-4. Design concept for disposal structures for remote-handled waste in Subalternative $1 \mathrm{~A}$

5-5. Surface water drainage control system for Subalternative $1 \mathrm{~A} \ldots \ldots \ldots \ldots \ldots$

5-6. Foundation blanket drain for Subalternative $1 \mathrm{~A} \ldots \ldots \ldots \ldots \ldots \ldots \ldots \ldots$

5-7. Dual-liner/leachate system for Subalternative $1 \mathrm{~A} \quad \ldots \ldots \ldots \ldots \ldots \ldots \ldots \ldots$. . . . 5 .29

5-8. Leachate collection and recovery system drain gallery and sump for

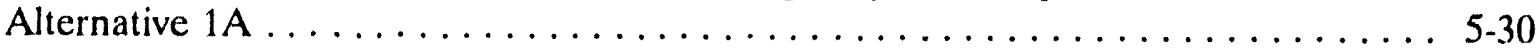

5-9. General design of multilayer final cover for waste disposal site in

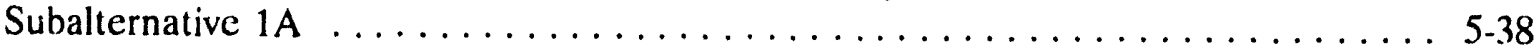


5-10. Areal extent of the final cover for Subalternative $1 \mathrm{~A} \ldots \ldots \ldots \ldots \ldots \ldots \ldots \ldots$

5-11. General disposal site layout for Suhalternative $1 \mathrm{~B} \ldots \ldots \ldots \ldots \ldots \ldots \ldots \ldots$

5-12. Surface water drainage control system for Subalternative $1 \mathrm{~A} \ldots \ldots \ldots \ldots \ldots$

5-13. Foundation blanket drain for Class $B$ and $C$ waste for Subalternative $1 B \ldots \ldots .47$

5-14. General site layout for Subalternative $1 \mathrm{C} \ldots \ldots \ldots \ldots \ldots \ldots \ldots \ldots \ldots \ldots \ldots$

5-15. Design concept for covered modules in Subalternative $1 \mathrm{C} \ldots \ldots \ldots \ldots \ldots \ldots$

5-16. Dual-liner/leachate collection system for Subalternative $1 \mathrm{C} \ldots \ldots \ldots \ldots \ldots \ldots$ 5-52

5-17. General site layout for Subalternative $1 \mathrm{D} \ldots \ldots \ldots \ldots \ldots \ldots \ldots \ldots \ldots \ldots$

5-18. Simplified sectional view of greater-depth burial pits for contact-handled waste in Subalternative 1D . . . . . . . . . . . . . . . . . . . . . . . 5-57

5-19. Simplified sectional view of disposal area with greater-depth boreholes for remote-handled waste in Subalternative $1 \mathrm{D} \ldots \ldots \ldots \ldots \ldots \ldots \ldots \ldots \ldots . \ldots \ldots$

5-20. The dual-liner/ieachate system for Subalternative $1 \mathrm{D} \ldots \ldots \ldots \ldots \ldots \ldots \ldots \ldots$

5-21. General disposal site layout for Subalternative $1 \mathrm{E} \ldots \ldots \ldots \ldots \ldots \ldots \ldots \ldots$

5-22. Simplified sectional view of shallow land burial pits for contact-handled waste

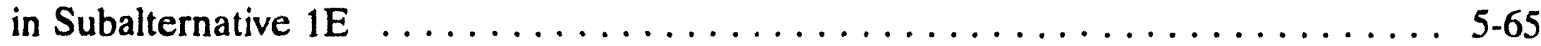

5-23. Simplified sectional view of disposal area with boreholes for remote-handled waste

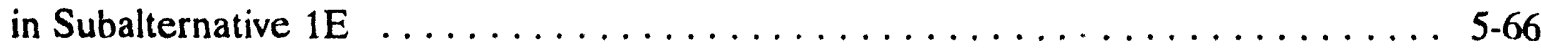

5-24. General disposal site layout for Subalternative $1 \mathrm{~F} \ldots \ldots \ldots \ldots \ldots \ldots$

5-25. General disposal site layout for Subalternative $1 \mathrm{G} \ldots \ldots \ldots \ldots \ldots \ldots \ldots \ldots \ldots$

5-26. General disposal site layout for Subalternative $1 \mathrm{H} \ldots \ldots \ldots \ldots \ldots \ldots \ldots \ldots$

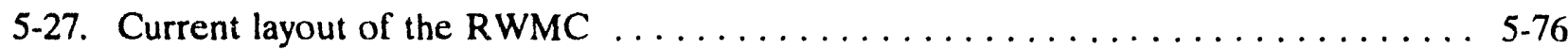

5-28. Disposal site layout for Alternative $2 \ldots \ldots \ldots \ldots \ldots \ldots \ldots \ldots \ldots \ldots \ldots \ldots$

5-29. The general site layout for Alternative $5 \ldots \ldots \ldots \ldots \ldots \ldots \ldots \ldots \ldots \ldots \ldots$

12-1. Overview schedule for MLLWDF Subalternatives 1A, 1B, 1C, 1F, 1G, and $1 \mathrm{H} \ldots 12-3$

12-2. Overview schedule for MLLVDF Subalternatives $1 \mathrm{D}$ and $1 \mathrm{E} \ldots \ldots \ldots \ldots \ldots$ 
12-3. Overview schedule for the RWMC alternative $\ldots \ldots \ldots \ldots \ldots \ldots \ldots \ldots \ldots$

12-4. Overview schedule for the offsite alternative $\ldots \ldots \ldots \ldots \ldots \ldots \ldots \ldots \ldots$

12-5. Overview schedule for the MLLWDF and offsite alternative $\ldots \ldots \ldots \ldots \ldots \ldots$

12-6. Overview schedule for the RWMC and offsite alternative $\ldots \ldots \ldots \ldots \ldots \ldots$

12-7. Overview schedule for the no action alternative $\ldots \ldots \ldots \ldots \ldots \ldots \ldots \ldots \ldots$

13-1. Life-cycle costs-unescalated costs, FY $1993 \mathrm{M} \$ \ldots \ldots \ldots \ldots \ldots \ldots \ldots$

\section{TABLES}

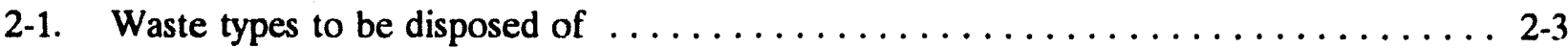

2-2. Sources/types of waste to be disposed of $\ldots \ldots \ldots \ldots \ldots \ldots \ldots \ldots \ldots \ldots \ldots \ldots$

2-3. Estimated waste volumes and characteristics from Large ER scenario $\ldots \ldots \ldots \ldots$ 2-13

2-4. Summary of waste types and volumes to be received at the disposal facility,

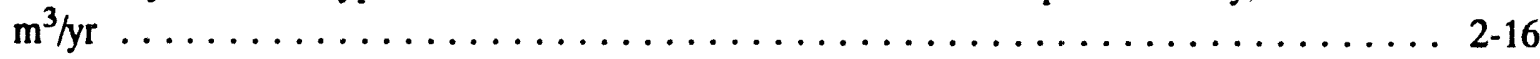

2-5. Levelized waste volumes disposed of, by source, for life of the disposal facility

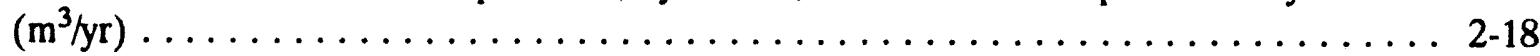

2-6. Summary of waste containers disposed of per year (levelized) $\ldots \ldots \ldots \ldots \ldots$

4-1. Levelized waste generation/waste treatment scenarios $\ldots \ldots \ldots \ldots \ldots \ldots \ldots$. . . . .

4-2. Combinations of alternatives selected for evaluation $\ldots \ldots \ldots \ldots \ldots \ldots \ldots$

4-3. Technologies considered for the disposal of INEL mixed waste and LLW . . . . . 4-21

4-4. Combinations of disposal technologies evaluated for the MLLWDF alternative . . . 4-22

4-5. Total annual volumes $\left(\mathrm{m}^{3}\right)$ of waste requiring disposal for various scenarios of, te

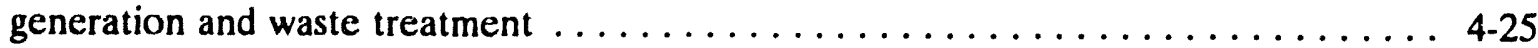

4-6. Subalternatives of Alternative 1 selected for evaluation, as well as other alternatives . 4-26

5-1. Comparison of waste-disposal design concepts in the literature that are relevant to

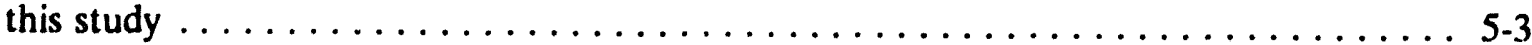

5-2. Waste-disposal design concepts selected for evaluation, in terms of successive

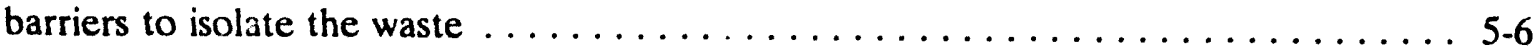


5-3. Number and size of concrete structures for each type of waste in

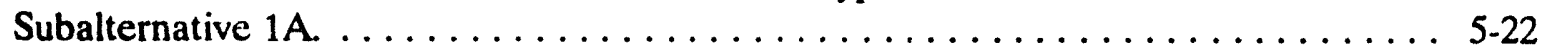

5-4. Number and size of concrete pads for each type of waste in Subalternative 1C. . . . 5-51

5-5. Number and size of disposal units for each type of waste in Subalternative 1D . . . . 5-56

5-6. Number and size of disposal units for each type of waste in Subalternative 1E . . . . 5-64

5-7. Number and size of disposal units for each type of waste in Subalternative $1 \mathrm{~F} \ldots \ldots$. . . 69

5-8. Number and size of concrete structures for each type of waste in

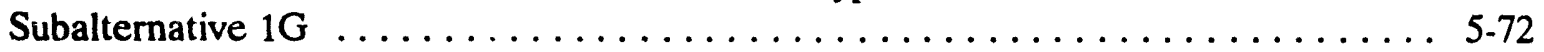

5-9. Number and size of concrete structures for each type of waste in

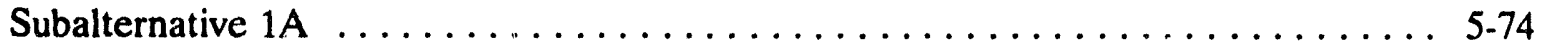

5-10. Rough estimates of the size of locations assumed to be available for future disposal

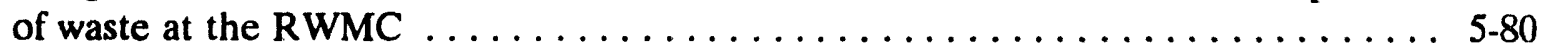

$5-11$. Detailed disposition of the various types of waste in Alternative $2 \ldots \ldots \ldots \ldots$

5-12. Dimensions are areas of disposal unit covers $\ldots \ldots \ldots \ldots \ldots \ldots \ldots \ldots \ldots$

5-13. Number of structures required for MLLWDF/Ship Offsite combination, compared with that for Subalternative $1 \mathrm{~A} \ldots \ldots \ldots \ldots \ldots \ldots \ldots \ldots \ldots . \ldots \ldots$. . . . . . . . . . . .

5-14. Detailed disposition of the various types of waste in the RWMC/Ship Offsite Alternative

5-15. Number and size of storage buildings for each type of waste in Alternative $5 \ldots \ldots$. . . 54

5-16. Summary of waste volumes to be disposed of, by disposal concept, for the life of the disposal facility $\left(\mathrm{m}^{3} / \mathrm{yr}\right)$.

5-17. Summary information on waste forms $\ldots \ldots \ldots \ldots \ldots \ldots \ldots \ldots \ldots \ldots$

5-18. Summary of the number of waste containers disposed of per year by concept $\ldots \ldots 5-99$

5-19. Summary of Alternative 1 (MLLWDF) disposal site and disposal unit physical dimensions

5-20. Summary of Alternative 2 (RWMC) disposal site and disposal unit physical dimensions

5-21. Summary of Alternative 4 (combinations of alternatives) disposal site and disposal unit physical dimensions 
5-22. Summary of Alternative 5 (no action alternative) storage site and storage unit

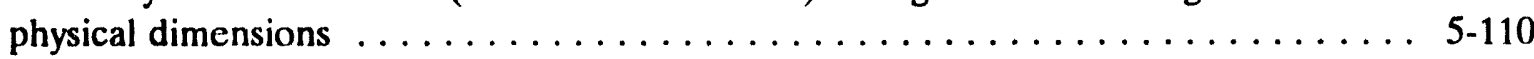

6-1. "Musts" and "wants" criteria for recommending an alternative $\ldots \ldots \ldots \ldots \ldots \ldots$

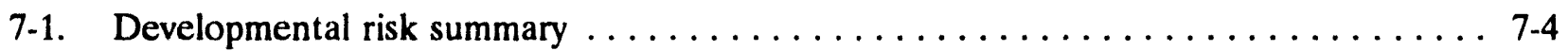

7-2. Relative difficulty of construction tasks $\ldots \ldots \ldots \ldots \ldots \ldots \ldots \ldots \ldots \ldots$

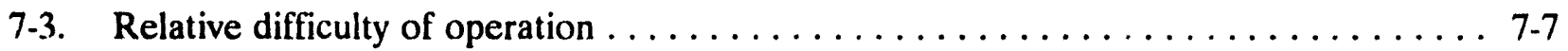

7-4. Summary of features requiring different levels of maintenance $\ldots \ldots \ldots \ldots \ldots \ldots$

7-5. Number and effectiveness over the long term $\ldots \ldots \ldots \ldots \ldots \ldots \ldots \ldots \ldots \ldots$

7-6. Number and quality of barriers to contact over the very long term $\ldots \ldots \ldots \ldots$ 7-14

7-7. Subjective evaluation of applicable "wants" $\ldots \ldots \ldots \ldots \ldots \ldots \ldots \ldots \ldots \ldots$ 7-16

7-8. Interim results for isolation performance $\ldots \ldots \ldots \ldots \ldots \ldots \ldots \ldots \ldots \ldots$

7-9. Interim results for constructability, operability, reliability, and maintainability . . . . 7-19

8-1. Subjective evaluation of performance on applicable "want" $\ldots \ldots \ldots \ldots \ldots \ldots$

10-1. Scope of safety and health evaluations $\ldots \ldots \ldots \ldots \ldots \ldots \ldots \ldots \ldots \ldots \ldots \ldots \ldots \ldots$

10-2. Fewest expected construction accidents ranking $\ldots \ldots \ldots \ldots \ldots \ldots \ldots \ldots \ldots$

10-3. Accidental release of radionuclide inventory $\ldots \ldots \ldots \ldots \ldots \ldots \ldots \ldots \ldots \ldots$

10-4. Radiation exposure ranking $\ldots \ldots \ldots \ldots \ldots \ldots \ldots \ldots \ldots \ldots \ldots \ldots \ldots \ldots$

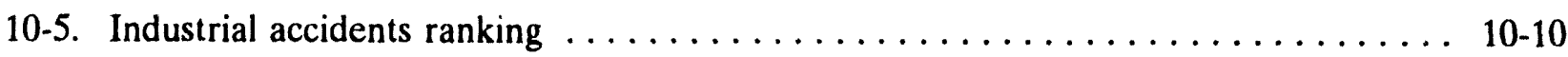

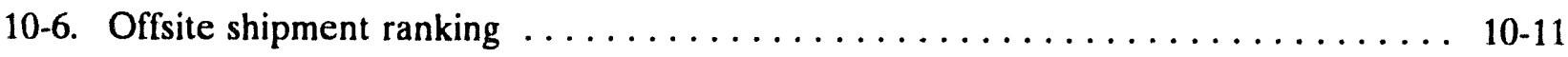

10-7. Subjective evaluation of performance on applicable "wants" $\ldots \ldots \ldots \ldots \ldots \ldots$ 10-12

11-1. Institutional interface-related observations for disposal of INEL mixed waste and

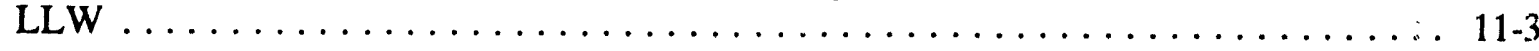

11-2. Subjective evaluation of performance on applicable "wants" $\ldots \ldots \ldots \ldots \ldots \ldots$

12-1. Subjective evaluation of performance on applicable "want. . . . . . . . . 12-13

13-1. Summary of life-cycle costs for the disposal alternatives-unescalated costs, FY 1993 M\$ . . . . . . . . . . . . . . . . . . . . . . 13-4 
13-2. Summary of life-cycle costs for the disposal alternatives-cost escalated to year of expenditure, $\mathrm{M} \$$

13-3. Subjective evaluation of performance on applicable "want" $\ldots \ldots \ldots \ldots \ldots \ldots \ldots$

15-1. Summary of performance of alternatives against "musts" $\ldots \ldots \ldots \ldots \ldots \ldots \ldots$ 15-2

15-2. Summary of performance of alternatives against "wants" $\ldots \ldots \ldots \ldots \ldots \ldots \ldots$

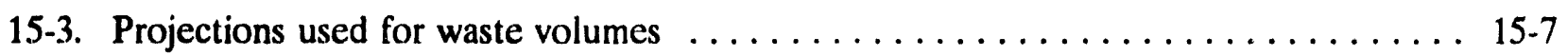

15-4. Potential suitability of disposal technologies at candidate sites $\ldots \ldots \ldots \ldots \ldots$ 


\section{SUMMARY}

\section{S-1 Background}

The Idaho National Engineering Laboratory (INEL) was established in southeastern Idaho in 1949 as a site where nuclear reactors, support facilities, and equipment could be built, tested, and operated. Today the INEL is one of the U.S. Department of Energy's (DOE's) principal centers for energy research and development, environmental restoration, and waste management.

Management of low-level ${ }^{a}$ radioactive waste and low-level mixed ${ }^{b, c}$ waste is addressed in this study. High-level waste, transuranic waste, hazardous waste, and nonhazardous nonradioactive waste are not addressed here.

Since 1952, low-level waste (LLW) generated at the INEL (and some LLW generated offsite) has been disposed of at the Radioactive Waste Management Complex (RWMC). The waste is disposed of in the Subsurface Disposal Area (SDA) of the RWMC by shallow land burial.

Although INEL low-level mixed "'aste also was disposed of previously by shallow land buria! in the SDA, that practice ceased several years ago. There is currently no permitted facility at the INEL for the disposal of low-level mixed waste. INEL mixed waste is being stored while awaiting the development of treatment and disposal capability.

Improved capability is needed for disposing of the INEL LLW and mixed waste for the following reasons:

- Progressive changes in the requirements governing waste disposal indicate the need for advanced disposal systems

- There is currently no permitted facility for the disposal of INEL mixed waste

- The remaining disposal space in the SDA is limited.

The Mixed and Low-Level Waste Disposal Facility (MLLWDF) project was initiated in 1992. The objective is to provide long-term disposal capability-whether onsite or offsite-for the INEL mixed waste, for which no disposition is currently available. The project will also provide long-term disposal capability for low-level waste. Low-level waste is currently being disposed of at the RWMC,

a. As defined by DOE Order 5820.2A, low-level radioactive waste is waste that contains radioactivity and is not classified as high-level waste, transuranic waste, or spent nuclear fuel or $11 \mathrm{e}(2)$ byproduct material.

b. Mixed waste is waste that contains both radioactive and hazardous components as defined by the Atomic Energy Act and the Resource Conservation and Recovery Act, respectively.

c. Hereafter, for brevity, the term "mixed waste" will often be used in this document without the modifier "low-level." It should be remembered, however, that the term applies only to the mixed waste that is low-level. 
which has limited remaining disposal capacity in its current configuration. Studies conducted to date on the MLLWDF project include environmental compliance plans, facility siting studies, and the development of functional and operational requirements. The present study continues the preconceptual design activities in support of the MLLWDF project.

\section{S-2 Purpose and Scope}

The objective of this study was to identify and evaluate, on a preliminary and overview basis, the alternatives for disposing of INEL-generated and -stored LLW and mixed waste. The results of the study were intended to provide information that will be useful for the selection of a preferred disposal alternative and for the conceptual design process. The results can also be used later as input to the alternatives evaluation required as part of the National Environmental Policy Act (NEPA) documentation to support selection of a waste disposal alternative.

The alternatives evaluated herein cover the full range from "no action" to constructing and operating a new facility, termed the MLLWDF.

The evaluations involved comparisons of the capabilities of disposal alternatives, based on a set of assumptions. The evaluations included comparisons of the projected isolation performance for each alternative. The evaluations also included facility, health and safety, environmental, institutional, schedule, and rough order-of-magnitude (ROM) life-cycle cost comparisons.

Detailed, engineering-level evaluations of the alternatives are outside the scope of this study. Except for cost and logistical considerations, the comparisons were performed on a qualitative basis. Evaluations were performed using data available at the time of analysis and are subject to change in the near future.

Figure S-1 is a flowchart that illustrates the sequence and logic for the various activities that constituted this study.

\section{S-3 Waste Streams and Required Waste Products}

To enable the development and comparison of alternatives, both the volumes and characteristics of the waste to be disposed of were evaluated if known, or assumed if unknown. Waste streams considered in this study included those generated from ongoing operations, waste from INEL Environmental Restoration (ER) activities, waste from the INEL Decontamination and Decommissioning (D\&D) Program, and waste currently in storage at the Transuranic Storage Area (TSA) and the Mixed Waste Storage Facility (MWSF). Because the alternatives evaluated in this study would begin operations about the year 2005 and operate for an assumed 40 years, the waste streams had to be considered relative to that time period.

This study assumed that a treatment facility or a combination of treatment facilities designed for INEL mixed waste and LLW will be on line, either onsite or offsite, during the time period of this study. It also assumed that the waste form produced at the treatment facilities will meet the waste acceptance criteria (WAC) of the disposal facility. 


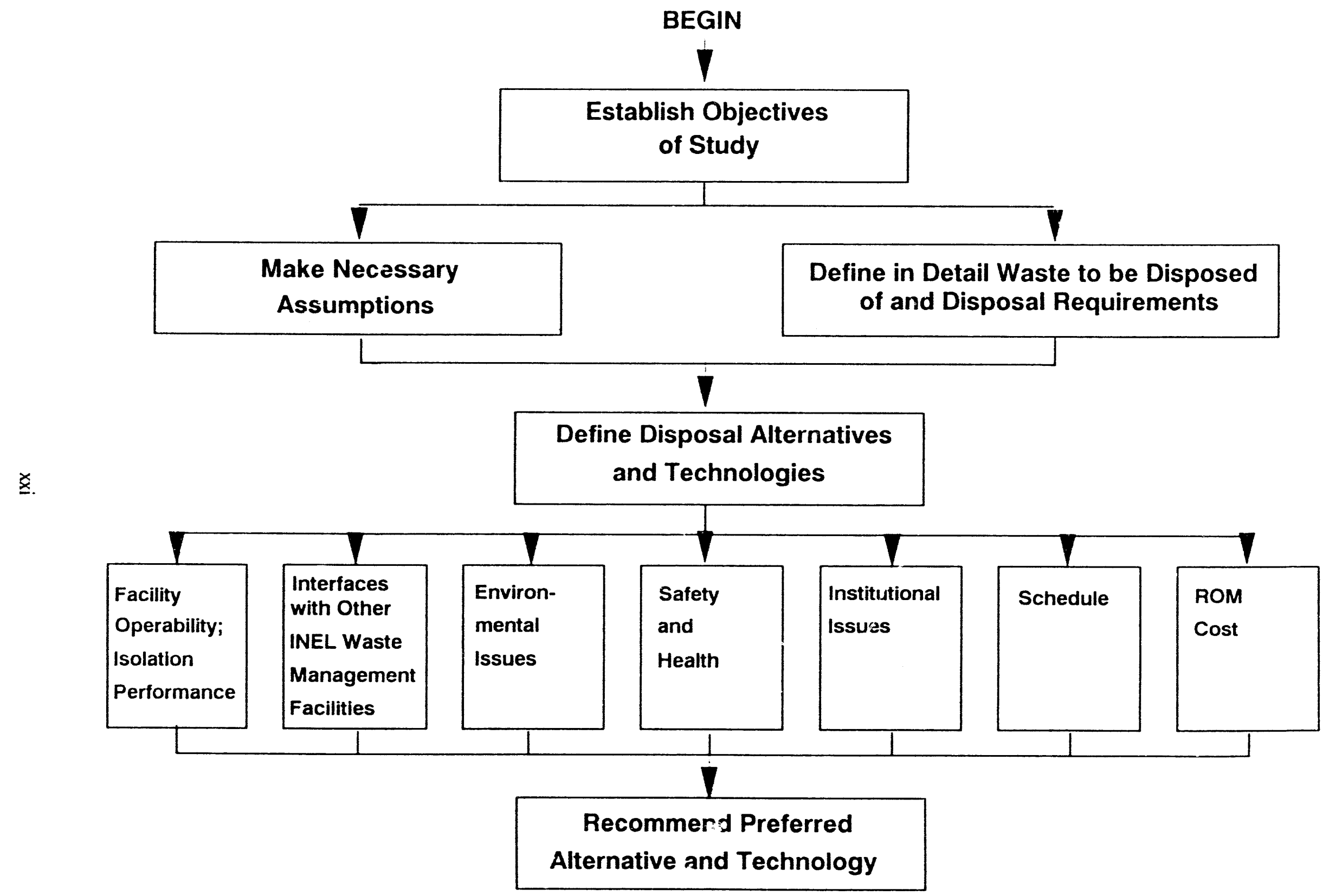

Figure S-1. Logic flowchart for current study. 
Two treatment scenarios were considered in this study: "maximum treatment," and "minimum treatment." Maximum treatment means that volume reduction is used for all applicable waste streams. Minimum treatment means that streams are treated only if required by regulation or DOE orders, and not for the purpose of volume reduction.

Two waste generation scenarios were also considered in this study. The ER and D\&D waste volumes currently being generated are quite small and were included in the waste streams from ongoing operations. This scenario was termed the "Small ER" and the "Small D\&D," or the minimum-generation scenario. These small volumes do not account for the large volumes of waste posuible as a result of expanding both programs in the future. This scenario was termed the "Large D\&D and "Large ER," or the maximum-generation scenario. It is noted that the "Large ER" cas' is a reasonable best estimate and not a true upper limit.

In order to comply with the regulations and DOE Orders (see Section S-4) governing the disposal of mixed waste and LLW, it was necessary to identify the types of waste to be disposed of. The Class A, B, C and greater than Class C LLW designations defined by the U.S. Nuclear Regulatory Commission (NRC) in 10 CFR 61 were used. Greater than Class $C$ waste is generally considered not acceptable for near-surface disposal and was not considered in the scope of this alternatives study. As a result, eight types of waste were considered for disposal. Table S-1 summarizes the waste sources and types of waste considered for disposal. The entries marked with an "X" in Table S-1 were addressed in this study. The simplifying assumption was made that all Class $A$ and Class $C \alpha$ waste are contact-handled, and all Class B and Class $C \beta$ - $\gamma$ waste are remotehandled.

Table S-1. Sources/types of waste to be disposed of.

\begin{tabular}{lcccc}
\hline \multicolumn{1}{c}{$\begin{array}{c}\text { Source } \\
\text { type }\end{array}$} & $\begin{array}{c}\text { Ongoing } \\
\text { operations }\end{array}$ & $\begin{array}{c}\text { "Large } \\
\text { D\&D" } \\
\text { scenario }\end{array}$ & $\begin{array}{c}\text { "Large ER" } \\
\text { scenario }\end{array}$ & $\begin{array}{c}\text { Stored } \\
\text { (TSA and } \\
\text { MWSF) }\end{array}$ \\
\hline Class A nonmixed & $\mathrm{X}$ & $\mathrm{X}$ & $\mathrm{X}$ & $\mathrm{O}$ \\
Class A mixed & $\mathrm{X}$ & $\mathrm{X}$ & $\mathrm{O}$ & $\mathrm{X}$ \\
Class B nonmixed & $\mathrm{X}$ & $\mathrm{X}$ & $\mathrm{O}$ & $\mathrm{O}$ \\
Class B mixed & $\mathrm{X}$ & $\mathrm{X}$ & $\mathrm{O}$ & $\mathrm{O}$ \\
Class C $\boldsymbol{\beta}-\boldsymbol{\gamma}^{\mathrm{a}}$ & $\mathrm{X}$ & $\mathrm{X}$ & $\mathrm{O}$ & $\mathrm{O}$ \\
Class C $\boldsymbol{\beta}-\boldsymbol{\gamma}$ mixed & $\mathrm{X}$ & $\mathrm{X}$ & $\mathrm{O}$ & $\mathrm{O}$ \\
Class C $\boldsymbol{\alpha}^{\mathrm{b}}$ & $\mathrm{O}$ & $\mathrm{X}$ & $\mathrm{X}$ & $\mathrm{O}$ \\
Class $\mathrm{C} \boldsymbol{\alpha}$ mixed & $\mathrm{O}$ & $\mathrm{O}$ & $\mathrm{O}$ & $\mathrm{X}$
\end{tabular}

a. The Class $C \beta-\gamma$ waste contains $<10 \mathrm{nCi} / \mathrm{g}$ of long-lived transuranic contamination but Class $C$ levels of $\beta-\gamma$ contamination.

b. The Class $\mathrm{C} \alpha$ waste contains 10 to $100 \mathrm{nCi} / \mathrm{g}$ of long-lived transuranic contamination. 
In support of the design of an INEL mixed and low-level waste treatment facility, information on the volumes and characteristics of mixed waste and LLW being generated by ongoing INEL operations was analyzed in another study (see Section 2.2.1). This same study provided information on the volumes and characteristics of mixed waste and LLW projected by the expanded D\&D program.

Based on input from remedial action managers, nearly all the ER activities are scheduled to be completed and the waste dispositioned before a new waste disposal facility will be operating (about 2005). Therefore, these streams were not addressed for disposal in this study. The major exception was the waste expected from remediation of the RWMC. Volumes and characteristics of the RWMC waste were estimated by those working in the ER effort, based on engincering judgement.

In support of the design of an INEL treatment facility for treating the contact-handled $\alpha$ contaminated LLW stored at the TSA, information on the volumes and characteristics of the waste was analyzed in another task (see Section 2.2.4). It is noted that the cited report assumed that all of the Class $\mathrm{C} \alpha$ waste stored at the TSA is mixed. In addition to the waste stored at the TSA, a small volume of mixed waste classified as listed (F-listed waste) and stored at the MWSF was considered for disposal.

The volumes and characteristics of waste generated from each source (as presented in the above resources) were evaluated under maximum- and minimum-treatment scenarios. Figures were developed, for each source, to illustrate the effect of treatment on the various waste streams and to determine the total volume and type of waste requiring disposal. As an example, Figure $\mathbf{S}-2$ was prepared for ongoing operations for the maximum-treatment scenario.

The posttreatment types and volumes of waste from each source and scenario were summarized in tables. The campaign length for each source of waste was estimated. The assumed campaign length for ongoing operations was 40 years (the length of disposal facility operations). The campaign length for D\&D operations was estimated to be 30 years. The campaign length for ER activities and for disposal of the waste stored at the TSA is 10 years. The campaign length for disposal of the waste stored at the MWSF is assumed to be 1 year.

Due to the complexities involved in estimating the cost of disposal facility operations over these various campaign lengths, the waste volumes used in the evaluation of alternatives and cost estimates were levelized over the expected life of the disposal facility (40 years). Table S-2 summarizes the levelized waste volumes to be disposed of by each source/type and scenario for the life of the disposal facility.

These levelized waste volumes for each of the four generation/treatment scenarios were then used to estimate the number of containers disposed of. Although many lorm" of waste packaging exist at the INEL, in order to simplify the study, only three forms of packaging were considered. The waste packaging included $4 \times 4 \times 6$-ft metal boxes, 71-gal square dítims, and $4 \times 4 \times 8$-ft concrete boxes. 


\section{LLW}

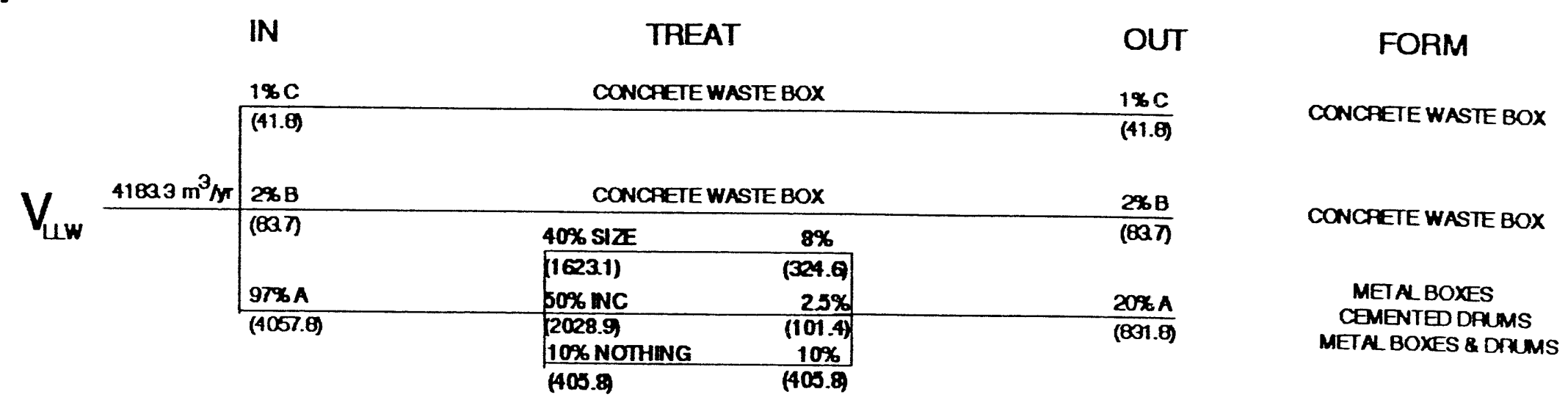

MW

IN

TREAT

OUT

FORM

$\underset{x}{x}$

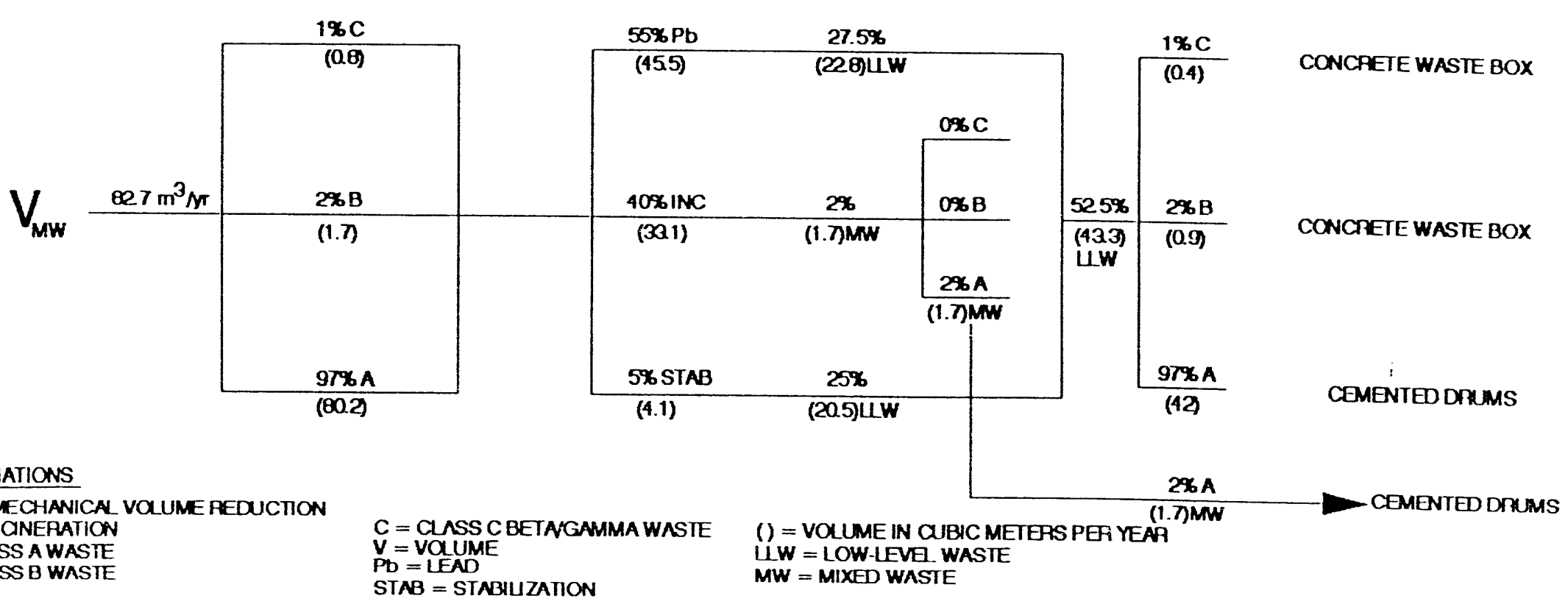

Figure S-2. Pretreatment and posttreatment types and volumes of waste for ongoing operations (maximum-treatment option).

\section{NBBREMATIONS}

STE = MECHANICA VOUME PEDUCTON INC = INONERATION

$A=$ C ASS A WASTE

$A=$ CLASSA WASIE
$\mathrm{B}=$ OASS B WASIE
$\mathrm{C}=$ CLASS CBETAKGAMA WASTE $\mathrm{PD}=\mathrm{LEAD}$

STAB = STABULZAIION OW-LEVE WASTE MW = MIXED WASTE 
Table S-2. Levelized waste volumes disposed of, by source, for life of the disposal facility $\left(\mathrm{m}^{3} / \mathrm{yr}\right)$.

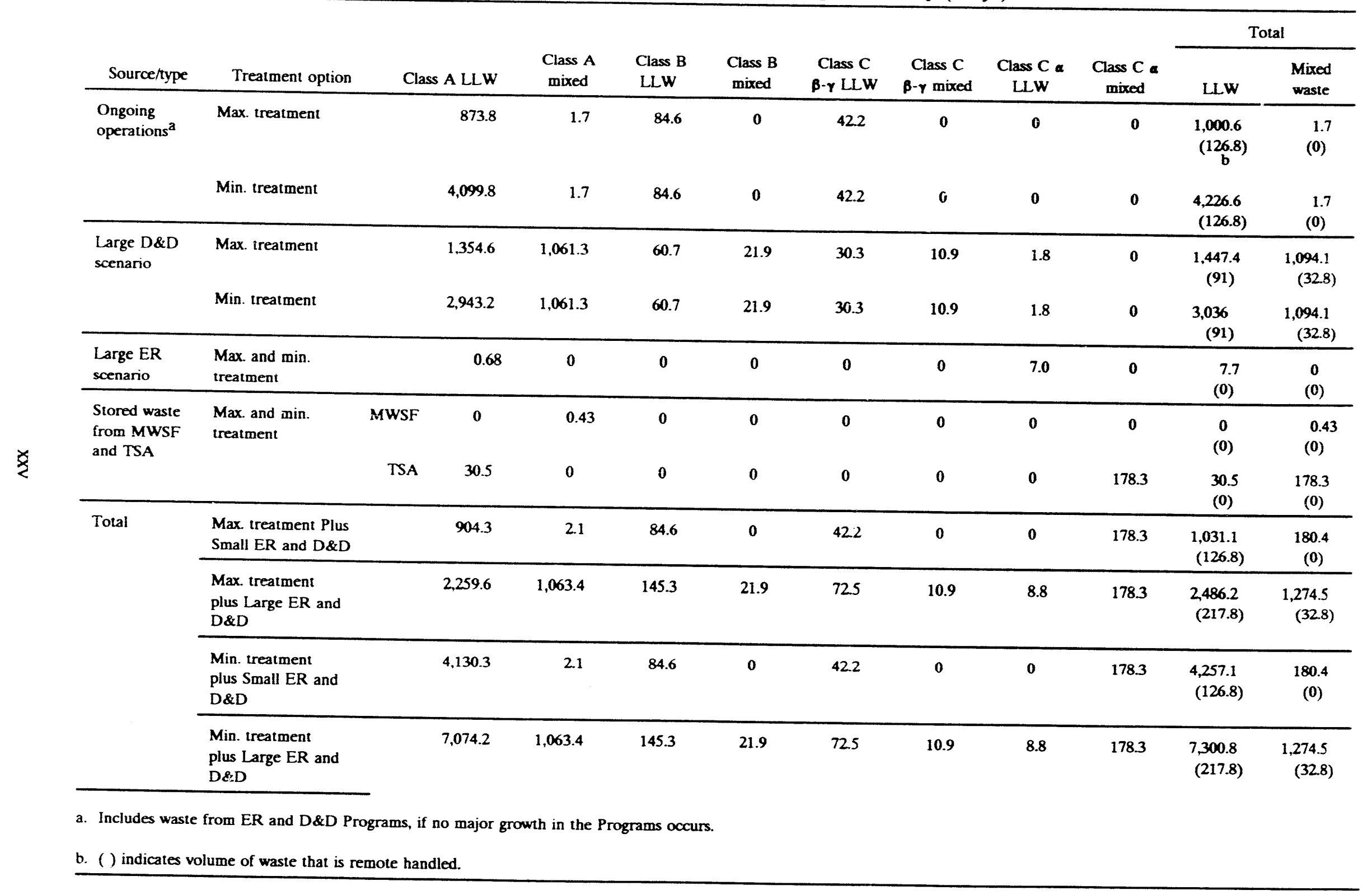




\section{S-4 Regulatory Requirements Expected to Govern Waste Disposal}

The development and evaluation of alternatives required an understanding of the regulatory requirements expected to apply to the disposal of LLW and mixed waste during the timeframe of this study. Because the timeframe of the study is many years in the future, the requirements cannot be known with certainty at present. The approach used in this study was to plan for compliance not only with current requirements, but also with applicable draft or proposed requirements considered likely to be issued.

The disposal of DOE LLW is currently controlled by DOE Order $5820.2 \mathrm{~A}$, "Radioactive Waste Management," particularly Chapter III, "Management of Low-Level Waste." There are also applicable requirements in DOE Order 6430.1A, "General Design Criteria," in Section 1324, "Radioactive Solid Waste Facilities."

DOE-HQ is currently preparing to make major revisions to DOE Order 5820.2A. The expected revisions to the Order that affect the disposal of mixed waste and LLW were included in the design requirements used in this study. These revised requirements and guidelines are expected to agree generally with NRC and U.S. Environmental Protection Agency (EPA) technical requirements.

The disposal-related regulatory requirements used in this study were based on the following sources:

- For LLW

- The current DOE Order 5820.2A, particularly Chapter III

- $\quad$ DOE Order 6430.1A, Section 1324

- The technical requirements of the NRC regulations at 10 CFR 61, plus (for guidance only) the technical interpretive guidance in Standard Format and Content of a License Application for a Low-Level Radioactive Waste Disposal Facility and Standard Review Plan for the Review of a License Application for a Low-Level Radioactive Waste Disposal Facility (see Section 2.3.1)

- The applicable environmental standards of draft EPA regulations 40 CFR 193, insofar as these performance standards are compatible with those in the above sources.

- For mixed waste

- The above requirements sources

- The U.S. EPA Resource Conservation and Recovery Act (RCRA) requirements for hazardous waste disposal, at 40 CFR 264 (in particular, 264.300-.317), supplemented by EPA guidance documents. 


\section{S-5 Assumptions and Uncertaintles}

Many items of information necessary to perform detailed comparisons of the alternatives were not available. The unknowns include gaps in today's knowledge, as well as the nature of possible future events, trends, policies, and decisions. Therefore, numerous assumptions were made about the missing items of information. To allow equitable comparisons between alternatives, assumptions were made in the following categories:

- Programmatic or general assumptions

- Waste assumptions

- Disposal facility assumptions

- Assumptions concerning auxiliary functions

- General regulatory assumptions

- Environmental regulatory assumptions

- Assumptions concerning interfaces with other INEL facilities

- Schedule assumptions

- Cost assumptions.

Several of the assumptions made are not only uncertain, but are also important enough to potentially affect the conclusions of the study. For this reason, "key uncertainties," which could change between now and the date of facility startup, were identified.

The key uncertainties fell into the following categories:

- Regulations

- DOE policies, philosophies, and programs

- Waste volumes and characteristics

- Status and missions of various waste management facilities

- Performance of waste management technologies

- Other.

\section{S-6 Disposal Alternatives}

Several preliminary alternatives for disposal of INEL mixed waste and LLW were formulated for evaluation and comparison. The alternatives form a relatively complete set of reasonable, upperlevel options. A list of the alternatives considered in this study is shown in Table S-3. 
Table S-3. Disposal alternatives and subalternatives.

\begin{tabular}{|c|c|c|}
\hline & Alternative or subalternative & Disposal technology \\
\hline \multicolumn{2}{|c|}{ 1A - Construct and operate the MLLWDF } & $\begin{array}{c}\text { Abovegrade } \\
\text { engineered structures }\end{array}$ \\
\hline $1 B-$ & $"$ & $\begin{array}{l}\text { Belowgrade } \\
\text { engineered structures }\end{array}$ \\
\hline $1 \mathrm{C}-$ & $"$ & Covered modules \\
\hline 1D - & $"$ & $\begin{array}{l}\text { Greater-depth } \\
\text { burial/boreholes }\end{array}$ \\
\hline $1 \mathrm{E}-$ & $"$ & $\begin{array}{c}\text { Shallow land burial/ } \\
\text { boreholes }\end{array}$ \\
\hline $1 \mathrm{~F}$ - & $"$ & $\begin{array}{c}\text { Shallow land burial/ } \\
\text { abovegrade engineered } \\
\text { structures }\end{array}$ \\
\hline $1 G$ - & t of large waste-generation scenario) & $\begin{array}{l}\text { Abovegrade } \\
\text { engineered structures }\end{array}$ \\
\hline $1 \mathrm{H}$ - & of minimum waste-treatment scenario) & $\begin{array}{c}\text { Abovegrade } \\
\text { engineered structures }\end{array}$ \\
\hline $2-\mathrm{Co}$ & disposal at RWMC & $\begin{array}{l}\text { Covered placement/ } \\
\text { shallow boreholes }\end{array}$ \\
\hline $3-$ Of & sposal & Shallow land burial/boreholes \\
\hline \multicolumn{3}{|c|}{4 - Combinations of alternatives } \\
\hline & and Offsite disposal & $\begin{array}{c}\text { Abovegrade } \\
\text { engineered structures// } \\
\text { shallow land burial/boreholes }\end{array}$ \\
\hline & RWMC and Offsite & $\begin{array}{c}\text { Covered placement/ } \\
\text { shallow boreholes// } \\
\text { shallow land burial/boreholes }\end{array}$ \\
\hline $5-\mathrm{No}$ & & Storage only \\
\hline
\end{tabular}




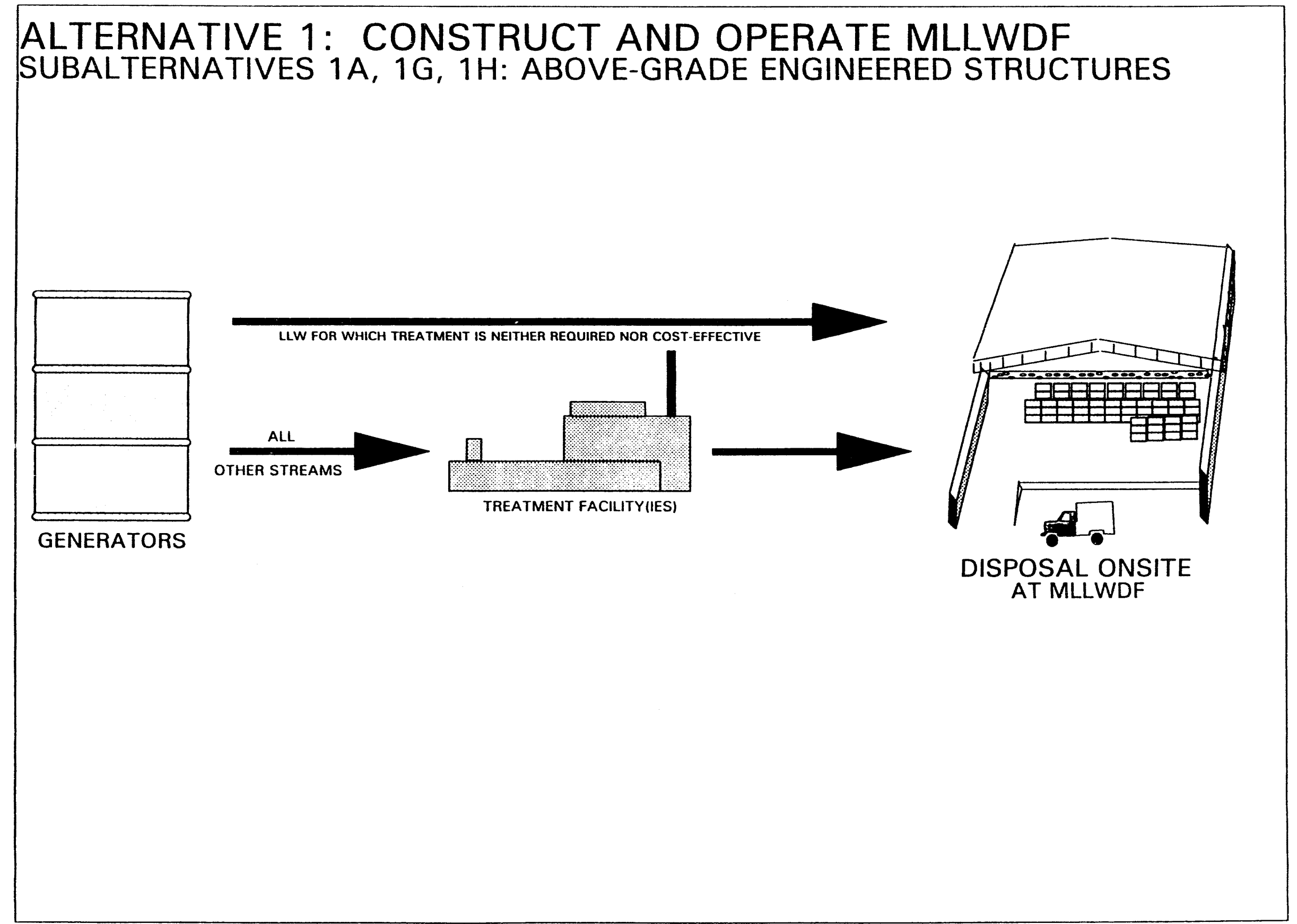

Figure S-3. Flow diagram for the MLLWDF Subalternatives $1 \mathrm{~A}, 1 \mathrm{G}$, and $1 \mathrm{H}$. 
Several DOE sites have operating disposal facilities for LLW. However, few if any DOE sites have operating disposal facilities that can accommodate mixed wastes, including $\alpha$-contaminated mixed waste and possibly remote-handled mixed waste. This study assumes that such disposal capabilities are available by the future time period of interest.

\section{Alternative 4: Combinations of Alternatives}

Combinations of the above alternatives were considered for evaluation. In each combination, some waste streams will be disposed of using one of the above alternatives, and the other waste streams will be disposed of by another alternative.

The matrix in Table S-4 indicates the two pairs of alternatives that were selected for consideration as combinations, marked with an "X." Pairs that were not selected are marked with a "O." Only the upper right half of the matrix was addressed because of symmetry. Also, the diagonal represents the combination of an alternative with itself, so it was not considered.

The descriptions and assumptions concerning the combinations of alternatives are generally the same as those for the individual alternatives comprising the combination. However, only part of the waste streams will be disposed of by each alternative. In both combinations, the mixed waste was shipped offsite for disposal and the LLW was disposed of onsite.

\section{Alternative 5: No Action}

In this alternative, no new action will be taken concerning disposal of INEL mixed waste and LLW. The MLLWDF will not be constructed. No other disposal alternative is implemented.

When the available disposal space in the RWMC is consumed, the newly generated mixed waste and LLW is stored indefinitely in buildings constructed for that purpose.

\section{S-7 Evaluations}

Seven areas were selected for evaluating the suitability of the alternatives on a preliminary overview basis. Each alternative was evaluated against the requirements and criteria established under each area. The following areas of comparison were selected:

Table S-4. Combinations of alternatives selected for evaluation.

\begin{tabular}{cccc}
\hline Alternative & MLLWDF & Continue RWMC & Ship Offsite \\
\hline MLLWDF & $\mathrm{O}$ & $\mathrm{X}$ \\
Continue RWMC & & $\mathrm{X}$ \\
Ship Offsite & & \\
\hline
\end{tabular}


- Operability and isolation performance evaluations

- These evaluations compared the alternatives on (a) the capability to handle and emplace all planned types of waste without excessive operational difficulty, (b) the use of proven technologies, (c) constructibility, operability, reliability, and maintainability of the handling and emplacement concept, and (d) postclosure performance of the isolation system.

- Evaluation of relationship to other INEL waste management facilities

- These evaluations compared the alternatives on the interfaces of the disposal alternatives with other major INEL waste management facilities and identified any potential interface problems that could have major programmatic or operational consequences.

- Environmental Evaluations

- These evaluations assessed the difficulties expected for each alternative to comply with the major bodies of environmental regulation.

- Safety and Health Evaluations

- These evaluations qualitatively compared the alternatives on the expected radiological and nonradiological risk to the workers, the public, and the environment during the construction and operations phases. (The isolation performance evaluations addressed the health and safety risks in the postoperations phase.)

- Institutional Evaluations

- These evaluations identified institutional issues that could negatively impact implementation and compared each disposal alternative on the basis of potential institutional problems.

- Schedule Evaluations

- These evaluations compared alternatives on the basis of the date when operations could be expected to start, as well as uncertainties in those dates. Schedules were prepared for each alternative. As an example, the schedule prepared for the MLLWDF Subalternative $1 \mathrm{~A}$ is shown in Figure S-4. Table S-5 summarizes the expected operational dates for each alternative. 


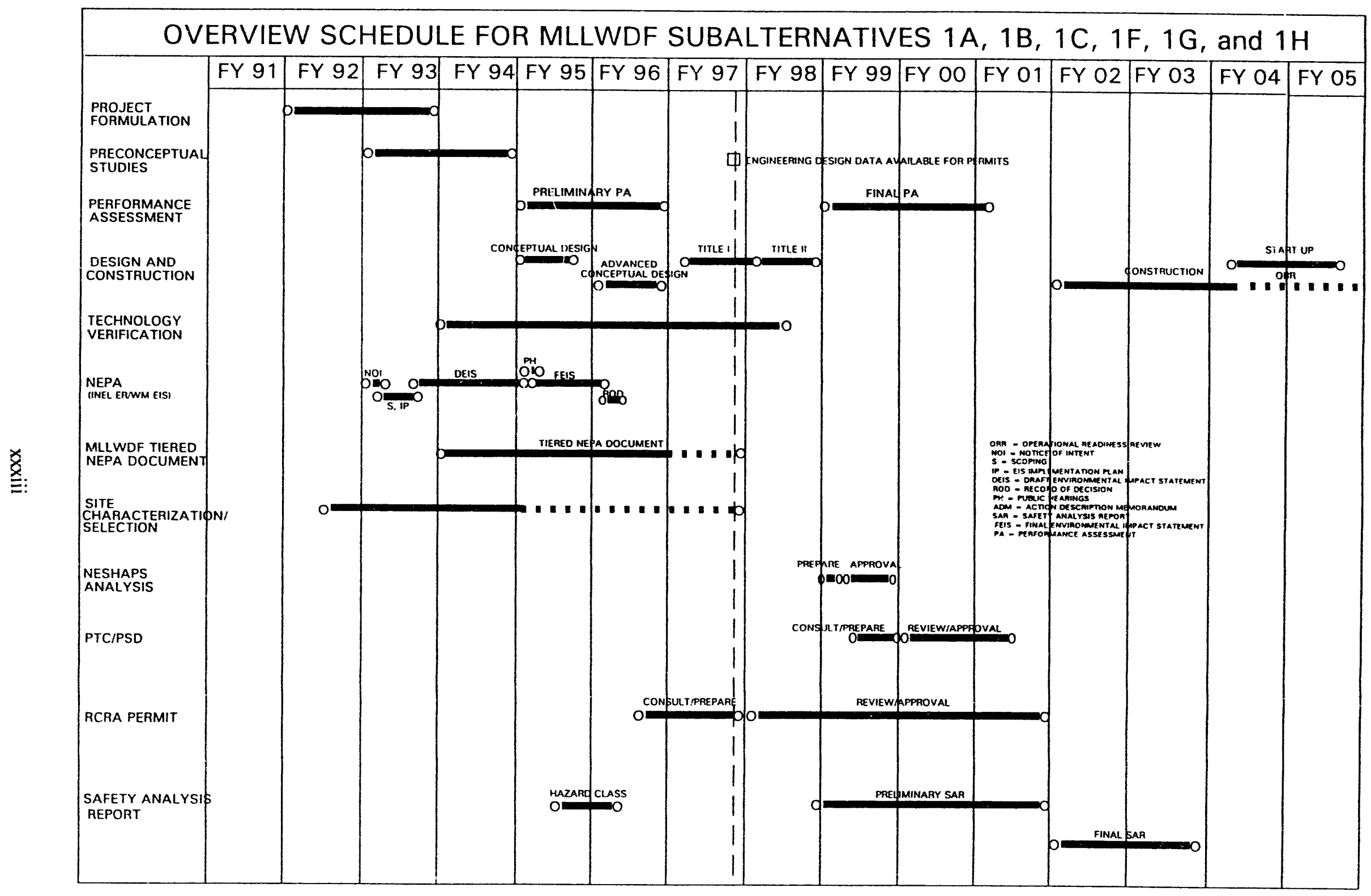

Figure S-4. Overview schedule for the MLLWDF Subalternative $1 \mathrm{~A}, 1 \mathrm{~B}, 1 \mathrm{C}, 1 \mathrm{~F}, 1 \mathrm{G}$, and $1 \mathrm{H}$. 
Table S-5. Start of facility operations for disposal alternatives.

\begin{tabular}{lc}
\hline \multicolumn{1}{c}{ Alternatives } & Start of facility operations \\
\hline MLLWDF Subalternatives 1A, 1B, 1C, 1F, & FY 2005 \\
1G, and 1H & FY 2004 \\
MLLWDF Subalternatives 1D and 1E & FY 2004 \\
Continue RWMC & FY 2000 \\
Offsite & \\
Combinations of alternatives & \\
$\quad$ MLLWDF and Offsite & FY 2003 \\
RWMC and Offsite & FY 2002 \\
No Action & FY 2002 \\
\hline
\end{tabular}

- $\quad$ Cost Evaluations

- These evaluations compared the ROM life-cycle costs of the alternatives. Both escalated and unescalated costs were estimated. See Table S-6 for the unescalated costs, which are the more meaningful because of the very long time periods involved.

In addition to discussing the performance of each alternative in the areas listed above, the performance was specifically evaluated against a list of "musts" and "wants."

The list of "musts" comprised the mandatory criteria for recommendation of a disposal alternative. To be considered viable, an alternative had to comply with every listed "must."

Alternatives were evaluated on the extent to which they satisfied the "wants." The list of "wants" represents criteria that will affect the recommendation of an alternative, but are not strict requirements. Each alternative was scored on each "want." Each "want" was assigned an importance weight. For each alternative, the scores on the various "wants" were multiplied by the importance weights of the respective "wants," then summed.

\section{S-8 Analysis of Results}

The results of the evaluations were analyzed, and other decision-making considerations were identified

Table S-i summarizes the "musts" and states whether each alternative passed or failed each criterion. 
Table S-6. Unescalated life-cycle costs for the disposal alternatives, M\$.

\begin{tabular}{cc}
\hline Alternatives & Life-cycle costs \\
\hline MLLWDF Subalternative 1A & 640 \\
Subalternative 1B & 650 \\
Subalternative 1C & 780 \\
Subalternative 1D & 500 \\
Subalternative 1E & 490 \\
Subalternative 1F & 488 \\
Subalternative 1G & 440 \\
Subalternative 1H & 970 \\
Continue RWMC & 510 \\
Offsite disposal & 295 \\
Combinations & \\
MLLWDF and Offsite & 677 \\
RWMC and Offsite & 601 \\
No Action & 533 \\
\hline
\end{tabular}

The no-action alternative failed the following "must" criteria and is not considered viable:

- Comply with all environmental and waste management regulatory requirements, including the requirements for long-term isolation.

- Provide all required disposal of mixed waste and LLW generated or currently stored at the INEL over a period of 40 years.

- Present no insurmountable institutional problems.

While Alternative 2, Continued Disposal at the RWMC and its combination (RWMC \& offsite), satisfies the "must" criteria for the scenario of maximum treatment and large generation, the use of available space is nearly at a maximum. These alternatives will not satisfy the criterion for disposal of all waste under the large-generation, minimum-treatment scenario.

The remaining alternatives satisfy all the "must" criteria and are considered viable.

The top row of Table S-8 summarizes the "wants;" the importance weights appear in parentheses. The remainder of Table S-8 presents the weighted scores. The MLLWDF Subalternatives $1 \mathrm{G}$ and $1 \mathrm{H}$ are shown in the master tables but were not considered in the overall 
Table S-7. Summary of performance of alternatives against "musts."

\begin{tabular}{|c|c|c|c|c|c|}
\hline \multirow[b]{2}{*}{ Alternative } & \multicolumn{5}{|c|}{ Musts } \\
\hline & $\begin{array}{l}\text { Is expected to comply with } \\
\text { all environmental and waste } \\
\text { management regulations, } \\
\text { including the requirements } \\
\text { for long-term isolation }\end{array}$ & $\begin{array}{l}\text { Present acceptable risk to } \\
\text { the workers, the public } \\
\text { and the environment } \\
\text { during the construction } \\
\text { and operations phases }\end{array}$ & $\begin{array}{l}\text { Over a period of } 40 \text { years, } \\
\text { provide all required disposal } \\
\text { of mixed waste and LLW } \\
\text { generated or currently stored } \\
\text { at the INEL, after any } \\
\text { necessary treatment has been } \\
\text { performed }\end{array}$ & $\begin{array}{l}\text { Present no potential } \\
\text { major conflicts with the } \\
\text { missions or operations } \\
\text { of other existing or } \\
\text { planned INEL waste } \\
\text { management facilities }\end{array}$ & $\begin{array}{l}\text { Present no } \\
\text { insurmountable } \\
\text { institutional problems }\end{array}$ \\
\hline MLLWDF - $1 \mathrm{~A}$ & $P$ & $\mathbf{P}$ & $\mathbf{P}$ & $\mathbf{P}$ & $\mathbf{P}$ \\
\hline MLLWDF - 1B & $\mathbf{P}$ & $\mathbf{P}$ & $\mathbf{P}$ & $\mathbf{P}$ & $\mathbf{P}$ \\
\hline MLLWDF - IC & $\mathbf{P}$ & $\mathbf{P}$ & $\mathbf{P}$ & $\mathbf{P}$ & $\mathbf{P}$ \\
\hline MLLWDF - 1D & $\mathbf{P}$ & $\mathbf{P}$ & $\mathbf{P}$ & $\mathbf{P}$ & $\mathbf{P}$ \\
\hline MLLWDF - 1E & $\mathbf{P}$ & $\mathbf{P}$ & $\mathbf{P}$ & $\mathbf{P}$ & $\mathbf{P}$ \\
\hline MLLWDF - 1F & $\mathbf{P}$ & $\mathbf{P}$ & $\mathbf{P}$ & $\mathbf{P}$ & $\mathbf{P}$ \\
\hline MLLWDF - 1G & $\mathbf{P}$ & $\mathbf{P}$ & $\mathbf{P}$ & $\mathbf{P}$ & $\mathbf{P}$ \\
\hline MLLWDF - $1 \mathbf{H}$ & $\mathbf{P}$ & $\mathbf{P}$ & $\mathbf{P}$ & $\mathbf{P}$ & $\mathbf{P}$ \\
\hline RWMC & $\mathbf{P}$ & $\mathbf{P}$ & $P^{b}$ & $\mathbf{P}$ & $\mathbf{P}$ \\
\hline Offsite & $\mathbf{P}$ & $\mathbf{P}$ & $\mathbf{P}$ & $\mathbf{P}$ & $\mathbf{P}$ \\
\hline MLLWDF/Offsite & $\mathbf{P}$ & $\mathbf{P}$ & $\mathbf{P}$ & $\mathbf{P}$ & $\mathbf{P}$ \\
\hline MLLWDF/RWMC & $P$ & $\mathbf{P}$ & $P^{b}$ & $\mathbf{P}$ & $\mathbf{P}$ \\
\hline No action ${ }^{\mathrm{a}}$ & $\mathrm{F}$ & $\mathbf{P}$ & $\mathbf{F}$ & $\mathbf{P}$ & F \\
\hline \multicolumn{6}{|c|}{$P=$ Passed the "must" criterion. } \\
\hline \multicolumn{6}{|c|}{ a. Alternatives that are no longer considered viable. } \\
\hline b. RWMC and the & nation with & $t$ pass the large-generation & nent & & \\
\hline
\end{tabular}


Table S-8. Summary of performance of alternatives against "wants."

Wants

\begin{tabular}{|c|c|c|c|c|c|c|c|c|c|}
\hline Alternative & $\begin{array}{l}\text { Minimize risk to } \\
\text { workers, the public, } \\
\text { and the environment } \\
\text { during the } \\
\text { construction and } \\
\text { operations phases } \\
\text { (5) }\end{array}$ & $\begin{array}{l}\text { Provide maximum } \\
\text { isolation of the } \\
\text { waste after the } \\
\text { operations period } \\
\text { (5) }\end{array}$ & $\begin{array}{l}\text { Minimize problems } \\
\text { in constructibility, } \\
\text { operability, } \\
\text { reliability, and } \\
\text { maintainability } \\
\text { (3) }\end{array}$ & $\begin{array}{l}\text { Minimize the } \\
\text { development risk } \\
\text { associated with } \\
\text { the isolation } \\
\text { system } \\
\text { technologies (3) }\end{array}$ & $\begin{array}{l}\text { Minimize the } \\
\text { schedule for } \\
\text { beginning disposal } \\
\text { operations } \\
\text { (2) }\end{array}$ & $\begin{array}{l}\text { Minimize } \\
\text { life-cycle } \\
\text { cost } \\
(2)\end{array}$ & $\begin{array}{l}\text { Minimize } \\
\text { potential } \\
\text { interface } \\
\text { problems with } \\
\text { other INEL } \\
\text { WM facilities } \\
\text { (1) }\end{array}$ & $\begin{array}{l}\text { Minimize } \\
\text { potential } \\
\text { institutional } \\
\text { problems } \\
\text { (1) }\end{array}$ & Sum \\
\hline MLLWDF - $1 \mathrm{~A}$ & 30 & 32.5 & 27 & 15 & 12 & 10 & 10 & 9 & 145.5 \\
\hline MLLWDF - 1B & 30 & 50 & 21 & 15 & 12 & 10 & 10 & 9 & 157 \\
\hline MLLWDF - IC & 45 & 27.5 & 30 & 24 & 12 & 8 & 9 & 9 & 164.5 \\
\hline MLLWDF - 1D & 35 & 47.5 & 24 & 27 & 14 & 12 & 10 & 7 & 176.5 \\
\hline MLLWDF - 1E & 40 & 42.5 & 27 & 27 & 14 & 12 & 10 & 5 & 177.5 \\
\hline MLLWDF - IF & 35 & 30 & 24 & 21 & 12 & 12 & 10 & 7 & 151 \\
\hline MLLWDF - 1G & 50 & 40 & 27 & 15 & 12 & 14 & 10 & 10 & 178 \\
\hline MLLWDF - $1 \mathrm{H}$ & 20 & 30 & 24 & 15 & 12 & 6 & 10 & 7 & 124 \\
\hline RWMC & 40 & 30 & 27 & 21 & 14 & 12 & 8 & 6 & 158 \\
\hline Offsite & 30 & 42.5 & 24 & 27 & 20 & 20 & 9 & 3 & 175.5 \\
\hline MLLWDF/Offsite & 30 & 35 & 21 & 18 & 16 & 8 & 9 & 5 & 142 \\
\hline RWMC/Offsite & 35 & 35 & 27 & 24 & 18 & 10 & 7 & 4 & 160 \\
\hline No Action $^{\mathrm{a}}$ & 25 & 15 & 30 & 30 & 18 & 12 & 10 & $\mathbf{0}$ & 140 \\
\hline
\end{tabular}

( ) = Importance weight.

a. Alternatives that failed a "must" and are not considered viable. 
ranking because they are scenarios not alternatives. These subalternatives are essentially the same as Subalternative $1 \mathrm{~A}$ with different assumed volumes of waste. The viable disposal alternatives are ranked as follows:

1. Subalternative 1E-Shallow land burial/boreholes

2. Subalternative $1 \mathrm{D}-$ Greater-depth burial/boreholes

3. Alternative 3-Ship waste offsite for disposal

4. Subalternative $1 \mathrm{C}-$ Covered modules

5. Alternative 4B-RWMC and ship offsite

6. Alternative 2-Continue disposal at RWMC

7. Subalternative 1B-Belowgrade engineered structures

8. Subalternative $1 \mathrm{~F}$-Shallow land burial/abovegrade engineered structures

9. Subalternative 1A-Abovegrade engineered structures

10. Alternative 4A-MLLWDF and ship offsite

Subalternatives $1 \mathrm{G}$ and $1 \mathrm{H}$ are not included in the above rankings because they are scenarios not alternatives.

It is noted that total scores within approximately 22 points of one another are covered within the uncertainty spread. Thus, the top 7 alternatives have scores within the uncertainty spread.

If the recommendation were based strictly on the "musts" and "wants," the preferred alternative for disposal of INEL mixed waste and LLW would be the onsite shallow land burial alternative. (The onsite greater-depth disposal alternative scores within one point of the leader, and the offsite shallow land burial alternative scores within two points.) However, selecting an alternative is extremely complex and involves many factors; some do not readily lend themselves to lists of "musts" and "wants." The decision is not completely determined by any evaluation of numbers. Several major factors that the decisionmaker needs to consider, in addition to the results of the "musts" and "wants," were identified and are listed below.

- Should a decision on enhanced disposal capability be pursued now or delayed?

Other than gaining additional regulatory clarifications, additional information on the ER and D\&D waste, and some insight about isolation performance in the INEL environment, no significant gain from delay was identified. (In fact, additional waste information can be obtained while implementation proceeds.) The resolution of the issues associated with the length of time required to implement a disposal alternative, the present lack of mixed waste disposal capacity, the limited amount of mixed waste 
storage space, and the limited disposal capacity at the RWMC will not be aided by delaying a decision.

- The effect of waste treatment on disposal requirements.

- DOE Order 5820.2A requires treatment of LLW only if cost effective or if needed to enable the disposal facility to meet its performance criteria. However, DOE Order 6430.1A states that all LLW must be treated if feasible. These issues impact the selection of a disposal alternative by (a) impacting the remaining life of the RWMC, and (b) by impacting the ability of a disposal facility to provide long-term isolation.

- Uncertainty of waste volume projections

- The major uncertainty involves the volume of waste produced by ER and D\&D activities. The issues associated with uncertainty in waste volume projections impact the selection of a disposal alternative by (a) impacting the remaining life of the RWMC, and (b) impacting the number of disposal units, space required, and funding necessary for design and construction.

- $\quad$ Site Selection

- The site for the proposed MLLWDF has not been selected yet. Consideration needs to be given to which disposal technologies are best suited for a particular site. For example, greater-depth burial concepts may not be suitable in a location with shallow surface sediments.

- Technology Risk

- No disposal concepts have been demonstrated to provide successful confinement for any time period approaching 500 years; therefore, implementation of any disposal technology involves some technology risk.

- Will other facilities be available?

- There is no assurance that several of the waste disposal or treatment facilities addressed in this study (offsite disposal of LLW and mixed waste, onsite treatment facilities, etc.) will exist.

- Public Perception

A major driver in the decisionmaking process is public perception. Disposal of LLW and mixed waste is expected to engender a high degree of public concern, making acceptance and implementation of an alternative a challenging process. 
- Offsite waste

- Consideration should be given to the question of whether to accept commercial mixed waste, as well as waste from other DOE sites, for disposal at the INEL. Although this study addresses disposal of only INEL-generated or -stored waste, the feasibility of accepting offsite waste for treatment, storage, and disposal is being addressed in other studies.

\section{S-9 Recommendations}

Based on analysis of the results, the following recommendations were made:

1. It is recommended that a detailed building-by-building study be performed as soon as possible to obtain the best estimate of future D\&D waste volumes and characteristics.

2. Based on the milestones in the final INEL Action Plan for Implementation of The Federal Facility Agreement and Consent Order, it is recommended that the environmental restoration personnel recvaluate periodically the projected long-term timing and volume of waste from remedial actions (including self-generated waste) and the need for treatment and disposal of such waste at the INEL.

3. Because of the uncertainties about isolation performance and the significance of the performance assessment, it is recommended that a preliminary performance assessment be conducted in FY 1995 and FY 1996 when conceptual design is performed. It is recommended that a detailed performance assessment be performed in FY 1999 and FY 2000 when Title II design has been completed.

4. Several performance-verification support studies are recommended in support of the selected disposal alternative. If the selected alternative is offsite disposal, these studies should be integrated with those performed by the host DOE site.

5. It is recommended that detailed design tradeoff studies be conducted.

6. The offsite alternative, the onsite shallow land burial alternative, and the onsite greater depth burial alternative are the conditionally recommended alternatives for the following reasons:

- They satisfy all "must" criteria

- They rank highest against the "wants" criteria

- They are the least expensive, or among the least expensive, viable alternatives in lifecycle cost 
The recommended alternatives are conditional on (a) the waste volume and characteristics projections, which would be confirmed in the studies discussed under Recommendations 1 and 2, (b) the results of the performance assessment that would be performed as discussed under Recommendation 3, (c) the results of the performance-verification support studies of Recommendation 4, (d) the successful development and operation of a suitable offsite facility, and (e) the willingness of the offsite facility to accept INEL waste.

7. It is recommended that activities begin on the recommended alternative(s) as soon as possible, while reevaluating the waste volume projections, so that additional time is not lost. This approach will help the INEL to develop the nocessary disposal capacity for INEL LLW and mixed waste as soon as feasible. 


\section{ACRONYMS}

\begin{tabular}{|c|c|}
\hline$\alpha$ & alpha \\
\hline ACGIH & American Conference of Government and Industrial Hygienists \\
\hline AEA & Atomic Energy Act \\
\hline AGES & abovegrade engineered structures \\
\hline ALARA & as low as reasonably achievable \\
\hline ASTM & American Society for Testing and Materials \\
\hline$\beta-\gamma$ & beta-gamma \\
\hline BDAT & best demonstrated available technology \\
\hline BGES & belowgrade engineered structures \\
\hline CAA & Clean Air Act \\
\hline CEQ & Council on Environmental Quality \\
\hline CERCLA & Comprehensive Environmental Response, Compensation, and Liability Act \\
\hline CFR & Code of Federal Regulations \\
\hline CQA & construction quality assurance \\
\hline CWA & Clean Water Act \\
\hline $\mathrm{D} \& \mathrm{D}$ & decontamination and decommissioning \\
\hline DOE & U.S. Department of Energy \\
\hline DOE-HQ & U.S. Department of Energy Headquarters \\
\hline DOE-ID & U.S. Department of Energy Idaho Operations Office \\
\hline DOT & U.S. Department of Transportation \\
\hline $\mathrm{EA}$ & environmental assessment \\
\hline EDE & effective dose equivalent \\
\hline $\mathrm{EDF}$ & engineering design file \\
\hline EIS & environmental impact statement \\
\hline EO & executive order \\
\hline EPA & U.S. Environmental Protection Agency \\
\hline ER & environmental restoration \\
\hline ER\&WM & Environmental Restoration and Waste Management \\
\hline FML & flexible membrane liner \\
\hline FONSI & finding of no significant impact \\
\hline FSAR & final safety analysis report \\
\hline FY & fiscal year \\
\hline HDPE & high-density polyethylene \\
\hline HEPA & high efficiency particulate air \\
\hline HP & Health Physicist \\
\hline HWSF & Hazardous Waste Storage Facility \\
\hline IAQB & Idaho Air Quality Bureau \\
\hline IDAPA & Idaho Administrative Procedures Act \\
\hline
\end{tabular}


INEL Idaho National Enginecring Laboratory

IWPF Idaho Waste Processing Facility

LCRS leachate collection and removal system

LDR Land Disposal Restrictions

LDS leak-detection system

LLW low-level waste

MLLWDF Mixed and Low-Level Waste Disposal Facility

MLLWTF Mixed and Low-Level Waste Treatment Facility

MW mixed waste

MWSF $\quad$ Mixed Waste Storage Facility

NEPA National Environmental Policy Act

NESHAP National Emission Standards for Hazardous Air Pollutants

NLLWP National Low-Level Waste Program

NOI notice of intent

NPDES National Pollutant Discharge Elimination System

NRC U.S. Nuclear Regulatory Commission

O\&M operations and maintenance

OSHA Occupational Safety and Health Act (or Administration)

ORNL Oak Ridge National Laboratory

OU operable unit

PSAR preliminary safety analysis report

PSD prevention of significant deterioration

PTC permit to construct

RAM reliability, availability, and maintainability

RCRA Resource Conservation and Recovery Act

RCT radiological control technician

ROD Record of Decision

ROM rough order-of-magnitude

RWMC Radioactive Waste Management Complex

SAR safety analysis report

SDA Subsurface Disposal Area

SRS Savannah River Site

SWEPP Stored Waste Experimental Pilot Plant

TBD to be determined

TRA Test Reactor Area

TRU transuranic

TSA Transuranic Storage Area

TSD treatment, storage, and disposal 
WAC waste acceptance criteria

WERF Waste Experimental Reduction Facility

WIPP

Waste Isolation Pilot Plant 


\section{REGULATORY SOURCES CITED}

All Federal and State statutes and regulations, Department of Energy (DOE) Orders, and Department of Energy Idaho Operations Office (DOE-ID) Orders and Supplemental Directives cited herein are listed below. Other references cited are listed at the end of the section where cited.

\section{DOE Orders, DOE-ID Orders, DOE-ID Supplemental Directives, and Secretary of Energy Notices}

DOE Order 4700.1, "Project Management System," March 6, 1987

DOE Order 5400.3, "Hazardous and Radioactive Mixed Waste Program," February 22, 1989

DOE Order 5440.1D, "National Environmental Policy Act Compliance Program," February 22, 1991

DOE Order 5480.3, "Safety Requirements for the Packaging and Transportation of Hazardous Materials, Hazardous Substances, and Hazardous Wastes," July 9, 1985

DOE Order 5480.4, "Environmental Protection, Safety, and Health Protection Standards," May 15,1984

DOE Order 5480.5, "Safety of Nuclear Facilities," September 23, 1986

DOE Order 5480.10, "Contractor Industrial Hygiene Program," June 26, 1985

DOE Order 5480.11, "Radiation Protection for Occupational Workers," December 21, 1988

DOE Order 5480.23, "Nuclear Safety Analysis," April 10, 1992

DOE Order 5820.2A, "Radioactive Waste Management," September 26, 1988

DOE Order 6430.1A, "General Design Criteria," April 6, 1989

DOE-ID SD 5820.2A, "Radioactive Waste Management (DRAFT)," June 27, 1991

DOE-ID Order 5480.3, "Safety Requirements for the Packaging and Transportation of Hazardous Materials, Hazardous Substances, and Hazardous Wastes," March 14, 1991

\section{Code of Federal Regulations}

Code of Federal Regulations, 10 CFR 61, "Licensing Requirements for Land Disposal of Radioactive Waste"

Code of Federal Regulations, 10 CFR 71, "Packaging and Transportation of Radioactive Material" 
Code of Federal Regulations, 29 CFR 1910, "Operational Safety and Health Standards"

Code of Federal Regulations, 29 CFR 1926, "Safety and Health Regulations for Construction"

Code of Federal Regulations, 40 CFR 193, "Environmental Radiation Protection Standards for Management and Land Disposal of Low-Level Radioactive Wastes"

Code of Federal Regulations, 40 CFR 261, "Identification and Listing of Hazardous Waste"

Code of Federal Regulations, 40 CFR 263, "Standards Applicable to Transporters of Hazardous Waste"

Code of Federal Regulations, 40 CFR 264, "Standards for Owners and Operators of Hazardous Waste Treatment, Storage, and Disposal Facilities"

Code of Federal Regulations, 40 CFR 270, "EPA-Administered Permit Programs-The Hazardous Waste Permit Program"

Code of Federal Regulations, 40 CFR 1500-1508, "National Environmental Policy Act Regulations"

Code of Federal Regulations, 49 CFR 173, "Shippers-General Requirements for Shipments and Packagings"

\section{Statutes}

Atomic Energy Act (AEA)

Clean Air Act (CAA)

Comprehensive Environmental Response, Compensation and Liability Act (CERCLA)

Endangered Species Act of 1973 (16 U.S.C. 1531 et seq.)

Energy Reorganization Act of 1974

Fish and Wildlife Coordination Act (16 U.S.C. 661 et seq.)

Hazardous and Solid Waste Amendments (HSWA) (1984) to the Resource Conservation and Recovery Act (RCRA)

Idaho Administrative Procedures Act (IDAPA) 16.01.1012, Procedures and Requirements for Permits to Construct and Operation Permits

National Historic Preservation Act of 1966 (16 U.S.C. 470 et seq.)

National Emission Standards for Hazardous Air Pollutants (NESHAP) 
National Environmental Policy Act (NEPA)

Resource Conservation and Recovery Act (42 U.S.C. 6901 et seq.)

Safe Drinking Water Act (SDWA)

xlix 


\section{A Preliminary Evaluation of Alternatives for Disposal of INEL Low-Level Waste and Low-Level Mixed Waste}

\section{INTRODUCTION}

\subsection{Purpose}

This study identifies and evaluates, on a preliminary and overview baisis, the alternatives for disposing of low-level radioactive waste (LLW) ${ }^{\mathrm{a}}$ and low-level mixed waste ${ }^{\mathrm{b}}$ generated or stored at the Idaho National Engineering Laboratory (INEL). This study is one of several preconceptual design activities in support of the Mixed ${ }^{c}$ and Low-Level Waste Disposal Facility (MLLWDF) project.

The results of the study are intended to provide information that will be useful for the

- Selection of a preferred disposal alternative

- Conceptual design process

- Alternatives evaluation required under the National Environmental Policy Act (NEPA) to support selection of a waste disposal alternative.

\subsection{Background}

\subsubsection{The Past and The Present}

Since 1952, LLW generated at the INEL (and some LLW generated offsite) has been disposed of at the Radioactive Waste Management Complex (RWMC). The RWMC is located in the southwestern part of the INEL. The waste is disposed of in the Subsurface Disposal Area (SDA) of the RWMC by shallow land burial.

a. As defined by DOE Order 5820.2A, low-level radioactive waste is waste that contains radioactivity and is not classified as high-level waste, transuranic waste, or spent nuclear fuel or $11 \mathrm{e}(2)$ byproduct material.

b. Mixed waste is waste that contains both radioactive and hazardous components as defined by the Atomic Energy Act and the Resource Conservation and Recovery Act, respectively.

c. Hereafter, for brevity, the term "mixed waste" will often be used in this document without the modifier "low-level." It should be remembered, however, that the term applies only to the mixed waste that is low-level. 
Although INEL low-level mixed waste also was disposed of previously by shallow land burial in the SDA, that practice ceased several years ago. There is currently no permitted facility at the INEL for the disposal of low-level mixed waste. INEL mixed waste is being stored while awaiting the development of treatment and disposal capability.

From 1954 through 1970, transuranic-contaminated waste, received primarily from offsite, also was buried in the SDA. Since 1970, such waste has been retrievably stored at the Transuranic Storage Area (TSA) of the RWMC.

For the stored waste in the TSA, the current plan is to prepare such waste that is transuranic (TRU) waste, per U.S. Department of Energy (DOE) Order 5820.2A, for eventual shipment to the proposed Waste Isolation Pilot Plant (WIPP) in New Mexico. (TRU waste is "waste that is contaminated with alpha-emitting transuranium radionuclides with half-lives greater than 20 years and concentrations greater than $100 \mathrm{nCi} / \mathrm{g}$ at the time of assay.") The TRU-contaminated stored waste that is not TRU waste (i.e., $<100 \mathrm{nCi} / \mathrm{g}$ ) will be managed as $\mathrm{LLW}$.

Environmental restoration studies are in progress to determine the disposition of the TRUcontaminated waste that is buried in the SDA.

Since the early 1980s, part of the INEL-generated LLW has been treated in the Waste Experimental Reduction Facility (WERF) to reduce the volume requiring disposal. WERF has also treated a small volume of low-level mixed waste.

\subsubsection{The Future}

LLW and mixed waste at the INEL are required to be managed in accordance with applicable Federal and State regulations and DOE Orders. These requirements provide for the protection of human health and the environment.

In response to these requirements, enhanced capability for treatment of INEL mixed waste and possibly LLW is being evaluated.

An improved disposal system also is needed for the INEL LLW and mixed waste for several reasons:

- Progressive changes in the requirements governing disposal of the waste demand the development of more advanced disposal systems

- There is currently no permitted facility for the disposal of INEL mixed waste

- The remaining disposal space in the SDA is limited.

DOE Order 5820.2A requires that "DOE low-level waste shall be managed on a systematic basis, using the most appropriate combination of waste generation reduction, segregation, treatment, and disposal practices so that the radioactive components are contained and the overall system costeffectiveness is maximized." The Order also requires that "DOE low-level waste operations shall be 
managed to ... ensure that no legacy requiring remedial action remains after operations have been terminated."

All of the above driving forces have led to the need to develop improved disposal capability for INEL LLW and mixed waste.

The MLLWDF project has been established to provide the necessary disposal capability discussed above, whether onsite or offsite. The project will provide disposal for the INEL mixed waste, for which no disposition is currently available. The project will also provide for disposal of LLW currently being performed at the RWMC. The remaining disposal capability for the RWMC in its current configuration is limited. This will be accomplished by (a) siting the facility in a location possessing characteristics that meet or exceed regulatory requirements, and (b) providing disposal technologies that comply with all applicable requirements and isolate the waste from the environment. Once the enhanced disposal capability is operational, disposal of LLW at the RWMC will cease and the SDA eventually will undergo closure activities.

Several preconceptual design studies are under way or planned to provide the basis for conceptual design of the MLLWDF. The studies include environmental compliance plans, facility siting studies, development of functional and operational requirements, and the present study of disposal alternatives.

Alternatives for providing disposal capability are evaluated here to arrive at a preliminary recommendation for the preferred alternative. Not only is the comparison of alternatives a NEPA requirement, but it is also good project management practice. Hence, this overview study of the alternatives for disposing of INEL LLW and mixed waste is needed. (The current document, however, is not a NEPA document.)

\subsection{Scope}

The scope of this study is to identify and evaluate, on a preliminary and overview basis, the alternatives for disposing of INEL-generated or -stored LLW and mixed waste.

The alternatives evaluated are to cover the full range from "no action" to constructing and operating a new facility, termed the MLLWDF.

The evaluations are io include qualitative comparisons of the projected isulation performance of disposal alternatives. The evaluations are also to include facility, health and safety, environmental, institutional, schedule, and rough order-of-magnitude (ROM) cost comparisons.

Many items of information necessary to perform detailed, final comparisons of the disposal alternatives are not available at this early date in the MLLWDF project. For example, the treatment processes that would be applied to the waste prior to disposal have not yet been determined by other INEL projects. Therefore, numerous assumptions were made about the missing items of information in order for this study to proceed. 
Many items of information used in this study are subject to change at any time. For example, the projections of waste streams are periodically updated. Information used in this study reflects the best data available at the time of the analysis and may change significantly in the future.

Detailed, engineering-level evaluations of the alternatives are outside the scope of this study. The present evaluations are performed at the overview level. Furthermore, except for cost and logistical estimates, quantitative comparisons of the alternatives are generally outside the scope of this study.

\subsection{Flowchart for Study}

Figure 1-1 is a flowchart that illustrates the sequence and logic structure for the various activities in this study. 


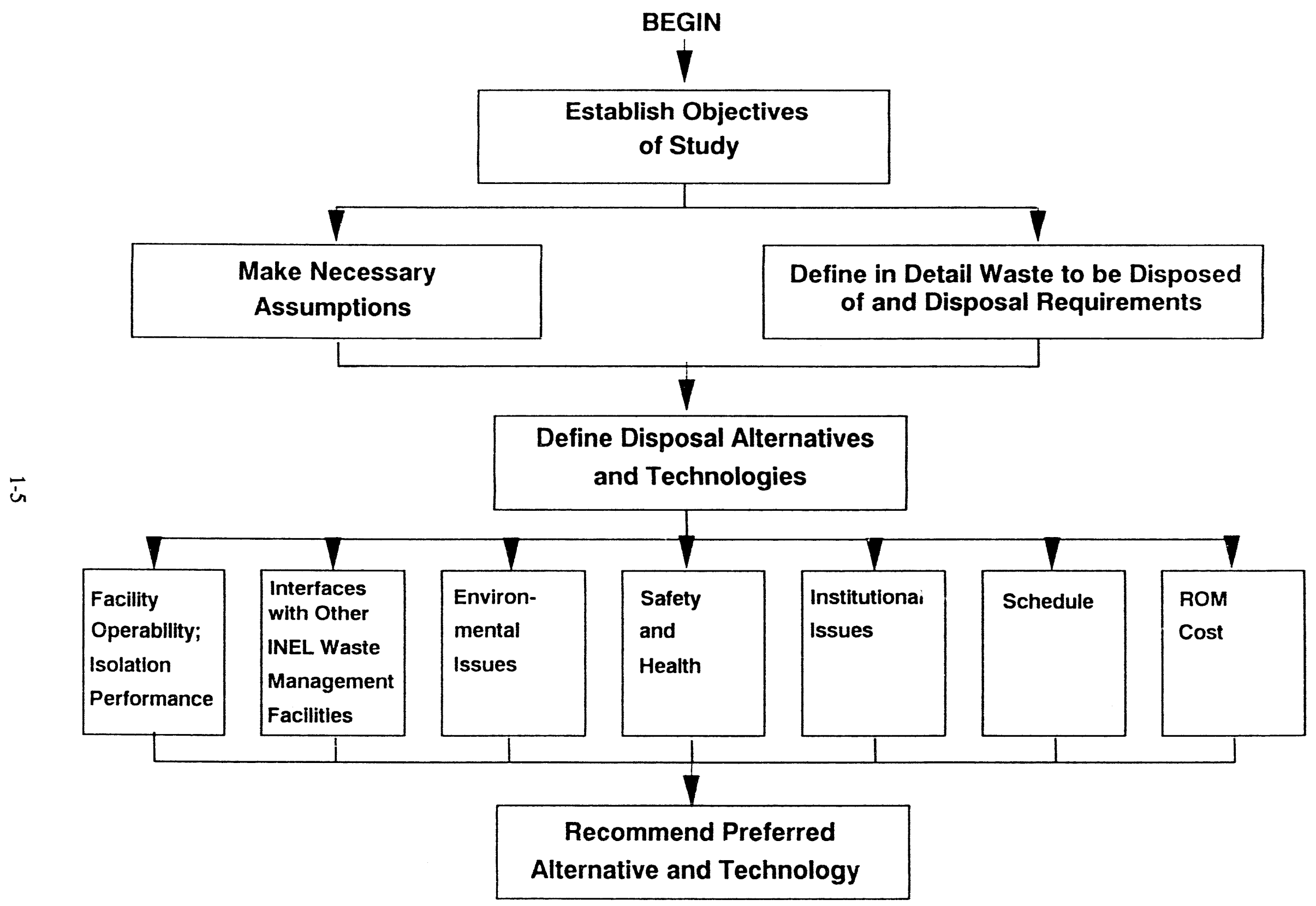

Figure 1-1. Logic flowchart for current study. 


\section{DESCRIPTION OF WASTE STREAMS AND REQUIREMENTS AFFECTING DISPOSAL}

To enable development and comparison of alternatives, the volumes and characteristics of the waste to be disposed of must be known or assumed. In order to develop an understanding of the volumes and characteristics of waste to be disposed of, the potential INEL waste generators, the characteristics of the waste, and volumes of the waste had to be identified. Although changes in INEL waste-generating, treatment, and disposal activities will occur between the present and the startup of the disposal facility, the waste characteristics and volumes must be fixed in order to perform comparisons of alternatives. The waste characteristics and volumes to be used in this study are defined in this section.

This section also identifies the requirements governing the disposal of the waste.

\subsection{Sources and Types of Waste To Be Disposed of}

\subsubsection{Sources of Waste to be Disposed of}

To accurately address the waste characteristics and volumes, the sources of waste must first be identified. The four potential sources of mixed waste and LLW at the INEL are shown in Figure 2-1. The potential sources include waste from ongoing operations, waste from INEL Environmental Restoration (ER) activities, waste from the INEL Decontamination and Decommissioning (D\&D) Program, and waste currently in storage at the TSA and the Mixed Waste Storage Facility (MWSF). For each source, some of the waste generated will not necessarily be mixed waste and LLW (e.g., some may be TRU waste), and will fall outside the scope of this study.

This study assumes that a treatment facility or a combination of treatment facilities designed for INEL mixed waste and LLW will be on line, either onsite or offsite, during the time period of the study. It also assumes that the waste form produced at the treatment facilities will meet the waste acceptance criteria (WAC) of the disposal facility.

Two treatment options are considered in this study. The first is "maximum treatment," which means that volume reduction is used for all applicable waste streams. In order to bound the range of designs for the MLLWDF, a "minimum-treatment" option is also considered. Minimum treatment means that waste streams are treated only if required by regulation or DOE orders, and not for the sole purpose of volume reduction.

\subsubsection{Types of Waste to Be Disposed of}

In order to comply with the regulations and DOE orders governing the disposal of mixed waste and LLW, it is necessary to identify the types of waste to be disposed of. The Class A, B, C and greater than Class C designations in Table 2-1 are the LLW categories defined by the U.S. Nuclear Regulatory Commission (NRC) in 10 Code of Federal Regulations (CFR) 61. (The justification for using this classification system is discussed in Section 2.3.) Greater than Class $C$ waste is generally considered not acceptable for near-surface disposal and will not be considered in the scope of this alternatives study. For such waste, the entries in Table 2-1 are marked with an "O." 


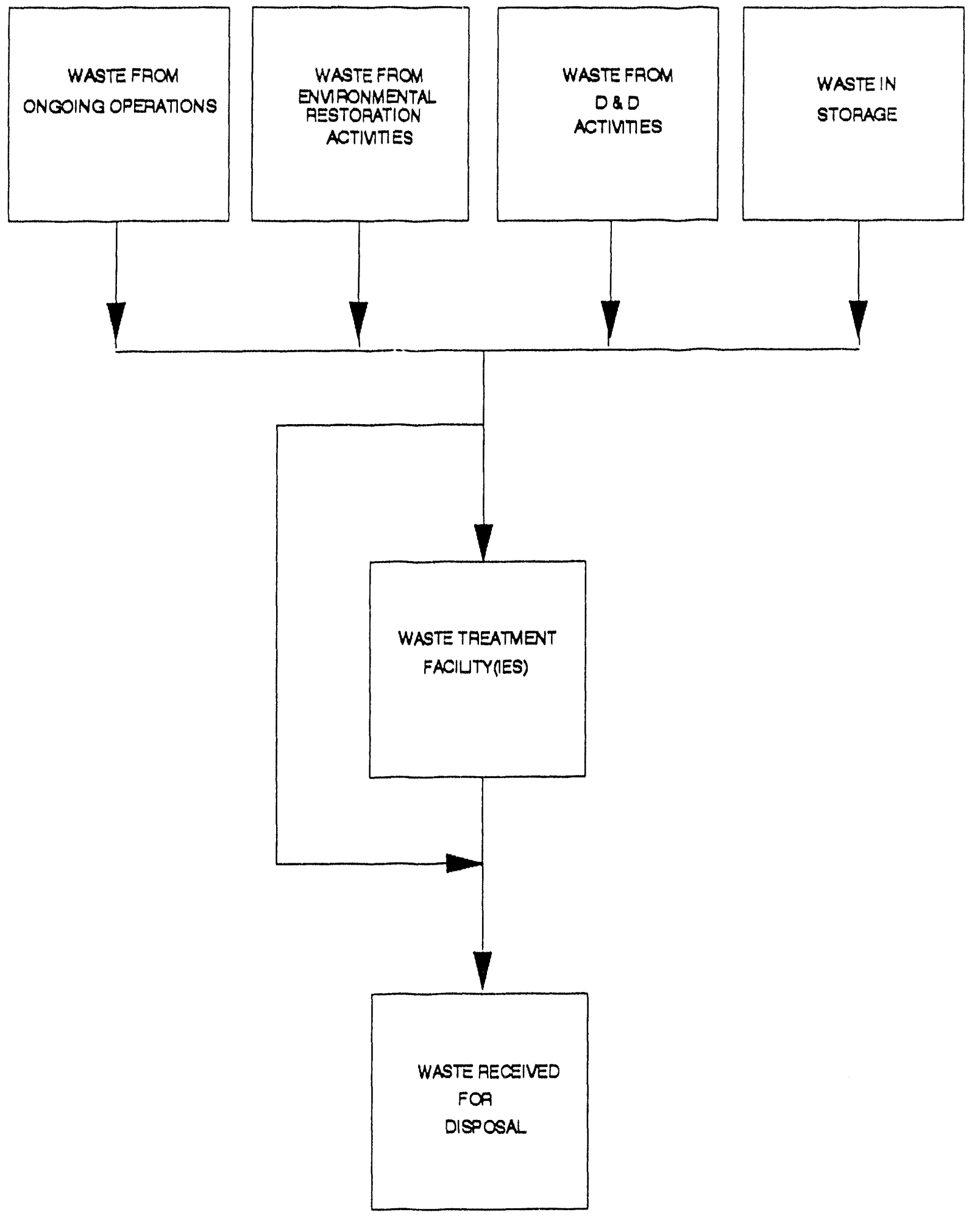

Figure 2-1. The four potential sources of mixed waste and LLW at the INEL. 
Table 2-1. Waste types to be disposed of.

\begin{tabular}{lcccc}
\hline \multicolumn{1}{c}{ Waste type } & Class A & Class B & Class C & > Class C \\
\hline Nonmixed $\beta-\gamma$ & $\mathrm{X}$ & $\mathrm{X}$ & $\mathrm{X}$ & 0 \\
Mixed $\beta-\gamma$ & $\mathrm{X}$ & $\mathrm{X}$ & $\mathrm{X}$ & 0 \\
$\alpha$ & $\mathrm{N} / \mathrm{A}$ & $\mathrm{N} / \mathrm{A}$ & $\mathrm{X}$ & 0 \\
$\alpha$ mixed & $\mathrm{N} / \mathrm{A}$ & $\mathrm{N} / \mathrm{A}$ & $\mathrm{X}$ & $\mathrm{O}$ \\
\hline
\end{tabular}

Each of the categories (across the top of Table 2-1) is subdivided into four additional categories (down the left side of the table). The first category, called nonmixed, identifies radioactive waste containing no Resource Conservation and Recovery Act (RCRA) hazardous constituents. The second category, mixed, is for radioactive waste containing hazardous constituents. The division between nonmixed and mixed is made because of the additional disposal requirements for mixed waste. The third and fourth categories, alpha $(\alpha)$ and $\alpha$ mixed, are for Class $C$ waste containing concentrations of long-lived, $\alpha$-emitting, TRU radionuclides in the range from 10 to $100 \mathrm{nCi} / \mathrm{g}$. The division between $\alpha$ and non- $\alpha$ waste is made because it is expected that the performance assessment for the long-lived radionuclides will indicate the requirement for additional isolation.

In accordance with 10 CFR 61, there is no Class $B \alpha$ waste; therefore, the two combinations of $\alpha$ waste and Class B waste are marked N/A in Table 2-1. Class A $\alpha$ waste does exist, but since the disposal requirements for Class $A \alpha$ and Class $A$ beta-gamma $(\beta-\gamma)$ waste are expected to be the samc, the division is not required and the combinations of Class $A$ waste and $\alpha$ waste are marked with an N/A in Table 2-1. The eight entries marked with an "X" in Table 2-1 are addressed in this study.

The simplifying assumption was made that all Class $\mathrm{A}$ and Class $\mathrm{C} \alpha$ waste are contact-handled, and all Class $B$ and Class $C \beta-\gamma$ waste are remote-handled.

In order to define the volume of waste to be disposid of, it is necessary to identify which waste types are generated by each source shown in Figure 2-1. The matrix in Table 2-2 indicates which sources generate the eight types of waste under consideration. The combinations marked with an "X" are those waste types requiring disposal. At this point, an evaluation of the sources and waste types can be conducted to estimate the volumes of waste to be disposed of. The entries in Table 2-2 will be explained in Section 2.2 .

\subsection{Volumes and Characteristics of Waste To Be Disposed of}

\subsubsection{Waste from Ongoing Operations}

In support of the design of an INEL Mixed and Low-Level Waste Treatment Facility (MLLWTF), information on the volumes and characteristics of mixed waste and LLW being generated by ongoing INEL operations was analyzed in another study (Smith et al. 1992).

Figure 2-2 summarizes the pretreatment and posttreatment types and volumes of waste from ongoing operations for the maximum-treatment option. 
Table 2-2. Sources/types of waste to be disposed of.

\begin{tabular}{lcccc}
\hline $\begin{array}{c}\text { Source } \\
\text { type }\end{array}$ & $\begin{array}{c}\text { Ongoing } \\
\text { operations }\end{array}$ & $\begin{array}{c}\text { "Large } \\
\text { D\&D" } \\
\text { scenario }\end{array}$ & $\begin{array}{c}\text { "Large ER" } \\
\text { scenario }\end{array}$ & $\begin{array}{c}\text { Stored } \\
\text { (TSA and } \\
\text { MWSF) }\end{array}$ \\
\hline Class A nonmixed & $\mathrm{X}$ & $\mathrm{X}$ & $\mathrm{X}$ & $\mathrm{O}$ \\
Class A mixed & $\mathrm{X}$ & $\mathrm{X}$ & $\mathrm{O}$ & $\mathrm{X}$ \\
Class B nonmixed & $\mathrm{X}$ & $\mathrm{X}$ & $\mathrm{O}$ & $\mathrm{O}$ \\
Class B mixed & $\mathrm{X}$ & $\mathrm{X}$ & $\mathrm{O}$ & $\mathrm{O}$ \\
Class C $\boldsymbol{\beta}-\boldsymbol{\gamma}^{\mathrm{a}}$ & $\mathrm{X}$ & $\mathrm{X}$ & $\mathrm{O}$ & $\mathrm{O}$ \\
Class C $\boldsymbol{\beta}-\boldsymbol{\gamma}$ mixed & $\mathrm{X}$ & $\mathrm{X}$ & $\mathrm{O}$ & $\mathrm{O}$ \\
Class C $\boldsymbol{\alpha}^{\mathrm{b}}$ & $\mathrm{O}$ & $\mathrm{X}$ & $\mathrm{X}$ & $\mathrm{O}$ \\
Class C $\boldsymbol{\alpha}$ mixed & $\mathrm{O}$ & $\mathrm{O}$ & $\mathrm{O}$ & $\mathrm{X}$ \\
\hline
\end{tabular}

a. The Class $C \beta-\gamma$ waste contains $<10 \mathrm{nCi} / \mathrm{g}$ of long-lived transuranic contamination but Class $C$ levels of $\beta-\gamma$ contamination.

b. The Class $\mathrm{C} \alpha$ waste contains 10 to $100 \mathrm{nCi} / \mathrm{g}$ of long-lived transuranic contamination.

The cited report and this study both use data (1/1/88 through 6/30/91) for waste from ongoing operations. Waste generators' projections of future volumes have proven not to be particularly accurate.

Two other sources of waste were addressed in the studies. Those sources are the INEL ER activities and the D\&D Program activities. As used in this document, the remedial actions deal principally with the remediation of areas outside of buildings and the D\&D Program deals with the remediation of the buildings and their contents. The ER and D\&D waste volumes currently being generated are quite small and are included in the waste from ongoing operations.

If the ER and D\&D Programs were to remain very small generators of waste, as they are today, the projections of waste from ongoing operations would satisfactorily encompass the ER and D\&D waste. This scenario is termed the "Small ER" and the "Small D\&D" case. These small volumes do not account for the large volumes of waste anticipated as a result of expanding both programs in the future. This scenario is called the "Large D\&D" and "Large ER" case and is addressed in Sections 2.2 .2 and 2.2 .3 , respectively.

Using the information in Smith et al. (1992), the currently generated waste streams were divided into mixed waste and LLW. As indicated in Figure 2-2, there are $82.7 \mathrm{~m}^{3} / \mathrm{yr}$ of mixed waste to be treated and $4,183.3 \mathrm{~m}^{3} / \mathrm{yr}$ of LLW to be treated. These volumes are equivalent to $2 \%$ and $98 \%$, respectively, of the waste volume $\left(4,266 \mathrm{~m}^{3} / \mathrm{yr}\right)$ to be treated. 
LLW

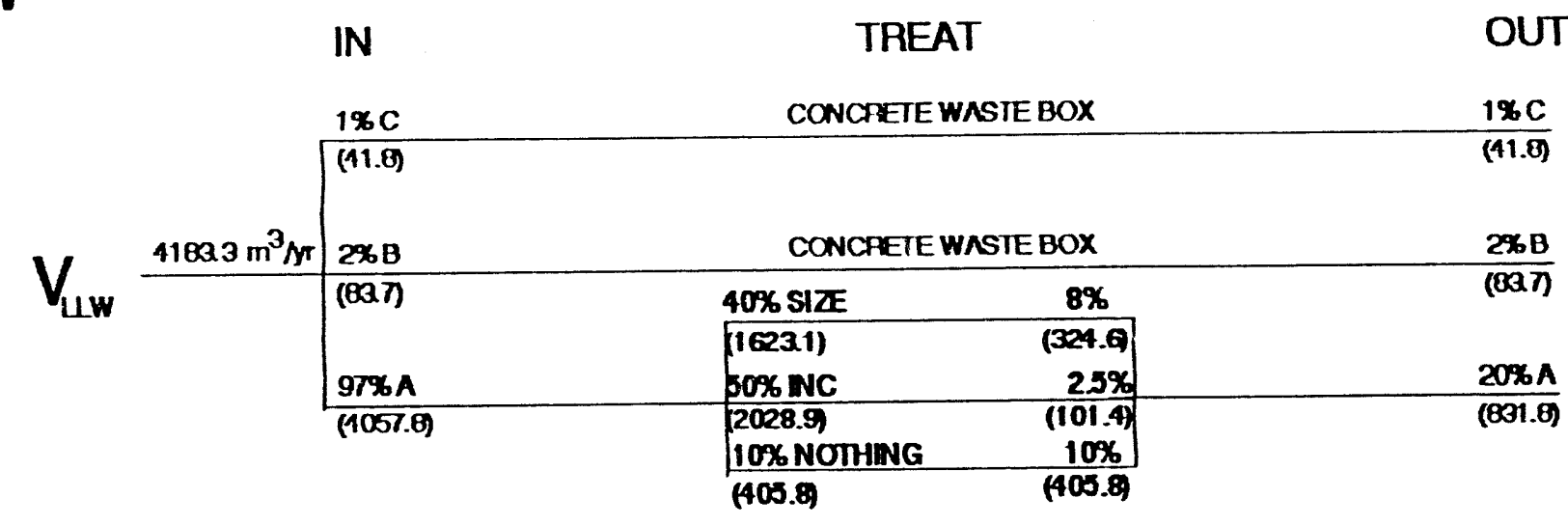

\section{FORM}

CONCREIE WNSTE BOX

CONCAETE WASTE BOX

METN BOXES

CEMENTED DFUMS

METNLBOXES \& DTMMS

\section{MW}

IN

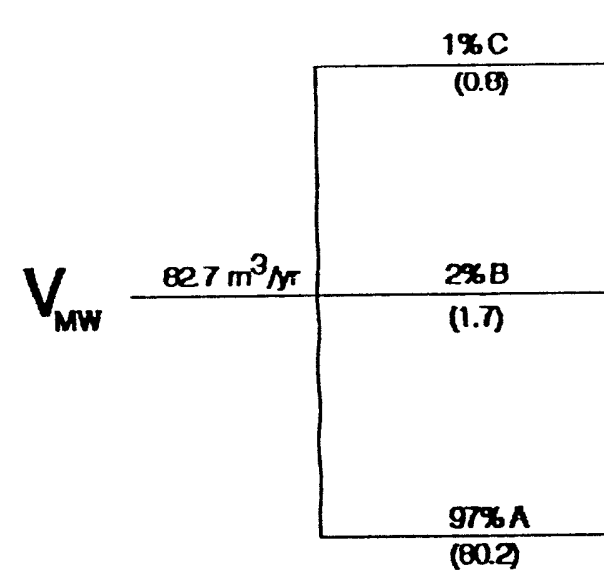

TREAT

OUT

$27.5 \%$

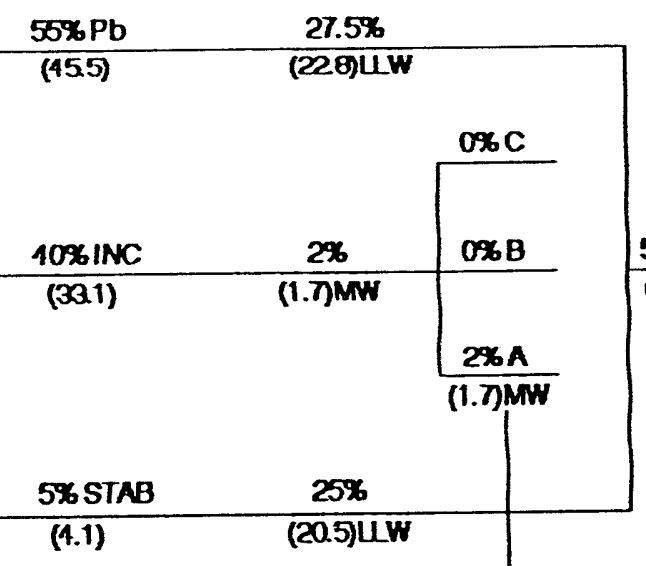

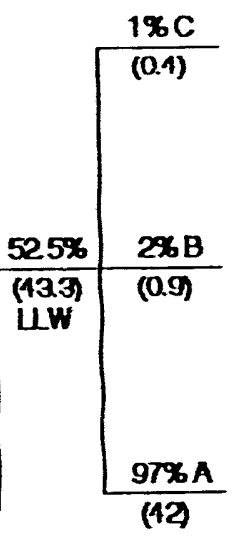

(42)

\section{FORM}

CONCPETE WNSTE BOX

CONCPETE WASTE BOX

CEMENTED DINMMS
ABBREVATIONS

SIZE = MECHANICA VOUME REDUCTION INC = INANEPATION $A=$ CLASS A WASTE
$B=$ CASSB WASTE

\section{C = CLASS CBETNGMMMA WASTE $\mathrm{V}=$ VOUME
$\mathrm{P}=\mathrm{W}=\mathrm{MA}$} STAB = STABIUZATION
2\%A $(1.7 \mathrm{MW}$

0 = VOLUME IN OUBIC METERS PER YENA UW = LOW-LEVE WASTE $M W=$ MIXED WASTE

Figure 2-2. Pretreatment and posttreatment types and volumes of waste for ongoing operations (maximum-treatment option). 
Both the mixed waste and LLW streams were then divided into Class A, B, and C streams. Because the waste streams in Smith et al. (1992) were not broken down into these classifications, further investigation was necessary. Waste classification breakdowns were assumed based on an evaluation of the waste streams currently treated and those not treated at the INEL WERF (EG\&G Idaho 1992). Approximately $97 \%$ of the generated LLW stream was identified as Class A waste, $2 \%$ as Class $\mathrm{B}$ waste, and $1 \%$ as Class $\mathrm{C}$ waste.

Applying this breakdown to the LLW streams before treatment, $4,057.8 \mathrm{~m}^{3} / \mathrm{yr}$ was defined as Class A waste, $83.7 \mathrm{~m}^{3} / \mathrm{yr}$ was defined as Class B waste, and $41.8 \mathrm{~m}^{3} / \mathrm{yr}$ was defined as Class $C$ waste. Following this same approach with the mixed waste streams, $80.2 \mathrm{~m}^{3} / \mathrm{yr}$ was defined as Class A waste, $1.7 \mathrm{~m}^{3} / \mathrm{yr}$ was defined as Class $B$ waste, and $0.8 \mathrm{~m}^{3} / \mathrm{yr}$ was defined as Class $C$ waste.

The Class $\mathrm{B}$ and $\mathrm{C}$ waste streams are primarily nonincinerable, noncompactible wastes that are assumed to be packaged by the generators into concrete waste boxes. Direct disposal of this waste in concrete waste boxes is considered to be the preferred management method.

The Class $\mathrm{A}$ waste is divided into streams requiring incineration, stabilization, lead treatment, or mechanical volume reduction. The following broad assumptions were used to determine output volumes:

- Incinerated-and-stabilized waste is reduced by a factor of $20: 1$

- Mechanically volume-reduced waste is reduced by a factor of 5:1

- $\quad$ Stabilized waste is conservatively assumed to increase by a factor of 1:5

- Lead recovery results in $10 \%$ waste that is then stabilized with an increase by a factor of $1: 5$

- $\quad$ Treated mixed waste that was classified as characteristic (D-listed waste) can be disposed of as LLW. Treated mixed waste that was classified as listed (F-listed waste) must be disposed of as mixed waste.

- $\quad$ No waste changes from one LLW type to another (e.g., Class A waste becoming Class B waste) result from the volume and mass changes associated with treatment.

See Smith et al. (1992) for further information on waste-treatment strategies.

For the maximum-treatment option, Figure 2-2 shows that the total volume of LLW from ongoing waste streams that will be sent for disposal includes $42.2 \mathrm{~m}^{3} / \mathrm{yr}$ of Class C waste, $84.6 \mathrm{~m}^{3} / \mathrm{yr}$ of Class B waste, and $873.8 \mathrm{~m}^{3} / \mathrm{yr}$ of Class A waste. The total volume of mixed waste to be disposed of from ongoing waste streams is $1.7 \mathrm{~m}^{3} / \mathrm{yr}$ of Class $A$ waste.

Class B waste and Class $C \beta-\gamma$ waste are assumed to be remote handled. As Table $2-2$ shows, no Class $\mathrm{C} \alpha$ waste (whether mixed or not) is expected from ongoing operations. Thus, the total amount of remote-handled waste is estimated to reflect the $1 \%\left(41.8 \mathrm{~m}^{3} / \mathrm{yr}\right)$ Class $\mathrm{C}$ waste and the $2 \%\left(83.7 \mathrm{~m}^{3} / \mathrm{yr}\right)$ Class B waste resulting from the LLW stream and the $1 \%\left(0.4 \mathrm{~m}^{3} / \mathrm{yr}\right)$ Class C 
waste and the $2 \%\left(0.9 \mathrm{~m}^{3} / \mathrm{yr}\right)$ Class B waste resulting from the original mixed waste stream. The total is $126.8 \mathrm{~m}^{3} / \mathrm{yr}$, of which none is mixed waste. This estimate is based on WERF waste stream data in Mixed and Low-Level Waste Treatment Facility Project (EG\&G Idaho 1992) and on the radioactive components of these waste types.

The packaging assumed for Class $B$ and Class $C$ waste is $4 \times 4 \times 8$-ft concrete waste boxes. Class $A$ waste is assumed to be packaged in 71-gal square drums and $4 \times 4 \times 6$ - $\mathrm{ft}$ metal boxes.

Figure 2-3 summarizes the ongoing waste types and volumes for the minimum-treatment option. In this option, only the mixed waste receives treatment. The LLW is sent directly to disposal. In the minimum-treatment option, the total volume from ongoing waste streams that will be disposed of includes $42.2 \mathrm{~m}^{3} / \mathrm{yr}$ of Class C waste, $84.6 \mathrm{~m}^{3} / \mathrm{yr}$ of Class B waste, and $4,099.8 \mathrm{~m}^{3} / \mathrm{yr}$ of Class A waste. The total volume of mixed waste from ongoing waste streams $\left(1.7 \mathrm{~m}^{3} / \mathrm{yr}\right.$ of Class $A$ waste) remains the same as in the maximum-treatment option.

The total amount of remote-handled waste for the minimum-treatment option is the same $\left(126.8 \mathrm{~m}^{3} / \mathrm{yr}\right)$ as for the maximum-treatment option because the Class $\mathrm{C}$ and $\mathrm{B}$ volumes do not change from one option to another.

\subsubsection{Waste from Large D\&D Scenario}

The waste to be generated by the D\&D Program, in the scenario in which the Program expands greatly in the future, is addressed here. Because no detailed, comprehensive projections of the D\&D waste have been made, the volumes and characteristics of the D\&D waste were estimated using information provided by those working in the D\&D effort, and based on engineering judgment (Smith et al. 1992). The $D \& D$ waste projections represent the maximum amount of $D \& D$ waste expected to be generated, treated, and disposed of when the new waste-disposal facility would be in operation.

Figure 2-4 summarizes the Large D\&D scenario waste types and volumes for the maximumtreatment option. Preliminary projections for this D\&D scenario are that $442 \mathrm{~m}^{3} / \mathrm{yr}$ of mixed waste will be produced and $3,341.6 \mathrm{~m}^{3} / \mathrm{yr}$ of LLW will be produced. These volumes are equivalent to $12 \%$ and $88 \%$, respectively, of the total pretreatment D\&D waste volume.

Applying the same classification breakdown to the D\&D LLW before treatment, as discussed above for ongoing operations, $33.3 \mathrm{~m}^{3} / \mathrm{yr}$ is Class $C \beta-\gamma$ waste, $66.7 \mathrm{~m}^{3} / \mathrm{yr}$ is Class $B$ waste, and $3,234 \mathrm{~m}^{3} / \mathrm{yr}$ is Class $\mathrm{A}$ waste. Following this same approach with the mixed waste streams, $4.4 \mathrm{~m}^{3} / \mathrm{yr}$ is Class $C \beta-\gamma$ waste, $8.8 \mathrm{~m}^{3} / \mathrm{yr}$ is Class B waste, and $428.8 \mathrm{~m}^{3} / \mathrm{yr}$ is Class A waste.

Of the $3,341.6 \mathrm{~m}^{3} / \mathrm{yr}$ of LLW generated by the D\&D program, $7.6 \mathrm{~m}^{3} / \mathrm{yr}$ is $\mathrm{C} \alpha$ waste $(<1 \%)$. The Class $C \alpha$ waste is assumed to receive treatment similar to that discussed below for the Class $A$ waste. An overall volume reduction factor of 3:1 was assumed for the Class $\mathrm{C} \alpha$ waste.

Again the Class C and B waste streams are primarily nonincinerable, noncompactible wastes and are directly disposed of in concrete waste boxes. 
LLW

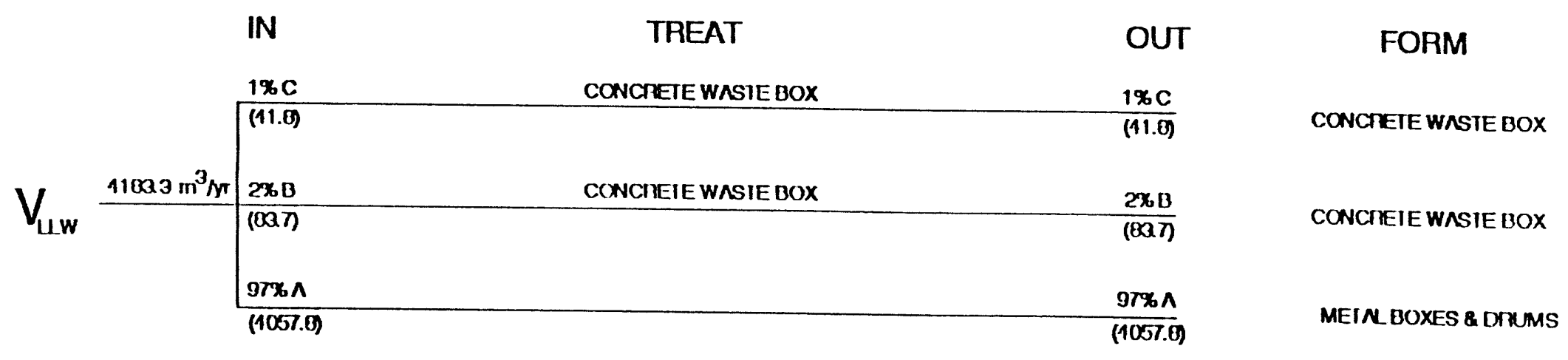

\section{MW}
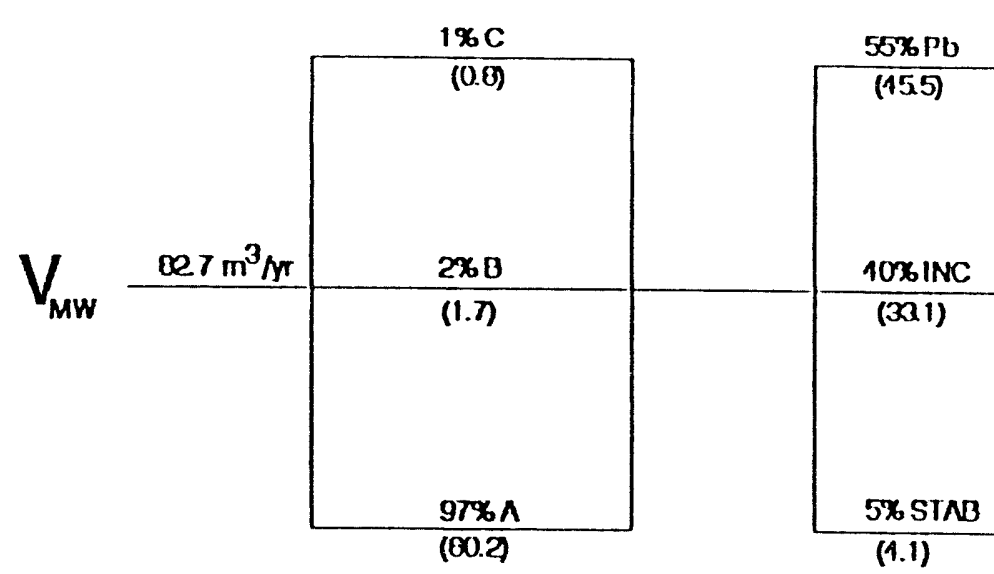

(A.I)

\begin{tabular}{|c|c|c|}
\hline (3ai) & $(1.7) \mathrm{MW}$ & $\frac{28, n}{(1.7 \mathrm{MW}}$ \\
\hline $5 x \operatorname{STNB}$ & $25 \%$ & \\
\hline (1.1) & (20.5)UW & \\
\hline
\end{tabular}
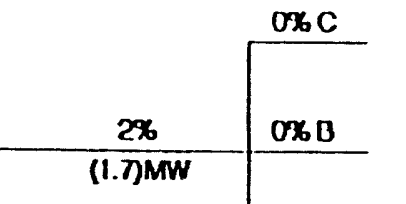

(00.2)

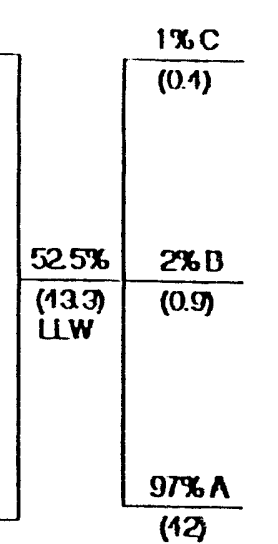

CONCTEIE WASIE BOX

CONCRETE WASIE BOX

NBBREVATIONS

SIIE = MECIINICN VOUME REDUCIION INC = INANETUTION

$\Lambda=$ CIASS $\Lambda$ WASIE

$B=$ aASS B WASIE

\section{$\mathrm{C}=$ CLASS C BETNGMMMA WASIE} $v=$ VOUME $\mathrm{PB}=$ LEND
SINB = SINHUZNIION
(20.5) UW 0 = VOLUME IN QUUIC MEIETSSPEA VEN MW = LOW-LEVE WASIE

Figure 2-3. Ongoing operations waste types and volumes for the minimum-treatment option. 
LLW

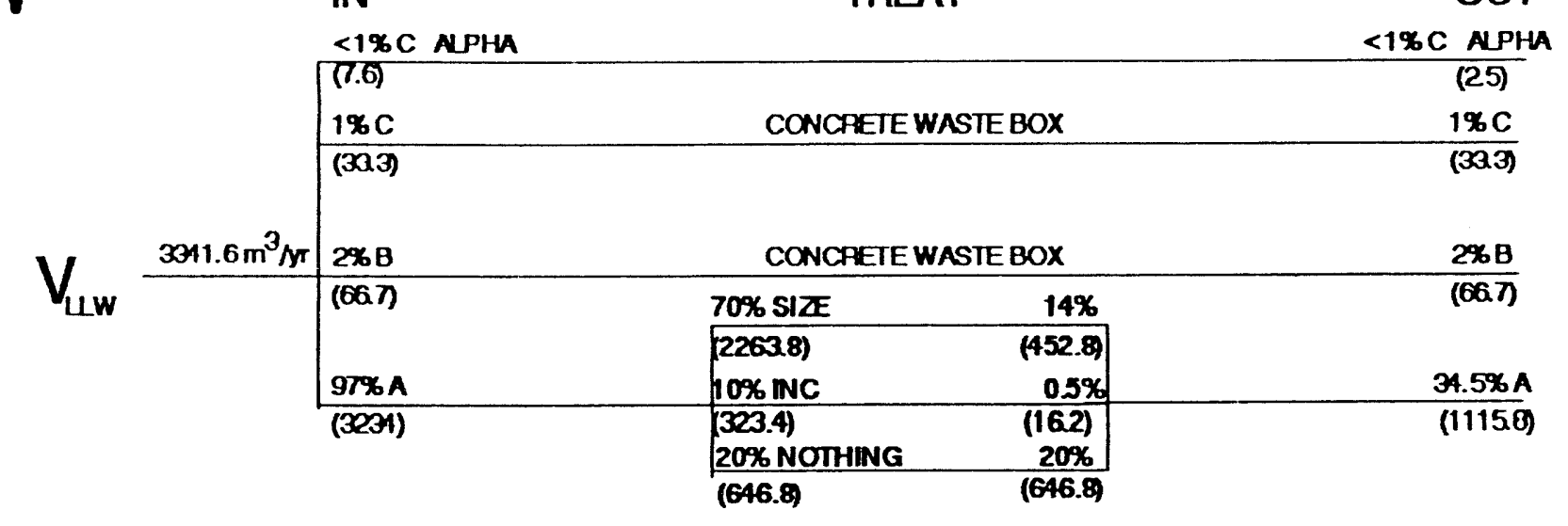

FORM

DPUMS OF IEB

CONCRETE WASTE BOX

CONCAETE WASTE BOX

METALBOXES

CEMENTED DANMS

METN BOXES \& DTUMS

\section{MW}

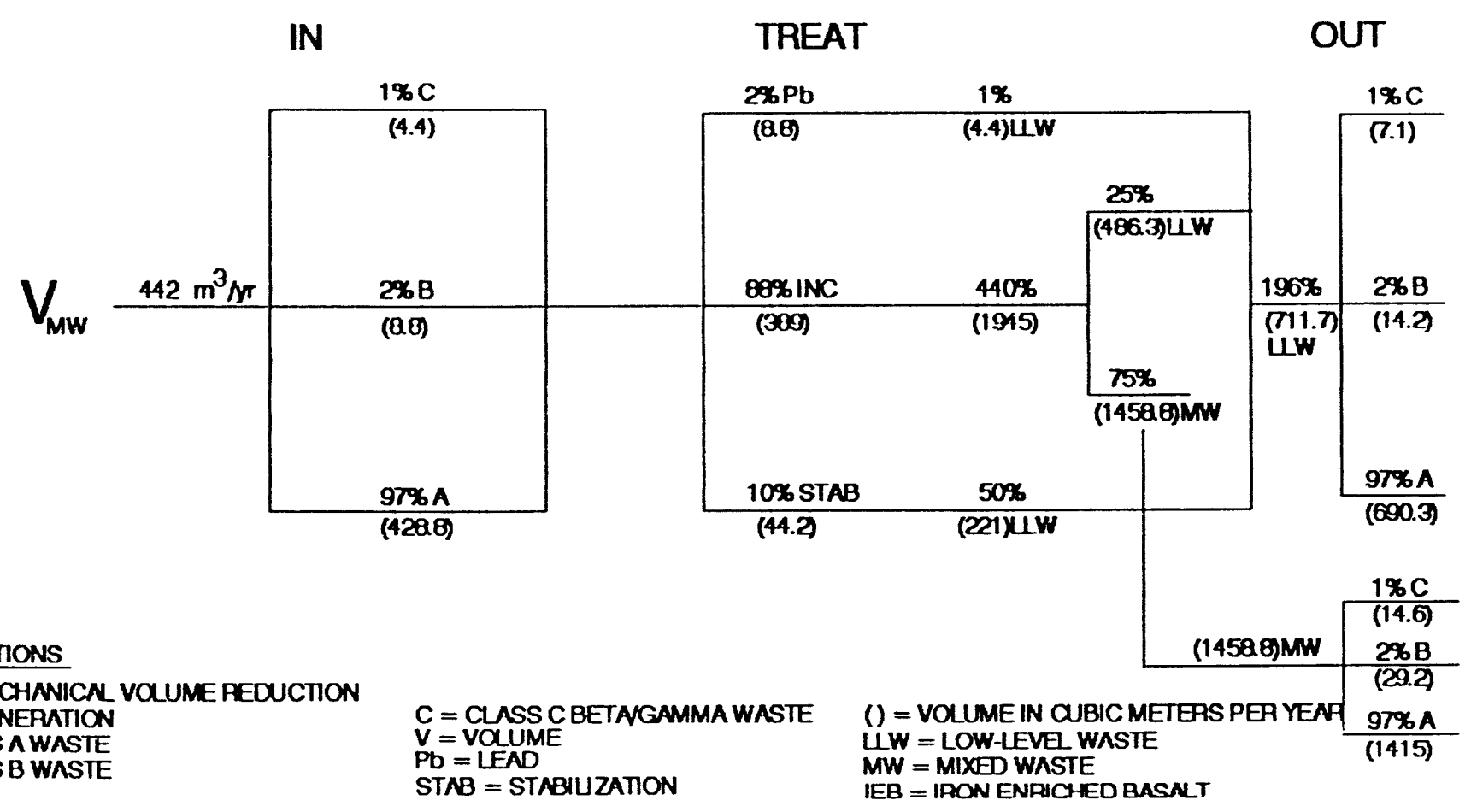

CONCRETE WASTE BOX

CEMENTED DARMS

CONCRETE WASTE BOX CONCRETE WASTE BOX

SIZE = MECHANICN VOLUME REDUCTION INC = INONERUTION

$\hat{A}=$ CLASS A WASTE
$\mathrm{B}=$ QANSB WASTE $\mathrm{V}=$ VOUUM STAB = STABIUZATION

CEMENTED DFUMS

Figure 2-4. D\&D waste types and volumes for the maximum-treatment option. 
The Class A LLW and mixed waste was divided into streams requiring incineration, stabilization, lead treatment, or mechanical volume reduction, as discussed for the waste streams from ongoing operations in Section 2.2.1. However, due to the nature of this waste, the mixed waste requiring incineration is not reduced in volume. Following stabilization, the volume of the waste increases by a factor of $1: 5$. Approximately $25 \%$ of this incinerable mixed waste is characteristic waste and therefore is no longer considered hazardous following treatment. The remaining fraction of the incinerable stream is listed waste and is therefore considered mixed waste.

The total posttreatment volume of LLW from the D\&D Program, maximum-treatment option, includes $2.5 \mathrm{~m}^{3} / \mathrm{yr}$ of Class $\mathrm{C} \alpha$ waste, $40.4 \mathrm{~m}^{3} / \mathrm{yr}$ of Class $\mathrm{C} \beta-\gamma$ waste, $80.9 \mathrm{~m}^{3} / \mathrm{yr}$ of Class B waste, and $1,806.1 \mathrm{~m}^{3} / \mathrm{yr}$ of Class $A$ waste. The total volume of mixed waste from the D\&D Program includes $14.6 \mathrm{~m}^{3} / \mathrm{yr}$ of Class $\mathrm{C}$ waste, $29.2 \mathrm{~m}^{3} / \mathrm{yr}$ of Class B waste, and $1,415 \mathrm{~m}^{3} / \mathrm{yr}$ of Class A waste.

The amount of remote-handled waste $\left(165.1 \mathrm{~m}^{3} / \mathrm{yr}\right)$ is estimated to reflect the $1 \%\left(33.3 \mathrm{~m}^{3} / \mathrm{yr}\right)$ Class $C \quad \beta-\gamma$ waste and the $2 \%\left(66.7 \mathrm{~m}^{3} / \mathrm{yr}\right)$ Class B waste resulting from the LLW stream; the $1 \%\left(7.1 \mathrm{~m}^{3} / \mathrm{yr}\right)$ Class $C \beta-\gamma$ waste and the $2 \%\left(14.2 \mathrm{~m}^{3} / \mathrm{yr}\right)$ Class B waste resulting from the original mixed waste stream; and the $1 \%\left(14.6 \mathrm{~m}^{3} / \mathrm{yr}\right)$ Class $\mathrm{C}$ waste and $2 \%\left(29.2 \mathrm{~m}^{3} / \mathrm{yr}\right)$ Class B waste resulting from the final mixed waste stream. The discussion for this basis is presented in Section 2.2.1.

As with the waste from ongoing operations, the waste containers for Class B and Class $C \beta-\gamma$ waste are assumed to be concrete waste boxes. The Class $C \alpha$ waste is assumed to be packaged in 71-gal square drums. Class $A$ waste is again assumed to be in 71-gal square drums and metal boxes.

Figure 2-5 summarizes the D\&D waste types and volumes for the minimum-treatment option. In the minimum-treatment option, the volume of Class $A$ waste changes from the maximum-treatment option. Under this option, $3,924.3 \mathrm{~m}^{3} / \mathrm{yr}$ of Class A LLW will be disposed of. The total volume of mixed waste from the D\&D minimum-treatment option remains the same as in the maximumtreatment option.

The total amount of remote-handled waste for the minimum-treatment option $\left(165.1 \mathrm{~m}^{3} / \mathrm{yr}\right)$ is the same as for the maximum-treatment option because the Class $C \beta-\gamma$ and Class B volumes do not change from one treatment option to another.

\subsubsection{Waste from Large ER Scenario}

The waste to be generated by the ER Program is addressed here. As discussed in Section 3, based on the input from remedial action managers, nearly all the ER activities are scheduled to be completed and the waste dispositioned before a new waste disposal facility will be operating (2000 to 2005 , depending on the alternative). These waste streams are, therefore, not addressed for disposal in this study. The major exception is the waste expected from remediation of the RWMC. This section gives a reasonable best estimate for the waste to be disposed of, based on the assumption of retrieving much of the buried waste. (Larger amounts of waste are also possible, depending on the remediation approach.) If waste is not retrieved, the amount of RWMC waste requiring redisposal would be very small. 


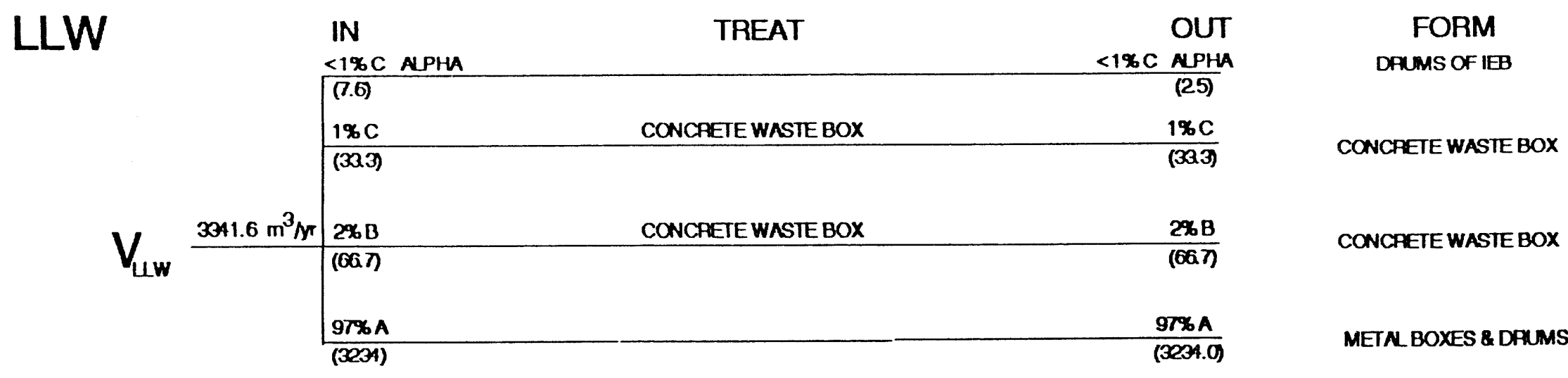

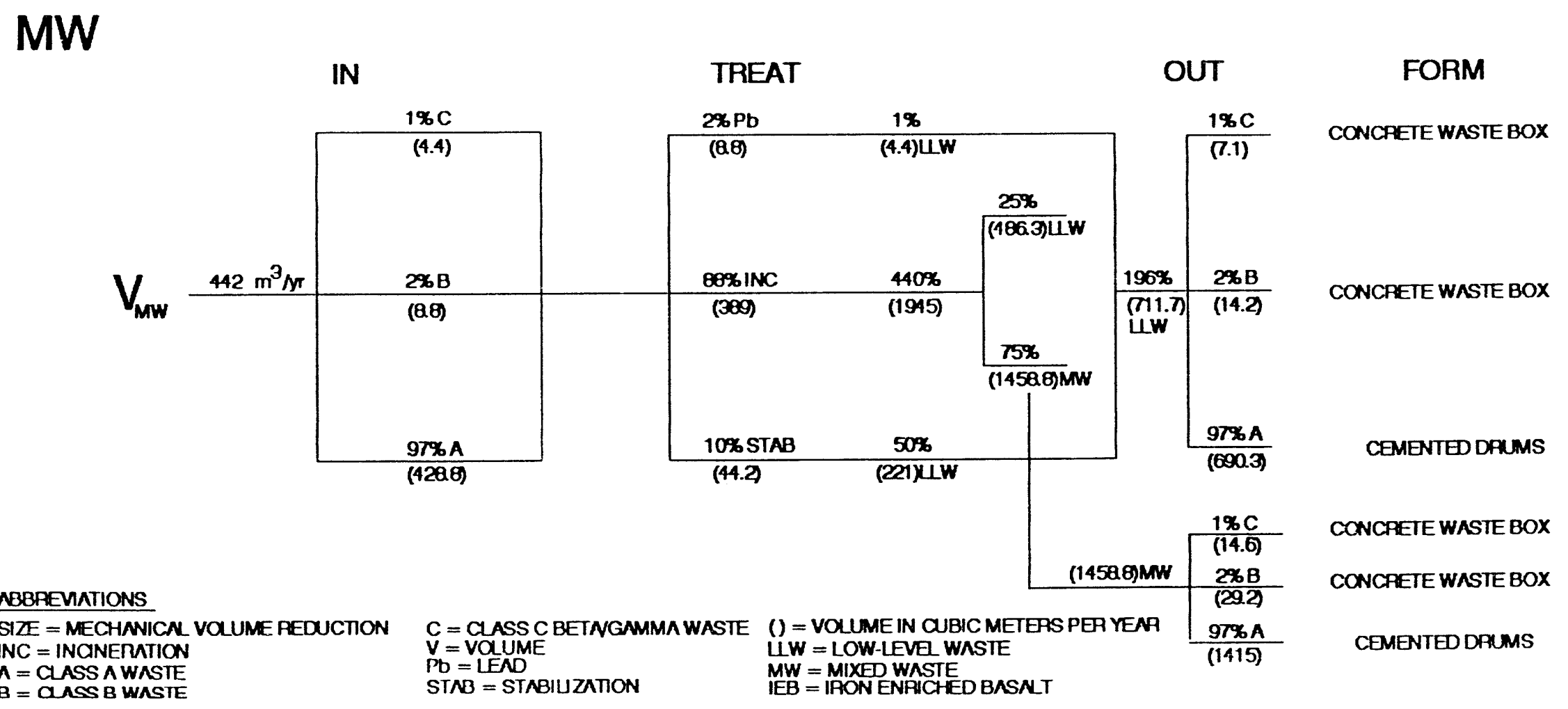

Figure 2-5. D\&D waste types and volumes for the minimum-treatment option, large D\&D senario. 
No comprehensive projections of the waste resulting from remediation of the RWMC have been made. No decision has been made on the remediation alternatives; any estimates of waste requiring redisposal would be uncertain and expected to change in the future. The volumes and characteristics of the waste were therefore estimated by those working in the ER effort, based on engineering judgement.

Table 2-3 summarizes the preliminary information on the expected waste volumes and characteristics from the RWMC by Operable Unit (OU).

The total estimated (posttreatment) volume of LLW resulting from ER activities that will require disposal is $279 \mathrm{~m}^{3}\left(365 \mathrm{yd}^{3}\right)$ of Class $C \alpha$ waste, $0 \mathrm{~m}^{3}$ of Class B waste, and $27 \mathrm{~m}^{3}\left(35 \mathrm{yd}^{3}\right)$ of Class A waste. No mixed waste is projected to be generated during the remediation of the RWMC.

The total volume of LLW (306 $\left.\mathrm{m}^{3}\right)$ generated by ER activities is expected to be contact handled. A small percentage of the TRU waste is expected to be remote handled, but the TRU waste is outside the scope of this study.

The projected waste containers from ER activities include 71 -gal square drums $(60 \%$, or $\left.183.6 \mathrm{~m}^{3}\right)$ and metal boxes $\left(40 \%\right.$, or $\left.122.4 \mathrm{~m}^{3}\right)$.

It is assumed that the ER waste resulting from remediation of RWMC will be treated at the remediation site by means other than a conventional (LLW and low-level mixed waste) treatment facility. For this reason, the volumes are considered to be the same for the maximum- and minimumtreatment options.

\subsubsection{Stored Waste}

The final source of waste to discuss (refer to Figure 2-1) is LLW and mixed waste currcintly stored at the INEL. Some of the mixed waste currently in storage at the INEL MWSF and all of the $\alpha$-contaminated LLW currently stored at the RWMC TSA are expected not to have been dispositioned by the time the selected disposal facility starts operations.

As discussed in Section 3, it is assumed that the stored LLW and most of the (non- $\alpha$ ) stored mixed waste currently backlogged due to the shutdown of WERF will have been eliminated when a new waste disposal facility begins operations. An exception to this is the mixed waste classified as listed (F-listed waste) (see Figure 2-6). This waste must be disposed of as mixed waste. Because no disposal facility is currently permitted for this waste, it is assumed that $1.7 \mathrm{~m}^{3} / \mathrm{yr}$ of mixed waste will remain in storage until a new waste disposal facility begins operations. This volume accumulated for an assumed 10 years (1992-2002) will amount to a total of $17 \mathrm{~m}^{3}$ of mixed waste to be received at the new disposal facility. This estimate is based on the mixed waste projections from ongoing waste operations, as discussed in Section 2.2.1. This entire volume of waste is assumed to be Class A mixed waste.

In support of the design of an INEL treatment facility for treating the contact-handled $\alpha$ contaminated LLW stored at the TSA, information on the characteristics of the waste was analyzed in another task (Roesener et al. 1992). Figure 2-6 summarizes the TSA stored waste types and 
Table 2-3. Estimated waste volumes and characteristics from Large ER scenario.

\begin{tabular}{|c|c|c|c|c|c|c|c|c|c|c|}
\hline $\begin{array}{l}\text { Operable } \\
\text { unit (OU) }\end{array}$ & $\begin{array}{l}\text { Total waste and } \\
\text { contaminated } \\
\text { material in OU }\end{array}$ & $\begin{array}{l}\text { Estimated volume } \\
\text { remaining or } \\
\text { returned to OU }\end{array}$ & $\begin{array}{l}\text { Estimated volume to } \\
\text { be disposed of }\end{array}$ & $\begin{array}{l}\text { Estimated volume } \\
\text { to be disposed by } \\
\text { class/type (these } \\
\text { will change when } \\
\text { information on } \\
\text { types comes in) }\end{array}$ & $\begin{array}{l}\text { Rad waste } \\
(\%)^{b}\end{array}$ & $\begin{array}{l}\text { Mixed } \\
\text { waste } \\
(\%)^{\mathrm{b}}\end{array}$ & $\begin{array}{l}\text { Volume TRU } \\
\text { waste }(>100 \\
\mathrm{nCi} / \mathrm{g})^{\mathrm{a}}\end{array}$ & $\begin{array}{l}\text { Volume } \propto \\
\text { LLW (10- } \\
100 \mathrm{nCi} / \mathrm{g})^{\mathrm{a}}\end{array}$ & 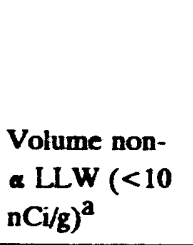 & $\begin{array}{l}\text { Waste } \\
\text { form }\end{array}$ \\
\hline $\begin{array}{l}7-3 \\
\text { Non-TRU } \\
\text { pits and } \\
\text { trenches }\end{array}$ & $\begin{array}{l}\text { Estimated } \\
99,000 \mathrm{yd}^{3} \text { of } \\
\text { waste and } \\
\text { contaminated } \\
\text { soil }\end{array}$ & $\begin{array}{l}89,100 \mathrm{yd}^{3} \\
\text { remaining in OU } \\
8,910 \mathrm{yd}^{3} \\
\text { returned to OU } \\
\text { as Class A waste }\end{array}$ & $\begin{array}{l}\text { Estimated } 9,900 \mathrm{yd}_{3} \\
\text { to be treated and } \\
\text { disposed of }(10 \% \text { of } \\
\text { total waste and } \\
\text { contaminated soil in } \\
\text { OU) }\end{array}$ & $\begin{array}{l}\text { A: } 8,910 \mathrm{yd}^{3} \\
\text { (returned to OU) } \\
\text { B: } 0 \mathrm{yd}^{3} \\
\text { C: } 50 \mathrm{yd}^{3} \\
\text { >C: } 940 \mathrm{yd}^{3}\end{array}$ & 100 & 0 & $940 \mathrm{yd}^{3}$ & $50 \mathrm{yd}^{3}$ & $5 \mathrm{yd}^{3}$ & $\begin{array}{l}60 \% \text { drums } \\
40 \% \text { metal } \\
\text { boxes }\end{array}$ \\
\hline $\begin{array}{l}7-13 \\
\text { TRU pits } \\
\text { and } \\
\text { trenches }\end{array}$ & $\begin{array}{l}\text { Estimated } \\
440,000 \mathrm{yd}^{3} \text { of } \\
\text { waste and } \\
\text { contaminated } \\
\text { soil }\end{array}$ & $\begin{array}{l}396,000 \mathrm{yd}^{3} \\
\text { remaining in OU } \\
35,200 \mathrm{yd}^{3} \\
\text { returned to OU } \\
\text { as Class } \mathrm{A}\end{array}$ & $\begin{array}{l}\text { Estimated } 44,000 \mathrm{yd}^{3} \\
\text { to be disposed of } \\
\text { (10\% of total waste } \\
\text { and contaminated soil } \\
\text { in OU) }\end{array}$ & $\begin{array}{l}\text { A: } 35,200 \mathrm{yd}^{3} \\
\text { (returned to OU) } \\
\text { B: } 0 \mathrm{yd}^{3} \\
\text { C: } 220 \mathrm{yd}^{3} \\
\text { >C: } 4,180 \mathrm{yd}^{3}\end{array}$ & 100 & 0 & $4,180 y^{3}$ & $220 \mathrm{yd}^{3}$ & $20 \mathrm{yd}^{3}$ & $\begin{array}{l}60 \% \text { drums } \\
40 \% \text { metal } \\
\text { boxes }\end{array}$ \\
\hline $\begin{array}{l}7-10 \\
\text { Pit } 9\end{array}$ & $\begin{array}{l}\text { Estimated } \\
18,500 \mathrm{yd}^{3} \text { of } \\
\text { waste and } \\
\text { contaminated } \\
\text { soil }\end{array}$ & $\begin{array}{l}16,650 \mathrm{yd}^{3} \\
\text { returned to OU } \\
\text { as Class } \mathrm{A}\end{array}$ & $\begin{array}{l}\text { Estimated } 18,500 \mathrm{yd}^{3} \\
\text { to be treated and } \\
\text { disposed of }(100 \% \text { of } \\
\text { total waste and } \\
\text { contaminated soil in } \\
\text { OU) }\end{array}$ & $\begin{array}{l}\text { A: } 16,650 \mathrm{yd}^{3} \\
\text { (returned to OU) } \\
\text { B: } 0 \mathrm{yd}^{3} \\
\text { C: } 95 \mathrm{yd}^{3} \\
>C: 1,755 \mathrm{yd}^{3}\end{array}$ & 100 & 0 & $1,755 y d^{3}$ & $95 \mathrm{yd}^{3}$ & $10 \mathrm{yd}^{3}$ & $\begin{array}{l}60 \% \text { drums } \\
40 \% \text { metal } \\
\text { boxes }\end{array}$ \\
\hline
\end{tabular}




\section{LLW and MW}

IN

TREAT

OUT

FORM

alphauw

$122 \mathrm{~m}^{3} \mathrm{Mr}$ UW Class A

EMPTY METAL DEUMS<smiles></smiles>

ISA

\section{MW}

IN

TREAT

OUT

FORM

$\mathbf{Y W W}_{\text {MWSF }}$

$100 \% \mathrm{~A}$

\section{ABBREMATIONS}

$\mathrm{C}=$ CLASS C BETNGMMMA WASTE $\mathbf{v}=$ VOLUME

$\Lambda=$ CASS A WASTE

0 = VOLUME IN QUBIC METERS PER YEAR

UW = LOW-LFVE WASTE

$M W=$ MIXFD WASTE

TSA = TRWSURWIC STORAGE AREA

MWSF = MIXED WASTE STORMGE FACLTIY

IEB = IRON ENHCI EDDBSN

Figure 2-6. Types and volumes of waste in storage for the maximum- and minimum-treatment options. 
volumes for the maximum and minimum-treatment options. Roesener et al. (1992) assumed that the waste would be addressed over a 10 -year campaign.

It is assumed that essentially $100 \%\left(1,725 \mathrm{~m}^{3} / \mathrm{yr}\right)$ of the $\alpha$-LLW retrieved from the TSA requires treatment due to F-listed RCRA constituents. Various containers (55-gal drums) undergo a rinsing/decontamination process and are therefore no longer considered hazardous. The estimated volume of this LLW is $122 \mathrm{~m}^{3} / \mathrm{yr}$, all of which is considered Class $A$.

The $\alpha$ mixed waste stream undergoes treatment processes in accordance with the best demonstrated available technology (BDAT) required by RCRA. The estimated volume of treated waste to be received by the new disposal facility is $713 \mathrm{~m}^{3} / \mathrm{yr}$. All of this treated waste is considered Class $\mathrm{C} \alpha$ mixed waste.

All the waste to be disposed of that is derived from waste stored in the INEL MWSF and the TSA is considered contact-handled waste.

\subsubsection{Total Waste}

The total waste volumes to be received at the disposal facility are given in Table 2-4.

The campaign length for each source of waste has been estimated and is shown in the timeline in Figure 2-7. The assumed campaign length for ongoing operations is 40 years (the assumed length of disposal facility operations). The campaign length for D\&D operations was estimated to be 30 years. The campaign length for ER activities and for disposal of the waste stored at the TSA is assumed to be 10 years. The campaign length for disposal of the waste stored at the MWSF is assumed to be 1 year.

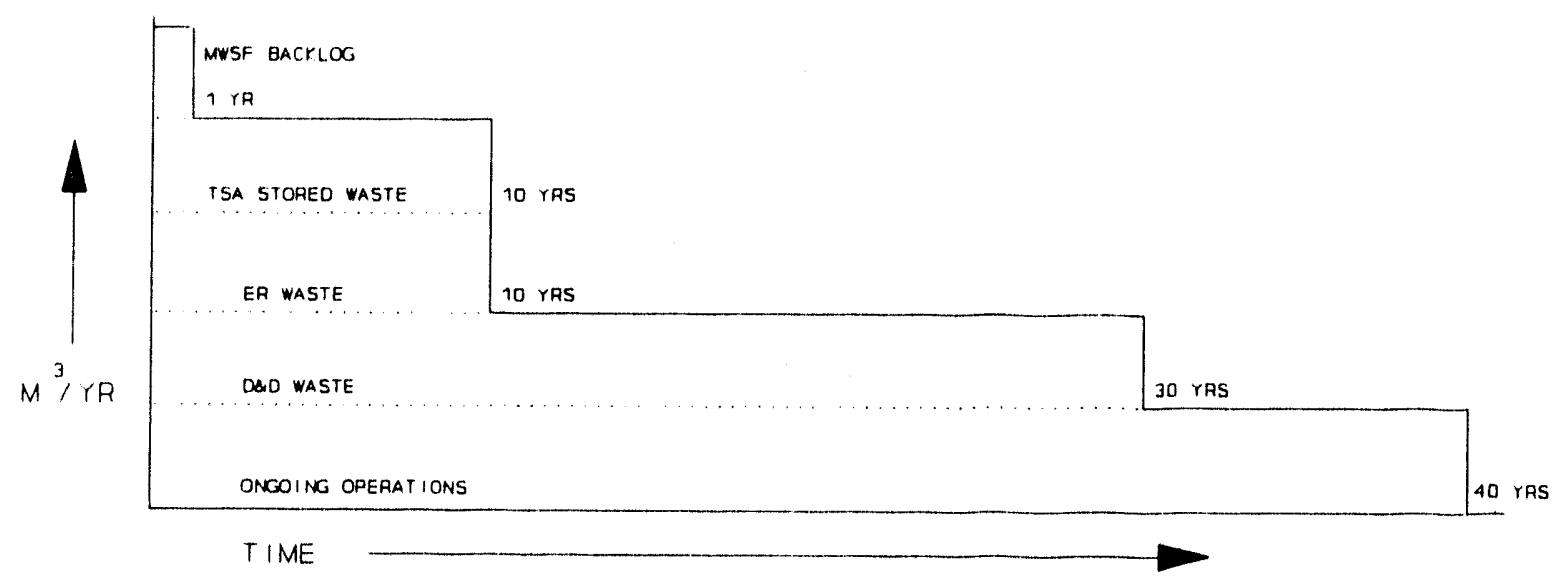

Figure 2-7. Estimated campaign length for each source of waste. 
Table 2-4. Summary of waste types and volumes to be received at the disposal facility, $\mathrm{m}^{3} / \mathrm{yr}$.

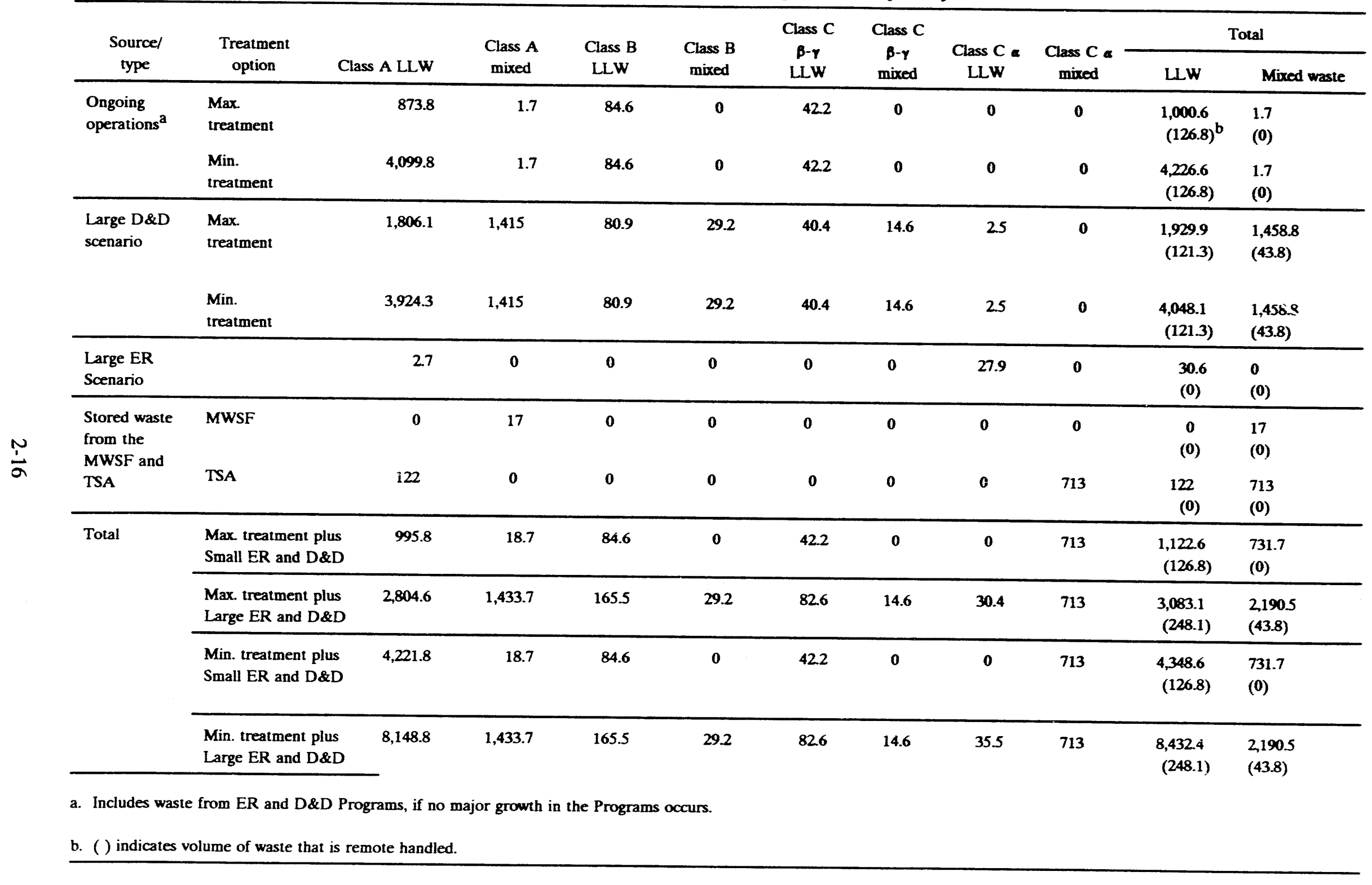


Due to the complexities involved in estimating the cost of disposal facility operations over these various campaign lengths, the waste volumes to be used in the cost estimates have been levelized over the expected life of the disposal facility ( 40 years). Table $2-5$ summarizes the levelized waste volumes to be disposed of by each source/type for the life of the disposal facility. The volumes of waste computed and shown in Table 2-4 were multiplied by the estimated campaign length for each waste source and subsequently divided by the estimated life of disposal facility operations. Because the campaign lengths are already quite uncertain, the additional uncertainty due to this approximation is considered acceptable.

The levelized waste volumes shown in Figure 2-5 were then used to estimate the number of containers disposed of for each of the four disposal scenarios (maximuin and minimum treatment combined with large ER/D\&D and small ER/D\&D) under consideration. A summary of waste containers disposed of per year is shown in Table 2-6.

Although many forms of waste packaging exist, in order to simplify this study, only three forms of packaging are considered. The assumed waste packaging includes $4 \times 4 \times 6$-ft metal boxes, 71 -gal square metal drums, and $4 \times 4 \times 8$-ft concrete boxes. INEL waste generators are currently using a variety of packaging for the remote-handled waste, depending upon the shielding required. However, in an attempt to reduce the number of variables in this study, the concrete waste box was selected for these waste types. The design of the concrete box can be varied to accommodate different levels of radiation and is considered adequate for the waste under consideration. The internal dimensions of the concrete waste box may be smaller than those used in this study to determine the number of waste containers disposed of per year. In consideration of the void spaces in today's packaging, it is felt that, by more efficient packaging, the number of waste containers disposed of per year is representative of that which will be disposed of.

As will be seen in Section 5, this set of waste packaging is not optimized in dimensions for disposal efficiency. This particular set of packagino dimensions represents recent thinking, but a systems analysis will be needed to optimally match packaging dimensions with disposal concept dimensions.

\subsection{Regulatory Requirements Assumed to Govern Waste Disposal}

\subsubsection{Introduction}

This section discusses the regulatory requirements assumed to apply to the disposal of LLW and mixed waste at the time the facilities discussed in this report are designed, constructed, and operated. Because that time is several years in the future, the requirements cannot be known with certainty at present. The approach used in this study was to plan for compliance not only with current requirements, but also with applicable draft or proposed requirements likely to be issued.

The disposal of DOE LLW is currently controlled by DOE Order 5820.2A, "Radioactive Waste Management," particularly Chapter III, "Management of Low-Level Waste." There are also) applicable requirements in DOE Order 6430.1A, "General Design Critcria," in Section 1324, 
Table 2-5. Levelized waste volumes disposed of, by source, for life of the disposal facility $\left(\mathrm{m}^{3} / \mathrm{yr}\right)$.

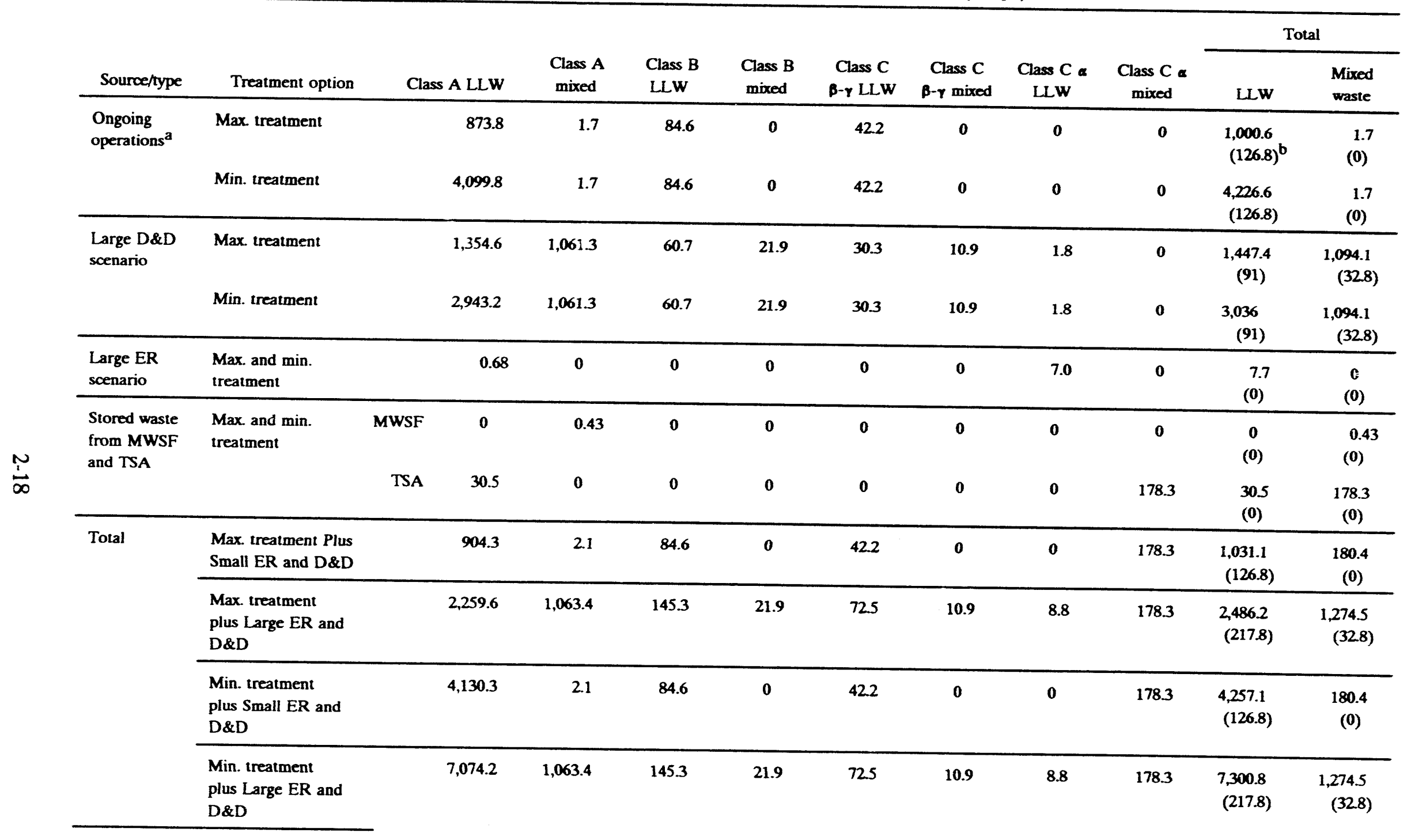

a. Includes waste from ER and D\&D Programs, if no major growth in the Programs occurs.

b. () indicates volume of waste that is remote handled. 
Table 2-6. Summary of waste containers disposed of per year (levelized).

\begin{tabular}{|c|c|c|c|c|c|c|c|c|c|c|c|}
\hline \multirow[b]{2}{*}{ Source/type } & \multirow[b]{2}{*}{ Container type } & \multirow[b]{2}{*}{$\begin{array}{c}\text { Class A } \\
\text { LLW } \\
\end{array}$} & \multirow[b]{2}{*}{$\begin{array}{l}\text { Class A } \\
\text { mixed }\end{array}$} & \multirow[b]{2}{*}{$\begin{array}{c}\text { Class B } \\
\text { LLW }\end{array}$} & \multirow[b]{2}{*}{$\begin{array}{c}\text { Class B } \\
\text { mixed }\end{array}$} & \multirow[b]{2}{*}{$\begin{array}{l}\text { Class C } \\
\beta-\tau \text { LLW }\end{array}$} & \multirow{2}{*}{$\begin{array}{l}\text { Class C } \\
\beta-\gamma \\
\text { mixed }\end{array}$} & \multirow[b]{2}{*}{$\begin{array}{l}\text { Class C } \\
\alpha \mathbf{L L W}\end{array}$} & \multirow[b]{2}{*}{$\begin{array}{l}\text { Class C } \alpha \\
\text { mixed }\end{array}$} & \multicolumn{2}{|c|}{ Total } \\
\hline & & & & & & & & & & LLW & $\begin{array}{l}\text { Mixed } \\
\text { waste }\end{array}$ \\
\hline \multirow{3}{*}{$\begin{array}{l}\text { Max. treatment plus } \\
\text { Small ER and D\&D }\end{array}$} & Metal boxes ${ }^{a}$ & 163 & $\mathbf{0}$ & 0 & 0 & $\mathbf{0}$ & $\mathbf{0}$ & 0 & 0 & 163 & 0 \\
\hline & Drums ${ }^{\mathbf{b}}$ & 1,714 & 8 & 0 & 0 & $\mathbf{0}$ & $\mathbf{0}$ & 0 & 663 & 1,714 & 671 \\
\hline & $\begin{array}{l}\text { Concrete waste } \\
\text { box }^{c}\end{array}$ & $\mathbf{0}$ & 0 & 24 & 0 & 12 & 0 & 0 & $\mathbf{0}$ & 36 & 0 \\
\hline \multirow{3}{*}{$\begin{array}{l}\text { Max. treatment plus } \\
\text { Large ER and D\&D }\end{array}$} & Metal boxes & 357 & 0 & 0 & $\mathbf{0}$ & 0 & $\mathbf{0}$ & 2 & 0 & 359 & 0 \\
\hline & Drums & 4,787 & 3,953 & $\mathbf{0}$ & $\mathbf{0}$ & 0 & 0 & 21 & 663 & 4,808 & 4,616 \\
\hline & $\begin{array}{l}\text { Concrete waste } \\
\text { box }\end{array}$ & 0 & 0 & 40 & 6 & 20 & 3 & 0 & 0 & 60 & 9 \\
\hline \multirow{3}{*}{$\begin{array}{l}\text { Min. treatment plus } \\
\text { Small ER and D\&D }\end{array}$} & Metal boxes & 578 & 0 & 0 & 0 & 0 & 0 & 0 & 0 & 578 & 0 \\
\hline & Drums & 9,519 & 8 & 0 & 0 & 0 & 0 & 0 & 663 & 9,519 & 671 \\
\hline & $\begin{array}{l}\text { Concrete } \\
\text { waste box }\end{array}$ & 0 & 0 & 24 & 0 & 12 & 0 & 0 & $\mathbf{0}$ & 36 & 0 \\
\hline \multirow{3}{*}{$\begin{array}{l}\text { Min. treatment plus } \\
\text { Large ER and D\&D }\end{array}$} & Metal boxes & 937 & 0 & 0 & 0 & 0 & 0 & 2 & 0 & 939 & 0 \\
\hline & Drums & 16,828 & 3,953 & $\mathbf{0}$ & 0 & 0 & 0 & 21 & 663 & 16,849 & 4,616 \\
\hline & $\begin{array}{l}\text { Concrete } \\
\text { waste box }\end{array}$ & 0 & 0 & 40 & 6 & 20 & 3 & $\mathbf{0}$ & $\mathbf{0}$ & 60 & 9 \\
\hline \multirow{2}{*}{\multicolumn{12}{|c|}{$\begin{array}{l}\text { Metal boxes are } 4 \times 4 \times 6 \mathrm{ft}=96 \mathrm{ft}^{3}=2.719 \mathrm{~m}^{3} \\
\text { Drums are 71-gal square drums }=9.5 \mathrm{ft}^{3}=0.269\end{array}$}} \\
\hline & & & & & & & & & & & \\
\hline \multicolumn{12}{|c|}{ c. Concrete waste boxes are $4 \times 4 \times 8 \mathrm{ft}=128 \mathrm{ft}^{3}=3.625 \mathrm{~m}^{3}$} \\
\hline
\end{tabular}


Radioactive Solid Waste Facilities. However, most of these requirements stem from DOE Order 5820.2A.

DOE-HQ is currently preparing to make major revisions to DOE Order 5820.2A (Duggan 1992). The planned completion date for the revision is late 1994. That date is before the design phase of the MLLWUF Project is expected to be completed. It seems prudent, therefore, to include the likely revisions to DOE Order 5820.2A in the design requirements used in this study, even though the revised Order has not yet been issued.

The expected revisions to the Order include the following items that will affect the disposal of mixed waste and LLW:

- The revised requirements and guidelines will agree with NRC and EPA requirements, as appropriate.

- $\quad$ For LLW, the NRC requirements are found in 10 CFR 61, "Licensing Requirements for Land Disposal of Radioactive Waste." [DOE is exempt from NRC regulations per se, except as specified in Section 202 of the Energy Reorganization Act of 1974 (i.e., DOE facilities that accept commercial high-level waste are licensed by the NRC)]. The NRC has proposed minor revisions to 10 CFR 61. Among other things, the revisions would clarify that the regulations, which were originally stipulated as applying to near-surface disposal, apply also to disposal in engineered facilities, which may be built totally or partially abovegrade, provided there is a protective earthen cover.

- The U.S. Environmental Protection Agency (EPA) has not yet issued its environmental standards governing LLW disposal. Drafts of the EPA regulations (40 CFR 193, "Environmental Radiation Protection Standards for Management and Land Disposal of Low Level Radioactive Wastes") have existed for several years. The EPA regulations are targeted for publishing in the Federal Register in the next few months. The EPA regulations will apply to DOE activities, regardless of whether the regulations are reflected in revised DOE Order 5820.2A.

- The revised Order will cover both radioactive waste and mixed waste.

- The revised Order will address the management of waste from contaminated sites and facilities.

- The possible expansion of the performance assessment-based DOE system for LLW-to include low-level mixed waste-will be addressed.

Based on these plans for revising DOE Order 5820.2A, the disposal-related regulatory requirements used in this document are based on the following sources: 
- For LLW

- The current DOE Order 5820.2A, particularly Chapter III

- $\quad$ DOE Order 6430.1A, Section 1324

- The technical requirements of the NRC regulations at 10 CFR 61, plus (for guidance only) the technical interpretive guidance in Standard Format and Content of a License Application for a Low-Level Radioactive Waste Disposal Facility (NRC 1991) and Standard Review Plan for the Review of a License Application for a Low-Level Radioactive Waste Disposal Facility (NRC 1987)

- The applicable environmental standards of draft 40 CFR 193, insofar as these performance standards are compatible with those in the above sources.

- $\quad$ For mixed waste

- The above requirements sources

- The EPA RCRA requirements for hazardous waste disposal, at 40 CFR 264 (in particular, 264.300-.317), supplemented by EPA guidance documents.

The remainder of Section 2.3 summarizes the key requirements from the above sources as they are expected to affect the disposal facilities studied herein. The RCRA permitting requirements are discussed in Section 9.2.4.1, rather than here.

\subsubsection{Requirements in DOE Order 5820.2A}

Chapter III of the current Order contains the requirements that apply to the disposal of LLW and (per Section 2.d of Chapter III) to low-level mixed waste.

2.3.2.1 Performance Objectives and Performance Assessments. Chapter III states the following performance objectives:

- Ensure that external exposure to the waste and concentrations of radioactive material that may be released into surface water, groundwater, soil, plants, and animals results in an effective dose equivalent (EDE) that does not exceed $25 \mathrm{mrem} / \mathrm{yr}$ to any member of the public

- Releases to the atmosphere shall meet the requirements of 40 CFR 61

- Reasonable efforts should be made to maintain releases of radioactivity in effluents to the general environment as low as reasonably achievable (ALARA) 
- Ensure that the committed EDEs received by individuals who inadvertently may intrude into the facility after the loss of active institutional control (100 years) will not exceed 100 $\mathrm{mrem} / \mathrm{yr}$ for continuous exposure or $500 \mathrm{mrem}$ for a single acute exposure

- Protect groundwater resources, consistent with Federal, State, and local requirements.

LLW disposal operations must be supported by a radiological performance assessment that demonstrates compliance with the above performance objectives.

2.3.2.2 Disposal Requirements. DOE Order 5820.2A requires that LLW be disposed of by methods appropriate to achieve the performance objectives, using engineering modifications as necessary.

The use of site-specific waste classification limits is optional.

Disposition of waste designated as greater-than-Class C, as defined in 10 CFR 61.55 (see Section 2.3.3.2 below), must be handled as special cases. Such waste is outside the scope of the MLLWDF Project.

All waste must meet the following general requirements:

- Waste must not be packaged for disposal in cardboard or fiberboard boxes. For all types of containers, void spaces within the waste and between the waste and its packaging shall be reduced as much as practical.

- Liquid wastes, or wastes containing free liquid, must be converted into a form that contains as little freestanding and noncorrosive liquid as is reasonably achievable, but, in no case, shall the liquid exceed $1 \%$ of the volume of the waste when the waste is in a disposal container, or $0.5 \%$ of the volume of the waste processed to a stable form.

- Waste must not be readily capable of detonation, or of explosive decomposition, or reaction at normal pressures and temperatures, or of explosive reaction with water.

- Waste must not contain, or be capable of generating, quantities of toxic gases, vapors, or fumes harmful to persons transporting, handling, or disposing the waste. This does not apply to radioactive gaseous waste packaged at a pressure that does not exceed $1.5 \mathrm{~atm}$ at $20^{\circ} \mathrm{C}$.

- Waste in a gaseous form must be packaged at a pressure that does not exceed $1.5 \mathrm{~atm}$ at $20^{\circ} \mathrm{C}$.

- Waste must not be pyrophoric. Pyrophoric materials contained in waste shall be treated, prepared, and packaged to be nonflammable. 
Disposal facility design must be based on design criteria that reflect the features of the proposed site, so that the performance objectives can be met. These criteria also must reflect projected waste volumes and characteristics.

Other requirements of interest in Chapter III relate to generator characterization of waste packages to be disposed of, WAC, disposal site selection, disposal operations, site closure and postclosure, environmental monitoring, and records. Chapter III also discusses waste storage to allow for nuclides to decay or until disposal by approved methods is available. Perpetual storage of waste, with no intent to dispose of it, is not listed as a possible reason for storage.

Chapter III requires treatment of LLW only if (a) treatment is needed so that the disposal site can meet the performance objectives (see Section 2.3.2.1 above), or (b) if waste treatment to increase the life and performance of the disposal facility is cost effective.

The present INEL LLW acceptance criteria are set forth in the INEL Low-Level Radioactive Waste Acceptance Criteria (EG\&G Idaho 1991). This reference states the requirements that waste generators must satisfy in order to process LLW at WERF and to dispose of LLW at the RWMC. The basic requirements are similar to those already discussed.

\subsubsection{Requirements in 10 CFR 61}

2.3.3.1 Performance Objectives. The NRC regulations in 10 CFR 61 state that a license application for a LLW disposal site must include analyses and other information to demonstrate that the performance objectives of Part $\mathrm{C}$ of the regulations will be met. In assessing performance, site characteristics should be considered in terms of the indefinite future and evaluated for at least a 500year timeframe. The performance objectives include the following:

- Concentrations of radioactive material that may be released to the general environment in groundwater, surface water, air, soil, plants, or animals must not result in an annual dose exceeding an equivalent of 25 mrem to the whole body, 75 mrem to the thyroid, and 25 mrem to any other organ of any member of the public.

- Reasonable effort should be made to maintain any releases of radioactivity in effluents to the general environment ALARA.

- The facility must ensure protection of any individual inadvertently intruding into the disposal site and occupying the site or contacting the waste at any time after active institutional controls over the disposal site are removed.

Institutional controls may not be relied upon for more than 100 years.

The similarity of the above performance objectives to those in DOE Order $5820.2 \mathrm{~A}$ is evident.

2.3.3.2 Technical Requirements. The NRC performed generic performance assessments of LLW disposal and derived the concentration limits for three classes of LLW, based on applying different disposal requirements to each class. The classes are referred to as A, B, and C. Class A 
waste has the lowest concentrations of radionuclides; Class $C$, the highest. Subpart D of the regulations includes tables specifying the maximum concentrations of several radionuclides for Class $\mathrm{A}, \mathrm{B}$, and $\mathrm{C}$ waste. [If the concentration exceeds the maximum values for Class $\mathrm{C}$, the (greaterthan Class C) waste is not generally acceptable for near-surface disposal.] The classification scheme provides the basis for the technical requirements in Subpart D of the regulations, as discussed immediately below.

Minimum technical requirements were established for all three classes of LLW. The requirements are nearly identical to the general requirements in DOE Order 5820.2A, as listed above in Section 2.3.2.2.

The technical requirements place additional restrictions on the disposal methods for each waste class. The requirements differ significantly from those in DOE Order 5820.2A.

Class $\mathrm{A}$ waste must meet the following additional requirements:

- Be segregated from higher classes of waste in separate disposal units unless the waste meets the stability requirements for Class $B$ and $C$ wastes.

Class $\mathrm{B}$ waste must meet the following requirement in addition to those for Class $\mathrm{A}$ waste:

- The waste must have structural stability for at least 300 years. It must be able to maintain its physical dimensions and form under expected disposal conditions. Stability can be provided by the original waste form, by processing to a stable form, or by placing waste in a disposal container or structure that provides stability after disposal.

Class $\mathrm{C}$ waste must meet the following requirement in addition to those for Class $\mathrm{B}$ waste:

- Be disposed of so that the top of the waste is a minimum of $5 \mathrm{~m}$ below the top surface of the cover or within an intruder barrier designed to protect against an inadvertent intrusion for at least $500 \mathrm{yr}$.

The NRC regulations include some general requirements for disposal design and operations. The requirements include topics such as buffer zones, cover design, surface-water drainage features, the need to fill voids between waste packages, etc. The regulations also include site suitability requirements, closure requirements, environmental monitoring requirements, etc. Closure and stabilization must be carried out as each disposal unit (e.g., trench) is filled and covered.

\subsubsection{Requirements in Draft 40 CFR 193}

For the most part, the EPA requirements in draft 40 CFR 193 are consistent with those of the foregoing sources. The performance requirements include the following:

- No member of the public in the general environment shall receive an annual whole body EDE of more than 25 mrem from all routes of exposure combined. 
- Performance assessments shall not consider any contributions from active institutional controls beyond 100 years after the facility is closed.

There is also a draft requirement related to increases in the levels of radioactivity of various classes of groundwaters as a result of LLW disposal. However, the application of the requirement is unclear, and it may change significantly when the regulations are finalized. Therefore, it was not used to guide this study.

\subsubsection{Requirements in 40 CFR 264}

The regulations in 40 CFR 264 establish minimum national standards that define the acceptable management of hazardous waste. The standards apply to owners and operators of all treatment, storage, and disposal (TSD) facilities for hazardous waste. These regulations apply to the constituents of mixed waste that are hazardous per 40 CFR 261.

Some of the subparts of 264 address items that apply to all types of TSD facilities, such as general facility standards, preparedness and prevention, contingency plan and emergency procedures, closure and postclosure, etc. Other subparts apply only to specific types of TSD facilities (e.g., incinerators).

One subpart of specific application is Subpart $\mathrm{N}$ on Landfills, 264.300-.317. A landfill is "a disposal facility or part of a facility where hazardous waste is placed in or on land." These regulations are directly applicable to the current study. Requirements governing design and operation, monitoring and inspection, closure and postclosure, etc. are provided in 40 CFR 264.300-.317. Specific requirements applicable to the facilities evaluated in this study are discussed in Section 5.

Another subpart of specific application is Subpart F on Releases from Solid Waste Management Units, 264.90-.101. The subpart presents requirements for groundwater monitoring. In addition, 40 CFR 264.92-.94 discusses the allowable concentration limits for the hazardous constituents to be disposed of, in order to protect the groundwater. The limits for contaminants in the groundwater will be specified in the RCRA permit for the facility. The limits may match those in the regulations for the Safe Drinking Water Act (see Section 9), or they may be different values based on EPA's consideration of all the factors involved for the particular facility.

\subsubsection{Conclusion}

At this time, no final performance assessment is available for LLW disposal at the RWMC. No performance assessments are available for the proposed new sites for the MLLWDF. Therefore, the waste packaging and disposal methods necessary to produce isolation that will result in projected compliance with the performance objectives in DOE Order 5820.2A and the other sources is not known. For this reason, and because of the planned incorporation of NRC and EPA requirements, as appropriate, into a revised DOE Order 5820.2A, this study conservatively assumed that all of the applicable requirements discussed in Section 2.3 will apply to the MLLWDF Project. 


\subsection{References}

Duggan, G. J., 1992, "Planned Revision to DOE Order 5820.2A, Radioactive Waste Management," Fourteenth Annual U.S. DOE Low-Level Radioactive Waste Management Conference, Phoenix, Arizona, November 1992.

EG\&G Idaho, 1992, Mixed and Low-Level Waste Treatment Facility Project, EGG-PD-92-006, April 1992.

EG\&G Idaho, 1991, INEL Low-Level Radioactive Waste Acceptance Criteria, DOE/ID-10112, Rev. 4, October 1991.

NRC, 1992, Standard Format and Content of a License Application for a Low-Level Radioactive Waste Disposal Facility, NUREG-1199, Rev. 2, January 1991.

NRC, 1987, Standard Review Plan for the Review of a License Application for a Low-Level Radioactive Waste Disposal Facility, NUREG-1200, January 1987.

Roesener, W. S., N. R. Soelberg, A. L. Ayers, Jr., 1992, Identification of a Treatment Process for the Idaho Waste Processing Facility Project - A Preconceptual Design Study, WM-PD-92-009, September 1992.

Smith, T. H., W. S. Roesener, M. J. Jorgensen-Waters, C. R. Edinborough, 1992, A Preliminary Evaluation of Alternatives for Treatment of INEL Low-Level Waste and Low-Level Mixed Waste, EGG-WMO-10321, June 1992. 


\section{ASSUMPTIONS FOR STUDY OF ALTERNATIVES}

\subsection{Background}

Many unknowns could impact the suitability of each alternative for disposal of INEL mixed waste and LLW. The unknowns include gaps in today's knowicdge, as well as the nature of possible future events, trends, policies, and decisions.

To evaluate the alternatives on a meaningful and equitable basis, many assumptions must be made about these unknowns. The assumptions cover items such as the waste streams to be disposed of, the regulatory requirements affecting the disposal facility, the timing of various events, etc.

A set of simplifying assumptions was developed as a basis for the evaluations of the disposal alternatives. As events unfold, some assumptions may prove to be accurate. Others may need to be revised. The present assumptions are the best set of guidelines that the study team could assemble to allow the evaluations to proceed with equitable comparisons.

\subsection{Assumptions}

\section{A. Programmatic or General Assumptions}

1. An enhanced disposal capability will be selected and implemented expeditiously.

2. At least one offsite DOE installation will agree to accept the INEL mixed waste and LLW for disposal.

3. The INEL LLW will not be disposed of at an offsite commercial facility. (Such disposal would require an exemption from the requirement in DOE Order 5820.2A, Chapter III, paragraph 2.c.)

4. The INEL mixed waste will not be disposed of at an offsite commercial facility. (Such disposal would require an exemption from the requirements in DOE Order $5820.2 \mathrm{~A}$, Chapter III, paragraphs 2.c. and 2.d.)

B. Waste Assumptions (Detailed waste assumptions are given in Section 2.)

1. The information given in Smith et al. (1992) for posttreatment characteristics and quantities accurately and completely represents both current waste streams and future extrapolations of non- $\alpha$-contaminated mixed waste and LLW from ongoing operations. [Smith et al. (1992) contains an evaluation of the alternatives for treatment of such waste.]

NOTE: Some waste-generating activities at the INEL may terminate before the future time period addressed in this study. On the other hand, new waste-generating activities may start. No attempt is made here to predict which projects or facilities may startup or shutdown. An assumption is made that the waste streams of the 
ongoing operations of the future are adequately represented by the waste streams of the current ongoing operations.

2. Some small waste streams generated by INEL environmental remedial actions are included in the waste streams from ongoing operations, discussed in item B.1 above. (As used in this report, remedial actions refer to cleanup conducted principally outside of buildings.) Future remedial action activities may result in large waste streams. However, remedial action managers indicate that nearly all their activities are scheduled to be completed and the waste dispositioned before a new waste disposal facility will be operating. Such waste streams are not addressed for disposal in this study. The major exception is the potentially large amount of waste from environmental remediation of the RWMC. Details of the assumptions concerning waste from the RWMC remediation are given in Section 2.

NOTE: The uncertainties in ER remediation waste may have a significant impact on the volumes of INEL mixed waste and LLW requiring disposal at the INEL. Therefore, the uncertainties in this item are a major uncertainty for this study.

3. Some small waste streams generated by the activities of the INEL D\&D Program are included in the waste streams from ongoing operations, discussed in item B.1 above. (As used in this report, D\&D refers to activities conducted principally to remediate buildings and their contents.) A major increase in INEL D\&D activities may begin in approximately 10 years and last approximately 30 years. The quantity of D\&D waste could increase significantly, if the increase in D\&D activities materializes. The time period of the possible increase in $D \& D$ waste generation matches closely the expected time period for operation of the new disposal facility. Accurate estimates of the nature and volume of D\&Dgenerated waste in that time period are not available. Nevertheless, a rough estimate of the upper limit of the increased D\&D waste is included in the waste streams addressed in this study. This was done because of the potentially large magnitude of the streams and the advisability of the disposal facility being able to accommodate the maximum waste flow projected in the time period of interest. The detailed assumptions are listed in Section 2. As an alternative scenario, the continuation of the D\&D program without a major expansion is also evaluated from a waste-generation standpoint in Section 2.

NOTE: The uncertainties in D\&D waste may have a major impact on the volumes of INEL mixed waste and LLW requiring treatment and disposal. Therefore, the uncertainties in this item are a major uncertainty for this study.

4. With one qualifier, the information given in Roesener et al. (1992) for posttreatment characteristics and quantities accurately and completely represents $\alpha$-contaminated mixed waste and LLW derived from contact-handled waste currently stored at the TSA of the RWMC. [Roesener et al. (1992) contains an evaluation of the alternatives for treatment of such waste.] The qualifier is that the waste volumes given in Roesener et al. (1992) were too small, because of the incompleteness of the most detailed current database, and have been multiplied by a factor of 1.15 for use in the present report. 
5. Characteristic mixed waste that has been treated and no longer displays the characteristics of a hazardous waste per the RCRA regulations will be disposed of as LLW, assuming it satisfies all the other WAC. Listed mixed waste that has been treated as necessary to satisfy the RCRA Land Disposal Restrictions (LDRs) will be disposed of as mixed waste.

6. With one exception, the (non- $\alpha$ ) stored mixed waste and stored LLW that are currently backlogged due to the temporary shutdown of WERF will have been treated as necessary and disposed of before a new waste disposal facility begins operations. The exception is $1.7 \mathrm{~m}^{3} / \mathrm{yr}$ of mixed waste that will require disposal in a mixed wastc disposal facility, even after treatment. If a 10-year accumulation (1992 to 2002) of such waste is assumed before opening the new disposal facility, then the backlog of mixed waste will be $17 \mathrm{~m}^{3}$.

7. Hazardous (nonmixed) waste is excluded from the scope of this study.

8. TRU waste ( $>100 \mathrm{nCi} / \mathrm{g}$ transuranics) is excluded from the scope of this study.

9. Beyond that which has been received already at the INEL, no waste will be received from offsite for disposal.

10. Waste received for disposal will satisfy the WAC of the disposal facility.

\section{Disposal Facility Assumptions}

1. Waste will be emplaced in the disposal units with the intent of final disposition.

2. Facilities shall be designed to limit maximum radiation exposure to an individual worker to less than one tenth of the annual occupational external dose-equivalent limits $(1 / 10$ of 5 rem).

3. For purposes of completeness, disposal alternatives will be designed to include Class $\mathrm{C} \alpha$ waste (whether mixed or not), even though adequate long-term isolation of such waste in some alternatives has not yet been proved.

\section{Assumptions Concerning Auxiliary Functions}

1. All onsite disposal alternatives will include a minimal waste-analysis laboratory capability on premises.

\section{E. General Regulatory Assumptions}

1. Except as indicated to the contrary, the regulatory environment of the future will include the current requirements and all proposed requirements as currently drafted.

2. Draft ID Supplemental Directive 5820.2A, "Radioactive Waste Management," will not be considered in this study because plans to issue it are not clear as of this writing. The technical requirements in the Supplemental Directive are very similar to the technical requirements in 10 CFR 61, which are being used in this study. 
3. Regulatory restrictions will not preclude the shipment of mixed waste or LLW to an offsite DOE facility for disposal.

\section{F. Environmental Regulatory Assumptions}

1. The waste management facilities and operations will be required to comply with all environmental and waste management regulatory requirements.

2. In particular, the waste disposal facilities will comply with all emissions standards of Federal, State of Idaho, and DOE regulations.

3. An environmental impact statement (EIS) will be required for the MLLWDF.

4. No "Below Regulatory Concern" provisions concerning radioactive or mixed waste will be in effect.

5. It will be possible to obtain regulatory approvals for a suitable disposal site and facility on the INEL.

\section{G. Assumptions Concerning Interfaces with Other INEL Facilities}

1. Either the MLLWDF or the RWMC will be operational at all times during the future time period studied.

2. The WAC of the MLLWDF will reflect the requirements of DOE Order 5820.2A, RCRA, and the technical requirements of 10 CFR 61. The WAC will also incorporate any other pertinent requirements related to those in the current WAC of the RWMC.

3. Treatment capability will be available for the INEL mixed waste and LLW.

\section{H. Schedule Assumptions}

1. The facility operating period for all disposal alternatives will be 40 years, at the end of which all disposal units that have not already been closed will be closed.

2. The period required to accomplish closure (including D\&D of waste-handling and support facilities) may depend on the alternative and technology, but is expected to be approximately 5 to 10 years.

3. Although the intent would be to continue indefinitely the institutional control over the disposed waste, the conservatively assumed institutional control period following facility closure is 100 years, in accordance with regulatory requirements.

\section{Cost Assumptions}

1. Cost estimates will be life-cycle costs. They will include design and construction costs, program costs, operations and maintenance (O\&M) costs, costs of shipping waste offsite, 
closure costs of disposal facilities, D\&D costs for waste-handling and support facilities, and costs of postclosure monitoring and security during the institutional control period.

2. For purposes of estimating shipping costs, the offsite DOE waste disposal facility is assumed to be located $1,000 \mathrm{mi}$ away.

3. Costs will be presented (a) as unescalated (constant FY 1993) dollars and (b) as escalated dollars to the years of expenditure. 


\subsection{References}

Roesener, W. S., N. R. Soelberg, A. L. Ayers, Jr., 1992, Identification of a Treatment Process for the Idaho Waste Processing Facility Project - A Preconceptual Design Study, WM-PD-92-009, September 1992.

Smith, T. H., W. S. Roesener, M. J. Jorgensen-Waters, C. R. Edinborough, 1992, A Preliminary Evaluation of Alternatives for Treatment of INEL Low-Level Waste and Low-Level Mixed Waste, EGG-WMO-10321, June 1992. 


\section{OVERVIEW OF ALTERNATIVES}

\subsection{Introduction}

Several preliminary alternatives for disposing of INEL mixed waste and LLW were formulated for evaluation and comparison. This section provides an overview description of each alternative. The description is aimed at the concepts embodied in the alternative, rather than at the details of the particular disposal technique. A more detailed description of each alternative is presented in Section 5 .

A very large number of alternatives and of variations on each alternative (hereafter called subalternatives) could have been formulated for evaluation. However, only a limited number could be evaluated due to practical limits on the magnitude of this study. Selecting the optimum set of alternatives and subalternatives for evaluation was a challenging task. This section explains the process by which the alternatives and subalternatives were screened down to a manageable number for evaluation.

The disposal methods actually implemented may not exactly match those evaluated in this study. However, the alternatives and subalternatives that were evaluated form a relatively complete set of reasonable, bounding options from which interpolation can probably give reliable, broad estimates concerning the effects of implementing other options.

Numerous assumptions were made about the alternatives and subalternatives so the evaluations could proceed. Top-level assumptions unique to a particular alternative are generally discussed here. Assumptions that apply to more than one alternative are generally listed in Section 3.

\subsection{Phases of Activity}

A disposal facility will generally pass through several phases of activity, as depicted in Figure 4-1. Some phases may overlap one another.

The construction phase comes first. The operations phase comes next and is assumed to last 40 years, or from about the year 2005 through 2045 (depending on the alternative), as stated in the assumptions of Section 3. For some disposal technologies, constructing new disposal units will be an ongoing activity throughout the operations phase. In such cases, the construction phase is defined as including construction of all necessary waste-handling and support facilities and sufficient disposal units to accommodate waste received during approximately the first 10 years. Additional disposal units will be constructed during the operating phase, while waste is being received.

After the operations phase comes the closure and stabilization phase, which may last 5 to 10 years, depending on the disposal alternative and technology. The intent of this phase is to achieve long-term stability of the disposal site and eliminate the need for active maintenance to the extent possible. This phase includes all operational and regulatory activities related to closure and stabilization although some activities can be started near the end of the operations phase. D\&D of waste-handling and support facilities is included in this phase. 


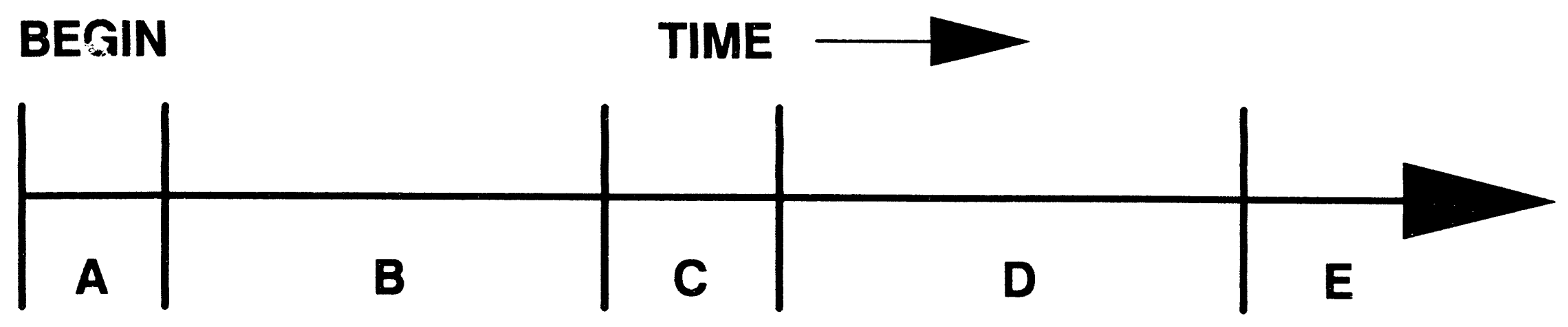

i $\quad A=$ Construction Phase

B = Operations Phase (assumed to last 40 years)

C $=$ Closure and Stabilization Phase (assumed to last 5 to 10 years)

$D=$ Institutional Control Phase (assumed to last 100 years)

$E=$ Post-Institutional Control Phase

Figure 4-1. Phases in the life of a waste-disposal facility. 
The next phase is the institutional control phase, during which human institutions are relied upon to control the site and to monitor its performance. In accordance with DOE Order 5820.2A, the institutional control phase is assumed to last the maximum of 100 years. During this phase, security provisions restrict public access to the closed site. The condition of the accessible portions of the disposal units is evaluated regularly by trained personnel, and corrective action is taken as necessary. The performance of the disposal units in maintaining waste confinement is monitored by means of a comprehensive environmental sampling and analysis program. Any performance deficiencies are identified, and corrective action is taken.

The final phase is the postinstitutional control phase, which has no assumed end point. In this phase, it is conservatively assumed that institutional control of access to the disposal site is lost. In performance assessments, it is conservatively assumed that the general population can then freely intrude upon the site. Reliance is placed solely on the passive isolation capabilities of the disposal facility and site to limit the radiological and nonradiological consequences of the disposed waste on the intruders.

\subsection{Overview Descriptions of Alternatives}

Figure 4-2 presents a highly simplified flow diagram for each disposal alternative and subalternative.

\subsubsection{Alternative 1: Construct and Operate MLLWDF}

In this alternative, a new disposal facility, termed the MLLWDF, will be constructed and operated (see Figure 4-2). The MLLWDF project will proceed essentially as planned in the project documentation and the draft Technical Project Plan (Sherick and Winston 1992).

The facility will provide complete capabilities for receiving, handling, and disposing of the waste. The facility will be sited, designed, and constructed so as to comply with all applicable requirements in Federal and State regulations and in DOE Orders.

In Section 4.4, several subalternatives for implementing this alternative are described briefly. The subalternatives involve various disposal technologies that were selected for evaluation, as well as various scenarios related to the waste volumes and waste forms to be received for disposal. To limit the number of subalternatives evaluated, no subalternatives were formulated for the other basic alternatives.

Siting studies for the proposed MLLWDF are under way. For cost or other evaluations in the current study, the location of the MLLWDF is assumed to be away from existing facilities, so the full range of siting activities will be required.

The schedule for the construction and operation of the MLLWDF generally follows that indicated in project documentation. The start date for radioactive operations is projected in Section 12 to be calendar year 2005. The operating life of the facility is assumed to be 40 years. Thus, for the purposes of this study, the MLLWDF will operate through calendar year 2045. 


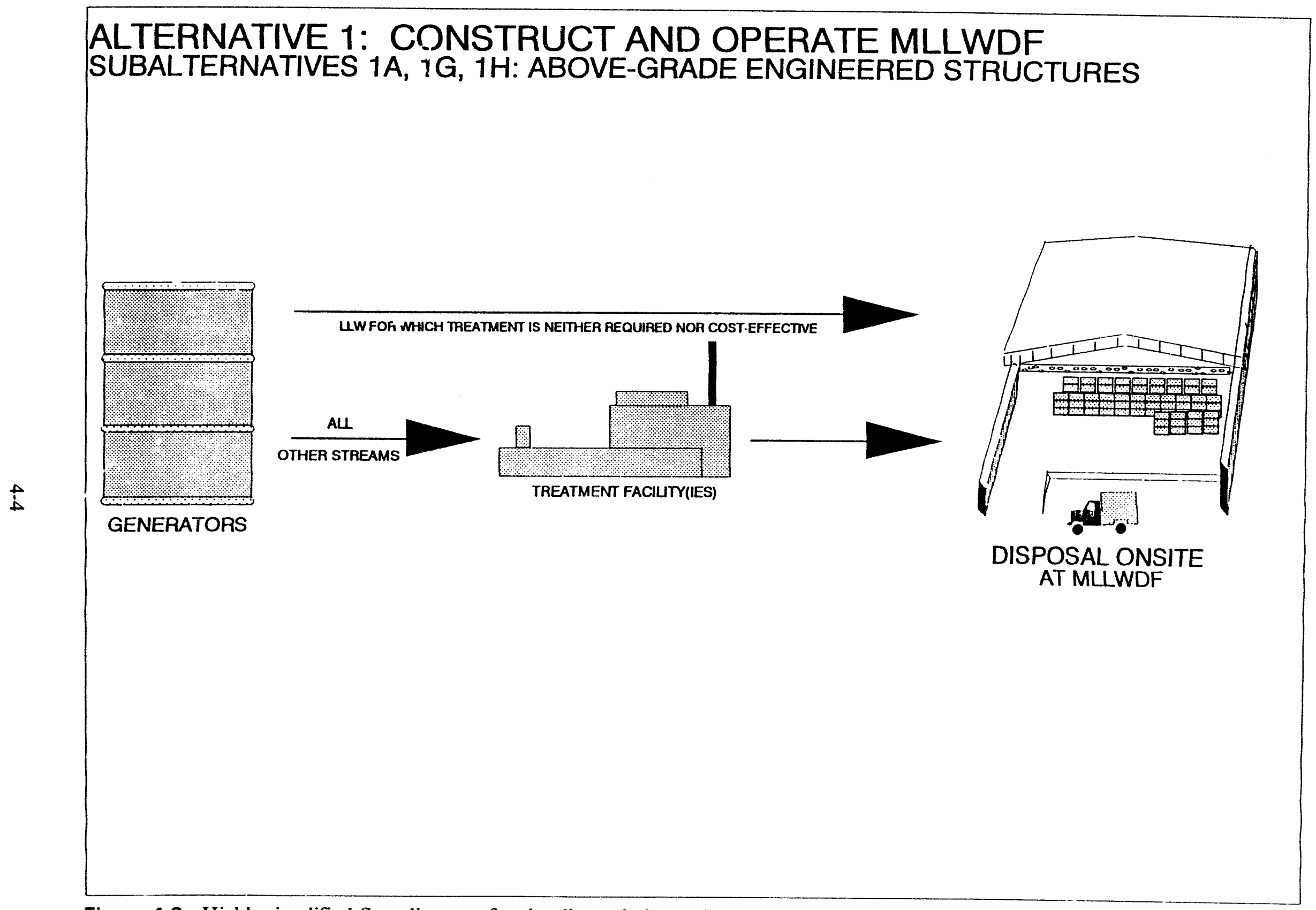

Figure 4-2. Highly simplified flow diagrams for the disposal alternatives and subalternatives evaluated. 


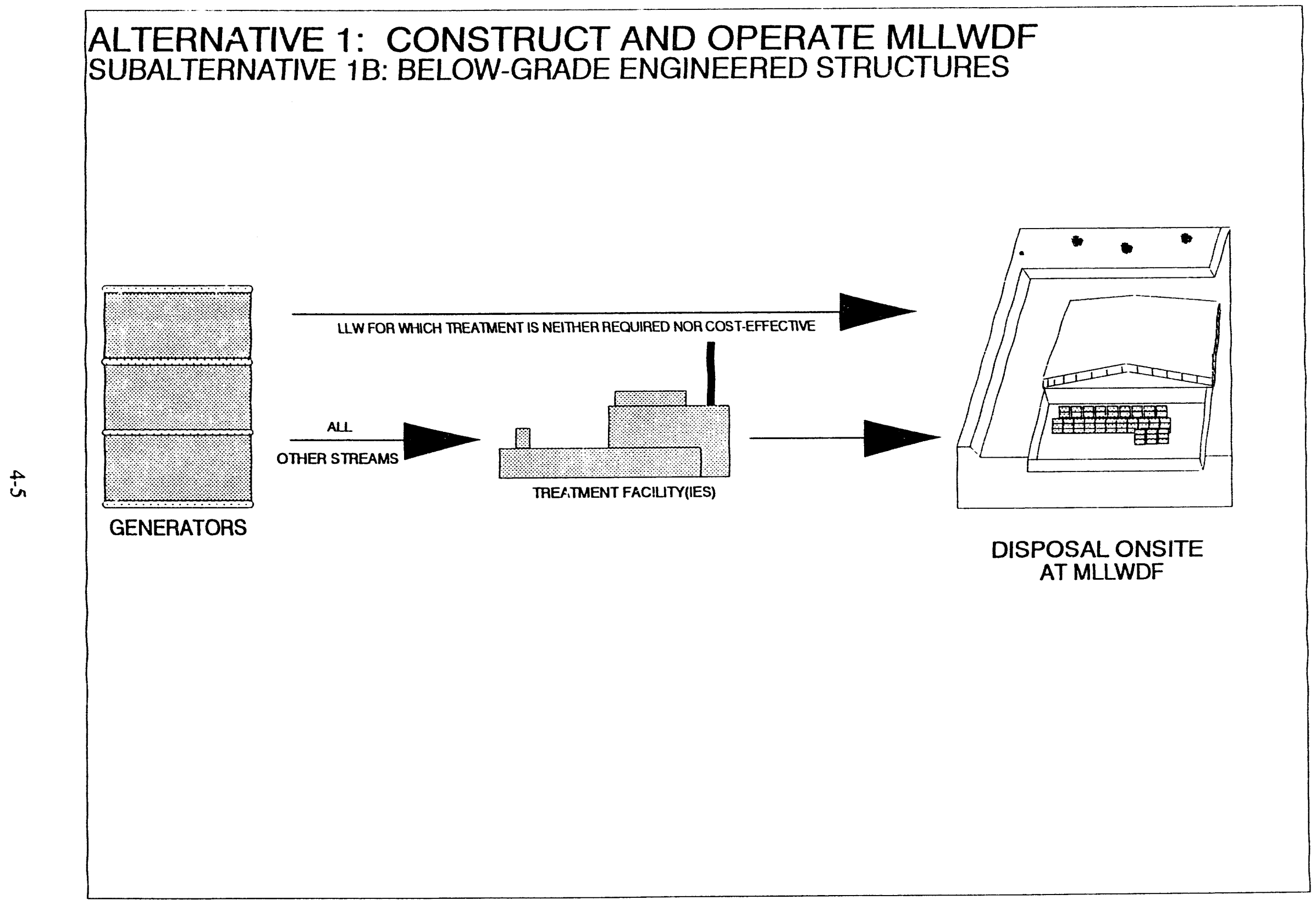

Figure 4-2. (continued). 


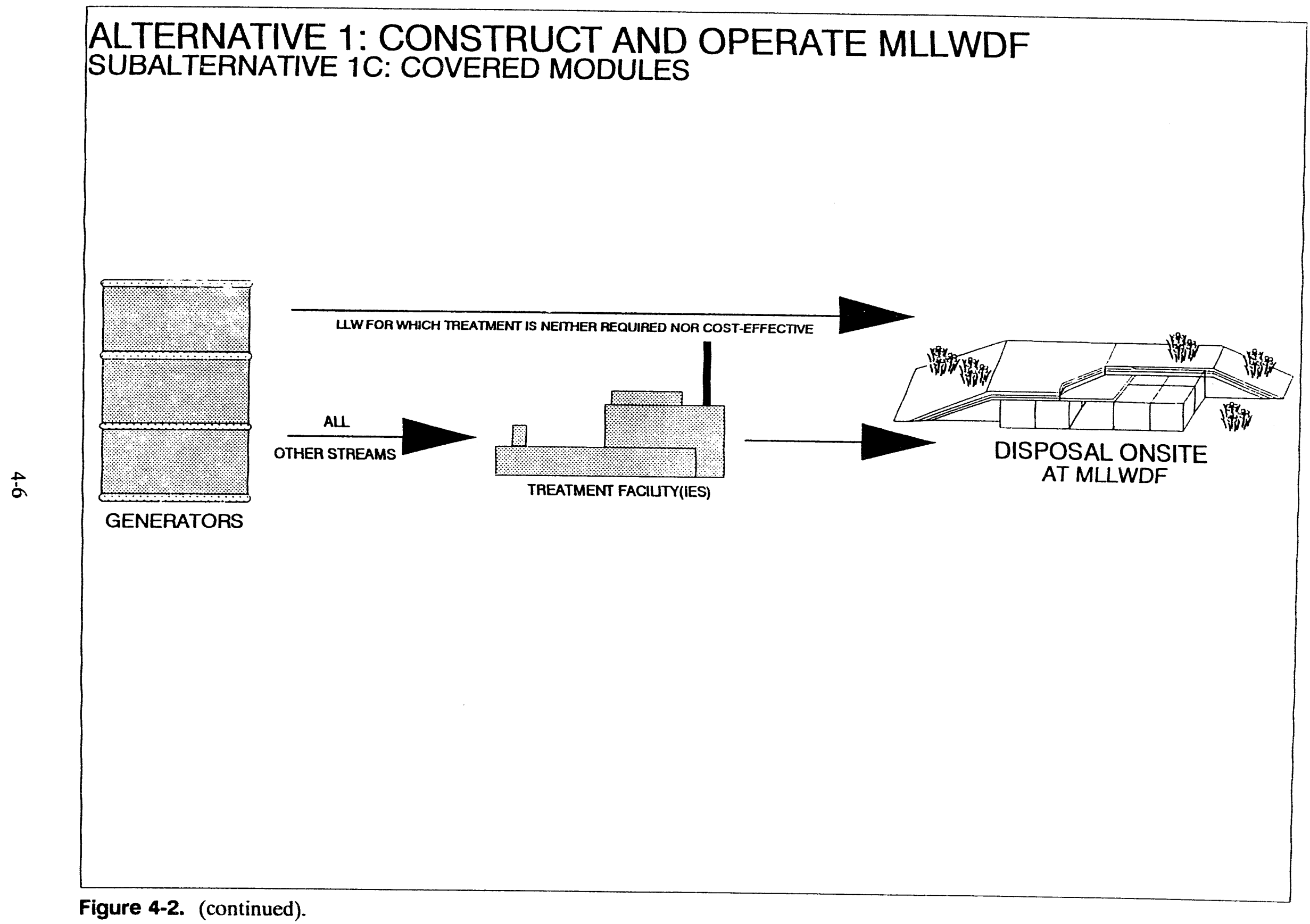

Figure 4-2. (continued). 


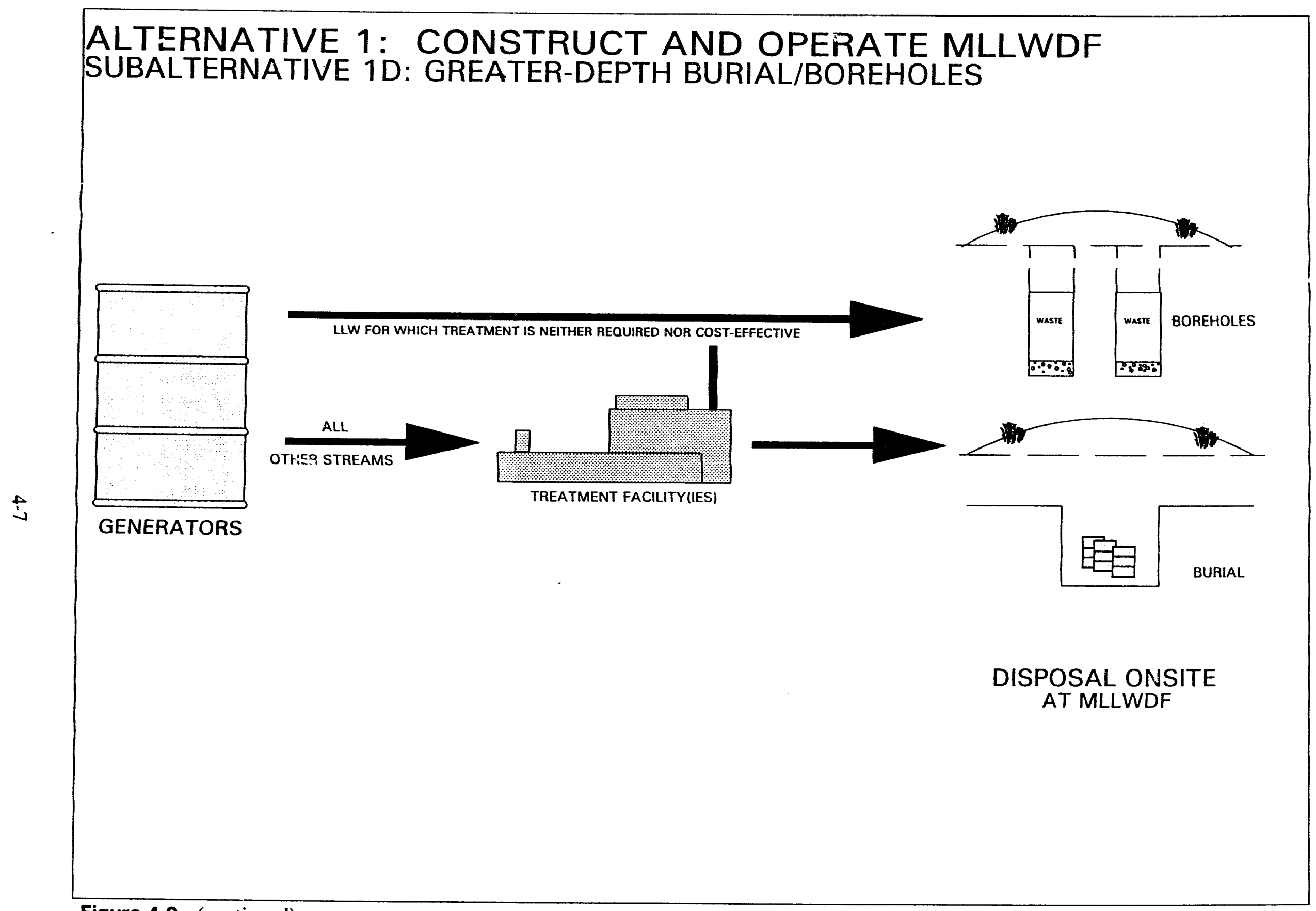

Figure 4-2. (continued). 


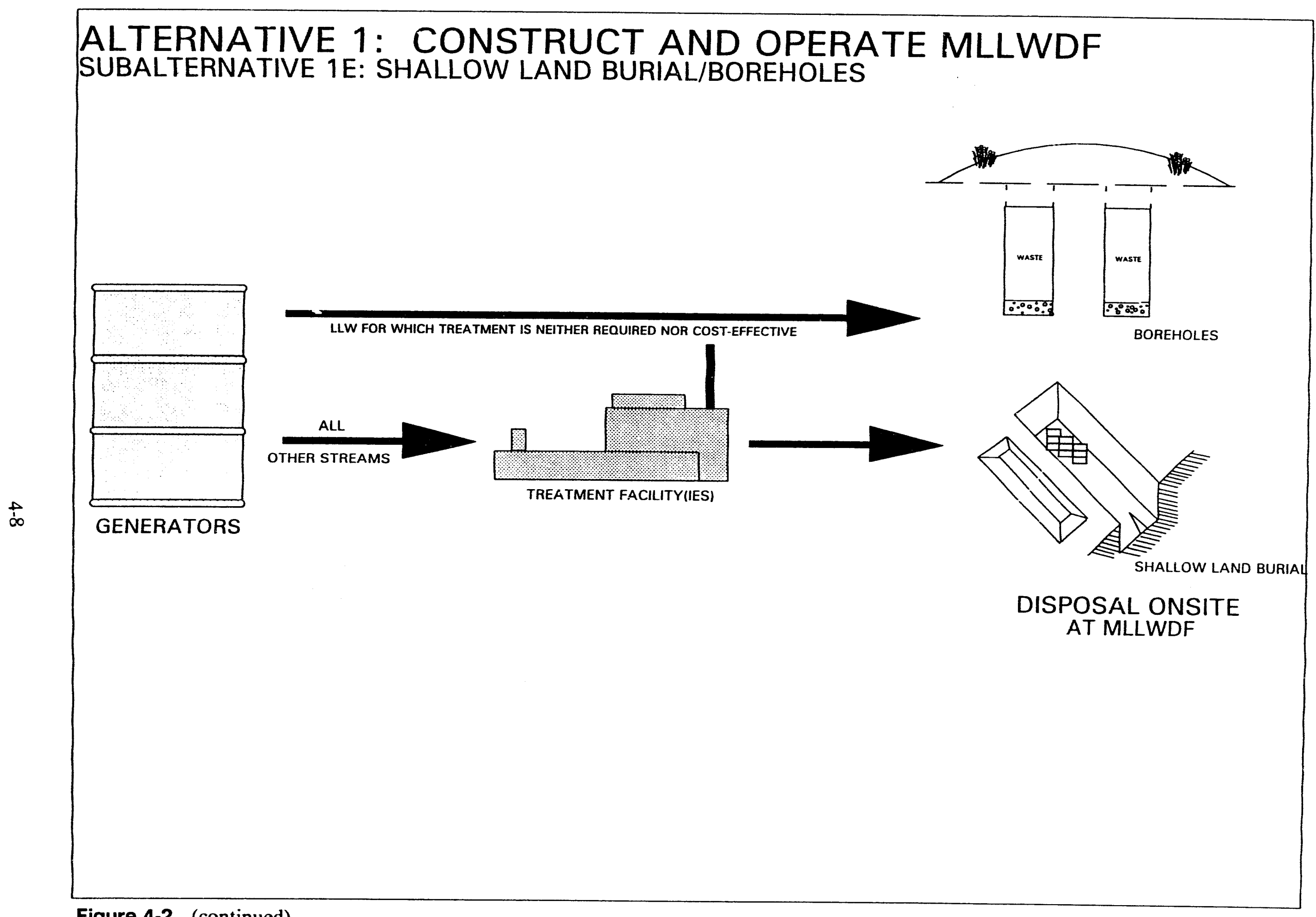

Figure 4-2. (continued). 


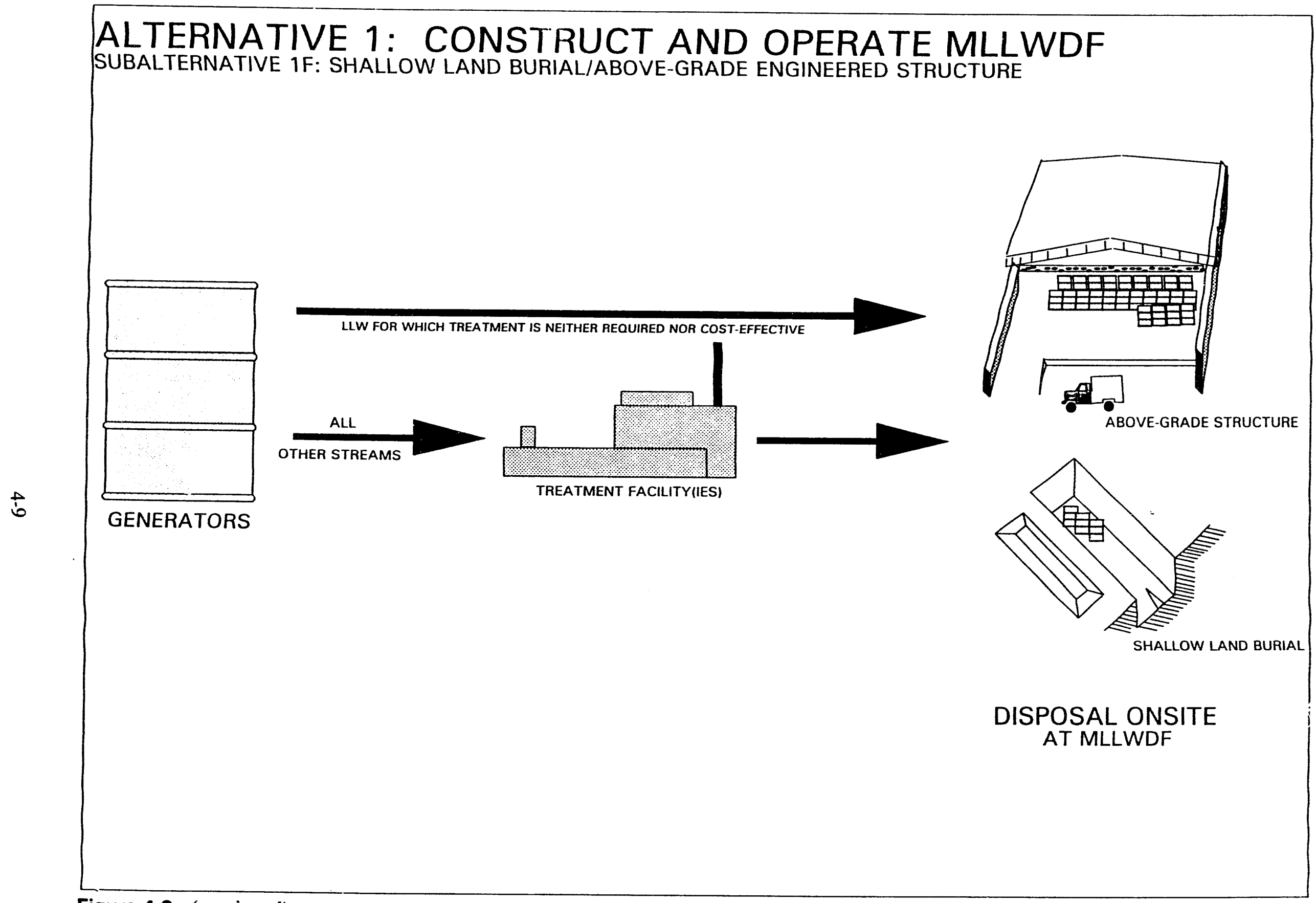

Figure 4-2. (continued). 


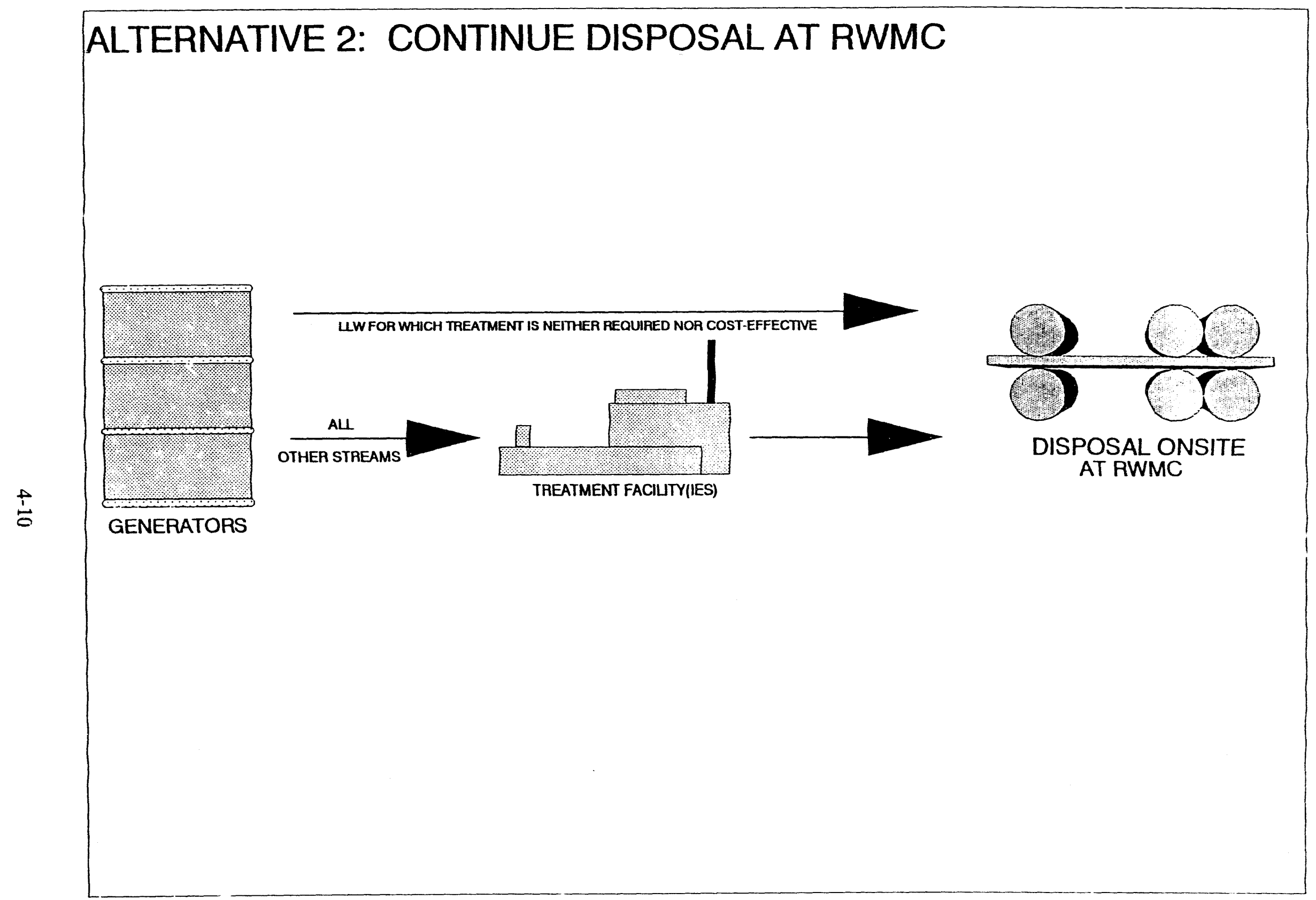

Figure 4-2. (continued). 


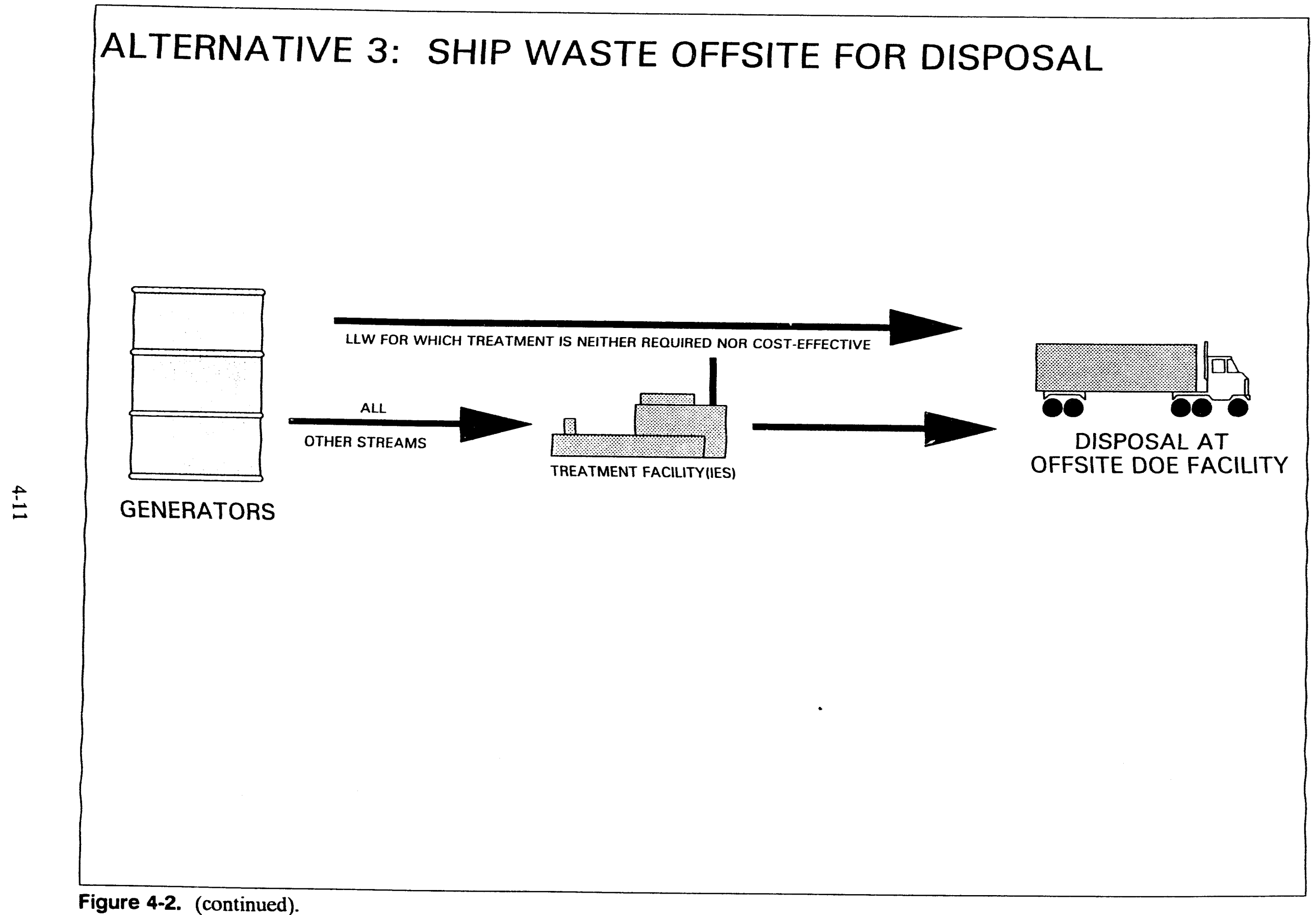

Figure 4-2. (continued). 


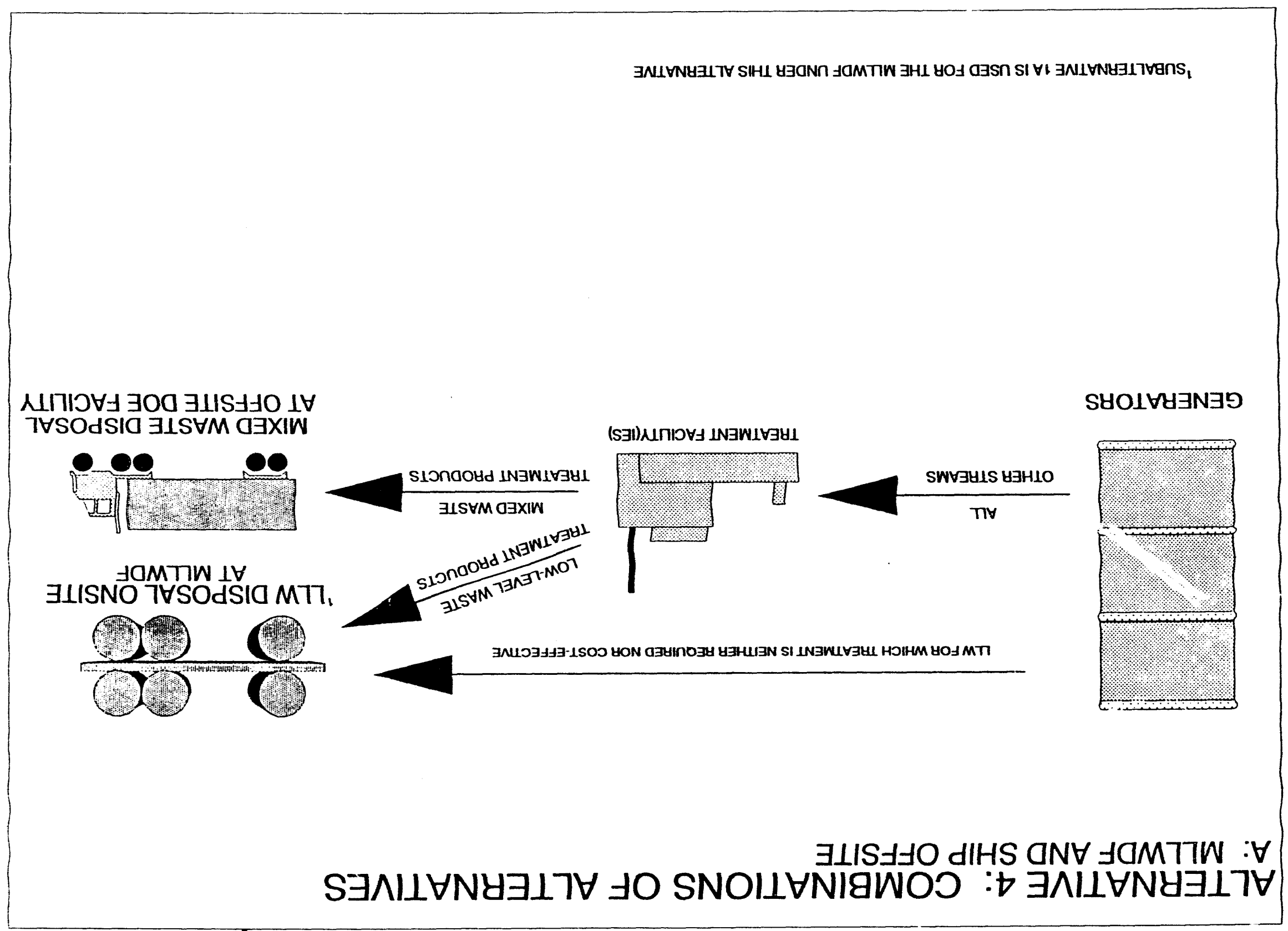




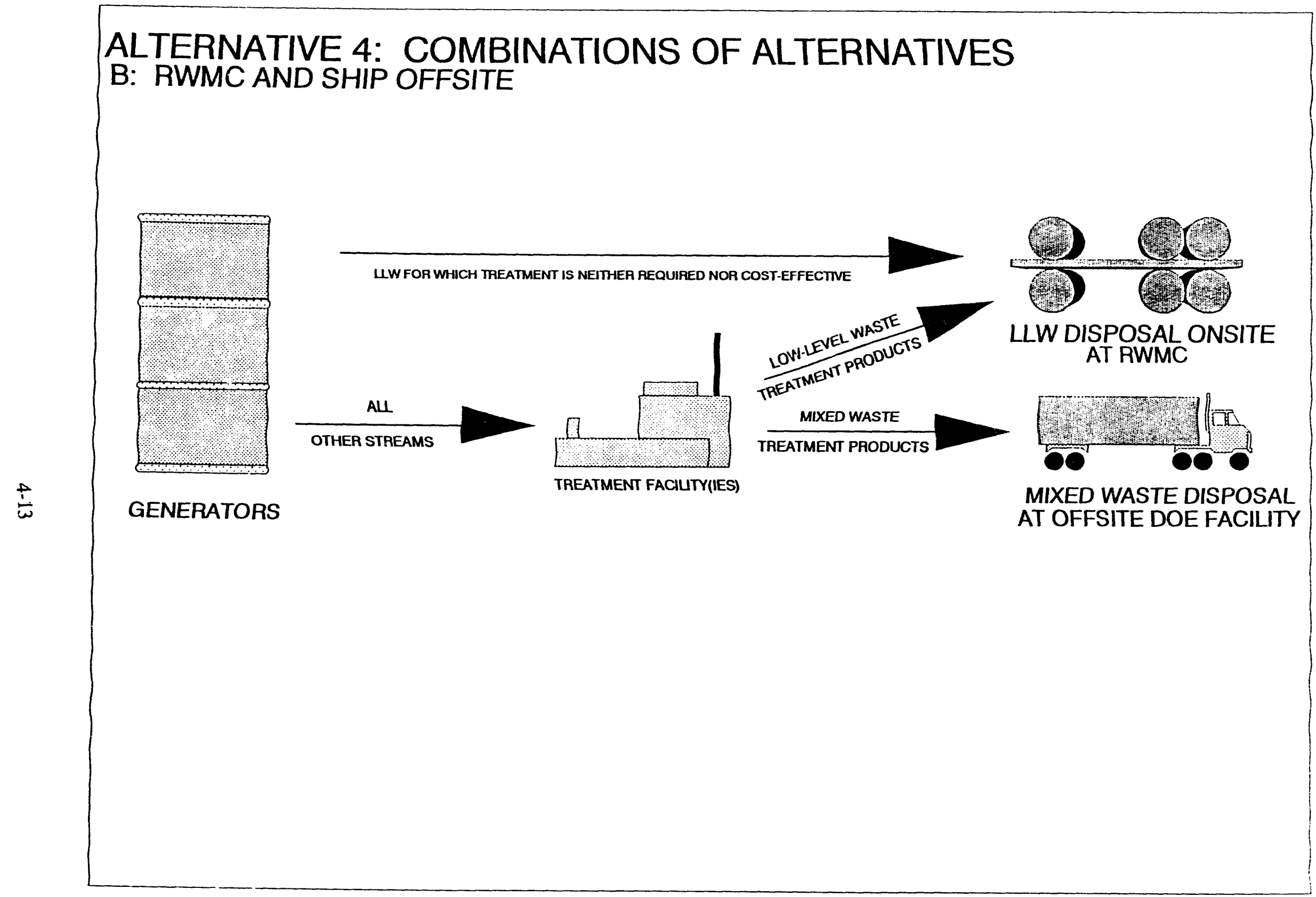

Figure 4-2. (continued). 


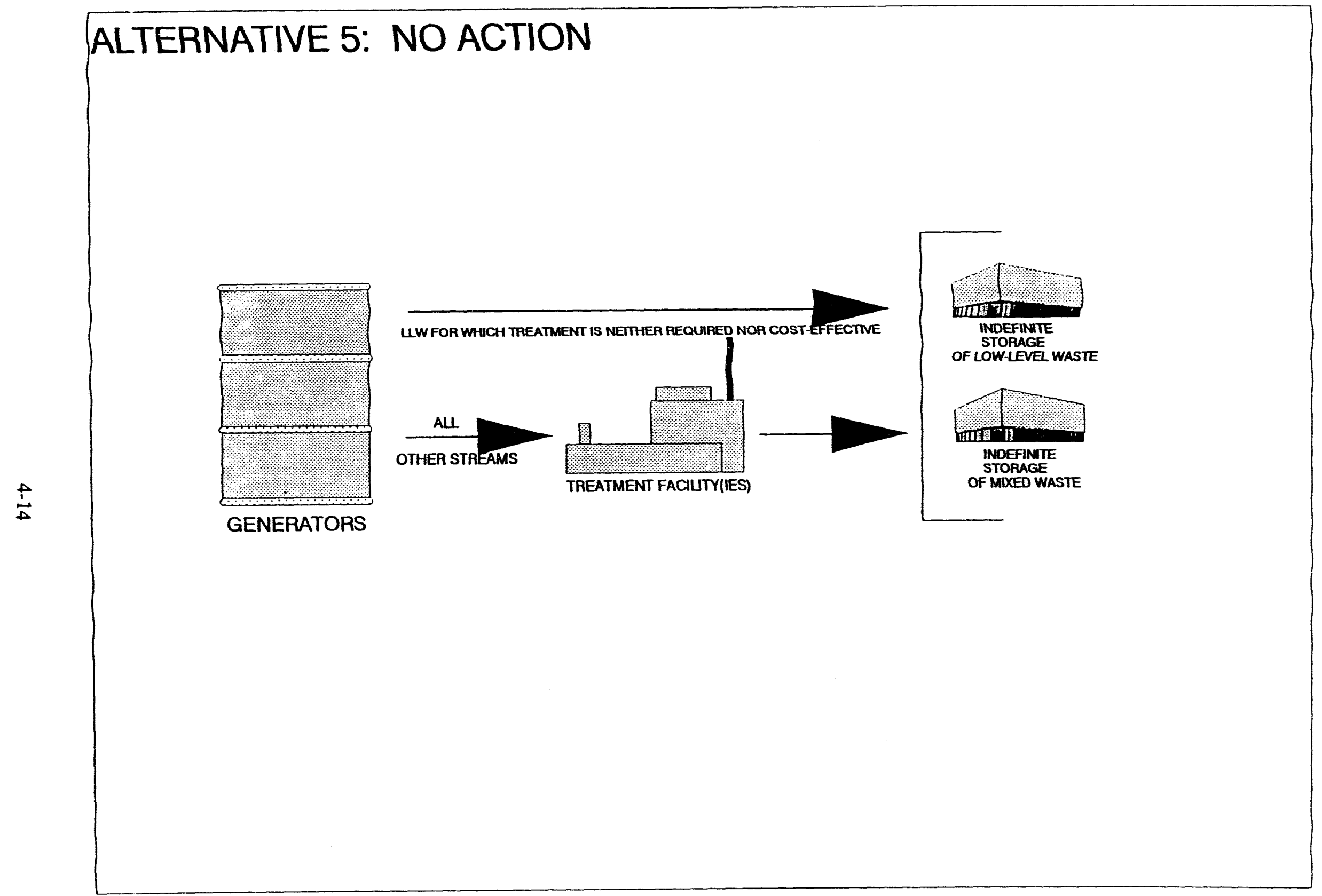

Figure 4-2. (continued). 


\subsubsection{Alternative 2: Continue Disposal at RWMC}

In this alternative, waste disposal will continue at the RWMC through the end of the study period (see Figure 4-2).

Assuming the current disposal methods are continued, the remaining capacity for pit disposal of LLW at the RWMC is limited to about 1.4 million $\mathrm{ft}^{3}$. RWMC personnel have projected that the capacity will be consumed by 2013 (20 years in the future). The projection is based on LLW generation similar to that in the Small ER and D\&D scenario and on waste treatment in WERF. When operational, WERF treats approximately inalf of the LLW that is generated.

The RWMC closure date cannot be projected with certainty because of large uncertainties in waste-generation rates and in the availability of waste-treatment capability. For example, the closure date would be considerably sooner if the possible major growth in the volume of waste generated by the D\&D Program takes place.

For perspective, simplified calculations of the remaining lifetime under several scenarios were performed for the present study. The four levelized waste generation/waste treatment scenarios of Table 2-5 were used, along with an assumed stacking efficiency in the pit of $95 \%$. The results were as follows in Table 4-1 (the remaining lifetimes have been rounded downward to the next lower whole year.

Because WERF treats approximately half of the LLW, the projections by RWMC personnel (which addressed disposal of LLW only) would be expected to fall between the 8 years for the minimum-treatment scenario and the 36 years for the maximum-treatment scenario. The projected lifetime of 20 years does indeed fall near the middle of that range.

For the purposes of convenience and conservatism in this study, it is assume 1 for all alternatives that disposal at the RWMC using present methods ends approximately 2004 (11 years in the future). Picking a different year would not significantly impact the recommendation of one alternative over another as the preferred waste disposal method after the RWMC closes. What is important is that the assumption concerning the RWMC termination date be essentially the same for all of the alternatives evaluated, so that they can be compared on a consistent basis.

Table 4-1. Levelized waste generation/waste treatment scenarios.

\begin{tabular}{lc}
\hline \multicolumn{1}{c}{ Scenario } & $\begin{array}{c}\text { Lifetime } \\
\text { (LLW disposal only) }\end{array}$ \\
\hline Maximum treatment/Small ER and D\&D & 36 years \\
Maximum treatment/Large ER and D\&D & 15 years \\
Minimum treatment/Small ER and D\&D & 8 years \\
Minimum treatment/Large ER and D\&D & 5 years \\
\hline
\end{tabular}


Once the LLW disposal space is depleted, a new method for disposing of LLW is assumed to be implemented at the RWMC. Several methods for disposing of additional LLW at the RWMC have been evaluated over the years. None of the methods is particularly attractive. Any sizeable unused areas of the RWMC SDA have only shallow sediments above the underlying basalt; that is the reason why the spaces are unused. Therefore, abovegrade stacking of waste, rather than shallow land burial, is necessary to make use of such areas.

The combination of methods selected for use in this study involves the following:

- Stacking the additional contact-handled, non- $\alpha$ LLW (whether mixed or not) on pads on top of filled disposal pits or unused areas, then covering the additional waste with a suitable cap or cover

- Disposing of remote-handled LLW and mixed waste by preparing additional soil vaults in remaining spaces

- Disposing of contact-handled $\alpha$ LLW (whether mixed or not) by processing it into a longlived waste form and stacking the waste containers on pads in remaining spaces, then emplacing a 5-m cover as an intruder barrier.

\subsubsection{Alternative 3: Ship Waste Offsite for Disposal}

In this alternative, the INEL mixed waste and LLW are shipped offsite for disposal (see Figure 4-2). As stated in Section 3, DOE Order 5820.2A requires an exemption for disposal of DOE LLW or mixed waste at commercial disposal facilities, so the waste is assumed here to be disposed of at another DOE site.

Several DOE sites have operating disposal facilities for LLW. However, few if any DOE sites have operating disposal facilities that can accommodate mixed wastes, including $\alpha$-contaminated mixed waste and possibly remote-handled mixed waste. This study assumes that such disposal capabilities are available by the future time period of interest. The method of disposal is assumed to be shallow land burial.

In this alternative, the only disposal-related facility required at the INEL is a building to stage the waste and load it for offsite shipment.

\subsubsection{Alternative 4: Combinations of Alternatives}

In this subsection, several combinations of the above alternatives are considered for evaluation. In each combination, some types of waste are disposed of using one of the above alternatives, and the other types of waste are disposec of by another alternative.

The descriptions and assumptions concerning the combinations of alternatives are generally the same as those for the individual alternatives comprising the combination. However, only some of the waste types are disposed of via each alternative. 
Some possible combinations of the above three alternatives are attractive and were judged worth considering in this study; some were not. In general, combinations are more attractive if their respective disposal capabilities are complementary, rather than duplicative.

The matrix in Table 4-2 indicates the pairs of alternatives that were selected for evaluation as combinations (marked with an "X"). Pairs not considered further are marked with an "O." Only the upper right half of the matrix needs to be addressed because of symmetry. Also, the diagonal represents the combination of an alternative with itself, so it need not be considered.

Two combinations of alternatives were selected for analysis. Overview flow charts for the selected combinations appear in Figure 4-2.

No combinations of other alternatives with the "no-action" alternative discussed in Section 4.3.5 were selected, for obvious reasons.

The following paragraphs provide a brief rationale for evaluating only the two combinations indicated.

The combination of the MLLWDF alternative with the Continue RWMC alternative involves continuing to dispose of LLW at the RWMC and constructing a new facility (MLLWDF) to dispose of only mixed waste. This combination would have some limited appeal because of the reduced cost of disposing of LLW by continuing to use the RWMC. However, the isolation performance for the disposal of LLW at the RWMC would be inferior to the performance at a new facility, so the combination was not pursued.

The combination of the MLLWDF alternative with the Ship Ofisite alternative would involve shipping the mixed waste offsite for disposal and constructing a new facility at the INEL to dispose of the LLW. This combination of alternatives was selected for evaluation. The opposite breakdown (shipping LLW offsite and retaining mixed waste onsite) would not be attractive because the volume of LLW to be shipped could be considerably larger than the volume of mixed waste.

The combination of the Continue RWMC alternative with the Ship Offsite alternative would involve shipping the mixed waste offsite for disposal and continuing to dispose of the LLW at the RWMC. The isolation performance deficiencies of long-term continuation of disposal at the RWMC would exist again in this combination. Nevertheless, this combination of alternatives was selected for evaluation because it would not require the construction of a new disposal facility at the INEL, nor would a new RCRA Part B permit be required.

Table 4-2. Combinations of alternatives selected for evaluation.

\begin{tabular}{|c|c|c|c|}
\hline Alternative & MLLWDF & Continue RWMC & Ship Offsite \\
\hline MLLWDF & & $\mathrm{O}$ & $\mathrm{X}$ \\
\hline Continue RWMC & & & $X$ \\
\hline Ship Offsite & & & \\
\hline
\end{tabular}


For simplicity, the combination involving the MLLWDF and Ship Offsite alternatives was evaluated only for the baseline subalternative under the MLLWDF. The baseline subalternative is explained in Section 4.4.

\subsubsection{Alternative 5: No Action}

This alternative is basically the same as the "no-action" alternative required by NEPA regulations. In this alternative, no new action is taken to dispose of INEL mixed waste and LLW. The MLLWDF is not constructed. No other disposal alternative is implemented.

Assuming that waste continues to be generated, the disposition of such waste in this alternative is as follows. When the available disposal space in the RWMC is consumed (assumed for simplicity to be approximately 2004), the newly generated LLW is stored indefinitely in buildings constructed for that purpose. Mixed waste also is stored indefinitely in buildings constructed for that purpose (see Figure 4-2).

For both types of waste, the initial construction will include the necessary handling and support capabilities, as well as the storage buildings required for the first 2 or 3 years of operation. Thereafter, additional storage buildings will be constructed annually or as needed.

\subsection{Subalternatives for Alternative 1}

\subsubsection{Introduction}

Alternative 1, Construct and Operate the MLLWDF, is a very broad alternative. To provide specific concepts for evaluation, several subalternatives were formulated. Some of the subalternatives were selected for evaluation.

The subalternatives were generated by listing the major factors that affect the design and operation of the proposed MLLWDF, and then considering how the major factors could be varied.

The major factors considered were as follows:

- Location of the MLLWDF

- Disposal technology

- Combinations of different disposal technologies to dispose of different types of waste

- Volume of waste to be disposed of

- Extent of treatment performed on the waste prior to receipt at the MLLWDF.

Examination of these major factors under a range of assumptions provides an indication of their impacts on the siting requirements, facility design, isolation performance, and cost of the MLLWDF. 
Some possible combinations of the above three alternatives are attractive and were judged worth considering in this study; some were not. In general, combinations are more attractive if their respective disposal capabilities are complementary, rather than duplicative.

The matrix in Table 4-2 indicates the pairs of alternatives that were selected for evaluation as combinations (marked with an "X"). Pairs not considered further are marked with an "O." Only the upper right half of the matrix needs to be addressed because of symmetry. Also, the diagonal represents the combination of an alternative with itself, so it need not be considered.

Two combinations of alternatives were selected for analysis. Overview flow charts for the selected combinations appear in Figure 4-2.

No combinations of other alternatives with the "no-action" alternative discussed in Section 4.3.5 were selected, for obvious reasons.

The following paragraphs provide a brief rationale for evaluating only the two combinations indicated.

The combination of the MLLWDF alternative with the Continue RWMC alternative involves continuing to dispose of LLW at the RWMC and constructing a new facility (MLLWDF) to dispose of only mixed waste. This combination would have some limited appeal because of the reduced cost of disposing of LLW by continuing to use the RWMC. However, the isolation performance for the disposal of LLW at the RWMC would be inferior to the performance at a new facility, so the combination was not pursued.

The combination of the MLLWDF alternative with the Ship Offsite alternative would involve shipping the mixed waste offsite for disposal and constructing a new facility at the INEL to dispose of the LLW. This combination of alternatives was selected for evaluation. The opposite breakdown (shipping LLW offsite and retaining mixed waste onsite) would not be attractive because the volume of LLW to be shipped could be considerably larger than the volume of mixed waste.

The combination of the Continue RWMC alternative with the Ship Offsite alternative would involve shipping the mixed waste offsite for disposal and continuing to dispose of the LLW at the $\mathrm{RWMC}$. The isolation performance deficiencies of long-term continuation of disposal at the RWMC would exist again in this combination. $\mathrm{N}$ vertheless, this combination of alternatives was selected for evaluation because it would not require the construction of a new disposal facility at the INEL, nor would a new RCRA Part B permit be required.

Table 4-2. Combinations of alternatives selected for evaluation.

\begin{tabular}{|c|c|c|c|}
\hline Alternative & MLLWDF & Continue RWMC & Ship Offsite \\
\hline MLLWDF & & $\mathrm{O}$ & $\mathrm{X}$ \\
\hline Continue RWMC & & & $\mathbf{X}$ \\
\hline Ship Offsite & & & \\
\hline
\end{tabular}


For simplicity, the combination involving the MLLWDF and Ship Offsite alternatives was evaluated only for the baseline subalternative under the MLLWDF. The baseline subalternative is explained in Section 4.4 .

\subsubsection{Alternative 5: No Action}

This alternative is basically the same as the "no-action" alternative required by NEPA regulations. In this alternative, no new action is taken to dispose of INEL mixed waste and LLW. The MLLWDF is not constructed. No other disposal alternative is implemented.

Assuming that waste continues to be generated, the disposition of such waste in this alternative is as follows. When the available disposal space in the RWMC is consumed (assumeo for simplicity to be approximately 2004), the newly generated LLW is stored indefinitely in buildings constructed for that purpose. Mixed waste also is stored indefinitely in buildings constructed for that purpose (see Figure 4-2).

For both types of waste, the initial construction will include the necessary handling and support capabilities, as well as the storage buildings required for the first 2 or 3 years of operation. Thereafter, additional storage buildings will be constructed annually or as needed.

\subsection{Subalternatives for Alternative 1}

\subsubsection{Introduction}

Alternative 1, Construct and Operate the MLLWDF, is a very broad alternative. To provide specific concepts for evaluation, several subalternatives were formulated. Some of the subalternatives were selected for evaluation.

The subalternatives were generated by listing the major factors that affect the design and operation of the proposed MLLWDF, and then considering how the major factors could be varied.

The major factors considered were as follows:

- Location of the MLLWDF

- Disposal technology

- Combinations of different disposal technologies to dispose of different types of waste

- Volume of waste to be disposed of

- Extent of treatment performed on the waste prior to receipt at the MLLWDF.

Examination of these major factors under a range of assumptions provides an indication of their impacts on the siting requirements, facility design, isolation performance, and cost of the MLLWDF. 
For each of the five major factors, a range of assumptions was considered that could result in generating subalternatives. The rationale by which each major factor was examined is explained below.

To limit the number of subalternatives to a manageable number, a baseline subalternative was formulated, then the five major factors generally were varied only one at a time to result in additional subalternatives. Thus, not every combination of major factors was used to formulate subalternatives.

\subsubsection{Location}

The first major factor, location, is related to siting the MLLWDF at the INEL. The location affects items such as the suitability of various disposal technologies, onsite shipping distances, and cost.

Separate studies are addressing the siting of the MLLWDF (Hillary 1992a, Hillary 1992b). The siting decision for the MLLWDF includes two issues: (a) whether to collocate the MLLWDF with a proposed new waste-treatment facility; and (b) if collocation is not pursued, where to locate the MLLWDF. Eight INEL sites are being investigated. Based on the results of previous siting studies, three sites were selected for use in this alternatives study.

For the current study, it was assumed that the MLLWDF is located separately from the new treatment facility. The MLLWDF was assumed to be constructed at one of the three sites, which is located in the central part of the INEL. That site, called Well Site 14, is used here as the baseline location. To limit the scope of the effort, no separate subalternative involving a different location was evaluated. Rather, at the completion of this study, a table was developed to indicate the potential suitability of the evaluated disposal technologies at the two other sites.

\subsubsection{Disposal Technology}

The second major factor is the disposal technology, that is, the method used to provide isolation of the waste. The technologies that could conceivably be used in the MLLWDF cover a broad range, from very basic and traditional shallow land burial to advanced, highly engineered structures.

To arrive at a set of technologies for evaluation, the current study considered the available technologies in a systematic manner. The approach used is patterned after that used (Cook 1987) at the DOE Savannah River Site in a technology alternatives study for the storage and disposal of LLW. For clarity in the present report, the terminology used in Cook (1987) has been changed slightly.

In the Savannah River study, potential storage and disposal technologies were considered to be described generically by four basic attributes:

- Facility has structure, has no structure, or waste package is modular

- Facility is abovegrade or belowgrade

- Facility has a thin cover (less than $5 \mathrm{~m}$ ), has no cover, or has a thick cover (at least $5 \mathrm{~m}$ ) 
- Waste is emplaced with or without fill.

The complete set of potential technologies, based on all possible combinations of these four attributes totaled $36(3 \times 2 \times 3 \times 2)$, of which 17 were considered feasible. Seven disposal technologies from among these 17 were chosen for study by the Savannah River personnel.

The current study modified the Savannah River approach somewhat. All four attributes listed above were considered, but the attribute of fill versus no fill was dropped because fill of some sort is planned for all technologies being given serious consideration. Table 4-3 lists the technologies that were examined here in the initial screening. The seven surviving disposal technologies from the Savannah River study were included here, along with two additional technologies.

To provide a broad range of concepts, six of the nine technologies categorized in Table 4-3 were selected for evaluation for the MLLWDF. (Not all waste types would be disposed of by a given technology, however. As discussed in Section 4.4.4, the technologies were combined so as to provide a disposal method for each type of waste.)

One technology selected for evaluation is shallow land burial (including shallow boreholes), representative of past and most current LLW disposal at DOE sites, as well as (with the addition of liners, and leak-detection and leachate collection and removal systems) most current hazardous waste disposal. Another technology selected for evaluation is greater-depth burial, in which the top of the uppermost waste package is at least $5 \mathrm{~m}$ belowgrade. A related technology is greater-depth boreholes. The fourth and fifth technologies selected for evaluation are engineered structures, both abovegrade (covered structure) and belowgrade (buried structure). The abovegrade engineered structure (AGES) is used as the baseline technology for purposes of comparison. In no way does this designation assume the superiority of this technology. The last technology selected for evaluation is covered modules.

The other three technologies listed in Table 4-3 were not evaluated for Alternative 1. Covered placement does not provide highly reliable waste isolation over long time periods. Greater-depth structures were considered "overkill," in that redundant intruder protection would be provided by the greater depth of burial and by the engineered structures. To limit the scope of the study, buried modules were considered to be adequately addressed by the evaluation of buried structures and covered modules.

\subsubsection{Combinations of Technologies}

As stated above, different technologies can be used to dispose of different types of waste. For each of the eight types of waste discussed in Section 2, disposal is governed by a somewhat different set of regulatory and operational requirements. Assigning the six technologies listed above to dispose of the eight types of waste leads to the generation of numerous subalternatives. This combining of technologies is the third major factor cited in Section 4.4.1. 
Table 4-3. Technologies considered for the disposal of INEL mixed waste and LLW (see text for explanation of entries).

\begin{tabular}{lccl}
\hline Generic name of technology & Structure & Above/belowgrade & Cover \\
\hline Covered placement & No & Above & Thin \\
Shallow land burial & No & Below & Thin \\
Greater-depth ${ }^{\text {a burial }}$ & No & Below & Thick \\
Greater-depth ${ }^{\mathrm{a}}$ boreholes & No & Below & Thick \\
Covered structure & Yes & Above & Thin \\
Buried structure & Yes & Below & Thin \\
Greater-depth ${ }^{\text {a structure }}$ & Yes & Below & Thick \\
Covered modules & Modular & Above & Thin \\
Buried modules & Modular & Below & Thin
\end{tabular}

a. As used in this document, "greater-depth" refers to disposal such that the top of the uppermost waste package is at least $5 \mathrm{~m}$ belowgrade.

It is conceivable to use one technology at the MLLWDF to dispose of all eight types of waste. It is also conceivable: to use a different technology to dispose of each of the eight types of waste. The number of possible combinations of technologies is extremely large. Only a few combinations could be evaluated in this study.

Table 4-4 lists the combinations of disposal technologies that were selected for evaluation. The technology for disposing of each of the eight types of waste is indicated, using a set of abbreviated designators for the six technologies selected from Table 4-3.

The rationale for pursuing these six combinations is as follows. The first combination, indicated in the first row of Table 4-4, is based on achieving compliance by use of the minimum technology and by the minimum investment of resources.

The technology combination in the second row is similar to that in the first row, except shallow land burial is replaced by greater-depth burial to provide an improvement in waste isolation.

The third and fourth rows involve using, respectively, abovegrade and belowgrade engineered structures for all types of waste. These combinations provide the ultimate in waste confinement. The argument can be made that using engineered structures for Class $\mathrm{A}$ and Class $\mathrm{A}$ mixed waste is overkill. On the other hand, these combinations have an advantage in improved public perception because the lower hazards represented in Class $\mathrm{A}$ and $\mathrm{A}$ mixed waste are not apparent to some members of the public. 
Table 4-4. Combinations of disposal technologies evaluated for the MLLWDF alternative. ${ }^{a}$

\begin{tabular}{|c|c|c|c|c|c|c|c|c|}
\hline $\begin{array}{c}\text { Designator } \\
\text { for } \\
\text { combination }\end{array}$ & $\begin{array}{c}\text { Class } \\
\text { A }\end{array}$ & $\begin{array}{c}\text { Class } \\
\text { B }\end{array}$ & $\begin{array}{c}\text { Class } \\
\mathrm{C} \\
\beta-\gamma\end{array}$ & $\begin{array}{c}\text { Class } \\
\text { C } \\
\alpha\end{array}$ & $\begin{array}{c}\text { Class } \\
\text { A } \\
\text { mixed }\end{array}$ & $\begin{array}{c}\text { Class } \\
\text { B } \\
\text { mixed }\end{array}$ & $\begin{array}{c}\text { Class } \\
C \\
\beta-\gamma \\
\text { mixed }\end{array}$ & $\begin{array}{c}\text { Class } \\
\mathrm{C} \\
\alpha \\
\text { mixed }\end{array}$ \\
\hline $\begin{array}{l}\text { Shallow land } \\
\text { burial }^{\mathrm{b}} / \\
\text { (shallow) }^{\text {boreholes }}{ }^{\mathrm{b}}\end{array}$ & SLB & SBH & SBH & SLB & SLB & SBH & SBH & SLB \\
\hline $\begin{array}{l}\text { Greater- } \\
\text { depth burial/ } \\
\text { (greater- } \\
\text { depth) } \\
\text { boreholes }\end{array}$ & GDB & GDBH & GDBH & GDB & GDB & GDBH & GDBH & GDB \\
\hline $\begin{array}{l}\text { Abovegrade } \\
\text { engineered } \\
\text { structures }\end{array}$ & AGES & AGES & AGES & AGES & AGES & AGES & AGES & AGES \\
\hline $\begin{array}{l}\text { Belowgrade } \\
\text { engineered } \\
\text { structures }\end{array}$ & BGES & BGES & BGES & BGES & BGES & BGES & BGES & BGES \\
\hline $\begin{array}{l}\text { Covered } \\
\text { modules }\end{array}$ & $\mathrm{CM}$ & $\mathrm{CM}$ & $\mathrm{CM}$ & $\mathrm{CM}$ & $\mathrm{CM}$ & $\mathrm{CM}$ & $\mathrm{CM}$ & $\mathrm{CM}$ \\
\hline $\begin{array}{l}\text { Shallow land } \\
\text { burial/ } \\
\text { abovegrade } \\
\text { engineered } \\
\text { structures }\end{array}$ & SLB & AGES & AGES & AGES & SLB & AGES & AGES & AGES \\
\hline
\end{tabular}

a. Key to abbreviated designators for technologies:

$$
\begin{array}{ll}
\text { SLB }=\text { Shallow land burial } & \text { AGES }=\text { Abovegrade engineered structures } \\
\text { SBH }=\text { Shallow boreholes } & \text { BGES }=\text { Belowgrade engineered structures } \\
\text { GDB }=\text { Greater-depth burial } & \mathrm{CM}=\text { Covered modules } \\
\text { GDBH }=\text { Greater-depth boreholes } &
\end{array}
$$

b. In this combination, all types of Class $\mathrm{C}$ waste are buried such that the top of the uppermost waste package is $5 \mathrm{~m}$ below the top surface of the cover of the disposal unit. Because of the considerable thickness of the required abovegrade cover, the disposal practice can still be considered as shallow land burial. 
The fifth row in Table 4-4 involves the use of covered modules for all types of waste. This technology uses smaller waste-confinement modules rather than large structures.

The last combination indicated in Table 4-4 uses shallow land burial again for the Class $\mathrm{A}$ and Class A mixed waste, and AGES for the remaining waste types.

This set of technology combinations spans the full range of considerations: minimum technology and maximum technology; abovegrade and belowgrade structures; use of single technologies and use of multiple technologies; etc.

\subsubsection{Volume of Waste and Extent of Waste Treatment}

The fourth and fifth major factors are considered simultaneously because they interact with each other.

The fourth major factor is the volume of waste to be disposed of. It affects the disposal cost, the required surface area for the disposal site, and other items. In extreme cases, the volume could even affect the choice of a disposal technology, with high-specific-cost technologies becoming less favorable as the volume of waste increases.

The volume of waste to be disposed of depends on two parameters: the volume of waste generated and the effect of any waste treatment on the volume.

As discussed in Section 2, there are considerable uncertainties in the volumes of waste to be generated. In particular, the volume of waste generated as a result of the remediation of facilities (D\&D) and of outside areas (ER) is quite uncertain. A scenario involving a major expansion in the D\&D program and increases in waste from the ER activities was evaluated. A major expansion in the D\&D program, however, is considered unlikely.

The future treatment of INEL waste is not known at this time. The extent of treatment assumed for the waste requiring disposal affects not only the volume of waste requiring disposal, but also the isolation performance of a disposal system that includes the waste form as one of the isolation barriers. One treatment philosophy is the "maximum-treatment" philosophy, in which all waste is treated that is practical to treat. The intent is to maximize isolation performance and minimize disposal volume. In the "minimum-treatment" philosophy, treatment is performed only where clearly required by regulations or DOE Orders. The intent is to minimize treatment cost while complying with the waste-product requirements.

In evaluating conceptual disposal facilities, good engineering practice would call for the majority of the evaluations to be performed on a conservative design that would accommodate all the waste that would reasonably be expected for disposal. Brief, bounding evaluations would be performed on worst-case, upper limit waste volumes, even though the scenario might be highly unlikely. Also, brief, bounding evaluations would be performed on the most favorable scenario of having to provide disposal space for the smallest potential volume of waste. As explained below, this approach was followed in selecting waste generation/waste treatment scenarios for evaluation, since limited resources did not allow the full evaluation of all possible scenarios. 
Table 2-5 presents the waste volumes requiring disposal under the various waste

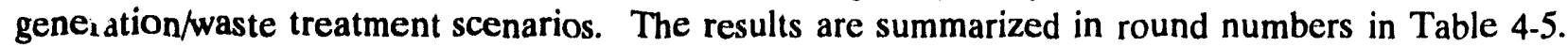
Only the total annual volumes of all types of waste are indicated.

The minimum-generation/maximum-treatment scenario results in the smallest volume of waste for disposal, $1,211 \mathrm{~m}^{3} / \mathrm{yr}$. It is the most optimistic scenario in terms of placing the smallest requirements on the disposal facility. It will be evaluated as a best-case, bounding scenario.

The maximum-generation/minimum-treatment scenario results in the largest volume of waste for disposal, $8,575 \mathrm{~m}^{3} / \mathrm{yr}$. (The large uncertainty in the amount of waste requiring disposal is evident by comparing this upper-limit number with the lower-limit number just discussed; the two numbers differ by a factor of approximately seven.) This scenario is the most pessimistic in terms of placing the greatest requirements on the disposal facility. It is considered to be an unlikely scenario but will be evaluated as a worst-case, bounding scenario.

The two remaining scenarios are intermediate in the volume of waste requiring disposal. Either one could be chosen as the baseline case for use in all of the evaluations other than the bounding scenarios. The maximum-generation/maximum-treatment scenario $\left(3,761 \mathrm{~m}^{3}\right)$ was selected for evaluation. The other scenario (minimum generation/minimum treatment) will not be evaluated. Both scenarios would result in similar volumes of waste requiring disposal, well within the uncertainties of this preconceptual study.

The scenarios that will be evaluated enable a comparison of the effects of waste generation on disposal requirements, by comparing the results for scenarios representing the two entries in the lcft column. The effects of waste treatment on disposal requirements will be evident by comparing the results for scenarios representing the two entries in the bottom row. Thus, the approach allows the basic comparisons of major factors to be made without evaluating all possible combinations in depth.

\subsubsection{Complete Set of Subalternatives}

The complete set of subalternatives selected for study is listed and categorized in Table 4-6. The assumptions made concerning the major factors are indicated for each subalternative.

Subalternatives $1 \mathrm{~A}$ through $1 \mathrm{~F}$ were formulated by using the six combinations of technologies listed in Table 4-4 (albeit in revised sequence with the baseline AGES disposal technology first). These subalternatives are all based on the high projections of waste volume and on the maximumtreatment philosophy. The intent of Subalternative $1 \mathrm{G}$ is to illustrate the effects of waste generation on disposal. Subalternative $1 G$ also results in the minimum waste volume expected for disposal. The intent of Subalternative $1 \mathrm{H}$ is to illustrate the effects of waste treatment. Subalternative $1 \mathrm{H}$ also results in the maximum volume of waste expected for disposal.

For convenience, the corresponding parameters for Alternatives 2 through 5 are also listed in Table 4-6. 
Table 4-5. Total annual volumes $\left(\mathrm{m}^{3}\right)$ of waste requiring disposal for various scenarios of waste generation and waste treatment.

\begin{tabular}{ccc}
\hline $\begin{array}{c}\text { Waste generation/waste } \\
\text { treatment scenario }\end{array}$ & $\begin{array}{c}\text { Maximum treatment } \\
\left(\mathrm{m}^{3} / \mathrm{yr}\right)\end{array}$ & $\begin{array}{c}\text { Minimum treatment } \\
\left(\mathrm{m}^{3} / \mathrm{yr}\right)\end{array}$ \\
\hline $\begin{array}{c}\text { Minimum Generation (Small } \\
\text { ER and D\&D) }\end{array}$ & 1,211 & 4,437 \\
$\begin{array}{c}\text { Maximum Generation (Large } \\
\text { ER and D\&D) }\end{array}$ & 3,761 & 8,575 \\
\hline
\end{tabular}


Table 4-6. Subalternatives of Alternative 1 selected for evaluation, as well as other alternatives.

\begin{tabular}{|c|c|c|c|c|}
\hline $\begin{array}{l}\text { Alt. or } \\
\text { Subalt. }\end{array}$ & $\begin{array}{l}\text { Description, or } \\
\text { purpose of } \\
\text { subalternative }\end{array}$ & Disposal technologies & $\begin{array}{c}\text { Waste } \\
\text { generation }\end{array}$ & $\begin{array}{c}\text { Waste } \\
\text { treatment } \\
\text { philosophy }\end{array}$ \\
\hline $1 \mathrm{~A}$ & Baseline case & AGES & High & Maximum \\
\hline $1 B$ & Different technology & BGES & High & Maximum \\
\hline $1 \mathrm{C}$ & Different technology & Covered modules & High & Maximum \\
\hline $1 \mathrm{D}$ & Different technology & $\begin{array}{l}\text { Greater- } \\
\text { depth burial/ } \\
\text { borcholes }\end{array}$ & High & Maximum \\
\hline $1 E$ & Different technology & $\begin{array}{c}\text { Shallow land burial/ } \\
\text { boreholes }\end{array}$ & High & Maximum \\
\hline $1 \mathrm{~F}$ & $\begin{array}{l}\text { Different technology } \\
\text { combination }\end{array}$ & $\begin{array}{c}\text { Shallow land burial/ } \\
\text { AGES }\end{array}$ & High & Maximum \\
\hline $1 G$ & $\begin{array}{c}\text { Effect of waste } \\
\text { generation (minimum- } \\
\text { volume scenario) }\end{array}$ & AGES & Low & Maximum \\
\hline $1 \mathrm{H}$ & $\begin{array}{l}\text { Effect of waste } \\
\text { treatment (maximum- } \\
\text { volume scenario) }\end{array}$ & AGES & High & Minimum \\
\hline 2 & $\begin{array}{c}\text { Continue disposal at } \\
\text { RWMC }\end{array}$ & $\begin{array}{l}\text { Covered placement/ } \\
\text { shallow boreholes }\end{array}$ & High & Maximum \\
\hline 3 & Ship offsite & $\begin{array}{l}\text { Shallow land } \\
\text { burial/boreholes }\end{array}$ & High & Maximum \\
\hline $4 \mathrm{~A}$ & $\begin{array}{l}\text { MLLWDF and ship } \\
\text { offsite }\end{array}$ & $\begin{array}{c}\text { AGES// } \\
\text { shallow land } \\
\text { burial/boreholes }\end{array}$ & High & Maximum \\
\hline $4 B$ & $\begin{array}{c}\text { Continue RWMC and } \\
\text { ship offsite }\end{array}$ & $\begin{array}{c}\text { Covered placement/ } \\
\text { shallow boreholes// } \\
\text { shallow land } \\
\text { burial/boreholes }\end{array}$ & High & Maximum \\
\hline 5 & No action & Storage only & High & Maximum \\
\hline
\end{tabular}




\subsection{References}

Cook, J. R., et al., 1987, Environmental Information Document: New Low-level Radioactive Waste Storage/Disposal Facilities at the Savannah River Plant, E.I. DuPont de Nemours \& Co., DPST85-862, April 1987.

Hillary, J. M., 1992a, Regulatory Siting Requirements for the Proposed Mixed and Low-Level Waste Disposal Facility at the INEL, EGG-WMO-10250, May 1992.

Hillary, J. M., 1992b, Siting Criteria for the Proposed Mixed and Low-Level Waste Disposal Facility at the INEL, EGG-WMO-10440, September 1992.

Sherick, M. J. and R. A. Winston, 1992, INEL Mixed and Low-Level Waste Disposal Facility Technical Project Plan and Schedule, Draft, EGG-WM-10479, September 1992. 


\section{DESCRIPTION OF ALTERNATIVES}

\subsection{Introduction}

In order to provide a reasonable basis for evaluating the alternatives summarized in Section 4, they are described in more detail in this section.

For each alternative and subalternative, this section describes how the waste would be handled and emplaced and how isolation would be provided. The facilities that would be constructed to provide these functions are described first. Then the operations to emplace the waste are described. Finally, the closure and postclosure activities are described. Figure 4-1 shows the time phases of waste disposal.

The basis for the choice of waste isolation concepts is discussed briefly in Section 5.2. Then, starting with Section 5.3, the alternatives are described in alphanumerical sequence.

It is stressc 1 that an in-depth engineering analysis of any alternative or subalternative will likely result in changes in the details of the waste handling and waste isolation concepts presented here. These designs are highly simplified and are intended only to be feasible designs to implement a concept. The designs are considered to provide an adequate basis for overview comparisons of alternatives at the preconceptual stage.

For brevity, Alternative 1, Construct and Operate MLLWDF, was used as a baseline in the descriptions of the subsequent alternatives. Descriptive material that applies to subsequent alternatives is not repeated in detail in the text. The same approach was used for the subalternatives within Alternative 1. Subaltcrnative 1A, Abovegrade Engineered Structures, was used as a baseline for the descriptions of the subsequent subalternatives.

In the description of Subalternative $1 \mathrm{~A}$, key design or other requiremenis are highlighted. It is frequently stated therein that the facility or the operations will incorporate some feature required by a particular citation in DOE Order $5820.2 \mathrm{~A}$ or 40 CFR 264 or 10 CFR 61. The citations from 10 CFR 61 are listed simply to indicate why the feature is included in the design. Section 2.3 discussed DOE's plans to include technical requirements of 10 CFR 61 in a future revision of DOE Order 5820.2A. There is no intent here to imply that DOE is required to comply with NRC regulatory requirements, nor that the revised DOE Order will exactly match the technical requirements of 10 CFR 61.

For simplicity, the alternatives are described in the present tense although they are only preconceptual at this time.

\subsection{Basis for Disposal Design Concepts Evaluated}

Disposal of LLY' generally has been performed by shallow land burial in pits, trenches, and shallow boreholes. How ... r, in 1969, a disposal facility began operation in France using engineered disposal structures. In the U.S., interest in enhanced disposal technologies for LLW increased in the mid-1980s. 
No permitted disposal facilities for low-level mixed waste of significant activity exist in this country. Hazardous waste disposal technology has been based on shallow land burial with liners and leachate detection and collection systems.

To provide perspective on the status of disposal technology for LLW and mixed waste, a brief literature review was performed. The review covered both U.S. and European technologies, both proposed and operating facilities, and both DOE and commercial activities. The review was not intended to provide a complete compendium of the available technologies. Rather, it provides a snapshot of enhanced technologies in use or under development.

The results of the brief literature survey appear in Table 5-1. For each waste-disposal design concept, the following information is given, as available in the literature: types of waste accommodated, type of disposal concept, the barriers that provide isolation (containers, intermcdiate barrier, outer barrier, and cover), and the referenced documents that provided the information.

The choice of disposal design concepts for evaluation in this study was based on the desire to evaluate a broad spectrum of current and proposed technologies potentially suitable for INEL waste. The choice reflects the information compiled in Table 5-1. In addition, many of the design concepts proposed in this section are adapted from the sources ciled in Table 5-1. The selection of preliminary values for disposal isolation parameters (c.g., structural wall thicknesses) was based on engineering judgment in adapting the information of Table 5-1 for use with the INEL waste and the INEL site conditions.

For convenience in reading the remainder of this section, Table 5-2 summarizes the key design parameters of the various alternatives and subalternatives. The emphasis is on the isolation barriers chosen. The format of Table 5-2 closely follows that of Table 5-1, to facilitate easy comparisons.

The performance requirements and other requirements that the alternatives must meet are discussed primarily in Section 2.3. Those requirements relate to two functions each alternative must perform: (a) safely handle and emplace the waste, (b) isolate the waste from populations and the environment. None of the alternatives can guarantee total containment of the waste for as long as it remains radioactive. Instead, the established regulatory requirements lead to a high degree of confidence that the risk to populations and the environment from compliant facilitics will be acceptably small.

Three key isolation needs affect the selection of disposal system designs. Two of the needs are requirements from $10 \mathrm{CFR} 61$. They are the requirement for 300 -year structural stability for Class $\mathrm{B}$ and Class $\mathrm{C}$ waste, and the requirement for 500 -year intruder protection for Class $\mathrm{C}$ waste. The third need is not a regulatory requirement, but reflects the fact that the dose-controlling radionuclides in Class $C \alpha$ waste have half-lives much longer than the 500 -year period cited above. It is difficult to provide convincing evidence that engin ered barriers can provide the additional protection, if needed per the performance assessment, for is lation periods of tens of thousands of years. Enhanced waste forms may have to be relied upon to provide isolation over such long time periods, or concentrations of long-lived radionuclides may be limited such that the risk is acceptable without accounting for engineered barriers. 
Table 5-1. Comparison of waste-disposal design concepts in the literature that are relevant to this study. ${ }^{\mathrm{a}}$

\begin{tabular}{|c|c|c|c|c|c|c|c|c|}
\hline References & Organization & $\begin{array}{l}\text { Type of } \\
\text { waste }\end{array}$ & $\begin{array}{l}\text { Type of } \\
\text { disposal } \\
\text { concept }\end{array}$ & Containers & Intermediate barrier & Outer barrier & Cover materials ${ }^{b}$ & Additional notes \\
\hline $\begin{array}{l}\text { Darnell } 1990 ; \\
\text { Darnell et al. } \\
1990\end{array}$ & $\begin{array}{l}\text { EG\&G } \\
\text { Idaho }\end{array}$ & $\begin{array}{l}\text { LLW; } \\
\text { mixed } \\
\text { waste } \\
\text { added } \\
\text { later }\end{array}$ & AGES & $\begin{array}{l}\text { Sulfur-polymer } \\
\text { cemented or } \\
\text { vitrified waste } \\
\text { in } 12 \text {-gauge } \\
\text { cubic carbon } \\
\text { steel boxes, } 1 \\
\mathrm{~m} \text { on a side }\end{array}$ & None & $\begin{array}{l}\text { Grouted reinforced } \\
\text { concrete vaults } \\
\text { (Type V Portland } \\
\text { cement), } 72 \times 16.5 \\
\times 9.6 \mathrm{~m} \text {; roof } 91- \\
122 \mathrm{~cm} \text {, floor and } \\
\text { walls } 107 \mathrm{~cm}\end{array}$ & $\begin{array}{l}\text { 12-in. topsoil } \\
24 \text {-in. rocky soil } \\
20 \text {-in. fine sand } \\
12 \text {-in. fine gravel } \\
80 \text {-in. clay/sand } \\
\text { 12-in. sand } \\
\text { imp. membrane; } \\
\text { then, on sides, } \\
\text { gravel } \\
\text { imp. membrane } \\
\text { gravel }\end{array}$ & $\begin{array}{l}\text { All waste treated } \\
\text { and grouted; } \\
\text { impervious } \\
\text { membrane on } \\
\text { waste stacks in } \\
\text { vault }\end{array}$ \\
\hline Dodge et al. 1989 & $\begin{array}{l}\text { REECO } \\
\text { (Nevada Test } \\
\text { Site) }\end{array}$ & $\begin{array}{l}\text { Mixed } \\
\text { waste }\end{array}$ & SLB & NS & $\begin{array}{l}\text { None in reference; } 2 \\
\text { membrane liners } \\
\text { added in current } \\
\text { plans }\end{array}$ & $\begin{array}{l}\text { Geologic barriers } \\
\text { surrounding pit } \\
1,080 \times 380 \times 30 \mathrm{ft}\end{array}$ & 9 to $13-\mathrm{ft}$ soil & \\
\hline $\begin{array}{l}\text { Van Cote 1982; } \\
\text { Convert } 1992\end{array}$ & $\begin{array}{l}\text { France } \\
\text { (Centre de la } \\
\text { Manche) }\end{array}$ & LLW & $\begin{array}{l}\text { BGES } \\
\text { (bottom } \\
\text { layer) }\end{array}$ & NS & $\begin{array}{l}\text { Some drums are } \\
\text { concrete-grouted in } \\
\text { concrete boxes with } \\
\text { walls } 10,20 \text {, or } \\
30 \mathrm{~cm} \text { thick }\end{array}$ & $\begin{array}{l}\text { Reinforced-concrete } \\
\text { trench vaults, } \\
\text { backfilled with } \\
\text { concrete }\end{array}$ & $\begin{array}{l}\text { Second tier of } \\
\text { waste containers } \\
\text { (see next row } \\
\text { entry below) } \\
\text { forms the cover }\end{array}$ & $\begin{array}{l}\text { "Intermediate- } \\
\text { level" waste is } \\
\text { disposed of in this } \\
\text { manner }\end{array}$ \\
\hline $\begin{array}{l}\text { Van Cote 1982; } \\
\text { Convert } 1992\end{array}$ & $\begin{array}{l}\text { France } \\
\text { (Centre de la } \\
\text { Manche) }\end{array}$ & LLW & $\begin{array}{l}\text { Covered } \\
\text { placement, } \\
\text { or tumuli } \\
\text { (top layer) }\end{array}$ & NS & $\begin{array}{l}\text { Some drums are } \\
\text { concrete-grouted in } \\
\text { concrete boxes with } \\
\text { walls } 10,20 \text {, or } \\
30 \mathrm{~cm} \text { thick }\end{array}$ & $\begin{array}{l}\text { Cover; see entry at } \\
\text { right }\end{array}$ & $\begin{array}{l}\text { topsoil } \\
\text { backfill } \\
\text { sand } \\
\text { clay } \\
\text { sand } \\
\text { geomembrane } \\
\text { sand } \\
\text { bactffill; } \\
\text { total thickness is } \\
4 \mathrm{~m}\end{array}$ & $\begin{array}{l}\text { Lower-radiation } \\
\text { waste, and waste } \\
\text { grouted in } \\
\text { concrete boxes } \\
\text { are disposed of in } \\
\text { this manner }\end{array}$ \\
\hline
\end{tabular}


Table 5-1. (continued).

\begin{tabular}{|c|c|c|c|c|c|c|c|c|}
\hline References & Organization & $\begin{array}{l}\text { Type of } \\
\text { waste }\end{array}$ & $\begin{array}{l}\text { Type of } \\
\text { disposal } \\
\text { concept }\end{array}$ & Containers & Intermediate barrier & Outer barrier & Cover materials ${ }^{b}$ & Additional notes \\
\hline $\begin{array}{l}\text { Anderson et al. } \\
1990 \text {; Anderson } \\
\text { et al. } 1992\end{array}$ & $\begin{array}{l}\text { Chem- } \\
\text { Nuclear } \\
\text { Systems, Inc. } \\
\text { (commercial } \\
\text { LLW sites in } \\
\text { IL, NC, PA) }\end{array}$ & LLW & AGES & Drums & $\begin{array}{l}\text { Cement-grouted in } 7 \\
\text { to } 10 \text {-in. thick, } \\
\text { reinforced concrete } \\
\text { overpacks, } 150 \text { to } \\
400 \mathrm{ft}^{3} \text { each; } \\
\text { cylindrical with } 10 \text { to } \\
12 \text {-mm plastic liner } \\
\text { for Class B and C } \\
\text { waste, rectangular } \\
\text { for Class A }\end{array}$ & $\begin{array}{l}\text { Plastic-coated, } \\
\text { trick-walled } \\
\text { reinforced concrete } \\
\text { vaults, } 50,000 \text { to } \\
100,000 \mathrm{ft}^{3} \text { each; } \\
\text { backfilled with } \\
\text { sand; sealed with } \\
\text { concrete }\end{array}$ & $\begin{array}{l}\text { Topsoil } \\
\text { drainage layer } \\
\text { membrane liner } \\
\text { clay liner } \\
\text { backfill; } \\
\text { total thickness is } \\
7 \text { to } 10 \mathrm{ft}\end{array}$ & $\begin{array}{l}\text { Engineered } \\
\text { barriers required } \\
\text { by law in the } 3 \\
\text { States; Pozzo!anic } \\
\text { cement used; } \\
\text { epoxy coating on } \\
\text { rebar }\end{array}$ \\
\hline $\begin{array}{l}\text { Wells and Bailey } \\
1991\end{array}$ & $\begin{array}{l}\text { Savannah } \\
\text { River Si:e }\end{array}$ & $\begin{array}{l}\text { Liquid } \\
\text { mixed } \\
\text { waste }\end{array}$ & AGES & $\begin{array}{l}\text { Liquid mixed } \\
\text { with } \\
\text { cementitious } \\
\text { solids to form } \\
\text { grout }\end{array}$ & $\begin{array}{l}\text { Double membrane } \\
\text { liner, } 80 \text { mil and } 60 \\
\text { mil with drainage } \\
\text { net between; } \\
\text { protected by } 2 \text { in. of } \\
\text { concrete }\end{array}$ & $\begin{array}{l}\text { Reinforced } \\
\text { concrete vaults, } \\
\text { floor } 24 \text { in. thick, } \\
\text { walls } 18 \text { in. thick; } \\
200 \times 50 \times 25 \mathrm{ft}\end{array}$ & $\begin{array}{l}24 \text { in. of soil } \\
\text { geotextile } \\
12 \text { in. of sand } \\
60 \text { mil liner/ } \\
23 \text { in. of clay }\end{array}$ & \\
\hline $\begin{array}{l}\text { Wells and Bailey, } \\
1991 \text {; Freitag et } \\
\text { a!. } 1992\end{array}$ & $\begin{array}{l}\text { Savannah } \\
\text { River Site }\end{array}$ & $\begin{array}{l}\text { Solid } \\
\text { mixed } \\
\text { waste and } \\
\text { hazardous } \\
\text { waste }\end{array}$ & AGES & $\begin{array}{l}\text { Concrete } \\
\text { boxes, } 6 \times 4 \times \\
4 \mathrm{ft} \text {; some } \\
\text { palletized } \\
\text { drums }\end{array}$ & $\begin{array}{l}\text { Double membrane } \\
\text { liner, } 80 \text { mil and } 60 \\
\text { mil with drainage } \\
\text { net between; } \\
\text { protected by } 2 \text { in. of } \\
\text { concrete (Wells and } \\
\text { Bailey } 1991 \text { ) or } 12 \\
\text { in. of sand (Freitag } \\
\text { et al. 1992) }\end{array}$ & $\begin{array}{l}\text { Reinforced } \\
\text { concrete vaults, } \\
\text { floor } 24 \text { in. thick, } \\
\text { walls } 30 \text { in. thick; } \\
200 \times 50 \times 25 \mathrm{ft}\end{array}$ & $\begin{array}{l}24 \text { in. of soil } \\
\text { geotextile } \\
12 \text { in. of sand } \\
60 \text { mil liner/ } \\
23 \text { in. of clay }\end{array}$ & \\
\hline $\begin{array}{l}\text { Deold and } \\
\text { Coleman 1991; } \\
\text { Gunning et al. } \\
1992\end{array}$ & $\begin{array}{l}\text { U.S. Ecology } \\
\text { (Nebraska } \\
\text { commercial } \\
\text { LLW site) }\end{array}$ & LLW & AGES & NS & $\begin{array}{l}\text { Class A: None, Class } \\
B \text { and } C: \text { NS }\end{array}$ & $\begin{array}{l}\text { Reinforced } \\
\text { concrete vaults, } 280 \\
\times 60 \times 20 \mathrm{ft} \text { for } \\
\text { Class A, } 300 \times 60 \times \\
30 \mathrm{ft} \text { for Class B } \\
\text { and C; base } 4.5 \mathrm{ft} \\
\text { thick, walls and } \\
\text { roofs } 3 \mathrm{ft} \text { thick for } \\
\mathrm{A} \text { and } 3.5 \mathrm{ft} \text { for B } \\
\text { and C }\end{array}$ & $\begin{array}{l}\text { Erosion-control } \\
\text { layer (riprap) } \\
5 \mathrm{ft} \text { of soil } \\
\text { geotextile } \\
6 \text { in. of sand } \\
1.5 \text {-ft reinforced } \\
\text { concrete } \\
3 \mathrm{ft} \text { of clay } \\
2 \mathrm{ft} \text { of sand } \\
\text { synthetic liner }\end{array}$ & $\begin{array}{l}\text { State regulations } \\
\text { preclude use of } \\
\text { SLB }\end{array}$ \\
\hline
\end{tabular}


Table 5-1. (continued).

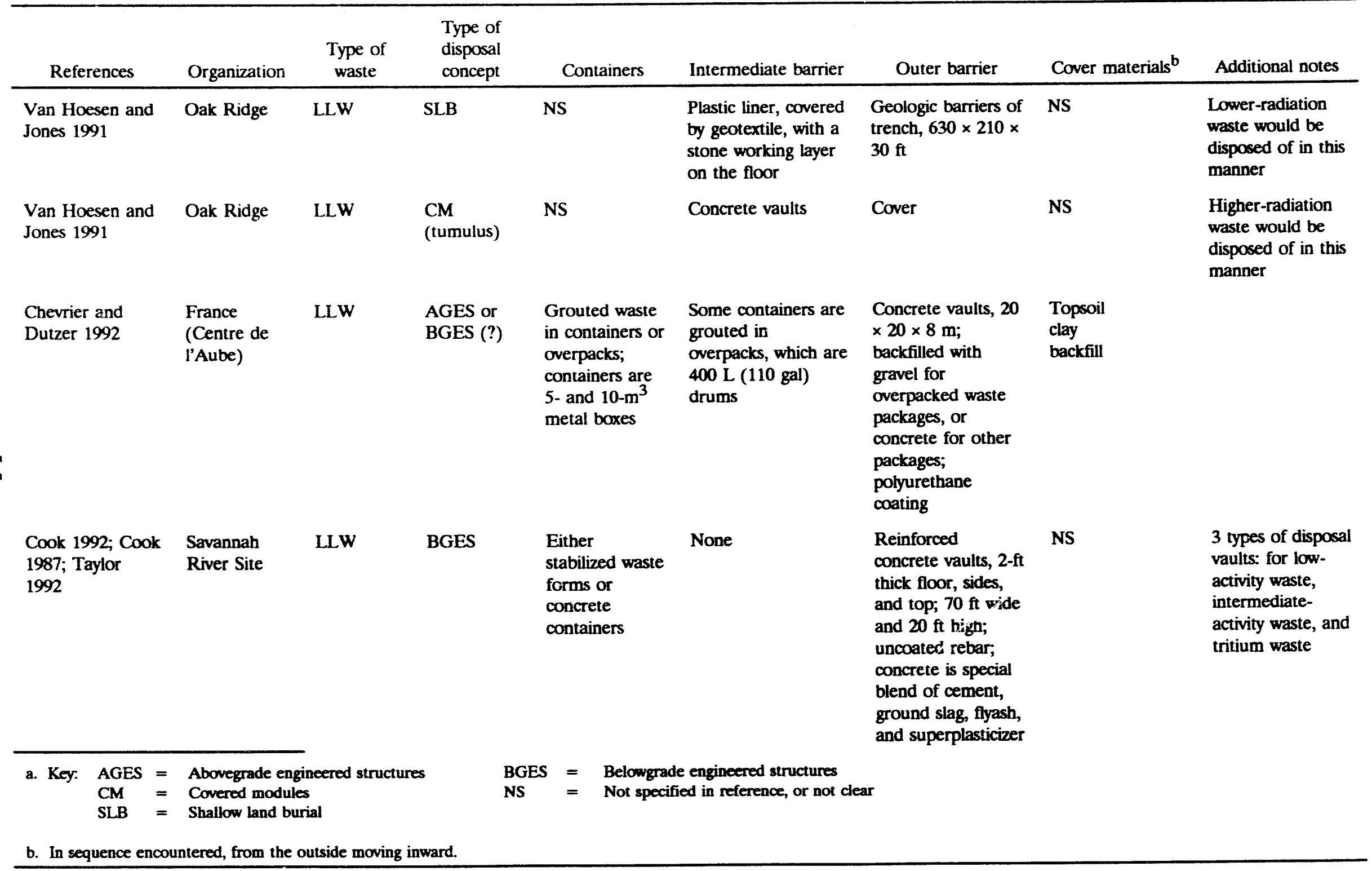


Table 5-2. Waste-disposal design concepts selected for evaluation, in terms of successive barriers to isolate the waste. ${ }^{\text {a }}$

\begin{tabular}{|c|c|c|c|c|c|c|c|}
\hline $\begin{array}{l}\text { Alt. or } \\
\text { subalt. }\end{array}$ & $\begin{array}{l}\text { Types of } \\
\text { waste }\end{array}$ & $\begin{array}{l}\text { Type of } \\
\text { disposal } \\
\text { concept }\end{array}$ & Containers & $\begin{array}{c}\text { Intermediate } \\
\text { barrier }\end{array}$ & Outer barrier $^{\text {b }}$ & $\begin{array}{c}\text { Cover } \\
\text { materials }\end{array}$ & Additional notes \\
\hline \multirow[t]{6}{*}{$1 \mathrm{~A}$} & $\mathbf{A}$ & AGES & $\begin{array}{l}\text { Standard containers, } \\
\text { as described in } \\
\text { Section } 2\end{array}$ & 2 membrane liners & $\begin{array}{l}\text { Grouted, reinforced concrete } \\
\text { vaults; 3-ft thick floor, walls, } \\
\text { and roof }\end{array}$ & Standard ${ }^{c}$ & \\
\hline & $\begin{array}{l}\mathrm{B}, \\
C_{B-\gamma}\end{array}$ & AGES & $"$ & $n$ & $n$ & " & \\
\hline & $\mathrm{Ca}$ & AGES & $"$ & " & $n$ & $n$ & $\begin{array}{l}\text { Assume waste form provides isolation } \\
\text { beyond life of other isolation barriers } \\
\text { (i.e., use IEB or equivalent waste form) }\end{array}$ \\
\hline & A mixed & AGES & $n$ & " & 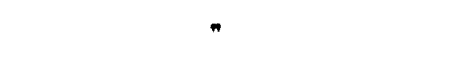 & n & \\
\hline & $\begin{array}{l}\text { B mixed, } \\
C \beta-\gamma \\
\text { mixed }\end{array}$ & AGES & $"$ & $n$ & $n$ & $n$ & \\
\hline & $\begin{array}{l}\mathrm{C} \alpha \\
\text { mixed }\end{array}$ & AGES & 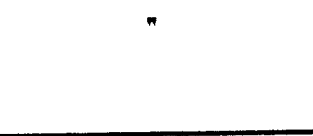 & " & $"$ & 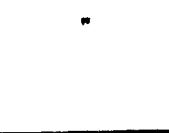 & $\begin{array}{l}\text { Assume waste form provides isolation } \\
\text { beyond life of other isolation barriers } \\
\text { (i.e., use IEB or equivalent waste form) }\end{array}$ \\
\hline \multirow[t]{6}{*}{$1 \mathrm{~B}$} & $\mathbf{A}$ & BGES & " & " & $\begin{array}{l}\text { Grouted, reinforced concrete } \\
\text { vaults; 3-ft thick foor, walls, } \\
\text { and roof, and geologic barriers }\end{array}$ & $"$ & \\
\hline & $\begin{array}{l}\mathrm{B} \\
\mathrm{C}_{\beta-\gamma}\end{array}$ & BGES & $n$ & " & $n$ & 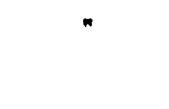 & \\
\hline & $\mathrm{C} \alpha$ & BGES & $n$ & " & $"$ & $n$ & $\begin{array}{l}\text { Assume waste form provides isclation } \\
\text { beyond life of other isolation barriers } \\
\text { (i.e., use IEB or equivalent waste form) }\end{array}$ \\
\hline & A mixed & BGES & $"$ & $n$ & $"$ & $n$ & \\
\hline & $\begin{array}{l}\text { B mixed, } \\
C \text { B-7 } \\
\text { mixed }\end{array}$ & BGES & " & " & $n$ & $"$ & \\
\hline & $\begin{array}{l}\mathrm{C} a \\
\text { mixed }\end{array}$ & BGES & " & $n$ & $"$ & $"$ & $\begin{array}{l}\text { Assume waste form provides isolation } \\
\text { beyond life of other isolation barriers } \\
\text { (i.e., use IEB or equivalent waste form) }\end{array}$ \\
\hline
\end{tabular}


Table 5-2. (continued).

\begin{tabular}{|c|c|c|c|c|c|c|c|}
\hline $\begin{array}{l}\text { Alt. or } \\
\text { subalt. }\end{array}$ & $\begin{array}{l}\text { Types of } \\
\text { waste }\end{array}$ & $\begin{array}{l}\text { Type of } \\
\text { disposal } \\
\text { concept }\end{array}$ & Containers & $\begin{array}{c}\text { Intermediate } \\
\text { barrier }\end{array}$ & Outer barrier ${ }^{b}$ & $\begin{array}{c}\text { Cover } \\
\text { materials }\end{array}$ & Additional notes \\
\hline \multirow[t]{6}{*}{$1 \mathrm{C}$} & A & $\mathbf{C M}$ & $"$ & $\begin{array}{l}\text { Grouted, } \\
\text { reinforced } \\
\text { concrete modules }\end{array}$ & 2 membrane liners & $"$ & \\
\hline & $\begin{array}{l}\mathrm{B} \\
\mathrm{C}_{B-\gamma}\end{array}$ & $\mathrm{CM}$ & 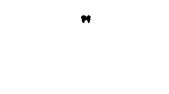 & $"$ & $"$ & " & \\
\hline & $\mathrm{C} \alpha$ & $\mathrm{CM}$ & $n$ & 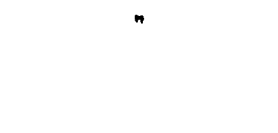 & $n$ & $"$ & $\begin{array}{l}\text { Assume waste form provides isolation } \\
\text { beyond life of other isolation barriers } \\
\text { (i.e., use IEB or equivalent waste form) }\end{array}$ \\
\hline & A mixed & $\mathrm{CM}$ & $"$ & $"$ & $"$ & $"$ & \\
\hline & $\begin{array}{l}\text { B mixed, } \\
C \text { B- } \\
\text { mixed }\end{array}$ & $\mathbf{C M}$ & $n$ & 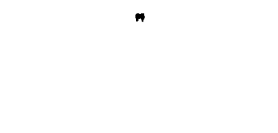 & $"$ & $n$ & \\
\hline & $\begin{array}{l}\mathrm{C} a \\
\text { mixed }\end{array}$ & $\mathrm{CM}$ & $n$ & $"$ & $n$ & $n$ & $\begin{array}{l}\text { Assume waste form provides isolation } \\
\text { beyond life of other isolation barriers } \\
\text { (i.e., use IEB or equivalent waste form) }\end{array}$ \\
\hline \multirow[t]{5}{*}{ 1D } & $\mathbf{A}$ & GDB & $"$ & 2 membrane liners & $\begin{array}{l}\text { Geologic barriers of greater- } \\
\text { depth burial }\end{array}$ & $"$ & \\
\hline & $\begin{array}{l}B \\
C_{B-7}\end{array}$ & GDBH & $*$ & $"$ & " & $n$ & \\
\hline & $\mathrm{Ca}$ & GDB & " & $"$ & $n$ & $n$ & $\begin{array}{l}\text { Assume waste form provides isolation } \\
\text { beyond life of other isolation barriers } \\
\text { (i.e., use IEB or equivalent waste form) }\end{array}$ \\
\hline & A mixed & GDB & $"$ & $"$ & 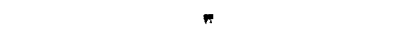 & $n$ & \\
\hline & $\begin{array}{l}B \text { mixed, } \\
C_{B-1} \\
\text { mixed }\end{array}$ & GDBH & $n$ & $n$ & $n$ & $n$ & \\
\hline
\end{tabular}


Table 5-2. (continued).

\begin{tabular}{|c|c|c|c|c|c|c|c|}
\hline $\begin{array}{l}\text { Alt. or } \\
\text { subalt. }\end{array}$ & $\begin{array}{l}\text { Types of } \\
\text { waste }\end{array}$ & $\begin{array}{l}\text { Type of } \\
\text { disposal } \\
\text { concept }\end{array}$ & Containers & $\begin{array}{c}\text { Intermediate } \\
\text { barrier }\end{array}$ & Outer barrier ${ }^{b}$ & $\begin{array}{c}\text { Cover } \\
\text { materials }\end{array}$ & Additional notes \\
\hline & $\begin{array}{l}C a \\
\text { mixed }\end{array}$ & GDB & $n$ & $n$ & $n$ & $"$ & $\begin{array}{l}\text { Assume waste form provides isolation } \\
\text { beyond life of other isolation barriers } \\
\text { (i.e., use IEB or equivalent waste form) }\end{array}$ \\
\hline \multirow[t]{6}{*}{$1 \mathrm{E}$} & $\mathbf{A}$ & SLB & $"$ & $"$ & Geologic barriers & $"$ & \\
\hline & $\begin{array}{l}\mathrm{B} \\
\mathrm{C}_{B-7}\end{array}$ & SBH & 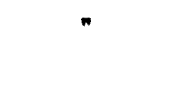 & $n$ & $n$ & 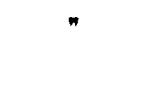 & \\
\hline & $\mathrm{C} \alpha$ & SLB & $"$ & $"$ & $"$ & $"$ & $\begin{array}{l}\text { Assume waste form provides isolation } \\
\text { beyond life of other isolation barriers } \\
\text { (i.e., use IEB or equivalent waste form) }\end{array}$ \\
\hline & $\begin{array}{l}\text { A } \\
\text { mixed }\end{array}$ & SLB & $n$ & $"$ & $"$ & $n$ & \\
\hline & $\begin{array}{l}B \text { mixed, } \\
C \text { Br } \\
\text { mixed }\end{array}$ & SBH & $n$ & " & $"$ & $n$ & \\
\hline & $\begin{array}{l}\mathrm{C} \alpha \\
\text { mixed }\end{array}$ & SLB & $"$ & - & $"$ & $"$ & $\begin{array}{l}\text { Assume waste form provides isolation } \\
\text { beyond life of other isolation barriers } \\
\text { (i.e., use IEB or equivalent waste form) }\end{array}$ \\
\hline \multirow[t]{4}{*}{$1 F$} & $\mathbf{A}$ & SLB & $"$ & $"$ & Geologic barriers & " & \\
\hline & $\begin{array}{l}\mathrm{B} \\
\mathrm{C} B-\gamma\end{array}$ & AGES & " & $n$ & $\begin{array}{c}\text { Grouted, reinforced concrete } \\
\text { vaults; } 3 \text { ft thick floor, walls, } \\
\text { and roof }\end{array}$ & $*$ & \\
\hline & $\mathrm{C} \alpha$ & AGES & $"$ & $"$ & $"$ & $"$ & $\begin{array}{l}\text { Assume waste form provides isolation } \\
\text { beyond life of other isolation barriers } \\
\text { (i.e., use IEB or equivalent waste form) }\end{array}$ \\
\hline & $\begin{array}{l}\text { A } \\
\text { mixed }\end{array}$ & SLB & $n$ & $n$ & Geologic barriers & " & \\
\hline
\end{tabular}


Table 5-2. (continued).

\begin{tabular}{|c|c|c|c|c|c|c|c|}
\hline $\begin{array}{l}\text { Alt. or } \\
\text { subalt. }\end{array}$ & $\begin{array}{l}\text { Types of } \\
\text { waste }\end{array}$ & $\begin{array}{l}\text { Type of } \\
\text { disposal } \\
\text { concept }\end{array}$ & Containers & $\begin{array}{c}\text { Intermediate } \\
\text { barrier }\end{array}$ & Outer barrier ${ }^{\mathbf{b}}$ & $\begin{array}{c}\text { Cover } \\
\text { materials }\end{array}$ & Additional notes \\
\hline & $\begin{array}{l}\text { B mixed, } \\
\text { C } \beta-\gamma \\
\text { mixed }\end{array}$ & AGES & $"$ & $"$ & $\begin{array}{l}\text { Grouted, reinforced concrete } \\
\text { vaults; 3-ft thick floor, walls, } \\
\text { and roof }\end{array}$ & $n$ & \\
\hline & $\begin{array}{l}\text { C } \alpha \\
\text { mixed }\end{array}$ & AGES & $"$ & $"$ & $"$ & 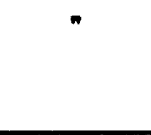 & $\begin{array}{l}\text { Assume waste form provides isolation } \\
\text { beyond life of other isolation barriers } \\
\text { (i.e., use IEB or equivalent waste form) }\end{array}$ \\
\hline $\mathbf{1 H}$ & All & $\begin{array}{l}\text { See } \\
\text { 1A }\end{array}$ & " & $\begin{array}{l}\text { See } \\
1 \mathrm{~A}\end{array}$ & $\begin{array}{l}\text { See } \\
\text { 1A }\end{array}$ & " & \\
\hline \multirow[t]{4}{*}{2} & $\mathbf{A}$ & CP & " & 2 membrane liners & See cover described at right & $n$ & \\
\hline & A mined & $\mathbf{C P}$ & $"$ & " & See cover described at right & $n$ & \\
\hline & $\begin{array}{l}\text { B mixed, } \\
\text { C } \beta-T \\
\text { mined }\end{array}$ & SBH & " & $\begin{array}{l}\text { Grouted concrete } \\
\text { liner in borehole; } \\
2 \text { membrane liners }\end{array}$ & Geologic barriers & $"$ & \\
\hline & $\begin{array}{l}\text { Ca } \\
\text { mived }\end{array}$ & $\mathbf{C P}$ & $"$ & 2 membrane liners & See cover described at right & $n$ & $\begin{array}{l}\text { Assume waste form provides isolation } \\
\text { beyond life of other isolation barriers } \\
\text { (i.e., use IEB or equivalent waste form) }\end{array}$ \\
\hline
\end{tabular}


Table 5-2. (continued).

\begin{tabular}{|c|c|c|c|c|c|c|c|}
\hline $\begin{array}{l}\text { Alt. or } \\
\text { subalt. }\end{array}$ & $\begin{array}{l}\text { Types of } \\
\text { waste }\end{array}$ & $\begin{array}{l}\text { Type of } \\
\text { disposal } \\
\text { concept }\end{array}$ & Containers & $\begin{array}{c}\text { Intermediate } \\
\text { barrier }\end{array}$ & Outer barried & $\begin{array}{c}\text { Cover } \\
\text { materials }\end{array}$ & Additional notes \\
\hline \multirow[t]{6}{*}{3} & $\mathbf{A}$ & SLB & $n$ & None & Geoiogic barriers & " & $\begin{array}{l}\text { Asoumes offite disposal technologies } \\
\text { are identical to those ued in } \\
\text { Subahernative 1E, ereept for bet of } 2 \\
\text { membrane liners. }\end{array}$ \\
\hline & $\begin{array}{l}\mathrm{B} \\
\mathrm{C}_{\beta-\gamma}\end{array}$ & SBH & $"$ & " & " & 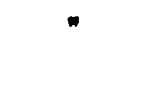 & n \\
\hline & $\mathrm{C} \alpha$ & SLB & $n$ & * & " & " & $n$ \\
\hline & A mixed & SLB & $"$ & 2 membrane liners & $"$ & - & $\begin{array}{l}\text { Ascumes offsite dispocal technologies } \\
\text { are identical to those used in } \\
\text { Subakernative IE }\end{array}$ \\
\hline & $\begin{array}{l}B, \\
C B-\gamma \\
\text { mixed }\end{array}$ & SBH & $n$ & " & $"$ & * & * \\
\hline & $\begin{array}{l}\mathrm{C} a \\
\text { mixed }\end{array}$ & SLB & " & $"$ & - & " & $n$ \\
\hline 4 & All & $\begin{array}{l}\text { Combine } \\
\text { individual } \\
\text { entries } \\
\text { from } \\
\text { respective } \\
\text { waste } \\
\text { types }\end{array}$ & " & $\begin{array}{l}\text { Combine } \\
\text { individual } \\
\text { entries } \\
\text { from } \\
\text { respective } \\
\text { waste } \\
\text { types }\end{array}$ & $\begin{array}{l}\text { Combine } \\
\text { individual } \\
\text { entries } \\
\text { from } \\
\text { respective } \\
\text { waste } \\
\text { types }\end{array}$ & " & \\
\hline
\end{tabular}


Table 5-2. (continued).

\begin{tabular}{|c|c|c|c|c|c|c|c|c|c|}
\hline $\begin{array}{l}\text { Alt. or } \\
\text { subalt. }\end{array}$ & $\begin{array}{l}\text { Type: } \\
\text { was }\end{array}$ & & $\begin{array}{l}\text { Type of } \\
\text { disposal } \\
\text { Concept }\end{array}$ & Containers & $\begin{array}{r}\text { Inte } \\
b\end{array}$ & $\begin{array}{l}\text { ermediate } \\
\text { barrier }\end{array}$ & Outer barrier $^{b}$ & $\begin{array}{c}\text { Cover } \\
\text { materials }\end{array}$ & Additional notes \\
\hline \multirow[t]{5}{*}{5} & $\mathrm{~A}_{\text {mix }}$ & & Storage & " & & None & Butler-type metal building & None & \\
\hline & $\begin{array}{l}\mathrm{B} \\
\mathrm{C}_{\beta-\gamma}\end{array}$ & & $\begin{array}{l}\text { Storage } \\
\text { in AGES }\end{array}$ & $n$ & \multicolumn{2}{|c|}{2 membrane liners } & $\begin{array}{l}\text { Grouted reinforced concrete } \\
\text { vaults; } 3 \text {-ft thick floor, } \\
\text { walls, and roof }\end{array}$ & None & $\begin{array}{l}\text { Assume waste form provides isolation } \\
\text { beyond life of other isolation barriers } \\
\text { (i.e., use IEB or equivalent waste form) }\end{array}$ \\
\hline & $\mathrm{Ca}$ & & $\begin{array}{l}\text { Storage } \\
\text { in AGES }\end{array}$ & " & \multicolumn{2}{|r|}{$"$} & $"$ & None & $n$ \\
\hline & $\begin{array}{l}\text { B mix } \\
C_{B-\gamma}\end{array}$ & & $\begin{array}{l}\text { Storage } \\
\text { in AGES }\end{array}$ & 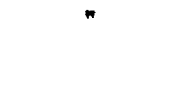 & \multicolumn{2}{|r|}{$"$} & " & None & " \\
\hline & $\begin{array}{l}\mathrm{C} a \\
\text { mixed }\end{array}$ & & $\begin{array}{l}\text { Storage } \\
\text { in AGES }\end{array}$ & $"$ & \multicolumn{2}{|r|}{ " } & " & None & \multirow[t]{2}{*}{$n$} \\
\hline a. Key: & $\begin{array}{l}\text { AGES } \\
\text { CM } \\
\text { GDB } \\
\text { SLB }\end{array}$ & $\begin{array}{l}= \\
= \\
=\end{array}$ & \multicolumn{2}{|c|}{$\begin{array}{l}\text { Abovegrade engineered structures } \\
\text { Covered modules } \\
\text { Greater-depth burial } \\
\text { Shallow land burial }\end{array}$} & \multicolumn{3}{|c|}{$\begin{array}{l}\text { BGES }=\text { Belowgrade engineered structures } \\
\text { CP } \\
\text { GDBH }=\text { Covered placement } \\
\text { IEB }=\text { Greater-depth boreholes } \\
=\text { Iron-enriched basalt }\end{array}$} & & \\
\hline
\end{tabular}




\subsection{Alternative 1: Construct and Operate MLLWDF}

This section provides a description of Alternative 1, in which a new MLLWDF would be constructed and operated. Sections 5.3.1 through 5.3.3 apply to all of the subalternatives for Alternative 1. Beginning with Section 5.3.4, the various subalternatives are individually described. Full detail is provided for Subalternative 1A. For brevity in discussing the subsequent subalternatives, features that would be the same as those for Subalternative $1 \mathrm{~A}$ are not discussed again; only features that differ from those for $1 \mathrm{~A}$ are discussed.

The facilities are designed to meet the performance and other requirements specified in Section 2.3. The facilities are also designed in accordance with DOE Order 6430.1A, "General Design Criteria," and the applicable codes and standards cited therein. In addition, guidance contained in several NRC and EPA publications (Thompson and Porter 1987; Denson et al. 1987; NRC 1991a; NRC 1991b; EPA 1989) is incorporated into many of the design features discussed in Section 5.

\subsubsection{Defining Assumptions}

This alternative involves the disposal of waste at a newly constructed facility, the MLLWDF. The defining assumptions are as follows:

- The MLLWDF is built

- The RWMC is shut down permanently when the MLLWDF begins operations

\subsubsection{Overview of Disposition of Waste Types}

Based on the detailed waste information in Section 2, an overview of the waste disposition in this alternative is as follows:

\section{Waste Disposed of:}

(For all subalternatives except for $1 \mathrm{G}$ and $1 \mathrm{H}$, Large ER and D\&D and Maximum Treatment is assumed)

$$
\begin{array}{ll}
\text { LLW } & 2,486.2 \mathrm{~m}^{3} / \mathrm{yr} \\
\text { Mixed Waste } & 1,274.5 \mathrm{~m}^{3} / \mathrm{yr}
\end{array}
$$

(For Subalternative 1G, Small ER and D\&D and Maximum Treatment are assumed)

$$
\begin{array}{lc}
\text { LLW } & 1,031.1 \mathrm{~m}^{3} / \mathrm{yr} \\
\text { Mixed Waste } & 180.4 \mathrm{~m}^{3} / \mathrm{yr}
\end{array}
$$

(For Subalternative 1H, Large ER and D\&D and Minimum Treatment are assumed)

$\begin{array}{ll}\text { LLW } & 7,300.8 \mathrm{~m}^{3} / \mathrm{yr} \\ \text { Mixed Waste } & 1,274.5 \mathrm{~m}^{3} / \mathrm{yr}\end{array}$


Waste Stored:

None

\subsubsection{Location}

As discussed in Section 4.4.2, the location of the MLLWDF affects a number of items such as the suitability of various disposal technologies, onsite shipping distances, and cost. Site selection for the MLLWDF is being addressed in separate studies (Hillary 1992a, Hillary 1992b). Various sites located on the $890 \mathrm{mi}^{2}$ of the INEL are being considered as potential candidate sites for the MLLWDF.

For the current study, three of the potential candidate sites are addressed. The baseline site is located in the central portion of the INEL. To limit the scope of the current effort, no separate subalternatives involving a different location for the MLLWDF are evaluated. Rather, at the completion of this study, a table is developed to indicate the potential suitability of the evaluated disposal technologies at the two other candidate sites.

5.3.3.1 Overview Description of Region of Interest. The region of interest, the INEL, lies in a closed topographic depression on the upper Snake River Plain. The depression is characterized as a cool, high desert that experiences relatively hot summers and cold winters. The INEL climate is semiarid, with an average annual total precipitation of $22 \mathrm{~cm}$ (Case et al. 1990).

The surface of the INEL is relatively flat-lying, sagebrush desert, with predominant relief being manifested either as volcanic buttes jutting up out of the desert floor or as unevenly surfaced basalt flows and/or flow vents and fissures. Elevations on the INEL range from 1,585 $\mathrm{m}(5,200 \mathrm{ft})$ in the northeast to $1,450 \mathrm{~m}(4,750 \mathrm{ft})$ in the southwest, with the average being $1,525 \mathrm{~m}(5,000 \mathrm{ft})$. A broad topographic ridge extends northward through the INEL. This ridge effectively separates the drainage of mountain ranges northwest of the INEL from the Snake River (Bowman et al. 1984).

In the western and northern portions of the INEL, intermittently flowing waters from the Big Lost River have created a flood plain consisting primarily of sands and gravels derived from the mountain ranges to the northwest. The Big Lost River, Little Lost River, and Birch Creek flow from the valleys between the northwestern mountain ranges to the Lost River Sinks in the northwest portion of the INEL, which acts as a closed drainage basin. There the water recharges the Snake River Plain Aquifer (Bowman et al. 1984).

The aquifer is approximatcly $320 \mathrm{~km}$ long and 48 to $97 \mathrm{~km}$ wide. It has a total estimated volume of 2,500 billion $\mathrm{m}^{3}$ of water. The direction of flow is roughly northeast to southwest, at measured rates ranging from 1.5 to $6.1 \mathrm{~m} /$ day, with an average of $3 \mathrm{~m} /$ day (Case et al. 1990).

5.3.3.2 Overview Description of Potential Disposal Sites. The locations of the three candidate sites currently of primary interest in the siting study are shown in Figure 5-1. Site A was used as the basis for the disposal design concepts developed in this study. 
DI S S

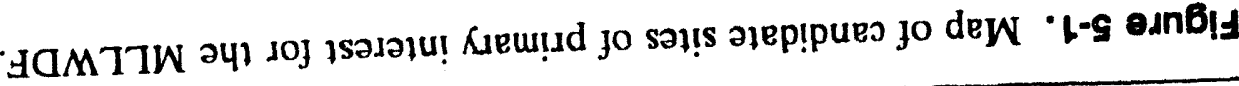

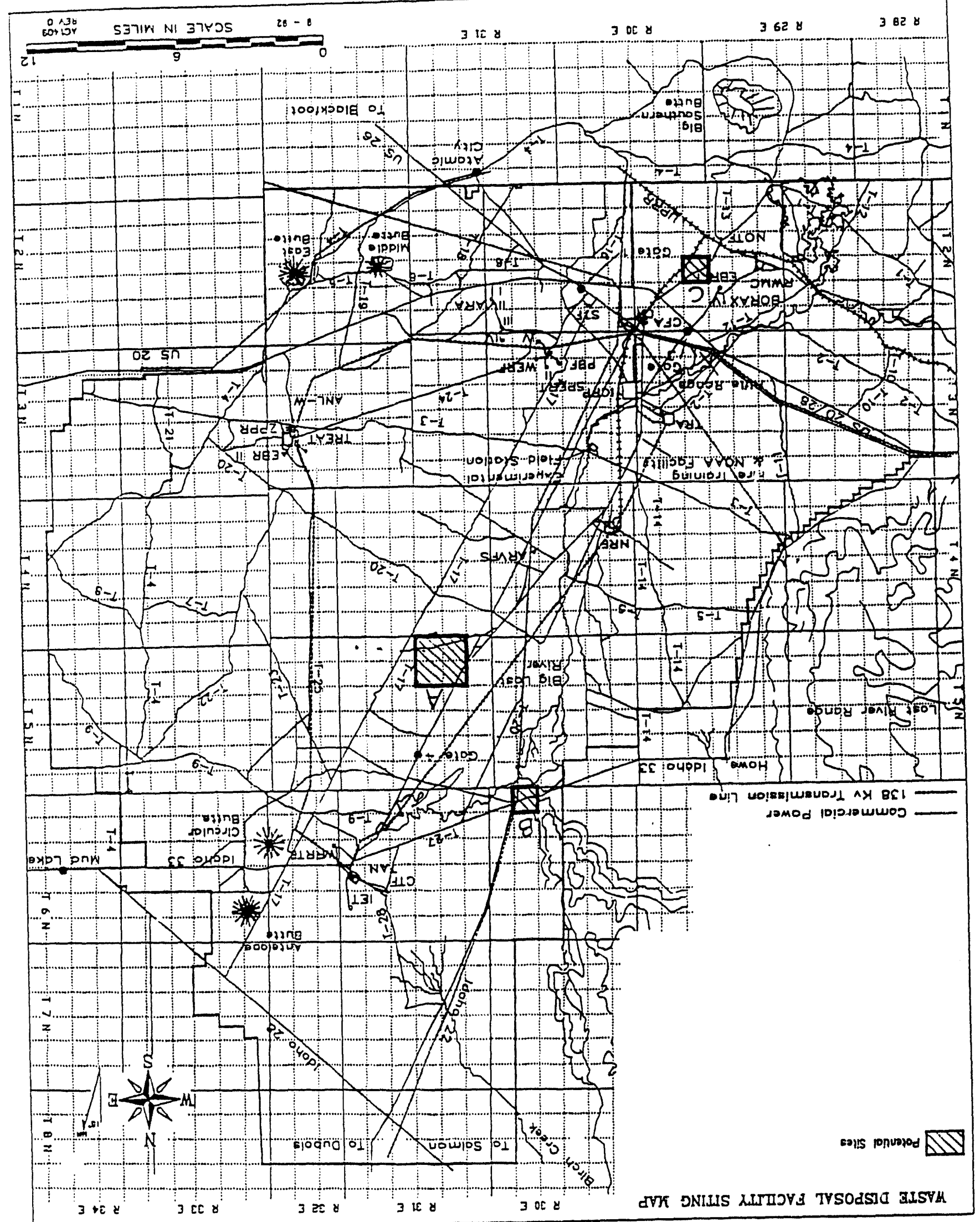


5.3.3.2.1 Site A-Site A, the baseline site for the MLLWDF, was identified in earlier siting studies. $^{\text {d }}$ Site $A$ is otherwise known as well Site 14 . Site characteristics presented here are based on the earlier studies, which consisted of (a) an office evaluation using available geohydrologic data and (b) limited field inspection. Further site characterization activities are planned to ensure that Site $A$ is suitable for the MLLWDF.

Site $A$ is located on the INEL at Township 5 North, Range 31 East, Section 28. The site is located in the central portion of the INEL and thus affords very good security. The site is located $1 \mathrm{mi}$ east of Lincoln Boulevard.

Site A has desirable natural features for a disposal site. The surface sedimentary beds, which inhibit migration of contaminants to the aquifer, extend to roughly $300 \mathrm{ft}$ before the first basalt layer is encountered. These are among the thickest continuous sedimentary beds on the INEL. The depth to the Snake River Plain Aquifer in the area ranges from 275 to $320 \mathrm{ft}$. It is estimated that contaminant migration from the site would have long flow paths to the southern INEL boundary, a distance of about $17 \mathrm{mi}$ along groundwater flow paths. Thick sedimentary beds indicate that lava flows have not covered the area of Sitc A for many thousands of years.

A potential drawback with Site $A$ is the proximity to the Big Lost River. The site is located approximately $5 \mathrm{mi}$ east of the main channel of the Big Lost River. The presence of the fine-grained sediments in the area suggests vulnerability to flooding. It has been suggested that berms could be constructed that would preclude potential flood waters of the Big Lost River from impacting the disposal site.

5.3.3.2.2 Site B-The second candidate site considered here for the MLLWDF, Site B, is located at the southern tip of the Lemhi Range, in the northwest corner of the INEL. An advantage of this location is that it is not above the Snake River Plain Aquifer, although it may not be hydraulically uncoupled from the aquifer.

The near-surface and surlace rocks are silicic volcanics. In places, the silicics are covered by shallow sediments. Limestone and quartzite rocks outcrop in the area of the tip of the Lemhi Range (Taylor 1993).

Potential drawbacks with Site B include the potential for flooding from local runoff and the presence of nearby faulting. Site B includes slight to relatively severe slopes. Many erosion channels are noted at this site. Control of surface water runon and runoff would be difficult, and much of the runoff could eventually find its way into the Snake River Plain Aquifer. Another drawback is that Site B is very close to the northwest boundary of the INEL and is near State Highways 22 and 28 (Taylor 1993).

d. Personal communication from J. T. Barraclough, U.S. Geological Survey, to C. A. Pelletier, Environmental Branch, Atomic Energy Commission, National Reactor Testing Station, November 26, 1969. 
5.3.3.2.3 Site C-The third candidate site considered here for the MLLWDF is located approximately $3 \mathrm{mi}$ east of the RWMC at Township 2 North, Range 30 East, Section 15.

Site $\mathrm{C}$ contains several contiguous expanses of relatively flat land suitable for construction. No evidence of flooding or accumulation of surface water is evident at the site. The site is more than $15 \mathrm{~km}$ from a known capable fault. Estimated depth to the aquifer at site $\mathrm{C}$ is $595 \mathrm{ft}$ (Taylor 1993).

Drawbacks with site $\mathrm{C}$ include thin, coarse-textured surface sediments, probably only 8 to $10 \mathrm{ft}$ thick, and the presence of basalt outcroppings scattered throughout the area. Also, the site is located in the area identified to be most vulncrable to lava inundation (Taylor 1993). In addition, the site is close to the site boundary.

\subsubsection{Subalternative 1A: Abovegrade Engineered Structures}

This section briefly describes the facilities constructed and the operations conducted for Subalternative $1 \mathrm{~A}$. In this subalternative, all types of waste received are disposed of in AGES.

To clarify why certain design features are provided, the principal regulatory requirements that affect the design of each feature are listed in italics at the beginning of each writeup. Some of the cited requirements apply only to the mixed waste. The other cited requirements apply to LLW, and hence apply to all the waste (whether mixed or not). In most cases, the requirements apply to all of the subalternatives and alternatives, but they are listed here for convenience. The list of requirements is not exhaustive. Provision for obtaining exemptions from particular design requirements, if the necessary approvals are obtained, is included in the regulations.

\subsubsection{Description of Facilities.}

5.3.4.1.1 General Site Layout and Preparation-Requirements: The principal requirement related to site layout is as follows:

- $\quad$ A buffer zone of land must be maintained between any buried waste and the disposal site boundary and beneath the disposed waste (10 CFR 61.52).

The general site layout used for Subalternative $1 \mathrm{~A}$ is presented in Figure 5-2. The total land area requirement for the disposal concept is 115 acres. The site includes a controlled access area where the disposal units are located and disposal operations are carried out, support facilities required for the conduct of the disposal operations, and a buffer zone.

Site preparation includes removal of sage brush, grading, and construction of access roads, drainage areas, fencing, security gates, and lighting. Site preparation would also include the installation of monitoring facilities (discussed in a later subsection), including air and water monitoring stations.

Access to the MLLWDF is from Lincoln Boulevard. A paved $1 \mathrm{mi}$ road connects the MLLWDF to Lincoln Boulevard. A patrol road is located immediately outside the outer facility fence. The road provides clear viewing of the interior of the disposal area, and provides access to 


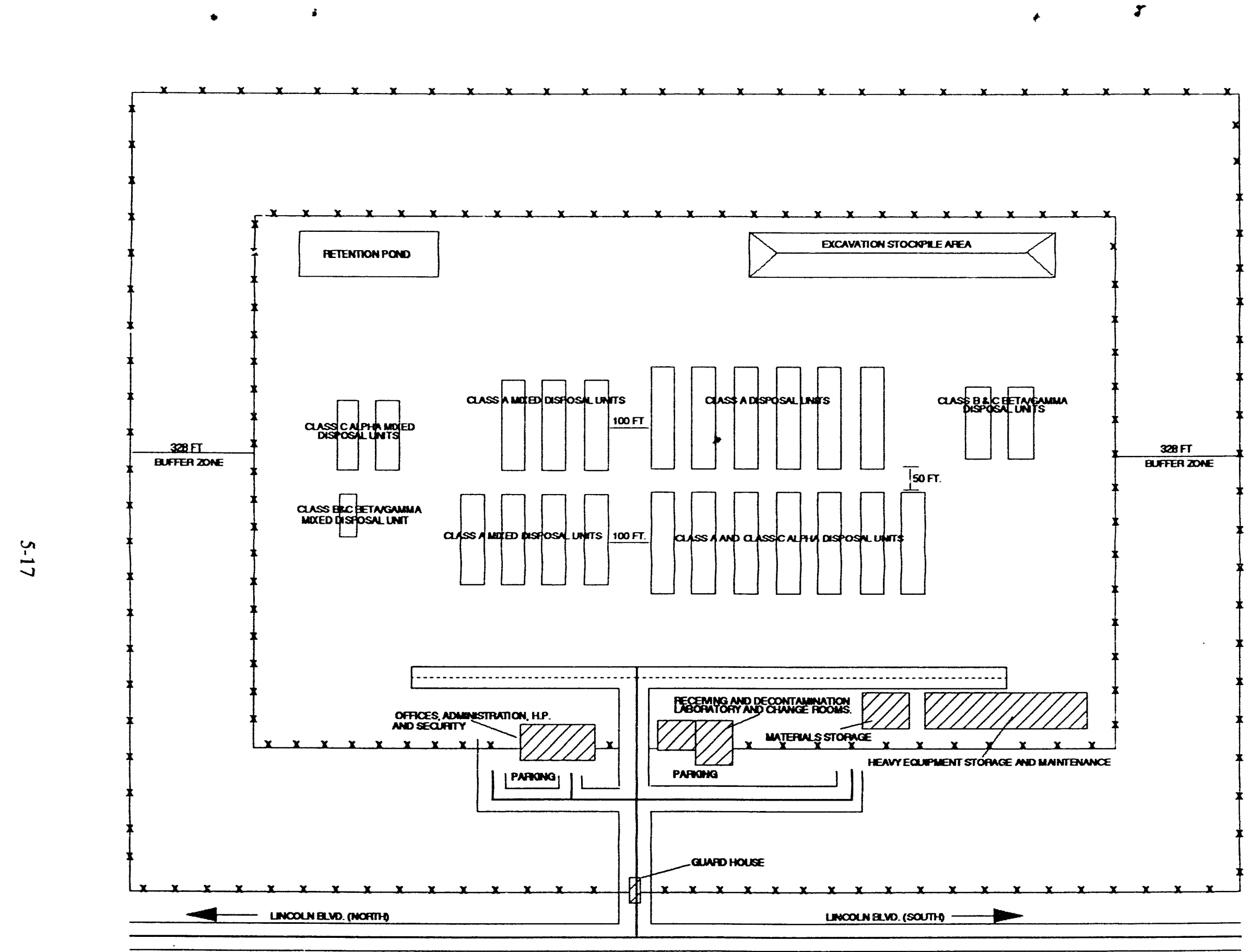

Figure 5-2. General disposal site layout for Subalternative $1 \mathrm{~A}$ (not to scale). 
perimeter air and water monitoring stations. Paved service roads are provided for access to the disposal locations of the facility. Areas are provided for parking government vehicles and evacuation buses, private vehicles, bus loading and unloading, and evacuation staging. These parking areas are located near the administrative and support areas.

Access to the area is through one main gate controlled by operating personnel. Two additional gates are provided; however, these remain locked during normal operation. External loudspeakers are installed to enable communication with drivers arriving at the main access gate.

In accordance with DOE Order $6430.1 \mathrm{~A}$, security fencing is provided to serve as a physical demarcation of the facility and an obstruction to illegal entry. Tall lighting poles are installed to make the entire area visible at night. Patrol personnel can observe clearly all areas of the disposal facility.

Support facilities include a guard house, office and administration building, support building, materials storage building, and heavy equipment storage and maintenance building. See the discussion on Support Facilities for further details.

The disposal area is a controlled access area surrounded by another security fence constructed in accordance with requirements of DOE 6430.1A. Access to the area is restricted to authorized personnel. Facilities located in the disposal area include the following:

- The disposal units for Class $\mathrm{A}$ waste and for Class B and $\mathrm{C}$ waste

- Support building

- Materials storage building

- Heavy equipment storage and maintenance building

- Disposal area drainage system

- Retention pond

- $\quad$ Fencing and gates.

The layout of the disposal units allows sufficient space between units for the movement of equipment and personnel during operations and for surface water drainage.

Disposal units for LLW and mixed waste are separated from each other by a distance of $100 \mathrm{ft}$. Also, the Class $B$ and $C$ disposal units are physically separated from disposal units for Class $A$ waste. (The minute amount of Class $\mathrm{C} \alpha$ waste is codisposed with the Class $\mathrm{C} \alpha$ mixed waste.) Refer to the section below on disposal unit construction for further details.

The Class A LLW disposal area consists of 13 separate side-loaded concrete structures in two rows. The structures are separated from each other by $20 \mathrm{ft}$. The rows are separated by $50 \mathrm{ft}$. 
The Class B and C $\beta-\gamma$ LLW disposal area consists of two separate top-loaded concrete structures. The two structures are separated from each other by $20 \mathrm{ft}$.

The Class A mixed waste disposal area consists of seven separate side-loaded concrete structures in two rows. The structures are separated from each other by $20 \mathrm{ft}$, and the rows are separated by $50 \mathrm{ft}$.

The Class B and C mixed waste disposal area consists of three separate top-loaded concrete structures. Two structures are for disposal of the Class $\mathrm{C} \alpha$ mixed waste, including the minute amount of Class $\mathrm{C} \alpha$ waste. One structure is for disposal of the Class B and C $\beta-\gamma$ mixed waste.

In accordance with 10 CFR 61.52 , a buffer zone is provided between unrestricted land areas and the disposal area. The width of the buffer zone $(100 \mathrm{~m}$ or $328 \mathrm{ft})$ will impact the results of the performance assessment. The width is based on a conceptual design report for LLW disposal (EG\&G Idaho 1987). In order to limit entry to authorized personnel and control exposures to radiation, access to the buffer zone is controlled by a security fence and a guard station.

5.3.4.1.2 Disposal Unit Construction-In this subalternative, all of the disposal units are massive, reinforced concrete structures (see Figures 5-3 and 5-4). A general lack of long-term experience with concrete structures makes it difficult to project the performance of this material beyond 50 to 100 years. Because of the challenge of producing concrete and other materials that will isolate the waste for 500 years (see the performance requirements in Section 2.3) under site-specific climatic conditions, a materials research and development program is conducted in connection with this subalternative.

This concept is a highly conservative approach to isolation of the waste. The individual waste containers and any inner liners are the first isolation barrier. The second isolation barrier is a double membrane liner for all types of waste, not just mixed waste. For simplicity, the membrane liners are not shown in the figures but are discussed later. The massive concrete structures are the third isolation barrier.

The concrete structures are intended to be the barrier to inadvertent intrusion for all the types of waste, not just Class $\mathrm{C}$ waste. Therefore, the structures are designed to provide a high degree of assurance of protection for 500 years. In addition, the concrete structures are designed to resist settling, so that the final cover will not be disrupted and possibly breached.

To help achieve this performance, the optimum formulation for the concrete is determined as a result of a development and testing program. The baseline formulation consists of Type V Portland cement, water, coarse and fine aggregate, and appropriate admixtures. An alternative is to use Type Il cement plus pozzolan and/or ground slag to protect against sulfate and toxic material attack on the concrete and chloride attack on the reinforcing steel.

At the joints of the concrete structure, joint sealants such as epoxy resins or polyurethane are used. Moisture barriers are emplaced on the outside and inside surfaces of the concrete to protect against water migration and alkali soils on the outside and against contamination and moisture on the inside (Denson et al. 1987). 


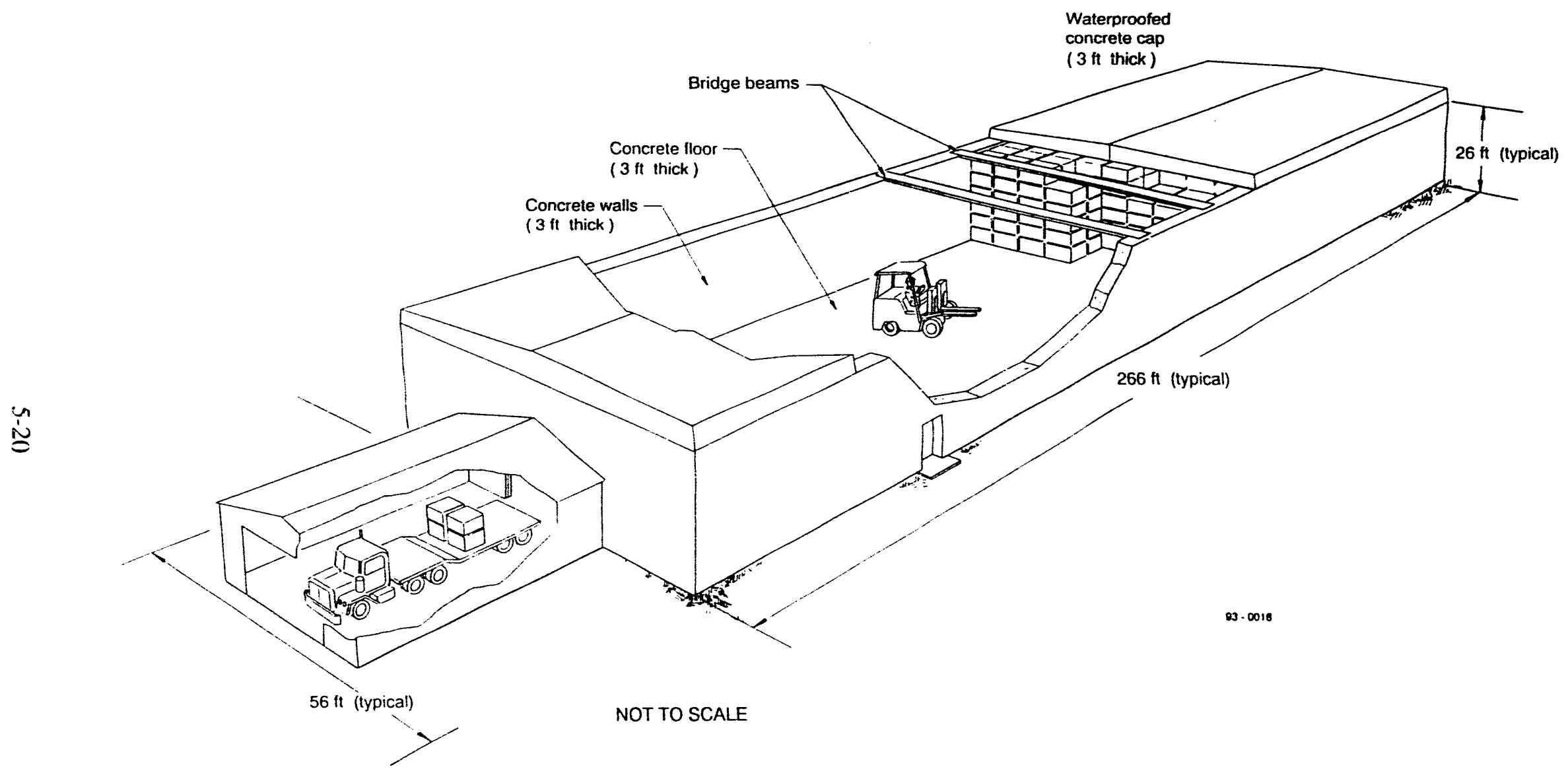

Figure 5-3. Design concept for disposal structures for contact-handled waste in Subalternative 1A.

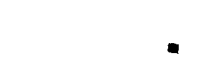




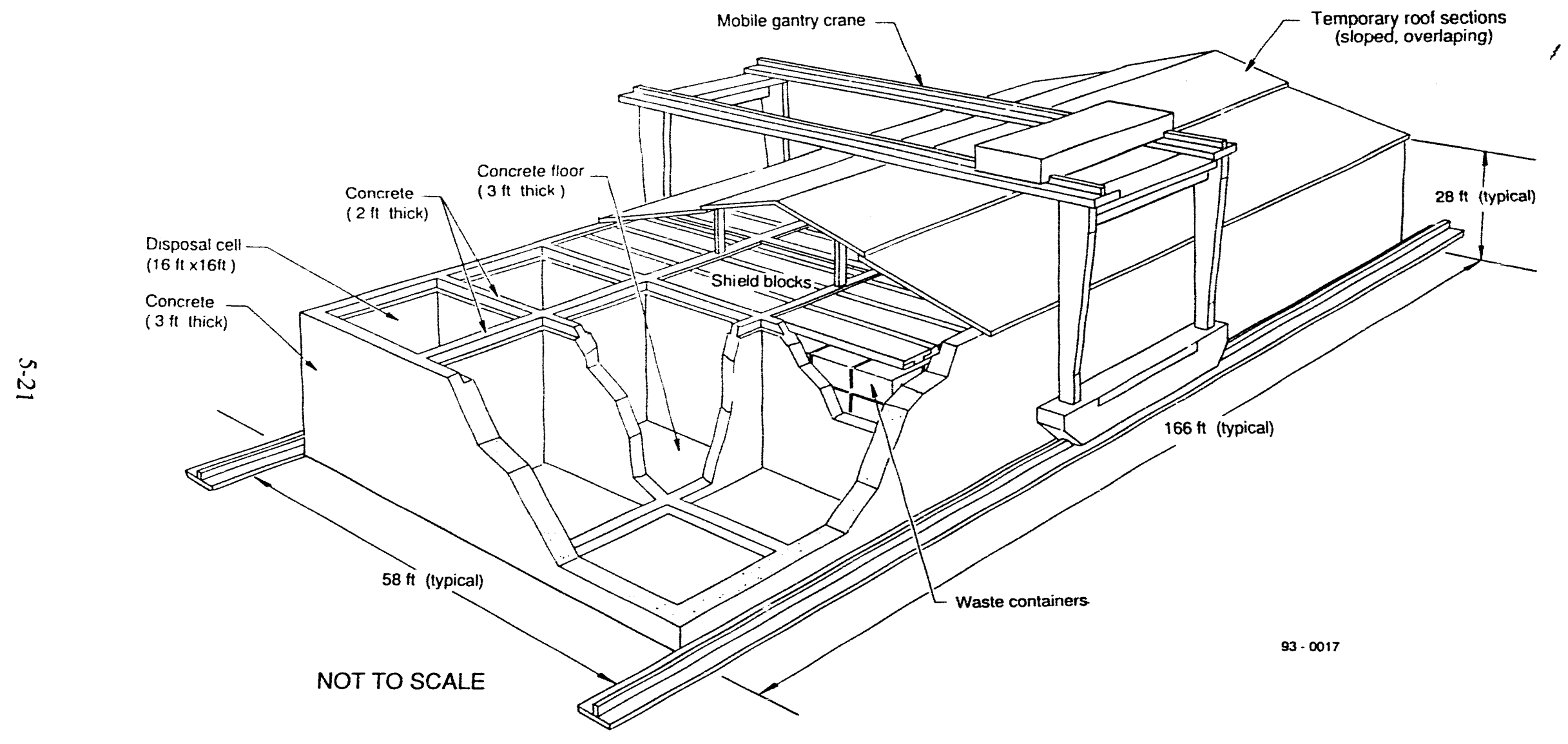

Figure 5-4. Design concept for disposal structures for remote-handled waste in Subalternative 1A. 
The design of the concrete structures is similar to the designs for the following facilities: (a) the Hazardous Waste/Mixed Waste Disposal Facility under development for the DOE Savannah River Site (Wells and Bailey 1991; Freitag et al. 1992), (b) the LLW disposal facility proposed for the Central Interstate Compact (Deold \& Coleman 1991; Gunning et al. 1992), and (c) a conceptual facility evaluated previously for the INEL LLW (Darnell 1990; Darnell et al. 1990). The floor, walls, and roof are all $3 \mathrm{ft}$ thick. The top of the floor is situated at grade level for ease in loading.

Based on the various regulatory requirements discussed in Section 2.3, it was determined that the eight types of waste can be disposed of in five groups of structures. The structures for each group of waste types were sized based on the numbers and sizes of waste containers in Table 2-6. The sizing of the structures is approximate, being based on rounded-off dimensions and without detailed consideration of facility support items (e.g., instrumentation, utilities). Neither are items such as backfill, liners, and leachate collection and removal systems and leak detection systems reflected in the basic dimensions given in this or other writeups in Section 5.

The results of the sizing are listed in Table 5-3. In cases where no significant penalty in disposal efficiency results, the dimensions are as similar as possible (e.g., same width and/or same height) from one group of structures to another. For Class $B$ and $C \beta-\gamma$ waste and also for $C \alpha$ mixed waste, one long structure could have been designed. However, to maintain redundant operational capability, two shorter structures were designed in each case. The structure for Class B mixed and $C \beta-\gamma$ mixed waste is already so small that the redundancy was not incorporated.

The internal dimensions of the structures were selected based in part on producing a minimum of empty space when all containers have been emplaced. Typically, a 2-ft space is left on top of the stack during operations to provide access and to accommodate ceiling-mounted instrumentation and utilities.

The structures incorporate all of the required instrumentation systems-constant air monitors, radiation area monitors, toxic chemical monitors, etc., as well as the necessary alarms and annunciators. In addition, instrumentation is emplaced in or on the walls of the structure to facilitate long-term monitoring of its condition, as discussed later.

Table 5-3. Number and size of concrete structures for each type of waste in Subalternative 1A.

\begin{tabular}{lccc}
\hline \multicolumn{1}{c}{ Type(s) of waste } & $\begin{array}{c}\text { Total number of } \\
\text { structures required } \\
\text { over 40-year period }\end{array}$ & $\begin{array}{c}\text { Interior } \\
\text { dimensions } \\
(\mathrm{W} \times \mathrm{L} \times \mathrm{H}, \mathrm{ft})\end{array}$ & $\begin{array}{c}\text { Exterior } \\
\text { dimensions } \\
(\mathrm{W} \times \mathrm{L} \times \mathrm{H}, \mathrm{ft})\end{array}$ \\
\hline A & 13 & $50 \times 260 \times 26$ & $56 \times 266 \times 29$ \\
B and $\mathrm{C} \beta-\gamma$ & 2 & $52 \times 160 \times 28$ & $58 \times 166 \times 31$ \\
A mixed & 7 & $50 \times 250 \times 26$ & $56 \times 256 \times 29$ \\
B mixed and C $\beta-\gamma$ mixed & 1 & $34 \times 70 \times 29$ & $40 \times 76 \times 32$ \\
C $\alpha$ mixed, and the minute & 2 & $50 \times 151 \times 26$ & $56 \times 157 \times 29$ \\
amount of $\mathrm{C} \alpha$ & & & \\
\hline
\end{tabular}


For contact-handled waste containers-Class $A$ and Class $C . \alpha$, whether mixed or not-the floor space in the concrete structures is all one open area. The roof of the structure is supported by concrete bridge-beams (sec Figure 5-3). Waste containers are emplaced by forklifts operating at grade level. Access to the interior is via large, interlocked dual rollup doors. The inner door is attached to the concrete structure. The outer door is located in an entry tunnel enclosure of Butlertype construction that is atlached to the concrete structure.

The floor space of the concrete structures that receive remote-handled waste containers-Class B and Class $C \beta-\gamma$, whether mixed or not-is divided into cells (see Figure 5-4). The cells, which are $16 \mathrm{ft}$ square, are divided by 2 -fit concrete walls. Access to the cells, by a mobile overhead gantry crane that straddles the structure, is provided via individually removable steel roof panels and concrete shield blocks with lifting lugs and with gaskets to limit water infiltration. The steel panels serve as weather shiclds to keep water out of the cells. Additional clearance beneath the roof is allowed for seating of the roof panels. This emplacement approach reduces the radiation fields from containers already received while additional containers are emplaced. Use of a crane with remote and/or shiclded operating capability also reduces exposure to personnel. When the concrete structure is filled to capacity, the 3 -ft thick reinforced concrete roof is emplaced.

For waste types needing more than two structures, only the structures needed for the first 10 years are available at the beginning of operations. Additional structures are constructed as needed, subject to maintaining one extra structure for operational redundancy.

During operations, the innermost level of confinement is the individual waste containers. To provide a second level of conlinement during operations, each concrete structure is provided with a ventilation system. Air from the interior of the structure is drawn by fans through a set of high efficiency particulate air (HEPA) filters and charcoal filters. The ventilation system ensures that airborne contamination levels within the structure are maintained within acceptable levels for wasteplacement personnel and that emissions to the environment are minimized and monitored. The concrete structures are designed to accommodate all normal operational loads, as well as all credible accident loads.

\subsection{Site Surface Water Management System-Requirements: The principal} requirements related to surface water management are as follows:

- Upstream /upgrade] drainage areas must be minimized to decrease the amount of runoff which could erode or inundate waste disposal units (10 CFR 61.50).

- Surface features must direct surface water drainage away from disposal units at velocities and gradients which will not result in erosion that will require ongoing active maintenance in the future (10 CFR 61.51).

- The disposal site must be designed to minimize to the extent practicable the contact of water with waste during storage, the contact of standing water with waste during disposal (10 CFR 61.51).

- A run-on control system is required capable of preventing flow onto active portions of the facility from at least a 25-year storm (40 CFR 264.;01). 
- A run-off management system is required to collect and control at least the water volume resulting from a 24-hour, 25-year storm (40 CFR 264.301).

A key consideration in the design of a waste disposal system is the prevention of water contact with the waste. AGES are particularly well suited for prevention of water-waste contact since the waste is completely enclosed in vaults that are situated above the ground surface. Additional design features that enhance the isolation of the waste from surface water are discussed in this section.

For the purposes of this section, surface water management will include control of waste contact with precipitation (snow and rain before it hits the ground), onsite run-off or offsite run-on (rain and melted snow running across the ground), and snow accumulation (snow that accumulates or drifts onto the site).

Rain and snow cannot fall directly on the waste as long as the concrete enclosure is intact. For side-loaded vaults that are loaded through double doors, only one door is allowed to be open at a time; thus, the enclosure is always intact. Any water entering when a waste transport vehicle is pulled in through the first door will drain to the lloor and be removed by the drain system. The first door will be shut before the second door is opened.

For vaults that are loaded via the roof, protection from precipitation is achieved by prohibiting disposal during inclement weather. Operation remains practical because the number of days of sunshine is high and the rate of waste receipt for the top-loaded vaults is low.

The roofs of both types of vaults are slightly pitched to shed water and melted snow. The temporary covers on the top-loaded vaults are designed to facilitate snow removal (the covers are not removed until they are dry and snow free).

Run-on from offsite is prevented by the construction of a site perimeter ditch that intercepts all water from offsite and diverts it around the site. The ditch is designed and graded to allow gravity flow (see Figure 5-5). The grade is gentle in order to minimize erosion.

Onsite run-off is controlled by grading the site to shed water to two sets of ditches. The buffer zone of the site is graded to shed water to the site perimeter ditch. A second ditch encompasses the controlled access area, or vault area, of the site (see Figure 5-5). The vault area is graded such that the rain falling on the area is directed away from the vaults and to the vault-area perimeter ditch. Snow melt also follows this path. The vault-area perimeter ditch is graded so that it drains by gravity to a retention pond. The grade is gentle enough to minimize erosion. Water in the pond is periodically sampled. If it is found to be free of contaminants, it is released to the site perimeter ditch. It is expected that the water will be released without exception from the drain pond. If the water is found to be contaminated, it will be necessary to treat it before release.

It may not be feasible to design the site perimeter ditch to control run-on from a large, designbasis storm (25-year storm). Since the site is located on a broad, flat plain, a low dike should be adequate to divert design-basis flood waters. If such a dike is required, the site will be graded such that the base of the vaults is located above the top of the dike. This will prevent flooding of the vaults even if the dike fails. 


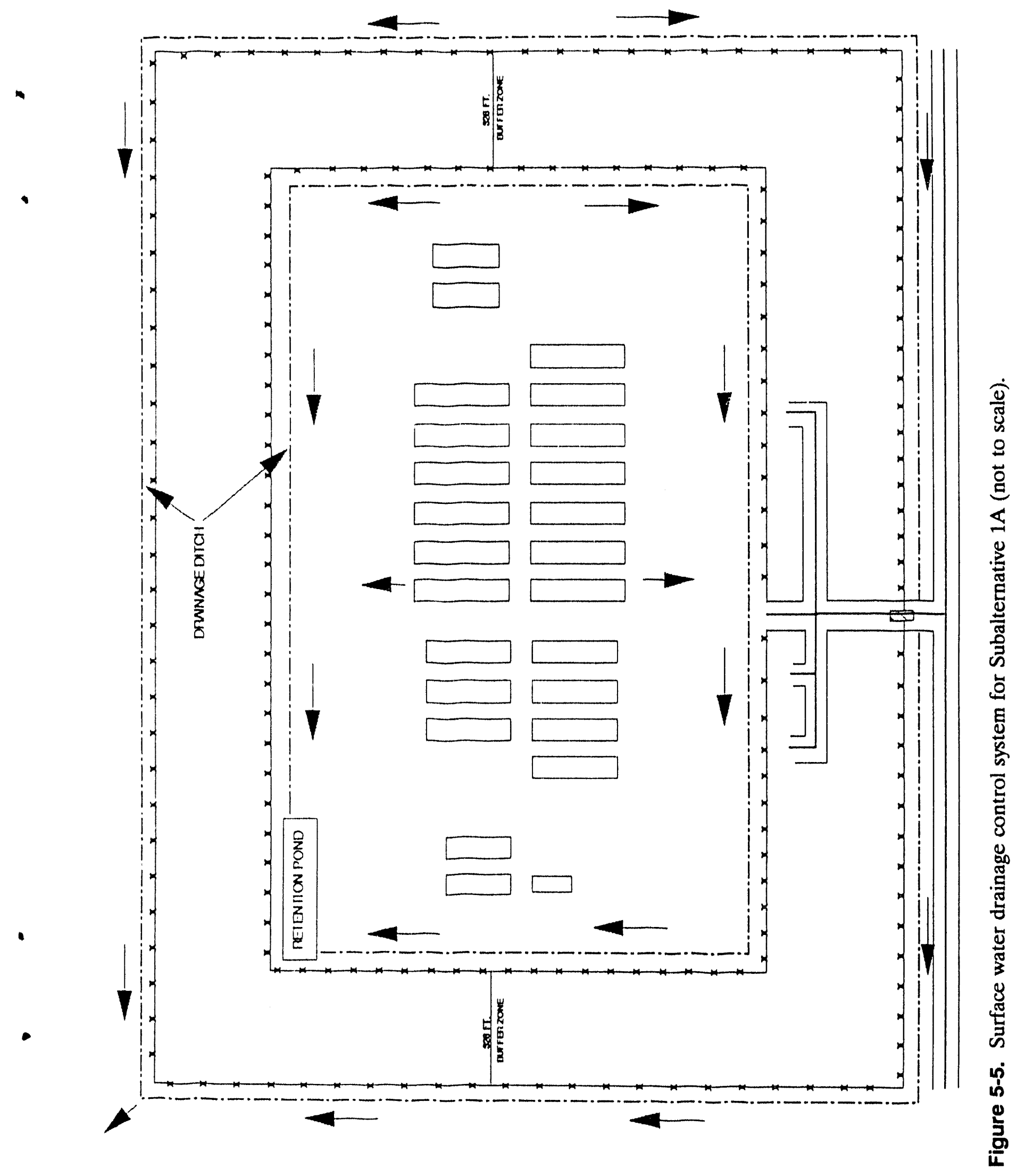


The site is not located in the expected flood plain associated with a break of the nearest dam (the Mackay Reservoir Dam).

Each vault is placed on a foundation drainage blanket as a final protection against water-waste contact (see Figure 5-6). The drainage blanket has a sloped foundation such that water runs to a drain collector pipe. The water from each drain collector is directed to a common sump. The water in the sump is periodically pumped to the retention pond. The purpose of the foundation blanket is not to collect moisture from the vault, but to prevent contact of the base of the vault with water in the soil. A moisture barrier is installed as part of the construction of the base pad. The moisture barrier is installed below and on the sides of each vault base pad. While it is unexpected, if water seeps through the concrete vault, the inner leachate collection systems are an additional barrier to water-waste contact.

Protection from snow accumulation is provided by snow removal, moisture seals, and strategically placed snow fences. Snow is frequently removed from around the vaults to reduce the impact of snow melt. The lower sides of all the vaults and the roofs of the side-loaded vaults are covered with a moisture barrier to protect against snow melt. The moisture barrier on the sides is protected from damage by snow removal equipment and normal operation by a fiber-board cover. Snow fences are placed around the site to help prevent drifting around the vaults. Removed snow is placed at the upper edge of the lined pond.

5.3.4.1.4 Liners, and Leachate Collection and Removal Systems and Leak Detection System-Requirements: The principal requirement related to liners is as follows:

- Two or more liners must be installed. The top liner must be constructed of materials (e.g., a geomembrane) to prevent the migration of hazardous constituents into the liner. The lower liner must consist of at least a geomembrane on top of a compacted soil (clay) liner at least $3 \mathrm{ft}$ thick and with a permeability of no more than $1 \times 10^{-7} \mathrm{~cm} / \mathrm{sec}$. Alternative designs are possible if approved by the EPA (40 CFR 264.301).

Requirements: The principal requirements related to leachate collection and removal systems are as follows:

- Leachate collection and removal systems must be emplaced above and between the two liners (40 CFR 264.301).

- $\quad$ The lower leachate collection and removal system (LCRS) also must function as the leak detection system (40 CFR 264.301).

- $\quad$ Alternative designs possible if approved by EPA.

Liners and LCRS are designed to work as a unit. The primary purpose of the combined systems is to prevent leachate from reaching the environment (the liners) and to direct the leachate to a colleciiun system where it can be monitored and treated for release or disposal, as appropriate (the leachate collection and removal system).

Dual-liner/leachate systems are required by the EPA for hazardous waste disposal and will be used for all mixed waste disposal units. Liner/leachate systems are not required for disposal of radioactive waste; however, use of such systems provides a backup to waste forms, containers, and 


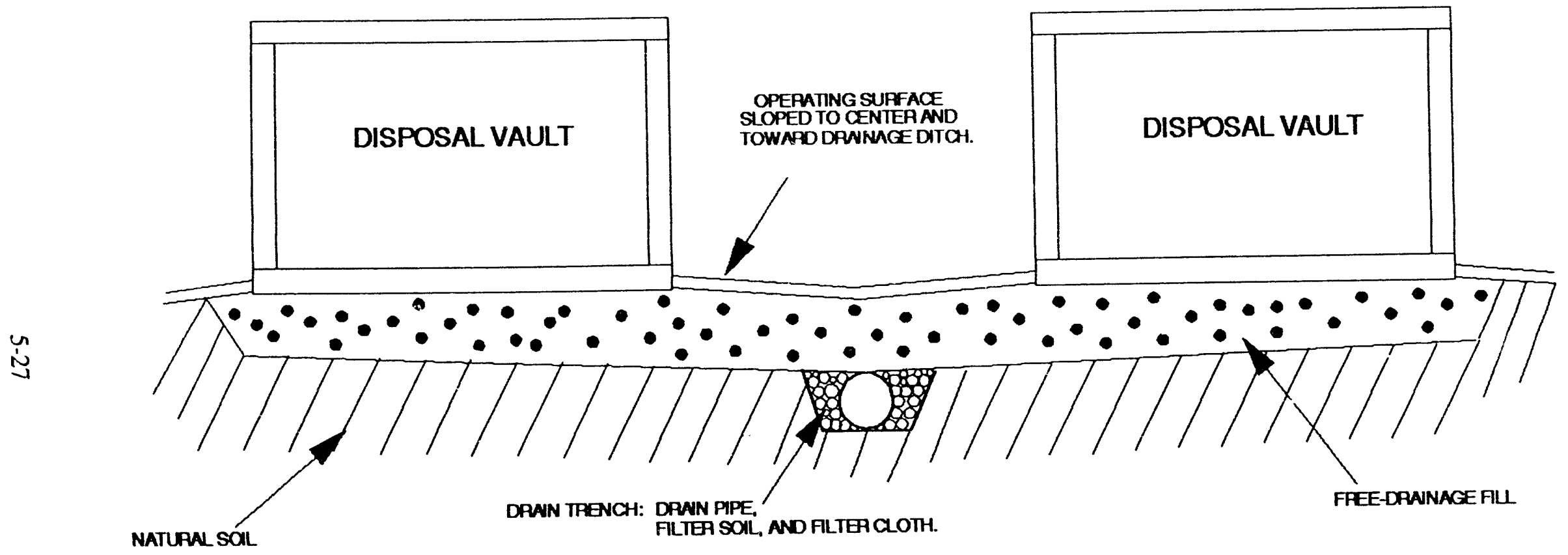

Figure 5-6. Foundation blanket drain for Subalternative 1A (not to scale). 
site characteristics. In addition, the use of liner/leachate systems allows monitoring of performance and early detection of problems. Therefore, a liner/leachate system will also be used for nonmixed waste disposal units.

The dual-liner/leachate system is shown in Figure 5-7. The general system is described below, starting at the vault floor and moving up to the waste.

The vault floor is sloped and lined with a 60-mil-thick high density polyethylene (HDPE) liner referred to as the secondary liner. The performance of the secondary liner combined with the concrete vault has been proven to be equivalent to that of a composite clay layer and liner (Freitag et al. 1992). The secondary liner extends up the sides of the vault on all sides. For Class $A$ and $C \alpha$ vaults, the liner need extend only $1 \mathrm{ft}$ above the concrete pad on which the waste is situated; for the other vaults, the liner extends up the entire side. Any leachate that comes through from the primary liner is collected above the secondary liner.

The secondary leachate collection and removal system consists of a drainage net that sits directly on top of the secondary liner. The net extends up the sides with the secondary liner. Any leachate that leaks through the primary liner is collected above the secondary liner and flows by gravity through the drainage net to a drain. The drain is an HDPE pipe that is embedded in the concrete floor of the vault. The drain pipe directs the leachate to a secondary drain sump where it is collected for sampling and treatment.

The primary liner is an 80-mil-thick layer of HDPE. The primary liner sits on top of the secondary LCRS. The primary liner extends up the sides of the wall with the secondary LCRS and liner. Any leachate that comes from the waste will collect above the surface of the primary liner.

The primary LCRS sits on top of the primary liner. The primary LCRS consists of a gravel drainage layer overlain with a sand filter. Any leachate collected above the primary liner flows by gravity through the primary LCRS to a drain. The drain is an HDPE pipe tha extends through the secondary LCRS and liner and is embedded in the concrete floor of the vault. The drain pipe directs the leachate to a primary drain sump where it is collected for sampling and treatment.

The primary LCRS is constructed such that when it is compacted, it still allows leachate flow, but will also support the weight of the concrete pad poured on its surface. The sand filter overlay protects the gravel drainage bed from plugging when the concrete pad is poured. The drainage layer, sand filter, and concrete pad are all sloped to improve drainage.

A concrete pad is poured on top of the primary LCRS. The pad is sloped to drains that are routed to the primary LCRS. The top of the concrete pad is lower than the liners on the side walls. The pad bears the weight of the waste and allows the use of fork lifts for waste emplacement.

The drainage pipes from each LCRS are made of double-walled HDPE and are set directly in the cement vault floor. The primary and secondary collection sumps are small, double-walled tanks located in a concrete drain gallery (Figure 5-8). The gallery is adjacent to the vault and is belowgrade. The drain pipes enter the sumps near the top. The sumps have top penetrations for leak-detection devices and for hookup of sampling/pumping equipment. 


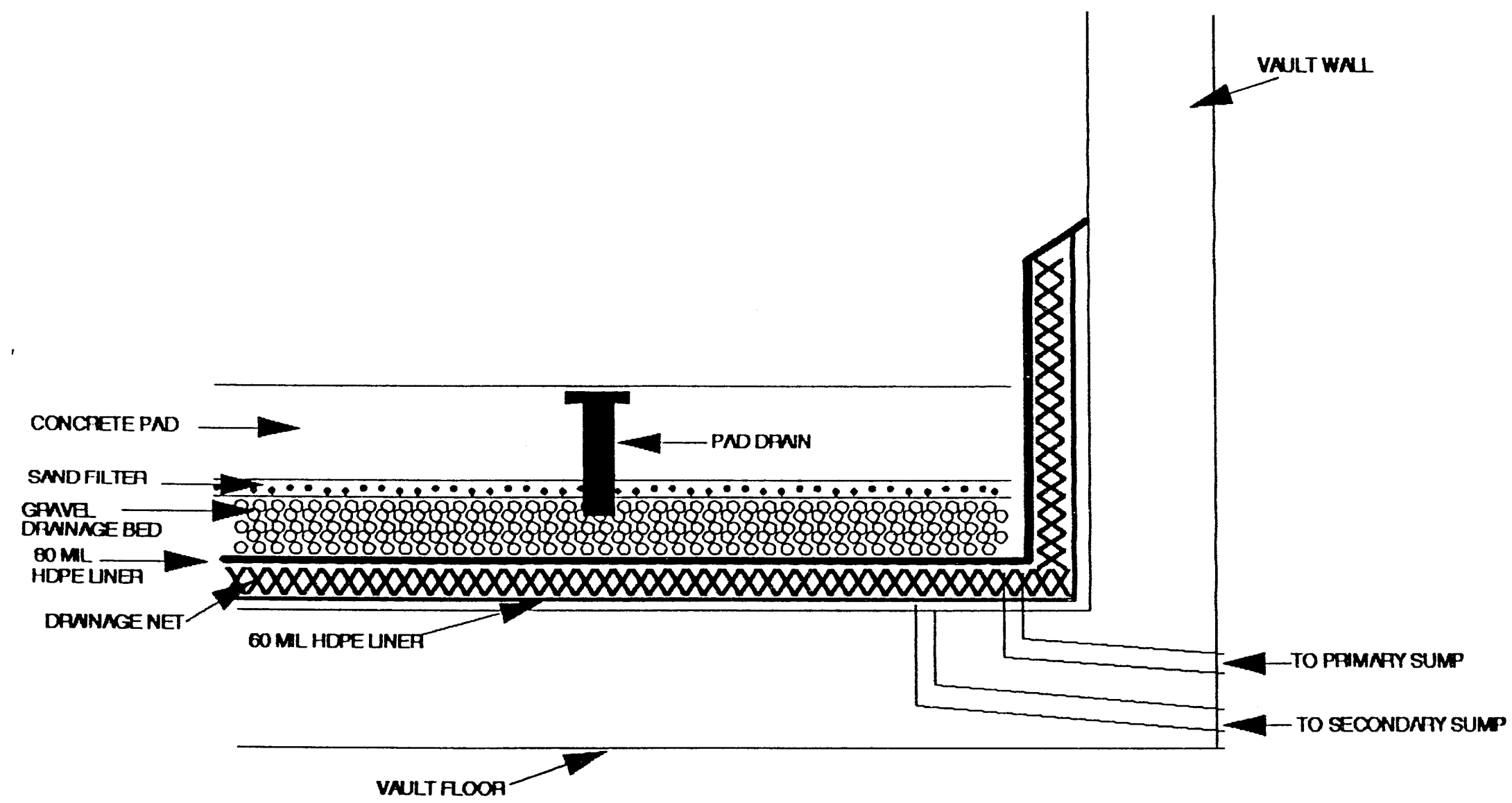

Figure 5-7. Dual-liner/leachate system for Subalternative $1 \mathrm{~A}$ (not to scale). 


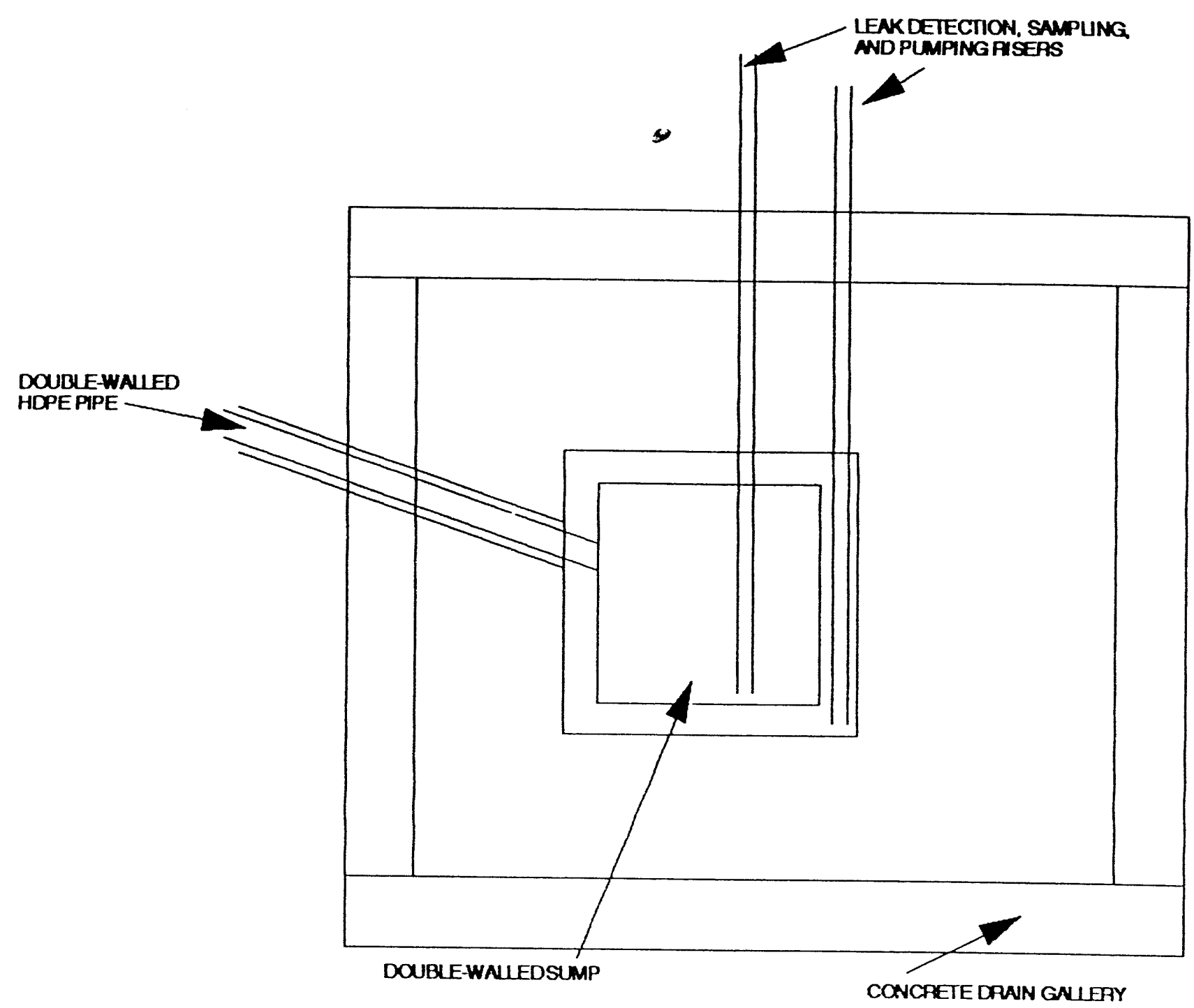

Figure 5-8. Leachate collection and recovery system drain gallery and sump for Alternative 1A (not to scale). 
Detailed design of the dual-liner/leachate collection system ensures that the combination of LCRS material, thickness, and slope is sufficient to detect a specified minimum leakage rate. Thus, the LCRS includes the required provisions for leak detection. Detailed design also ensures that plugging of the LCRS does not occur.

5.3.4.1.5 Support Facilities-The following support facilities are provided in this subalternative. The MLLWDF is manned by uniformed guards 24 hours a day, 7 days a week. A small guard house is located on the road connecting Lincoln Boulevard to the MLLWDF, the main access road. It is manned by uniformed security guards during normal working hours. During off shifts, the guards make routine inspection tours several times a shift to ensure that any existing offnormal conditions are detected. Any deviations from normal conditions are repotted and appropriate actions taken.

The office and administration building provides controlled entry of personnel and vehicular traffic into the facility. All personnel report to the access control office within the administration building for radiological dosimetry prior to entering radiation areas and for survey prior to leaving. The building also provides office facilities for personnel including management, administration, enginecring, security, salety, quality, industrial hygiene, health physics (HP), and records management personnel and provides a lunch room. The building is a preengineered metal building, sealed to its foundation. The building is $10,000 \mathrm{ft}^{2}$ and is one story high.

The support building provides several capabilities. Space is providec for personnel change areas, a decontamination arca, a warchouse storage area, and an analytical laboratory for confirmatory sampling of incoming waste. Ventilation for the laboratory is provided with HEPA filters, and stack monitoring is conducted for radionuclides. The building is a preengineered metal structure, similar to the administration facility. The support building is a $8,000 \mathrm{ft}^{2}$ and is one story high.

The materials storage building provides storage capacity for materials used in disposal facility construction and operation. The building is a $2,500 \mathrm{ft}^{2}$ single story, preengineered metal structure, serving as a weather cover for the stored materials.

The heavy equipment storage and maintenance building provides a covered structure for storage and maintenance of heavy equipment and for decontamination of transport vehicles. Electric power for headbolt heaters is provided to minimize cold weather startup problems. The facility is a 7,500 $\mathrm{ft}^{2}$, preengineered metal structure.

Utilities supporting operations are provided at the facility due to its remote location. The site utilities include water for domestic (potable) and industrial use, irrigation applications, electrical power, communications including telecommunications and automatic processing equipment connections, sanitary wastewater treatment, and stormwater management systems.

5.3.4.1.6 Construction Quality Assurance-Requirements: The principal requirements related to Construction Quality Assurance (CQA) are as follows:

- A CQA Program is required addressing dikes, liners, LCRS, LDS, and covers to ensure that all design criteria and requirements in the permit are satisfied in the constructed unit. ACQA Plan, detailed inspection, etc. must be included (40 CFR 264.19 and .303). 
- A quality control program is required covering design, construction, operation, and closure of the facility (10 CFR $61.12(j))$.

The need to provide a high degree of assurance that the (Class $C$ ) waste will remain isolated from the environment and potential intruders for at least 500 years brings a new dimension to the CQA program. To meet the waste disposal performance requirements in Section 2.3, CQA activities are required that go well beyond those typically used to ensure facility performance during a normal 30- to 40-year operating life. Therefore, to satisfy the CQA requirements above, a very substantial CQA program is conducted under this subalternative and all other subalternatives. The CQA program is described here because of the significant impact of the program on construction costs.

The nature of the CQA program is not simply to collect more and more documentation to meet the (NQA-1) requirements (NRC 1991a). Rather, the CQA program is a comprehensive, integrated, technically derived set of parameters and measurements that are rigorously obtained and documented to provide a high degree of confidence that the 500-year and other performance objectives are met.

The CQA program includes the activities of all construction subcontractors whose work could affect the performance of the facility. The program extends in time from the beginning of the project (e.g., characterizing the geologic setting) through closure and postclosure monitoring.

The CQA program applies to any activity, structure, system, or component that could affect attaining the performance objectives in Section 2.3. Two major segments of the program are testing of construction materials and inspections of completed work. Lists of tests to be performed are prepared as part of the (CQA) Plan, and only approved laboratories are used.

Chief among the materials to be tested is the concrete. Even a very small crack can increase the hydraulic transport properties of a concrete wall by orders of magnitude. Taylor (1992) and Denson et al. (1987) discuss the complexity of producing concrete with a high assurance of meeting the 500-year performance requirement. Concrete testing includes determinations of slump, air content, unit weight, chlorine content, compressive strength, and numerous other parameters. Each batch is sampled to ensure all parameters are within established limits. All lots of reinforcing or structural steel items are sampled, tested, and certified. Chemical mixtures are sampled, as are curing compounds, joint materials, and coatings.

The construction work inspections include activities such as detailed inspections of the concrete forms and of the emplaced reinforcing steel, observation of concrete emplacement to guard against problems such as over-vibration that induces segregation of the mix, overseeing of the curing process to minimize thermally induced cracking, inspections of the cured concrete for surface checks or drying shrinkage cracks, or other "loss of moisture" defects.

The guidance in EPA (1989) on the CQA requirements in 40 CFR 264 provides great detail on the CQA of liners, as well as covers and the LCRS and LDS. This guidance is assumed to be complied with as applicable in this subalternative.

The entire CQA program is thoroughly documented, from the detailed CQA Plan (which lists all tests and inspections to be conducted) through the collection and maintenance of the records. In particular, thorough records are kept of any nonconformances and the corrective actions taken. 


\subsubsection{Description of Operations.}

5.3.4.2.1 Waste Receipt-All facilities that intend to dispose of waste at the MLLWDF submit general estimates of waste volumes and characteristics and are trained with respect to the WAC for the facility. Training includes the necessary documentation required and the form of packaging that must be used.

When a waste package is ready for disposal, the generator or treatment facility, as appropriate, calls the MLLWDF to arrange for transfer of the waste. An electronic record is sent to the MLLWDF for review. The review includes a preliminary check of the waste against WAC. Preliminary selection of disposal location is also made at this time. Assuming the waste meets the WAC, arrangements are made for transfer. Unless operations are curtailed for inclement weather or maintenance, it is expected that approval will be given for same-day or next-day transfer.

When the waste arrives at the front gate of the MLLWDF, the transport vehicle is parked on an inspection pad located just outside the site perimeter fence. The waste shipment is inspected to ensure that radiation levels are as expected, that no damage has occurred during shipment, and that the waste packages match those recorded on the shipping manifest. The manifest is also checked against the list of waste that was preapproved for shipment. Any discrepancies are immediately resolved. Noncompliant packages are immediately returned to the treatment or generation facility.

Assuming that receipt of the waste is approved, the shipment enters the MLLWDF and is directed to the appropriate vault for disposal. The status of the shipment is updated in the electronic record. The preapproval system of waste reccipt minimizes the number of shipments that must be returned to the treatment or generation facilities and helps to speed up the process of inspection at the front gate.

5.3.4.2.2 Waste Transfer-Once a shipment of waste is accepted, it is immediately taken to the appropriate disposal vault. Since a treatment facility is used for most waste, it is expected that each shipment will consist of only a single type of waste (e.g., Class $\mathrm{C} \alpha$ mixed or Class A nonmixed); therefore, the shipment can be directed to a specific vault. At this point, two possible scenarios exist, one for side-loaded vaults and one for top-loaded vaults.

For side-loaded vaults, the shipment is driven into the tunnel enclosure adjacent to the vault. Inside, the shipment is unloaded and the waste packages are placed in a staging area for a final inspection. Waste coming from the treatment facility that is suitable for disposal in side-loaded vaults is prepackaged in either standard boxes or sets of drums, the drums being banded together on pallets. The waste packages are moved using forklifts.

The final inspection involves surveys, visual confirmation of identity, and comparison to shipping and electronic records. If sampling of the waste is needed as part of the waste acceptance plan, the waste container is moved into the sampling area of the enclosure. After completion of inspection and sampling, the electronic record is updated as nccessary and each package is assigned a specific location inside the vault.

After offloading of all waste, the transport vehicle is surveyed to ensure it is not contaminated and is then returned to the treatment or generator facility. 
For top-loaded vaults, the shipment is driven to a position adjacent to the end of the appropriate vault. The shipment then receives a second inspection. Generally speaking, the inspection is much abbreviated compared with that for the side-loaded vaults because the higher radiation levels outside the packaging makes inspections less safe. In most cases, the waste package is contained in a shipping cask, so the inspection includes only a confirmation of waste shipment identification number, an update of the electronic record, and the assignment of a specific vault location.

5.3.4.2.3 Waste Emplacement-Requirements: The principal requirement governing waste emplacement includes the following:

- $\quad$ Void spaces between waste packages must be filled with earth or other material to reduce future subsidence within the fill (10 CFR 61.52).

For side-loaded vaults, waste packages are moved by forklift from the staging area to assigned locations in the vault. The scheme for waste placement is to stack waste from floor to ceiling, beginning at the back and working toward the front. The back is the short wall of the vault furthest from the staging area. This approach is used to limit exposure to workers. The waste is stacked as close together as possible to limit the void space. A corridor a little over $4 \mathrm{ft}$ wide is left along the walls and back to allow inspection of these areas. Just prior to closure, when the center of the vault is full, the corridors are filled from back to front.

Waste that is suitable for side-loaded vaults is generally low in activity (i.e., contact-handled). A limited number of packages approach the limits for safe contact handling. These packages are stacked toward the center of the waste in order to take advantage of shielding from other lower activity waste packages.

Once the final disposal location of each package is confirmed, the electronic record is updated.

For top-loaded vaults, the temporary cover and shielding blocks associated with the cell where the waste is to be placed are removed and placed to the side. A gantry crane is used to remove the cover and shielding blocks. The gantry crane is then used to hoist the waste package and its protective shielded cask off the transport vehicle. The waste is positioned over the appropriate vault position and the waste is lowered into place. The gantry crane is then moved back over the transport vehicle, and the shielded cask lowered into place for return to the treatment or generator facility. The operation of the gantry crane is performed from a remote and/or shiclded platform to protect operators from excessive cxposure.

Once the waste is in place, its identification number and condition are confirmed and the electronic record is updated. The shielding blocks and temporary cover are replaced as soon as possible to reduce potential for exposure of the waste to the elements.

The transport vehicle and shipping cask are surveyed to ensure they are not contaminated and are then released for return to the treatment or generator facility.

Periodically, backfill is placed around and on top of the waste in the top-loaded vaults. The fill, a coarse sand, serves in the short-term as a shield and in the long-term to minimize subsidence. The 
side-loaded vaults do not require backfill until they are filled with waste because the radiation levels are relatively low.

5.3.4.2.4 Monitoring-Requirements: $A$ monitoring program is required by DOE Order 5820.2A (Section III.3.k), 10 CFR 61.53, and 40 CFR 264.303 and .310 with the following objectives:

- Measure operational effluent releases, migration of radionuclides, disposal unit subsidence, and changes in disposal facility and disposal site parameters which may affect long-term site performance

- Provide data to evaluate the potential health and environmental impacts and ... to enable the evaluation of long-term effects and the need for mitigative measures.

- Provide early warning of releases of radionuclides from the disposal site before they leave the site boundary.

A monitoring program meeting the above requirements is conducted. There are three phases to the program.

In the first phase, a preoperational monitoring program is conducted before construction begins. The program focuses on obtaining data related to (a) the hydrology, geology, geochemistry, seismology, ecology, and meteorology of the disposal site, and (b) the preexisting concentrations of contaminants at the disposal site.

In the second phase, the monitoring program is continued during construction and expanded during operations. The focus shifts to the determination of contaminant concentrations in air, surface water, ground water, soil, plants, and animal tissuc. Radiation levels are also measured. Although most of the monitoring points are within or at the boundary of the disposal site, some points are located outside the disposal site. Design of the program and evaluation of the results are integrated with monitoring programs conducted by other organizations at the INEL.

Also, during operations, operational monitoring is conducted at least weekly, including the status of the surface water management system, the leachate collection and removal system, and the leak detection system.

During construction and operations, the condition and structural performance of the isolation barriers (e.g., settling, strains, joint and seal behavior, cracking) are monitored at suitable frequencies and locations to verify design assumptions. The limiting acceptable values of parameters are specified, and remedial action plans are implemented if the values are exceeded.

The third phase of monitoring occurs after closure of the disposal units. The monitoring program continues as long as institutional control of the site continues (assumed, per DOE Order $5820.2 \mathrm{~A}$ and 10 CFR 61.59 to be 100 years). More details on postclosure monitoring are given in Section 5.3.4.3. 


\subsubsection{Closure and Postclosure Activities.}

5.3.4.3.1 Disposal Unit Closure-Requirements: The principal requirements related to closure of disposal units are as follows:

- Closure and stabilization measures ... must be carried out as each disposal unit (e.g., each trench) is filled and covered (10 CFR 61.52).

Closure of side-loaded vaults will take place as each vault is filled. Closure entails a preclosure inspection, filling of inspection and access aisles with waste, backfilling the vault with sand, sealing of penetrations, and removal of the portable entry tunnel. The penetrations that will require sealing are the ventilation ports and the waste access door (rollup door). The ports and door are designed for sealing with concrete. It is intended that the vaults never again be entered once they are sealed. After the rollup door is sealed, the entry tunnel will be removed and placed adjacent to the next vault to be loaded. Installation of the multilayer engineered cover common to all the vaults is discussed in the following section.

Closure of the top-loaded vaults will take place as each vault is filled. Closure entails backfilling each waste cell with sand and pouring of a permanent concrete roof. Backfilling is performed remotely using the gantry crane and engineered attachments. The backfilling procedure includes shaking to ensure voids are filled. The gantry crane can be moved to the next vault when the backfilling operation is complete. Filling of the vault cells with waste is controlled such that when the backfill sand is in place, the dose rate at the surface is low enough to allow final surface preparation and pouring of the concretc roof to be done locally (as opposed to remotely). The construction of the roof is similar to the construction of the sides and bottom of the vault. Installation of the multilayer engineered cover common to all the vaults is discussed in the following section.

The leachate collection and removal system associated with each vault will be maintained operable to allow postclosure monitoring. At the end of the institutional control period, the LCRS drain pipes will be plugged, and the void volumes in the sump and drain gallery backfilled with sand.

5.3.4.3.2 Cover-Requirements: The principal design requirements related to cover design, from 10 CFR 61.51 and 40 CFR 264.310, are as follows:

- Covers must be designed to minimize to the extent practical water infiltration, to direct percolating or surface water away from the disposed waste, and to resist degradation by surface geologic processes and biotic activity.

- The disposal site must be designed to minimize to the extent practicable ... the contact of percolating or standing water with wastes after disposal.

- The cover must function with minimum maintenance.

- The cover must accommodate settling and subsidence while maintaining its integrity. 
- The cover must have a permeability no more than that of any bottom liner or natural subsoils present.

The joint guidance of the EPA and the NRC (Thompson and Porter 1987) for the layers constituting an acceptable cover for disposed mixed waste is as follows: starting from the outside, (a) outer rock or vegetative layer, (b) filter and drainage layer, (c) impervious flexible membrane liner, (d) compacted, low-permeability clay layer, (e) filter and drainage layer. If the waste does not have an engineered vault roof structure, an additional compacted clay layer is placed next to the waste.

One of the last steps in closure of the waste disposal facility is emplacement of the final cover over the site. Several regulatory guidance documents exist (Denson et al. 1987; EPA 1989; NRC 1991c) for cover designs. Those documents, along with O'Donnell et al. (1992), show how multilayer covers can, with proper design and construction, give an increased assurance of meeting the above requirements. All of these documents were used in the design of the cover described herein.

Site-specific conditions strongly affect cover performance. In addition, experience has shown the difficulty of constructing a multilayer cover that will perform as intended. Therefore, a waste cover research and development program, including laboratory and field tests, is conducted in connection with this or any other subalternative. The selection of particular soils and other materials for the cover is made by knowledgeable soils scientists and geologists.

Before describing the preconceptual design of the cover, the functions of the cover are briefly discussed.

The primary function of the cover is to minimize the contact of water with the disposed waste by (a) minimizing infiltration and (b) promoting drainage. These two aspects of the primary function can lead to a search for a cover material with conflicting properties. Minimizing infiltration requires low-permeability media, while promoting drainage requires high-permeability media. The solution is to use a multilayer cover. One layer provides low permeability to minimize infiltration, while another layer provides high permeability to promote drainage.

Other functions or constraints of the cover include the following:

- Minimize surface erosion due to wind and water

- Minimize differential settlement and subsidence

- Minimize shrink/swell behavior and cracking

- Provide resistance to freeze/thaw damage

- Minimize intrusion by burrowing animals and root penetration.

Figure 5-9 illustrates the general aspects of the cover design developed for this subalternative. Starting at the top of the cover, the successive layers and their functions are as follows. The top layer consists of selected native vegetation rooted in approximately $2 \mathrm{ft}$ of compacted topsoil. This layer protects against erosion of the cover. (It is assumed that protection against design basis floods is provided by the water run-on and run-off control system discussed elsewhere.) The layer provides evapotranspiration that reduces the water available for infiltration. The top layer also protects the 


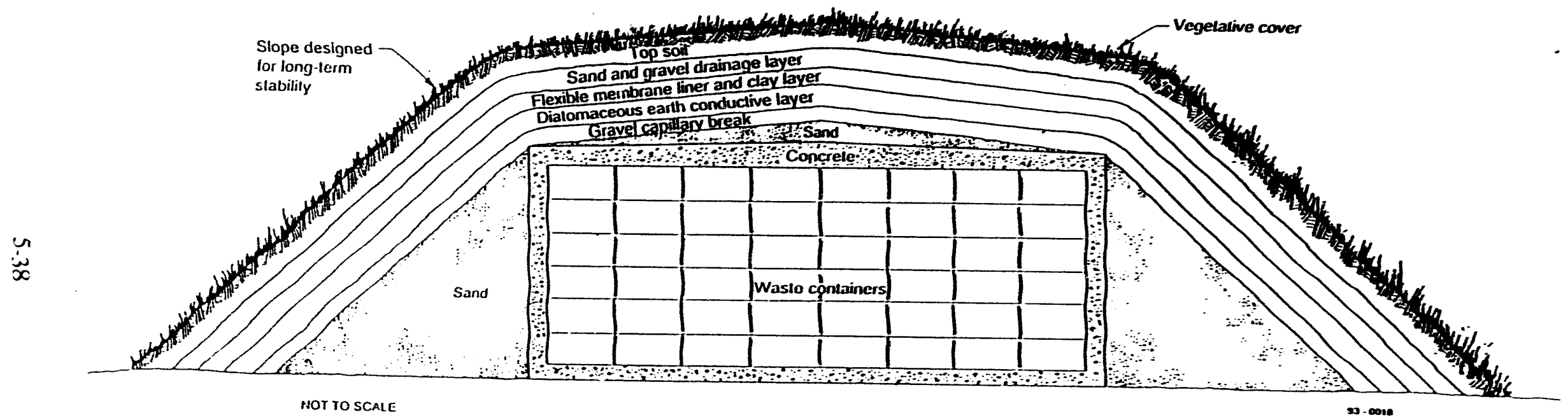

Figure 5-9. General design of multilayer final cover for waste disposal site in Subalternative 1A (not to scale). 
underlying low-permeability layer against freezing and desiccation. Additional research and development is needed on the long-term performance of vegetative barriers at the INEL.

The next layer is a graded filter and drainage layer. It consists of sand, underlain by pea gravel, underlain by gravel, underlain by sand. Each segment of this layer is approximately $1 \mathrm{ft}$ thick. This 4-ft layer minimizes the potential for a standing head of water above the low-permeability layer below. The layer provides adequate permeability and capacity for drainage of infiltrating water. It also provides protection against erosion, freezing, and desiccation. Finally, the layer serves as a biotic intrusion barrier. The sand and gravel, for example, will not support stable burrows for burrowing animals.

The low-permeability layer must be below the maximum depth of frost penetration because fine soils are susceptible to frost heave. The top two layers of the cover are a total of approximately $6 \mathrm{ft}$ in thickness. Freezing depths at the INEL are typically 5 to $6 \mathrm{ft}$.

The next layer is a low-permeability moisture barrier. The recommended design of this layer, per the EPA requirements, is a composite barrier. The top portion of the barrier is a flexible membrane liner (FML), fabricated and emplaced in accordance with American Society for Testing and Materials (ASTM) standards. Immediately below and in intimate contact with the FML is a clay liner. The clay liner complements the FML by providing an additional barrier to water infiltration behind any small holes that may form in the FML. The FML helps minimize moisture content changes in the clay layer, thereby reducing the possibility of shrinking, swelling, and cracking. The clay liner is $3 \mathrm{ft}$ thick to avoid problems with cracking. (A minimum thickness of $2 \mathrm{ft}$ is required, and $3 \mathrm{ft}$ is recommended by the NRC.) Being a natural material, the clay provides a high degree of assurance of long-term integrity, whereas the integrity of the man-made FML over time periods excecding a few decades has not been proven. Thick clay is difficult to compact properly, and improperly compacted clay may settle, disrupting the liner. The best equipment for compacting the clay is a sheepsfoot roller. It helps tie together adjacent passes (lifts) of clay. Lifts are limited in thickness to 6 in., to allow sulficient compaction. Measurements as required by ASTM tests are made after each lift. The clay layer is crowned to improve drainage.

The innermost layer provides the final protection against moisture infiltration. The layer consists of a conductive matcrial and a capillary break or discontinuity (O'Donnell et al. 1992). The layer relies on the fundamental physical properties of groundwater to make any further infiltration virtually impossible. The upper portion of the layer is $1 \mathrm{ft}$ of diatomaceous earth or some appropriate substitute material. The properties of the diatomaceous earth are such that it acts as a wicking layer for moisture. It attracts any moisture that is present, by virtue of matric potential, or capillary forces. The lower part of this layer is $1 \mathrm{ft}$ of gravel. The same matric potential provides a force that tends to counter water movement into the gravel. Thus, the properties of the two materials in this layer are such that, under "appropriate conditions," any water that reaches this point in the cover is under tension and will not enter the gravel. A capillary discontinuity is formed at the interface between the diatomaceous earth and the gravel, thereby preventing leakage downward under the influence of gravity. Instead, the water is scavenged by the diatomaceous earth and conducted around the concrete structure to drains. The "appropriate condition" is that soil water tension be maintained, which in turn requires unsaturated llow conditions. Small- and large-scale tests (O'Donnell et al. 1992) indicate that such conditions can be maintained if the low-permeability clay layer above has a 
hydraulic conductivity of less than $10^{-6} \mathrm{~cm} / \mathrm{sec}$. This value is readily achieved by even a moderateperformance clay layer.

The cover includes penetrations for instrumentation and leads used for monitoring purposes during the period of institutional control. Special measures are taken to seal these penetrations during use and to close them before final closure, so that the infiltration resistance of the cover is not compromised.

Some subsidence of the cover will occur, although it is expected to be small. To minimize subsidence, the layers of the cover are not all emplaced at the same time. Lower layers are allowed to compress for a period of time before following layers are emplaced. The optimum emplacement times are determined through special studies.

The total thickness of the cover is approximately $11 \mathrm{ft}$. This value might seem excessive. However, the necessary thickness is driven by (a) the need for the cover to fulfill many functions, which in turn requires several different materials with widely varying properties, and (b) the fact that thin layers are not recommended (NRC 1991c) because they cannot tolerate large settlements without impacting cover performance. Planned covers for commercial LLW facilities (Anderson et al 1990; Deold and Coleman 1991; Anderson et al. 1992; Gunning et al. 1992) are typically 7 to $12 \mathrm{ft}$ thick. The cover scheduled to be emplaced at a French LLW disposal facility (Van Cote 1982; Convert $1992)$ is $4 \mathrm{~m}(13 \mathrm{ft})$ thick.

The slopes of the various layers and areas of the cover are important considerations. On the top portion of the cover, the inner layers must have slopes of at least 2 degrees, and the top layer must have a slope of 3 to 5 degrees. All top slopes are assumed here to be 3 degrees. This slope results in the center of the top portion of the cover being $15 \mathrm{ft}$ higher than the edge of the top portion. To bring about the required slope for all layers of the cover, the dome effect is achieved by placing a tapered layer of sand directly on top of the structural units. (A tapered layer of sand is also placed around the sides of the structures, below the cover.)

Excessive slopes on the sides of the cover would result in erosion of the vegetation/topsoil outside layer. Final selection of the slope depends on the soil characteristics and the denseness of the vegetation, and is based on the results of tests. In this study, a slope of 12 degrees is assumed as a rough estimate for the sides of the cover.

With the slopes established, the overall size of the cover can be calculated. Figure 5-10 indicates the areal extent of the cover (the dashed-line boundary) in relation to the disposal site area. For Subalternative 1A, the final cover is $1,722 \mathrm{ft}$ long by $972 \mathrm{ft}$ wide ( 38 acres).

5.3.4.3.3 Maintenance and Monitoring During Institutional Control PeriodRequirements: The principal requirements related to postclosure maintenance and monitoring are as follows:

- $\quad$ The disposal facility must ... eliminate to the extent practicable the need for ongoing active maintenance of the disposal site following closure so that only surveillance, monitoring, or minor custodial care are required (10 CFR 61.44). 


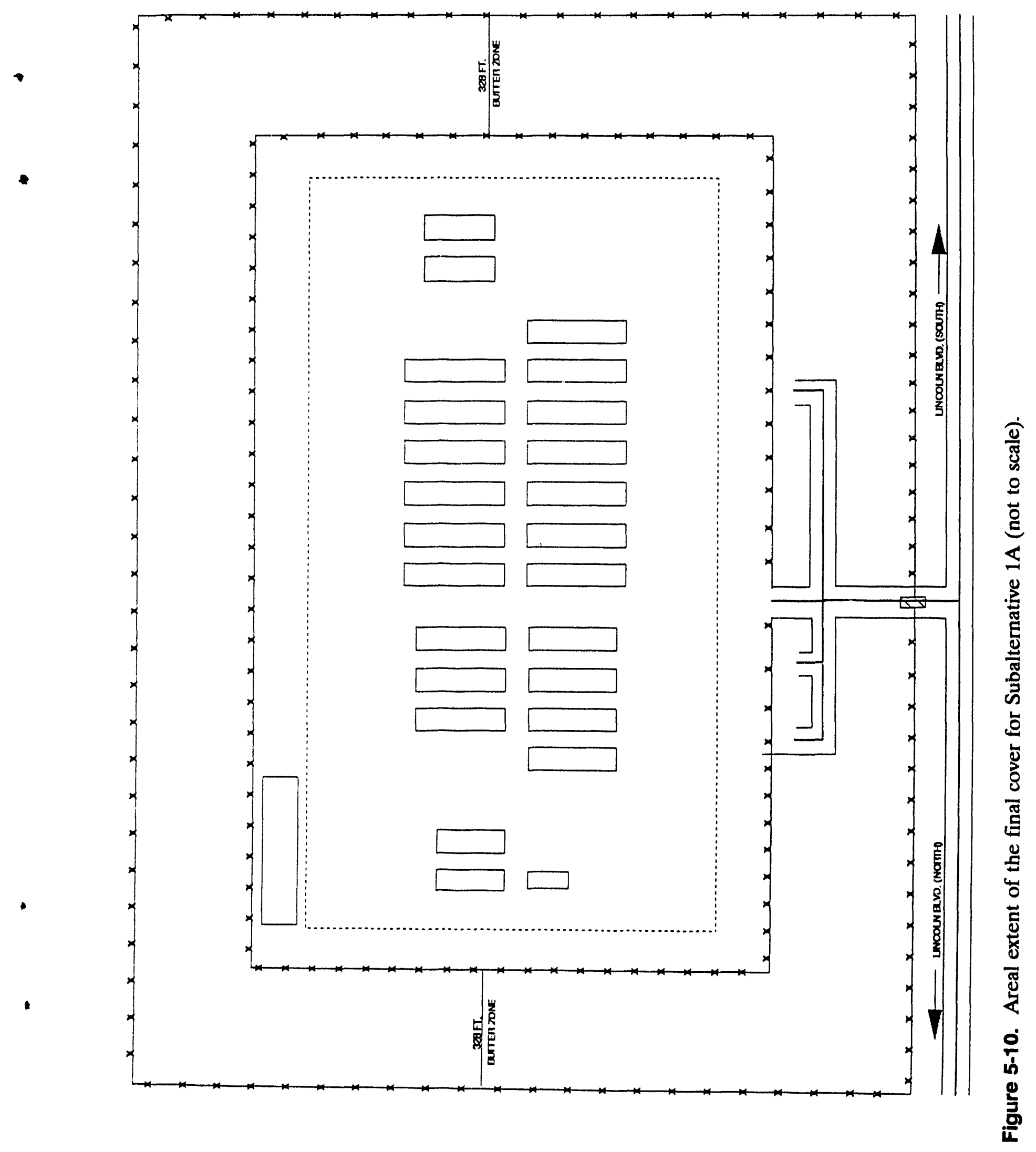


- The final cover must be repaired as necessary to correct the effects of settling, erosion, etc. The leachate collection and removal system must be operated until leachate is no longer detected. The runon and runoff systems must be maintained (40 CFR 264.310).

- $\quad$ Postclosure monitoring shall continue for 30 years (40 CFR 264.117).

- $\quad$ After the disposal site is closed, the licensee responsible for postoperational surveillance of the disposal site shall maintain a monitoring system based on the operating history and the closure and stabilization of the disposal site (10 CFR 61.53(d)).

The disposal site is actively monitored and maintained during the periods of operations and site closure, and to a lesser extent during the institutional control period (assumed to be 100 years) following site closure. The aim of the maintenance is to limit, to the extent practicable, the effects of such degradation processes as erosion, concrete cracking, subsidence, biological intrusion, and inadvertent human intrusion. In the long term, custodial care is limited to minor custodial activities such as security, grounds care, painting, and fence maintenance and repair.

Postclosure maintenance of the disposal site includes a monitoring program capable of providing early warning of releases of hazardous and radioactive constituents from the disposal site before they leave the site boundary.

The environmental monitoring program discussed in Section 5.3.4.2 continues throughout the institutional control period. The number of sampling locations and sampling frequency is the same during operations and the first 10 years of institutional control. If all performance indications are positive, the extent of the monitoring program is gradually reduced.

Monitoring of the status of the surface water management system, the leachate collection and removal systems, and leak detection system continues during this period. The leachate collection and removal system requires sampling and analysis of liquids collected as long as leachate is detected. The run-on and run-off systems continue to be maintained. Samples of retention pond water are also analyzed as necessary.

During the institutional control period, the condition and structural performance of the isolation barriers and the final cover (e.g., settling, strains, joint and seal behavior, cracking) are monitored at suitable frequencies and locations to verify design assumptions. The limiting acceptable values of parameters are specified, and a remedial action plan is implemented if the values are exceeded.

\subsubsection{Subaiternative 1B: Belowgrade Engineered Structures}

This section briefly describes the facilities constructed and the operations conducted for Subaltcrnative 1B. In this subalternative, all types of waste received are disposed of in BGES.

Hereafter in Section 5.3, only topics in which this subalternative differs significantly from Subalternative $1 \mathrm{~A}$ are discussed. 
5.3.5.1 Description of Facilities. The liners, waste handling facilities, support facilities, and CQA for this subalternative are essentially the same as for Subalternative 1A, and are not discussed further.

5.3.5.1.1 General Site Layout and Preparation-The general site layout used for Subalternative 1B is presented in Figure 5-11. The total land area requirement for the disposal concept is 112 acres. The discussion of site layout and preparation for Subalternative 1A applies identically to Subalternative $1 \mathrm{~B}$ except that the disposal unit configuration has been changed slightly to allow truck access to the belowgrade Class A LLW and mixed waste disposal units.

One row of the Class A LLW and mixed waste disposal units is available for disposal at a given time. An area approximately $200 \mathrm{ft}$ wide and extending the length of the row $(896 \mathrm{ft})$ is excavated to allow truck access to the disposal units. A ramp approximately $400 \mathrm{ft}$ long ( $7.5 \%$ grade) provides the truck access to this area. When the disposal units reach their design capacity, the excavated area is backfilled.

The Class $B$ and $C$ waste disposal units are located in a separate shallow, backfilled pit. An access ramp is provided to the working area $2 \mathrm{ft}$ belowgrade.

5.3.5.1.2 Disposal Unit Construction-In this subalternative, all of the disposal units are massive, reinforced concrete structures. The structures are identical in design and size to those described for Subalternative 1A (Figures 5-3 and 5-4 and Table 5-3). The isolation barriers are basically the same as in Subalternative 1A. The only difference is that the structures in this subalternative are constructed below the natural grade. The geologic barriers around the buried structures provide still another (partial) barrier.

The disposal units are constructed so that the top of the roof, when completed, is approximately at grade level. For the structures housing Class A waste, whether mixed or not, truck access to the structure is via a ramp (sec Figure 5-11) that descends to the bottom of the excavated area. Waste offloading and emplacement is accomplished by means of forklifts. For the structures housing Class B and Class $\mathrm{C}$ waste, whether mixed or not, the waste is emplaced by means of a gantry crane similar to that for Subalternative $1 \mathrm{~A}$.

5.3.5.1.3 Site Surface Water Management System-The principles of the site surface water management system are similar to that for Subalternative 1A. However, because the waste is buried belowgrade, an additional set of trenches is required around the vaults, and pumping is necessary to remove water from belowgrade sumps to the retention pond. The essential differences are described below.

The Class A mixed waste disposal units and the Class A disposal units are placed on the floor of a pit. The floor of the pit is surrounded by a trench that runs along the base of the pit wall (see Figure 5-12). The floor is graded such that the water that falls on the vault area of the pit drains by gravity to the pit perimeter trench. The vaults are placed on foundation drainage blankets that discharge to a common sump. The drainage blankets are similar in design to those used in Subalternative 1A (sce Figure 5-6). The perimeter trench is graded to allow gravity flow to a sump at one corner. Both the foundation drainage blanket sump and the trench sump are equipped with a pump to move water to the retention pond. 
$t b-s$

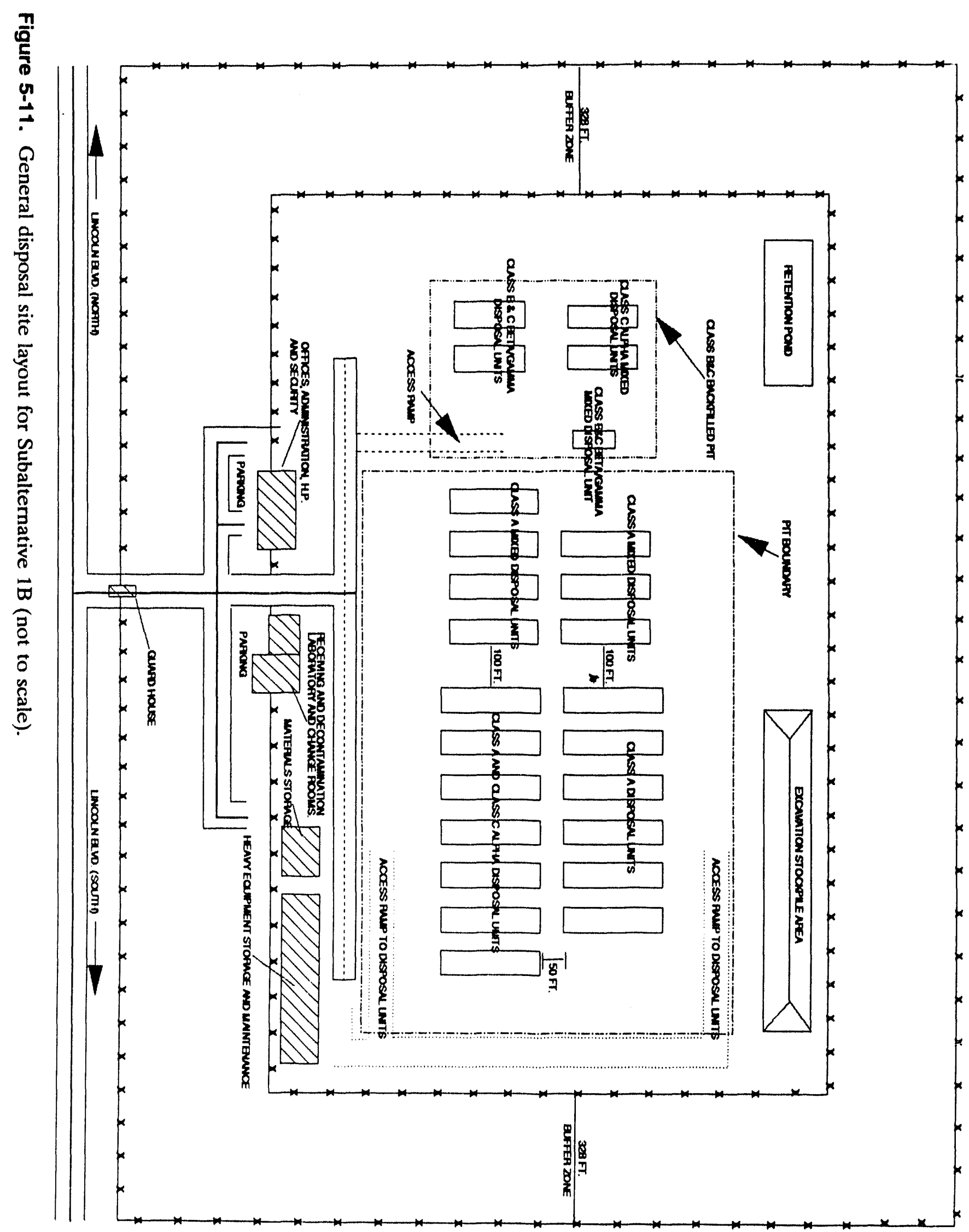




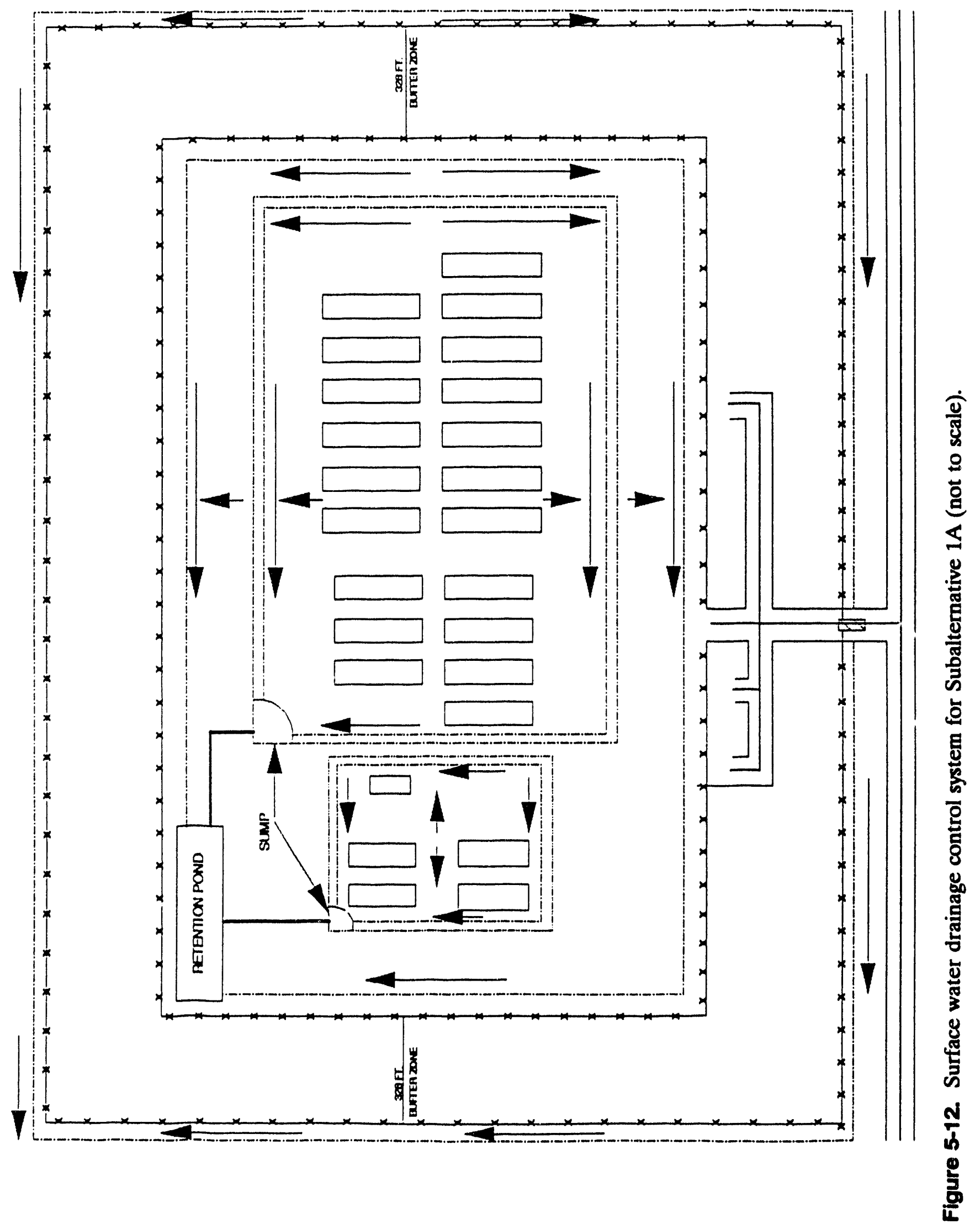


The remainder of the disposal units are also placed belowground, but the area around the vaults is backfilled such that the operating area is just a few feet belowgrade (see Figure 5-13). A ditch runs around the perimeter of the operating area (see Figure 5-12). The surface of the area is sloped such that water falling on the operating area is collected in the perimeter ditch. Water in the ditch flows by gravity to a sump at one corner. The sump is equipped with a pump to move water to the retention pond. A drainage blanket, similar to the one used in Alternative $1 \mathrm{~A}$, is installed under each vault. The blankets are modified such that they extend up the sides of the vaults (see Figure 5-13). Water that is collected in the vault drainage blankets is directed to a single underground sump. From the sump, the waste is pumped to the retention pond.

Water falling in the vault area, but not falling in the pit or the operating areas, is directed toward the vault area perimeter ditch as before.

5.3.5.2 Description of Operations. All operations for Subalternative 1B are essentially the same as those for Alternative $1 \mathrm{~A}$, except for the fact that the disposal structures are belowgrade, rather than above.

5.3.5.3 Closure and Postclosure Activities. All closure and postclosure activities for Subalternative 1B are essentially the same as those for Subalternative $1 \mathrm{~A}$, except for the fact that the sub-grade location of the disposal structures affects the elevation and area of the final cover.

5.3.5.3.1 Cover-The cover again consists of the same material layers, thicknesses, and slopes as shown in Figure $5-9$ and discussed in the accompanying text. However, to ensure total coverage, it is desirable that the entire thickness of the cover extend a short distance (c.g., $20 \mathrm{ft}$ ) beyond the edge of the structures. To accomplish this, a minimum of $4 \mathrm{ft}$ of sand is placed on the roof at the edge, with the centerline thickness of the sand layer being increased from $15 \mathrm{ft}$ to $19 \mathrm{ft}$, to maintain the required minimum slope of 3 degrees. With this adjustment, the cover dimensions are $1,474 \mathrm{ft}$ long by $724 \mathrm{ft}$ wide ( 24 acres).

\subsubsection{Subalternative 1C: Covered Modules}

This section briefly describes the facilities constructed and the operations conducted for Subalternative 1C. In this subalternative, all types of waste received are disposed of in concrete containers, or modules, on abovegrade concrete pads. A multilayer cover is then emplaced.

\subsubsection{Description of Facilities.}

5.3.6.1.1 General Site Layout and Preparation-The general site layout used for Subalternative $1 \mathrm{C}$ is presented in Figure 5-14. The total land area requirement for the disposal concept is 132 acres. The discussion of site layout and preparation for Subalternative $1 \mathrm{~A}$ applies identically to Subalternative $1 C$, except that the waste is stacked on abovegrade concrete pads rather than in abovegrade disposal units.

Disposal pads for LLW and mixed waste are separated from each other by a distance of $100 \mathrm{ft}$. The LLW disposal area consists of three separate concrete pads. The pads are separated from each other by $50 \mathrm{ft}$. The mixed waste disposal area also consists of three separate concrete pads, separated by $50 \mathrm{ft}$. 


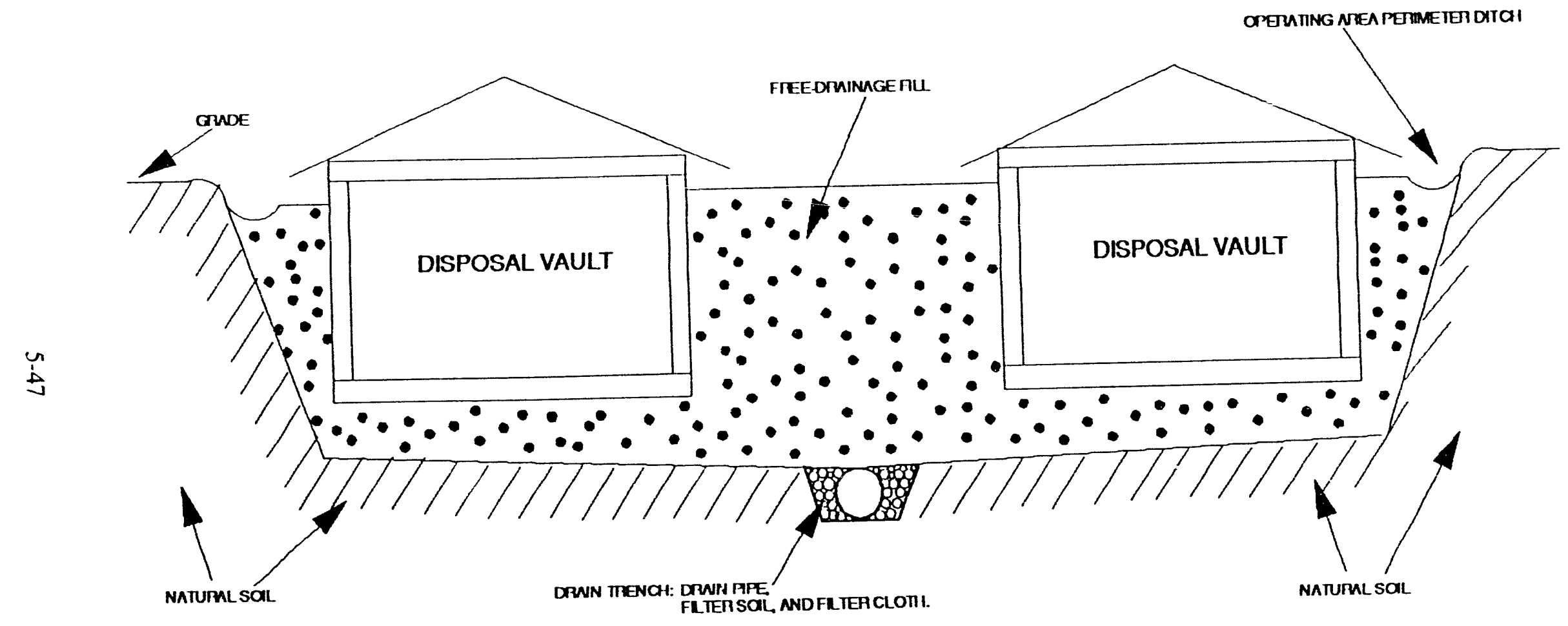

Figure 5-13. Foundation blanket drain for Class B and C waste for Subalternative 1B (not to scale). 


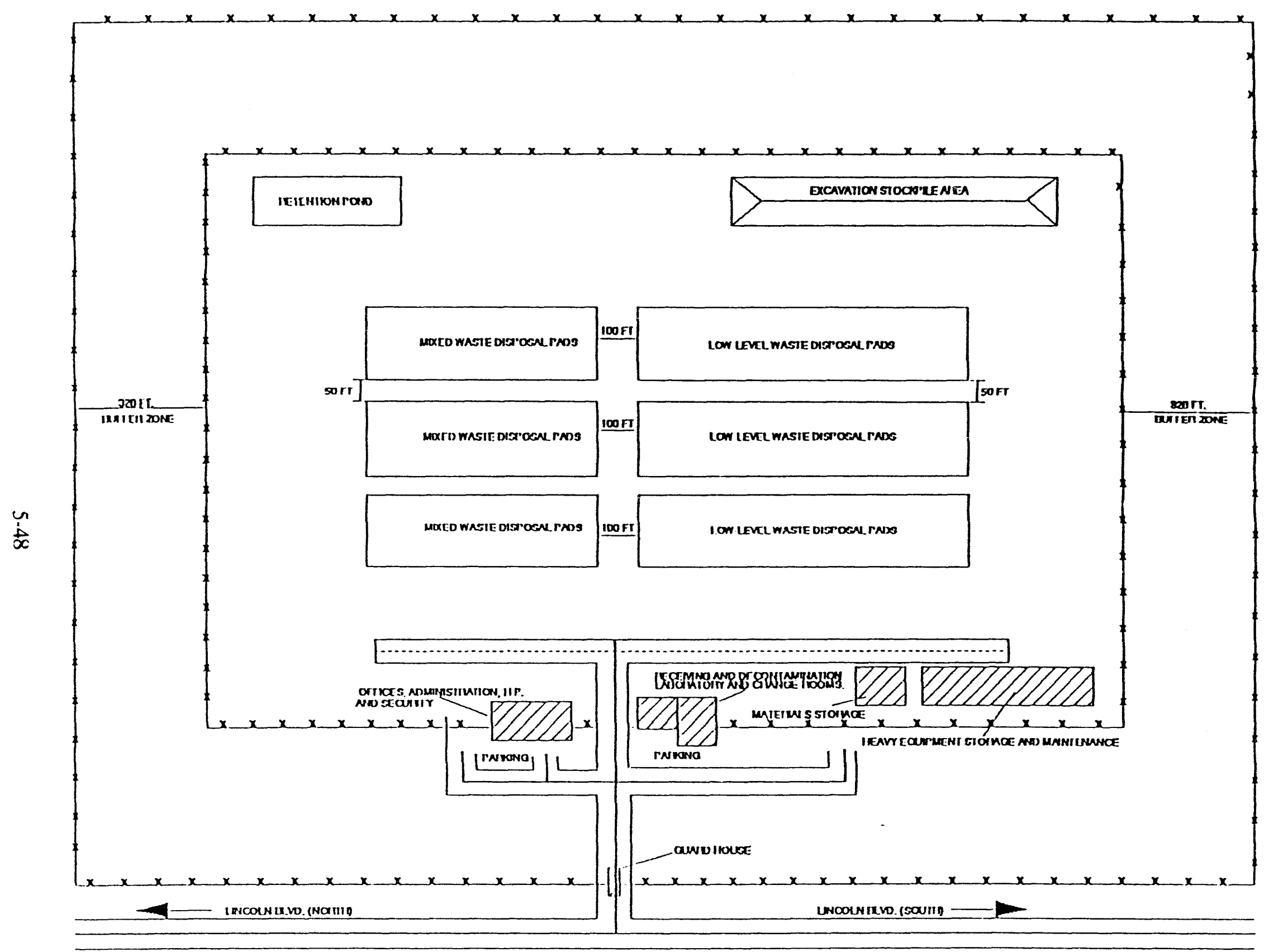

Figure 5-14. General site layout for Subalternative 1C (not to scale). 
5.3.6.1.2 Disposal Unit Construction-In this subalternative, all of the disposal units are reinforced concrete containers, or modules, on concrete pads (see Figure 5-15).

The concrete containers provide stability and a barrier against intruders. Because of the challenge of producing concrete and other materials that will isolate the waste for 500 years (see the performance requirements in Section 2.3) under site-specific climatic conditions, a materials research and development program is conducted in connection with this subalternative, as with Subalternative $1 \mathrm{~A}$.

The individual waste containers and any inner liners are the first isolation barrier. The second isolation barrier is the concrete modules. The modules are designed to maintain their integrity under normal operational loads, as well as many credible accident loads. The third isolation barrier is a double membrane liner placed under the concrete pad, for all types of waste, not just mixed waste. For simplicity, the membrane liners are not shown in Figure 5-15 but are discussed later. The cover emplaced over the modules is also part of the third isolation barrier.

The design of this concept borrows from the Interim Waste Management Facility, the current version of the concrete vault/tumulus disposal technology used at DOE's Oak Ridge site for the past 5 years (Van Hoesen and Jones 1991). Concepts were also borrowed from the French waste disposal facility, Centre de la Manche (Van Cote 1982).

In this subaltcrnative, waste packaged in $4 \times 4 \times 8$-ft concrete boxes is grouted into the container at the generator or treatment facility and the boxes are sealed shut. The boxes' top, sides, and bottom are approximately 6 in. thick. Waste packaged in metal drums is overpacked at the generator or treatment facility in $4 \times 4 \times 6$-ft concrete boxes. These boxes also have tops, walls, and bottoms that are approximately $6 \mathrm{in}$. thick, resulting in outside dimensions of $5 \times 5 \times 7 \mathrm{ft}$. Waste packaged in $4 \times 4 \times 6$-ft metal boxes is overpacked in the same $4 \times 4 \times 6$-ft concrete boxes, having outside dimensions again of $5 \times 5 \times 7 \mathrm{ft}$. In all cases, the waste is grouted in the concrete box by the generator or treatment facility.

This family of waste packagings is not optimized for disposal-space efficiency, ease of handling, minimum transport costs, elc. A tradeoff study of various packaging sizes is needed during conceptual design, but is beyond the scope of this study.

Based on the experience of Oak Ridge, the concrete pads in this subalternative are $2 \mathrm{ft}$ thick. This thickness allows them to withstand the large loads imposed by the stacked concrete modules and the final cover. The pads are sloped slightly to allow collection of any leaking or infiltrating liquid.

To simplify this subalternative, it was determined that the eight types of waste can be disposed of on two groups of pads, one group for mixed waste and one for nonmixed waste. An assumption was made, based on an interpretation of 10 CFR 61.52 and .56 (b)(1), that the Class A waste can be disposed of on the same pads as the other classes of waste, by depending on the concrete modules to provide the required stability for the Class $A$ waste. If desired, curbs are erected between types of waste on a pad to allow separate monitoring of infiltrating water. 


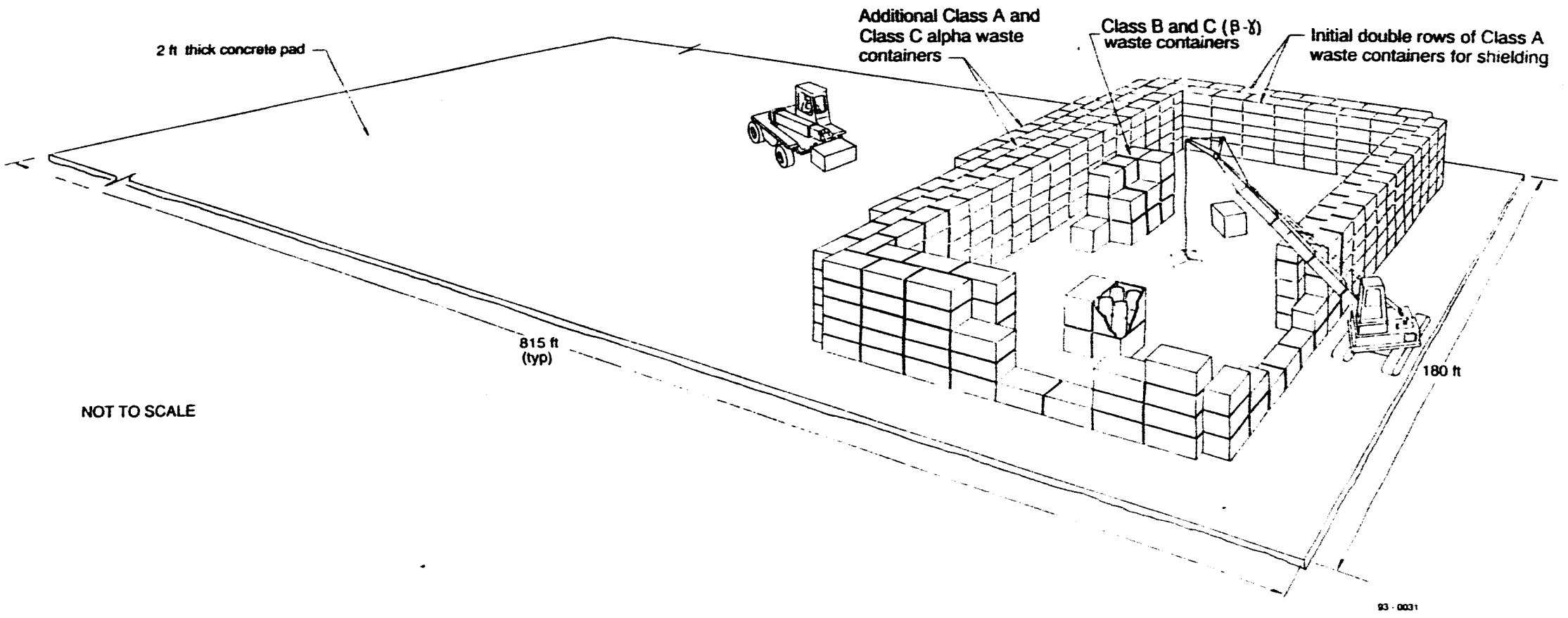

Figure 5-15. Design concept for covered modules in Subalternative 1C (for clarity, Butler-type building covers are not shown). 
The pads were sized based on the numbers and sizes of waste containers in Table 2-6. The results of the sizing are listed in Table 5-4. The dimensions include an allowance of $15 \mathrm{ft}$ around the circumference of the pads, between the edge of the waste stack and the edge of the pad, for operations of the cranc and other equipment. If separate pads are desired for each class of waste, no major impact results on the layout. More and smaller pads result, rather than fewer and larger pads.

5.3.6.1.3 Site Surface Water Management System-The site surface water management system is essentially the same as that for Subalternative 1A. Rain and snow cannot fall directly on the waste because all the waste is contained in concrete modules. Additionally, Butler-type buildings are used as interim covers over lilled sections of the pads. Water from rain and snow melt is directed to drains along one edge. The drains are directed to a sump. Water collected in the sump is pumped periodically to the retention pond.

There are no foundation drainage beds in this subalternative because a dual liner/leachate system is placed underneath the pads.

5.3.6.1.4 Liners and Leachate Collection and Removal Systems-The principles governing the design of the liners and LCRSs are the same for this alternative as for Subalternative 1A. However, the dual-liner/leachate system is installed underneath the disposal units, instead of inside the units.

The dual-liner/leachate system is shown in Figure 5-16. The general system is described below, starting at the natural soil base and moving up to the concrete pad.

The region below each pad is excavated to a depth of about $6 \mathrm{ft}$. A 3-ft-thick clay liner is placed on the bottom of the hole. Directly on top of the clay liner is a 60-mil HDPE liner. The combination of the clay and the HDPE liners is referred to as the secondary liner. The secondary liner extends to the surface on all sides of the pad. Any leachate that comes through from the primary liner is collected above the secondary liner.

The secondary leachate collection and removal system consists of a drainage net that sits directly on top of the secondary liner. The nct extends up the sides to the surface, along with the secondary liner. Any leachate that leaks through the primary liner is collected above the secondary liner and flows by gravity through the drainage net to a drain. The drain is an HDPE pipe that is embedded in the clay liner. The drain pipe directs the leachate to a secondary drain sump where it is collected for sampling and treatment.

Table 5-4. Number and size of concrete pads for each type of waste in Subalternative 1C.

\begin{tabular}{ccc}
\hline Type(s) of waste & $\begin{array}{c}\text { Total number of } \\
\text { pads required over } \\
\text { 40)-year period }\end{array}$ & $\begin{array}{c}\text { Dimensions } \\
(\mathrm{W} \times \mathrm{L}, \mathrm{ft})\end{array}$ \\
\hline Nonmixed & 3 & $180 \times 815$ \\
Mixed & 3 & $180 \times 486$ \\
\hline
\end{tabular}




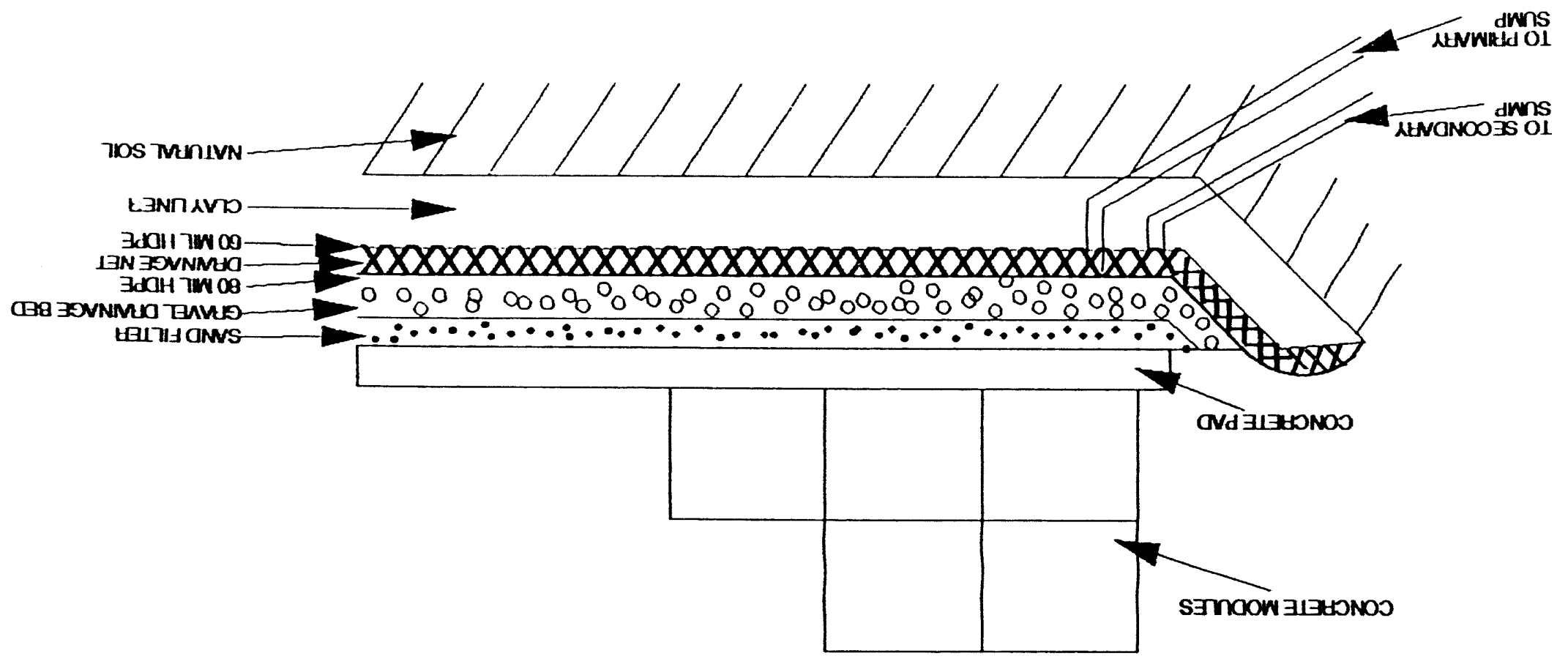


The primary liner is an 8()-mil layer of HDPE. The primary liner sits on top of the secondary LCRS. The primary liner extends up to the surface on all sides of the pad with the secondary LCRS and liner. Any leachate that comes from the waste will collect above the surface of the primary liner.

The primary LCRS sits on top of the primary liner. The primary LCRS consists of a gravel drainage layer overlain with a sand filter. The gravel/sand filter combination is about $3 \mathrm{ft}$ thick. Any leachate collected above the primary liner flows by gravity through the primary LCRS to a drain. The drain is an HDPE pipe that extends through the secondary LCRS and liner and is embedded in the clay liner. The drain pipe directs the leachate to a primary drain sump where it is collected for sampling and treatment.

The primary LCRS is constructed such that, when it is compacted, it still allows leachate flow, but will also support the weight of the concrete pad poured on its surface. The sand filter overlay protects the gravel drainage bed from plugging when the concrete pad is poured. The drainage layer, sand filter, and concrete pad are all sloped to improve drainage.

The concrete pad that is poured on top of the primary LCRS is discussed in the previous section on site surface-water management.

The drainage pipes from each LCRS are made of double-walled HDPE. The pipes penetrate the liners and gravity drain to separate sumps. The primary and secondary collection sumps are small, double-walled tanks located in a concrete drain gallery (Figure 5-8). The gallery is adjacent to the pad and belowgrade. The drain pipes enter the sumps near the top. The sumps have top penetrations for leak delection devices and for hookup of sampling/pumping equipment.

Detailed design of the dual-liner/leachate collection system ensures that the combination of LCRS material, thickness, and slope is sufficient to detect a specified minimum leakage rate. Detailed design also ensures that plugging of the LCRS does not occur.

\subsubsection{Description of Operations.}

5.3.6.2.1 Waste Emplacement-Class $A$ and Class $C \alpha$ waste is placed on the pads using forklifts. Class $B$ and $C \beta-\gamma$ waste, because of its higher radiation levels, is placed on the pads using a boom crane (see Figure 5-15). The Class $A$ and Class $C \alpha$ waste is stacked such that hollow spaces are left to accept the Class $B$ and $C \beta-\gamma$ waste. Once the higher radiation level $\beta-\gamma$ waste is in place, a layer of Class $A$ or Class $C \alpha$ waste is placed on top using a boom crane. All other aspects of waste emplacement are similar to those discussed in Subalternative $1 \mathrm{~A}$.

\subsubsection{Closure and Postclosure Activities.}

5.3.6.3.1 Disposal Unit Closure-The concrete modules are resistant to weather damage; however, Butler-type buildings are used as interim covers over filled sections of the pads. Using an interim cover reduces the amount of contact of waste modules with rain and snow, and also reduces the amount of water that must be handled by the various water collection systems. The interim cover buildings are removed when the final cover is installed. 
5.3.6.3.2 Cover-The cover again consists of the same material layers, thicknesses, and slopes as shown in Figure 5-9 and discussed in the accompanying text. However, the height of the covered modules is a maximum of only $21 \mathrm{ft}$. The dimensions of the cover are 1,705 $\mathrm{ft}$ long by $944 \mathrm{ft}$ wide ( 37 acres).

\subsubsection{Subalternative 1D: Greater-depth Burial/Boreholes}

This section brielly describes the facilities constructed and the operations conducted for Subalternative 1D. In this subalternative, Class A waste, Class A mixed waste, and Class $C \alpha$ mixed waste (and the very small amount of Class $\mathrm{C} \alpha$ waste) are disposed of by greater-depth burial in pits. The other types of waste are disposed of in greater-depth boreholes.

\subsubsection{Description of Facilities.}

5.3.7.1.1 General Site Layout and Preparation-The general site layout used for Subalternative 1D is presented in Figure 5-17. The total land area requirement for the disposal concept is 112 acres. The discussion of site layout and preparation for Subalternative 1A applies identically to Subalternative 1D, except that the disposal area configuration has been changed to disposal by greater-depth burial and greater-depth boreholes.

The layout of the disposal area includes three separate greater-depth disposal pits. These pits are designed for disposal of Class A LLW, Class A mixed waste, and Class C $\alpha$ LLW and mixed waste. The disposal pits are separated from each other by a distance of $100 \mathrm{ft}$.

Also included in the disposal area is a pit to allow disposal of the Class B and C $\beta$ - $\gamma$ waste in greater-depth borcholes. The borehole disposal area is separated from the burial pits by an access road and a total distance of $150 \mathrm{ft}$.

From the access road, ramps are constructed to allow for truck access into the working areas of each pit. The ramps extend between $500 \mathrm{ft}$ and $520 \mathrm{ft}$ in length (7.5\% grade) and $50 \mathrm{ft}$ in width. The disposal pit dimensions are increased by $50 \mathrm{ft}$ to allow for construction of these ramps.

5.3.7.1.2 Disposal Unit Construction-Three points of design philosophy are discussed before the designs are presented. First, greater-depth burial is still within what the NRC terms "nearsurface disposal," per 10 CFR 61, because the waste is disposed of in the upper $30 \mathrm{~m}$ of the earth's surface. As used in this document, the term applies to disposal in which the top of the uppermost waste container is at least $5 \mathrm{~m}$ belowgrade, for all classes of waste. The specific criterion used for the designs herein is that the top of the uppermost container be $10 \mathrm{~m}(33 \mathrm{ft})$ below the surface. The $33 \mathrm{ft}$ of materials over the waste includes the $11 \mathrm{ft}$ thick linal cover. Thus, the top of the waste stack is typically $22 \mathrm{ft}$ belowgrade.

Second, the burial could take place in pits, trenches (long, narrow pits), or borcholes. The types of waste representing the largest volumes are the contact-handled types: Class A, Class A mixed, and Class $C \alpha$ mixed. For each of these types, disposal in a separate pit was selected for evaluation here. (The very small amount of Class $C \alpha$ waste is codisposed with the $C \alpha$ mixed waste.) Pits were selected rather than trenches because of the greater convenience of container handling and emplacement. These types of waste are very low in radiation level, so the improved shielding offered 


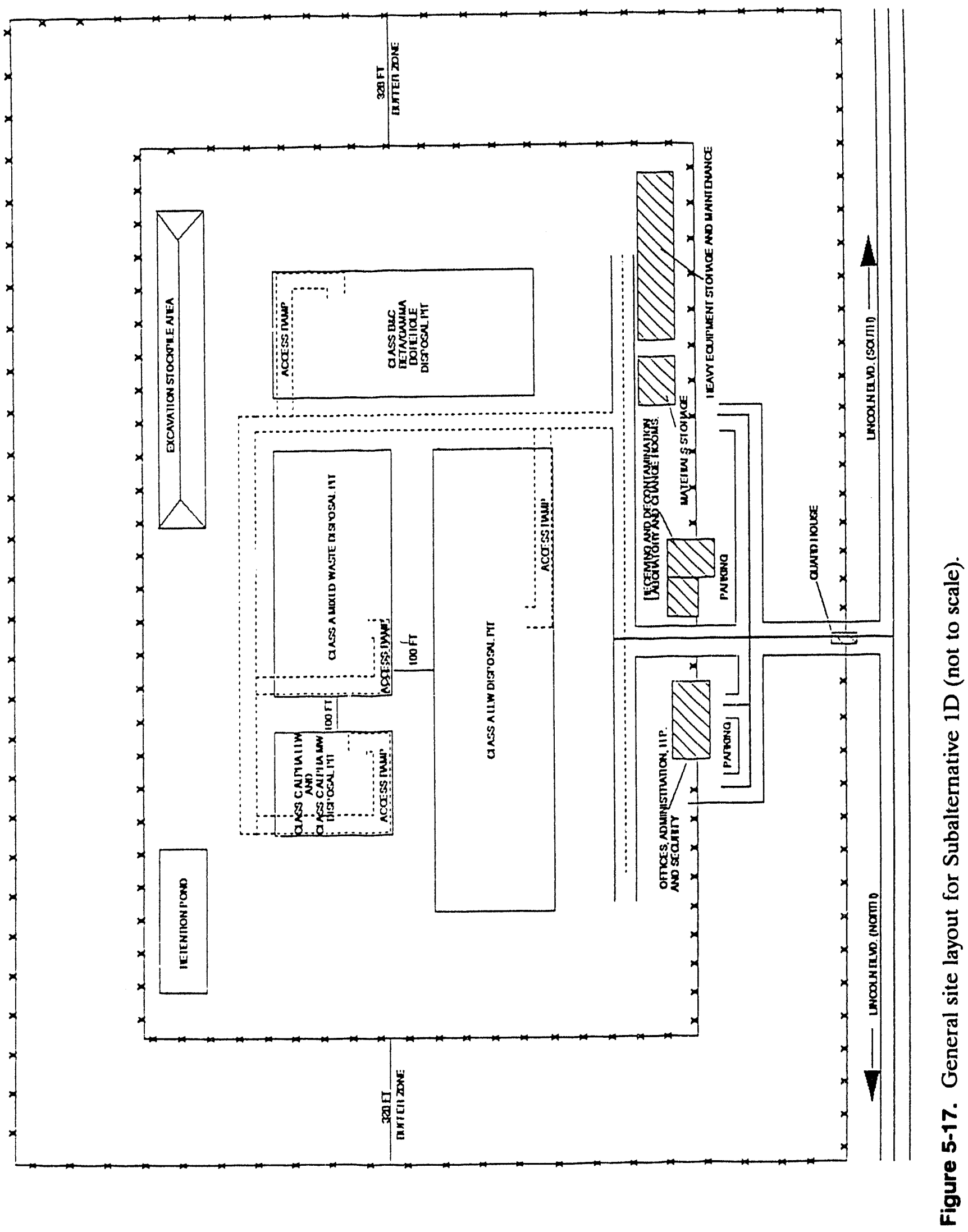


by trench disposal is not necessary in this instance, as demonstrated by the successful use of large disposal pits for Class A waste at the RWMC and elsewhere. Certain refinements in stacking arrangements and interim cover can be used with pit disposal to reduce radiation exposures if necessary. For the remote-handled waste types-Classes B, B mixed, C $\beta-\gamma$, and C $\beta-\gamma$ mixed-disposal in borcholes was selected. This method offers the best radiation shielding of the three disposal methods listed, and such shielding is important for these types of waste.

Third, the design details selected for evaluation here and in Subalternative $1 \mathrm{E}$ are intended to illustrate basic principles (e.g., the use of disposal at a greater-than-normal depth). Certain improvements could be made to the designs. For example, an improved borchole design is being developed at the RWMC. However, the intent here is to illustrate the basic principle, not develop the optimum design. If this subalternative is selected for implementation, detailed studies will be conducted to optimize the design.

The individual waste containers and any inner liners are the first isolation barrier. The second isolation barrier is a dual membrane liner, for all types of waste, not just mixed waste. The third isolation barrier is the greater-depth geologic disposal, plus the final cover.

The numbers and sizes of the burial pits and boreholes are listed in Table 5-5.

Figure 5-18 provides a simplified sectional view of the greater-depth burial pits for the contacthandled types of waste. All containers of these types of waste are square steel drums or steel boxes. Not shown in the figure are a double liner, a leachate collection and removal system, and a leak detection system. These items are located beneath the stack of waste and are protected by a compacted gravel working surface.

Figure 5-19 provides a simplified sectional view of the disposal arrangement for the greaterdepth boreholes. Aside from the greater depth of the emplacement, this concept is quite similar to

Table 5-5. Number and size of disposal units for each type of waste in Subalternative 1D.

\begin{tabular}{|c|c|c|}
\hline Type(s) of waste & $\begin{array}{l}\text { Total number of } \\
\text { units required } \\
\text { over } 40 \text {-year } \\
\text { period }\end{array}$ & $\begin{array}{c}\text { Dimensions during } \\
\text { operations, } \mathrm{ft} \\
(\mathrm{W} \times \mathrm{L}, \text { bottom }) / \\
(\mathrm{W} \times \mathrm{L}, \text { top }) / \\
\text { (depth, grade tc bottom) }\end{array}$ \\
\hline A & 1 pit & $\begin{array}{l}180 \times 1,248 / \\
248 \times 1,316 / 40\end{array}$ \\
\hline A mixed & 1 pit & $\begin{array}{l}180 \times 617 / \\
248 \times 685 / 40\end{array}$ \\
\hline $\mathrm{C} \alpha$ mixed and $\mathrm{C} \alpha$ & 1 pit & $\begin{array}{l}180 \times 110 / \\
248 \times 178 / 40\end{array}$ \\
\hline $\begin{array}{l}B, B \text { mixed, } C \beta-\gamma, C \beta-\gamma \\
\text { mixed }\end{array}$ & 1,380 boreholes & 6 (diameter)/38 \\
\hline
\end{tabular}




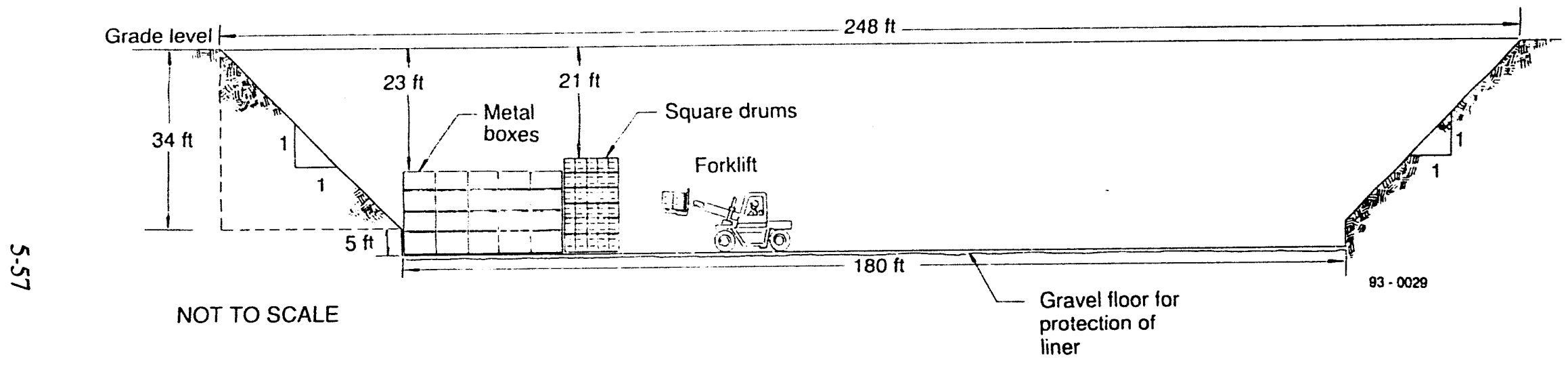

Figure 5-18. Simplified sectional view of greater-depth burial pits for contact-handled waste in Subalternative 1D. 


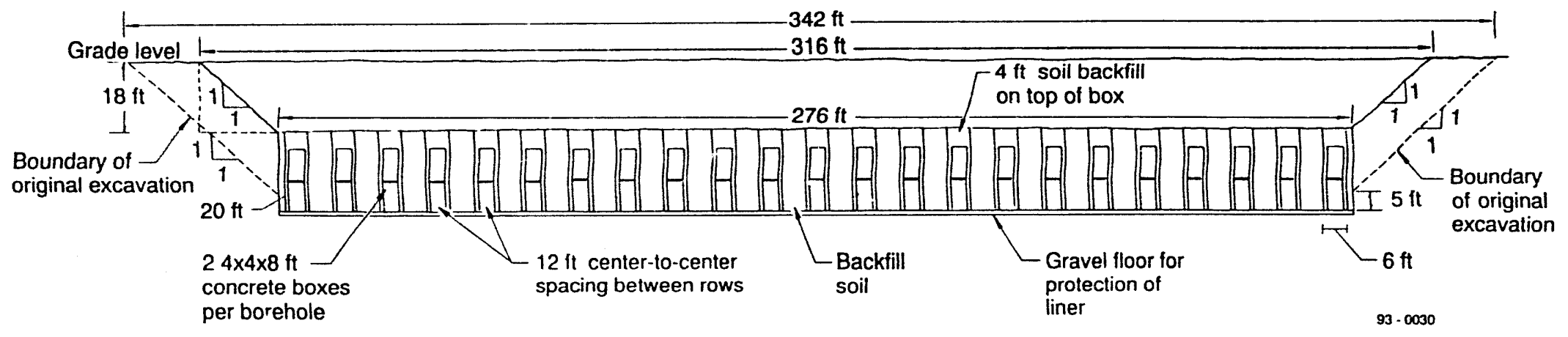

NOT TO SCALE

Figure 5-19. Simplified sectional view of disposal area with greater-depth boreholes for remote-handled waste in Subalternative 1D. 
the soil vault disposal method that has been used for several years at the RWMC. Each 6-ft-diameter borehole is excavated $20 \mathrm{ft}$ deep in backfilled soil. [The backfilled soil, along with a protective layer of compacted gravel, covers the double liner, the leachate collection and removal systems, and the leak detection system (not shown in the figure).] The top of the borehole is located $18 \mathrm{ft}$ belowgrade. All waste disposed of in boreholes is in $4 \times 8 \times 4$-ft concrete boxes. Each borchole accommodates 2 concrete boxes on end. The boxes are assumed to have a lifting appurtenance on the end and to be emplaced vertically in the borcholes. A metal sleeve is used during emplacement to assist in alignment of the container and in stabilization of the borehole until emplacement is completed. The slecve is then removed. The hole is backfilled, including $4 \mathrm{ft}$ of earth on top for shielding. The boreholes are in rows, with $10 \mathrm{ft}$ center-to-center spacing within rows and 12-ft centerto-center spacing between rows. The boreholes consume an overall area of $600 \times 276 \mathrm{ft}$ at the bottom of a pit. Each of the 4 types of remote-handled waste is placed in a separate area of the array, with the most highly radioactive Class $C \beta-\gamma$ waste being placed near the center.

5.3.7.1.3 Site Surface Water Management System-The site surface water management system is essentially the same as that for Subalternative 1A. Rain and snow that falls directly in the waste disposal pits will be collected by the dual liner/leachate system discussed in the following section.

5.3.7.1.4 Liners and Leachate Collection and Removal Systems-The principles governing the design of the liners and LCRSs are the same for this alternative as for Subalternative 1A. However, the dual/liner leachate system is installed underneath the disposal units, instead of inside the units.

The dual-liner/leachate system is shown in Figure 5-20. The general system is described below starting at the natural soil base and moving up to the waste containers.

A $3-\mathrm{ft}$ thick clay liner is placed on the bottom of each pit. Directly on top of the clay liner is a 60-mil HDPE liner. The combination of the clay and the HDPE liner is referred to as the secondary liner. The secondary liner extends to the surface on all sides of the pad. Any leachate that comes through from the primary liner is collected above the secondary liner.

The secondary leachate collection and removal system consists of a drainage net that sits directly on top of the secondary liner. The net extends up the sides to the surface, along with the secondary liner. Any leachate that leaks through the primarv liner is collected above the secondary liner and flows by gravity through the drainage net to a drain. The drain is an HDPE pipe that is embedded in the clay liner. The drain pipe directs the leachate to a secondary drain sump where it is collected for sampling and treatment.

The primary linei is also a clay/HDPE combination. The clay is $3 \mathrm{ft}$ thick; an 80 -mil HDPE liner is used. The primary liner sits on top of the secondary LCRS. A filter layer separates the primary liner and secondary LCRS to prevent plugging of the LCRS with clay. The primary liner extends up to the surface on all sides of the pad with the secondary LCRS and liner. Any leachate that comes from the waste will collect above the surface of the primary liner.

The primary LCRS sits on top of the primary liner. The primary LCRS consists of a gravel drainage layer overlain with a sand filter. The gravel/sand filter combination is about $3 \mathrm{ft}$ thick. Any 


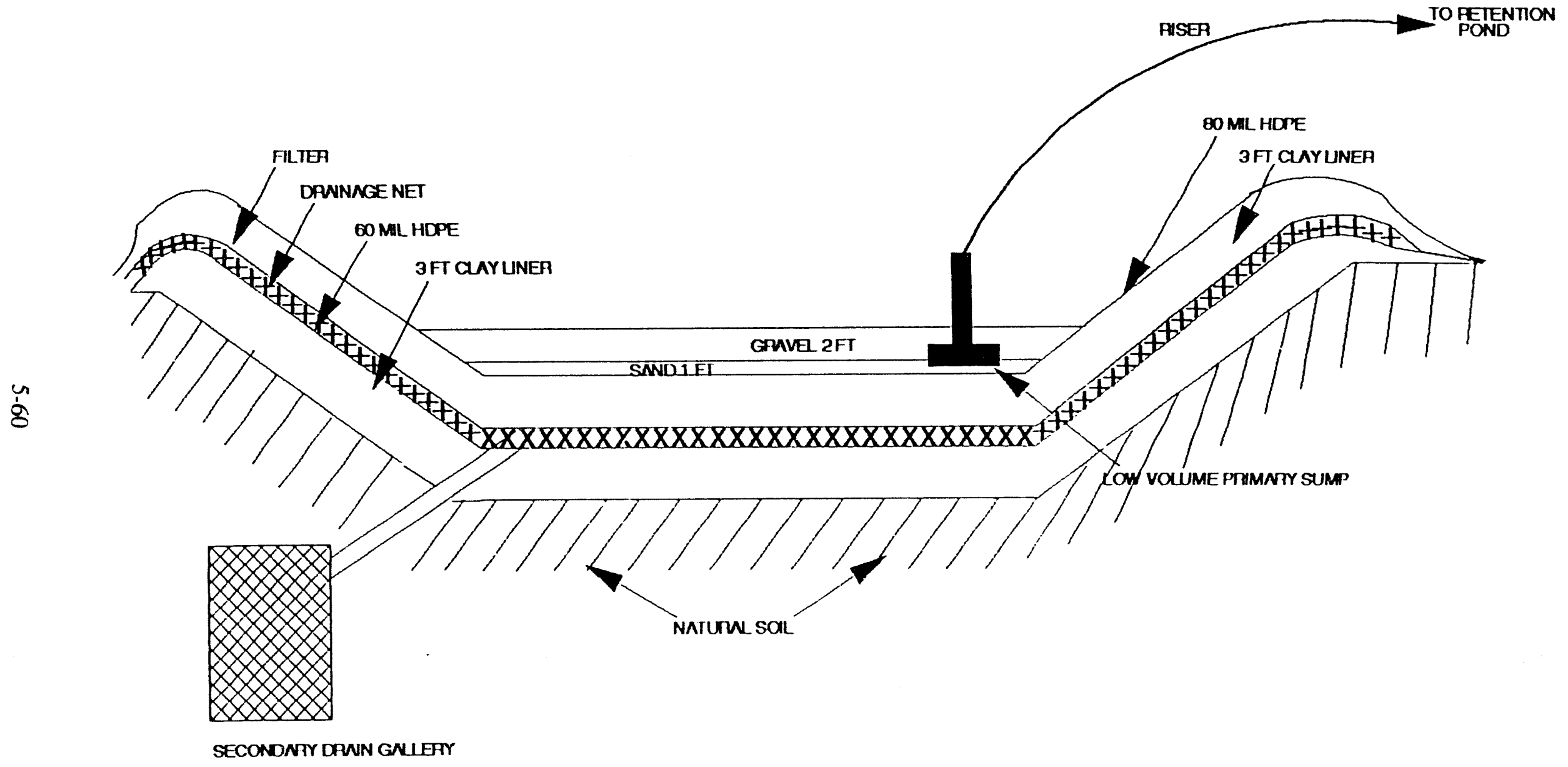

Figure 5-20. The dual-liner/leachate system for Subalternative 1D (not to scale). 


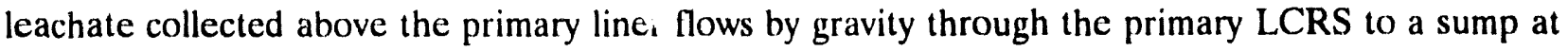
one corner of the pit.

The operating surface is placed on top of the primary LCRS. The operating surface for the pits is a compacted gravel that allows drainage while supporting the waste and the waste handling vehicles. The operating surface for the greater-depth boreholes is the backfill layer in which the holes are drilled. The sand filter overlay protects the primary LCRS gravel drainage bed from plugging. The drainage layer, sand filter, and operating surface are all sloped to improve drainage. Water that flows through the operating surface is collected in the primary LCRS.

The drainage pipe from the secondary LCRS is made of double-walled HDPE. The pipe penetrates the secondary liner and drains by gravity to a sump. The secondary collection sump is a small, double-walled tank located in a concrete drain gallery. The gallery is adjacent to the pad and

is belowgrade. The drain pipe enters the sump near the top. The sump has top penetrations for leak detection devices and for hookup of sampling/pumping equipment.

The primary LCRS sump is a shallow, low-volume sump filled by gravity draining of the primary LCRS. The sump has top penetrations for sampling/pumping equipment. Because the pit is open to the atmosphere, it is expected that the primary sump will need to be pumped frequently to remove rain and snow melt. The sump will be sampled before pumping. If the water is clean, as is expected, then the water will be pumped to the retention pond.

Detailed design of the dual-liner/leachate collection system ensures that the combination of LCRS material, thickness, and slope is sufficient to detect a specified minimum leakage rate. Detailed design also ensures that plugging of the LCRS does not occur.

\subsubsection{Description of Operations.}

5.3.7.2.1 Waste Emplacement-Following waste receipt and transfer operations similar to those described for Subalternative 1A, the waste containers are emplaced in pits using forklifts and in the greater-depth borcholes using a boom crane. The scheme for waste placement in a pit is to stack waste at the end of the pit furthest from the access ramp, and then move toward the ramp. Packages that approach the limits of sale contact handling are placed toward the center of the stack in order to take advantage of shielding from other lower activity waste packages. Periodically, the stacked waste is covered with fill material.

The first step in disposal of waste in greater-depth boreholes is to drill a 6-ft diameter hole in the previously prepared pit. A cover unit with a metal extension sleeve is placed over each hole. The cover supplies shielding while placing waste. The extension tube fits inside the hole and prevents collapse of the upper sides during waste emplacement. Waste containers are moved from transfer vehicles to the borehole using a boom crane. Once two containers are placed, the space around the waste containers is filled with grout. Then, the cover unit and extension sleeve are removed and the top of the hole is backfilled with soil. 


\subsubsection{Closure and Postclosure Activities.}

5.3.7.3.1 Disposal Unit Closure-Waste placed in greater-depth boreholes is covered as a part of normal operations. During final closure of the pit, the entire pit is backfilled to grade level in preparation for installation of the final cover.

Waste placed in the greater-depth burial pits is periodically covered as a normal part of waste operations. At the end of the useful life of a pit (when it is full), the last waste emplaced and the access ramp are covered with fill to grade level, in preparation for installation of the final cover.

Following the institutional control period, the LCRS drain pipes are plugged, and the void volumes in the drain galleries are backfilled with sand.

5.3.7.3.2 Cover-The cover again consists of the same material layers, thicknesses, and slopes as shown in Figure 5-9 and discussed in the accompanying text. However, the tops of the burial pits are at grade level, so the edge of the cover does not have to descend from an elevation down to grade level, except at the ends. The dimensions of the cover are approximately $1,932 \mathrm{ft}$ long by $800 \mathrm{ft}$ wide ( 36 acres).

\subsubsection{Subalternative 1E: Shallow Land Burial/Boreholes}

This section briefly describes the facilities constructed and the operations conducted for Subalternative $1 \mathrm{E}$. In this subalternative, Class $A$ waste and Class $A$ mixed waste are disposed of by shallow land burial in pits. Class $\mathrm{C} \alpha$ mixed waste (and the very small amount of Class $\mathrm{C} \alpha$ waste) are disposed of in slightly deeper pits for intruder protection. The other types of waste are disposed of in borcholes.

\subsubsection{Description of Facilities.}

5.3.8.1.1 General Site Layout and Preparation-The general site layout used for Subalternative $1 \mathrm{E}$ is presented in Figure 5-21. The total land area requirement for the disposal concept is 108 acres. The discussion of site layout and preparation for Subalternative 1D applies identically to Subalternative $1 \mathrm{E}$, except that the disposal pit dimensions have been changed for disposal by shallow land burial and boreholes. See the discussion on disposal unit construction for further details.

In this Subalternative, the access ramps extend between $265 \mathrm{ft}$ and $310 \mathrm{ft}$ in length (7.5\% grade) and $50 \mathrm{ft}$ in width. The disposal pit dimensions have been increased by $50 \mathrm{ft}$ to allow for construction of these ramps.

5.3.8.1.2 Disposal Unit Construction-The designs of the various disposal units for this subaltcrnative are almost identical to those for Subalternative 1D, except that the depth of disposal here is considerably less. The depth of disposal for the various types of Class $C$ waste is such that, for either pit disposal or borehole disposal, $5 \mathrm{~m}$ (16 ft) of earthen cover is emplaced over the uppermost waste container. Since the $16 \mathrm{ft}$ includes the final cover, which is $11 \mathrm{ft}$ thick, the top of the Class $\mathrm{C}$ containers is $5 \mathrm{ft}$ belowgrade. For the pits containing Class $\mathrm{A}$ waste and Class $\mathrm{A}$ mixed 


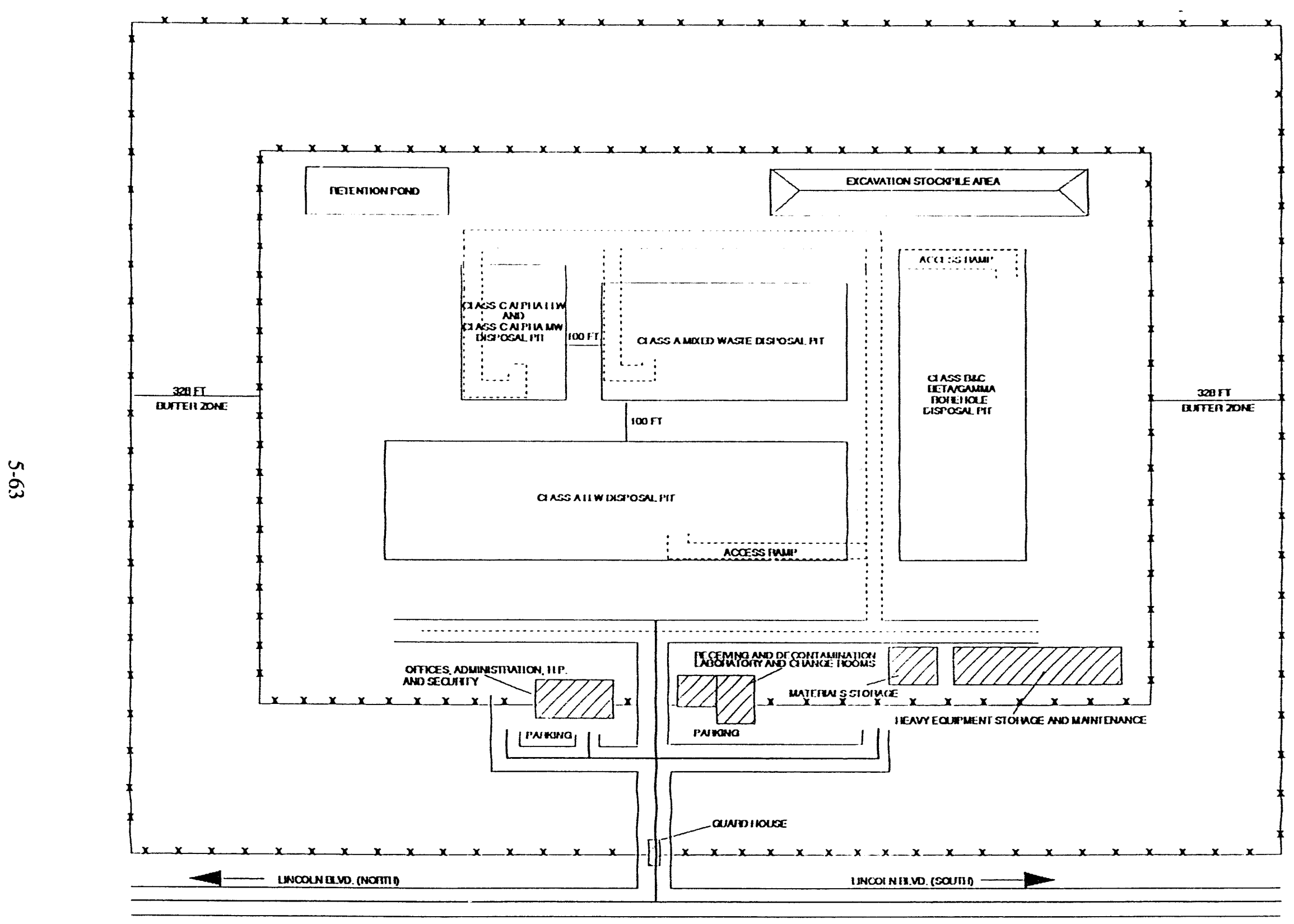

Figure 5-21. General disposal site layout for Subalternative 1E (not to scale). 
waste, the uppermost containers are 2 li belowgrade, simply to provide a minimal amount of radiation shielding.

The individual waste containers and any inner liners are the first isolation barrier. The second isolation barrier is a dual membrane liner, for all types of waste, not just mixed waste. The third isolation barrier is the geologic disposal, plus the final cover.

The numbers and sizes of the burial pits and boreholes are listed in Table 5-6.

Figure 5-22 provides a simplified sectional view of the burial pits for the contact-handled types of waste. All containers of these types of waste are square steel drums or steel boxes. Not shown in the figure are a double liner, a leachate collection and removal system, and a leak detection system. These items are located beneath the stack of waste and are protected by a compacted gravel working surface.

Figure 5-23 provides a simplified sectional view of the disposal arrangement for the boreholes. This concept is quite similar to the soil vault disposal method that has been used for several years at the RWMC. Each 6-ft-diameter borchole is excavated $21 \mathrm{ft}$ deep in backfilled soil. [The backfilled soil, along with a protective working surface of compacted gravel, covers the double liner, the leachate collection and removal systems, and the leak detection system (not shown in the figure)]. The top of the borehole is located at grade. All of the waste disposed of in the boreholes is in $4 \times 8 \times 4$ - $\mathrm{ft}$ concrete boxes. Each borehole accommodates 2 concrete boxes on end. The boxes are assumed to have a lifting appurtenance on the end and to be emplaced vertically in the boreholes. A metal sleeve is used during emplacement to assist in aligning the container and in stabilizing the borehole until emplacement is completed. The sleeve is then removed. The hole is backfilled, including $5 \mathrm{ft}$ of earth on top for shielding. The boreholes are in rows, with 10-ft center-to-center spacing within rows and 12 -ft center-to-center spacing between rows. The borcholes consume an overall area of $600 \times 276 \mathrm{ft}$ at the bottom of a pit. Each of the four types of remote-handled waste

Table 5-6. Number and size of disposal units for each type of waste in Subalternative 1E.

\begin{tabular}{|c|c|c|}
\hline Type(s) of waste & $\begin{array}{l}\text { Total number } \\
\text { of units } \\
\text { required over } \\
40 \text {-year period }\end{array}$ & $\begin{array}{c}\text { Dimensions during } \\
\text { operations, } \mathrm{ft} \\
(\mathrm{W} \times \mathrm{L}, \text { bottom }) / \\
(\mathrm{W} \times \mathrm{L}, \text { top }) / \\
\text { (depth, grade to bottom) }\end{array}$ \\
\hline$A$ & 1 pit & $\begin{array}{l}180 \times 1,248 / \\
210 \times 1,278 / 20\end{array}$ \\
\hline A mixed & 1 pit & $\begin{array}{l}180 \times 617 / \\
210 \times 647 / 20\end{array}$ \\
\hline$C \alpha$ mixed and $C \alpha$ & 1 pit & $\begin{array}{l}180 \times 110 / \\
216 \times 146 / 23\end{array}$ \\
\hline $\begin{array}{l}B, B \text { mixed, } C \beta-\gamma, C \beta-\gamma \\
\text { mixed }\end{array}$ & 1,380 boreholes & 6 (diameter)//21 \\
\hline
\end{tabular}




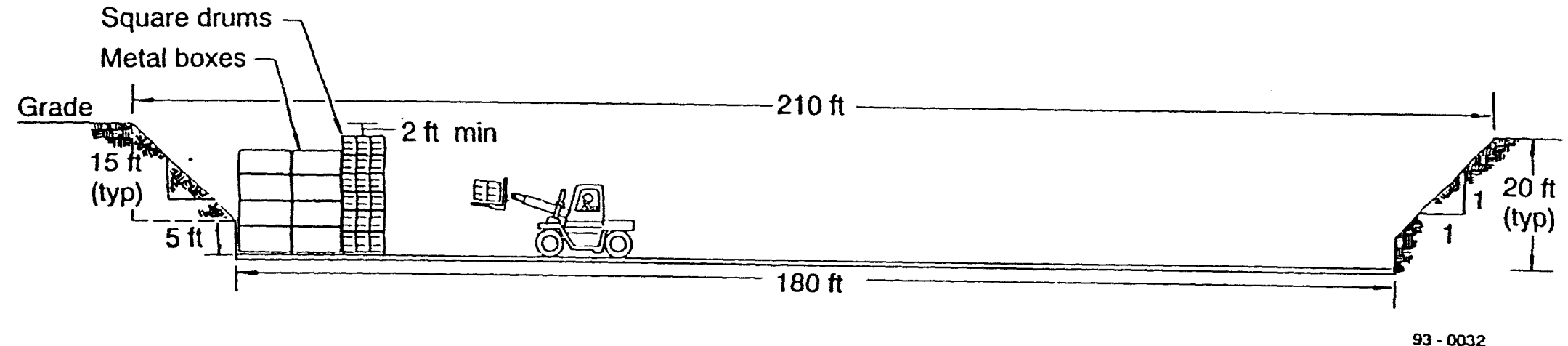

Figure 5-22. Simplified sectional view of shallow land burial pits for contact-handled waste in Subalternative 1E (not to scale). 


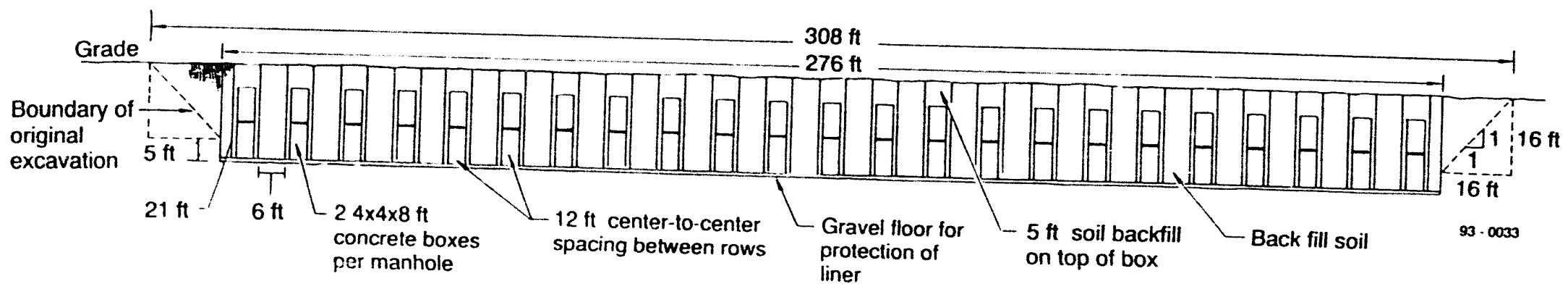

NOT TO SCALE

Figure 5-23. Simplified sectional view of disposal area with boreholes for remote-handled waste in Subalternative 1E. 
is placed in a separate area of the array, with the most highly radioactive Class $C \beta-\gamma$ waste being placed near the center.

5.3.8.2 Description of Operations. The operations in Subalternative $1 \mathrm{E}$ are the same as those in Subalternative ID except for the elevation at which they are performed.

5.3.8.3 Closure and Postclosure Activities. Closure for this subalternative is the same as for Subalternative 1D.

\subsubsection{Subalternative 1F: Shallow Land Burial/Abovegrade Engineered Structures}

This section briefly describes the facilities constructed and the operations conducted for Subalternative 1F. In this subalternative, Class $A$ waste and Class $A$ mixed waste are disposed of by shallow land burial. The other types of waste are disposed of in AGES.

\subsubsection{Description of Facilities.}

5.3.9.1.1 General Site Layout and Preparation-The general site layout used for Subalternative $1 \mathrm{~F}$ is presented in Figure 5-24. The total land area requirement for the disposal concept is 122 acres. The discussion of site layout and preparation for Subalternative $1 \mathrm{~A}$ applies identically to Subalternative $1 \mathrm{~F}$, except that the disposal area configuration has been changed for disposal in AGES and shallow land burial pits.

The shallow land burial pits are separated from the AGES by an access road and a total distance of $250 \mathrm{ft}$, which allows sufficient space between areas for disposal unit covers and for the movement of equipment and personnel during operations.

The Class A LLW and mixed waste disposal area consists of two separate shallow land burial pits, one for each type of waste. The pits are separated from each other by $100 \mathrm{ft}$. Ramps $267 \mathrm{ft}$ long $(7.5 \%$ grade) and $50 \mathrm{ft}$ in width allow truck access into the two burial pits. The disposal pit dimensions have been increased by $50 \mathrm{ft}$ to allow for construction of these ramps.

The Class B and C $\beta-\gamma$ LLW, Class B and C $\beta-\gamma$ mixed waste, and Class $C \alpha$ LLW and mixed waste disposal area consists of five separate AGES units in three rows, one row for each type of waste. The structures are separated from each other by $20 \mathrm{ft}$. The rows are separated by $100 \mathrm{ft}$.

5.3.9.1.2 Disposal Unit Construction-As explained in Section 4, this subalternative is a combination of the disposal technologies used in Subalternatives 1A (AGES) and 1E (shallow land burial). Shallow land burial is used for Class $A$ and Class A mixed waste. AGES is used for the other types of waste, which have a greater radiological hazard. The same disposal unit design and construction are used as discussed previously. The same isolation barriers are included as discussed previously.

The numbers and sizes of the various disposal units are listed in Table 5-7. The disposal units in this subalternative are the same as the corresponding portions of Subalternatives $1 \mathrm{~A}$ and $1 \mathrm{E}$. 


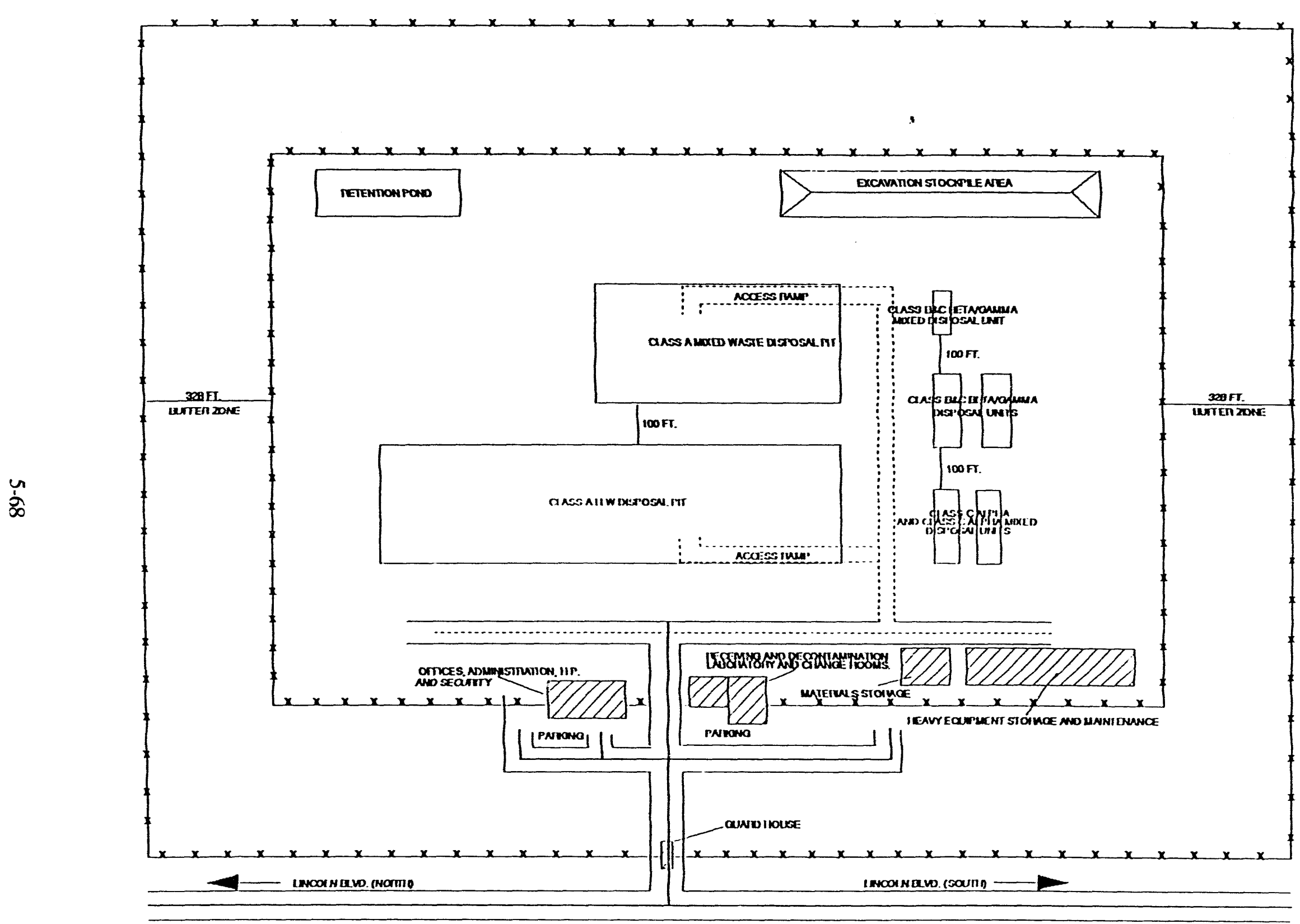

Figure 5-24. General disposal site layout for Subalternative 1F (not to scale). 
Table 5-7. Number and size of disposal units for each type of waste in Subalternative 1F.

A. Shallow land burial units

\begin{tabular}{|c|c|c|c|}
\hline Type(s) of waste & $\begin{array}{l}\text { Total number of units } \\
\text { required over } 40 \text {-year } \\
\text { period }\end{array}$ & \multicolumn{2}{|c|}{$\begin{array}{l}\text { Dimensions during operations, } \mathrm{ft} \\
(\mathrm{W} \times \mathrm{L}, \text { bottom }) / \\
(\mathrm{W} \times \mathrm{L}, \text { top }) / \\
(\text { depth, grade to bottom })\end{array}$} \\
\hline A & 1 pit & \multicolumn{2}{|c|}{$\begin{array}{l}180 \times 1,248 / \\
210 \times 1,278 / \\
20\end{array}$} \\
\hline A mixed & 1 pit & \multicolumn{2}{|c|}{$\begin{array}{l}180 \times 617 / \\
210 \times 647 / \\
20\end{array}$} \\
\hline \multicolumn{4}{|c|}{ B. Abovegrade engineered structures } \\
\hline Type(s) of waste & $\begin{array}{c}\text { Total number of } \\
\text { structures required } \\
\text { over } 40 \text {-year period }\end{array}$ & $\begin{array}{c}\text { Interior } \\
\text { dimensions } \\
(\mathrm{W} \times \mathrm{L} \times \mathrm{H}, \mathrm{ft})\end{array}$ & $\begin{array}{c}\text { Exterior } \\
\text { dimensions } \\
(\mathrm{W} \times \mathrm{L} \times \mathrm{H}, \mathrm{ft})\end{array}$ \\
\hline$B$ and $C \beta-\gamma$ & 2 & $52 \times 160 \times 28$ & $58 \times 166 \times 31$ \\
\hline $\begin{array}{l}B \text { mixed and } C \beta-\gamma \\
\text { mixed }\end{array}$ & 1 & $34 \times 70 \times 29$ & $40 \times 76 \times 32$ \\
\hline $\begin{array}{l}\mathrm{C}_{\alpha} \text { mixed, and the } \\
\text { minute amount of } \\
\mathrm{C}_{\alpha}\end{array}$ & 2 & $50 \times 151 \times 26$ & $56 \times 157 \times 29$ \\
\hline
\end{tabular}

5.3.9.2 Description of Operations. The operations in this subalternative are a logical combination of those for Subalternative $1 \mathrm{~A}$ and $1 \mathrm{E}$.

5.3.9.3 Closure and Postclosure Activities. The closure and postclosure activities in this Subalternative are a logical combination of those for Subalternative $1 \mathrm{~A}$ and $1 \mathrm{E}$.

5.3.9.3.1 Cover-Two separate covers are used in this subalternative because of the layout and the differing heights of the AGES and shallow land burial disposal units. The same material layers, thicknesses, and slope are used as shown in Figure 5-9. The cover for the AGES disposal units is $989 \mathrm{ft}$ long by $526 \mathrm{ft}$ wide, an area of 12 acres. The cover over the shallow land burial units is $7: 4 \mathrm{ft}$ wide. It is $1,382 \mathrm{ft}$ long over the Class $\mathrm{A}$ waste disposal pit and $751 \mathrm{ft}$ long over the adjacent Class A mixed waste disposal pit, covering an area of 18 acres. Thus, the total area of the two covers is 30 acres. 


\subsubsection{Subalternative 1G: Effect of Waste Volume}

This section briefly describes the facilities constructed and the operations conducted for Subalternative 1G. This subalternative is identical to Subalternative :A, in which all types of waste received are disposed of in AGES, except that the volume of waste is reduced duc: to assuming the small ER and D\&D scenario occurs.

\subsubsection{Description of Facilities.}

5.3.10.1.1 General Site Layout and Preparation-The general site layout used for Subalternative $1 \mathrm{G}$ is presented in Figure 5-25. The total land area requirement for the disposal concept is 86 acres, compared with 115 acres in Subalternative $1 \mathrm{~A}$. The discussion of site layout and preparation for Subalternative $1 \mathrm{~A}$ applies identically to Subalternative $1 \mathrm{G}$, except that fewer disposal units are required.

The Class A LLW disposal area consists of five separate concrete structures in two rows. The Class B and C $\beta-\gamma$ LLW disposal area consists of two separate structures. The Class $A$ and Class $C$ $\beta-\gamma \alpha$ mixed waste disposal area consists of two scparate concrete structures in a single row. No Class $B$ and $C \beta-\gamma$ mixed waste requires disposal in this subalternative, nor is there any Class $C \alpha$ waste.

5.3.10.1.2 Disposal Unit Construction-The results of sizing the concrete structures are listed in Table 5-8. The same approach was used as in sizing the structures for Alternative 1A. For all types of waste except Class $C \alpha$ mixed, fewer or smaller structures are required than for Subalternative 1A. No structures are required for Class B mixed and $C \beta-\gamma$ mixed waste. The volume of Class $A$ mixed waste is extremely small, so the few containers of that type of waste are assumed to be disposed of in the same structures as the containers of Class $C \alpha$ mixed waste.

Except for the volumes of waste and its effects, the facilities for this subalternative are the same as those for Alternative 1A. The site layout is unchanged except for the number of disposal structures. The site surface water management system, liners, leachate collection and removal systems, leak detection systems, support facilities, and CQA are the same as those for Alternative 1A, except for reducing the size where necessary.

5.3.10.2 Description of Operations. All operations for Subalternative $1 \mathrm{G}$ are identical to those for Subalternative $1 \mathrm{~A}$.

5.3.10.3 Closure and Postclosure Activities. All closure and postclosure activities for Subalternative $1 \mathrm{G}$ are identical to those for Subaltcrnative $1 \mathrm{~A}$, except for the size of the final cover, which is $1,066 \mathrm{ft}$ long by $972 \mathrm{ft}$ wide (24 acres).

\subsubsection{Subalternative 1H: Effect of Waste Treatment}

This section briefly describes the facilities constructed and the operations conducted for Subalternative $1 \mathrm{H}$. This subalternative is identical to Subalternative $1 \mathrm{~A}$, in which all types of waste received are disposed of in AGES, except that the volume of waste is increased due to assuming the minimum waste treatment scenario occurs. 


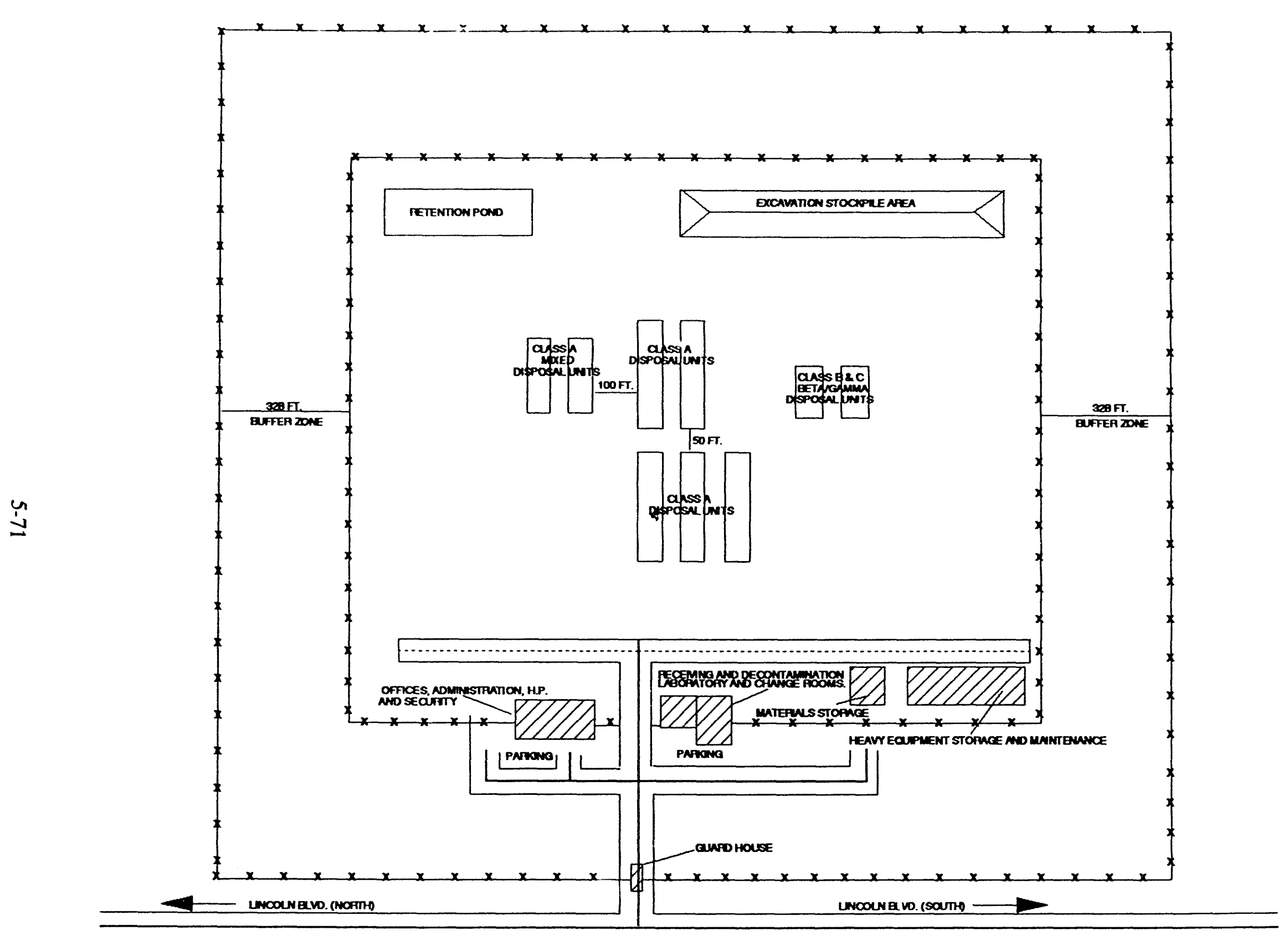

Figure 5-25. General disposal site layout for Subalternative 1G (not to scale). 
Table 5-8. Number and size of concrete structures for each type of waste in Subalternative 1G.

\begin{tabular}{|c|c|c|c|}
\hline Type(s) of waste & $\begin{array}{c}\text { Total number of } \\
\text { structures required over } \\
\text { 40-year period }\end{array}$ & $\begin{array}{l}\text { Interior dimensions } \\
(\mathrm{W} \times \mathrm{L} \times \mathrm{H}, \mathrm{ft})\end{array}$ & $\begin{array}{l}\text { Exterior dimensions } \\
(\mathrm{W} \times \mathrm{L} \times \mathrm{H}, \mathrm{ft})\end{array}$ \\
\hline A & 5 & $50 \times 260 \times 26$ & $56 \times 266 \times 29$ \\
\hline$B$ and $C \beta-\gamma$ & 2 & $52 \times 88 \times 28$ & $58 \times 94 \times 31$ \\
\hline A mixed & $\begin{array}{l}\text { Due to extremely small } \\
\text { volume of Class A } \\
\text { mixed waste, combine } \\
\text { with Class } C \alpha \text { mixed } \\
\text { waste }\end{array}$ & N/A & N/A \\
\hline $\begin{array}{l}\text { B mixed and C } \beta-\gamma \\
\text { mixed }\end{array}$ & 0 & N/A & N/A \\
\hline $\begin{array}{l}\mathrm{C} \alpha \text { mixed (plus } \mathrm{A} \\
\text { mixed) }\end{array}$ & 2 & $50 \times 143 \times 26$ & $56 \times 149 \times 29$ \\
\hline
\end{tabular}

\subsubsection{Description of Facillties.}

5.3.11.1.1 General Stte Layout and Preparation-The general site layout used for Subalternative $1 \mathrm{H}$ is presented in Figure 5-26. The total land area requirement for disposal is 166 acres, compared with 115 acres in Subalternative 1A. The discussion of site layout and preparation for Subalternative $1 \mathrm{~A}$ applies identically to Subalternative $1 \mathrm{H}$, except that more disposal units are required.

The Class A LLW disposal area consists of $\mathbf{4 0}$ separate concrete structures in four rows. The Class B and C $\beta-\gamma$ LLW disposal area consists of two separate structures. The Class A mixed waste disposal area consists of seven separate concrete structures in two rows. The Class B and C mixed waste disposal area consists of three separate concrete structures. Two structures are for disposal of the Class $\mathrm{C} \alpha$ mixed waste, including the very small amount of nonmixed Class $\mathrm{C} \alpha$ waste. One structure is for disposal of the Class $B$ and $C \beta-\gamma$ mixed waste.

5.3.11.1.2 Disposal Unit Construction-The results of sizing the concrete structures are listed in Table 5-9. For all types of waste except Class $A$, the same number and size of structures are required as for Subalternative $1 \mathrm{~A}$. This situation results because only Class $\mathrm{A}$ waste would not undergo the volume reduction in maximum treatment that is performed in minimum treatment.

Except for the volumes of waste and its effects, the facilities for this subalternative are the same as those for Alternative 1A. The site layout is unchanged except for the number of disposal structures. The site surface water management system, liners, leachate collection and removal systems, leak detection systems, support facilities, and CQA are the same as those for Alternative $1 \mathrm{~A}$, except for increasing the size where necessary. 

Table 5-9. Number and size of concrete structures for each type of waste in Subalternative 1A.

\begin{tabular}{|c|c|c|c|}
\hline Type(s) of waste & $\begin{array}{c}\text { Total number of } \\
\text { structures required } \\
\text { over } 40 \text {-year period }\end{array}$ & $\begin{array}{c}\text { Interior dimensions } \\
(\mathrm{W} \times \mathrm{L} \times \mathrm{H}, \mathrm{ft})\end{array}$ & $\begin{array}{l}\text { Exterior dimensions } \\
\quad(\mathrm{W} \times \mathrm{L} \times \mathrm{H}, \mathrm{ft})\end{array}$ \\
\hline$A$ and $C \alpha$ & 40 & $50 \times 260 \times 26$ & $56 \times 266 \times 29$ \\
\hline$B$ and $C \boldsymbol{\beta}-\boldsymbol{\gamma}$ & 2 & $52 \times 160 \times 28$ & $58 \times 166 \times 31$ \\
\hline A mixed & 7 & $50 \times 250 \times 26$ & $56 \times 256 \times 29$ \\
\hline$B$ mixed and $C \beta-\gamma$ mixed & 1 & $34 \times 70 \times 29$ & $40 \times 76 \times 32$ \\
\hline $\begin{array}{l}\mathrm{C} \alpha \text { mixed, and the minute } \\
\text { amount of } \mathrm{C} \alpha .\end{array}$ & 2 & $50 \times 151 \times 26$ & $56 \times 157 \times 29$ \\
\hline
\end{tabular}

5.3.11.1.3 Support Facilities-The support facilities provided for in Subalternative 1A are also provided for in Subalternative $1 \mathrm{H}$; however, they are sized somewhat larger to accommodate the increase in workers, materials, and equipment necessary for facility operations.

5.3.11.2 Description of Operations. All operations for Subalternative $1 \mathrm{H}$ are identical to those for Subalternative $1 \mathrm{~A}$.

5.3.11.3 Closure and Postclosure Activities. All closure and postclosure activities for Subalternative $1 \mathrm{H}$ are identical to those for Subalternative $1 \mathrm{~A}$, except for the size of the final co\%er, which is $1,950 \mathrm{ft}$ long by $1,604 \mathrm{ft}$ wide (72 acres).

\subsection{Alternative 2: Continue Disposal at RWMC}

This section provides a description of Alternative 2, in which waste continues to be disposed of at the RWMC through the year 2044. After the space available for disposal by the current methods is totally consumed (assumed for purposes of this study to occur in 2004), modified disposal techniques are implemented to make use of remaining areas.

The intent of this alternative is to identify a method for disposing of the waste through 2044 that does not require constructing a new facility or shipping the waste offsite.

\subsubsection{Defining Assumptions}

This alternative involves the continued disposal of waste at the RWMC using modified techniques, after space suitable for waste disposal using the current methods is consumed. The defining assumptions are

- The MLLWDF is not built 
- The disposal methods at the RWMC are modified to accommodate more waste after the space available by current disposal methods is consumed, assumed for purposes of this study to occur in 2004

- The large-volume waste generation scenario applies, as does the maximum waste treatment scenario.

\subsubsection{Overview of Disposition of Waste Types}

Based on the detailed waste information in Section 2, an overview of the waste disposition in this alternative is as follows:

Waste disposed of:

$$
\begin{array}{ll}
\text { LLW } & 2,486.2 \mathrm{~m}^{3} / \mathrm{yr} \\
\text { Mixed Waste } & 1,274.5 \mathrm{~m}^{3} / \mathrm{yr}
\end{array}
$$

Waste Stored:

None

\subsubsection{Location}

The location of the waste disposal in this alternative is at the RWMC (Figure 5-27), a facility that is currently disposing of LLW and storing TRU waste.

The location of the RWMC has been characterized thoroughly. Detailed descriptions are available in numerous reports.

An important assumption made in developing this alternative is that no change is made in the boundaries of the RWMC. No area is added to the RWMC by annexing land outside the present boundaries. Annexation would require a NEPA review, perhaps including an Environmental Assessment. In addition, the land immediately outside the current boundaries generally has only shallow surface sediments and, therefore, would not be attractive for constructing additional disposal pits.

\subsubsection{Description of Facilities}

5.4.4.1 General Site Layout and Preparation, and Disposal Unit Construction. Because of the close interaction between site layout and disposal unit construction for this alternative, the two topics are addressed together.

The general site layout used for Alternative 2 is presented in Figure 5-28. Similar to the MLLWDF alternative, the RWMC alternative includes controlled access areas where the disposal pits are located and where disposal operations are carried out, and administrative and operational support areas required for the conduct of the disposal operations. 


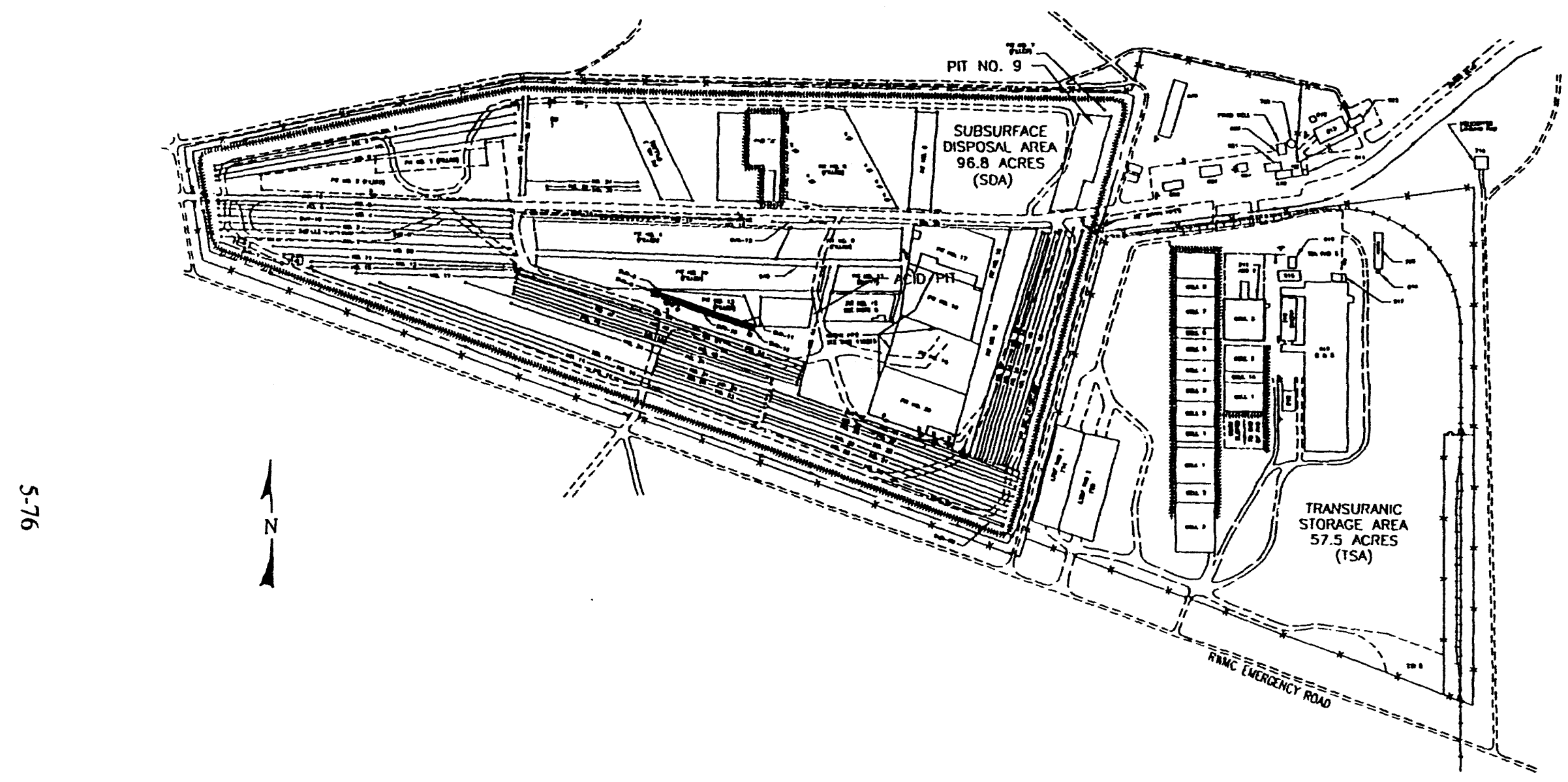

AC1501

Figure 5-27. Current layout of the RWMC. 


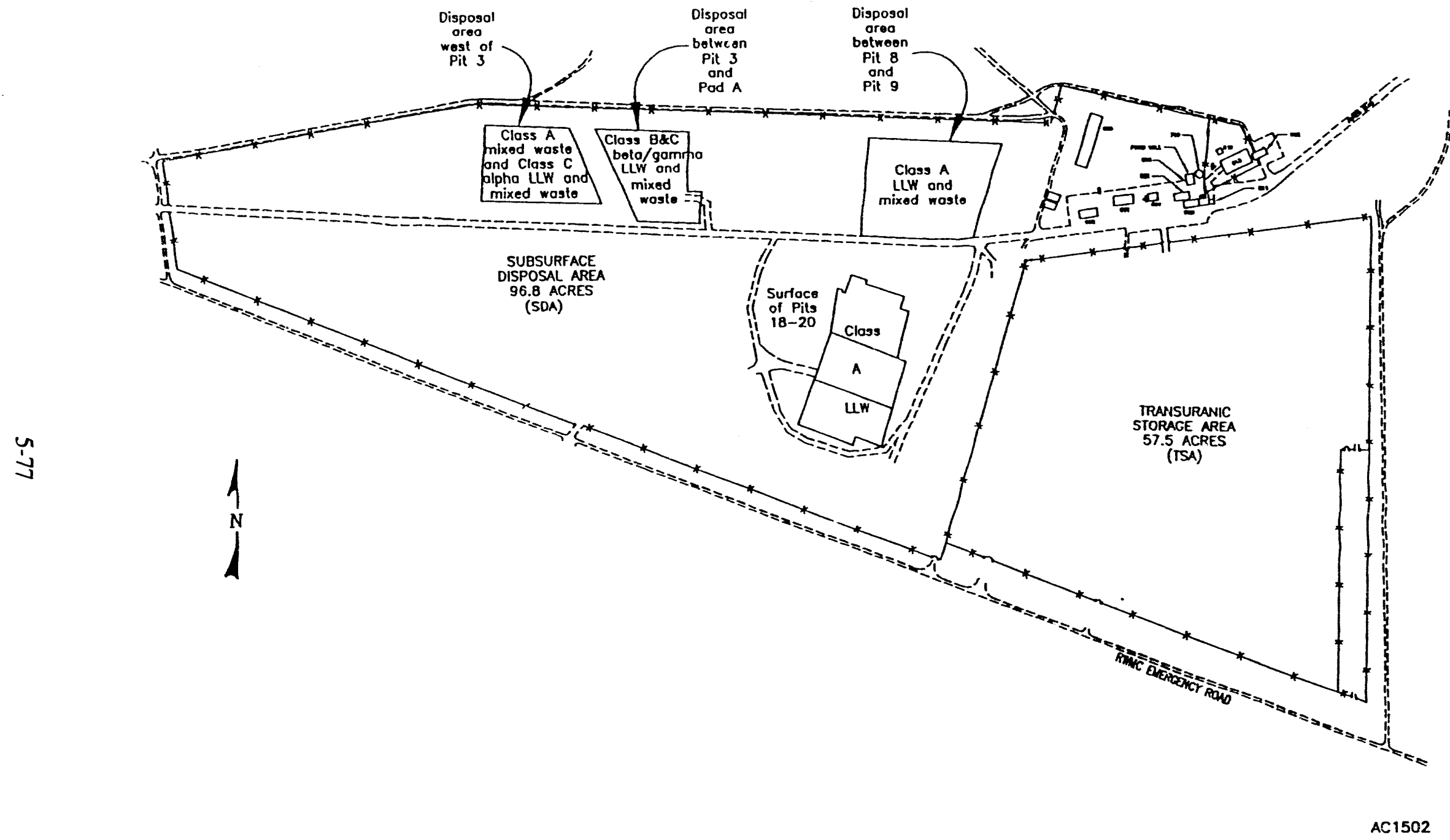

Figure 5-28. Disposal site layout for Alternative 2. 
The RWMC is an active facility at the INEL with access roads, drainage areas, monitoring facilities, fencing, security gates, and lighting already in place. Therefore, site preparation activities will be minimal for this alternative.

The main access road to the RWMC is via Van Buren Blvd. and Adams Rd. Both are paved, all-weather roads intended for heavy truck use. Entrance to the RWMC is through one main gate controlled by operating personnel. A patrol road is located immediately outside the facility fence. The road provides clear viewing of the interior of the disposal area, and provides access to emergency evacuation routes. Graded, graveled roads are provided for access to the disposal locations of the RWMC. Areas are provided for parking government vehicles and evacuation buses, private vehicles, bus loading and unloading, and evacuation staging. These parking areas are located near the administrative area.

The administrative and operational support areas at the RWMC include the following:

- Radiological Control Technician Building (WMF-601)

- RWMC Highbay (WMF-602)

- $\quad$ Pumphouse (WMF-603)

- Change room and lunch room Building (WMF-604)

- Heavy-equipment storage shed (WMF-609)

- $\quad$ Security guardhouse (WMF-611)

- $\quad$ Office Building (WMF-613)

- Communications and Alarm Building (WMF-619)

- Work Control Trailers and Access Control Office (WMF-620 and 621)

- $\quad$ RWMC Office Building (WMF-622)

- Construction Support Buildings (WMF-645 and WMF-646)

- Water Storage Tank (WMF-709).

See the discussion on Support Facilities for further details.

The disposal area is a controlled access area surrounded by a barbed wire fence that serves to discourage unauthorized access and to keep large animals from entering the SDA. The fence is approximately $1.1 \mathrm{~m}(3.6 \mathrm{ft})$ high, with steel posts on $3-\mathrm{m}(9.8-\mathrm{ft})$ centers.

The assumption of being restricted to the current boundaries of the RWMC imposes severe limitations on the selection of waste disposal methods and disposal area layout after the current 
disposal methods are terminated. For this alternative, it is assumed that all the current pit areas are filled and that interim closure of each pit has taken place.

Although the useful life of the RWMC for disposing of waste by current methods is assumed in this alternative to have ended, several areas of the RWMC could be available for waste disposal by modified methods not requiring disposal pits. Such areas were identified and evaluated, as discussed below.

The TSA was ruled out as an area to provide space for disposal of LLW and low-level mixed waste. Several new facilities for managing the stored TRU waste are already planned for the TSA. The proposed Waste Characterization Facility and the Storage Modules will consume nearly all of the remaining sizeable areas of the TSA. In addition, the TSA has historically been used only for activities related to the storage of TRU waste. Using the TSA for the disposal of LLW would be a major redirection of land use and could lead to several programmatic, operational, and regulatory difficulties.

Within the SDA, the sizcable areas with relatively deep surface sediments (about 12 to $25 \mathrm{ft}$ thick) have already been used lor pit, trench, or soil vault disposal of waste. The remaining areas generally have shallow surface sediments and are relatively small in size. Therefore, the search for disposal areas and the consideration of feasible disposal techniques was necessarily limited to these less desirable areas.

Another limitation in seeking additional space in the SDA stems from the fact that most of the SDA is being addressed under CERCLA remediation. (Note: Because the final remediation of the operable units has not been determined, no credit was taken for possible reuse of disposal space made available by the retrieval of buried waste.) It is possible that remediation activities could be underway in any of the affected areas in the year 2004 or afterward. The use of such areas for modified disposal methods could result in a regulatory or operational conflict. Such areas were dropped from further consideration. The remedial action has the potential to affect all of the SDA pits except Pits 18 through 20 (see Figure 5-28), as well as all of the trenches. Thus, only (the surface of) Pits 18 through 20 and the unused areas are candidates for future disposal.

The largest unused areas identified as potential locations for further disposal were as follows (see Figure 5-28): (a) the area between Pits 8 and 9 (currently used as a lay-down area for Pit 9 activities, but such activities are expected to be completed before 2004); (b) the area between Pad A and $\mathrm{Pit} 3$; and (c) the area just west of Pit 3. These three areas and the surface of Pits 18 through 20 were the areas pursued for additional disposal.

The surface areas of these locations were estimated, allowing a perimeter strip of at least $20 \mathrm{ft}$ between the operations and the adjacent areas (e.g., areas that could be undergoing remediation). The rough estimates of the dimensions and areas are given in Table 5-10.

The disposal methods chosen for this alternative are as follows. The contact-handled types of waste (Class $A$ and Class $C \alpha$, whether or not either type is mixed) are disposed of by covered placement abovegrade, through stackin on top of unused areas or closed pits. The amount of surface area available for disposal of $v$ 'ste is quite limited, so the metal boxes are stacked five high $(20 \mathrm{ft}$ ), instead of four high as in St' alternatives $1 \mathrm{D}$ and $1 \mathrm{E}$, to conserve space. The square drums are still stacked six high (18ft). F r r the Class $C \alpha$ waste (whether mixed or not), the standard 11-ft thick cover is preceded by a 5 - $\mathrm{ft}$ inickness of sand. The result is a cover $16 \mathrm{ft}(5 \mathrm{~m})$ thick that meets the requirement for intruder protection for Class $\mathrm{C}$ waste. 
Table 5-10. Rough estimates of the size of locations assumed to be available for future disposal of waste at the RWMC.

\begin{tabular}{|c|c|c|c|}
\hline Location & $\begin{array}{l}\text { Average width } \\
\text { (east-west) } \\
\text { (ft) }\end{array}$ & $\begin{array}{l}\text { Average length } \\
\text { (north-south) } \\
(\mathrm{ft})\end{array}$ & $\begin{array}{l}\text { Area } \\
\left(\mathrm{ft}^{2}\right)\end{array}$ \\
\hline $\begin{array}{l}\text { Surface of Pits } 18 \\
\text { through } 20\end{array}$ & 241 & 589 & 142,000 \\
\hline Between Pits 8 and 9 & 423 & 324 & 137,000 \\
\hline West of Pit 3 & 357 & 241 & 86,000 \\
\hline $\begin{array}{l}\text { Between Pit } 3 \text { and } \\
\text { Pad A }\end{array}$ & 191 & 357 & 68,000 \\
\hline
\end{tabular}

For the contact-handled types of waste, the individual waste containers and any liners are the first isolation barrier. The second isolation barricr is a dual membrane liner for all types of waste, not just mixed waste. The third isolation barrier is the final cover.

The remote-handled types of waste (Class B and Class $C \beta-\gamma$, whether mixed or not) are disposed of in closely nested vaults similar to those that the RWMC personnel are starting to construct. The envisioned vaults consist of 16-ft long, 6-ft ID sleeves of 6 in thick concrete, nested tightly together in a vertical orientation. The sleeves rest on $1-\mathrm{ft}$ thick concrete vault bases that are hexagonal in shape, $7 \mathrm{ft}$ across, and nested tightly together. The sleeves are capped by concrete plugs that are the same shape and size as the bases, but approximately $4 \mathrm{ft}$ thick to provide radiation shielding. The small areas between the sleeves are backfilled with sand. When supported by soil on the sides, the resulting array provides a solid working surface (atop the nested plugs) sufficiently stable during operations to support the vehicles used for emplacing waste in the vaults.

For the remote-handled types of waste, the individual waste containers and any liners are the first isolation barrier. The second isolation barrier is a dual membrane liner and the grouted concrete liner in the boreholes. The third isolation barrier is the geologic media and the final cover.

Given the disposal configurations just discussed and the numbers of waste containers of various sizes to be disposed of, the necessary surface area to dispose of each type of waste was estimated. Table 5-11 lists the various classes of waste to be disposed of, the required surface area for disposal, and which of the four disposal areas it is disposed in. When the required surface area for disposal is compared with the available surface area for disposal, the conclusion is that sufficient space is available but with little to spare.

The area where nested-vault disposal is contemplated in this alternative (between Pad A and Pit 3) is expected to have surface sediments only about 6 to $15 \mathrm{ft}$ in thickness. This is insufficient to allow emplacement of the concrete sleeves totally belowgrade, assuming no drilling into the underlying basalt. The construction of the disposal unit is therefore envisioned as follows. The surface sediments are excavated to the basalt (assumed to be about $10 \mathrm{ft}$ belowgrade), then backfilled 
Table 5-11. Detailed disposition of the various types of waste in Alternative 2.

\begin{tabular}{|c|c|c|c|}
\hline Type(s) of waste & $\begin{array}{l}\text { Required surface } \\
\text { area } \\
\left(\mathrm{ft}^{2}\right)\end{array}$ & $\begin{array}{l}\text { Disposal } \\
\text { method }\end{array}$ & Disposal location \\
\hline Class A & 206,524 & $\begin{array}{l}\text { Covered } \\
\text { placement }\end{array}$ & $\begin{array}{l}\text { Surface of Pits } 18 \\
\text { through } 20 \text {, plus part } \\
\text { in area between Pits } 8 \\
\text { and } 9\end{array}$ \\
\hline Class A mixed & 110,961 & $\begin{array}{l}\text { Covered } \\
\text { placement }\end{array}$ & $\begin{array}{l}\text { Part in area between } \\
\text { Pits } 8 \text { and } 9, \text { plus part } \\
\text { in area west of Pit } 3\end{array}$ \\
\hline Class B and Class $C \beta-\gamma$ & 50,880 & Nested vaults & $\begin{array}{l}\text { Area between Pit } 3 \\
\text { and Pad A }\end{array}$ \\
\hline $\begin{array}{l}\text { Class } B \text { mixed and } \\
\text { Class } C \beta-\gamma \text { mixed }\end{array}$ & 7,632 & Nested vaults & $\begin{array}{l}\text { Area between Pit } 3 \\
\text { and Pad A }\end{array}$ \\
\hline Class $\mathrm{C} \alpha$ & 994 & $\begin{array}{l}\text { Covered } \\
\text { placement }\end{array}$ & Area west of Pit 3 \\
\hline Class $\mathrm{C} \alpha$ mixed & 18,611 & $\begin{array}{l}\text { Covered } \\
\text { placement }\end{array}$ & Area west of Pit 3 \\
\hline
\end{tabular}

with about $2 \mathrm{ft}$ of sediments. The bases are emplaced, followed by the sleeves and plugs. Soil is mounded around the nested vaults, up to the level of the top of the plugs, to form a working surface. The height of the working surface is approximately 13-ft abovegrade.

5.4.4.2 Site Surface Water Management System. The overall concept for site surface drainage is much the same as for previous alternatives (i.e., precipitation is directed away from the disposal site, contact of waste and precipitation falling on the site is minimized, and the precipitation that does fall on the site is collected for sampling before release).

Precipitation is directed away from the disposal site with a system of dikes and ditches that are placed around the boundary of each of the four new disposal areas. The water in the ditches is directed by gravity flow to the surface water collection system presently installed at the RWMC.

Management of precipitation falling on the waste disposal areas for contact-handled waste is similar to that described in Subalternative 1C. Management of precipitation falling on the waste disposal area for remote-handled waste is similar to that described in Subalternative 1E.

5.4.4.3 Liners and Leachate Collection and Removal Systems. The dual-liner/leachate system for the contact-handled waste disposal areas is similar to that described in Subalternative 1C. The system for the remote-handled saste disposal area is similar to that described in Subalternative $1 \mathrm{E}$. 
5.4.4.4 Support Facilities and Activities. The following support facilities and activities are currently provided in this alternative.

The Radiological Control Technician (RCT) Building (WMF-601) is a single-story, stcel-frame metal building, with a mezzanine, situated on a concrete pad. The structure provides offices, restrooms, change rooms, and lockers for RCT, and a radiological control laboratory.

The RWMC Highbay (Building WMF-602) is a 2,400 $\mathrm{ft}^{2}$, single-story, metal building on a concrete pad. It is a multipurpose operational support building, equipped with a laboratory hood. WMF-602 is used for technology development, thawing containers in cold weather, temporary storage, vehicle monitoring, equipment maintenance, and other similar activities.

The Pumphouse (WMF-603) is a single-story, steel-frame, metal building on a concrete pad. The building houses the fire, potable water supply pumps, and the propane emergency generator for RWMC.

The Change House and Lunch Room Building (WMF-604) is a steel-frame metal building on a concrete pad. This building provides space for operational personnel change areas and a lunch room, and has a restroom, and showers.

Building WMF-609 is the Heavy Equipment Storage Building. This structure is an $11,000 \mathrm{ft}^{2}$, 11-bay metal building enclosed on three sides, except for the two south bays that are totally enclosed and serve as a repair shop.

Building WMF-611 is the Guardhouse. This structure provides security control and access control to the RWMC area.

The Office Facility (WMF-613) is a single-story, 7,000 $\mathrm{ft}^{2}$ steel-frame metal building on a concrete pad. This building provides a conference room and office space.

The Communications Building (WMF-619) is a steel-frame metal building on a concrete pad. It houses telephone switch gear and electrical alarm equipment.

WMF-620 is the Work Control Building. It is a double-wide, $16.9 \times 8.4 \mathrm{~m}$ trailer containing a hard wall office, conference room, several cubicles, and restrooms.

The Office Building (WMF-621) is a double-wive, $16.9 \times 8.4 \mathrm{~m}$ trailer annexed to WMF-620, divided into office spaces with movable partitions.

WMF-622 is the RWMC Office Building. It is a double-wide, $11.7 \times 8.4 \mathrm{~m}$ trailer, annexed to WMF-613.

The Construction Support Building (WMF-646) is a double-wide $17.1 \times 8.5 \mathrm{~m}$ trailer, used as an office and meeting place.

WMF-709 is the Water Storage Tank with a capacity of 946,000$) \mathrm{L}(250,000 \mathrm{gal})$. 
Utilities supporting operations are provided at the RWMC Facility. The site includes water for domestic and industrial use, electrical power, communications including telecommunications, sanitary wastewater treatment, and stormwater management systems.

New facilities at the RWMC are required to meet current operational needs. In order to provide this space, facilities will be upgraded or expanded and new facilities will be constructed. Based on the RWMC estimated projections for the year 2013, buildings WMF-611, WMF-620, WMF-621, and WMF-622 will be demolished or excessed. New facilities planned for the administrative area at the RWMC include the Operation Control Building (WMF-637), Maintenance Facility (WMF-TBD), and Material Handling Facility (WMF-TBD).

The Operations Control Building is scheduled for construction in fiscal year 1993. It is a $24,000 \mathrm{ft}^{2}$ preengineered metal building and will be located adjacent to Building 622 . This building is intended to house an emergency control center, the main guard desk, a vehicle monitoring station, a personnel monitoring area, office space for operational support personnel and administrative personnel, showers, and a training/unch room.

The Maintenance Facility $\left(5,000 \mathrm{ft}^{2}\right)$, and the Material Handling Facility $\left(3,500 \mathrm{ft}^{2}\right)$ will provide additional maintenance and storage capacity at the RWMC. These facilities are scheduled for construction in fiscal year 1994.

Utility upgrades are also ; lanned at the RWMC in the near future. A Sewage Treatment Lagoon is planned to replace the individual sewer systems currently being used. An additional water storage tank and a new distribution system to upgrade the current fire/potable water system are also planned to meet future needs.

It is assumed for the purpose of cost estimating that the existing and planned facilities, and utilities will, for the most part, be adequate for the modified disposal operations. However, it is assumed that a like replacement for the new facilities (Operations Control Building, Maintenance Facility and Material Handling Facility) would be necessary approximately $30 \mathrm{yr}$ after construction. Although the RWMC projections for the year 2013 show no replacement for the Heavy Equipment and Storage Facility, it is assumed that a like replacement would be necessary around fiscal year 2015.

5.4.4.5 Construction Quality Assurance. The CQA program for Alternative 2 is similar in approach to that discussed for Subalternative $1 \mathrm{~A}$ in Section 5.3.4.1. However, because the magnitude of construction is greatly reduced for Alternative 2, the magnitude of the CQA program is proportionally reduced.

\subsubsection{Description of Operations}

5.4.5.1 Waste Reccipt, Transfer, and Emplacement. Waste receipt, transfer, and emplaccment operations for contact-handled waste are similar to those described for Subalternative $1 \mathrm{C}$, with the exception that aisles are not left between the waste packages. Operations for remote-handled waste are the same as those described in Subalternative 1E, except for the elevation at which they are performed. 
5.4.5.2 Monitoring. A monitoring program, as discussed for Subalternative $1 \mathrm{~A}$ in Section 5.3.4.1, is conducted at the RWMC. The monitoring program and evaluation of the results are integrated with monitoring programs conducted by other organizations at the INEL. The current RWMC monitoring program will have provided much of the first-phase data required prior to construction and operation of the modified facility.

In the second phase, the current monitoring program is continued with minor modification throughout construction and operation of the new activities at the RWMC. The monitoring program measures contaminant concentrations in air, surface water, ground water, soil, plants, and animal tissue. Several low-volume air samplers located at the RWMC determine concentrations of airborne radionuclides in ambient air in the vicinity of the RWMC and help to evaluate the airborne transport of radionuclide migration from RWMC operations. Surface water runoff sampling is conducted at the RWMC if sufficient water is available. Radionuclide concentrations in runoff water are analyzed to determine if radionuclide transport from the area is possible during runoff conditions. The groundwater in the Snake River Plain Aquifer, approximately $177 \mathrm{~m}(580 \mathrm{ft})$ beneath the RWMC is monitored to help determine whether radionuclides have migrated from the waste to the groundwater below the facility. (Note that it would be extremely difficult, if not impossible, to differentiate between contamination from previous and new waste disposal.) Routine soil surveillance is conducted at the RWMC. In addition, periodic soil monitoring outside the facility is conducted to determine if RWMC operations contribute to soil contamination, and to determine long-term trends. Routine sampling of small mammals and vegetation is also conducted at the RWMC to aid in detection of potential biological radionuclide transport.

Modification to the existing program for this alternative includes environmental monitoring of nonradiological contaminants and operational monitoring to be conducted at least weekly to evaluate the status of the surface water management system, and the leachate collection, removal, and detection systems.

Also during construction and operations for this alternative, the condition and structural performance of isolation barriers are monitored at suitable frequencies and locations to verify design assumptions. The limiting acceptable values of parameters are specified, and remedial action plan are implemented if the values are exceeded.

The third phase of monitoring in this alternative occurs after closure of the disposal units. The monitoring program continues as long as institutional control of the site continues.

\subsubsection{Closure and Postclosure Activities}

5.4.6.1 Disposal Unit Closure. The closure of contact-handled disposal areas is the same as for Subalternative 1C. The closure of the remote-handled disposal area is the same as for Subaltcrnative 1E.

5.4.6.2 Cover. If Alternative 2 were implemented, the final cover would be integrated with that for the other disposal units at the RWMC. However, the form of the final cover for the RWMC can not be projected with confidence. One reason for the uncertainty is the unknown final disposition of the TRU waste pits and trenches in the SDA. Those pits and trenches may be reopened and the waste containers retrieved, or the disposal units may be left in place and covered. 
To provide some basis for the cost estimates and isolation assessments for Alternative 2, assumptions had to be made regarding the cover. For simplicity, the cover for the four disposal units in this alternative was designed independently of the other disposal units in the SDA. That is, any potential cover for the other disposal units was disregarded.

The cover again consists of the same material layers, thicknesses, and slopes as shown in Figure 5-9 and discussed in the accompanying text. The dimensions and areas of the covers over each disposal unit are as follows in Table 5-12.

5.4.6.3 Maintenance and Monitoring During Institutional Control Period. Maintenance and monitoring during the institutional control period for Alternative 2 is the same as provided in Section 5.3.4.3 for Subalternative 1A.

\subsection{Alternative 3: Ship Waste Offsite for Disposal}

This section briefly describes the facilities constructed and the operations conducted for Alternative 3, in which an offsite DOE facility is used for disposal of all INEL LLW and mixed LLW. While the specific offsite DOE facility to be used is not known, assumptions are made and facilities and operations described based on the most common types of disposal facilities currently available or expected to become available in the near future.

\subsubsection{Defining Assumptions}

This alternative involves the disposal of waste at an offsite DOE facility. The defining assumptions are as follows:

- $\quad$ MLLWDF is not built

- RWMC is shut down permanently

- Large-volume waste generation scenario applies

- Maximum waste treatment scenario applies.

Table 5-12. Dimensions are areas of disposal unit covers.

\begin{tabular}{lcc}
\hline \multicolumn{1}{c}{ Disposal unit } & $\begin{array}{c}\text { Cover dimensions } \\
(\mathrm{ft})\end{array}$ & $\begin{array}{c}\text { Area } \\
(\text { acres })\end{array}$ \\
\hline Surface of Pits 18 through 20 & $537 \times 885$ & 11 \\
Between Pit 8 and Pit 9 & $719 \times 620$ & 10 \\
West of Pit 3 & $653 \times 537$ & 8 \\
Between Pit 3 and Pad A (soil vaults) & $419 \times 585$ & 6 \\
\hline
\end{tabular}




\subsubsection{Overview of Disposition of Waste Types}

Based on the detailed waste information in Section 2, an overview of the waste disposition in this alternative is as follows:

Waste Disposed of onsite:

None

Waste Disposed of offsite:

$$
\begin{array}{ll}
\text { LLW } & 2,486.2 \mathrm{~m}^{3} / \mathrm{yr} \\
\text { Mixed Waste } & 1,274.5 \mathrm{~m}^{3} / \mathrm{yr}
\end{array}
$$

Waste Stored:

None

\subsubsection{Location}

The actual offsite DOE facility to be used for disposal is not known. For the purposes of this evaluation, it is assumed that the disposal site is located $1,000 \mathrm{mi}$ away from the INEL. This is a rough average of the distances to the DOE sites that are developing disposal capability for mixed waste to supplement their current disposal capability for LLW.

\subsubsection{Description of Facilities}

For the purposes of this evaluation, it is assumed that the waste is disposed of using technologies similar to those described in Subalternative 1E. In this alternative, shallow land burial is used for mixed and nonmixed Class $\mathrm{A}$ and Class $\mathrm{C} \alpha$ waste and shallow bore-hole technology is used for disposal of mixed and nonmixed Class $B$ and $C \beta-\gamma$ waste. These disposal technologies were chosen because they represent the most common technologies currently in use or planned for use to dispose of LLW and mixed waste at DOE sites.

Only two disposal related facilities are required at the INEL: (a) a central shipping facility for gathering the waste for shipping; and (b) a maintenance area for transport vehicles. In the previous subalternatives, it was assumed that the facility providing treatment prior to disposal had the capacity to store 1 month's volume of treated waste. The 1-month storage building at the treatment facility is assumed to be adequate to serve as the central shipping facility for gathering both treated and untreated waste for shipping. In the previous alternatives, the truck maintenance area was assumed to be part of the disposal site (c.g., the heavy equipment storage and maintenance area in Alternative 1). A similar facility, at about half the size, is constructed at the treatment site. 


\subsubsection{Description of Operations}

5.5.5.1 Waste Receipt. Treated and untreated waste is col'ected in the INEL central shipping facility associated with the treatment facility. Here, the waste characteristics are recorded, shipping strategies are developed, and shipping paperwork is prepared.

5.5.5.2 Waste Transfer. When a sufficient quantity of waste is accumulated, personnel from the central shipping facility at the INEL make arrangements to transfer the waste to the offsite disposal facility. As part of the arrangements, an electronic description of the waste shipment is sent to the disposal facility for review. Upon receipt of approval to ship, the waste is loaded onto transfer vehicles and secured for transport. Waste is loaded using forklifts or overhead cranes as appropriate. After securing the waste, final inspections are performed to confirm that all shipping regulations are met; for example, radiation and contamination conditions are checked. Shipping paperwork and electronic records are updated as appropriate.

The remainder of the waste transfer is specific to the offsite disposal facility. The offsite facility performs a receipt inspection to ensure that radiation levels are as expected, that no damage has occurred during shipment, and that the waste packages match those recorded on the shipping manifest. Any discrepancies are immediately resolved.

5.5.5.3 Waste Emplacement. Waste emplacement is also site specific. Generally, the transport vehicle is driven to the disposal area, the waste unloaded and placed according to disposal site procedures, and the electronic record updated to note the final disposal location of the waste.

The transport vehicle and any associated transport containers are checked to ensure that they are not contaminated and are then released for return to the INEL.

\subsubsection{Closure and Postclosure Activities}

Closure and postclosure activities are specific to the offsite location and are not discussed. For the purposes of this study, such activities were assumed to be similar to those for Subalternative 1E.

\subsection{Alternative 4: Combinations of Alternatives}

This section provides a description of Alternative 4, in which combinations of other alternatives are used to dispose of the waste. Two combinations are described. The MLLWDF/Ship Offsite combination uses portions of Alternatives 1 and 3: the LLW is disposed of onsite at the MLLWDF, using Subalternative 1A (AGES), and the mixed waste is shipped offsite for disposal. The RWMC/Ship Offsite combination uses portions of Alternatives 2 and 3: the LLW is disposed of by modified techniques at the RWMC, and the mixed waste is shipped offsite for disposal. Since mixed waste is shipped offsite in both combinations, there is no need for a RCRA permit for INEL disposal.

\subsubsection{Defining Assumptions}

For the MLLWDF/Ship Offsite alternative, the defining assumptions are as follows: 
- MLLWDF is built to dispose of LLW only

- $\quad$ RWMC is shut down permanently when the MLLWDF begins operations

- Offsite DOE disposal facility disposes of the INEL mixed waste

- Maxinıum-generation scenario applies, as does the maximum-treatment scenario.

For the RWMC/Ship Offsite alternative, the defining assumptions are as follows:

- $\quad$ MLLWDF is not built

- Disposal methods at the RWMC are modified to accommodate more LLW after the space available by current disposal methods is consumed, assumed for purposes of the study to occur in 2004

- Offsite DOE disposal facility disposes of the INEL mixed waste

- Maximum-generation scenario applies, as does the maximum-treatment scenario.

\subsubsection{Overview of Disposition of Waste Types}

Based on the detailed waste information in Section 2, an overview of the waste disposition in both combinations is as follows:

Waste disposed of onsite:

$\begin{array}{ll}\text { LLW } & 2,486.2 \mathrm{~m}^{3} / \mathrm{yr} \\ \text { Mixed waste } & \text { None }\end{array}$

Waste disposed of offsite:

LLW None

Mixed waste $\quad 1,274.5 \mathrm{~m}^{3} / \mathrm{yr}$

Waste Stored:

None

\subsubsection{Location}

The locations of waste disposal are the same as those discussed in connection with the alternatives comprising these combinations.

\subsubsection{Description of Facilities}

For the combinations of alternatives, the facilities are generally simple combinations of the facilities discussed under the respective constituent alternatives. Only significant points of difference 
are discussed here. Because the mixed waste is shipped offsite for disposal in both combinations, the required onsite disposal areas are somewhat smaller than for the corresponding constituent alternatives.

For the MLLWDF/Ship Offsite combination, Table 5-13 compares the number of structures against that required for Subalternative $1 \mathrm{~A}$. Only 15 structures, rather than 25 , are required. As a result, the total site area of the MLLWDF is only 89 acres, compared with 115 acres in Subalternative $1 \mathrm{~A}$.

For the RWMC/Ship Offsite combination, the onsite disposal locations of the nonmixed waste are listed in Table 5-14. The locations are unchanged from those for the corresponding waste types in Alternative 2, with one exception. The minute amount of Class $C \alpha$ waste, which was codisposed with the Class $\mathrm{C} \alpha$ mixed waste in Alternative 2, is now codisposed with the Class B and Class $\beta-\gamma$ waste in the concrete vaults. This change eliminates the need for a separate disposal area, with a 16- $\mathrm{ft}$-thick final cover. Only 3 of the 4 new disposal areas in the RWMC are needed for this combination; the area west of Pit 3 is no longer needed for disposal.

\subsubsection{Description of Operations}

Waste operations are generally the same as for the constituent alternatives. The amount of waste to be handled and emplaced cnsite is slightly reduced for both combinations because the mixed waste is shipped offsite for disposal.

Table 5-13. Number of structures required for MLLWDF/Ship Offsite combination, compared with that for Subalternative 1A.

\begin{tabular}{lcc}
\hline \multicolumn{1}{c}{ Types(s) of waste } & $\begin{array}{c}\text { Number of structures required } \\
\text { for MLLWDF/Ship Offsite } \\
\text { Combination }\end{array}$ & $\begin{array}{c}\text { Number of structures } \\
\text { required for } \\
\text { Subalternative 1A }\end{array}$ \\
\hline A & 13 & 13 \\
B and $C \beta-\gamma$ and, for \\
$\begin{array}{l}\text { Alternative } 4, \text { the minute } \\
\text { amount of } \mathrm{C} \alpha\end{array}$ & 2 & 2 \\
A mixed & 0 & 7 \\
B mixed and $C \beta-\gamma$ mixed & 0 & 2 \\
$\begin{array}{l}\text { C } \alpha \text { mixed and, for } \\
\text { Alternative } 1 \mathrm{~A}, \text { the minute } \\
\text { amount of } \mathrm{C} \alpha\end{array}$ & 0 & 25 \\
Total & 15 & \\
\hline
\end{tabular}


Table 5-14. Detailed disposition of the various types of waste in the RWMC/Ship Offsite Alternative.

\begin{tabular}{lcll}
\hline \multicolumn{1}{c}{ Type(s) of waste } & $\begin{array}{c}\text { Required surface } \\
\text { area } \\
\left(\mathrm{ft}^{2}\right)\end{array}$ & $\begin{array}{c}\text { Disposal } \\
\text { method }\end{array}$ & \multicolumn{1}{c}{ Disposal location } \\
\hline Class $\mathrm{A}$ & 206,524 & $\begin{array}{l}\text { Covered } \\
\text { placement }\end{array}$ & $\begin{array}{l}\text { Surface of Pits 18 through 20, } \\
\text { plus part in area between } \\
\text { Pits } 8 \text { and } 9\end{array}$ \\
Class B and Class C $\beta-\gamma$ & 50,880 & Nested vaults & $\begin{array}{l}\text { Area between Pit 3 and } \\
\text { Pad A }\end{array}$ \\
Class $\mathrm{C} \alpha$ & 994 & Nested vaults & $\begin{array}{l}\text { Area between Pit 3 and } \\
\text { Pad A }\end{array}$ \\
\hline
\end{tabular}

\subsubsection{Closure and Postclosure Activities}

Closure and postclosure activities are generally the same as for the constituent alternatives.

The final covers for the combinations are somewhat reduced in areal extent because of having to encompass fewer disposal units. For the MLLWDF/Ship Offsite combination, the cover dimensions are $1,138 \mathrm{ft} \times 972 \mathrm{ft}$ (25 acres, as opposed to 38 acres for Subalternative $1 \mathrm{~A}$ ). For the RWMC/Ship Offsite combination, the cover again will be integrated with the RWMC final cover. Eliminating the need to cover the area west of Pit 3 reduces the cover area for these disposal units to 27 acres, compared with 35 acres in Alternative 2.

\subsection{Alternative 5: No Action}

This section provides a description of Alternative 5, which is basically the same as the "no-action" alternative required by NEPA regulations. No new action is taken to dispose of INEL mixed waste and LLW. The MLLWDF is not constructed, and no other disposal alternative is implemented.

Some of the waste would already exist (c.g., the Class $\mathrm{C} \alpha$ mixed waste at the TSA) if and when this alternative is implemented. Additional waste would continue to be generated. Since the waste would not be disposed of, it would have to be stored indefinitely in buildings constructed for that purpose. This study evaluates storage through the year 2044. The storage operations would be conducted in ac-7rdance with requirements in DOE Order 5820.2A and, in the case of the mixed waste, the RCRA regulations in 40 CFR 264.

\subsubsection{Defining Assumptions}

This alternative involves the long-term storage of waste. The defining assumptions are as follows: 
- $\quad$ MLLWDF is not built

- No other disposal alternative is implemented

- RWMC stops accepting waste for disposal, as discussed under Alternative 2, in 2004

- Maximum-generation scenario applies, as does the maximum-treatment scenario.

\subsubsection{Overview of Disposition of Waste Types}

Based on the detailed waste information in Section 2, an overview of the waste disposition in this alternative is as follows:

Waste disposed of:

None

Waste Stored:

$\begin{array}{ll}\text { LLW } & 2,486.2 \mathrm{~m}^{3} / \mathrm{yr} \\ \text { Mixed waste } & 1,274.5 \mathrm{~m}^{3} / \mathrm{yr}\end{array}$

\subsubsection{Location}

The storage facility might be located at any of the three candidate sites of primary interest discussed in Section 5.3.3. The facility also might be located adjacent to an existing waste management facility. To provide a basis for cost estimates, the storage facility was assumed to be located adjacent to the WERF.

\subsubsection{Description of Facilities}

5.7.4.1 General Site Layout and Preparation. The general site layout used for Alternative 5 is presented in Figure 5-29. The total land area requirement for the alternative is $\mathbf{4 7}$ acres. The site includes a controlled access area where the storage units are located and where day-to-day operations are carried out, an area for construction laydown, and support facilities required for the proper conduct of storage operations.

Although the site is assumed to be adjacent to the WERF facility, development of new land area is required. Site preparation would include removal of sage brush, grading, and construction of access roads, drainage areas, fencing, sccurity gates, and lighting.

It is assumed that a paved $1 / 2-\mathrm{mi}$ road connects the "torage facility complex to the Power Burst Facility (PBF)/WERF area. A patrol road is located immediately outside the facility fence. Paved service roads are provided for access to the storage structures. Areas are provided for parking and are located near the administrative and support areas. 


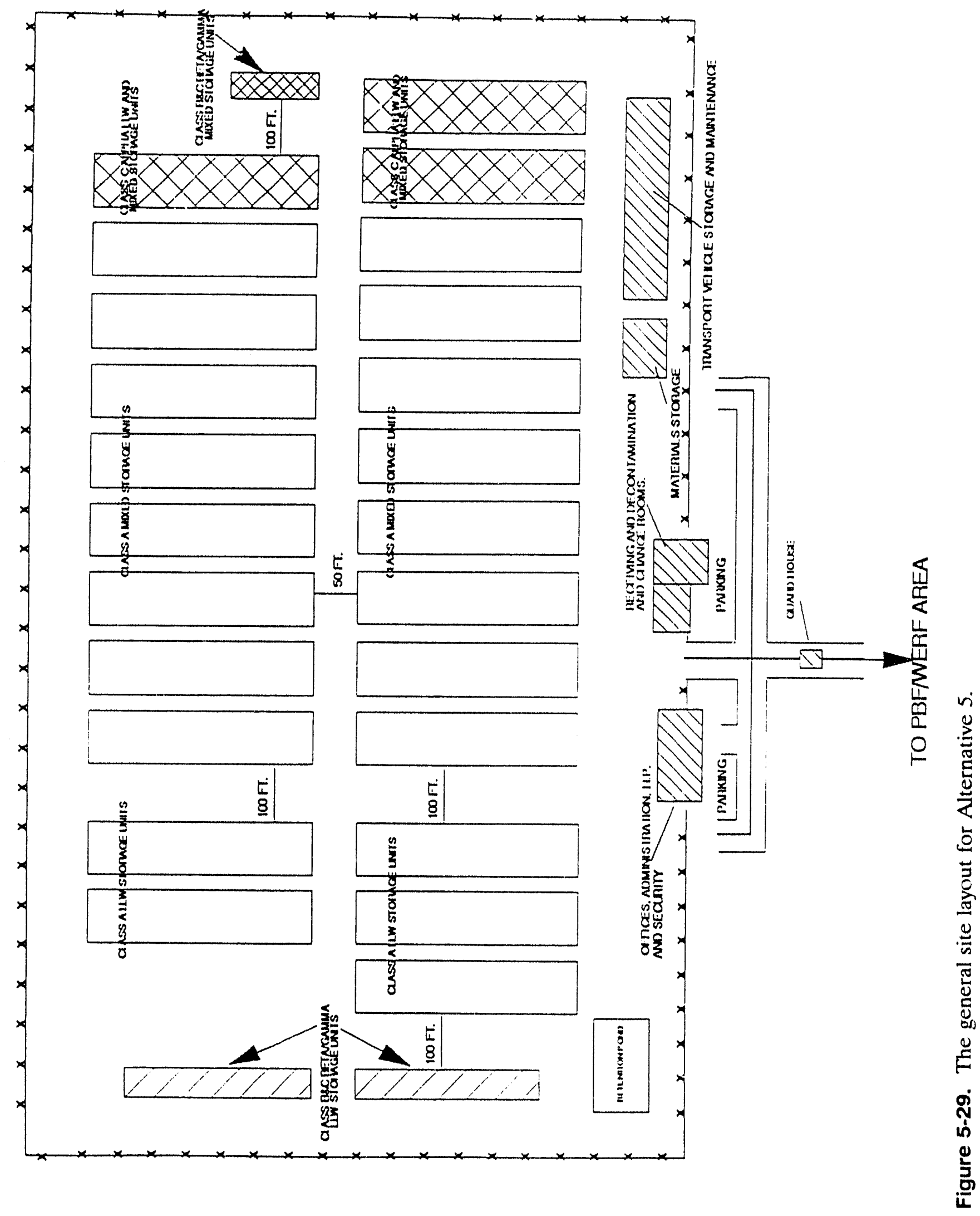


Access to the area is through one main gate controlled by security personnel. Security fencing is provided to serve as a physical demarcation of the facility and an obstruction for illegal entry. Tall lighting poles are installed to make the entire area visible at night.

Support facilities included at the storage facility are the same as those discussed for Subalternative 1A. See the discussion on Support Facilities for further details.

The storage areas for LLW and mixed waste are separated by a distance of $100 \mathrm{ft}$. Also, the storage units for remote-handled waste are separated from those for the contact-handled waste by a distance of $100 \mathrm{ft}$.

The Class A LLW storage area consists of five Butler-type structures in two rows. The structures are separated from each other by $20 \mathrm{ft}$. The rows are separated by $50 \mathrm{ft}$.

The Class B and Class C $\beta-\gamma$ LLW storage area consists of two concrete structures. The two structures are separated from each other by $50 \mathrm{ft}$.

The Class A mixed waste storage area consists of 16 Butler-type structures in two rows. The structures are separated from each other by $20 \mathrm{ft}$, and the rows are separated by $50 \mathrm{ft}$.

The Class B and Class $C \beta-\gamma$ mixed waste storage area consists of one concrete structure.

The Class $\mathrm{C} \alpha \mathrm{LLW}$ and mixed waste storage area consists of three Butler-type structures in two rows. The structures are separated from each other by $20 \mathrm{ft}$, and the rows are separated by $50 \mathrm{ft}$.

As shown in Figure 5-29, arcas have been allowed for one additional storage structure for the Class A and Class C LLW and mixed waste. This is to allow for construction and replacement of new structures throughout the 140-year period. These additional structures are denoted on the figure with dotted lines.

Unlike the MLLWDF subalternatives, a buffer zone is not included in this alternative. The NRC bufter zone requirement is not applicable to storage facilities.

5.7.4.2 Storage Unit Construction. In this alternative, the contact-handled types of waste (Class $A, A$ mixed, $C \alpha$, and $C \alpha$ mixed) are stored in Butler-type metal-sided buildings. The buildings are constructed on concrete pads about $1 \mathrm{ft}$ thick. The buildings are equipped with ventilation to allow personal access during emplacement, and for periodic inspections. The isolation barriers are the individual waste containers and any inner liners and the dual-liner/leachate system placed under the building.

Separate buildings are used for mixed waste and nonmixed waste. For the nonmixed waste, the waste containers are densely stacked as in Subalternative 1A. For the mixed waste, however, RCRA regulations require sufficient aisles to allow the inspection of containers and the movement of emergency equipment within the array of containers. The allowed array of containers and aisle spacing is negotiated on a casc-by-case basis with the regulators. The stack-and-aisle arrangement assumed here is the same as that recently negotiated for the new storage modules for mixed TRU 
waste at the RWMC. The arrangement is much less space-efficient than the dense stacking arrangement because of aisle spacing and the limited height for stacking containers.

The storage buildings for contact-handled waste were sized based on the numbers and sizes of waste containers in Table 2-6. The results are listed in the first part of Table 5-15. The very small amount of Class $C \alpha$ waste was combined with the Class $C \alpha$ mixed waste.

The remote-handled types of waste (Class $B$ and $C \beta-\gamma$, whether mixed or not), require shielding for handling and while in storage. The shielding could be provided in various ways. Examples are by casks, lead-lined buildings, concrete structures, belowgrade boreholes as in Subalternative 1D, or vaults as in Alternative 2. For the present alternative, abovegrade concrete structures were selected, because of the ease of handling and retricval, and the freedom from concern about groundwater intrusion.

The thickness of concrete needed is xpected to be 2 to $3 \mathrm{ft}$, based on shielding estimates from simple nomographs. For simplicity, the storage design used here for remote-handled waste is basically that of the AGES, with the same 3-ft thick walls. The differences are as follows: (a) the concrete does not undergo the elaborate CQA measures to ensure 500-year integrity, and (b) no final cover is emplaced. The size and number of storage buildings are listed in the second part of Table 5-15 and are identical to those for disposal of the remote-handled waste in Subalternative $1 \mathrm{~A}$.

Even without elaborate CQA, the AGES buildings are expected to survive the 40-year campaign and the subsequent 100-year institutional control period (see Figure 4-1). However, the Butler-type

Table 5-15. Number and size of storage buildings for each type of waste in Alternative 5.

A. Butler-type metal storage buildings for contact-handled waste

\begin{tabular}{lcc}
\hline \multicolumn{1}{c}{ Type(s) of waste } & $\begin{array}{c}\text { Total number of units } \\
\text { required over } 40 \text {-year period }\end{array}$ & $\begin{array}{c}\text { Dimensions } \\
(\mathrm{W} \times \mathrm{L} \times \mathrm{H}, \mathrm{ft})\end{array}$ \\
\hline $\mathrm{A}$ & 5 & $100 \times 224 \times 26$ \\
$\mathrm{~A}$ mixed & 16 & $104 \times 224 \times 18$ \\
$\mathrm{C} \alpha$ mixed, and the minute & 3 & $104 \times 224 \times 18$ \\
amount of $\mathrm{C} \alpha$ & & \\
\hline
\end{tabular}

B. AGES storage units for remote-handled waste

\begin{tabular}{lccc}
\hline Type(s) of waste & $\begin{array}{c}\text { Total number of units } \\
\text { required over 40-year } \\
\text { period }\end{array}$ & $\begin{array}{c}\text { Interior dimensions } \\
(\mathrm{W} \times \mathrm{L} \times \mathrm{H}, \mathrm{ft})\end{array}$ & $\begin{array}{c}\text { Exterior dimensions } \\
(\mathrm{W} \times \mathrm{L} \times \mathrm{H}, \mathrm{ft})\end{array}$ \\
\hline B and $\mathrm{C} \beta-\gamma$ & 2 & $52 \times 160 \times 28$ & $58 \times 166 \times 31$ \\
$\begin{array}{l}\text { B mixed and C } \beta-\gamma \\
\text { mixed }\end{array}$ & 1 & $34 \times 70 \times 29$ & $40 \times 76 \times 32$ \\
\hline
\end{tabular}


metal buildings are not expected to survive the 140-year total period. The maximum useful life of such buildings is estimated to be approximately 40 years. It is assumed in the cost estimates that, every 40 years, replacement buildings of this type are constructed and the waste in storage is transferred to the new buildings. It is assumed that, after the end of institutional control, no additional replacement of buildings takes place.

5.7.4.3 Site Surface Water Management System. The management of site surface water is similar to that described for Subalternative $1 \mathrm{~A}$ with two exceptions. First, there is no buffer zone. Therefore, the site perimeter ditch and the storage area (disposal area for Subaiternative 1A) perimeter ditch are adjacent to each other, separated by a dike that encircles the storage area. Second, the contact-handled waste units do not have a foundation drainage bed because a dual liner/leachate system is placed underneath the pads.

5.7.4.4 Liners and Leachate Collection and Removal Systems. Dual liner/leachate collection systems are used with all storage buildings to minimize the possibility of leachate contamination of the sitc. The contact-handled waste storage units have a dual-liner-leachate collection system installed below the concrete pad, similar to that described in Subalternative 1C. The vaults used for storage of remote-handled waste have an internal system identical to that described in Subalternative $1 \mathrm{~A}$.

5.7.4.5 Support Facilities and Activities. The support facilities provided for in the MLLWDF Subalternative $1 \mathrm{~A}$ are also provided for in Alternative 5.

Many of the utilities supporting operations can be provided from the PBF area. The site utilitics include water for domestic and industrial use, irrigation applications, electrical power, communications including telecommunications and automatic processing equipment connections, sanitary wastewater treatment, and stormwater management systems.

5.7.4.6 Construction Quality Assurance. The CQA program for Alternative 5 is simply that associated with normal construction of wasic storage facilities. No special measures analogous to those in Section 5.3.4.1 are taken. In the case of the concrete structures for the remote-handled waste, the elimination of the elaborate CQA program is expected to reduce the cost by approximately a factor of three. This observation is based on information obtained from two other DOE sites.

\subsubsection{Description of Operations}

5.7.5.1 Waste Receipt, Transfer, and Emplacement. Waste operations are similar to those described for Subalternative $1 \mathrm{~A}$ with the exception that the stacking pattern for the contact-handled mixed waste is altered to allow for inspection.

One other operational difference is that, after 40 years of use, the waste in one structure will have to be removed and placed in a newer structure. The basic transfer and emplacement operations associated with re-storing the waste are the same as those used when originally storing the waste.

5.7.5.2 Monitoring. A monitoring program is conducted for Alternative 5. The monitoring program and evaluation of the results are integrated with monitoring programs conducted by other 
organizations at the INEL. The current WERF monitoring program will have provided much of the initial data required prior to construction and opcration of the storage facility complex.

The monitoring program is continued with minor modification throughout construction and operation of the storage facility complex. Measurements and observations are made and recorded to provide data to evaluate the potential health and environmental impact during the construction and operation of the facility and to enable the evaluation of long-term effects and the need for mitigative measures. The monitoring system is capable of providing early warning of the releases of contaminants from the storage site before they leave the site boundary. The monitoring program measures contaminant concentrations in air, surface water, ground water, soil, plants, and animal tissue.

The monitoring program continues as long as institutional control of the site continues.

\subsubsection{Closure and Postclosure Activities}

5.7.6.1 Disposal Unit Closure. Because the waste is placed in indefinite storage, ready retrieval is required. Therefore, no final closure of the storage buildings is performed. As each building is filled to capacity, all openings are secured, but no permanent closure takes place.

5.7.6.2 Cover. Because the waste is placed in indefinite storagc, ready retrieval is required. Therefore, no final cover is placed over the storage buildings.

5.7.6.3 Maintenance and Monitoring During Institutional Control Period. Maintenance and monitoring during the institutional control period for Alternative 5 is the same as provided in Section 5.3.4.3 for Subalternative 1A, but is different in scope. Monitoring during the institutional control period for Subalternative $1 \mathrm{~A}$ will include monitoring the condition and structural performance of the isolation barriers and the final cover in addition to environmental monitoring. Monitoring conducted for Alternative 5 will primarily be environmental.

\subsection{Summary Charts}

This section provides a summary of information presented thus far. The waste volumes to be disposed of, by disposal concept, for the life of the disposal facility are summarized in Table 5-16. The waste forms disposed of by class are shown in Table 5-17. The number of waste containers disposed of per year, by concept, is summarized in Table 5-18.

Tables 5-19 through 5-22 provide a summary of the disposal site and disposal unit physical dimensions for each onsite alternative. Alternative 3, Offsite Disposal, is not listed but is likely to be similar to the MLLWDF Subalternative 1E, Shallow Land Burial/Boreholes. 
Table 5-16. Summary of waste volumes to be disposed of, by disposal concept, for the life of the disposal facility $\left(\mathrm{m}^{3} / \mathrm{yr}\right)$. $^{\mathrm{a}}$

\begin{tabular}{|c|c|c|c|c|c|c|c|c|c|c|c|}
\hline \multirow[b]{2}{*}{ Disposal alternative } & \multirow{2}{*}{$\begin{array}{l}\text { Disposal } \\
\text { concept }\end{array}$} & \multirow{2}{*}{$\begin{array}{c}\text { Class A } \\
\text { LLW }\end{array}$} & \multirow{2}{*}{$\begin{array}{c}\text { Class A } \\
\text { mixed }\end{array}$} & \multirow{2}{*}{$\begin{array}{l}\text { Class B } \\
\text { LLW }\end{array}$} & \multirow{2}{*}{$\begin{array}{c}\text { Class B } \\
\text { mixed }\end{array}$} & \multirow{2}{*}{$\begin{array}{c}\text { Class } C \beta-\gamma \\
\text { LLW }\end{array}$} & \multirow{2}{*}{$\begin{array}{l}\text { Class } C \beta- \\
\gamma \text { mixed }\end{array}$} & \multirow{2}{*}{$\begin{array}{l}\text { Class } C_{\alpha} \\
\text { LLW }\end{array}$} & \multirow{2}{*}{$\begin{array}{c}\text { Class } C_{a} \\
\text { mixed }\end{array}$} & \multicolumn{2}{|c|}{ Total } \\
\hline & & & & & & & & & & LLW & Mixed waste \\
\hline MLLWDF - 1A & AGES & $2,259.6$ & $1,063.4$ & 145.3 & 21.9 & 72.5 & 10.9 & 8.8 & 178.3 & $2,486.2$ & $1,274.5$ \\
\hline$\overline{M L L W D F}-1 B$ & BGES & $2,259.6$ & $1,063.4$ & 145.3 & 21.9 & 72.5 & 10.9 & 8.8 & 178.3 & $2,486.2$ & $1,274.5$ \\
\hline MLLWDF - 1C & $\mathrm{CM}$ & $2,259.6$ & $1,063.4$ & 145.3 & 21.9 & 72.5 & 10.9 & 8.8 & 178.3 & $2,486.2$ & $1,274.5$ \\
\hline \multirow[t]{2}{*}{$\overline{\text { MLLWDF - ID }}$} & GDB & $2,259.6$ & $1,063.4$ & 0 & $\overline{0}$ & 0 & 0 & 8.8 & 178.3 & $2,268.4$ & $1,241.7$ \\
\hline & GDBH & 0 & 0 & 145.3 & 21.9 & 72.5 & 10.9 & 0 & $\mathbf{0}$ & 217.8 & 32.8 \\
\hline \multirow[t]{2}{*}{$\overline{\text { MLLWDF - 1E }}$} & SLB & $2,259.6$ & $1,063.4$ & 0 & 0 & 0 & 0 & 8.8 & 178.3 & $2,268.4$ & $1,241.7$ \\
\hline & SBH & 0 & 0 & 145.3 & 21.9 & 72.5 & 10.9 & $\mathbf{0}$ & $\mathbf{0}$ & 217.8 & 32.8 \\
\hline \multirow[t]{2}{*}{$\overline{\text { MLLWDF - IF }}$} & $\overline{\text { SLB }}$ & $2,259.6$ & $1,063.4$ & $\overline{0}$ & $\overline{0}$ & 0 & 0 & 0 & 0 & $2,259.6$ & $1,063.4$ \\
\hline & AGES & 0 & 0 & 145.3 & 21.9 & 72.5 & 10.9 & 8.8 & i78.3 & 226.6 & 211.1 \\
\hline$\overline{M L L W D F-1 G}$ & AGES & 904.3 & 2.1 & 84.6 & 0 & 42.2 & 0 & 0 & 178.3 & $1,031.1$ & 180.4 \\
\hline MLLWDF - $1 \mathrm{H}$ & AGES & $7,074.2$ & $1,063.4$ & 145.3 & 21.9 & 72.5 & 10.9 & 8.8 & 178.3 & $7,300.8$ & $1,274.5$ \\
\hline \multirow[t]{2}{*}{$\overline{\mathrm{RWMC}}$} & $\overline{C P}$ & $2,259.6$ & $1,063.4$ & 0 & 0 & 0 & 0 & 8.8 & 178.3 & $2,268.4$ & $1,241.7$ \\
\hline & SBH & 0 & 0 & 145.3 & 21.9 & 72.5 & 10.9 & 0 & $\mathbf{0}$ & 217.8 & 32.8 \\
\hline \multirow[t]{2}{*}{ Offsite } & SLB & $2,259.6$ & $1,063.4$ & 0 & $\overline{0}$ & 0 & 0 & 8.8 & 178.3 & $2,268.4$ & $1,241.7$ \\
\hline & SBH & 0 & 0 & 145.3 & 21.9 & 72.5 & 10.9 & 0 & 0 & 217.8 & 32.8 \\
\hline \multirow[t]{3}{*}{ Offsite/MLLWDF } & AGES & $2,259.6$ & 0 & $14 \overline{5.3}$ & 0 & 72.5 & 0 & 8.8 & 0 & $2,486.2$ & $\overline{0}$ \\
\hline & SBH & 0 & 0 & 0 & 21.9 & 0 & 10.9 & $\mathbf{0}$ & 0 & 0 & 32.8 \\
\hline & SLB & 0 & $1,063.4$ & 0 & 0 & $\mathbf{0}$ & $\mathbf{0}$ & 0 & 178.3 & 0 & $1,241.7$ \\
\hline \multirow[t]{3}{*}{ Offsite/RWMC } & $\mathrm{CP}$ & $2,259.6$ & 0 & 0 & 0 & 0 & 0 & 0 & 0 & $2,259.6$ & 0 \\
\hline & SBH & 0 & 0 & 145.3 & 21.9 & 72.5 & 10.9 & 8.8 & 0 & 226.8 & 32.8 \\
\hline & SLB & $\mathbf{0}$ & $1,063.4$ & 0 & $\mathbf{0}$ & 0 & 0 & 0 & 178.3 & 0 & $1,241.7$ \\
\hline No Action & Storage & $2,259.6$ & $1,063.4$ & 145.3 & 21.9 & 72.5 & 10.9 & 8.8 & 178.3 & $2,486.2$ & $1,274.5$ \\
\hline $\begin{array}{ll}\text { a. Acronyms used in t } \\
\text { AGES } & = \\
\text { BGES } & = \\
\text { CM } & = \\
\text { CP } & =\end{array}$ & $\begin{array}{c}\text { his table are def } \\
\text { Abovegrade } \\
\text { Belowgrade } \\
\text { Covered mo } \\
\text { Covered plac }\end{array}$ & $\begin{array}{l}\text { d below: } \\
\text { zineered stru } \\
\text { jineered stru } \\
\text { es } \\
\text { lent }\end{array}$ & $\begin{array}{l}\text { ctures } \\
\text { tures }\end{array}$ & $\begin{array}{l}\text { GDB } \\
\text { GDBH } \\
\text { SBH }\end{array}$ & $\begin{array}{l}\text { Greater } \\
\text { Greater } \\
\text { Shallow }\end{array}$ & $\begin{array}{l}\text { r-depth burial } \\
\text { r-depth boreho } \\
\text { boreholes }\end{array}$ & oles & & & & \\
\hline
\end{tabular}


Table 5-17. Summary information on waste forms.

\begin{tabular}{|c|c|c|}
\hline IVaste class & $\begin{array}{l}\text { All alternatives except } 1 \mathrm{H} \\
\text { (maximum treatment) }\end{array}$ & $\begin{array}{c}1 \mathrm{H} \\
\text { (minimum treatment) }\end{array}$ \\
\hline A & $\begin{array}{l}\text { Stabilized (e.g., cement, sulfur-polymer } \\
\text { cement) incinerator ash; size-reduced } \\
\text { metals; compacted noncombustibles } \\
\text { (e.g., glass, wire); soil, rubble, concrete, } \\
\text { compacted or untreated }\end{array}$ & $\begin{array}{l}\text { Combustibles, untreated; metals, } \\
\text { untreated; noncombustibles (e.g., } \\
\text { glass wire), untreated; soil, rubble, } \\
\text { concrete, untreated; filters, untreated }\end{array}$ \\
\hline B & $\begin{array}{l}\text { Activated metals, unt:eated; stabilized } \\
\text { sludges and resins }\end{array}$ & $\begin{array}{l}\text { Activated metals, untreated; sludges } \\
\text { and resins, untreated }\end{array}$ \\
\hline $\mathrm{C} \alpha$ & Same as $C \alpha$ mixed & Same as $\mathrm{C} \alpha$ mixed \\
\hline$C \beta-\gamma$ & Activated metals, untreated & Same as maximum treatment \\
\hline A mixed & $\begin{array}{l}\text { Stabilized (e.g., cement, sulfur-polymer } \\
\text { cement) incinerator ash; stabilized } \\
\text { residues from lead and cadmium } \\
\text { recovery; stabilized solids; stabilized } \\
\text { soil and rubble }\end{array}$ & Same as maximum treatment \\
\hline$B$ mixed & $\begin{array}{l}\text { Stabilized incinerator ash; stabilized } \\
\text { sludge; stabilized size-reduced metal }\end{array}$ & Same as maximum treatment \\
\hline $\mathrm{C} \propto \boldsymbol{\alpha}$ mixed & Iron-enriched basalt & Same as maximum treatment \\
\hline$C \boldsymbol{\beta}-\boldsymbol{\gamma}$ mixed & $\begin{array}{l}\text { Stabilized incinerator ash; stabilized } \\
\text { sludge; stabilized size-reduced metal }\end{array}$ & Same as maximum treatment \\
\hline
\end{tabular}


Table 5-18. Summary of the number of waste containers disposed of per year by concept. ${ }^{\text {a }}$

\begin{tabular}{|c|c|c|c|c|}
\hline \multirow{2}{*}{$\begin{array}{c}\text { Disposal } \\
\text { alternative }\end{array}$} & \multirow[b]{2}{*}{ Disposal concept } & \multirow[b]{2}{*}{ Disposal container } & \multicolumn{2}{|c|}{ Number of containers } \\
\hline & & & LLW & Mixed waste \\
\hline \multirow[t]{3}{*}{ MLLWDF-1A } & AGES & Mctal boxes ${ }^{b}$ & 359 & 0 \\
\hline & & Drums ${ }^{c}$ & 4,808 & 4,616 \\
\hline & & Concrete waste boxes ${ }^{d}$ & 60 & 9 \\
\hline \multirow[t]{3}{*}{ MLLWDF-1B } & BGES & Metal boxes & 359 & 0 \\
\hline & & Drums & 4,808 & 4,616 \\
\hline & & Concrete waste boxes & 60 & 9 \\
\hline \multirow[t]{3}{*}{ MLLWDF-1C } & $\mathrm{CM}$ & Metal boxes & 359 & 0 \\
\hline & & Drums & 4,808 & 4,616 \\
\hline & & Concrete waste boxes & 60 & 9 \\
\hline \multirow[t]{6}{*}{ MLLWDF-1D } & GDB & Metal boxes & 359 & 0 \\
\hline & & Drums & 4,808 & 4,616 \\
\hline & & Concrete waste boxes & 0 & 0 \\
\hline & GDBH & Metal boxes & 0 & 0 \\
\hline & & Drums & 0 & 0 \\
\hline & & Concrete waste boxes & 60 & 9 \\
\hline \multirow[t]{6}{*}{ MLLWDF-1E } & $\overline{\text { SLB }}$ & Metal boxes & 359 & 0 \\
\hline & & Drums & 4,808 & 4,616 \\
\hline & & Concrete waste boxes & 0 & 0 \\
\hline & SBH & Metal boxes & 0 & 0 \\
\hline & & Drums & 0 & 0 \\
\hline & & Concrete waste boxes & 60 & 9 \\
\hline \multirow[t]{6}{*}{ MLLWDF-1F } & SLB & Metal boxes & 357 & 0 \\
\hline & & Drums & 4,787 & 3,953 \\
\hline & & Concrete waste boxes & 0 & 0 \\
\hline & AGES & Melal boxes & 2 & 0 \\
\hline & & Drums & 21 & 663 \\
\hline & & Concrete waste boxes & 60 & 9 \\
\hline \multirow[t]{3}{*}{ MLLWDF-1G } & AGES & Mctal boxes & 163 & 0 \\
\hline & & Drums & 1,714 & 671 \\
\hline & & Concrete waste boxes & 36 & 0 \\
\hline \multirow[t]{3}{*}{ MLLWDF-1H } & AGES & Mclal boxes & 939 & 0 \\
\hline & & Drums & 16,849 & 4,616 \\
\hline & & Concrete waste boxes & 60 & 9 \\
\hline \multirow[t]{6}{*}{ RWMC } & $\mathrm{CP}$ & Mctal boxes & 359 & 0 \\
\hline & & Drums & 4,808 & 4,616 \\
\hline & & Concrete waste boxes & 0 & 0 \\
\hline & SBH & Metal boxes & 0 & 0 \\
\hline & & Drums & 0 & 0 \\
\hline & & Concrete waste boxes & 60 & 9 \\
\hline
\end{tabular}


Table 5-18. (continued).

\begin{tabular}{|c|c|c|c|c|}
\hline \multirow{2}{*}{$\begin{array}{l}\text { Disposal } \\
\text { alternative }\end{array}$} & \multirow[b]{2}{*}{ Disposal concept } & \multirow[b]{2}{*}{ Disposal container } & \multicolumn{2}{|c|}{ Number of containers } \\
\hline & & & LLW & Mixed waste \\
\hline \multirow[t]{6}{*}{ Offsite } & SLB & Metal boxes & 359 & 0 \\
\hline & & Drums & 4,808 & 4,616 \\
\hline & & Concrete waste boxes & 0 & 0 \\
\hline & SBH & Metal boxes & 0 & 0 \\
\hline & & Drums & 0 & 0 \\
\hline & & Concrete waste boxes & 60 & 9 \\
\hline \multirow[t]{9}{*}{ Offsite/MLLWDF } & AGES & Metal drums & 359 & 0 \\
\hline & & Drums & 4,808 & 0 \\
\hline & & Concrete waste boxes & 60 & 0 \\
\hline & SBH & Metal drums & 0 & 0 \\
\hline & & Drums & 0 & 0 \\
\hline & & Concrete waste boxes & 0 & 0 \\
\hline & SLB & Metal drums & 0 & 0 \\
\hline & & Drums & 0 & 4,616 \\
\hline & & Concrete waste boxes & 0 & 0 \\
\hline \multirow[t]{9}{*}{ Offsite/RWMC } & $\overline{\mathrm{CP}}$ & Metal boxes & 357 & $\overline{0}$ \\
\hline & & Drums & 4,787 & 0 \\
\hline & & Concrete waste boxes & 0 & 0 \\
\hline & SBH & Metal boxes & 2 & 0 \\
\hline & & Drums & 21 & 0 \\
\hline & & Concrete waste boxes & 60 & 9 \\
\hline & SLB & Metal boxes & 0 & 0 \\
\hline & & Drums & 0 & 4,616 \\
\hline & & Concrete waste boxes & 0 & 0 \\
\hline \multirow[t]{3}{*}{ No action } & Storage & Metal boxes & 359 & 0 \\
\hline & & Drums & 4,808 & 4,616 \\
\hline & & Concrete waste boxes & 60 & 9 \\
\hline
\end{tabular}

a. The acronyms used in this table are defined as follows:
AGES = Abovegrade engineered structures
BGES = Belowgrade engineered structures
$\mathrm{CP} \quad=$ Covered placement
$\mathrm{CM}=$ Covered modules
$\mathrm{SBH}=$ Shallow boreholes
GDB = Greater-depth burial
$\mathrm{GDBH}=$ Greater-depth boreholes

b. Metal boxes are $4 \times 4 \times 6 \mathrm{ft}=96 \mathrm{ft}^{2}=2.719 \mathrm{~m}^{3}$

c. Drums are 71-gal square drums $=9.5 \mathrm{ft}^{2}=0.269 \mathrm{~m}^{3}$

d. Concrete waste boxes are $4 \times 4 \times 8 \mathrm{ft}=128 \mathrm{ft}^{2}=3.625 \mathrm{~m}^{3}$ 
Table 5-19. Summary of Alternative 1 (MLLWDF) disposal site and disposal unit physical dimensions. ${ }^{\text {a }}$

\begin{tabular}{|c|c|c|c|}
\hline Disposal alternative & Disposal concept & Parameter & Dimensions \\
\hline \multirow[t]{21}{*}{ MLLWDF-1A } & AGES & Site dimensions $(\mathrm{ft} \times \mathrm{ft})$ & $1,938 \times 2,588$ \\
\hline & & Total site area (acres) & 115 \\
\hline & & Disposal area dimensions $(\mathrm{ft} \times \mathrm{ft})$ & $1,282 \times 1,932$ \\
\hline & & Total disposal area (acres) & 57 \\
\hline & & Cover dimensions $(\mathrm{ft} \times \mathrm{ft})$ & $972 \times 1,722$ \\
\hline & & Total cover area (acres) & 38 \\
\hline & & Class A disposal units & \\
\hline & & Number of structures & 13 \\
\hline & & Exterior dimensions $(\mathrm{W} \times \mathrm{L} \times \mathrm{H}, \mathrm{ft})$ & $56 \times 266 \times 29$ \\
\hline & & Class $B$ and $C \beta-\gamma$ disposal units & \\
\hline & & Number of structures & 2 \\
\hline & & Exterior dimensions $(\mathrm{W} \times \mathrm{L} \times \mathrm{H}, \mathrm{ft})$ & $58 \times 166 \times 31$ \\
\hline & & Class A mixed disposal units & \\
\hline & & Number of structures & 7 \\
\hline & & Exterior dimensions $(\mathrm{W} \times \mathrm{L} \times \mathrm{H}, \mathrm{ft})$ & $56 \times 256 \times 29$ \\
\hline & & $\begin{array}{l}\text { Class } B \text { and } C \beta-\gamma \text { mixed disposal } \\
\text { units }\end{array}$ & \\
\hline & & Number of structures & 1 \\
\hline & & Exterior dimensions $(\mathrm{W} \times \mathrm{L} \times \mathrm{H}, \mathrm{ft})$ & $40 \times 76 \times 32$ \\
\hline & & $\begin{array}{l}\text { Class } C \alpha \text { and } C \alpha \text { mixed disposal } \\
\text { units }\end{array}$ & \\
\hline & & Number of structures & 2 \\
\hline & & Exterior dimensions $(\mathrm{W} \times \mathrm{L} \times \mathrm{H}, \mathrm{ft})$ & $56 \times 157 \times 29$ \\
\hline \multirow[t]{6}{*}{ MLLWDF-1B } & BGES & Site dimensions $(\mathrm{ft} \times \mathrm{ft})$ & $1,938 \times 2,528$ \\
\hline & & Total site area (acres) & 112 \\
\hline & & Disposal area dimensions $(\mathrm{ft} \times \mathrm{ft})$ & $1,282 \times 1,872$ \\
\hline & & Total disposal area (acres) & 55 \\
\hline & & Cover dimensions $(f t \times f t)$ & $724 \times 1,474$ \\
\hline & & Total cover area (acres) & 24 \\
\hline
\end{tabular}


Table 5-19. (continued),

\begin{tabular}{|c|c|c|c|}
\hline Disposal alternative & Disposal concept & Parameter & Dimensions \\
\hline & & Class A disposal units & \\
\hline & & Number of structures & 13 \\
\hline & & Exterior dimensions $(\mathrm{W} \times \mathrm{L} \times \mathrm{H}, \mathrm{ft})$ & $56 \times 266 \times 29$ \\
\hline & & Class $B$ and $C \beta-\gamma$ disposal units & \\
\hline & & Number of structures & 2 \\
\hline & & Exterior dimensions $(\mathrm{W} \times \mathrm{L} \times \mathrm{H}, \mathrm{ft})$ & $58 \times 266 \times 29$ \\
\hline & & Class A mixed disposal unite & \\
\hline & & Number of structures & 7 \\
\hline & & Exterior dimensions $(\mathrm{W} \times \mathrm{L} \times \mathrm{H}, \mathrm{ft})$ & $56 \times 266 \times 29$ \\
\hline & & $\begin{array}{l}\text { Class } B \text { and } C \beta-\gamma \text { mixed disposal } \\
\text { units }\end{array}$ & \\
\hline & & Number of structures & 1 \\
\hline & & Exterior dimensions $(\mathrm{W} \times \mathrm{L} \times \mathrm{H}, \mathrm{ft})$ & $40 \times 76 \times 32$ \\
\hline & & $\begin{array}{l}\text { Class } C \alpha \text { and } C \alpha \text { mixed disposal } \\
\text { units }\end{array}$ & \\
\hline & & Number of structures & 2 \\
\hline & & Exterior dimensions $(\mathrm{W} \times \mathrm{L} \times \mathrm{H}, \mathrm{ft})$ & $56 \times 157 \times 29$ \\
\hline \multirow[t]{4}{*}{ MLLWDF-1C } & $\mathrm{CM}$ & $\begin{array}{l}\text { Site dimensions }(\mathrm{ft} \times \mathrm{ft}) \\
\text { Total site area }(\text { acres }) \\
\text { Disposal area dimensions }(\mathrm{ft} \times \mathrm{ft} \text { ) } \\
\text { Total disposal area }(\text { acres }) \\
\text { Cover dimensions }(\mathrm{ft} \times \mathrm{ft}) \\
\text { Total cover area (acres) }\end{array}$ & $\begin{array}{l}1,996 \times 2,884 \\
1,32 \\
1,340 \times 2,228 \\
69 \\
944 \times 1,705 \\
37\end{array}$ \\
\hline & & Nonmixed waste disposal pads & \\
\hline & & Number of pads & 3 \\
\hline & & Dimensions $(\mathrm{W} \times \mathrm{L}, \mathrm{ft})$ & $180 \times 815$ \\
\hline
\end{tabular}


Table 5-19. (continued).

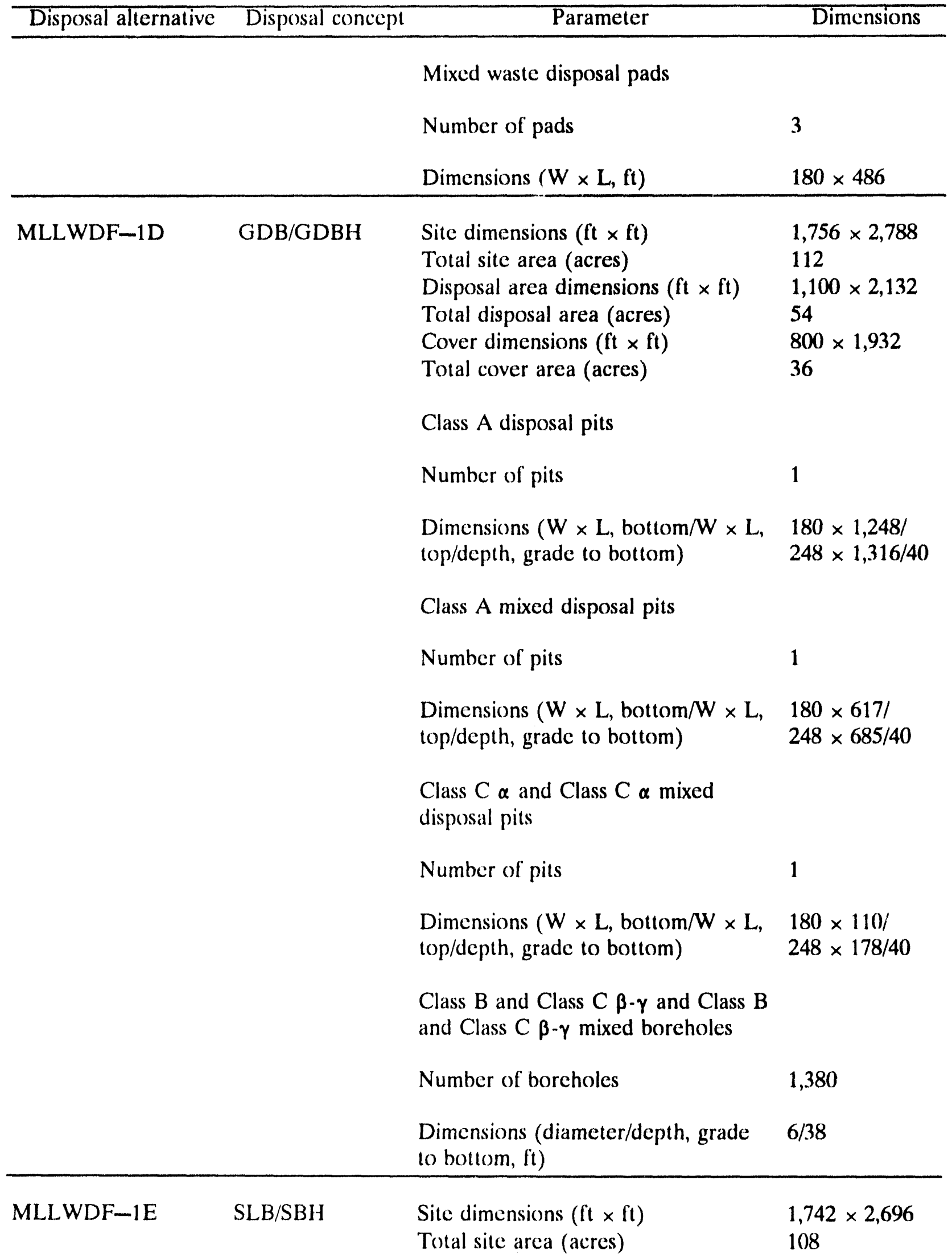


Table 5-19. (continued).

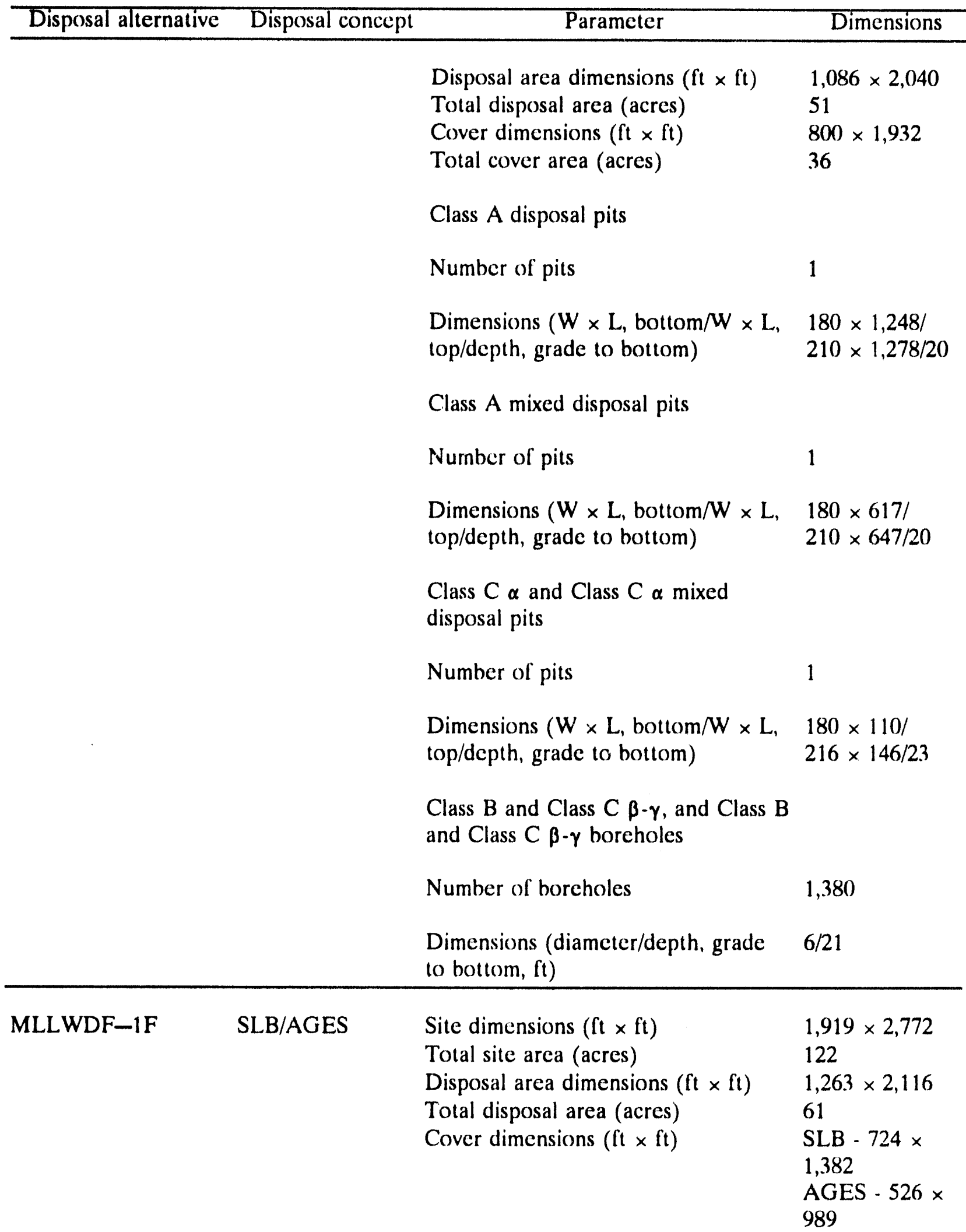


Table 5-19. (continued).

\begin{tabular}{|c|c|c|c|}
\hline Disposal alternative & Disposal concept & Parameter & Dimensions \\
\hline & & Total cover area (acres) & $\begin{array}{l}\text { SLB }-18 \\
\text { AGES - } 12 \\
\text { TOTAL - } 30\end{array}$ \\
\hline & & Class A disposal pits & \\
\hline & & Number of pits & 1 \\
\hline & & $\begin{array}{l}\text { Dimensions }(W \times L \text {, bottom } / W \times L \text {, } \\
\text { top/depth, grade to bottom, } f t)\end{array}$ & $\begin{array}{l}180 \times 1,248 / \\
210 \times 1,278 / 20\end{array}$ \\
\hline & & Class A mixed disposal pits & \\
\hline & & Number of pits & 1 \\
\hline & & $\begin{array}{l}\text { Dimensions }(W \times L \text {, bottom } / W \times L \text {, } \\
\text { top/depth, grade to bottom, } f t)\end{array}$ & $\begin{array}{l}180 \times 617 / \\
210 \times 647 / 20\end{array}$ \\
\hline & & $\begin{array}{l}\text { Class } B \text { and } C \beta-\gamma \text { disposal units } \\
\text { (AGES) }\end{array}$ & \\
\hline & & Number of units & 2 \\
\hline & & Exterior dimensions $(\mathrm{W} \times \mathrm{L} \times \mathrm{H}, \mathrm{ft})$ & $58 \times 166 \times 31$ \\
\hline & & $\begin{array}{l}\text { Class } B \text { and Class } C \beta-\gamma \text { mixed } \\
\text { disposal units (AGES) }\end{array}$ & \\
\hline & & Number of units & 1 \\
\hline & & Extcrior dimensions $(\mathrm{W} \times \mathrm{L} \times \mathrm{H}, \mathrm{ft})$ & $56 \times 157 \times 29$ \\
\hline & & $\begin{array}{l}\text { Class } C \alpha \text { and Class } C \alpha \text { mixed } \\
\text { disposal units (AGES) }\end{array}$ & \\
\hline & & Number of units & 2 \\
\hline & & Exterior dimensions $(\mathrm{W} \times \mathrm{L} \times \mathrm{H}, \mathrm{ft})$ & $56 \times 157 \times 29$ \\
\hline MLLWDF-1G & AGES & $\begin{array}{l}\text { Site dimensions }(\mathrm{ft} \times \mathrm{ft}) \\
\text { Total site area }(\text { acres }) \\
\text { Disposal area dimensions }(\mathrm{ft} \times \mathrm{ft} \text { ) } \\
\text { Total disposal area }(\text { acres }) \\
\text { Cover dimensions }(\mathrm{ft} \times \mathrm{ft}) \\
\text { Total cover area }(\text { acres })\end{array}$ & $\begin{array}{l}1,938 \times 1,932 \\
86 \\
1,282 \times 1,276 \\
38 \\
972 \times 1,066 \\
24\end{array}$ \\
\hline
\end{tabular}


Table 5-19. (continued).

\begin{tabular}{|c|c|c|c|}
\hline Disposal alternative & Disposal concept & Parameter & Dimensions \\
\hline & & \multicolumn{2}{|l|}{ Class A disposal units } \\
\hline & & Number of units & 5 \\
\hline & & Exterior dimensions $(\mathrm{W} \times \mathrm{L} \times \mathrm{H}, \mathrm{ft})$ & $56 \times 266 \times 29$ \\
\hline & & \multicolumn{2}{|l|}{$\begin{array}{l}\text { Class } B \text { and Class } C \beta-\gamma \text { disposal } \\
\text { units }\end{array}$} \\
\hline & & Number of units & 2 \\
\hline & & Exterior dimensions $(\mathrm{W} \times \mathrm{L} \times \mathrm{H}, \mathrm{ft})$ & $58 \times 94 \times 31$ \\
\hline & & \multicolumn{2}{|l|}{$\begin{array}{l}\text { Class B and Class } C \beta-\gamma \text { mixed } \\
\text { disposal units }\end{array}$} \\
\hline & & Number of units & 0 \\
\hline & & Exterior dimensions $(\mathrm{W} \times \mathrm{L} \times \mathrm{H}, \mathrm{ft})$ & N/A \\
\hline & & \multicolumn{2}{|l|}{$\begin{array}{l}\text { Class } \mathrm{C} \alpha \text { and Class } \mathrm{A} \text { mixed disposal } \\
\text { units }\end{array}$} \\
\hline & & Number of units & 2 \\
\hline & & Exterior dimensions $(\mathrm{W} \times \mathrm{L} \times \mathrm{H}, \mathrm{ft})$ & $56 \times 157 \times 29$ \\
\hline \multirow[t]{5}{*}{ MLLWDF-1H } & \multirow[t]{5}{*}{ AGES } & $\begin{array}{l}\text { Site dimensions }(\mathrm{ft} \times \mathrm{ft}) \\
\text { Total site area }(\text { acres }) \\
\text { Disposal area dimensions }(\mathrm{ft} \times \mathrm{ft})\end{array}$ & $\begin{array}{l}2,570 \times 2,816 \\
166 \\
1,914 \times 2,160\end{array}$ \\
\hline & & $\begin{array}{l}\text { Total disposal area (acres) } \\
\text { Cover dimensions }(\mathrm{ft} \times \mathrm{ft}) \\
\text { Total cover area (acres) }\end{array}$ & $\begin{array}{l}95 \\
1,604 \times 1,950 \\
72\end{array}$ \\
\hline & & Class A disposal units & \\
\hline & & Number of units & 40 \\
\hline & & Exterior dimensions $(\mathrm{W} \times \mathrm{L} \times \mathrm{H}, \mathrm{ft})$ & $56 \times 266 \times 29$ \\
\hline
\end{tabular}


Table 5-19. (continued).

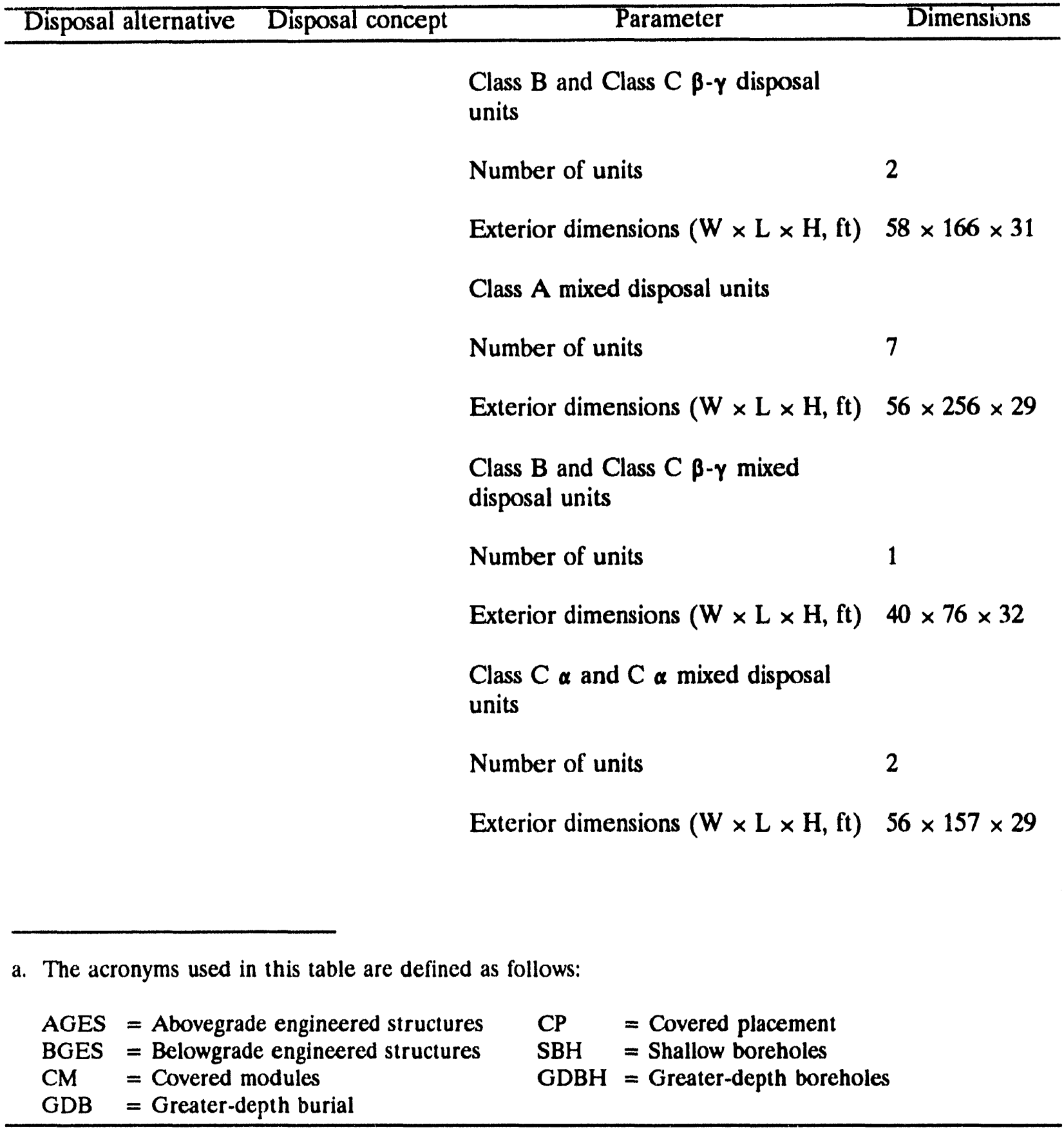


Table 5-20. Summary of Alternative 2 (RWMC) disposal site and disposal unit physical dimensions. ${ }^{\text {a }}$

\begin{tabular}{|c|c|c|c|}
\hline Disposal alternative & Disposal concept & Parameter & Dimensions \\
\hline \multirow[t]{21}{*}{ RWMC } & $\mathrm{CP} / \mathrm{SBH}$ & Total existing site arca (acres) & $\begin{array}{l}165(\mathrm{SDA} \\
+ \text { Administ } \\
\text { area) }\end{array}$ \\
\hline & & $\begin{array}{l}\text { Total existing disposal area } \\
\text { (acres) }\end{array}$ & 154 (SDA \\
\hline & & Total modified site area (acres) & 21 \\
\hline & & $\begin{array}{l}\text { Total modified disposal area } \\
\text { (acres) }\end{array}$ & 10 \\
\hline & & Surface of Pits 18 through 20 & \\
\hline & & $\begin{array}{l}\text { Disposal area } \\
\text { dimensions }(\mathrm{W} \times \mathrm{L}, \mathrm{ft})\end{array}$ & $241 \times 589$ \\
\hline & & Cover dimensions $(\mathrm{ft} \times \mathrm{ft})$ & $537 \times 885$ \\
\hline & & Cover area (acres) & 11 \\
\hline & & Between Pits 8 and 9 & \\
\hline & & $\begin{array}{l}\text { Disposal area dimensions } \\
(\mathrm{W} \times \mathrm{L}, \mathrm{ft})\end{array}$ & $423 \times 324$ \\
\hline & & Cover dimensions $(\mathrm{ft} \times \mathrm{ft})$ & $719 \times 620$ \\
\hline & & Cover area (acres) & 10 \\
\hline & & West of Pit 3 & \\
\hline & & $\begin{array}{l}\text { Disposal area dimensions } \\
(\mathrm{W} \times \mathrm{L}, \mathrm{ft})\end{array}$ & $357 \times 241$ \\
\hline & & Cover dimensions $(\mathrm{ft} \times \mathrm{ft})$ & $653 \times 537$ \\
\hline & & Cover area (acres) & 8 \\
\hline & & Between Pit 3 and Pad A & \\
\hline & & $\begin{array}{l}\text { Disposal arca dimensions } \\
(\mathrm{W} \times \mathrm{L}, \mathrm{ft})\end{array}$ & $191 \times 357$ \\
\hline & & Cover dimensions $(\mathrm{ft} \times \mathrm{ft})$ & $419 \times 585$ \\
\hline & & Cover area (acres) & 6 \\
\hline & & Total cover area (acres) & 35 \\
\hline
\end{tabular}

a. The acronyms used in this table are defined as follows:

$\mathrm{CP}=$ Covered placement $\quad \mathrm{SBH}=$ Shallow borcholes 
Table 5-21. Summary of Alternative 4 (combinations of alternatives) disposal site and disposal unit physical dimensions. ${ }^{a}$

\begin{tabular}{|c|c|c|c|}
\hline $\begin{array}{c}\text { Disposal } \\
\text { alternative }\end{array}$ & Disposal concept & Parameter & Dimensions \\
\hline \multirow{13}{*}{$\begin{array}{l}\text { MLLWDF/ } \\
\text { offsite }\end{array}$} & \multirow[t]{13}{*}{ AGES/SBH/SLB } & \multirow{2}{*}{$\begin{array}{l}\text { Site dimensions }(\mathrm{ft} \times \mathrm{ft}) \\
\text { Total site area (acres) }\end{array}$} & $1,938 \times 2,004$ \\
\hline & & & 89 \\
\hline & & Disposal area dimensions $(\mathrm{ft} \times \mathrm{ft})$ & $1,282 \times 1,348$ \\
\hline & & Total disposal area $(\mathrm{ft} \times \mathrm{ft})$ & 40 \\
\hline & & Cover dimensions $(\mathrm{ft} \times \mathrm{ft})$ & $972 \times 1,138$ \\
\hline & & Total cover area (acres) & 25 \\
\hline & & Class A disposal units & \\
\hline & & Number of structures & 13 \\
\hline & & $\begin{array}{l}\text { Exterior dimensions } \\
(\mathrm{W} \times \mathrm{L} \times \mathrm{H}, \mathrm{ft})\end{array}$ & $56 \times 266 \times 29$ \\
\hline & & $\begin{array}{l}\text { Class } B, C \alpha \text {, and } C \beta-\gamma \text { disposal } \\
\text { units }\end{array}$ & \\
\hline & & Number of structures & \\
\hline & & \multirow{2}{*}{$\begin{array}{l}\text { Exterior dimensions } \\
(\mathrm{W} \times \mathrm{L} \times \mathrm{H}, \mathrm{ft})\end{array}$} & 2 \\
\hline & & & $58 \times 166 \times 31$ \\
\hline \multirow{10}{*}{ RWMC/offsite } & \multirow{10}{*}{$\mathrm{CP} / \mathrm{SBH} / \mathrm{SLB}$} & & \\
\hline & & Total existing site area (acres) & $\begin{array}{l}165 \text { (SDA + TSA + } \\
\text { Administrative area) }\end{array}$ \\
\hline & & Total existing disposal area (acres) & $154(\mathrm{SDA}+\mathrm{TSA})$ \\
\hline & & Total modified site area (acres) & 17 \\
\hline & & Total modified disposal area (acres) & 6 \\
\hline & & $\begin{array}{l}\text { Surface of Pits } 18 \text { through } 20 \text { plus } \\
\text { part of area between Pits } 8 \text { and } 9 \\
\text { (Class A waste) }\end{array}$ & \\
\hline & & Disposal area required $\left(\mathrm{ft}^{2}\right)$ & 206,524 \\
\hline & & \multicolumn{2}{|l|}{$\begin{array}{l}\text { Area between Pit } 3 \text { and Pad } A \\
\text { (Class } B \text {, Class } C \alpha \text {, and Class } C \beta-\gamma \\
\text { waste) }\end{array}$} \\
\hline & & Disposal area required $\left(\mathrm{ft}^{2}\right)$ & 51,878 \\
\hline & & Total cover area (acres) & 27 \\
\hline \multicolumn{4}{|c|}{ a. The acronyms used in this table are defined as follows: } \\
\hline $\begin{array}{ll}\mathrm{CP} & =\mathrm{CO} \\
\mathrm{AGES} & =\mathrm{Ab}\end{array}$ & $\begin{array}{l}=\text { Covered placement } \\
=\text { Above-grade engineered structures }\end{array}$ & $\begin{array}{l}\text { SBH }=\text { Shallow boreholes } \\
\text { SLB }=\text { Shallow land burial }\end{array}$ & \\
\hline
\end{tabular}


Table 5-22. Summary of Alternative 5 (no action alternative) storage site and storage unit physical dimensions.

\begin{tabular}{|c|c|c|c|}
\hline Disposal alternative & Disposal concept & Parameter & Dimensions \\
\hline \multirow[t]{19}{*}{ No action } & Storage & Site dimensions $(\mathrm{ft} \times \mathrm{ft})$ & $798 \times 1934$ \\
\hline & & Total site area (acres) & 35 \\
\hline & & Storage area dimensions $(\mathrm{ft} \times \mathrm{ft})$ & $798 \times 1934$ \\
\hline & & Total storage area (acres) & 35 \\
\hline & & Class A storage units & \\
\hline & & Number of structures & 5 \\
\hline & & $\begin{array}{l}\text { Extcrior dimensions } \\
(\mathrm{W} \times \mathrm{L} \times \mathrm{H}, \mathrm{ft})\end{array}$ & $100 \times 224 \times 26$ \\
\hline & & Class A mixed storage units & \\
\hline & & Number of structures & 16 \\
\hline & & $\begin{array}{l}\text { Exterior dimensions } \\
(\mathrm{W} \times \mathrm{L} \times \mathrm{H}, \mathrm{ft})\end{array}$ & $104 \times 224 \times 18$ \\
\hline & & $\begin{array}{l}\text { Class } C \alpha \text { alpha and Class } C \alpha \\
\text { mixed storage units }\end{array}$ & \\
\hline & & Number of structures & 3 \\
\hline & & $\begin{array}{l}\text { Exterior dimensions } \\
(\mathrm{W} \times \mathrm{L} \times \mathrm{H}, \mathrm{ft})\end{array}$ & $104 \times 224 \times 18$ \\
\hline & & $\begin{array}{l}\text { Class } B \text { and Class } C \beta-\gamma \text { storage } \\
\text { units }\end{array}$ & \\
\hline & & Number of structures & 2 \\
\hline & & $\begin{array}{l}\text { Exterior dimensions } \\
(\mathrm{W} \times \mathrm{L} \times \mathrm{H}, \mathrm{ft})\end{array}$ & $58 \times 166 \times 31$ \\
\hline & & $\begin{array}{l}\text { Class } B \text { and Class } C \beta-\gamma \text { storage } \\
\text { units }\end{array}$ & \\
\hline & & Number of structures & 1 \\
\hline & & $\begin{array}{l}\text { Exterior dimensions } \\
(\mathrm{W} \times \mathrm{L} \times \mathrm{H}, \mathrm{ft})\end{array}$ & $40 \times 76 \times 32$ \\
\hline
\end{tabular}




\subsection{References}

Anderson, R. T., et al., 1992, "Development of New Low Level Radioactive Waste Disposal Sites: A Progress Report," Waste Management '92, Tucson, AZ, March 1992, pp. 1707-1711.

Anderson, R. T., et al., 1990, "Advanced Disposal Technologies for New Low-Level Waste Disposal Compact Sites," Waste Management '90, Tucson, AZ, March 1990, pp. 179-185.

Bowman, A. L., et. al., 1984, INEL Environmental Characterization Report, EGG-NPR-6688, September 1984.

Case, M. J., S. J. Maheras, M. A. McKenzie-Carter, M. E. Sussman, and P. Voilleque, 1990, Draft Radioactive Waste Management Complex Performance Assessment, EGG-WM-8773, June 1990.

Chevrier, G. and M. Dutzer, 1992, "The Centre de l'Aube Low-Level Waste Disposal Facility," Waste Management '92, Tucson, AZ, March 1992, pp. 1345-1351.

Convert, P., 1992, "Final Closure of the Centre de la Manche Radioactive Waste Disposal Facility," Waste Management '92, Tucson, AZ, March 1992, pp. 1773-1776.

Cook, J. R., et al., 1987, New Low-Level Radioactive Waste Storage/Disposal Facilities at the Savannah River Plant, DPST-85-862, April 1987.

Cook, J. R., 1992, "Radionuclide Limits for Vault Disposal at the Savannah River Site," Waste Management '92, Tucson, AZ, March 1992, pp. 1421-1428.

Darnell, G. R., 1990, "Integrated Treatment and Disposal of Mixed and Low-Level Radioactive Waste," Waste Management '90, Tucson, AZ, March 1990.

Darnell, G. R., R. Shuman, N. Chau, E. A. Jennrich, 1990, "Abovegrade Earth-mounded Concrete Vault-Structural and Radiological Performance," Emerging Technologies for Hazardous Waste Treatment, Atlantic City, NJ, June 4-7, 1990.

Denson, R. H., et al., 1987, Recommendations to the NRC for Review Criteria for Alternative Methods of Low-Level Radioactive Waste Disposal, Task 2a: Below-Ground Vaults, U.S. Army Engineer Waterways Experiment Station, NUREG/CR-5041, Vol. 1, December 1987.

Deold, J. H. and C. E. Coleman, 1991, "The Ncbraska Facility Engineered Barriers, Moisture Collection Systems and Environmental Monitoring," 13th Annual U.S. DOE Low-Level Waste Management Conference, CONF-911114-Proc, November 19-21, 1991, pp. 9-24.

Dodge, R. L., et al., 1989. "Development of a Mixed Waste Management Facility at the Nevada Test Site," Waste Management '89, Tucson, AZ, March 1989.

EG\&G Idaho, Inc., 1987, Conceptual Design Report: Alternative Concepts for Low-Level Radioactive Waste Disposal, DOE/LLE-60T, June 1987.

EPA, 1989, Requirements for Hazardous Waste Landfill Design, Construction, and Closure, prepared for the U.S. EPA by Eastern Research Group, Inc., EPA 625 4-89 022, August 1989. 
Freitag, A. A., et al., 1992, "Concrete Disposal Vaults-An Alternative to Hazardous Waste/Mixed Waste Earthen Landfills," Waste Management 92, Tucson, AZ, March 1992, Vol. 3, pp. 1249-1254.

Gunning, J. E., et al., 1992, "An Above-Grade, Low-Level Radioactive Waste Disposal Facility: The Design and Licensing Status of the Central Interstate Compact Facility," Waste Management '92, Tucson, AZ, March 1992, pp. 1729-1733.

Hillary, J. M., 1992a, Regulatory Siting Requirements for the Proposed Mixed and Low-Level Waste Disposal Facility at the INEL, EGG-WMO-10250, May 1992.

Hillary, J. M., 1992b, Siting Criteria for the Proposed Mixed and Low-Level Waste Disposal Facility at the INEL, EGG-WMO-10440, September 1992.

NRC, 1991a, Standard Format and Content of a License Application for a Low-Level Radioactive Waste Disposal Facility, U.S. Nuclear Regulatory Commission, NUREG-1199, Rev. 2, January 1991.

NRC, 1991b, Standard Review Plan for the Review of a License Application for a Low-Level Radioactive Waste Disposal Facility, U.S. Nuclear Regulatory Commission, NUREG-1200, Revision 2, January 1991.

NRC, 1991c, Recommendations to the NRC for Soil Cover Systems Over Uranium Mill Tailings and Low-Level Radioactive Wastes, U.S. Army Engineer Waterways Experiment Station, NUREG/CR-5432, February 1991.

O'Donnell, E., et al., 1992, "Control of Water Infiltration into Near-Surface LLW Disposal Units-Progress Report on Field Experiments at a Humid Region Site, Beltsville, Maryland," Waste Management '92, Tucson, AZ, March 1992.

Taylor, D. D., 1993, Preliminary Site Selection for the Idaho Waste Processing Facility and the Mixed and Low-Level Waste Treatment Facility at the Idaho National Engineering Laboratory, EGG-WM-10622, 1993.

Taylor, W. P., 1992, Development of High Integrity, Maximum Durability Concrete Structures for LLW Disposal Facilities," Waste Management '92, Tucson, AZ, March 1992, pp. 1479-1483.

Thompson, H. L., Jr. and Porter, J. W., 1987, "Joint NRC-EPA Guidance on a Conceptual Design Approach for Commercial Mixed Low-Level Radioactive and Hazardous Waste Disposal Facilities," letter to the States, Compact Regions, and all NRC licensees, August 3, 1987.

Van Cote, F., 1982, "12 Years of Experience of Shallow Land Disposal of Low and Intermediate Level Radioactive Waste in France," Proceedings of the Symposium on Low-Level Waste Disposal, NUREG/CP-0028, CONF-820911, Vol. 3, September 29-30, 1982, pp. 177-207.

Van Hoesen, S. D. and L. S. Jones, 1991, "Oak Ridge Low-Level Waste Disposal Facility Designs," 13th Annual U.S. DOE Low-Level Waste Management Conference, CONF-911114-Proc, November 19-21, 1991, pp. 25-35. 
Wells, M. N. and L. L. Bailey, 1991, "Mixed Waste Disposal Facilities at the Savannah River Site," 13th Annual U.S. DOE Low-Level Waste Management Conference, CONF-911114-Proc, November 19-21, 1991, pp. 36-48. 


\section{OVERVIEW OF EVALUATIONS}

\subsection{Objective}

The objective of this section is to list the areas in which the alternatives are compared, and to state why these particular areas of comparison were selected.

\subsection{Evaluations Selected}

Seven areas were selected for evaluation. The characteristics of the ideal alternative are discussed in each area, although no one alternative is expected to be top-rated in all seven areas.

\subsubsection{Operability and Isolation Performance Evaluations}

The ideal disposal alternative should provide the capability to (a) handle and emplace all planned types of waste without excessive operational difficulty and (b) isolate the waste for the necessary periods of time after closure.

For each disposal alternative, the evaluations address the constructibility, operability, and maintainability of the handling and emplacement concept. The evaluations also address the use of proven technology. Finally, the alternatives are evaluated qualitatively in terms of expected postclosure performance of the isolation system.

\subsubsection{Evaluation of Relationship to Other INEL Waste Management Facilities}

The ideal disposal alternative should interface with the other INEL waste management facilities with a minimum of redundancies and interface issues. Several waste management facilities already exist at the INEL. Major new facilities are projected in the time period of interest (approximately the years 2004 through 2044).

These evaluations examine the relationship of each disposal alternative to the other current and projected INEL facilities. The intent is to identify potential overlaps, redundancies, and interface problem areas.

\subsubsection{Environmental Evaluations}

The ideal disposal alternative should comply readily with all applicable environmental requirements, presenting as few environmental issues as possible.

These evaluations compare the alternatives on the difficulties expected in achieving compliance with environmental requirements.

\subsubsection{Safety and Health Evaluations}

The ideal disposal alternative should minimize health and safety risks to the workers, the public, and the environment during the construction and operations phases. (The isolation performance 
evaluations discussed in Section 6.2.1 will address the health and safety risks in the postoperations phase.)

These evaluations qualitatively compare the alternatives on the expected radiological and nonradiological risks to the workers, the public, and the environment during the construction and operations phases.

\subsubsection{Institutional Evaluations}

The ideal disposal alternative should be implementable with a minimum of potential disruptions due to institutional problems. Whereas the discussions in Subsection 6.2.2 related to interfaces with other INEL facilities, these discussions relate to interfaces with non-INEL institutions. Examples are the State of Idaho, local labor unions, local citizens' groups, and States through which waste might be shipped for offsite disposal.

These evaluations compare the alternatives on the basis of institutional issues that might arise during implementation.

\subsubsection{Schedule Evaluations}

The ideal disposal alternative should be implementable with a minimum of delay. The projected shutdown and closure of the RWMC is a driving force for prompt implementation of the preferred alternative.

These evaluations compare the dates when the disposal alternatives could be expected to begin operations, as well as uncertainties in those dates.

\subsubsection{Cost Evaluations}

The ideal disposal alternative should be implementable with a minimum of cost over its entire life cycle.

These evaluations compare the ROM life-cycle costs of the disposal alternatives.

\section{3 "Musts" and "Wants"}

In addition to discussing the performance of each alternative in the areas listed in Section 6.2, the performance will be specifically evaluated against a list of "musts" and "wants."

To be viable, an alternative must comply with every listed "must."

Alternatives are also evaluated on the extent to which they satisfy the "wants." Each alternative will be scored on each "want." Each "want" will be weighted in importance. For each alternative, the scores on the various "wants" will be multiplied by the importance weights of the respective "wants," then summed.

Table 6-1 lists the "musts" and "wants" used in the study. The section of this report in which each "must" and "want" is addressed is also stated in the table. 
Table 6-1. "Musts" and "wants" criteria for recommending an alternative.

"Musts"-To be worthy of recommendation, an alternative must be judged to satisfy all of the following "go/no go" criteria:

1. Comply with all environmental and waste management regulatory requirements, including the requirements for long-term isolation (Sections 7 and 9)

2. Present acceptable risk to the workers, the public, and the environment during the construction and operations phases (Section 10)

3. Over a period of 40 years, provide all required disposal of LLW and mixed waste generated or currently stored at the INEL, after any necessary treatment has been performed (Section 7)

4. Present no potential major conflicts with the missions or operations of other existing or planned INEL waste management facilities (Section 8)

5. Present no insurmountable institutional problems (Section 11).

"Wants"-Alternatives are also compared on how well they satisfy the following criteria:

1. Minimize risk to the workers, the public, and the environment during the construction and operations phases (Section 10)

2. Provide maximum isolation of the waste after the operations period (Section 7)

3. Minimize problems in constructibility, operability, reliability, and maintainability (Section 7)

4. Minimize the development risk associated with the isolation system technologies (Section 7)

5. Minimize the schedule for beginning disposal operations (Section 12)

6. Minimize the life-cycle cost (Section 13)

7. Minimize potential interface problems with other INEL waste management facilities (Section 8)

8. Minimize potential institutional prob'ems (Section 11)

9. Provide ready retrievability of waste during the operations phase (Section 7)

10. Allow for easy monitoring of isolation integrity and performance during the operations and institutional control phases (Section 7). 


\section{FACILITY AND PERFORMANCE EVALUATIONS}

\subsection{Introduction}

This section evaluates the alternatives on (a) the ability to dispose of all waste such that regulatory requirements are met, (b) the developmental risk associated with the technologies chosen for disposal, (c) constructability, operability, reliability, and maintainability, including an assessment of the ability to monitor isolation integrity and performance and to retrieve the waste if necessary, and (d) the ability to provide long-term isolation. The results of the evaluation are a determination of which alternatives comply with the "musts" and, therefore, are viable, and a relative ranking of the alternatives against a list of "wants." The approaches used for items (a) through (d) are summarized bricfly in the following paragrapiss.

The ability of each alternative to dispose of all wastes in accordance with regulations is evaluated. This part of the evaluation is based on the assumption that the disposal technologies will perform as expected.

Each alternative is evaluated to determine the risk of developing each technology to the point where it can be used. The evaluation is based on comparisons with technologies currently in use.

The alternatives are ranked, relative to each other, on the basis of constructability, operability, reliability, and maintainability. Because of the lack of operations data, reliability, availability, and maintainability (RAM) analyses, and the limited use of some of the chosen disposal technologies in radiological environments, the ranking of alternatives is limited to relative observations based on facility walk-throughs at other sites, limited site and technology-specific information, and engineering judgement. Facility walk-throughs included disposal areas at the Oak Ridge National Laboratory (ORNL), the Savannah River Site (SRS), and the Chemical Waste Management facility at Emmelle, Alabama. At ORNL, the "tumulus," or covered module, disposal sites (similar to Subalternative 1C) were viewed. At SRS, the low- and intermediate-activity vaults (similar to Subalternative 1B) were seen. At the Chemical Waste Management facility, a large site for commercial disposal of hazardous waste in lined shallow-land burial pits was toured.

The relative capabilities of the alternatives to provide long-term isolation of the waste are evaluated by means of a qualitative performance assessment.

\subsection{Requirements and Criteria}

\subsubsection{Disposal Standards}

The primary criterion for evaluation of an alternative is that the alternative dispose of all LLW and mixed waste and while complying with the various environmental and waste management regulations discussed in Section 2.3.

For disposal of LLW, the key element of the regulation is that isolation performance objectives must be met. Additional requirements are specilied to ensure that the performance objectives are 
met. The additional requirements address such items as waste form, waste containers, surface water drainage, stability, cover design, closure, postclosure, and environmental monitoring.

For the disposal of mixed waste, concentration and/or treatment limits are also set to ensure that groundwater is protected. Requirements are also included that cover the design and operation, monitoring and inspection, and closure and postclosure.

It was stated above that the disposal of all the waste is one of the criteria. In Section III.3.h(4) of DOE Order 5820.2A, it states that it is acceptable to store the waste to allow for nuclide decay or until disposal by approved methods is available. However, perpetual storage of waste, with no intent to dispose of it, is not listed as a possible reason for storage.

\subsubsection{Development Risk}

There are no specific regulatory requirements related to development risk.

Each alternative is evaluated to determine the technical risk of developing the confinement system, including the cover, the liner/leachate collection system, and the balance of the engineered barriers including the waste form, waste container, and structure (if applicable). The evaluation is based on comparisons between alternatives and on comparisons with similar technologies in current development or use. A technology that is essentially identical to a currently operational technology is considered to have no developmental risk. A technology that has been tested only at pilot scale or that involves a simple extension or combination of tested/operational technologies is considered to be a small risk. A technology that has as its base a known technology but that involves major nontested design modifications is considered a moderate risk. A technology that has never been tested is considered a major developmental risk.

\subsubsection{Constructability, Operability, Reliability, and Maintainability}

Each alternative is judged relative to the others on its constructability, operability, reliability and maintainability. For this cvaluation, constructability is defined as the relative ease of construction of the disposal system. Operability is defined as the ability to emplace waste without damaging isolation barriers. Operability also includes an assessment of the ease of monitoring of confinement integrity and isolation performance during both the operational and institutional control phases. Additionally, an assessment of the ease of retrievability during operations is included. It is not intended that the waste be retrieved; however, if a failure should occur, retrieval might be necessary. Reliability is defined as the freedom from unscheduled work outages, that is, periods when the waste cannot be accepted for disposal. [Reliability of isolation (i.c., performance) is evaluated in a separate section.] Maintainability is defined as the degree of freedom from, and the relative ease of, maintenance.

\subsubsection{Isolation Performance}

Long-term performance criteria, in the form of exposure and release limits, are specified in DOE Order 5820.2A and in 10 CFR 61 (sce Section 2.3).

To quantitatively evaluate an alternative against these specific performance criteria is a complex task requiring data not available at the present stage of the project. Radionuclide inventory data are 
required. Extensive data on the subsurlace environment are needed for contaminant transport calculations. In lieu of quantitative evaluation of each alternative against the performance objectives, each alternative is qualitatively assessed relative to the others on its expected ability to provide longterm isolation. This qualitative comparison of the alternatives is made based on the perceived effectiveness of the engineered barriers and other design features associated with each alternative. The effects of waste form, waste container, disposal technology (including liners, structures, and depth of disposal), cover design, and interactions between these parameters are considered.

\subsection{Evaluations of Alternatives Against Requiremenis/Criteria}

\subsubsection{Disposal Standards and the Ability to Dispose of All Waste}

The alternatives were developed based on the assumption that the disposal concept will have to meet the requirements defined in DOE 5820.2A and 10 CFR 61. The alternatives were also developeci to meet the various design criteria for controlling and monitoring water infiltration and leachate migration found in the DOE and NRC documents as well as in RCRA. It is assumed, therefore, that the designs lor Alternatives 1 through 4 can be implemented so as to meet all the regulatory requirements. Alternative 5, No Action, which involves indefinite storage of waste, does not meet the DOE requirement that discourages indefinite storage (as opposed to disposal) of LLW or mixed waste.

While Alternative 2, Continued Disposal at the RWMC, meets the requirements for the secenario of maximum treatment/maximum generation, the use of available space is nearly at a maximum. If Alternative 2 were implemented for the scenario of maximum generation/minimum treatment, all of the waste could not be disposed of at the RWMC.

\subsubsection{Developmental Risk}

For this evaluation, cach alternative is subdivided into three component technologies: the cover, the liner/leachate collection system, and the balance of the engineered barriers including waste form, waste container, and structure (if applicable). An evaluation of the developmental risk for cach component is presented below. A summary of the analysis is presented in Table 7-1.

7.3.2.1 Cover. The cover design is similar for all alternatives except Alternative 2 and Alternative 5. The bulk of the allernatives all use a design that is a modification/combination of the design features that have been tested at pilot scale at the Los Alannos National Laboratory or full scale at facilities such as the hazardous waste disposal facility at Emmelle, Alabama. There is a small risk associated with the cover design because the combination of features has never been tested.

Alternative 2 involves the same basic design, but, because it is necessary to design for interactions with other covers that may be constructed as a result of environmental restoration at the RWMC, the Alternative 2 cover is eonsidered to have additional risk compared to the other designs, and is given the classification of moderate developmental risk. A portion of Alternative $4 B$, which is a combination of Alternatives 2 and 3 also has a moderate risk. 
Table 7-1. Developmental risk summary.

\begin{tabular}{|c|c|c|c|c|c|c|c|c|c|c|c|c|}
\hline \multirow[b]{2}{*}{ Alternative } & \multicolumn{4}{|c|}{ Cover } & \multicolumn{4}{|c|}{ Liner/leachate system } & \multicolumn{4}{|c|}{ Balance of barriers } \\
\hline & None & Small & Moderate & Major & None & Small & Moderate & Major & None & Small & Moderate & Major \\
\hline $1 \mathrm{~A}$ & - & $x$ & - & - & - & - & $\mathrm{X}$ & - & - & - & $\mathrm{x}$ & - \\
\hline $1 \mathrm{~B}$ & - & $\mathrm{x}$ & - & - & - & - & $\mathrm{x}$ & - & - & - & $\mathrm{x}$ & - \\
\hline $1 \mathrm{C}$ & - & $\mathrm{X}$ & - & - & - & $\mathrm{X}$ & - & - & $\mathrm{X}$ & - & _- & - \\
\hline $1 \mathrm{D}$ & - & $\mathrm{X}$ & - & - & $\mathrm{x}$ & - & - & - & $\mathrm{X}$ & - & - & - \\
\hline $1 \mathrm{E}$ & - & $\mathrm{x}$ & - & - & $\mathrm{x}$ & - & - & - & $\mathrm{X}$ & - & - & - \\
\hline $1 \mathrm{~F}$ & - & $\mathrm{x}$ & - & - & $\mathbf{P}$ & - & $\mathbf{P}$ & - & $\mathbf{P}$ & - & $\mathbf{P}$ & - \\
\hline $1 \mathrm{G}$ & - & $x$ & - & - & - & - & $\mathrm{X}$ & - & - & - & $x$ & - \\
\hline $1 \mathrm{H}$ & - & $x$ & - & - & - & - & $\mathrm{X}$ & - & - & - & $x$ & - \\
\hline 2 & - & - & $\mathrm{x}$ & - & - & $\mathrm{x}$ & - & - & $\mathrm{x}$ & - & - & - \\
\hline 3 & - & $\mathrm{x}$ & - & - & $\mathrm{x}$ & - & - & - & $\mathbf{x}$ & - & - & - \\
\hline $4 \mathrm{~A}$ & - & $x$ & - & - & $\mathbf{P}$ & - & $\mathbf{P}$ & - & $\mathbf{P}$ & - & $\mathbf{P}$ & - \\
\hline 4B & - & $\mathbf{P}$ & $\mathbf{P}$ & - & $\mathbf{P}$ & $\mathbf{P}$ & - & - & $x$ & - & - & - \\
\hline 5 & $\mathrm{x}$ & - & - & - & $\mathrm{x}$ & - & - & - & $\mathrm{x}$ & - & - & - \\
\hline
\end{tabular}

$\mathrm{X}$. Indicates that all of the associated technologies used incur the same level of developmental risk.

P. Indicates that a portion of the associated technologies used incur one level of the developmental risk, while the balance incurs a different level. 
Alternative 5 has no cover, su no developmental risk is associated with the cover. (Developmental risk must not be confused with isolation performance. As stated in Section 7.3.4, the performance of Alternative 5 will be greatly impaired by the lack of a cover.)

7.3.2.2 Uner/leachate System. The liner/leachate system associated with the shallow-land burial and greater-depth disposal concepts is essentially identical to that which has been successfully used for hazardous waste disposal at locations such as Emmelle, Alabama. Therefore, no developmental risk is associated with Alternatives $1 \mathrm{D}, 1 \mathrm{E}, 3$, and a portion of $1 \mathrm{~F}, 4 \mathrm{~A}$, and $4 \mathrm{~B}$. The liner/leachate systems that are placed beneath the pads in Alternatives $1 \mathrm{C}, 2,5$, and a portion of $4 \mathrm{~B}$ are minor modifications to the liner system that has been successfully used at ORNL for the past several years. The development risk is expected to be small. The liner/leachate systems used in the vault alternatives are expected to be a greater risk because successful use has not yet been proven in operation. However, since the components of the system are similar to those used in the other liner/leachate systems, the developmental risk assigned is moderate and not major. The alternatives affected are $1 \mathrm{~A}, 1 \mathrm{~B}, 1 \mathrm{G}, 1 \mathrm{H}$, and portions of $1 \mathrm{~F}$ and $4 \mathrm{~A}$.

7.3.2.3 Additional Engineered Barriers. The basic waste form and containers are the same for all the alternatives with the exception of $1 \mathrm{H}$. Alternative $1 \mathrm{H}$ does not treat nonmixed $\mathrm{LLW}$ as do the other options; however, the nonmixed waste forms used in the balance of the options have all been created previously, so there is no relative developmental risk. Therefore, there is no relative difference in developmental risk among any of the alternatives with respect to waste form and waste containers, and these items are not evaluated.

The structures that house the waste are varied. For Alternative 5, the structure is a common Butler-type building that has no development risk. For the shallow-land and greater-depth borehole alternatives (1D, 1E, 3, and portions of $1 \mathrm{~F}, 2,4 \mathrm{~A}$, and $4 \mathrm{~B}$ ), there is no structure, so there is no associated development risk. (Development risk and isolation performance should not be confused.) For Alternative $1 \mathrm{C}$ and a portion of Alternatives 2 and $4 \mathrm{~B}$, the pad structure and the concrete modules are essentially the same as those used at ORNL, so there is expected to be no developmental risk. The options involving vaults are expected to involve a moderate development risk because of the extrapolations in size and roof support structure from the French designs that have been in use for several years. Thus, Alternatives 1A, 1B, 1G, and $1 \mathrm{H}$ and portions of Alternatives $1 \mathrm{~F}$ and $4 \mathrm{~A}$ have a moderate development risk.

\subsubsection{Relative Constructability, Operability, Reliability, and Maintainability}

The evaluation of relative constructability, operability, reliability, and naaintainability for the disposal alternatives is based on experience with similar facilities. For example, shallow-land burial has been used for many years for the disposal of various types of waste. Disposal in vaults and covered modules has been used for many years in France. Forklifts are commonly used in disposal operations, and cranes are sometimes used, particularly for highly radioactive waste. Hazardous waste has been disposed of in large pits with liner/leachate systems. The experience from similar facilities, combined with engineering judgement, was used to make the following evaluations.

7.3.3.1 Constructability. The basic disposal construction units are lined pits, both empty and backfilled (this includes the partial pit of Alternative 2), lined vaults, concrete pads, and Butler-type buildings. The construction of Butler buildings is a standard industry practice and is considered to 
be a relatively simple operation. The construction of cement pads is considered to be of small difficulty due to pad thickness (the concrete pad of Alternative 5 is not so thick as that of Alternatives $1 \mathrm{C}, 2$, or $4 \mathrm{~B}$ and is considered easy to construct). The construction of pits (Alternatives $1 \mathrm{~B}, 1 \mathrm{E}$, and 3 and portions of Alternatives $1 \mathrm{~F}, 2,4 \mathrm{~A}$, and $4 \mathrm{~B}$ ) is considered to be of small difficulty, due primarily to the installation of the liner. The deeper pit of Alternative $1 \mathrm{D}$ is considered slightly more difficult than the shallow pits and is classified as moderately difficult to construct. Construction of concrete vaults (either type) for Alternatives $1 \mathrm{~A}, 1 \mathrm{~B}, 1 \mathrm{G}$, and $1 \mathrm{H}$ and portions of $1 \mathrm{~F}, 4 \mathrm{~A}$, and 5 is considered to be a task of major difficulty. The types of construction units and the relative severity of the construction tasks are summarized in Table 7-2.

7.3.3.2 Operability. Each alternative is assessed in terms of its ability to be operated without damaging the liner system, the relative ease of monitoring, and the relative ease of retrieving the waste should such an action become necessary. A summary of the relative difficulty of operation of the alternatives is shown in Table 7-3.

Each alternative involves placement of the bulk of the waste using forklifts and placement of the balance of the waste using a boom or gantry cranc. As a result, the basic placement operations are

Table 7-2. Relative difficulty of construction tasks.

\begin{tabular}{|c|c|c|c|c|}
\hline Alternative & $\begin{array}{l}\text { Lined or } \\
\text { backfilled pits }\end{array}$ & Vaults & Pads & $\begin{array}{c}\text { Butler } \\
\text { buildings }\end{array}$ \\
\hline $1 \mathrm{~A}$ & N/A & Major & N/A & N/A \\
\hline $1 \mathrm{~B}$ & Small & Major & N/A & N/A \\
\hline $1 \mathrm{C}$ & N/A & N/A & Small & Easy \\
\hline $1 \mathrm{D}$ & Moderate & N/A & N/A & N/A \\
\hline $1 \mathrm{E}$ & Small & $\mathrm{N} / \mathrm{A}$ & $\mathrm{N} / \mathrm{A}$ & N/A \\
\hline $1 \mathrm{~F}$ & Small & Major & N/A & N/A \\
\hline $1 \mathrm{G}$ & $\mathrm{N} / \mathrm{A}$ & Major & N/A & N/A \\
\hline $1 \mathrm{H}$ & N/A & Major & N/A & N/A \\
\hline 2 & Small & $\mathrm{N} / \mathrm{A}$ & Small & Easy \\
\hline 3 & Small & N/A & N/A & N/A \\
\hline $4 \mathrm{~A}$ & Small & Major & N/A & N/A \\
\hline 4B & Small & $N / A$ & Small & Easy \\
\hline 5 & N/A & Major & Easy & Easy \\
\hline
\end{tabular}


Table 7-3. Relative difficulty of operation.

\begin{tabular}{|c|c|c|c|c|c|c|c|c|c|c|}
\hline \multirow[b]{2}{*}{ Alternative } & \multicolumn{3}{|c|}{ Seriousness of liner damage } & \multicolumn{3}{|c|}{ Difficulty of monitoring } & \multicolumn{4}{|c|}{ Difficulty of retrieval } \\
\hline & Very low & Small & Moderate & Very low & Small & Moderate & Very low & Small & Moderate & Major \\
\hline $1 \mathrm{~A}$ & - & $\mathbf{P}$ & $\mathbf{P}$ & - & - & $x$ & - & $\mathbf{P}$ & $\mathbf{P}$ & - \\
\hline 1B & - & $P$ & $\mathbf{P}$ & - & - & $\mathrm{X}$ & - & $\mathbf{P}$ & $\mathbf{P}$ & - \\
\hline $1 \mathrm{C}$ & $\mathrm{X}$ & - & - & - & $\mathbf{P}$ & $\mathbf{P}$ & - & $\mathrm{X}$ & - & - \\
\hline $1 \mathrm{D}$ & $\mathrm{P}$ & - & $\mathbf{P}$ & $\mathrm{X}$ & - & - & - & - & - & $\mathrm{X}$ \\
\hline $1 \mathrm{E}$ & $\mathbf{P}$ & - & $\mathbf{P}$ & $\mathrm{X}$ & - & - & - & - & - & $\mathrm{X}$ \\
\hline $1 \mathrm{~F}$ & - & - & $\mathrm{X}$ & $\mathbf{P}$ & - & $\mathbf{P}$ & - & - & $\mathbf{P}$ & $\mathbf{P}$ \\
\hline $1 \mathrm{G}$ & - & $\mathbf{P}$ & $\mathbf{P}$ & - & - & $\mathrm{X}$ & - & $\mathbf{P}$ & $\mathbf{F}$ & - \\
\hline $1 \mathrm{H}$ & - & $\mathbf{P}$ & $\mathbf{P}$ & - & - & $X$ & - & $\mathbf{P}$ & $\mathbf{P}$ & - \\
\hline 2 & $X$ & - & - & $\mathbf{P}$ & $\mathbf{P}$ & $\mathbf{P}$ & - & $\mathbf{P}$ & $\mathbf{P}$ & - \\
\hline 3 & $P$ & - & $\mathbf{P}$ & $X$ & - & - & - & - & - & $X$ \\
\hline $4 \mathrm{~A}$ & $\mathbf{P}$ & $\mathbf{P}$ & $\mathbf{P}$ & $\mathbf{P}$ & - & $\mathbf{P}$ & - & $\mathbf{P}$ & $\mathbf{P}$ & $\mathbf{P}$ \\
\hline $4 B$ & $\mathbf{P}$ & - & $\mathbf{P}$ & $P$ & $\mathbf{P}$ & $\mathbf{P}$ & - & $\mathbf{P}$ & $P$ & $P$ \\
\hline 5 & $\mathbf{P}$ & - & $\mathbf{P}$ & - & $\mathbf{P}$ & $\mathbf{P}$ & $\mathbf{P}$ & - & $\mathbf{P}$ & - \\
\hline
\end{tabular}

$\mathrm{X}$. Indicates that all of the associated technologies used incur the same level of developmental risk.

P. Indicates that a portion of the associated technologies used incur one level of developmental risk, while the balance incurs a different level. 
essentially the same for all the alternatives. The difference in operability is not found in these basic operations, but rather in the potential for a mistake that causes damage to the most vulnerable member of the confinement system, the liner.

The least potential for damage is in those alternatives that place waste on a pad or that place waste in a borehole that is backfilled before the placement of waste. In both of these cases, the liner is protected from the heavy equipment used to place the waste. In the case of the pad alternatives (1C and a portion of $2,4 \mathrm{~B}$, and 5 ), the concrete pad protects the liner. In the case of the backfilled boreholes (portions of 1D, 1E, 2, 3, 4A, and 4B), the dirt backfill protects the liner.

The alternatives that involve placement of waste in a vault using a forklift (portions of $1 \mathrm{~A}, 1 \mathrm{~B}$, $1 \mathrm{G}, 1 \mathrm{H}$, and $4 \mathrm{~A}$ ) involve the greatest potential for liner damage associated with accidentally running a forklift tine or the corner of a waste box through the liner. While the potential for damage is high, the repair should be simple since there is easy access to the damaged area, and since the damage will be detected easily. Because of the ease of repair, this is considered to be only a small operational problem.

The alternatives that involve placement of waste in a pit (excluding Alternative 1B, which is really placement in a vault for purposes of this discussion) all involve a moderate potential for damage to the liner. The damage could occur when placing the interim cover (backfill) over the waste. The damage is from the heavy equipment used to place the fill material over the waste. Repair of such damage is more difficult than for the vault options because the fill material must be removed to get at the damage. Therefore, alternatives that involve placement of waste in a pit (portions of 1D, 1E, $1 F, 3,4 A$, and $4 B$ ) are considered moderately difficult to operate.

The placement of waste in vaults using cranes is also a hazard. Dropping a waste container could result in significant liner damage. The potential for dropping the waste is low, but the repair is difficult due to the radiation levels of the waste. The waste cell, and perhaps adjacent cells, would have to be emptied to allow repair. The low probability of the event combined with extremely difficult repair results in a moderate operational problem for portions of Alternatives $1 \mathrm{~A}, 1 \mathrm{~B}, 1 \mathrm{~F}, 1 \mathrm{G}$, $1 \mathrm{H}, 4 \mathrm{~A}, 4 \mathrm{~B}$, and 5 .

Another area of interest with respect to operability is the relative ease of monitoring of confinement integrity and isolation performance. Monitoring confinement integrity involves monitoring the status of the confinement barriers, while monitoring isolation performance involves checking the dual/liner leachate collection system sumps and various air and water monitoring stations. Monitoring the integrity of the vaults (Alternatives $1 \mathrm{~A}, 1 \mathrm{~B}, 1 \mathrm{G}, 1 \mathrm{H}$, and portions of 1F, 4A, and 5) is the most difficult. Monitoring the vaults involves various status checks of the concrete, for example, visual checks for cracking and monitoring of strain gauge readings. The number of vaults, and the volume of concrete, makes the task moderately difficult compared to the monitoring involved in the nonvault alternatives. Monitoring of the integrity of the concrete pads of Alternatives $1 \mathrm{C}$ and portions of 2, 4B, and 5 is considered small in difficulty (far less concrete than in the alternatives using vaults). Monitoring the integrity of pits (Alternatives $1 \mathrm{D}, 1 \mathrm{E}, 3$, and portions of $1 \mathrm{~F}, 2,4 \mathrm{~A}$, and $4 \mathrm{~B}$ ) is performed by monitoring the dual/liner leachate system sumps. Monitoring of the sumps is considered as part of monitoring isolation performance, which is discussed in the following paragraphs. 
Monitoring the isolation performance involves sampling leachate in dual liner/leachate system sumps and sampling air and water at various locations on and adjacent to the site. The air and water sampling is essentially the same for all the alternatives. The differences in monitoring difficulty arise from the number of dual liner/leachate sumps that need to be sampled. The easiest alternatives to monitor are those involving large pits. The pits have just one or two sumps requiring monitoring. (Alternative 1B is an exception; it is in a large pit, but each vault has its own sump.) Alternatives in the easy category include $1 \mathrm{D}, 1 \mathrm{E}, 3$, and portions of $1 \mathrm{~F}, 2,4 \mathrm{~A}$, and $4 \mathrm{~B}$.

The remainder of the Alternatives (1A, 1B, 1C, 1G, 1H, 5, and portions of 1F, 2, 4A, and 4B) involve numerous sumps. Each separate vault, building, or portion of cement pad has its own sump. Therefore, these alternatives are moderately difficult to monitor.

A final area of concern relative to operations is the retrievability of the waste during the operational period. Waste stored in the Butler buildings of Alternative 5 is the easiest to retrieve because the aisle left to allow inspection can be easily negotiated by a forklift.

The alternatives using side-loaded vaults (portions of $1 \mathrm{~A}, 1 \mathrm{~B}, 1 \mathrm{G}, 1 \mathrm{H}$, and $4 \mathrm{~A}$ ) or concrete pads (1C and portions of 2 and 4B) pose only a small difficulty in retrieval since access is fairly simple and since forklifts can be used to move the waste. The pads are a little easier because the side of the Butler building is easily breached. The concrete vault, on the other hand, can be entered only from the side. Neither alternative is as simple as Alternative 5 because there are no aisles.

Waste retrieval from alternatives using top-loaded vaults (portions of $1 \mathrm{~A}, 1 \mathrm{~B}, 1 \mathrm{~F}, 1 \mathrm{G}, 1 \mathrm{H}, 4 \mathrm{~A}$, and 5) is moderately difficult. This is because access is impaired by the temporary roof and the movable shielding and because a gantry crane must be used to retrieve the waste. Waste retrieval from the lined boreholes of Alternatives 2 and $4 \mathrm{~B}$ is also considered moderately difficult because of the individual concrete tubes around each set of waste packages.

Waste retrieval from alternatives where the waste is buried is a major difficulty. This applies to Alternatives $1 \mathrm{D}, 1 \mathrm{E}$, and 3 and portions of $1 \mathrm{~F}, 4 \mathrm{~A}$, and $4 \mathrm{~B}$.

7.3.3.3 Reliability. Reliability is defined as the freedom from unscheduled outages. Outages are expected from extreme weather or equipment failures. Extreme weather will not affect gradelevel vault or pad disposal or building storage alternatives, that is portions of Alternatives $1 \mathrm{~A}, 1 \mathrm{C}, 1 \mathrm{G}$, $1 \mathrm{H}, 2,4 \mathrm{~A}$, and $4 \mathrm{~B}$, and 5 .

Heavy precipitation will prevent use of pit options due to wet ramps and floors. This will affect Alternatives 1B, 1D, 1E, and 3 and portions of Alternatives 1F, 4A, and 4B. The mounded pit portion of Alternative 2 also will be affected.

High winds or heavy rain will prevent operations of crane emplacement alternatives. This will affect portions of all alternatives. Therefore, there is no relative difference for this effect.

7.3.3.4 Maintainability. Maintenance is related to the quantity and type of equipment and structures to be maintained. Most options have the same equipment. The differences are the type of crane and the number of pumps. A summary of the features requiring different levels of maintenance is shown in Table 7-4. 
Table 7-4. Summary of ficatures requiring different levels of maintenance.

\begin{tabular}{clll}
\hline Alternative & \multicolumn{1}{c}{ Crane used } & $\begin{array}{c}\text { Extra pumping depth } \\
\text { and large water } \\
\text { collection area }\end{array}$ & $\begin{array}{c}\text { Use of } \\
\text { offsite } \\
\text { transport }\end{array}$ \\
\hline 1A & Gantry & No & No \\
1B & Gantry & Yes & No \\
1C & Boom & No & No \\
1D & Boom & Yes & No \\
1E & Boom & Yes-Deep & No \\
1F & Boom and gantry & Yes & No \\
1G & Gantry & No & No \\
1H & Gantry & No & No \\
2 & Boom & Yes-Shallow & No \\
3 & Boom & Yes & Yes \\
$4 \mathrm{~A}$ & Boom and gantry & Yes & Yes \\
$4 B$ & Boom & Yes & Yes \\
5 & Gantry & No & No \\
\hline
\end{tabular}

Gantry cranes are more complex than boom cranes and require more maintenance. Therefore, the crane portions of Alternatives $1 \mathrm{~A}, 1 \mathrm{~B}, 1 \mathrm{~F}, 1 \mathrm{G}, 1 \mathrm{H}$, and $4 \mathrm{~A}$ all involve more maintenance.

The alternatives with numerous sumps for collection of leachate use low-volume pumps that are not run continuously. These pumps are a low-maintenance item, and are not expected to be a problem. On the other hand, the alternatives that involve an extra high-volume pump to move water out of a pit and also involve large exposed areas where pumping must occur (Alternatives 1B, 1D, $1 \mathrm{E}, 1 \mathrm{~F}, 2,3,4 \mathrm{~A}$, and $4 \mathrm{~B}$ ) will require more maintenance. Alternative 2 is considered in this category because of the back-filled mounded pit.

Alternatives that involve offsite shipping require greater maintenance for the fleet of transportation vehicles. Offsite shipping is required for Alternatives 3, 4A, and 4B.

\subsubsection{Isolation Performance}

The key regulatory requirements related to isolation performance are the performance objectives. The performance objectives establish the minimum requirements for the protection of human health and safely and the environment. The objectives include specific dose limits and groundwater protection standards. The concept behind the objectives is to limit the contact of humans, animals, and plants with radioactive material, thus limiting the risk associated with the disposal of that 
material. The limiting of contact is accomplished by limiting contact with waste at the disposal site, and by limiting the release of contaminants in the waste that can travel away from the site. Along with inadvertent intrusion, the water pathway is the transport mechanism of primary concern for a reasonably designed site. (Windblown transport of radioactivity should not occur for any of the alternatives considered).

Quantitative evaluation of the dose received from contact at the site (the inadvertent intruder) and contact away from the site (ingestion of water-borne contaminants) is a complex task requiring detailcd information on both site characteristics and disposal technology. Such a quantitative analysis is not feasible at this stage of the project. However, a qualitative analysis, based on the relative ability of each alternative to prevent intrusion and to prevent water from coming into contact with and leaching contaminants from the waste, can be performed.

The approach for the qualitative evaluation is to consider and compare the various barriers to human and water contact provided by each alternative. The comparisons are done for a "long-term" period, defined as the first 500 years, and for a "very long-term" period, a period far into the future (i.e., tens of thousands of years or more) (see Sections 7.3.4.1 and 7.3.4.2, respectively). The logic for this approach is that, in the long-term period, exposure to any of the radionuclides disposed of can potentially occur; whereas, in the very long-term period, exposure is only to the "long-lived" radionuclides, those with half-lives on the order of 100 s of years or more. Generally speaking, the Class $C \alpha$ waste (whether mixed or not) is the only waste of concern beyond the 500 -year period. The other classes of waste will have decayed to the point where it is difficult to determine the difference in radiation exposure between that due to the remaining radioactivity in the waste and that due to natural radioactivity in the geologic media.

7.3.4.1 Long-term Performance. The qualitative comparisons are based on the isolation barriers provided in each alternative, as well as the comparative radionuclide inventory. The radionuclide inventory is the same for all alternatives except for Subalternative 1G. That subalternative represents the minimum-generation scenario and would have a smaller radionuclide inventory than the other alternatives. The pretreatment volume difference is a factor of approximately 3 . The radionuclide inventory difference could be greater than or less than a factor of 3 .

The barriers to human contact are the waste form, container, disposal structure, cover, and geologic media. The barriers to water contact/leaching include those for human contact and also liners. (Note that the leachate collection and removal system is assumed to be removed from service after the institutional control period and is therefore not a significant factor).

The waste forms are identical for all alternatives with the exception of Subalternative $1 \mathrm{H}$, which contains untreated Class A LLW. The containers are the same for all alternatives if the concrete modules of Subalternative $1 \mathrm{C}$ are considered part of the structure. Furthermore, waste containers are generally not expected to provide a significant isolation barrier by themselves for times longer than a few decades.

The structures and geologic barriers vary significantly from option to option. The cover is much the same from alternative to alternative with the exception of the thickness. The liners are, in 
principle, much the same from one alternative to another, and, in the absence of specific performance data, are assumed to perform the same.

It is assumed that the geologic media for the offsite disposal location provide equivalent isolation to that provided by the geologic media at the INEL disposal location.

In the long-term performance comparisons, all the barriers designed into the alternatives are assumed to be suitably designed and constructed and to perform their intended functions. The relative performance of the alternatives then depends on the total number and effectiveness of the barriers.

The number and effectiveness of the barriers for each alternative are summarized in Table 7-5. Containers and liners are not included because these are the same for all alternatives. Waste form is also not included because the only difference is as noted above for Subalternative $1 \mathrm{H}$. (This difference is considered in the evaluation later in this section.)

7.3.4.2 Very Long-term Performance. The evaluation is based on the barriers expected still to be present far into the future. At that time, only geologic barriers and waste-form barriers are considered to be effective. All engineered barriers, with the exception of waste form, are assumed to have lost their effectiveness. It is possible that the concrete barriers, covers, etc., might maintain some resistance, but their worth likely will have been severely compromised due to cracking, settling, erosion, and the general degradation associated with the passage of time. Geologic barriers are assumed to be able to withstand the passage of time (at least on the scale of long-lived radionuclides). It is also assumed that the iron-enriched basalt (IEB) waste form, which is an analog of the natural basalt found in the region, will resist degradation over the very long-term period. The number and quality (or effectiveness) of the barriers for each alternative are summarized in Table 7-6.

As stated previously, at these times far in the future, all radionuclides except the long-lived radionuclides in Class $\mathrm{C} \alpha$ waste (whether mixed or not) are assumed to have decayed to negligible levels, by comparison. Therefore, Class $\mathrm{C} \alpha$ is the only waste type listed in Table 7-6.

All of the Class $\mathrm{C} \alpha$ waste (whether mixed or not) for all of the alternatives is in an IEB form. The geologic barriers are of three types. The first, abovegrade location, is considered a low-quality barrier. The assumption is that, after a sufficiently long period, the IEB waste will be lying exposed at the surface. Surface water contact and direct human contact will be possible. The second type of geologic barrier is shallow burial. Shallow burial should be sufficient to prevent human contact, with the exception of drilling, and will provide significantly better protection against water contact compared with the abovegrade case. The final type of geologic barrier is greater depth burial. The protection against human contact is slightly improved over shallow burial, with the contact from drilling remaining the major concern. Contact with water is reduced significantly, compared with the abovegrade case, and is better than for the shallow burial case. 
Table 7-5. Number and effectiveness of barriers over the long term.

\begin{tabular}{|c|c|c|c|c|c|}
\hline \multirow[b]{2}{*}{ Alternative } & \multirow[b]{2}{*}{ Waste type } & \multicolumn{3}{|c|}{ Barriers } & \multirow[b]{2}{*}{ Comments } \\
\hline & & Structure & Cover ${ }^{4}$ & Geologic & \\
\hline $1 \mathrm{~A}$ & All & AGES & $11 \mathrm{ft}$ thick & Abovegrade & 3 ft thick concrete \\
\hline $1 B$ & All & BCil:S & 15 ft thick $^{b}$ & Shallow burial & 3 ft thick concrete \\
\hline $1 \mathrm{C}$ & All & $\mathrm{CM}$ & $11 \mathrm{ft}$ thick & Abovegrade & 6 in. thick concrete module \\
\hline \multirow[t]{2}{*}{$1 \mathrm{D}$} & A. $C *$ & GDB & 11 ft thick & Greater-depth burial & - \\
\hline & $B, C \beta-\gamma$ & GDBH & 11 ft thick & Greater-depth burial & - \\
\hline \multirow[t]{2}{*}{ IE } & A. $\mathrm{C} \alpha$ & SLBB & 11 ft thick $^{c}$ & Shallow burial & - \\
\hline & $B, C \beta-\gamma$ & SBH & $11 \mathrm{ft} \mathrm{thick}^{\mathrm{c}}$ & Shallow burial & - \\
\hline \multirow[t]{2}{*}{$1 F$} & A & SLBB & 11 ft thick & Shallow burial & - \\
\hline & $\mathrm{B}, \mathrm{C}$ & AGES & $11 \mathrm{ft}$ thick & Abovegrade & $3 \mathrm{ft}$ thick concrete \\
\hline $1 \mathrm{G}$ & All & AGES & $11 \mathrm{ft}$ thick & Abovegrade & $\begin{array}{l}3 \mathrm{ft} \text { ihick concrete } \\
\text { Less inventory of radionuclides }\end{array}$ \\
\hline 111 & All & AGES & $\| \mathrm{ft}$ thick & Abovegrade & $\begin{array}{l}3 \mathrm{ft} \text { thick concrete } \\
\text { Class A waste is not stabilized }\end{array}$ \\
\hline \multirow[t]{2}{*}{2} & A. C $\alpha$ & $\mathrm{CP}$ & $1 /$ ft thick $^{\mathrm{c}}$ & Abovegrade & - \\
\hline & $B, C \beta-\gamma$ & $\mathrm{SBH}$ & II ft thick ${ }^{c}$ & Shallow burial & Grouted concrete liner in hole \\
\hline \multirow[t]{2}{*}{3} & $\mathrm{~A}, \mathrm{C} \alpha$ & SLB & 11 ft thick ${ }^{c}$ & Shallow burial & - \\
\hline & $B, C \beta-\gamma$ & SBII & 11 It thick ${ }^{c}$ & Shallow burial & - \\
\hline \multirow[t]{4}{*}{$4 A$} & $\begin{array}{l}\text { A, C } \alpha \\
\text { LI.W }\end{array}$ & AGES & 11 ft thick & Abovegrade & 3 ft thick concrete \\
\hline & $\begin{array}{l}B, C \beta \cdot \gamma \\
1.1 . W\end{array}$ & AGl:S & $11 \mathrm{ft}$ thick & Ahovegrade & $3 \mathrm{ft}$ thick concrete \\
\hline & $\begin{array}{l}\text { A, Ca } \\
\text { Mixed } \\
\text { Waste }\end{array}$ & SI.B & 11 ft thick $^{c}$ & Shallow burial & - \\
\hline & $\begin{array}{l}\text { B, C } \beta-y \\
\text { Mixed } \\
\text { Waste }\end{array}$ & $\mathrm{SBH}$ & $11 \mathrm{ft}_{\text {thick }}^{\mathrm{c}}$ & Shallow burial & - \\
\hline \multirow[t]{4}{*}{413} & $\begin{array}{l}\text { A.C } \alpha \\
\text { LI.W }\end{array}$ & $(P$ & 11 li thick $^{c}$ & Abovegrade & - \\
\hline & $\begin{array}{l}B, C \beta-\gamma \\
\text { LI.W }\end{array}$ & $S B H$ & II fl thick & Shallow hurial & Grouled concrete liner in hole \\
\hline & $\begin{array}{l}\text { A,C } \alpha \\
\text { Mixed } \\
\text { Waste }\end{array}$ & SI.B & 11 fl hick $^{c}$ & Shallow burial & - \\
\hline & $\begin{array}{l}B, C \beta \cdot \gamma \\
\text { Mixed } \\
\text { Wasle }\end{array}$ & SBH & $11 \mathrm{fl}$ thick $\mathrm{c}$ & Shallow burial & - \\
\hline
\end{tabular}


Table 7-5. (continued).

\begin{tabular}{|c|c|c|c|c|c|}
\hline \multirow[b]{2}{*}{ Alternative } & \multirow[b]{2}{*}{ Waste type } & \multicolumn{3}{|c|}{ Barriers } & \multirow[b]{2}{*}{ Comments } \\
\hline & & Structure & Cover $^{A}$ & Geologic & \\
\hline \multirow[t]{2}{*}{5} & $A, C$ & $\begin{array}{l}\text { Storage in Butler } \\
\text { building }\end{array}$ & None & Abovegrade & - \\
\hline & $B, C$ B- $\boldsymbol{\gamma}$ & AGES & None & Abovegrade & $3 \mathrm{ft}$ thick concrete \\
\hline
\end{tabular}

a. Cover thickness at its thinnest point above the structure (or above grade level for buried structures).

Key:

$\begin{array}{ll}\text { AGES } & =\quad \text { Abovegrade engineered structures } \\ \text { BGES } & =\quad \text { Belowgrade engineered structures } \\ \mathrm{CM} & =\text { Covered modules } \\ \mathrm{CP} & =\text { Covered placement }\end{array}$

GDB $\quad=\quad$ Greater-depth burial

GDBH $=$ Greater-depth boreholes

SHB $=$ Shallow boreholes

SLB $\quad=\quad$ Shallow land burial

b. The slight increase in cover thickness is due to a slight areal extension of cover dimensions, as explained in Section 5 .

c. Five feet of additional fill are emplaced for Class B and C waste.

Table 7-6. Number and effectiveness of barriers to contact over the very long term.

Barriers

\begin{tabular}{llll}
\hline Alternative & Type of waste & Waste form & Geologic \\
\hline $1 \mathrm{~A}$ & $\mathrm{C} \alpha$ & IEB & Abovegrade \\
1B & $\mathrm{C} \alpha$ & IEB & Shallow burial \\
1C & $\mathrm{C} \alpha$ & IEB & Abovegrade \\
1D & $\mathrm{C} \alpha$ & IEB & Greater-depth burial \\
$1 \mathrm{E}$ & $\mathrm{C} \alpha$ & IEB & Shallow burial \\
1F & $\mathrm{C} \alpha$ & IEB & Abovegrade \\
1G & $\mathrm{C} \alpha$ & IEB & Abovegrade \\
$1 \mathrm{H}$ & $\mathrm{C} \alpha$ & IEB & Abovegrade \\
2 & $\mathrm{C} \alpha$ & IEB & Abovegrade \\
3 & $\mathrm{C} \alpha$ & IEB & Shallow burial \\
$4 \mathrm{~A}$ & $\mathrm{C} \alpha$ LLW & IEB & Abovegrade \\
& $\mathrm{C} \alpha$ mixed waste & IEB & Shallow burial \\
$4 \mathrm{~B}$ & $\mathrm{C} \alpha$ LLW & IEB & Abovegrade \\
& $\mathrm{C} \alpha$ mixed waste & IEB & Shallow burial \\
5 & $\mathrm{C} \alpha$ & IEB & Abovegrade \\
\hline
\end{tabular}




\subsection{Overall Conclusions}

\subsection{1 "Musts"}

Two of the "musts" listed in Table 6-1 apply to this section. Each must is repeated below, along with a summary of each allernative's ability to satisfy the requirement:

- Comply with all environmental and waste management regulatory requirements, including the requirements for long-term isolation

- Over a period of 40 years, provide all required disposal of LLW and mixed waste generated or currently stored at the INEL, after any necessary treatment has been performed.

Alternatives 1 through 4 satisfy both of the musts. Although the alternatives (except for $1 \mathrm{H}$ ) were evaluated only for the maximum-generation/maximum-treatment scenario, it is noted that Alternative 2 will not meet the disposal requirement of the second must for the scenario of maximum generation/minimum treatment. There is not enough disposal space available at the RWMC in that scenario.

Alternative 5, no action, involves short-term monitored storage and not disposal. While the shortterm storage can be designed to meet all other requirements in the short-term, it does not provide long-term isolation. Therefore, Alternative 5, does not satisfy either of the "musts."

\subsection{2 "Wants"}

Three of the "wants" listed in Table 6-1 are applicable to this section:

- Provide maximum isolation to the waste after the operations period.

- Minimize the problems of constructability, operability, reliability, and maintainability. Included in operability are an evaluation of the retrievability of the waste during the operations phase and the ease of monitoring of the confinement integrity and performance during the operations and institutional control phases.

- Minimize the developmental risk associated with the confinement system technologies.

The subjective evaluation of performance for these three wants is shown in Table 7-7. The information and tables in the previous section were used to develop the scores. The specific logic used is explained in the lollowing paragraphs.

The evaluation of the ability of each alternative to provide maximum isolation after the operations period was based on the number and effectiveness of barriers. Tables 7-5 and 7-6 and the associated text were used to develop the scores. Each alternative started with a score of 10 . The interim results, in terms of individual and total scores, are shown in Table 7-8. 
Table 7-7. Subjective evaluation of applicable "wants."

\begin{tabular}{cccc}
\hline Alternative & $\begin{array}{c}\text { Provide maximum } \\
\text { isolation for the waste } \\
\text { after the operation } \\
\text { period }\end{array}$ & $\begin{array}{c}\text { Minimize problems of } \\
\text { constructability, operability } \\
\text { including retrievability and } \\
\text { monitorability) reliability, } \\
\text { and maintainability }\end{array}$ & $\begin{array}{c}\text { Minimize the } \\
\text { development risk } \\
\text { associated with the } \\
\text { confinement system } \\
\text { technologies }\end{array}$ \\
\hline 1A & 6.5 & 9 & 5 \\
1B & 10 & 7 & 5 \\
1C & 5.5 & 10 & 8 \\
1D & 9.5 & 8 & 9 \\
1E & 8.5 & 9 & 9 \\
1F & 6 & 8 & 7 \\
1G & 8 & 9 & 5 \\
1H & 6 & 8 & 5 \\
2 & 6 & 9 & 7 \\
3 & 8.5 & 8 & 9 \\
4A & 7 & 7 & 6 \\
4B & 7 & 9 & 8 \\
5 & 3 & 10 & 10 \\
\hline
\end{tabular}

The barriers for the long-term performance are considered first. The waste form is the same between alternatives except for Subalternative $1 \mathrm{H}$. The score of Subalternative $1 \mathrm{H}$ was reduced one point because of the lack of stabilization of the Class A waste. Only one point was lost because Class A waste is comparatively low in radionuclide content. The waste containers are the same between all options, so no points are lost for this barrier.

The structures vary greatly. It is assumed that the thick-walled concrete structures are better than the thin-walled modules, which are better than the use of no concrete structures whatsoever. Therefore, Alternatives $1 \mathrm{~A}, 1 \mathrm{~B}, 1 \mathrm{G}$, and $1 \mathrm{H}$ rate the same and are the best with respect to structure. Alternatives 1D, 1E, and 3 are the worst, since they have no structure, and are penalized three points each. Alternatives $1 \mathrm{~F}$ and 5 each have structure for the highly radioactive Class $B$ and $C$ waste and are reduced only a single point for having no structure for Class $\mathbf{A}$ waste. The remainder of the alternatives have various combinations of structures that are intermediate in effectiveness; they are all reduced two points.

The cover varies only slightly from alternative to alternative and is not considered a critical item except for Alternative 5. Alternative 5 has no cover and is reduced 5 points. The option protects the bulk of the high-activity waste in a concrete structure, and therefore is not completely unacceptable. 
Table 7-8. Interim results for isolation performance.

\begin{tabular}{cccc}
\hline Alternative & Long term & Very long term & Total/2 \\
\hline 1A & 7 & 5 & 6 \\
1B & 9 & 9 & 9 \\
1C & 5 & 5 & 5 \\
1D & 7 & 10 & 8.5 \\
$1 \mathrm{E}$ & 6 & 9 & 7.5 \\
1F & 6 & 5 & 5.5 \\
$1 \mathrm{G}$ & 9 & 5 & 7 \\
$1 \mathrm{H}$ & 6 & 5 & 5.5 \\
2 & 6 & 5 & 5.5 \\
3 & 6 & 9 & 7.5 \\
$4 \mathrm{~A}$ & 6 & 7 & 6.5 \\
$4 \mathrm{~B}$ & 6 & 7 & 6.5 \\
5 & 1 & 5 & 3 \\
\hline
\end{tabular}

The geologic barriers are of three type, Alternative $1 \mathrm{D}$ with a deep barrier is the best alternative in this regard. Alternatives $1 \mathrm{~B}, 1 \mathrm{E}$, and 3 , with shallow barriers are the next best, and are reduced one point in comparison to $1 \mathrm{D}$. The fully abovegrade alternatives, $1 \mathrm{~A}, 1 \mathrm{C}, 1 \mathrm{G}, 1 \mathrm{H}$, and 5 , are the worst and are all reduced 3 points. The alternative that stores all of the high-activity Class $\mathrm{B}$ and $\mathrm{C}$ waste above ground, $1 \mathrm{~F}$, is also reduced three points. The remainder of the alternatives are somewhere in between the worst and Alternatives $1 \mathrm{E}$ and 3 ; they are reduced two points.

Subalternative $1 \mathrm{G}$ has less radionuclide inventory of the other alternatives and is given a two-point dividend.

For the very long-term period, the waste forms were all the same, so no points were lost. The best alternative with regard to geologic barriers is $1 \mathrm{D}$. The abovegrade alternatives had essentially no protection from contact of humans or water and were reduced 5 points. Ten points was not subtracted because the IEB waste form was assumed to prevent the waste contact from being totally unacceptable. The shallow burial alternatives were reduced a single point in comparison to the deep burial alternative. Both the combination alternatives have about half their waste abovegrade and half in shallow burial; therefore, each is reduced three points. The inventory of long-lived waste is not significantly different for any of the alternatives. (The major source, Class $\mathrm{C} \alpha$ mixed waste from the TSA, is present in all alternatives.) Therefore, no effect of inventory for Subalternative $1 \mathrm{G}$ is seen for long-term performance. 
The overall scores for the long- and very long-term performance evaluations are summed and divided by two, with equal weighting being applied to the two performance periods. The result is shown in the last column of Table 7-8. Each score was then adjusted by the ratio 10/9 to make the best score (that for Alternative 1B) equal to a 10. The results are shown in Table 7-7.

It is noted that all of the alternatives are assumed to be designed to comply with the 500-year isolation requirement. However, the scores indicate greater confidence in the performance of some alternatives than others to meet and exceed that requirement.

For developmental risk, Table 7-1 was used to develop the scoring. Each alternative started with a raw score of 10. Each time the alternative received an evaluation of small developmental risk, one point was lost. For a moderate risk, two points were lost. (No alternatives were given a major risk.) If an alternative received partial risk in a single category (e.g., cover), then the risk was weighted according to the amount of waste that was affected by each part of the cover.

Alternative 5 is the best option from the standpoint of developmental risk because it primarily makes use of common technologies. The vault options score the worst because of the development risks associated with the vault structures and the liners.

The relative constructability, operability, reliability, and maintainability are scored separately. Then the pieces are added together and divided by 4 to give the final result. The individual rankings and totals are shown in Table 7-9.

For construction, Table 7.2 was used. Each alternative started with a score of ten. The scores were adjusted down one point for a small difficulty, two for a moderate difficulty, and three for a major difficulty. Subalternative $1 \mathrm{G}$ was given an extra point because of the reduction in structures related to the reduced waste volume. Similarly, Subalternative $1 \mathrm{H}$ lost a point due to the larger number of structures required. Alternative 2 and $4 \mathrm{~B}$ lost only a total of one point each because each of the small rankings applied to different portions of the waste. Similar weighting was performed for Alternatives $1 \mathrm{~F}$ and $4 \mathrm{~A}$. In Alternatives $1 \mathrm{~F}$ and 5 , less than $10 \%$ of the waste is placed in vaults, so the use of vaults was scored as a loss of only one point. In Alternative $4 \mathrm{~A}$, two-thirds of the waste is placed in a vault, resulting in the loss of two points. Both $1 \mathrm{~F}$ and $4 \mathrm{~A}$ lose an additional point for the small problems with pits.

For operability, Table 7-3 was used. Each alternative started with a score of 10 . The scores were adjusted down by one point for each evaluation of small, by two points for each evaluation of moderate, and by three points for each evaluation of major. Partial evaluations were weighted according to amount of waste affected. Subalternative $1 \mathrm{G}$ gained an extra point because of its relatively low waste volume, and Subalternative $1 \mathrm{H}$ lost a point because of its high waste volume.

For reliability, the text in Section 7.3.3.3 was used. Each alternative started with a score of ten. Two points were lost by each alternative that was listed as being affected by heavy precipitation. Since Alternatives $2,4 \mathrm{~A}$, and $4 \mathrm{~B}$ were affected to only a small degree by the rain, they were each given a loss of only one point. 
For maintainability, Table 7.4 was used. Each alternative started with a score of ten. One point was lost if a gantry crane was used. Two points were lost if the alternative required extra pumping. One point was lost if the alternative involved offsite transportation. Subalternative 1D lost an additional point because the pits used were decp. Alternative 2 regained a point because the $\mathrm{pit} / \mathrm{mound}$ is fairly small.

The overall scores for the constructability, operability, reliability, and maintainability category were summed and divided by four. The results are shown in Table 7-9. Each score was then adjusted by the ratio of 10/9.25 to make the best score (that for Alternative 5) equal to a 10 . The results are shown in Table 7-7.

Table 7-9. Interim results for constructability, opcrability, reliability, and maintainability.

\begin{tabular}{ccccccc}
\hline Alternative & Constructability & Operability & Reliability & Maintainability & Total & Total/4 \\
\hline 1A & 7 & 6 & 10 & 9 & 32 & 8 \\
1B & 6 & 6 & 8 & 7 & 27 & 6.75 \\
1C & 9 & 7 & 10 & 10 & 36 & 9 \\
1D & 8 & 5 & 8 & 7 & 28 & 7 \\
1E & 9 & 5 & 8 & 8 & 30 & 7.5 \\
1F & 8 & 5 & 8 & 7 & 28 & 7 \\
1G & 8 & 7 & 10 & 9 & 34 & 8.5 \\
1H & 6 & 5 & 10 & 9 & 30 & 7.5 \\
2 & 9 & 7 & 9 & 9 & 34 & 8.5 \\
3 & 9 & 7 & 8 & 7 & 31 & 7.75 \\
4A & 7 & 5 & 9 & 6 & 27 & 6.75 \\
4B & 9 & 7 & 9 & 7 & 32 & 8 \\
5 & 10 & 8 & 10 & 9 & 37 & 9.25 \\
\hline
\end{tabular}




\section{EVALUATION OF INTERFACES WITH OTHER WASTE MANAGEMENT FACILITIES}

\subsection{Introduction}

This section evaluates the projected interfaces of the disposal alternatives with other major INEL or offsite waste management facilities, whether existing or planned. The intent is to identify any potential mismatches or other interface problems that could have major programmatic or operational consequences.

The projected interfaces of the disposal alternatives with the following current or proposed major facilities for managing INEL waste are evaluated: RWMC, MLLWTF, Idaho Waste Processing Facility (IWPF), and offsite treatment. With the exception of RWMC, all of these facilities are in the early planning stages. Many years are available in which to work the interfaces before operations begin. Therefore, this discussion of interfaces is oriented toward identifying potential problem areas, rather than listing actual incompatibilities in detail.

The WERF treatment facility is assumed to have terminated operations when the selected disposal alternative begins operations. Therefore, no operational interface with WERF is expected to occur.

The interface with the HWSF is expected to be minimal for all disposal alternatives. The disposal alternatives are not expected to dispose of waste that is generated as hazardous waste. Such waste would continue to be managed as at present (i.e., be staged in the HWSF and shipped offsite for disposal).

The interface with the MWSF that is operational at that time is expected to be minor. (A mixed waste storage facility was recently constructed adjacent to WERF.) Most mixed waste stored at the MWSF is expected to be treated at MLLWTF, IWPF, or offsite before the residue is transported to the MLLWDF.

\subsection{Requirements/Criteria}

Few if any regulatory requirements, per se, relate to the interfaces between DOE waste management facilities. Nevertheless, the interfaces can be critical to the overall success of multiplefacility waste management systems.

In the absence of regulatory requirements, potential interface issues between facilities were identified by the following approach. Flows of waste between facilities were considered in light of potential mismatches involving what? (waste streams), how much? (waste), when? (will the flow occur), and where? (will the flow occur).

Under the category of "what?" and "how much?," the respective issues include (a) the characteristics of the waste leaving one facility versus the WAC of the receiving facility, and (b) the flow rate of waste to the receiving facility and the capacity of the receiving facility. 
Under the category of "when?," the issues include the projected dates of startup and shutdown of the interfacing facilities, as well as the effects of interrupted operations.

Under the category of "where?," the issues include the location of the disposal facility in relationship to the generators and the treatment facilities.

The "how?" category of interface issues generally cannot be addressed now but will become important as the design of the new facilities progresses. Examples of this category of issue are (a) the compatibility of specific types of waste containers with specific types of handling equipment, and (b) considerations of volumetric efficiency in handling and stacking containers.

Another category of interface issues involves cost impacts of one waste management facility on another. For example, if one disposal alternative provides less isolation of the waste than another alternative does, the operating cost of the treatment facility may increase in order to provide improved waste form immobilization. This category of impacts is included in the cost evaluations of Section 13.

\subsection{Interface of Disposal Alternatives with RWMC}

\subsubsection{Introduction}

The INEL LLW is currently disposed of at the RWMC. The facility has performed this function since 1952, using shallow land burial. The unused disposal capacity of the RWMC, within its present boundaries, is limited. Furthermore, the RWMC is not in a favorable location with respect to local surface water drainage. Present plans are to eventually stop disposing of waste at the RWMC, and to begin disposing of both LLW and mixed waste in the selected disposal alternative.

\subsubsection{Evaluation of Interfaces}

Three items are of interest concerning the interface of the various disposal alternatives with the RWMC. The first item involves the timing of disposal facility shutdown and startup. If the RWMC were to shut down earlier than expected, the disposal alternatives with shorter implementation schedules would become more attractive. Such facilities would be operational sooner to accept waste for disposal. On the other hand, if the MLLWDF were delayed beyond the shutdown date of the RWMC, significant disruptions to INEL operations could occur. These considerations are strictly schedule-related, however, and are addressed in the schedule comparisons of Section 12.

Second, as the RWMC phases out, a close interface would be maintained with the staff of whatever replacement facility is constructed. RWMC personnel would transmit and discuss issues related to items such as WAC, operating procedures, etc., to help ensure the smooth startup and operation of the new facility. Alternatives 2 (Continue RWMC) and 4B (Continue RWMC/Ship Offsite) would have a slight advantage in improved interfacing because the RWMC personnel could become the implementers of the modified disposal methods.

The third item relates to potential disruptions caused by implementing Alternative 2 or 4B. In these alternatives, while RWMC operations and remediation continue, modified disposal methods 
would be prepared for operation. These simultaneous activities would have the potential to cause disruptions.

\subsection{Interface of Disposal Alternatives with MLLWTF and IWPF}

\subsubsection{Introduction}

The MLLWTF and IWPF are proposed facilities for treating INEL mixed waste and LLW. The waste forms resulting from treatment at either or both facilities will be disposed of at the MLLWDF.

The MLLWTF would treat INEL LLW and mixed LLW that are not contaminated with TRU radionuclides. It would treat waste of these types, whether generated by ongoing operations, D\&D activities, or ER activities. Final selection of waste treatment methods has not been made. The cstimated operations date is 2005 .

The IWPF would treat INEL waste contaminated with TRU radionuclides. Included in the potential feed streams are contact-handled and remote-handled stored TRU waste, recovered buried TRU waste, newly-generated TRU waste, and Class $C \alpha$ and Class $C \alpha$ mixed LLW. The final treatment requirements for some waste streams have not been determined. The requirements will depend on the final version of the WAC for the WIPP, the outcome of the CERCLA process for the RWMC, and other unknowns. For this reason, the current design concept for the IWPF entails phased implementation. Phase I will treat only well-characterized $\alpha$ LLW. Subsequent expansions or additions to IWPF in Phase II will allow treating the other waste streams when the treatment needs and WAC become better defined. The estimated operations dates for Phase I and Phase II are 2006 and 2008 , respectively.

The MLLWTF and IWPF projects are in the carly stages, having been started in 1991. Current activities include compiling regulatory requirements, identifying treatment strategies, and preparing siting critcria. Sites for the facilities haves not been selected. If judged advantageous overall, two or more of the IWPF, MLLWTF, and MLLWDF will be collocated.

\subsubsection{Evaluation of Interfaces}

Comparison of the projected startup dates of the MLLWTF and IWPF with that of the disposal alternatives indicates no incompatibilities. For all disposal alternatives, the disposal facility is expected to be available by the time the new treatment facilities begin operations.

The advantages of collocating the treatment and disposal facilities might not be available for Alternatives 2 and $4 \mathrm{~B}$ because the preferred site for the treatment facilities might be a location other than the RWMC. Several of the collocation advantages are monetary, such as reduced onsite shipping costs. Other advantages are not readily measured in monetary terms (e.g., improved communication).

Waste disposal throughput (receipt rates) would be adequate for all disposal alternatives. Disposal capacity would be adequate for all alternatives except for Alternatives 2 and $4 \mathrm{~B}$ under the maximum-generation, minimum-treatment scenario. That limitation is addressed in Section 7. 
The eventual MLLWDF requirements concerning the acceptability of various waste forms are an important interface with the treatment facilitics. The WAC for the MLLWDF have not yet been determined. The required waste form, packaging, and other criteria will reflect the regulations cited in Section 2.3 and the results of a preliminary performance assessment to be completed in FY-1996. Only general observations can be made at present concerning this aspect of the interface. The isolation performance of the various disposal concepts is addressed in Section 7.

Subalternative $1 \mathrm{C}$ involves grouting the waste into concrete containers because the concept provides less additional isolation than the other concepts (except for Alternatives 2 and 5). Some of the impacts of this requirement will appear in the increased cost to the treatment facility. In addition, however, this subalternative requires closer interfacing to ensure the grouting process is performing as intended, a slight negative consideration.

One other interface point involves the extent of waste volume reduction achieved through treatment. In Alternatives 2 and $4 \mathrm{~B}$, the modified (surface) disposal methods would consume almost all of the remaining sizeable surface areas that are available at the RWMC, even for the maximumgeneration, maximum-treatment scenario. If the maximum-generation, minimum-treatment scenario came about, there would not be sufficient space at the RWMC for surface disposal unless more extreme, less desirable methods were used (e.g., stacking waste containers still higher). If maxinumgeneration occurs, then the treatment facility would be required to perform maximum treatment in order to dispose of all the waste at the RWMC. The lack of flexibility of Alternatives 2 and $4 \mathrm{~B}$ on this point is a negative consideration.

\subsection{Interface of Disposal Alternatives with Offsite Treatment Facility}

\subsubsection{Introduction}

Offsite treatment is being considered for INEL mixed waste and LLW. The waste forms resulting from the treatment would be disposed of at the MLLWDF. The selection of waste treatment methods has not been made. The operations date could considerably be sooner than the 2005 and 2006 dates for operation of the MLLWTF and IWPF, respectively.

\subsubsection{Evaluation of Interfaces}

The principal interface issue identified here relates to the potential selection of offsite treatment, together with Alternative 3,4A, or $4 \mathrm{~B}$, all of which involve offsite disposal. One offsite facility would treat the waste, and another offsite facility (unless it is the same facility) would dispose of the waste. This situation would place the INEL in the position of negotiating waste forms, compliance with acceptance criteria, and possibly even schedules between two offsite organizations. Potentially significant interface problems and loss of some degree of INEL control could result, unless the same offsite facility both treats and disposes of the waste.

For Alternatives 2 and $4 \mathrm{~B}$, the concern about volume-reduction needs, discussed in Section 8.4.2, would apply here also. 


\subsection{Overall Conclusions}

\subsection{1 "Musts"}

One of the "musts" listed in Table 6-1 applies to this section:

- Present no potential major conflicts with the missions or operations of other existing or planned INEL waste management facilities.

The conclusion of this overview evaluation is that, for each alternative, the requirement will be satisfied. This conclusion is based on the assumption that the other waste management facilities are developed and operated as discussed above and as delineated in Section 3.

\subsection{2 "Wants"}

One of the "wants" listed in Table 6-1 applies to this section:

- Minimize potential interface problems with other INEL waste management facilities.

The subjective evaluation of performance on this "want" is as follows in Table 8-1. (A score of 10 is assigned to the best-performing alternative. Lesser scores as appropriate, down to zero, are assigned to the other alternatives.)

Reductions in scores were applied based on the following considerations for the indicated alternatives. The potential disruptions in RWMC operations caused by Alternatives 2 and $4 \mathrm{~B}$ were judged to be offset by the improved interfacing achieved by using the same staff at the same facility. Therefore, no net changes in the scores resulted from these two considerations. The need for a grouting process for all waste in Subalternative $1 \mathrm{C}$, and the resulting constraints and interfacing for the treatment facility led to reducing the score by one point. The limited collocation potential of Alternatives 2 and $4 \mathrm{~B}$ resulted in a loss of one point. The fact that Alternatives 2 and $4 \mathrm{~B}$ would constrain the treatment facility to maximum treatment if the maximum-generation scenario occurs resulted in another loss of one point each. The potential need for extensive interfacing between offsite treatment and disposal facilitics led to reducing the scores of Alternatives 3, 4A, and 4B by one point each. Some loss of INEL control over the treatment-disposal interface could also result. All of the listed point reductions were chosen to be small because the negative impacts of the interface considerations either were minor or were possibilities, not certainties. 
Table 8-1. Subjective evaluation of performance on applicable "want."

Minimize potential

Alternative

interface problems

\section{MLLWDF}

Abovegrade engineered structures

Belowgrade engineered structures

Covered modules

Greater-depth burial/boreholes

Shallow land burial/boreholes

Shallow land burial/abovegrade

engineered structures

AGES_effect of waste volume

AGES-effect of waste treatment

Continue RWMC

Ship offsite

Combinations

MLLWDF/Offsite

Continue RWMC/Offsite 


\section{ENVIRONMENTAL EVALUATIONS}

\subsection{Introduction}

The purpose of this section is to identify the major bodies of environmental regulation affecting the sclection and implementation of an alternative and to evaluate each alternative against the requirements established by those regulations. The approach is to evaluate and compare, qualitatively, the difficulties expected in achieving compliance with the environmental requirements for the disposal alternatives.

A survey of the applicable Federal, State of Idaho, and DOE environmental protection regulations and standards provides the basis for the requirements and criteria established for environmental comparisons. The topics considered are NEPA, siting requirements, monitoring, and permitting.

A survey of facility safety analysis reports, engineering design files (EDFs), environmental compliance plans, siting studies, and interviews with facility personnel provide the basis for the environmental comparisons.

\subsection{Requirements/Criteria}

\subsubsection{Overview of Requirements/Criteria}

DOE facilities are required to manage their radioactive wastes in accordance with DOE Orders developed to carry out the authority granted by the Atomic Energy Act (AEA). The disposal of INEL mixed waste and LLW is not subject to NRC regulations. The radioactive component of mixed waste and LLW is subject to the requirements of DOE Order 5820.2A. See Section 7 for a discussion of the requirements of $5820.2 \mathrm{~A}$.

Hazardous wastes must be managed in compliance with regulations promulgated by the EPA under RCRA Subtitle $C$, which sets the requirements for hazardous waste management by regulating generators and transporters of hazardous waste and owners and operators of TSD facilities. It is the policy of DOE, as stated in DOE Order 5400.3, to manage all departmental hazardous and radioactive mixed wastes according to the requirements of Subtitle C of RCRA, and the AEA. RCRA applies to the extent that it is not inconsistent with the AEA.

Executive Order (EO) 12088, amended by EO 12580, requires Federal agencies to comply with applicable administrative and procedural pollution control standards established by several Federal laws.

Listed below are the specific sources of requirements and criteria used in this study to evaluate the regulatory implications associated with each alternative.

- NEPA documentation

- $\quad$ Siting 
- $\quad$ Permitting

- RCRA

- National Emission Standards for Hazardous Air Pollutants (NESHAP)

- Permit to Construct/Prevention of Significant Deterioration (PTC/PSD)

- National Pollutant Discharge Elimination System (NPDES)

- Monitoring

\subsubsection{National Environmental Policy Act Requirements}

All potential projects involving any Federal agency must undergo a review pursuant to NEPA to identify and evaluate potential environmental impacts. The Council on Environmental Quality (CEQ) regulations implementing NEPA ensure that Federal agencies consider environmental information before making decisions on proposed actions. The NEPA process includes decision points at which the significance of environmental effects is considered, project alternatives are identified, and appropriate mitigation measures are identified and adopted.

The CEQ regulations recognize four distinct NEPA documents as "environmental documents" in 40 CFR 1508.10:

- Environmental Assessment (EA)

- $\quad$ Finding of no significant impact (FONSI)

- $\quad$ Notice of intent (NOI)

- Environmental Impact Statement (EIS).

The current strategy for NEPA compliance at the INEL includes preparation of a programmatic or dual-purpose programmatic and project-specific EIS to address existing and planned environmental restoration and waste management activities.

DOE NEPA regulations and discussions with coordinators of the current INEL NEPA strategy provided the basis for the NEPA requirements for each alternative. The actual decisions must be made by DOE-HQ, through submitting appropriate information on the proposed action.

\subsubsection{Siting Requirements/Criteria}

The physical location of a TSD facility directly influences the potential for impacting human health and the environment. Physical locations refer to the geologic, hydrologic, and pedologic characteristics of a sitc, as well as adjoining lands, surface water, and ground water that may be impacted if hazardous and/or radiological constituents are released from the facility. 
RCRA regulations include standards to control the location of TSD facilities in seismic zones and 100-year floodplains (see 40 CFR 264.18 and 40 CFR 270.14).

The EPA requires that other Federal laws that affect the location and permitting of TSD facilities be followed if applicable. These Federal laws include, but are not limited to

- National Historic Preservation Act of 1966 (16 U.S.C. 470 et seq.)

- $\quad$ Endangered Species Act of 1973 (16 U.S.C. 1531 et seq.)

- Fish and Wildlife Coordination Act (16 U.S.C. 661 et seq.) etc.

\subsubsection{Permitting Requirements}

This section describes Federal and State permits and approvals applicable to the disposal of INEL low-level and mixed waste.

9.2.4.1 Resource Conservation and Recovery Act. RCRA, as amended (42 U.S.C. 6901 et seq.) requires any facility that treats, stores (except in satellite and temporary accumulation areas), or disposes of hazardous or mixed waste to obtain a RCRA permit. The RCRA permit process consists of two parts: Part A, interim status, and Part B, final permit.

Construction or modification of a TSD facility requires the preparation of a new permit application or modification of the existing permit. No physical construction of a new facility at the INEL will begin without having submitted Parts A and B (together) of the RCRA permit application to the State of Idaho Hazardous Materials Bureau and having received an approved and effective RCRA permit. No modification can be made before the permit is approved.

9.2.4.2 National Emission Standards for Hazardous Air Pollutants. Under the Clean Air Act (CAA), the EPA has set national emission standards for certain hazardous air pollutants that have no national ambient standard, but which may cause adverse effects on public health. These standards regulate both new and existing emission sources and limit the amounts of pollutants.

The EPA requires a NESHAP document and approval for all new or modified sources of radionuclides from DOE facilities that will cause an EDE of greater than or equal to $0.1 \mathrm{mrem} / \mathrm{yr}$, to any member of the public.

It is possible that disposal structures will include a ventilation system consisting of a distributed fresh air supply and a filtered exhaust having HEPA filters and a charcoal filter. Air emissions from normal operations will be modeled although it is expected that emissions will be below values that typically require a NESHAP permit. No difficulties are anticipated in achieving environmental compliance with NESHAP requirements for any alternative; the subject is therefore not discussed further here.

9.2.4.3 State of Idaho Permit to Construct/Prevention of Significant Deterioration. The Idaho Administrative Procedures Act (IDAPA) 16.01.1012 establishes uniform procedures for the issuance of PTCs and operating permits. Anyone intending to construct, modify, or operate 
equipment that emits air pollutants into the atmosphere, or use equipment that results in the emissions of air pollutants, within the State of Idaho may need to obtain an air quality permit from the Idaho Air Quality Bureau (IAQB). A PTC must be obtained prior to construction or modifications except for listed classes of equipment.

PSD review by the State of Idaho is necessary for any radiological source. The PSD program requires a preconstruction review of all major facilities and major modifications. A major facility is a facility that has actual or allowable air emissions of 100 ton/yr or more of any air contaminant. The INEL is considered a major facility.

Analysis of air emissions from the disposal facility will be necessary to satisfy State of Idaho regulations. No difficultics are anticipated in achicving environmental compliance with PTC/PSD requirements. This discussion applies to all alternatives requiring construction of a new facility; the subject is therefore not discussed further here.

9.2.4.4 National Pollutant Discharge Elimination System. The Clean Water Act's (CWA) primary mechanism for imposing limitations on pollutant discharges is a nationwide permit program established under Section 402 and referred to as the NPDES.

No discharge to surface waterways or municipal water systems is anticipated for any alternative. However, as of October 1, 1992, storm water discharges (storm water point sources) are subject to the NPDES program. U.S. Department of Energy Idaho Operations Office (DOE-ID) plans to obtain a general NPDES permit for all existing INEL facilities. Any new facility will require a modification of this NPDES permit. No difficulties are anticipated in achieving environmental compliance with NPDES permit requirements. This discussion of NPDES permits applies to all alternatives requiring construction of a new facility; the subject is therefore not discussed further here.

\subsubsection{Monitoring}

A monitoring program for disposal facilities is required by Federal, State, and local regulations as well as DOE Orders. The regulations applicable to monitoring requirements for mixed waste and LLW disposal facilitics include RCRA, CAA, State of Idaho, and DOE Order 5820.2A.

This study assumes that the waste disposal facilities will be designed to comply with all applicable monitoring requirements (see Section 3). No unique problems are anticipated in meeting the required standards. Section 5 discusses the specific requirements for the monitoring program and the monitoring systems and programs during the facility operations and institutional control phases.

This discussion of monitoring applies to all alternatives; the subject is therefore not discussed further here. 


\subsection{Evaluations of Alternatives Against Requirements}

\subsubsection{Construct and Operate MLLWDF}

9.3.1.1 Subatternative 1A: Abovegrade Engineered Structure. The MLLWDF alternative is used as the baseline case against which the other alternatives are compared. For brevity in discussing the subsequent subalternatives, difficulties that would be the same as those for Subalternative $1 \mathrm{~A}$ are not discussed again; only areas that differ from those for $1 \mathrm{~A}$ are discussed.

9.3.1.1.1 NEPA-The construction and operation of disposal facilities for mixed waste and LLW are not specifically addressed in the NEPA regulations. In support of a decision regarding the level of NEPA documentation required for the MLLWDF, environmental documentation has been submitted for review and recornmendation. It has been assumed here that the MLLWDF will require the preparation of an EIS based on the potential for significant environmental impact and public controversy for such an action.

As of this writing (July 1993), the proposed NEPA strategy for the MLLWDF involves preparing an EIS tiered off the INEL Environmental Restoration and Waste Management (ER\&WM) EIS, although the strategy is still evolving. The current schedule (which is also evolving) for the INEL ER\&WM EIS calls for completion of the Final EIS in November 1995. A Record of Decision (ROD) is expected to be issued by the end of January 1996.

Construction of a mixed waste and LLW disposal facility at the INEL may be controversial and may have a high degree of public concern. These issues and concerns will arise throughout the EIS process, and may present some difficulty in finalizing the EIS and issuing a ROD. See Section 11 for further details.

Because NEPA requires evaluating all reasonable alternatives to a proposed action, the alternatives considered in this study will be evaluated in the MLLWDF EIS, providing thev remain viable options. For this reason, the NEPA requirements, schedules, and costs for each treatment alternative are assumed to be identical to the MLLWDF.

9.3.1.1.2 Siting-The exact location to be proposed for the MLLWDF is not determined yet. Implementation of the MLLWDF alternative will require siting studies to select possible locations. The EIS process will eventually identify the preferred location for the MLLWDF.

Siting studies require surveys, consultations, and modeling studies to ensure compliance with Federal, State, and DOE criteria. Extensive site characterization studies will be required to verify the location is environmentally sound.

Other difficulties associated with siting the MLLWDF include political and regulatory issues related to collocating the facility with the proposed treatment facilities. These issues are currently being evaluated.

9.3.1.1.3 Permitting/Approvals-Resource Conservation and Recovery Act-The INEL MLLWDF will be designed for storage and disposal of mixed waste and, therefore, requires a RCRA 
permit. Difficulties associated with RCRA permitting for the MLLWDF are associated with the cost and schedule impacts, and the potential for controversy and public opposition.

The INEL MLLWDF does not qualify for interim status; therefore, both a Part A and a Part B permit application must be submitted and approved prior to construction.

The RCRA permitting process requires public meetings and public comment periods similar to the NEPA process discussed above. Construction of a disposal facility for mixed and LLW may create controversy and public opposition, as discussed in Section 9.3.1.1.1. Public opposition may arise throughout the permitting process and may present some difficulty in issuing a permit.

9.3.1.1.4 Conclusions-Subalternative $1 \mathrm{~A}$ will comply with all environmental requirements. The difficulty associated with achieving environmental compliance duc to this alternative is expected to be among the smallest of the onsite disposal alternatives because the new facility will dispose of all INEL mixed and LLW in accordance with DOE orders, and State and Federal regulations. The MLLWDF will include new engineered technologies and controls to minimize any releases from the facility, which will facilitate the permitting process.

\subsubsection{Subalternative 1B: Belowgrade Engineered Structures.}

9.3.1.2.1 NEPA-The issues and concerns arising throughout the EIS process may again present some difficulty in finalizing the EIS and issuing a ROD. The degree of controversy associated with Subalternative 1B for long-term disposal of INEL mixed waste and LLW is difficult to assess, as is any potential environmental impact. Construction of a belowgrade structure may receive less criticism than the abovegrade structure because it would be less visible and have the added protection of the surrounding soil. On the other hand, an abovegrade structure may give the public a greater sense of security because the structure is further removed from the water table and more easily monitored. Overall, it is expected that the difficulties associated with NEPA compliance for subalternative 1B will be similar to those associated with Subalternative $1 \mathrm{~A}$.

9.3.1.2.2 Siting-The discussion above in Section 9.3.1.1.2 applies identically to Subalternative 1B. However, siting a belowgrade structure is likely to present more difficulties than an abovegrade structure due to the limited number of sites on the INEL that comply with all of the Federal, State, and DOE regulations and also have suffic:-nt, fine-grained sediments.

9.3.1.2.3 Permitting/Approvals-Resource Conservation and Recovery Act-Subalternative 1B is designed for disposal of mixed waste and, therefore, requires a RCRA permit. Difficulties associated with RCRA permitting for Subalternative $1 B$ are the same as those discussed above for Subalternative $1 \mathrm{~A}$.

The permit will require public meetings and public comment periods, as discussed above for Subalternative 1A. The potential public opposition associated with a belowgrade structure, as compared to an abovegrade structure is, again, difficult to assess.

9.3.1.2.4 Conclusions-Subalternative $1 \mathrm{~B}$ will comply with all environmental requirements. The difficulty associated with achieving environmental compliance duc to this alternative is expected to be similar to Subalternative 1A. Subalternative 1B will dispose of all INEL 
mixed and LLW in accordance with DOE orders, and State and Federal regulations. Subalternative 1B will include new engineered technologies and controls to minimize any releases from the facility.

\subsubsection{Subalternative 1C: Covered Modules.}

9.3.1.3.1 NEPA-The discussion above in Jection 9.3.1.1.1 applies identically to Subalternative 1C. The difficulties associated with NEPA compliance for this subalternative are expected to be the same as for Subalternative $1 \mathrm{~A}$.

9.3.1.3.2 Siting-The discussion above in Section 9.3.1.1.2 applies identically to Subalternative 1C. The difficulties associated with siting for this subaiternative are expected to be the same as for Subalternative $1 \mathrm{~A}$.

9.3.1.3.3 Permitting/Approvals-Resource Conservation and Recovery Act-Subalternative 1C will be designed for treatment of mixed waste and, therefore, requires a RCRA permit. The discussion above in Section 9.3.1.1.3 applies identically to Subalternative 1C. The difficultics associated with RCRA compliance for this subalternative are expected to be the same as for Subalternative $1 \mathrm{~A}$.

9.3.1.3.4 Conclusions-Subalternative 1C will comply with all environmental requirements. The difficulties associated with achieving environmental compliance due to this alternative are expected to be the same as for Subalternative $1 \mathrm{~A}$ because Subalternative $1 \mathrm{C}$ will dispose of all INEL mixed and LLW in accordance with DOE Orders, and State and Federal regulations. Subalternative $1 \mathrm{C}$ will include abovegrade disposal technologies similar to those included in Subalternative $1 \mathrm{~A}$ and engineering controls to control any releases from the facility, which will facilitate the permitting/approval processes.

\subsubsection{Subalternative 1D: Greater-depth Burial/Boreholes.}

9.3.1.4.1 NEPA-The difficulties associated with Subalternative 1D are the same as those discussed for Subalternative 1A. The degree of controversy and public opposition associated with Subalternative $1 \mathrm{D}$ is expected to be greater than for Subalternative $1 \mathrm{~A}$ because the greater-depth burial concept does not include any engineered structures. It is expected that public perception of this subalternative will be tied to perception of the current disposal methods at the INEL. The perception is expected to be negative even though burial is at greater depths and liners will be installed. The current disposal site contains CERCLA sites (where significant releases have occurred); therefore, the technology may be perceived as one that has "failed."

9.3.1.4.2 Siting-Siting studies will be needed for Subalternative 1D to ensure compliance with DOE orders, and Federal and State regulations. The discussion above in Section 9.3.1.2.2 applies to this subalternative; however, siting is expected to be slightly more difficult due to the need for burial at greater depths.

\subsection{Permitting/Approva/s-Resource Conservation and Recovery} Act-Subalternative 1D will be designed for disposal of mixed waste and, therefore, requires a RCRA permit. Difficulties associated with RCRA permitting for Subalternative 1D are expected to include those discussed above for Subalternative $1 \mathrm{~A}$. 
The degree of public controversy associated with Subalternative 1D is expected to be greater that associated with Subalternative $1 \mathrm{~A}$. Even though the design of the disposal technology will satisfy RCRA requirements, the public perception of this technology is expected to be negative (for those reasons discussed above under NEPA) and may cause difficulties during the public comment periods.

9.3.1.4.4 Conclusions-Subalternative $1 \mathrm{D}$ will comply with all environmental requirements. The difficulties associated with this subalternative, however, are expected to be among the greatest of the MLLWDF subalternatives because no engineered structures are used for greaterdepth burial.

\subsubsection{Subalternative 1E: Shallow Land Burial/Boreholes.}

9.3.1.5.1 NEPA-The difficulties associaled with NEPA compliance for Subalternative 1E are again primarily related to public controversy and opposition. The discussion above in Section 9.3.4.1 applies to this subalternative. The degree of controversy and public opposition is expected to be even greater than that for the previous subalternative (Subalternative 1D) because under this subaltcrnative, Class $A$ waste is disposed of by shallow land burial, as opposed to greater-depth burial.

9.3.1.5.2 Siting-The discussion above in Section 9.3.1.4.2 applies identically to Subalternative $1 \mathrm{E}$.

9.3.1.5.3 Permitting/Approvals-Resource Conservation and Recovery Act-Subalternative $1 \mathrm{E}$ will be designed for disposal of mixed waste and, therefore, requires a RCRA permit. The discussion above in Section 9.3.1.4.3 applies identically to Subalternative 1E.

9.3.1.5.4 Conclusions-Subalternative $1 \mathrm{E}$ will comply with all environmental requirements. The difficulties associated with this subalternative are expected to be the greatest of the MLLWDF subalternatives because no engineered structures are used for shallow land burial.

\subsubsection{Subalternative 1F: Shallow Land Burial/Abovegrade Engineered Structures..}

9.3.1.6.1 NEPA-The difficulties associated with NEPA compliance for Subalternative 1F are related to public perception. Again, the public may view shallow land burial as negative due to the problems associated with the current INEL disposal practices (shallow land burial). The difficulties are expected to be greater than for Subalternative $1 \mathrm{~A}$ but slightly less than for Subalternative $1 \mathrm{E}$.

9.3.1.6.2 Siting-Siting studies will be required for Subalternative $1 \mathrm{~F}$ and will involve difficulties similar to those discussed in Section 9.3.1.2.2 under Subalternative 1B.

9.3.1.6.3 Permitting/Approva/s-ResourceConservation and Recovery Act-Subalternative $1 F$ will he designed for disposal of mixed waste and, therefore, requires a RCRA permit. Difficulties associated with RCRA permitting for Subalternative $1 \mathrm{~F}$ include those discussed above for Subalternative 1A. However, greater opposition is expected due to the use of shallow land burial.

9.3.1.6.4 Conclusions-Subalternative $1 \mathrm{~F}$ will comply with all environmental requirements. The difficulty associated with achieving environmental compliance due to this 
alternative is expected to be greater than for Subalternative $1 \mathrm{~A}$ but smaller than for Subalternative $1 \mathrm{E}$.

\subsubsection{Subalternative 1G: Effect of Waste Volume.}

9.3.1.7.1 NEPA-The issues and concerns arising throughout the EIS process may present some difficulty in finalizing the EIS and issuing a ROD because disposal facilitics receive a great deal of public attention and associated controversy. The effect of waste volume on the MLLWDF (Subalternative 1G) will carry the same difficulties as those associated with Subalternative 1A. However, less waste will require less disposal capacity and less undisturbed area and may carry less potential for environmental impact.

9.3.1.7.2 Siting-The discussion above in Section 9.3.1.1.2 applies identically to Subalternative 1G. The lower volume of waste will again reduce the amount of land required to meet the necessary disposal capacity.

\subsection{Permitting/Approva/s-Resource Conservation and Recovery} Act-Subalternative $1 \mathrm{G}$ will require a RCRA permit just as Subalternative 1A. The difficulties associated with RCRA permitting for Subalternative $1 \mathrm{G}$ are the same as those discussed above for Subalternative $1 \mathrm{~A}$.

9.3.1.7.4 Conclusions-Subalternative $1 G$ will comply with all environmental requirements. The difficulty associated with achieving environmental compliance due to this alternative is expected to be among the smallest of the onsite disposal alternatives because the new facility will dispose of all INEL mixed waste and LLW in accordance with DOE orders, and State and Federal regulations. This subalternative will include engineered technologies and controls to minimize releases from the lacility. Subalternative $G$ will dispose of less waste than the other alternatives and will require less undisturbed area for development.

\subsubsection{Subalternative 1H: Effect of Waste Treatment.}

9.3.1.8.1 NEPA-Subalternative $1 \mathrm{H}$ will again require the same NEPA documentation as Subalternative $1 \mathrm{~A}$. The difficulties associated with NEPA compliance are expected to include those discussed above in Section 9.3.1.1.1. The effect of waste treatment (minimum treatment as opposed to maximum treatment) however, is expected to increase the difficulties associated with public perception. Minimum treatment is expected to result in a less stable waste form that would be less acceptable to the public and the State of Idaho than the maximum treatment option.

9.3.1.8.2 Siting-The discussion above in Section 9.3.1.1.2 applies identically to Subalternative $1 \mathrm{H}$. The minimum treatment option in comparison with the maximum-treatment option, considered in Subalternative 1A, will increase the actual volume of waste to be disposed of, thus requiring more disposal capacity.

9.3.1.8.3 Permitting/Approvals-Resource Conservation and Recovery Act-Subalternative $1 \mathrm{H}$ will again require a RCRA permit. The difficulties associated with RCRA permitting for Subalternative $1 \mathrm{H}$ are the same as these discussed above for Subalternative $1 \mathrm{~A}$. 
9.3.1.8.4 Conclusions-Subalternative $1 \mathrm{H}$ will comply with all environmental requirements. The difficulty associated with achieving environmental compliance due to this alternative is expected to be among the largest of the onsite disposal alternatives because the new facility will dispose of waste th $t$ has not been treated (volume reduced/stabilized) and will require the greatest disposal capacity.

\subsubsection{Continue Disposal at RWMC}

9.3.2.1 NEPA. The alternative of continuing disposal at the RWMC will be one of the alternatives in the EIS evaluated for the MLLWDF. The difficulties associated with NEPA compliance for the RWMC alternative will, therefore, be the same as for the MLLWDF Subalternative $1 \mathrm{~A}$.

The degree of controversy associated with continued disposal at the RWMC is expected to be greater than for the MLLWDF baseline alternative. The proposed disposal technology for the Class A waste and the Class $\mathrm{C} \alpha$ waste is to continue the current practice of stacking on the existing filled pits. No engineered barriers are considered for these waste types under this alternative. Because the RWMC contains sites having previous releases of hazardous substances, continued operation of this facility may not be vicwed favorably by the public and the State of Idaho. In order to continue operations at the RWMC site, only those sites not designated as inactive CERCLA sites will be available for disposal.

9.3.2.2 Siting. The RWMC is located in the southwest corner of the INEL. Because the current location of the RWMC is located in a depression, it has been subject to flooding in the past. $\mathrm{A}$ berm has been constructed around the facility to divert surface water runoff. The RWMC currently is not permitted for disposal of mixed waste. Disposal of mixed waste requires a new RCRA permit and compliance with the siting criteria set forth in those regulations. Therefore, the RWMC is subject to the mandatory siting criteria discussed above for the MLLWDF.

9.3.2.3 Permitting/Approvals. Resource Conservation and Recovery Act-The RWMC alternative will be designed for disposal of mixed waste and, therefore, requires a RCRA permit. RWMC has Interim Status for storage of mixed waste and hazardous waste.

The RCRA Part B documentation for storage of these wastes has been submitted to the State. Continued operations at the RWMC will include disposal of mixed wastes. Because the RWMC is not currently permitted for disposal of RCRA wastes, a new RCRA permit application and issuance will be required before any waste could be placed. Difficulties associated with RCRA permitting for the RWMC are the same as those discussed above for the MLLWDF.

The new RCRA permit will require public meetings and public comment periods. The potential controversy and public opposition associated with the continued operation of RWMC is anticipated to be greater than for the MLLWDF for reasons discussed above in Sections 9.3.2.1 and 9.3.2.2.

9.3.2.4 Conclusions. Continued operations at the RWMC will comply with all environmental requirements. The difficulties associated with achieving environmental compliance due to this alternative are expected to be greater than for the MLLWDF because of the past history of the facility. The RWMC alternative will not include engineered disposal technologies like those included 
in the MLLWDF; however, engineering controls will be included to control any releases from the facility.

\subsubsection{Offsite Treatment}

9.3.3.1 NEPA. The offsite disposal alternative will be an alternative evaluated in the EIS for the MLLWDF. The difficulties associated with NEPA compliance for the offsite disposal alternative will primarily involve public perception.

The potential exists for political controversy and public opposition to arise as a result of shipping radioactive and mixed wastes across several states. Institutional factors associated with this alternative are presented in Section 11, and other issues associated with this alternative are discussed in Section 14.

9.3.3.2 Permitting. All licensing and permitting required for disposal of INEL wastes will be the responsibility of the offsite TSD facility. The involved offsite facility is assumed to be permitted to handle the INEL wastes. Difficulties related to licensing and permitting will be addressed by that facility. However, DOE will audit the offsite TSD facility and ensure that the appropriate permits are in place.

9.3.3.3 Shipping Regulations. Shipping must comply with DOT requirements per 49 CFR 173, 10 CFR 71, RCRA requirements per 40 CFR 263, and the applicable requirements of the State of Idaho and any Tribal parties or states through which the waste must be transported. Compliance with these regulations will not present any insurmountable difficulties.

Recent revisions to the DOT regulations, referred to as HM-181, present performance-based packaging requirements. The new packaging requirements present a potential regulatory difficulty. HM-181 requires all transitional provisions for continued use of pre-181 packaging to expire October 1, 1996.

This alternative will require compliance with these regulations for offsite shipments of mixed and LLW. Mixed waste will be required to be shipped in a package meeting the performance requirements for both radioactive waste and mixed waste. These packaging requirements are not necessarily compatible. In addition, any wastes packaged prior to 1996 may require repackaging prior to transporting offsite. The majority of the waste volume considered in this study will be generated after 1996. The repackaging requirement is therelore applicable only to a small volume of waste stored at the MWSF; therefore, the overall impact of this requirement is expected to be minor.

9.3 3.4 Conclusions. The offsite alternative will comply with all environmental requirements. The difficulty associated with achieving environmental compliance due to this alternative is expected to be small. This alternative will transfer many regulatory difficulties associated with waste treatment from the operators of the onsite disposal facility to the operators of the offsite disposal facility.

\subsubsection{Combinations of Alternatives}

The environmental requirements and the difficulties expected in complying for the selected combinations of alternatives will generally correspond to the requirements and difficulties associated 
with the individual contributing alternatives. However, the combined alternatives remove the need for disposal of mixed wastes at the INEL, thus eliminating the need for acquiring a RCRA permit for the onsite facilities.

For brevity, the two combinations selected for study are not evaluated here in detail. The conclusions for the combinations of alternatives are given in Section 9.4.

\subsubsection{No Action}

9.3.5.1 NEPA and Siting. The no-action alternative will be one of the alternatives in the EIS evaluated for the MLLWDF. Implementing the no-action alternative requires that additional storage facilitics be constructed at the INEL. The difficultics anticipated in achieving environmental compliance with NEPA requirements for the storage facilities are expected to be similar to those discussed for the MLLWDF. Although the controversy surrounding disposal of mixed and LLW won't be an issue, the indefinite storage of these wastes in a less adequate facility may be cause for criticism.

The construction of new storage facilitics for INEL mixed and LLW will require compliance with Federal, State, and DOE siting criteria discussed above under the MLLWDF Subalternative $1 \mathrm{~A}$. The studies and difficulties associated with siting a new storage facility will be similar to siting the MLLWDF.

9.3.5.2 Permitting/Approvals. Resource Conservation and Recovery Act-Construction of an additional storage facility for mixed waste will require a Part A and a Part B permit. Although the indefinite storage of treated waste will be in compliance with RCRA, it is not considered good management practice. Therefore, permitting these facilities may be difficult.

9.3.5.3 Conclusions. The no-action alternative will comply with all environmental requirements. Although indefinite storage of mixed waste is not encouraged, it is not prohibited by Federal or State regulation or DOE order.

\subsection{Overall Conclusions}

\subsection{1 "Musts"}

One of the "musts" listed in Table 6-1 applies to this section:

- Comply with all environmental and waste management regulatory requirements, including the requirements for long-term confinement.

The conclusion of the overview evaluation is that all of the disposal alternatives will comply with all environmental requirements. The disposal options will be designed and permitted in accordance with Federal, State and DOE regulations. The additional criterion for long-term confinement was evaluated in Section 7. 


\subsection{2 "Wants"}

None of the "wants" listed in Table 6-1 applies to this section. 


\section{SAFETY AND HEALTH EVALUATIONS}

\subsection{Introduction}

This section evaluates the alternatives on the safety and health risk they will present, up through the time of final closure of the disposal site. The projected post-closure isolation performance of the alternatives was compared in Section 7.

Detailed, quantitative comparisons of the safety and health risk of the disposal alternatives require that a rigorous safety analysis, per DOE Order 5480.23 , be available for each alternative. Safety analyses do not exist for any of the alternatives. The safety analysis for the MLLWDF, for example, will not be prepared for several years. Thus, insufficient information exists to allow quantitative evaluations, such as calculated radiation doses from accidental releases. Because of the lack of safety documentation, of radionuclide and hazardous material inventory information, and of even preconceptual designs, the present safety and health evaluations are limited to overview observations. The observations are based on judgments derived from the current safety analysis report (SAR) of the RWMC (EG\&G Idaho 1986), knowledge of the general hazards of the waste and the disposal activities, and a generic safety evaluation of similar disposal concepts in Conceptual Design Report: Alternatives Concept for Low-Level Radioactive Waste Disposal (1987). Because of the incomplete state of knowledge, the observations are subject to later refinement as the detailed design is developed and detailed safety evaluations are performed for the selected alternative.

It is assumed that all of the alternatives will be designed and operated to comply with all applicable requirements. Thus, the comparative margin of safety and health performance is addressed here.

\subsection{Requirements/Criteria}

The major requirements/criteria that relate to safety and health protection in DOE facility operations are found in several DOE Orders and in other standards sources cited therein. Some of the criteria are specific safety criteria (e.g., limits on radiation dose).

DOE Order 6430.1A governs the design of DOE facilities. Many design criteria in 6430.1A stem from other DOE Orders or national standards. Thousands of the design criteria in $6430.1 \mathrm{~A}$ are safety-related. The criteria cover everything from the seismic resistance of buildings and the redundancy of utilities, to the adequacy of ventilation systems and the confinement capabilities of process vessels.

DOE Order 5480.23 contains the requirement to prepare a SAR for a nuclear facility. (All of the disposal alternatives would fall under the definition of a nuclear facility.) A SAR is a comprehensive document, the preparation of which involves considerable time and funding. The required content of a SAR is also found in DOE Order 5480.23.

DOE Order 5480.5 governs the operations of all of the disposal alternatives located at DOE sites. 
Radiological safety in DOE facilities is governed by DOE Orders 5400.5 and 5480.11 . Concentration limits and exposure limits are specified.

Nonradiological salety is governed by DOE Orders 5480.10 and 5480.4. The latter Order cites the Occupational Safety and Health Act (OSHA) standards listed in 29 CFR 1910 (operations) and 29 CFR 1926 (construction) as mandatory standards. Exposure limits for toxic materials are cited in the OSHA standards and in publications of the American Conference of Government and Industrial Hygienists (ACGIH).

Offsite shipments are governed by the regulations of the NRC, the EPA, and the U.S. Department of Transportation (DOT).

\subsection{Hazards Associated with Storing, Shipping, and Disposing of INEL Mixed Waste and LLW}

Certain hazards are inherent with constructing and operating the facilities and equipment to store, ship, or dispose of INEL mixed waste and LLW. Other than common industrial hazards, the hazards result from the radioactive and/or toxic nature of the waste, combined with potential events internal or external to the facility or equipment.

In most cases, the inherent hazards do not vary from one alternative to another. Except for the waste generation and waste treatment scenarios in Subalternatives $1 \mathrm{G}$ and $1 \mathrm{H}$, the same waste with the same radiological and nonradiological hazards will be handled and disposed of. However, the various alternatives involve different ways of handling and isolating the waste.

The principal hazards associated with the waste were identified, based on waste stream characterizations and on safety evaluations of existing disposal facilities. The principal hazards associated with the waste are expected to be as follows:

- Direct radiation

- Radioactive contamination resulting from accidental loss of isolation

- Toxic chemical fumes, vapors, or fibers (e.g., volatile organics, lead, mercury, asbestos), resulting from accidental loss of confinement; this hazard is assumed to have been greatly reduced as a result of treatment.

The principal types of events by which control of the hazards could be lost include fires, explosions, earthquakes, equipment failures, accidental breach of isolation, and shipping accidents.

\subsection{Approach}

The first steps in developing an approach to the safety and health evaluations were to (a) identify the scope and (b) determine simplifying assumptions to reduce the number of required comparisons. 
Table 10-1 indicates the scope of the evaluations. The various phases of the life cycle of a disposal facility, from construction through final closure, are listed across the top of Table 10-1. All of the construction activities are included under the construction phase, even if they occur during operations. The postclosure isolation performance of the alternatives was compared in Section 7. Postclosure monitoring involves comparatively insignificant hazards and is deleted from consideration.

Various considerations that might or might not apply to each phase are listed down the left side of Table 10-1. Potential receptors are listed first: workers, public, and environment. Broad classes of hazards are listed next: radiological, chemical, and industrial. Finally, acute and chronic types of impacts are listed.

Several items in Table 10-1 are of comparatively minor importance and need not be pursued. These items are indicated by dashes on the table.

- At the remote INEL site, impacts on the public from disposal activities up through the time of closure are expected to be minuscule, based on past experience at the RWMC. (Releases during offsite shipment could impact the public, however.)

- No routine releases of radionuclides or hazardous chemicals are planned. Therefore, the only chronic impacts expected are external doses to workers (and to the public during shipping).

Table 10-1. Scope of safety and health evaluations.

\begin{tabular}{|c|c|c|c|c|}
\hline Phase $\rightarrow$ & Construction & Operations & $\begin{array}{c}\text { Offsite } \\
\text { shipping }\end{array}$ & Final closure \\
\hline \multicolumn{5}{|l|}{ Receptors: } \\
\hline Workers & $\mathrm{X}$ & $\mathbf{X}$ & $\mathbf{X}$ & $\mathbf{X}$ \\
\hline Public & - & - & $\mathbf{X}$ & - \\
\hline Environment & - & $X$ & $\mathbf{X}$ & $\mathbf{X}$ \\
\hline \multicolumn{5}{|l|}{$\begin{array}{l}\text { Type } \\
\text { of hazard: }\end{array}$} \\
\hline Radiological & - & $\mathrm{X}$ & $\mathbf{X}$ & $\mathbf{X}$ \\
\hline Chemical & - & - & - & - \\
\hline Industrial & $\mathrm{X}$ & $\mathrm{X}$ & $\mathbf{X}$ & $\mathbf{X}$ \\
\hline \multicolumn{5}{|l|}{$\begin{array}{l}\text { Type of } \\
\text { impact: }\end{array}$} \\
\hline Acute & $X$ & $X$ & $X$ & $\mathbf{X}$ \\
\hline Chronic & - & $X^{a}$ & $X^{\mathrm{a}}$ & $X^{a}$ \\
\hline
\end{tabular}

a. The chronic impacts are expected to consist only of external radiation doses. 
- As a result of treatment to meet LDRs, most of the hazardous chemicals in the waste will have been either eliminated or stabilized to a form that is not readily dispersible. Therefore, the hazard from accidental release of the chemicals during operations, closure, or shipment is small compared with the hazard from release of the radioactive materials in the waste.

- During construction, no waste would be present (except for Alternative 2, Continue RWMC) so no radiological or significant chemical hazards would exist.

These simplifications led to the following potential impacts to evaluate, each of which is marked with an $X$ in Table 10-1:

- During construction

- Industrial accidents impacting workers

- During operations or closure

- Accidental radiological releases impacting workers or the environment

- External doses to the workers during routine operations

- Industrial accidents impacting workers

- During offsite shipment

Accidental radiological releases impacting transportation workers, the public, or the environment

- External radiation doses to transportation workers and the public during routine shipping conditions

- Industrial (shipping) accidents impacting workers or the public.

For each of these impacts, the disposal alternatives are compared in the following section.

\subsection{Evaluations of Alternatives Against Requirements/Criteria}

Based on the foregoing discussion of requirements/criteria, expected hazards, and approach, the disposal alternatives were evaluated in a qualitative, overview sense. The discussions deal only with differences among the alternatives and subalternatives.

Simplifying assumptions were made as follows:

- All facilities and equipment will be designed and operated in accordance with the applicable requirements cited in Section 10.2. 
- The attention devoted to salety programs such as ALARA is comparable among the alternatives.

- Hazards at the offsite disposal facility are evaluated in the same manner as for the INEL facilities, even though INEL workers would not be subject to the hazards.

- For combinations of alternatives or subalternatives, the performance is weighted more toward that of the constituent representing the larger fraction of the waste or the larger fraction of the hazard.

\subsubsection{Safety and Health During Construction}

The National Safety Council publishes data (1992) for occupational injuries, illnesses, and deaths per labor hour in various industries. The construction industry is included and is one of the more hazardous industries. For a given type of construction, projects requiring a larger number of labor hours can be statistically expected to have a larger number of injuries and fatalities (hereafter termed "accidents," for simplicity).

In the present comparisons, an estimate was made of the relative magnitude of the construction effort for each alternative. The construction costs, which are estimated in detail in Section 13, provide a general confirmation of the relative rankings of labor-hours for the various alternatives. Any special hazards expected to be encountered during construction of a given alternative were also considered.

Subalternative $1 \mathrm{~A}$ is used as the baseline case against which the other alternatives and subalternatives are compared. It requires one of the largest construction efforts, in terms of labor hours. In addition, it involves the handling of massive components, such as emplacing concrete components and installing the gantry crane. Therefore, Subalternative $1 \mathrm{~A}$ would have one of the largest expected numbers of construction accidents. Subalternative $1 \mathrm{G}$ is the same concept, but with fewer structures and fewer expected construction accidents. Subalternative $1 \mathrm{H}$, with more structures, would have more expected accidents. Subalternative 1B is the same concept as $1 \mathrm{~A}$, but it is located belowgrade, and therefore would be expected to have slightly more accidents.

Subalternative $1 \mathrm{C}$ involves a reduced magnitude of construction-only the drainage system, liners, and a concrete pad. The expecied number of construction accidents would be among the lowest.

Subalternative $1 \mathrm{E}$ involves excavation of the shallow land burial pits and soil vaults, and construction of the drainage system and working surface. Based on the magnitude of the construction effort and the hazards involved, the number of accidents would be expected to fall between those for Subalternative $1 \mathrm{~A}$ and Subaltcrnative 1C. Subalternative $1 \mathrm{D}$ involves basically the same construction as $1 \mathrm{E}$, but at a greater depth belowgrade. The number of accidents expected is slightly higher than that for Subalternative $1 \mathrm{E}$.

Subalternative $1 \mathrm{~F}$ is a combination of Subalternatives $1 \mathrm{~A}$ and $1 \mathrm{E}$; it would fall between the two in terms of expected accidents. 
Construction for Alternative 2 would be similar to that for Subalternative 1C. However, an additional hazard is associated with excavation and construction activities in an area where waste has been disposed of previously.

For Alternative 3, the construction activities would occur at a non-INEL site. Judging from the facilities already constructed or planned, the offsite facility is assumed to be a variant of Subalternative $1 \mathrm{E}$. However, the basic construction of the facility would have been completed already for the disposal of waste from the host site or from other DOE sites. Only the additional disposal units required for the INEL waste would have to be constructed. Therefore, the expected number of construction accidents is reduced compared with that for Subalternative $1 \mathrm{E}$.

Alternative 4 involves combinations of the other alternatives. The expected numbers of construction accidents would reflect the numbers for the constituent alternatives.

Alternative 5 involves construction of a considerable number of Butler-type storage buildings on concrete pads. The construction is fairly substantial in magnitude but routine in nature. However, Alternative 5 also requires the construction of several massive concrete structures, like those in Subalternative $1 \mathrm{~A}$, for storing the remote-handled waste. Therefore, an intermediate number of construction accidents would be expected.

Based on the above comparisons, a rough judgmental ranking of the alternatives was developed. The alternative with the fewest expected construction accidents was awarded 10 points and the alternative with the most was awarded 0 points (see Table 10-2):

Table 10-2. Fewest expected construction accidents ranking.

\begin{tabular}{cc} 
Alternative & Points \\
\hline 1A & 2 \\
1B & 1 \\
1C & 9 \\
1D & 6 \\
1E & 7 \\
1F & 7 \\
1G & 5 \\
$1 \mathrm{H}$ & 4 \\
2 & 0 \\
3 & 7 \\
$4 \mathrm{~A}$ & 10 \\
$4 \mathrm{~B}$ & 3 \\
5 & 8 \\
\end{tabular}




\subsubsection{Safety and Health During Operations and Closure}

Safety and health during operations and closure are addressed here in three parts: (a) accidental releases of radionuclides, (b) routine radiation exposure, and (c) industrial accidents.

10.5.2.1 Accidental Releases of Radionuclides. It is assumed that the likelihood of releases during linal closure and cover emplacement is small compared with that during the wasteemplacement phase.

The impacts of radiological releases during operations will tend to increase with increasing radionuclide inventory and mobility. Except for Subalternatives $1 \mathrm{G}$ and $1 \mathrm{H}$, the same waste volume with the same radionuclide inventory and mobility is handled in each alternative. (Refer to the maximum-generation/maximum-treatment scenario in Table 4-5.) Subalternative 1 H involves the same total radionuclide inventory as the other alternatives (maximum-generation scenario); the waste volume is larger, however, because minimum treatment (e.g., minimum-volume reduction) is performed. Because $1 \mathrm{H}$ involves minimum treatment, the mobility of the radionuclides has not been reduced by stabilization. Subalternative $1 G$ (minimum-generation/maximum-treatment scenario) involves approximately one-third the waste volume of the other alternatives. The radionuclide inventory is also reduced, although the fractional reduction in radionuclide inventory may be greater than or less than one-third.

Differences in impacts from accidental releases arise also because of differences in the following items: handling methods, isolation provided by disposal units in their preclosure condition, and the degree of isolation provided by the waste package itself. These three items are discussed in sequence below.

The alternatives and subalternatives do not differ profoundly in waste handling methods. All of them use a forklift to emplace the contact-handled waste containers and either a gantry crane or a boom crane to emplace the remote-handled waste containers. However, two minor differences were judged in the safety of the handling methods. First, emplacement of the remote-handled waste in the concrete structures was judged slightly more likely to experience difficulties than is emplacement in other disposal units because of the possibility of dropping a heavy shielding block on a waste container. Second, concepts involving belowgrade operations are also projected to have a slightly higher frequency of accidents than concepts involving operations at grade level. In addition, Alternative 5 would require reemplacing the waste every 40 years, as new store storage facilities are constructed, and was downgraded on this point.

All of the alternatives and subalternatives provide adequate isolation, prior to final closure, to waste containers that have been emplaced. The isolation affords protection against accidents such as fires, explosions, floods, earthquakes, high winds, and operational errors. Superior isolation is provided by Subalternatives $1 \mathrm{~A}, 1 \mathrm{~B}, 1 \mathrm{G}$, and $1 \mathrm{H}$ because of the massive concrete structures and the presence of a ventilation system with HEPA filters. The disposal methods in Alternatives 1C, 2, 4B, and 5 provide the least protection, assuming that waste containers in Subalternatives $1 \mathrm{D}$ and $1 \mathrm{E}$ are covered frequently after emplacement.

The as-received waste packages are the same in all alternatives and subalternatives except 1C, in which the primary waste containers are grouted into concrete boxes. These containers would be 
highly resistant to breach by dropping or puncture during handling. The containers also would be expected to exhibit a lower release fraction in all types of accidents.

The following simplified Table 10-3 compares the alternatives on the items discussed in this subsection. The terms "std.," "less," and "more" represent a characteristic that is judged to be about the same as, less than, or more than the average. Except for the inventory and mobility differences involving Subalternatives $1 \mathrm{G}$ and $1 \mathrm{H}$, and the enhanced waste package of Subalternative $1 \mathrm{C}$, the differences are generally quite small. A rough relative score was assigned to each concept, based on the foregoing discussion. A score of 10 represents the most favorable alternative on this evaluation. Each increment (e.g., a change from "std." to "less") in the inventory and mobility and packaging columns was assigned a value of four points. Increments in the other columns were assigned a value of two points.

10.5.2.2 Routine Radiation Exposure. The approach for comparing routine radiation exposures to workers for the various alternatives borrows extensively from DOE (1987). In that document, worker doses were evaluated in detail for disposal concepts similar to several concepts in the current study. A standardized methodology developed by the Electric Power Research Institute was used. The methodology is based on a computer model. Waste inventory source terms, packaging information, waste handling flowsheets, worker job classifications (e.g., RCT, crane operator, rigger),

Table 10-3. Accidental release of radionuclide inventory.

\begin{tabular}{llllll}
\hline & \multirow{2}{*}{$\begin{array}{l}\text { Inventory } \\
\text { alternative }\end{array}$} & and mobility & Protection provided by: & & \\
1A & std. & Handling method & Disposal unit & Package & Score \\
1B & std. & less & more & std. & 6 \\
1C & std. & more & more & std. & 4 \\
1D & std. & ave. & less & more & 8 \\
1E & std. & ave. & std. & std. & 4 \\
1F & std. & ave. & std. & std. & 4 \\
1G & better & ave. & more & std. & 6 \\
1H & worse & ave. & more & std. & 10 \\
2 & std. & more & more & std. & 2 \\
3 & std. & ave. & less & std. & 4 \\
4A & std. & less & std. & std. & 4 \\
4B & std. & ave. & more & std. & 4 \\
5 & std. & less & less & std. & 2 \\
\hline
\end{tabular}


and specific operations are input to the model. Worker doses, in person-rem/yr, are then calculated by the model. The worker doses include those arising from operations, from construction of new disposal units during operations, and from closure.

The numerical values for worker doses in DOE (1987) were adjusted to (a) eliminate the doses from certain operational steps not included in the current concepts, and (b) estimate the doses for disposal concepts not addressed in the reference. The results, on a comparative basis, were converted into scores and are given below in Table 10-4. Scores of 10 and 0 were assigned to the concepts having, respectively, the smallest and largest worker dose. For alternatives that are combinations, the estimated dose and score were assumed to be controlled primarily by the concept that would be implemented for the remote-handled waste because most of the operational dose is expected to arise from handling the remote-handled waste.

Alternative 5 was downgraded rather severely based on considerations of routine radiation exposure because stored containe 13 of mixed waste must be inspected weekly, per RCRA regulations in 40 CFR 264.174. The inspectors and other workers are subject to routine radiation exposure indefinitely, not simply during the emplacement of the waste containers.

Table 10-4. Radiation exposure ranking.

\begin{tabular}{cc} 
Alternative & Score \\
\hline 1A & 4 \\
1B & 4 \\
1C & 6 \\
1D & 8 \\
$1 \mathrm{E}$ & 8 \\
$1 \mathrm{~F}$ & 5 \\
$1 \mathrm{G}$ & 5 \\
$1 \mathrm{H}$ & 10 \\
2 & 4 \\
3 & 6 \\
$4 \mathrm{~A}$ & $8^{\mathrm{a}}$ \\
$4 \mathrm{~B}$ & $5^{\mathrm{a}}$ \\
5 & $7^{\mathrm{a}}$
\end{tabular}

a. Score does not reflect the radiation dose during offsite shipment, which is addressed in Section 10.5.3. 
10.5.2.3 Industrial Accidents. Industrial accidents in construction activities during operations were addressed in Section 10.5.1.

Several factors were identified that could tend to increase the frequency of industrial accidents for one alternative compared with that for the other alternatives. The following assumptions and observations resulted from considering those factors:

- The frequency of industrial accidents during operations would tend to be related to the number of personnel required for the operating crew. The operating-crew sizes would be similar for all concepts except for Subalternatives $1 \mathrm{G}$ and $1 \mathrm{H}$, which involve handling different volumes of waste.

- Belowgrade operations would tend to have a slightly higher frequency of accidents than would the corresponding abovegrade operations.

- All of the alternatives and subalternatives involve the use of a crane and forklifts.

- Alternative 5 would require reemplacing the waste every 40 years, as new storage facilities are constructed.

- The likelihood of industrial accidents during closure is related to the size of the final cover over the disposal units.

The following simplified Table 10-5 compares the alternatives on the items discussed above. A rough qualitative score is given for each concept, based on the other entries in the table and the foregoing discussion. A score of 10 represents the most favorable alternative on this evaluation. Each increment (each change from a "yes" to a "no," or from a "std." to a "large") in any of the columns was assigned a value of two points.

Table 10-5. Industrial accidents ranking.

\begin{tabular}{lllll}
\hline Alternative & Crew size & Belowgrade & Cover size & Score \\
\hline $1 \mathrm{~A}$ & std. & no & std. & 6 \\
$1 \mathrm{~B}$ & std. & yes & small & 6 \\
$1 \mathrm{C}$ & std. & no & std. & 6 \\
$1 \mathrm{D}$ & std. & yes & std. & 4 \\
$1 \mathrm{E}$ & std. & yes & std. & 4 \\
$1 \mathrm{~F}$ & std. & yes & std. & 4 \\
$1 \mathrm{G}$ & small & no & small & 10 \\
$1 \mathrm{H}$ & large & no & large & 2 \\
2 & std. & no & std. & 6 \\
3 & std. & yes & std. & 4 \\
$4 \mathrm{~A}$ & std. & no & std. & 6 \\
$4 \mathrm{~B}$ & std. & no & std. & 6 \\
5 & reemplace & no & none & 8 \\
\hline
\end{tabular}




\subsubsection{Safety and Health During Offsite Shipment}

Only the offsite disposal alternative and, to a lesser extent, its combinations with other alternatives include offsite shipment of waste. In Alternative 3, all the waste is shipped offsite for disposal. In Alternative 4, only the mixed waste (about $1 / 3$ of the total posttreatment waste volume) is shipped offsite for disposal.

Shipment of the waste to the disposal facility will be conducted in accordance with the packaging and transport regulations of NRC, EPA, and DOT, as well as DOE and DOE-ID Orders 5480.3. Nevertheless, oflsite shipment will involve a residual risk of accidental releases during shipment. The risk will be small, but the publicity accompanying even a minor incident would be unfavorable. In addition, the public located along the shipping route and the drivers of vehicles carrying waste shipments will receive small routine exposures to radiation during shipment. The exposures are not expected to exceed the regulatory limits.

Based on the above comparisons, a very rough ranking was developed for the alternatives, from the ones with the fewest expected impacts from shipping (awarded 10 points) to the one with the most impacts ( 0 points). Scores of 7 points were assigned to the combination alternatives $4 \mathrm{~A}$ and 4B because only $1 / 3$ of the total waste would be shipped in those alternatives (see Table 10-6).

One net result of the alternatives involving offsite disposal is to shift the risk from the onsite INEL workers to the workers at the of tsite facility (and the transport vehicle drivers and public along the shipping route).

\subsection{Overall Conclusions}

\subsection{1 "Musts"}

One of the "musts" listed in Table 6-1 applies to this section:

- Present acceptable risk to the workers, the public, and the environment during the construction and operations phases.

The conclusion of this overview evaluation is that, for each of the alternatives, the 1 isk will be acceptable (in terms of compliance with the applicable DOE Orders) and the "must" requirement will be satisfied.

Table 10-6. Offsite shipment ranking.

Alternative Points

$\begin{array}{ll}\text { All except } 3 \text { and } 4 & 10 \\ 4 A \text { and } 4 B & 7 \\ 3 & 0\end{array}$




\subsection{2 "Wants"}

One of the "wants" listed in Table 6-1 applies to this section:

- Minimize risk to the workers, the public, and the environment during the construction and operations phases.

The subjective evaluation of performance on this "want" is as indicated in Table 10-7. (A score of 10 is assigned to the best-performing alternative. Lesser scores as judged appropriate, down to zero, are assigned to the other alternatives.)

The overall scores were obtained by averaging the scores for each alternative on the five rankings given earlier in this section, then normalizing so that the highest score was 10 .

Table 10-7. Subjective evaliation of performance on applicable "wants."

\begin{tabular}{ll} 
Alternative & Score \\
\hline $1 \mathrm{~A}$ & 6 \\
$1 \mathrm{~B}$ & 6 \\
$1 \mathrm{C}$ & 9 \\
$1 \mathrm{D}$ & 7 \\
$1 \mathrm{E}$ & 8 \\
$1 \mathrm{~F}$ & 7 \\
$1 \mathrm{G}$ & 10 \\
$1 \mathrm{H}$ & 4 \\
2 & 8 \\
3 & 6 \\
$4 \mathrm{~A}$ & 6 \\
$4 \mathrm{~B}$ & 7 \\
5 & 5
\end{tabular}




\subsection{References}

DOE (U.S. Department of Energy), 1987, Conceptual Design Report: Alternative Concepts for LowLevel Radioactive Waste Disposal, DOE/LLW-60T, June 1987.

EG\&G Idaho, Inc, 1986, Safety Analysis for the Radioactive Waste Management Complex at the Idaho National Engineering Laboratory, WM-PD-86-011, Rev. 2, July 1986.

National Safety Council, 1992, Accident Facts, 1992 edition. 


\section{INSTITUTIONAL EVALUATIONS}

\subsection{Introduction}

Implementation of an alternative for disposal of INEL mixed waste and LLW would involve interfacing with various institutions. Ideally, the selected alternative would minimize potential interfacing problems. The purpose of this section is to identify institutional issues that could negatively impact the implementation of a disposal alternative and to compare each disposal alternative on the basis of potential institutional problems.

An institution is defined as an organization or an establishment devoted to the promotion of a particular purpose. An institutional issue is defined as a point, matter, or dispute, the outcome of which is of special importance to an institution.

The institutions discussed in these evaluations are considered to be "stakeholders" because they would have an interest in, and would be involved with, the implementation of a particular disposal alternative. The evaluations in this section relate to interfaces with the following high-level institutions:

- $\quad$ EPA Region 10

- State of Idaho

- Local citizen groups

- Indian tribes

- Other states

- Local labor unions

- Environmental groups.

Although the listed institutions include regulatory agencies, the intent of this section is not to discuss regulators and compliance issues. Regulatory compliance issues are addressed in Section 7 and Section 9.

It is difficult to assess all the potential institutional issues that could be a problem in the future. Therefor 2 , these evaluations are only preliminary observations based on engineering judgment and on interviews with experienced individuals, and are not provable conclusions.

\subsection{Criteria}

Few regulatory requirements relate to the interface between high-level institutions. Nevertheless, potential interfacing problems can significantly impact the implementation of an alternative. 
Institutional issues of two types could arise. One type includes the issues of the "mechanics" category. These issues could impact the implementation of an alternative because an institution might not have the ability, procedures, or resources to deal with the potential impacts (e.g., inadequate regulatory personnel for reviewing permit applications). No major issues of this type are expected. They are therefore not considered further.

The other type of issue relates to the emotional, technical, and political aspects of implementing an alternative. These "acceptance" issues could impact the implementation of an alternative because of an overall dislike of a disposal alternative and the resulting impacts (e.g., a negative public perception of a particular disposal method).

\subsection{Evaluations of Alternatives Against Criteria}

Table 11-1 summarizes the institutional interface-related observations that can be made, based on the criteria in Section 11.2. The marked interfaces indicate the potential for a negative impact (either in Idaho or in the state where the offsite disposal facility would be located) on the implementation of a disposal alternative due to institutional issues related to acceptance. The interfaces not marked indicate that no potential negative impact was identified.

\subsubsection{Construct and Operate MLLWDF}

Potential institutional issues associated with implementation of the MLLWDF disposal alternative relate to lack of acceptance by several institutions. The State of Idaho, local citizens groups, Indian tribes, and environmental groups may not readily accept construction and operation of a new disposal facility for mixed waste and LLW. All parties might be concerned with the potential environmental impacts due to operating the disposal facility. They might also assume that a new facility with excess disposal capacity could be used to dispose of waste generated at other DOE sites, making Idaho a "national DOE waste disposal center."

Indian tribes could be concerned with construction in previously undisturbed areas and the potential to disturb sacred lands. This discussion of Indian tribes applies to all other alternatives requiring construction of new facilities in previously undisturbed areas.

For the minimum-treatment subalternative $(1 \mathrm{H})$, issues can also be expected to develop with State, local, tribal, and environmental groups because LLW would be disposed of without prior treatment. Disposal without treatment might be viewed negatively because it could increase the potential for adverse environmental impacts and increases the required disposal capacity. The maximum-treatment subalternatives $(1 \mathrm{~A}, 1 \mathrm{~B}, 1 \mathrm{C}, 1 \mathrm{D}, 1 \mathrm{E}, 1 \mathrm{~F}, 1 \mathrm{G})$ would not have these added issues.

Those subalternatives $(1 \mathrm{D}, 1 \mathrm{E}, 1 \mathrm{~F})$ using conventional, nonengineered disposal technologies are expected to receive additional criticism by institutional groups. Even those disposal facilities using highly engineered disposal technologies in the commercial world have had negative encounters with institutional groups (The Radioactive Exchange 1992).

The institutional issues associated with Subalternative 1G (low waste volume) are expected to be the same as those previously discussed. The lower volume of waste, however, will require less disposal capacity and would therefore be preferred. 
Tabie 11-1. Institutional interface-related observations for disposal of INEL mixed waste and LLW.

\begin{tabular}{|c|c|c|c|c|c|c|c|c|}
\hline $\begin{array}{l}\text { Alternative or } \\
\text { suhalternative }^{\mathbf{a}}\end{array}$ & $\begin{array}{l}\text { State of } \\
\text { Idaho }\end{array}$ & $\begin{array}{l}\text { Local } \\
\text { citizens } \\
\text { groups }\end{array}$ & $\begin{array}{l}\text { Indian } \\
\text { tribes }\end{array}$ & $\begin{array}{c}\text { EPA } \\
\text { Region } 10 \\
\end{array}$ & DOT & $\begin{array}{l}\text { Other } \\
\text { states }\end{array}$ & $\begin{array}{c}\text { Local labor } \\
\text { unions }\end{array}$ & $\begin{array}{c}\text { Environmental } \\
\text { groups }\end{array}$ \\
\hline \multicolumn{9}{|l|}{ MLLWDF } \\
\hline Subalt. $1 \mathrm{~A}$ & $\mathbf{x}$ & $\mathbf{x}$ & $\mathrm{x}$ & - & - & - & - & $\mathbf{x}$ \\
\hline Subalt. 1B & X & $\mathbf{X}$ & $\mathrm{x}$ & - & - & - & - & $\mathbf{x}$ \\
\hline Subalt. 1C & X & $\mathbf{x}$ & $\mathbf{X}$ & - & - & - & - & $\mathbf{x}$ \\
\hline Subalt. 1D & $\mathbf{X}$ & $\mathbf{X}$ & $\mathbf{x}$ & - & - & - & - & $\mathbf{x}$ \\
\hline Subalt. 1E & $\mathbf{x}$ & $\mathbf{X}$ & $\mathbf{x}$ & - & - & - & - & $\mathbf{x}$ \\
\hline Subalt. 1F & $\mathbf{x}$ & $\mathbf{x}$ & $\mathbf{x}$ & - & - & - & - & $\mathbf{x}$ \\
\hline Subalt. 1G & $\mathbf{x}$ & $\mathbf{x}$ & $\mathbf{x}$ & - & - & - & - & $\mathbf{x}$ \\
\hline Subalt. $1 \mathrm{H}$ & $\mathbf{x}$ & $\mathbf{x}$ & $\mathbf{x}$ & - & - & - & - & $\mathbf{x}$ \\
\hline Continue RWMC & $\mathbf{x}$ & $\mathrm{X}$ & $\mathbf{X}$ & - & - & - & - & $\mathbf{x}$ \\
\hline Offsite & $\mathbf{x}$ & $\mathbf{x}$ & $\mathbf{x}$ & - & $\underset{\text { (minor) }}{\mathrm{X}}$ & $\mathbf{x}$ & $\mathbf{X}$ & $\mathbf{x}$ \\
\hline MLLWDF and offsite & $\mathbf{x}$ & $\mathbf{x}$ & $\mathbf{x}$ & - & $\underset{\text { (minor) }}{\mathrm{X}}$ & $\mathbf{X}$ & $\mathbf{X}$ & $\mathbf{X}$ \\
\hline RWMC and offsite & $\mathbf{x}$ & $\mathbf{x}$ & $\mathbf{x}$ & - & $\underset{\text { (minor) }}{\mathrm{X}}$ & $\mathbf{x}$ & $\mathbf{x}$ & $\mathbf{x}$ \\
\hline No action & $\mathbf{x}$ & $\mathbf{X}$ & $\mathbf{x}$ & $\mathbf{x}$ & - & - & - & $\mathbf{x}$ \\
\hline $\begin{array}{l}X=\text { Institutional issues } n \\
\text { a. Description of subalter }\end{array}$ & $\begin{array}{l}\text { ively impact } \\
\text { ves is found }\end{array}$ & $\begin{array}{l}\text { an alterna } \\
\text { Section } 5 .\end{array}$ & ve due to & otential difficr & ties with "a & eptance." & & \\
\hline
\end{tabular}




\subsubsection{Continue Disposal at RWMC}

Potential issues associated with implementation of the RWMC alternative relate to lack of acceptance by several institutions. The institutional issues associated with this alternative are expected to be similar to those discussed above under the MLLWDF alternative. The State of Idaho, local citizens groups, Indian tribes, and environmental groups might not readily accept continued operation of the RWMC. All parties might be concerned with the potential environmental impacts from extending the life of the disposal facility, based on a controversy concerning the adequacy of past disposal practice at the RWMC and on the perception that future practices might not be substantially improved in concept.

\subsubsection{Offsite Disposal}

Shipment of mixed waste and LLW offsite could raise issues with most institutions. This alternative could receive considerable criticism by State, local, tribal, and environmental groups. Transportation of radioactive waste is often perceived as dangerous due to a lack of accurate information and an abundance of misinformation available to the public. DOE public affairs organizations would be necessary in the State of Idaho and other states along the transportation route to address transportation issues.

Transportation of mixed waste and LLW across Indian lands could create institutional problems. Indian tribes might be concerned with the potential environmental, health, and safety risks of transporting these wastes to an offsite disposal facility. Transportation-related uribal concerns will need to be addressed and cooperative agreements established.

Institutional issues involving local labor unions could potentially disrupt implementation of the offsite alternative. Shipping wastes offsite for disposal would reduce the INEL labor force currently involved in waste disposal. This labor force reduction could cause union grievances.

Shipment of LLW and mixed waste will require interface with the DOT to ensure conformance to packaging and shipping requirements. Minor institutional issues could be expected; however, no insurmountabie problems are anticipated.

\subsubsection{Combinations}

The institutional issues expected in implemienting the selected combinations of alternatives would generally correspond to the institutional issues associated with the individual contributing alternatives. However, the combined alternatives remove the institutional issues associated with the need for disposal of mixed wastes at the INEL, as required under the onsite disposal alternatives.

For brevity, the two combinations selected for study are not evaluated here in detail. The conclusions for the combinations of alternatives are given in Section 11.4.

\subsubsection{No Action}

Potential institutional issues associated with the no-action disposal alternative would be expected to be the greatest among the disposal alternatives. The State of Idaho, EPA Region 10, local citizens 
groups, Indian tribes, and environmental groups are not likely to accept a position of "no action" and indefinite waste storage at the INEL. These parties are likely to feel that the Federal government produced the waste and, therefore, should assume full responsibility for adequate and safe treatment and disposal.

\subsection{Overall Conclusions}

\subsection{1 "Musts"}

One of the "musts" listed in Table 6-1 applies to this section:

- $\quad$ Present no insurmountable institutional problems.

The conclusion of this overview evaluation is that the no-action alternative would present insurmountable institutional problems. (Insurmountable is defined as opposition so strong that the project will not be able to proceed.) Disposal capability for mixed and LLW would not be present under this alternative, requiring indefinite storage of these wastes. The institutional issues associated with the lack of DOE's assuming responsibility for adequately disposing of its wastes are expected to be insurmountable. This alternative fails this "must" criterion. All other alternatives would satisfy this requirement.

\subsection{2 "Wants"}

One of the "wants" listed in Table 6-1 applies to this section:

- Minimize potential institutional problems.

The subjective evaluation of performance on these "wants" is as follows in Table 11-2. (A score of 10 is assigned to the best-performing alternative. Lesser scores as appropriate, down to zero, are assigned to the other alternatives.)

The rationale for the scores, by reference to Table 11-2, is as follows:

The score of the MLLWDF subalternative $1 G$ was left at 10 because its institutional issues were judged to be the smallest. This disposal alternative uses engineered disposal technologies to provide maximum confinement. Under this alternative, all waste is treated, providing a stable waste form and maximum volume reduction.

The score for the MLLWDF, Subalternatives 1A, 1B, 1C, 1D, 1E, 1F, and $1 \mathrm{H}$ were all reduced by one because the high-volume option is considered here. Subalternative $1 \mathrm{H}$ was further reduced by two because the minimum-treatment option is considered. Subalternatives 1D, 1E and 1F were further reduced because the disposal technologies considered included those without engineered barriers. Subalternative $1 \mathrm{E}$ was then reduced to 5 because of the potentiai for institutional problems associated with shallow land burial. 
Table 11-2. Subjective evaluation of performance on applicabie "wants."

Alternative

MLLWDF

1A-AGES

1B-BGES

$1 \mathrm{C}-\mathrm{CM}$

1D-GDB/GDBH

1E-SLB/SBH

1F-SLB/AGES

1G-AGES/low waste volume

1H-AGES/maximum treatment

Continue RWMC

Offsite

Combinations

MLLWDF/offsite

5

RWMC/offsite

No action
Minimize Potential Institutional Problems
9

9

9

7

5

7

10

7

6

3

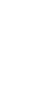

.

列

.

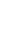


The scores of the combinations of alternatives were reduced according to the strengths and weaknesses of their constituent parts. However, the combined alternatives remove the need for onsite disposal of mixed wastes, decreasing the potential for related institutional issues.

The no-action alternative involves insurmountable institutional issues associated with indefinite storage of INEL mixed waste and LLW and was rated the lowest of the alternatives (a zero). 


\subsection{References}

The Radioactive Exchange, 1992, "Illinois Siting Commission Slammed in Analysis by Martinsville Geologist," The Radioactive Exchange, Volume 11, No. 20, November 16, 1992. 


\section{SCHEDULE EVALUATIONS}

\subsection{Introduction}

This section identifies the requirements and criteria affecting the schedule for each alternative. Alternitives are compared on the basis of the date when operations are expected to start, as well as uncertiinties in those dates. It is noted that implementation of an alternative would involve integration of the regulatory compliance activities with design, construction, and operation phases of the project. This section includes timelines for each alternative that show the anticipated dates for the various stages of design and construction, as integrated with NEPA, siting, permitting, and safety analysis processes.

\subsection{Requirements and Criteria}

The major requirements and criteria that relate to schedule development for DOE programs and projects are found in several DOE Orders and Federal and State regulations. The requirements prohibit certain design, construction, and startup activities from proceeding until certain regulatory steps have been completed, as discussed below.

DOE Orders 4700.1 and $6430.1 \mathrm{~A}$ require that safety and environmental protection be considered in program planning and in the acquisition of projects. Specifically, these orders require that a preliminary safety analysis report (PSAR) be initiated during the conceptual design phase of a prrject and further developed during preliminary (Title I) design and detailed (Title II) design phases. The PSAR must be completed and approved prior to the start of construction. The final safety analysis report (FSAR) must be completed and approved prior to the initiation of facility operations.

In order to meet the requirements of DOE Order 5440.1D, DOE Order 4700.1, and the appropriate implementing DOE-ID Orders, the environmental effects of all proposed Federal actions must be identified and assessed in the early planning stages of a project. NEPA approval is required before resources are unalterably committed and before work commences to implement the proposed action. This generally means that NEPA document approval should occur prior to Title II design.

No physical construction of a new RCRA facility at the INEL will begin without having a RCRA permit, and no facility modification can be made before the modified permit is approved. Detailed design information is required for the RCRA Part B permit application. The new or modified permit application must therefore be submitted during the Title II design phase.

State of Idaho regulations require that a PTC be obtained prior to construction or modification of any stationary source, major facility, or major modification. In order to perform the analysis for a permit to construct/prevention of significant deterioration (PTC/PSD) permit, some Title II design parameters would be required. The analysis could be performed as soon as Title II design is approved or as soon as the needed Title II design parameters are bounded. Construction must then begin within 1 year after obtaining the PTC. 
Construction or modification of an emissions source of radionuclides may not commence until either a NESHAP approval to construct has been issued by the EPA, or the application meets all the NESHAP requirements and an approval to construct is not required. NESHAP documentation preparation could start as soon as the specificd Tille ll design parameters are complete or as soon as the needed Title II design parameters are bounded. The NESHAP analysis must be submitted to the State of Idaho, along with the PTC/PSD application. This means the NESHAP analysis must be approved in time to obtain the Idaho PTC/PSD.

For most projects of this magnitude, the critical path on the schedule would thus include (a) the NEPA process, (b) the NESHAP/PTC/PSD process, (c) the RCRA permitting process, and (d) the process for obtaining line item funding. Thus, the traditional design, construction, and startup steps constitute only part of the critical path.

\subsection{Evaluations of Alternatives Against Requirements/Criteria}

Based on the foregoing discussion of requirements, schedules for the alternatives were developed and are shown in Figures 12-1 through 12-7. The waste disposal alternatives were then evaluated on an overview basis to determine which alternative would begin production operations in the least amount of time and present the least amount of uncertainty.

Surveys of project TDDs, environmental compliance plans, and interviews with project management, permitting, safety analyses, and facility experts provide the basis for the dates.

\subsubsection{Construct and Operate the MLLWDF}

The MLLWDF Subalternative $1 \mathrm{~A}$ is used as the baseline against which other alternatives are compared. For all alternatives, and subalternatives except for $1 \mathrm{G}$ and $1 \mathrm{H}$, the large-generation and maximum-treatment scenario applies.

12.3.1.1 Subalternative 1A: MLLWDF operations for Subalternative 1A are estimated to begin in the fourth quarter of FY 2005 (Figure 12-1). The estimated time for preconceptual studies, design activities, and technology verification is based on project management knowledge of current studies and projections of future activities, derived from experience and engineering judgment.

The schedule for construction activities is based on project management and construction management experience and consideration of the extensive CQA required for the concrete structures. The scheduled time for construction (represented by the solid black line in Figure 12-1) includes construction of all the support facilities, monitoring systems, and enough disposal structures to hold approximately 10 years capacity of waste. The disposal units constructed during the initial phase would include four Class $\mathrm{A}$ and Class $\mathrm{C} \propto$ disposal units, two Class $\mathrm{A}$ mixed disposal units, one Class $B$ and Class $C \beta-\gamma$ disposal unit, one Class $C \alpha$ mixed disposal units, and one Class B and Class $C \beta-\gamma$ mixed disposal unit. The dotted black line for construction in the figure represents ongoing construction of additional disposal units as required. 


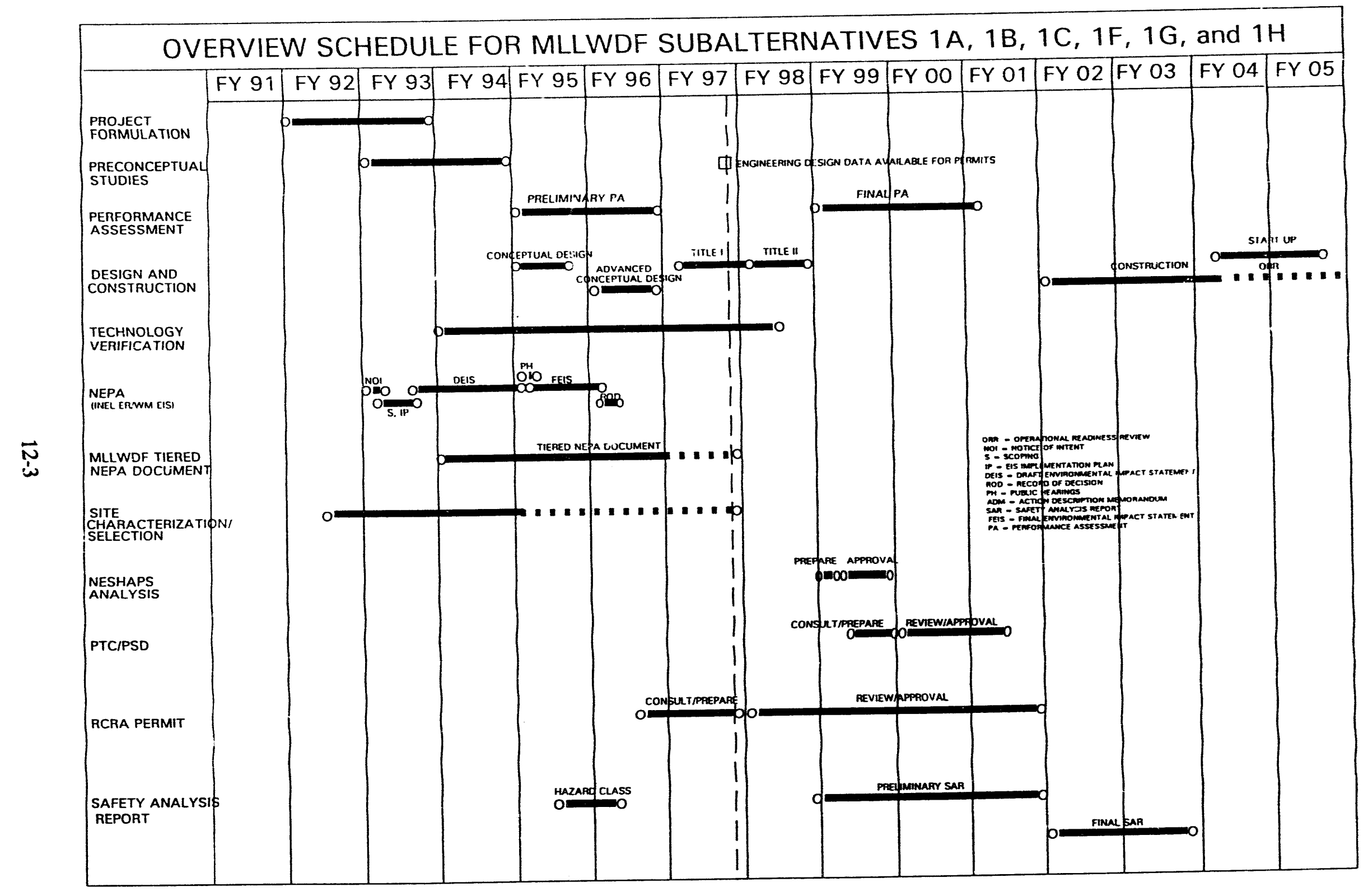

Figure 12-1. Overview schedule for MLLWDF Subalternatives 1A, 1B, 1C, 1F, 1G, and 1H. 


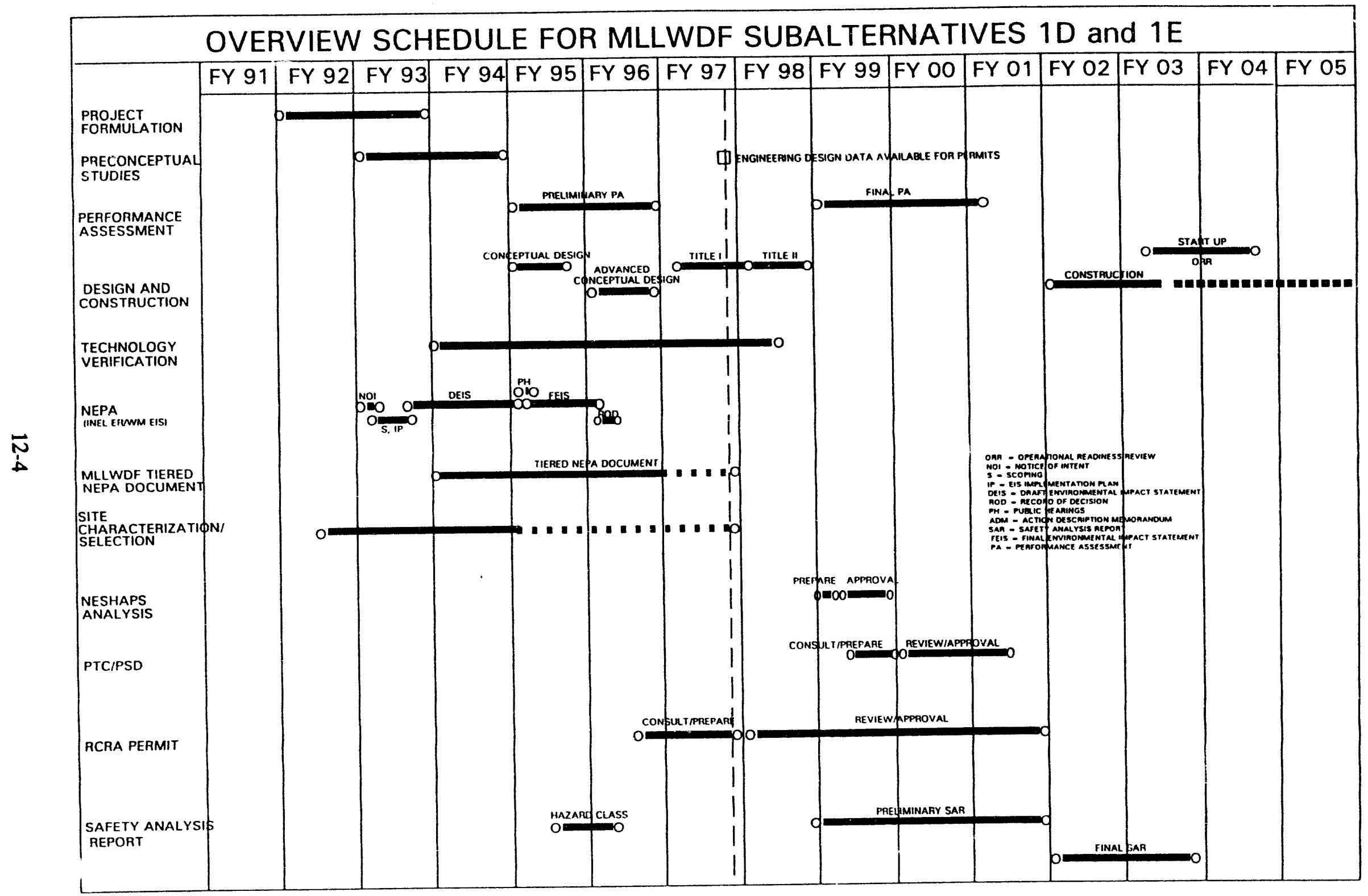

Figure 12-2. Overview schedule for MLLWDF Subalternatives $1 \mathrm{D}$ and $1 \mathrm{E}$. 


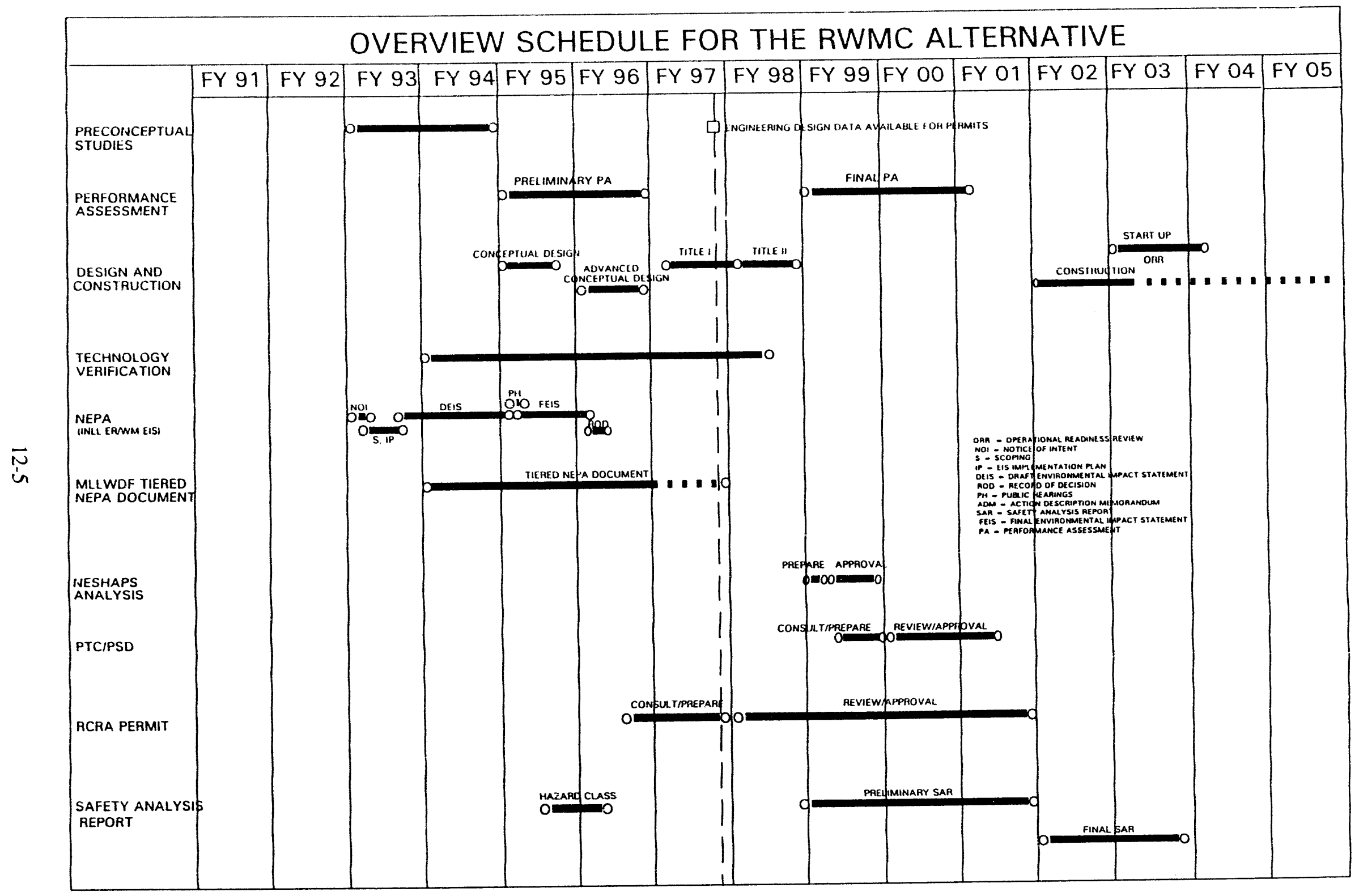

Figure 12-3. Overview schedule for the RWMC alternative. 


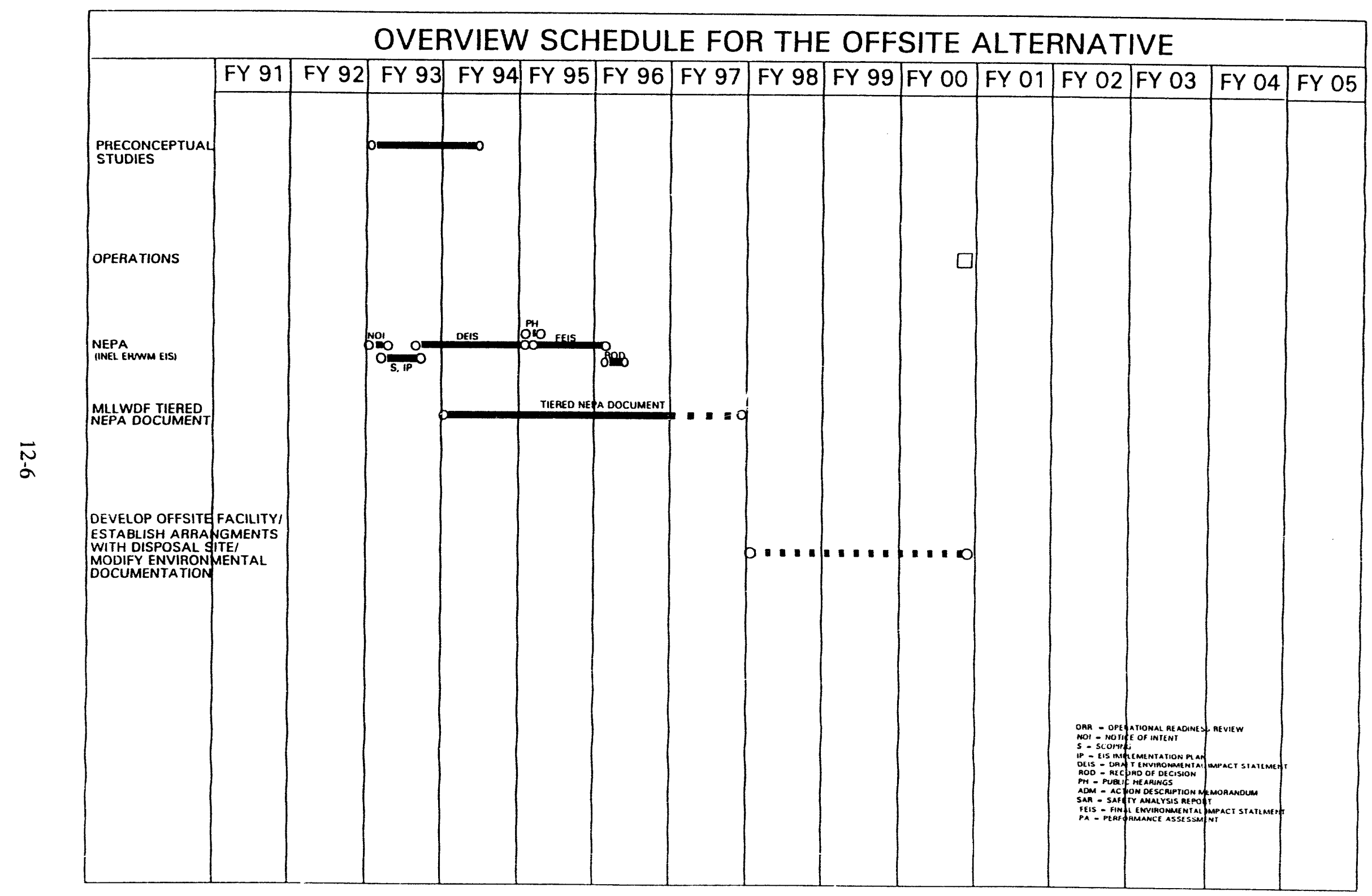

Figure 12-4. Overview schedule for the offsite alternative. 


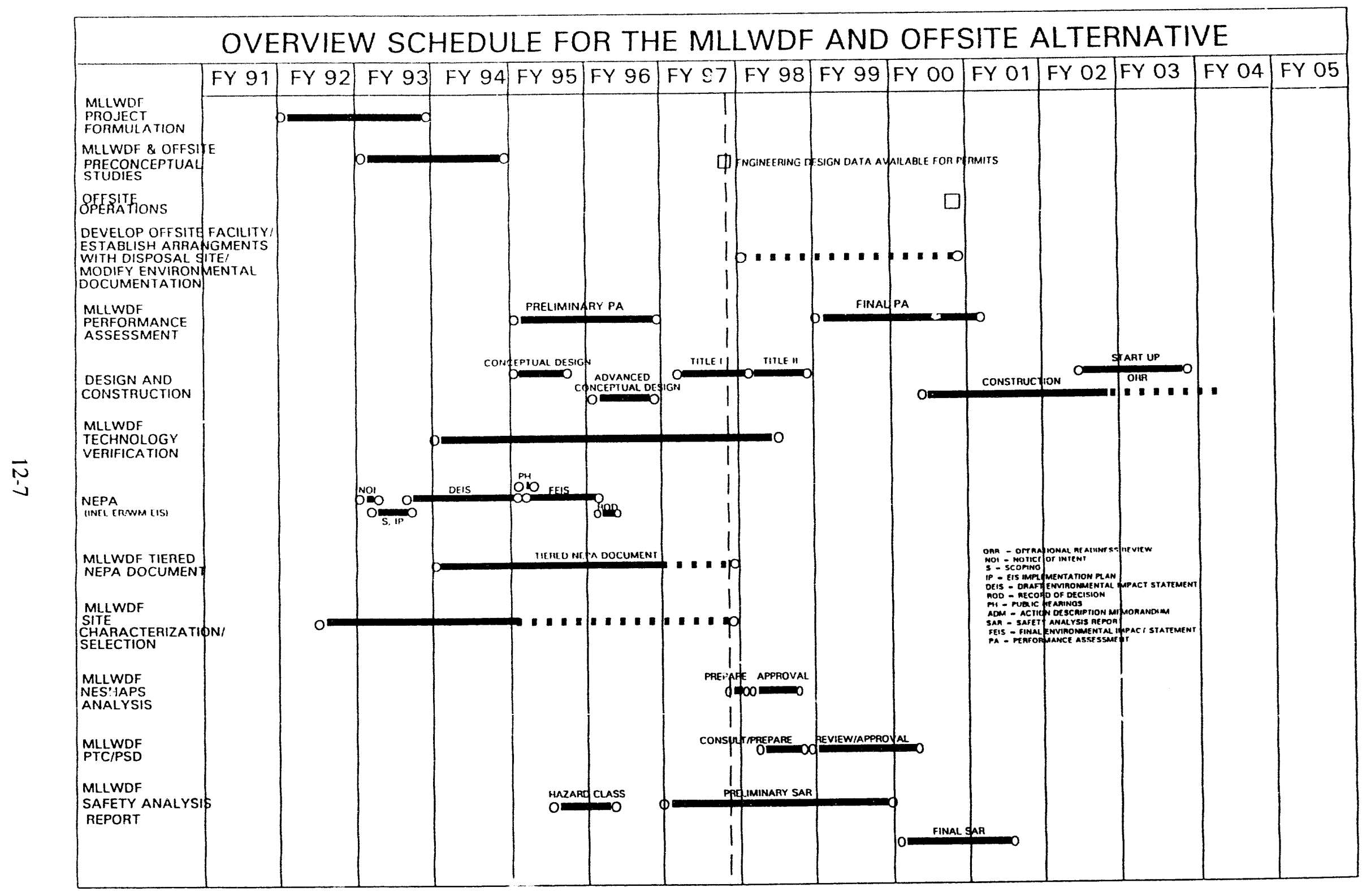

Figure 12-5. Overview schedule for the MLLWDF and offsite alternative. 


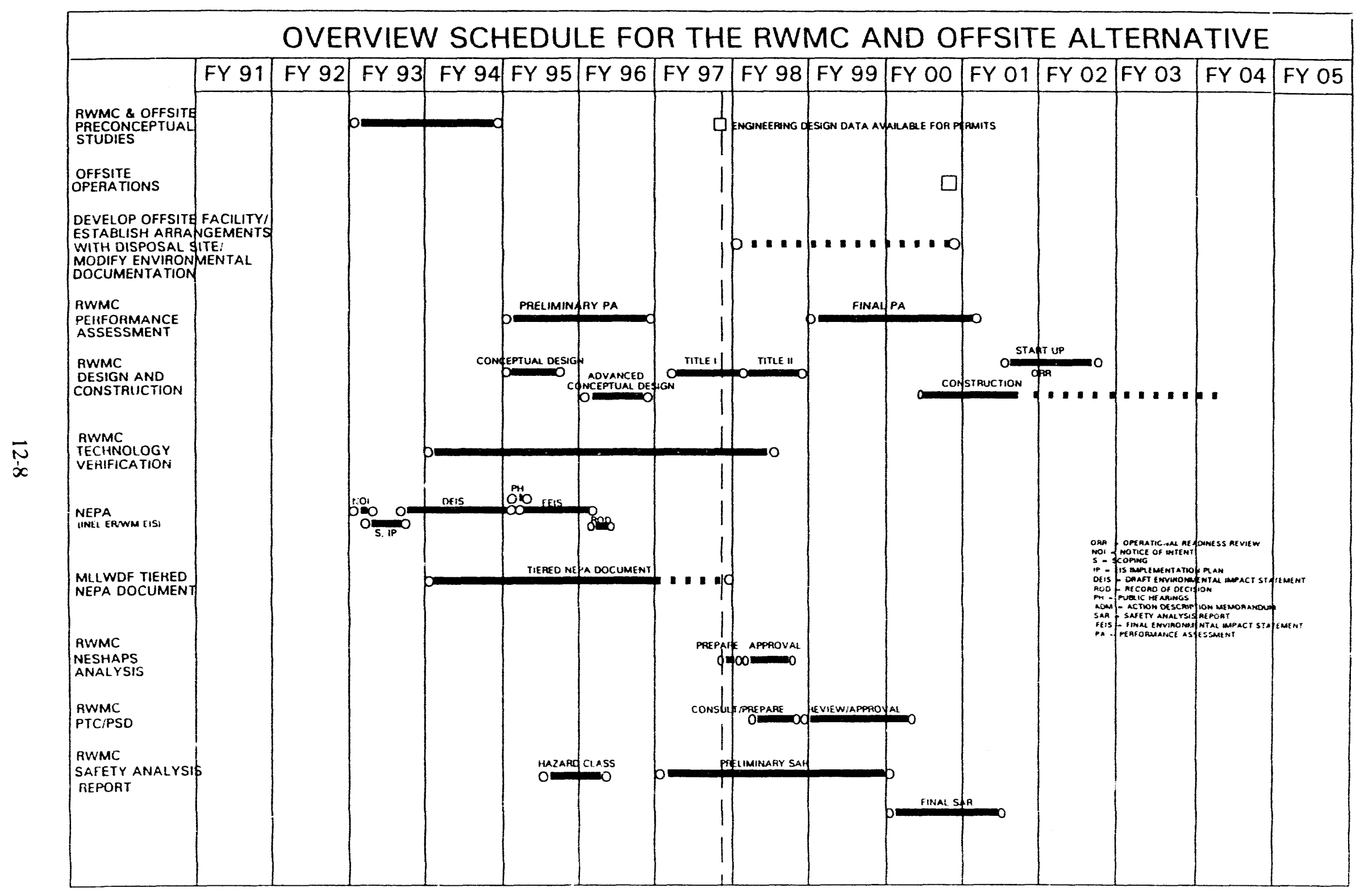

Figure 12-6. Overview schedule for the RWMC and offsite alternative. 


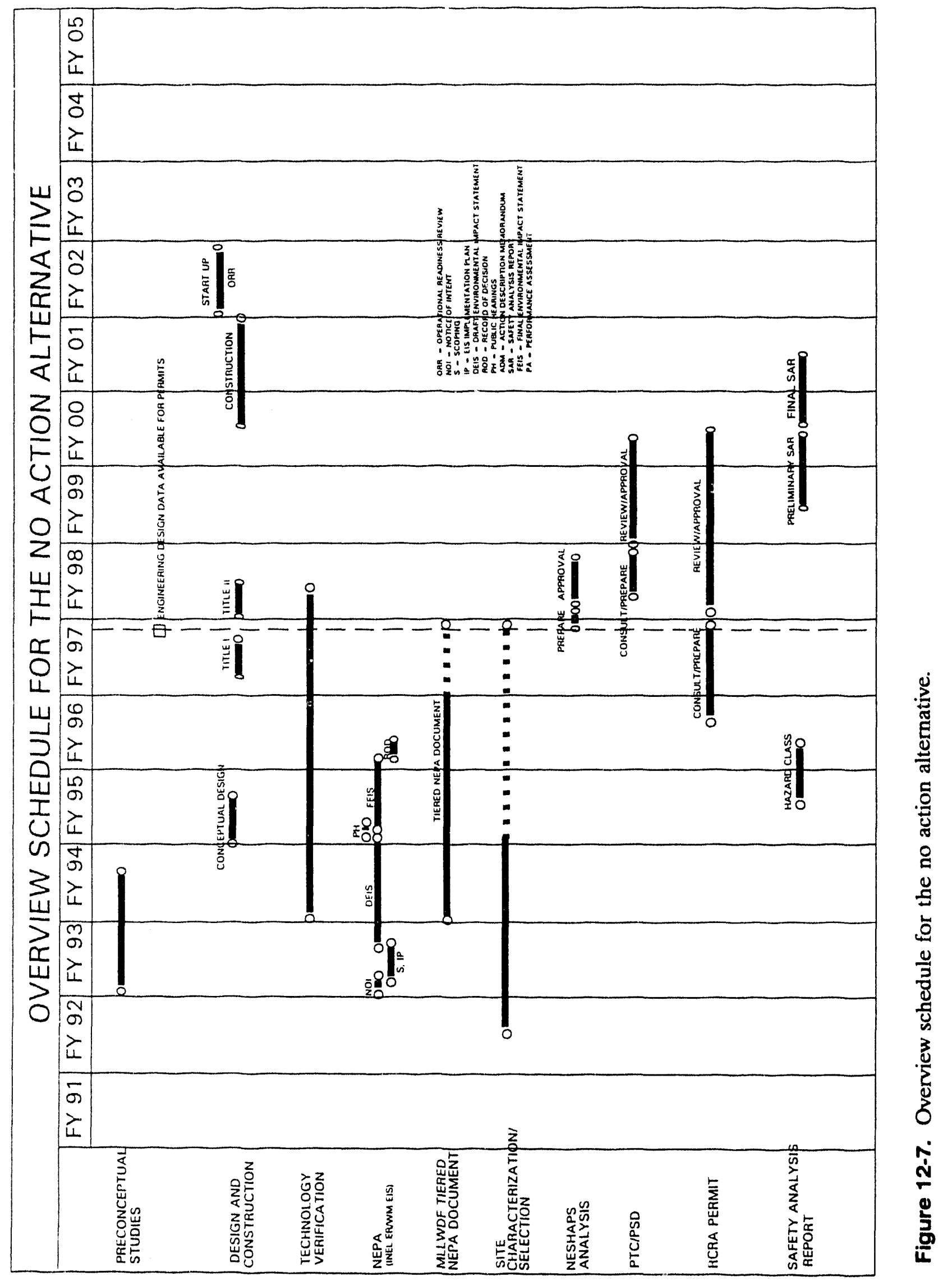


The rationale behind the estimated time for preparation and approval of NEPA documentation is based on the activity schedule for the INEL ER\&WM EIS. Delays in the INEL ER\&WM EIS schedule may delay the ticred NEPA process. A dotted black line is shown to represent the potential for delay and to identify the time available for completion of NEPA prior to the start of Title II design.

Site selection is dependent on the NEPA schedulc. Preliminary siting activities are necessary to determine candidate sites for evaluation in the draft NEPA document. This process is shown by the solid black line in the figure. The preferred site would be determined by the end of the NEPA process and is represented by the dotted black line in the figure. This discussion of NEPA applies to all alternatives and is not repeated.

Permitting and SAR timelines are based on requirements discussed in Scction 12.2, interviews with the permit coordinators, SAR preparation and review experience, and project management experience.

Uncertainty is associated with the MLLWDF schedule due to dependence on the INEL ER\&WM EIS and the evolving NEPA strategy for the MLLWDF. Because the MLLWDF tiered document depends on the INEL ER\&WM EIS, slippage of 1 year or more in the baseline EIS could impact the schedule for start of MLLWDF Title II design. A delay in the start of Title II design would delay the permitting processes and potentially the start of construction.

Uncertainty is associated with the length of time required for the RCRA permitting process. The schedule shows 4 years for the RCRA permit review and approval process. It could, however, take 3 to 5 years for approval. Since the start of construction depends on approval of the RCRA permit, an increase or decrease in the time required for the permitting process would increase or decrease the start of construction and consequently the start date for disposal facility operations.

The schedule for the MLLWDF hinges on the timely approval of key decisions as defined in DOE Order 4700.1, and the justified and supported need for the project. Delays in the approval of these key decisions would delay the disposal alternative schedulc.

Schedule delays may also result due to the potential for problems encountered during the startup phase of the project. These schedule uncertainties for the MLLWDF apply identically to all the onsite alternatives and will not be discussed further here.

12.3.1.2 Subalternatives $1 \mathrm{~B}, 1 \mathrm{C}, 1 \mathrm{~F}, 1 \mathrm{G}$ and $1 \mathrm{H}$ : Operations for Subalternatives $1 \mathrm{~B}, 1 \mathrm{C}$, $1 \mathrm{~F}, 1 \mathrm{G}$, and $1 \mathrm{H}$ are estimated to begin in the fourth quarter of FY 2005 (Figure 12-1), the same as for Subalternative $1 \mathrm{~A}$.

The timelines for all activities are essentially the same for these subalternatives as for Subalternative 1A. All the subalternatives require preparation of the same environmental and safety documentation. The slight variation in disposal concepts among these subalternatives is not expected to significantly impact the time required to complete these activities.

The timelines for preconceptual studies, design and construction activities, and technology verification are the same as for Subalternative $1 \mathrm{~A}$ because all of the disposal concepts include 
engineered structures. The engineered structures are constructed of special reinforced concrete requiring material behavior studies and special CQA requirements. These CQA requirements are expected to make the construction processes slower than normal for these subalternatives.

12.3.1.3 Subalternatives 1D, 1E: Operations for Subalternatives $1 D$ and $1 E$ are estimated to begin in the fourth quarter of FY 2004 (Figure 12-2), 1 year sooner than for Subalternative 1A.

Subalternatives $1 \mathrm{D}$ and $1 \mathrm{E}$ will dispose of the same waste as Subalternative $1 \mathrm{~A}$ and, therefore, are subject to the same environmental and safety requirements. The timelines for preparation and approval of environmental and safe.y documentation are the same as for Subalternative $1 \mathrm{~A}$. The variation in disposal concept is not expected to significantly impact the time required to complete these activities.

The timelines for preconceptual studies and design are also expected to be the same as for Subalternative $1 \mathrm{~A}$. The primary difference in the schedules lies in the time required for construction. Construction of disposal areas in Subalternatives $1 \mathrm{D}$ and $1 \mathrm{E}$ primarily involves soil excavation and no use of concrete structures. Therefore, the time required for construction is estimated at $60 \%$ of that required for Subalternative $1 \mathrm{~A}$.

\subsubsection{Continue Disposal at RWMC}

Disposal operations at the modified RWMC are estimated to begin in the second quarter of FY 2004 (Figure 12-3), 1.5 year sooner than for the MLLWDF Subalternative 1A.

The timelines for most activities are the same for the RWMC alternative as for the MLLWDF Subalternative $1 \mathrm{~A}$ because the proposed changes to the facility are considered significant.

The timelines tor environmental and safety documentation are the same as for Subalternative 1A. The new disposal operations at RWMC will be different in scope from current operations, requiring new permits and new safety documentation.

The time required for preconceptual studies and design of the modified facility are expected to be the same as for Subalternative 1A. It is assumed that the modified RWMC would be a line item construction project and subject to the lengthy funding cycle. Also, the changes anticipated for the RWMC will require design of concrete pads, double liners, and leachate collection systems.

Once again, the primary difference in the schedules lies in the time required for construction. Construction of the RWMC disposal areas is not expected to require as much time as that required for Subalternative 1A. In addition, the RWMC alternative does not require extensive site preparation activities and construction of new support facilities. Therefore, the time required for construction is estimated at $50 \%$ of that required for Subalternative $1 \mathrm{~A}$.

\subsubsection{Offsite Disposal}

Offsite operations are estimated to begin at the end of FY 2000 (Figure 12-4). The estimated operational date is 5 years earlier than for the MLLWDF Subalternative $1 \mathrm{~A}$. 
The projected start of operations is based on informal discussions with personnel involved in development of mixed waste disposal facilities at various DOE sites, and the estimated length of time required to establish arrangements with an offsite facility, to modify the offsite facility to meet INEL waste disposal needs, and to modify and prepare any necessary environmental documentation.

The schedule for this alternative is very uncertain and primarily dependent upon the offsite facility and the NEPA strategy. The uncertainties and ramifications are discussed in Section 14.

\subsubsection{Combinations of Alternatives}

The scheduling requirements and dates expected in achieving operations for the selected combinations of alternatives will generally correspond to the requirements and dates associated with the individual contributing alternatives (Figures $12-5$ to 12-6). A given combination would carry the uncertainties of both alternatives and the operational date of the later alternative. Shipment of mixed waste offsite for disposal, however, eliminates the need for the onsite alternatives to obtain a RCRA permit. This shortens the operational date of the onsite portion of the alternatives by approximately 2 years.

For brevity, the two combinations are not evaluated here in detail. The conclusions for the combinations of alternatives are given in Section 12.4 .

\subsubsection{No Action}

Because the no-action alternative requires indefinite storage of mixed waste, the schedule shown represents construction of a storage facility complex. Storage facility operations are estimated to begin at the end of FY 2002 (Figure 12-7).

\subsection{Overall Conclusions}

\subsection{1 "Musts"}

None of the "musts" listed in Table 6-1 applies to this section.

\subsection{2 "Wants"}

One of the "wants" listed in Table 6-1 applies to this section:

- Minimize the schedule for beginning radioactive operations.

The subjective evaluation of performance on this "want" is ss shown in Table 12-1. (A score of 10 is assigned to the best-performing alternative. Lesser scores as appropriate, down to zero, are assigned to the other alternatives.)

Reductions in scores were applied to the indicated alternatives, based on which alternative is expected to achieve operations first. The degree of uncertainty associated with each disposal alternative schedule correlates with the length of schedule and was not a major factor in the ranking process. 
Table 12-1. Subjective evaluation of performance on applicable "want."

\begin{tabular}{|c|c|}
\hline Alternative & $\begin{array}{l}\text { Minimize schedule for } \\
\text { disposal operations }\end{array}$ \\
\hline \multicolumn{2}{|l|}{ MLLWDF } \\
\hline Subalternative $1 \mathrm{~A}$ & 6 \\
\hline Subalternative 1B & 6 \\
\hline Subalternative $1 \mathrm{C}$ & 6 \\
\hline Subalternative $1 \mathrm{D}$ & 7 \\
\hline Subalternative $1 \mathrm{E}$ & 7 \\
\hline Subalternative $1 \mathrm{~F}$ & 6 \\
\hline Subalternative $1 \mathrm{G}$ & 6 \\
\hline Subalternative $1 \mathrm{H}$ & 6 \\
\hline Disposal at RWMC & 7 \\
\hline Olisite Disposal & 10 \\
\hline \multicolumn{2}{|l|}{ Combinations } \\
\hline MLLWDF/Offsite & 8 \\
\hline RWMC/Offsite & 9 \\
\hline No action & 9 \\
\hline
\end{tabular}

The offsite alternative achieved operations faster than any other alternative and was given a score of 10 . All other alternatives were ranked relative to the offsite alternative. The scores for the combinations of alternatives were reduced according to the date for which the combined alternative would be fully operational. 


\section{COST EVALUATIONS}

\subsection{Introduction}

This section evaluates the ROM life-cycle cost associated with each alternative. The result of the evaluation is a ranking of alternatives on the basis of cost.

Many methods are used to express the cost of a waste-disposal alternative. Not all methods include the same cost elements. The guiding principle used for this cost evaluation was to develop cost estimates that are comprehensive, fair, and on a consistent basis among the disposal alternatives. This principle was followed in determining which cost elements to include in the estimate.

The cost method chosen was the life-cycle cost of each alternative. Life-cycle cost, as defined in DOE Order 4700.1, is

"The sum total of the direct, indirect, recurring, non-recurring and other related costs incurred or estimated to be incurred in the design, development, production, operation, maintenance, support and final disposition of a major system over its anticipated useful life span. Where system or project planning anticipates use of existing sites or facilities, restoration and refurbishment costs should be included."

The life-cycle cost method is the most comprehensive of cost methods.

The life-cycle costs developed herein are the summations of seven categories of costs:

- Project management, siting, environmental permitting, NEPA, safety analyses, technology development, and other "up front" costs

- Design-related costs

- Construction costs, including the building, the equipment, etc. The costs of all required facilities are included

- Startup and readiness review costs

- Operating costs-labor, materials, utilities, etc.

- D\&D costs

- Closure and postclosure costs, including the cover materials and construction and long-term monitoring.

Also included in the total cost are reserves and contingency.

The cost estimates in this section are not of budget quality; they are considered to be of planning quality. The stage of development for all the alternatives is very early. Detailed information about the alternatives is not available, and many simplifying approximations are made. For example, the cover for Alternative 2 does not take into account other covers that may be present at the RWMC as a result of CERCLA remedial actions, and the covers for all alternatives do not consider 
the interpenetration/overlapping of edges and corners of adjacent disposal units. Very limited preconceptual design work has been done. Therefore, only ROM costs can be developed at present.

The costs were estimated by a team composed of personnel who represented cost estimating, process engineering, environmental compliance, and program management. The costs were based primarily on engineering judgment applied to corresponding costs that had been incurred for the RWMC, Stored Waste Examination Pilot Plant (SWEPP), and other similar projects and facilities. Those earlier costs were escalated to 1993 dollars and modified to reflect today's more stringent regulatory environment. In some instances, current costs were used where available from vendors, recent INEL projects, projects at other DOE sites, or other cost studies. The building costs were based on parametric analysis of INEL construction costs on recent projects.

In many instances, determination of equitable costs among the various alternatives was very difficult due to differing implementation schedules, differing compliance levels, and many other factors. Nevertheless, fair comparison of alternatives was the goal of the cost estimates.

\subsection{Requirements/Criteria}

It is required by DOE Order $6430.1 \mathrm{~A}$ that a life-cycle cost estimate, "be performed during site selection for TSD facilities," and that, "design decisions for all projects shall be based on consideration of life-cycle cost." Therefore, for the purposes of this study, life-cycle cost estimates are used for evaluation. No other unique requirements related to the cost of disposal alternatives were identified.

The criterion used in these evaluations is to minimize life-cycle costs.

\subsection{Evaluations of Alternatives Against the Requirements/Criteria}

\subsubsection{Results}

The summarized results of the cost evaluations are presented in Tables 13-1 and 13-2. The life-cycle cost for each alternative is given, as well as the subtotals for the seven cost categories listed and for reserves and contingency.

The costs are given in two forms. One form (Table 13-2) is escalated costs (year-ofexpenditure). This form is used for budget purposes although, as stated earlier, the current estimates are not of budget quality. The other form (Table 13-1) is unescalated costs. The unescalated costs are presented because the costs that are escalated over the long duration of the disposal facility's operating and monitoring life (approximately 140 years) assume enormous proportions and can make meaningful comparisons difficult.

The unescalated costs are graphically displayed in Figure 13-1. Costs from all seven categories are included, as well as reserves and contingency. Decontamination and decommissioning costs are relatively small and are barely visible at the chart scale.

Detailed cost breakdowns are given in Appendix A.

\subsubsection{Bases}

The bases for the cost estimates are given in Appendix A. 


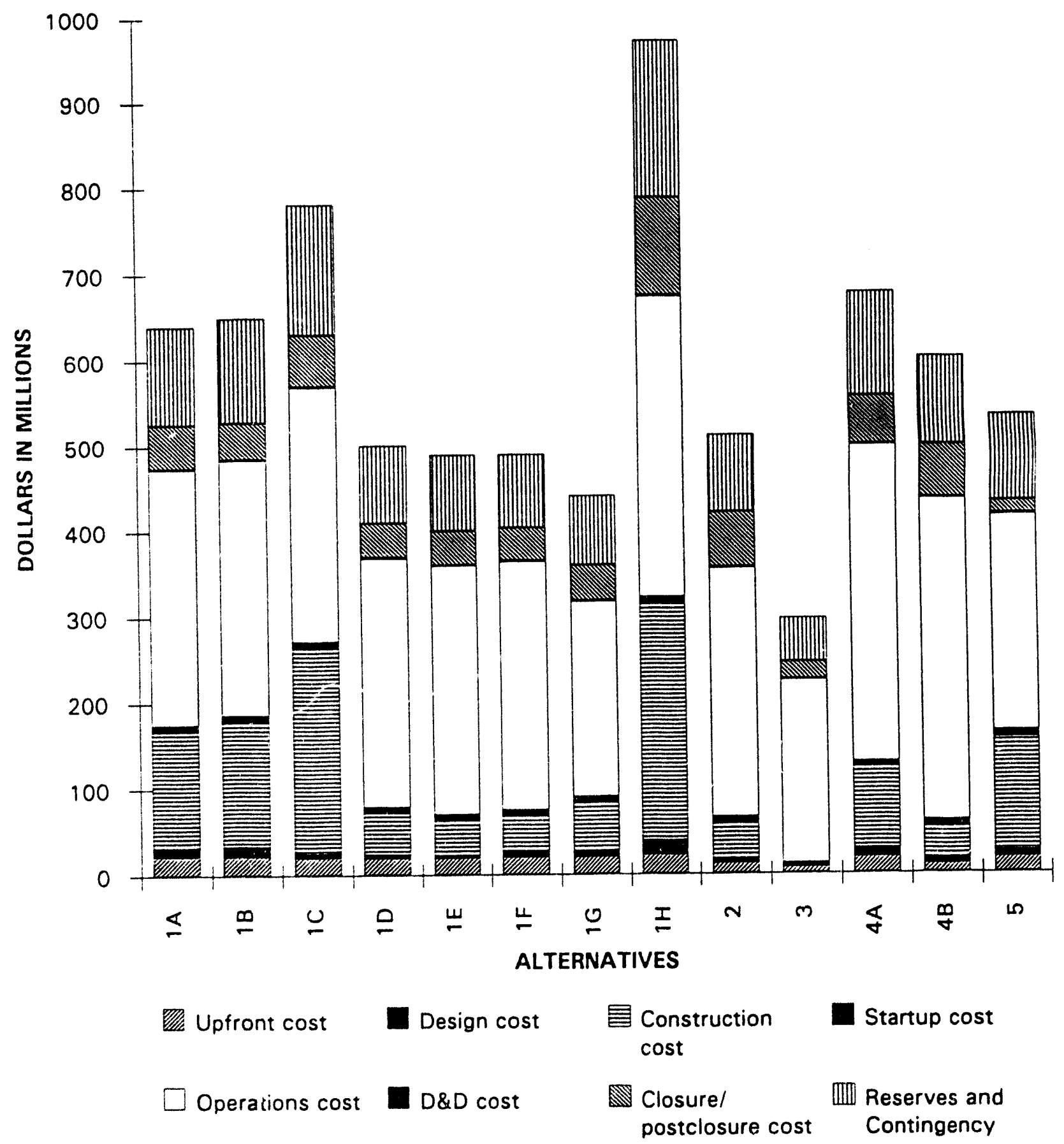

Figure 13-1. Life-cycle costs-unescalated, FY-93 MS. 
Table 13-1. Summary of life-cycle costs for the disposal alternatives-unescalated costs, FY-93 M\$.

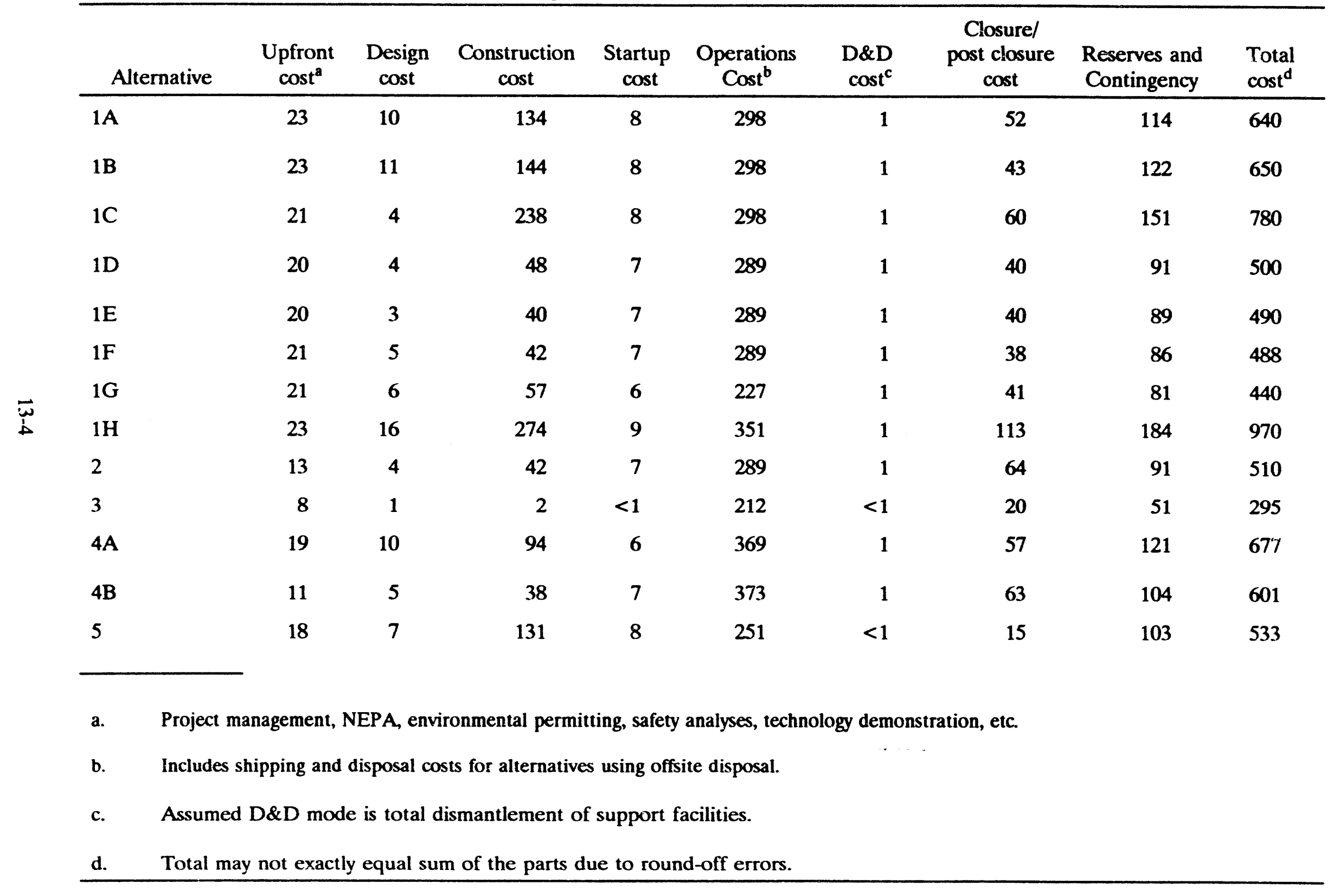


Table 13-2. Summary of life-cycle costs for the disposal alternatives-cost escalated to year of expenditure, M\$.

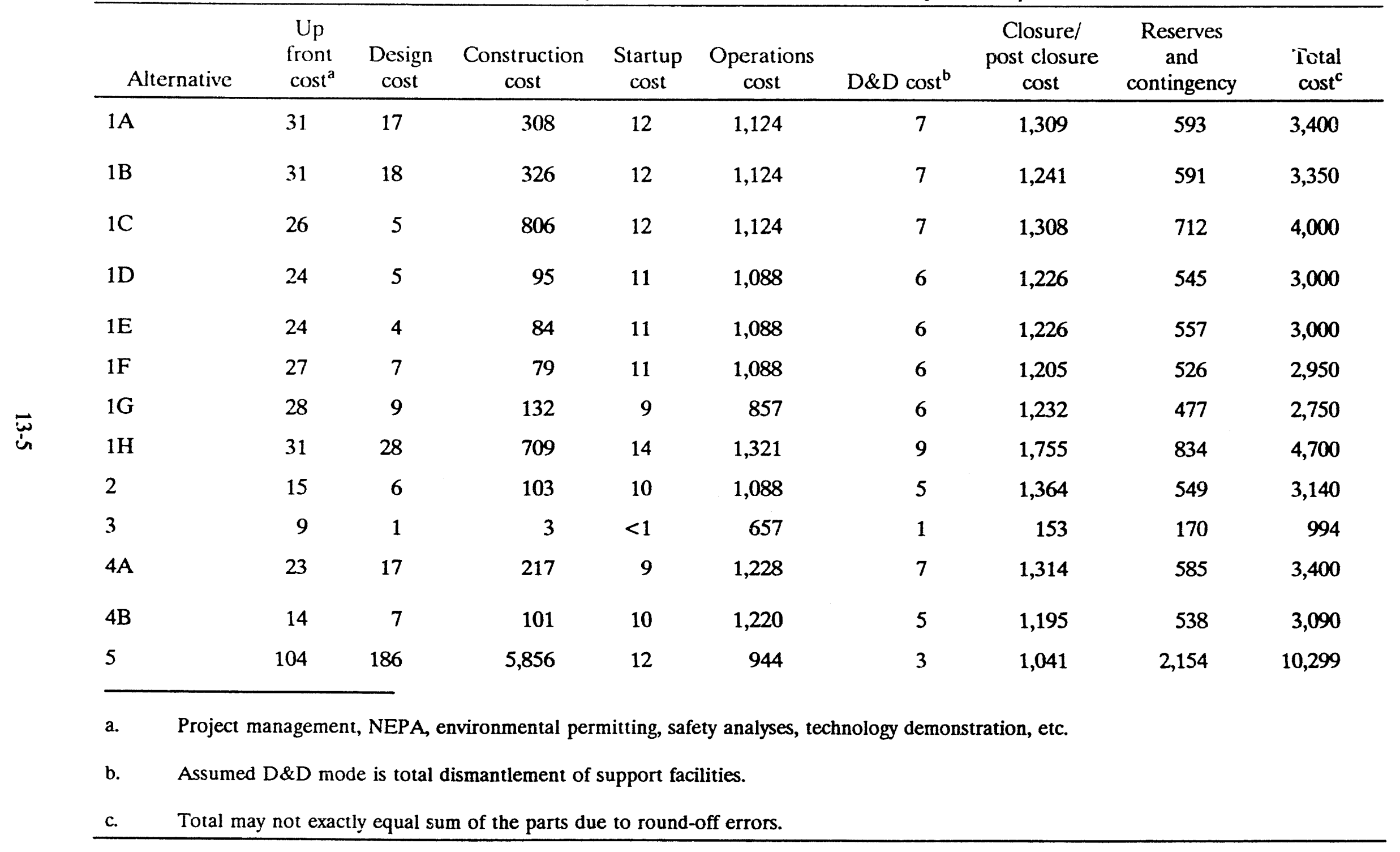




\subsubsection{Discussion of Results}

For simplicity, the results are discussed in terms of comparisons with Subalternative $1 \mathrm{~A}$. In addition, only the results for unescalated costs are discussed. The escalated costs are shown, but are not as useful (see discussion in Section 13.4.3).

Subalternative 1A, AGES, has a total cost of $\$ 640$ million. Not including reserves and contingency, the operations cost, at $\$ 298$ million, is $57 \%$ of the total, the construction cost, at $\$ 134$ million, is $25 \%$ of the total, and the closure/postclosure cost, at $\$ 52$ million, is $10 \%$ of the total. Upfront, design, startup, and D\&D cosıs, at $\$ 42$ million, account for the remaining $8 \%$.

Subalternative 1B, BGES, is essentially the same cost as Subalternative 1A. Construction is slightly larger because of the cost associated with excavation. However, the placement underground results in an offsetting reduction in the cost of the closure cover.

Subalternative 1C, Covered Modules, is $\$ 140$ million more expensive than Subalternative $1 \mathrm{~A}$. The major difference is in the construction cost. While it is $\$ 56$ million cheaper to build the pads than the vaults, the concrete modules cost $\$ 160$ million, for a total increase of $\$ 104$ million. The $\$ 37$ million change in reserves and contingency accounts for most of the rest of the cost difference.

Subalternative 1D, Greater Depth Burial, is $\$ 140$ million less expensive than Subalternative 1A. The primary difference is in the construction cost. Since no vaults must be built, construction is only $\$ 48$ million, which is $\$ 86$ million less than construction in Subalternative $1 \mathrm{~A}$. The operations staff is slightly smaller for this option, resulting in a savings of $\$ 9$ million. Since 1D is a belowground alternative, the closure cover is $\$ 12$ million less expensive. Reserves and contingency cost is $\$ 2.3$ million less.

Subalternative 1E, Shallow Burial, is $\$ 150$ million less than Subalternative $1 \mathrm{~A}$. The discussion for Subalternative 1D is applicable. The cost of construction is slightly less than for Subalternative $1 \mathrm{D}$ because the burial is more shallow.

Subalternative $1 \mathrm{~F}$, a combination of $1 \mathrm{~A}$ and $1 \mathrm{D}$, is $\$ 152$ million less expensive than Subalternative 1A. While the alternative might be expected to cost somewhere in between Subalternative $1 \mathrm{~A}$ and $1 \mathrm{D}$, it was found that disposal using shallow land burial was the least expensive: for Class $\mathrm{A}$ and Class $\mathrm{C} \alpha$ waste, and that disposal using AGES was the least expensive for Class $\mathrm{B}$ and $C \beta-\gamma$ waste. Therefore, the combination of the two ends up being a relatively inexpensive alternative. The primary savings are in construction cost, $\$ 92$ million, operations cost, $\$ 9$ million, closure cover cost, $\$ 14$ million, and reserves and contingency, $\$ 28$ million.

Subalternative $1 G$, the minimum-generation/maximum-treatment scenario, is $\$ 200$ million less expensive than Subalternative 1A. The savings is a reflection of the factor of 3.1 lower volume of waste. There are at least small savings in every category. The major savings are $\$ 77$ million in construction, $\$ 71$ million in operations, $\$ 11$ million in closure/post-closure, and $\$ 33$ million in reserves and contingency.

Subalternative $1 \mathrm{H}$, the maximum-generation/minimum-treatment scenario, is the most expensive alternative. It is $\$ 330$ million more than Subalternative $1 \mathrm{~A}$. The additional expense is a reflection of the factor of 2.3 increase in volume of waste. There is at least a small increase in every 
category. The major increases are $\$ 140$ million in construction, $\$ 53$ million in operations, $\$ 61$ million in closure/post-closure, and $\$ \mathbf{\$ 7}$ million in reserves and contingency. ${ }^{\mathrm{e}}$

Alternative 2, RWMC, is $\$ 130$ million less expensive than Subalternative $1 \mathrm{~A}$. The major savings is $\$ 92$ million dollars in construction cost. The construction of pads versus vaults is the reason for the savings. Note that this alternative does not use concrete modules; therefore, the savings in construction is not offset by the cost of modules as it was in Subalternative 1C.

Alternative 3, offsite disposal, is the least expensive alternative. It is $\$ 345$ million less than Subalternative 1A. This option is similar to $1 \mathrm{E}$, but additional savings are realized because of the large amount of sunk cost that will not be charged for offsite disposal. This sunk cost is primarily a reflection of the cost of employees at the offsite disposal facility whose salaries are already paid (for example, manager, record keepers). Only the additional salaries incurred by the actual waste disposal operations need to be paid. The savings are reduced slightly by the need to build and staff the facility that will package and ship the waste.

Alternative $4 \mathrm{~A}$, the combination of Subalternatives $1 \mathrm{~A}$ and 3 , is $\$ 37$ million more expensive than Subalternative 1A. Some savings are seen in construction and onsite disposal operations cost. However, these savings are offset by the cost of construction and operation of the onsite shipping facility and the charge for shipping to and disposal at the offsite facility.

Alternative $4 \mathrm{~B}$, the combination of Subalternatives $1 \mathrm{~A}$ and 2 , is $\$ 39$ million less expensive than Subalternative $1 \mathrm{~A}$. The savings are a reflection of the benefits gained from using the RWMC as discussed previously.

Alternative 5, no action, is $\$ 107$ million less expensive than Subalternative $1 \mathrm{~A}$. The major cost savings are in operations, a savings of $\$ 47$ million due to reduction of staff, and in closure/postclosure cost, a savings of $\$ 37$ million due to the elimination of a cover. There is no significant savings in construction because, over the lifetime of the alternative, numerous old Butlertype storage buildings must be replaced by similar new storage buildings.

\subsection{Overall Conclusions}

\subsection{1 "Musts"}

None of the "musts" listed in Table 6-1 apply to this section. No absolute limit has been established for the allowable cost of a disposal alternative.

e. Note that Subalternative $1 \mathrm{H}$ involves an indirect effect on the cost of treatment. This indirect effect was not considered in the cost estimate. A previous study on treatment alternatives (Smith et al. 1992), indicated that the savings realized by downsizing the treatment facility were offset by the increased cost of disposal containers and of disposal itself. Therefore, the indirect effects tend to be cancelling, and the comparison is valid. 


\subsection{2 "Wants"}

One of the "wants" listed in Table 6-1 applies to this section:

- Minimize the life-cycle cost.

The subjective evaluation of performance on this "want" is as follows in Table 13-3. (The scores are based on the unescalated costs.) The rationale for these scores, by reference to the results presented in Table 13-1, is as follows. The lowest cost alternative, offsite shipment, was assigned the score of 10. All other alternatives were assigned a score based on ten times the ratio to the lowest cost alternative. For example, the most expensive alternative, the maximum-generation/minimumtreatment scenario, at $\$ 970$ million, was assigned the score of 3 , based on the calculation:

$(295 / 970) \times 10=3.0$

Thus, the most expensive alternative is given a 3 and the scores for the remainder of the alternatives fall between 3 and 10 , inclusive.

\subsubsection{Escalation and Discount Factors}

As a simplification, unescalated costs were used for comparison instead of either escalated or present-worth costs. Over the long time period involved, the escalated costs (dollars that will be spent when work is performed) become enormous. As a result, small expenditures in the later years can totally mask expenditures in earlier years. For example, the fully burdened annual cost of one monitoring person, which today would be $\$ 125,000$, would be over $\$ 303,000,000$ in the year 2133 ! Thus, a single year's salary in the distant future would be about one-tenth of the entire escalated life cycle cost for the alternative.

A more useful estimation would be to use an escalation rate combined with a discount rate to calculate the present worth of the money to be spent in future years. The escalation and discount rates can be fairly well estimated over the short run of 5 to 10 years. Over the long run, the values of escalation and discount are not known, however; the relative values are generally a few percent different, with discount being higher than escalation. This means that money spent near the front end of a project will have a greater impact on present-worth than money spent in the later years. For example, assuming an escalation of $4 \%$ and a discount of $6 \%$, a salary of $\$ 125,000$ in 1993 dollars would be $\$ 303,000,000$ in the year 2133 and would require the setting aside (investing at $6 \%$ ) of approximately $\$ 9,000$ in 1093 , that is, the present-worth cost is $\$ 9,000$. The same equivalent salary in 2033 would be $\$ 600,000$ and would require the setting aside of $\$ 60,000$ in 1993 .

Present-worth costs yield the present value of future expenditures, and their use allows the comparison of vastly different options which represent significantly different time-series of investment. In the case of the comparison of alternatives performed in this report, the time-series of investment is very similar, with most tasks operating for the same period. Because of the similarity of timing of expenditures among the alternatives, and because the bulk of the expenditures occur in the first 60 years of the project (in FY-93 dollars, the 100-year institutional control phase is only $2 \%$ of the total cost), the use of unescalated costs is expected to provide an accurate comparison of relative costs. 
Table 13-3. Subjective evaluation of performance on applicable "want."

\begin{tabular}{cc}
\hline Alternative & Minimize life-cycle cost \\
\hline 1A & 5 \\
1B & 5 \\
1C & 4 \\
1D & 6 \\
1E & 6 \\
1F & 6 \\
1G & 7 \\
1H & 3 \\
2 & 6 \\
3 & 10 \\
4A & 4 \\
4B & 5 \\
5 & 6 \\
\hline
\end{tabular}




\subsection{References}

Smith, T. H., W. S. Roesener, M. J. Jorgensen-Waters, C. R. Edinborough, 1992, A Preliminary Evaluation of Alternatives for Treatment of INEL Low-Level Waste and Low-Level Mixed Waste, EGG-WMO-10321, June 1992. 


\section{KEY UNCERTAINTIES}

Sections 7 through 13 evaluated the disposal alternatives. Before proceeding to the analysis of the results (Section 15) and the recommendations (Section 16), this section discusses the key uncertainties affecting the recommendations.

Although the study encountered several key uncertainties, the following points need to be considered:

- Uncertainties exist in all projects at all stages.

- The uncertainties should not be construed as cause to do nothing or to delay making a decision, unless delay will improve the situation.

- The time required to develop a new LLW disposal site is so long that the remaining disposal capacity at the RWMC may be consumed by about that same time. Thus, the decision about additional LLW disposal capacity should not be delayed unnecessarily.

\subsection{Introduction}

Some of the assumptions in Section 3 cover areas that are not only uncertain, but also important enough to potentially affect the conclusions of the study. Such assumptions, along with some other factors, are termed here the "key uncertainties" of the study. The key uncertainties are items

- That are not yet known

- Whose nature nevertheless had to be assumed

- That could change significantly between now and the date of facility startup.

The length of lime required to implement a disposal alternative helps convey the significance of the key uncertainties. This study is being conducted in 1993. The alternative would begin operations about $20(0)-2005,7-12$ years later. In the intervening 7-12 years, events could occur that could

- Significantly change the altractiveness of one alternative relative to another

- Signilicantly change the viability of the selected alternative.

The key uncertainties focus on the major unknowns, not on the many lower-level uncertainties (e.g., the size of the operating staff for a given disposal facility). The lower-level uncertainties would not be expected to result in either of the bulleted outcomes above.

The key uncertainties, some of which are interlocking, tend to fall into several categories:

- Regulations (uncertainty 14.2.1 in the following subsection) 
- DOE policies, philosophies, and programs (uncertainties 14.2.2 through 14.2.5)

- Waste volumes and characteristics (uncertainties 14.2.3 and 14.2.4)

- Status and missions of various waste management facilities (uncertainties 14.2.6 and 14.2.7)

- Performance of waste management technologies (uncertainties 14.2.8 and 14.2.9)

- Other (uncertainties 14.2.10 through 14.2.13).

\subsection{Key Uncertaintles}

\subsubsection{Shifting Regulations}

One assumption in the study involved complying with not only all existing applicable regulations, but also all potentially applicable regulations that have been proposed or drafted. Even with this expanded vision of compliance, regulatory changes will impact the evaluations of this study during the 7 to 12-year period before operations begin. Potential Federal regulations already being formulated but not yet proposed include the RCRA reauthorization and planned restrictions on the siting of TSD facilities. The likely inclusion of many of the NRC technical requirements of 10 CFR 61 in a revised DOE Order $5820.2 \mathrm{~A}$ is also of interest.

Another potential source of new regulations or policies that could impact the development of new disposal capability is the State of Idaho.

\subsubsection{Stablity of DOE Policies and Practices}

The long-term disposal alternative could require as long as 12 or more years to achieve operations. During the interim, significant changes are likely in DOE policies and practices, as different Administrations and different DOE executives come and go. These changes could result in redirection, or termination of projects to develop new disposal capability, before the projects are completed.

\subsubsection{Volumes and Characteristics of Waste that Will Be Generated}

The projections of the volumes and characteristics of the waste that will be generated are uncertain. The waste from ongoing operations is uncertain because many activities that generate waste on an ongoing basis either will or may be shut down. An example is the Idaho Chemical Processing Plant (ICPP) reprocessing mission. Waste generation reduction may also impact the volume of waste.

The waste to be generated by remedial actions and D\&D activities is highly uncertain. The waste uncertainties are due primarily to programmatic uncertainties about the remediation approach for each cleanup, as well as the magnitude of the D\&D activities. The current magnitude of the D\&D program is such that several decades would be required to accomplish D\&D of all surplus contaminated facilities at the INEL. It is unknown whether an acceleration of the program, with a resulting increase in waste generation, will occur. 


\subsubsection{Volume Reduction of LLW}

As discussed in Section 2, DOE Order 5820.2A [Chapter III, paragraph 3.f(2)] requires volume reduction of LLW only "to the extent that it is cost effective." However, another DOE document implies a stronger driving force for volume reduction. DOE Order 6430.1A, Division 1300-8.1, states, "volume reduction equipment for both liquid and solid wastes shall be required where feasible." Strict application of this requirement (or other driving forces) could lead to implementation of maximumtreatment type of facilities, resulting in reduction of the volume of LLW requiring disposal. Maximum treatment would also result in reduced mobility of the waste product and less dependence on the disposal facility to provide long-term isolation.

\subsubsection{Outcome of Privatization Initiatives}

The INEL Private Sector Participation Initiative and related activities could impact the results of the present study. EG\&G Idaho is evaluating the feasibility of near-term offsite shipment of INEL LLW and mixed waste for treatment at commercial facilities. In addition, a commercial treatment operator has proposed construction and operation of a new facility to treat the INEL LLW and mixed waste, including the Class $C \alpha$ waste. Offsite treatment in an existing commercial facility could conceivably begin as soon as 1 or 2 years hence. Construction of an offsite commercial facility would take several years.

It is conceivable that waste disposal could be added to the scope of the offsite activities, particularly with the new treatment facility. (Disposal of DOE LLW at a commercial facility would require an exemption from the requirement in DOE Order 5820.2A, Chapter III, paragraph 2.c.) The near-term offsite commercial treatment (and possibly disposal) could become the de facto alternative for long-term treatment and disposal. The eventual outcomes of these near-term actions, and their impacts on the long-term treatment and disposal alternatives, cannot be predicted at this time.

\subsubsection{Assumptions Concerning the Future of Other INEL Waste Management Facilities}

In order to focus on alternatives for disposing of INEL mixed waste and LLW, assumptions were made that other INEL waste management facilities would be constructed or operated as planned. Included are the MLLWTF and IWPF (or suitable treatment alternatives), and the RWMC. One or more of these facilities might not be constructed or operated, at least not on the planned schedule. A higher-level alternatives study than this one would be required to examine the impacts of various facilities not being available as planned.

\subsubsection{Uncertainties of Disposal at Another DOE Site}

One of the disposal alternatives and two of the combinations involve disposal of INEL waste at another DOE site. At least four other DOE sites are further along than the INEL in pursuing onsite disposal for mixed waste. However, there is no assurance that the other sites will be successful in developing their sites, nor that a successful site would accept INEL waste for disposal. 


\subsubsection{Impacts of the Performance Assessment of the MLLWDF}

The selection of a disposal concept will be based, in part, on a determination that the concept provides the necessary degree of waste isolation. The isolation is also affected by the form of the waste, following any treatment that is performed. These interrelated factors are evaluated in a performance assessment, as required by DOE Order 5820.2A and 10 CFR 61 .

A draft performance assessment of the RWMC has been performed (Maheras et al. 1993). The final performance assessment of the RWMC is planned for completion by September 30, 1993. The final RWMC assessment will provide valuable general insights about isolation-performance parameters in the INEL environment.

As shown in Section 12, a preliminary performance assessment is planned for the MLLWDF in FY 1995 and 1996. The results of the preliminary assessment will provide the basis for selecting a combination of waste treatment and waste disposal technologies that provide the required isolation. The results will be confirmed in the final performance assessment in FY 1999 and 2000.

The foregoing information indicates that the required isolation to be provided by the disposal concept will not be known until FY 1996. Significant uncertainties about isolation performance of specific concepts will persist until that time. If interim judgments about isolation performance prove too pessimistic, a disposal concept that is unnecessarily conservative and expensive could be pursued during conceptual design in FY 1995 and 1996. If interim judgments prove too optimistic, the disposal concept pursued during conceptual design could be under-designed and could, if implemented, result in inadequate isolation. The fact that the extent of LLW treatment is interrelated with isolation performance further complicates the matter.

\subsubsection{Use of Technologies that Are Not Totally Proven}

The disposal concepts were identified while recognizing the following "want" from Section 6:

- Minimize the developmental risk associated with the isolation system technologies.

However, this restriction does not remove all technological risk. The long time periods over which isolation must be maintained make engineering demonstrations of performance all but impossible. None of the concepts has been demonstrated to provide successful confinement for any time period approaching 500 years. Decisions related to long-term isolation will have to be made based on extrapolation of short-term test results, projections of mathematical models, consideration of longerterm results from other facilities, and technical judgment. Thus, there is an element of uncertainty in the long-term performance of the disposal concepts.

\subsubsection{Lack of Preconceptual Designs}

Preconceptual designs do not exist for any of the disposal alternatives. Also, the family of waste containers to be used when the disposal facility is in operation has not been determined. This lack of information affects the evaluations and comparisons of alternatives. The approach taken in this study was to develop only enough overview design information to allow approximate calculation of 
waste logistics (e.g., numbers of waste containers), planning-basis (ROM) life-cycle cost estimates, and qualitative safety comparisons.

\subsubsection{Schedule Uncertainties}

Experience has shown that long-range schedules of the type developed here have substantial uncertainties. However, the uncertainties would tend to affect most of the alternatives similarly (e.g., stretching out NEPA schedules). Significant changes in schedule ranking, from one alternative to another, would not be expected.

As discussed in Section 9. two major schedule uncertainties relate to (a) the RCRA permitting and (b) the link in NEPA conipliance between this project and the INEL ER\&WM EIS. If the ROD for that EIS is delayed more than about one year, all steps in implementing any disposal alternative, from Title II design on, will be correspondingly delayed.

Another schedule uncertainty relates to assuming that DOE-HQ approval is received by July 1994 for the start of conceptual design on October 1, 1994. Delays in receiving the approval will be expected to delay the entire project.

\subsubsection{Cost Uncertainties}

Because of the lack of design information, the life-cycle cost estimates have substantial uncertainties. For alternatives whose estimated total costs are close to one another, these uncertainties could result in some change of the cost ranking.

\subsubsection{Subjective Rankings}

The alternatives were evaluated in several areas and then compared against several "musts" and weighted "wants." The "must" comparisons are generally objective. The "want" comparisons are subjective, as is any multivariable qualitative comparison, to some extent. For alternatives whose scores on the "wants" are close to one another, the relative ranking could change. 


\subsection{References}

Maheras, S. J., et al., 1993, Radioactive Waste Management Complex Low-Level Waste Radiological Performance Assessment, draft report, EGG-WM-8773, April 1993. 


\section{ANALYSIS OF RESULTS}

\subsection{Introduction}

Sections 7 through 13 presented overview evaluations of the alternatives for disposal of INEL mixed waste and LLW. The evaluations included facility operations, and isolation performance, safety, environmental, institutional, schedule, cost, and facility interface comparisons, based on a set of simplifying assumptions. The purpose of this section is to (a) analyze the results presented in those sections and (b) identify other decision making considerations.

Sections 7 through 13 also evaluated the alternatives on the performance against a list of "musts" and "wants." This section compiles the rankings into one master table for "musts" and another for "wants." The MLLWDF Subalternatives $1 G$ and $1 \mathrm{H}$ are included in the master tables but are not considered in the overall ranking. These subalternatives are essentially the same as Subalternative $1 \mathrm{~A}$ with different assumed volumes of waste.

\subsection{Results on "Musts"}

The list of "musts" comprises the mandatory criteria for recommendation of a disposal alternative. To be considered viable, an alternative must comply with every listed "must." Table 15-1 summarizes the "musts" and whether each alternative passed or failed each criterion.

The no-action alternative failed the following "must" criteria and is not considered viable:

- Comply with all environmental and waste management regulatory requirements, including the requirements for long-term isolation.

- Over a period of 40 years, provide all required disposal of LLW and mixed waste generated or currently stored at the INEL, after any necessary treatment has been performed.

- Present no insurmountable institutional problems.

While Alternative 2, Continued Disposal at the RWMC and its combination (RWMC and offsite), satisfies the "must" criteria for the scenario of maximum treatment and large generation, the use of available space is nearly at a maximum. These alternatives will not satisfy the criterion for disposal of all waste under the large-generation/minimum-treatment scenario.

The remaining alternatives satisfy all "must" criteria and are considered viable.

\subsection{Results On "Wants"}

The list of "wants" comprises criteria that would impact the recommendation of an alternative, but are not strict requirements. Each "want" was assigned an importance weight, based on perceived priority to DOE and on engineering judgment. 
Table 15-1. Summary of performance of alternatives against "musts."

Musts

\begin{tabular}{|c|c|c|c|c|c|}
\hline Alternative & $\begin{array}{l}\text { Is expected to comply with } \\
\text { all environmental and waste } \\
\text { management regulations, } \\
\text { including the requirements } \\
\text { for long-term isolation }\end{array}$ & $\begin{array}{l}\text { Present acceptable risk to } \\
\text { the workers, the public } \\
\text { and the environment } \\
\text { during the construction } \\
\text { and operations phases }\end{array}$ & $\begin{array}{l}\text { Over a period of } 40 \text { years, } \\
\text { provide all required disposal } \\
\text { of mixed waste and LLW } \\
\text { generated or currently stored } \\
\text { at the INEL, after any } \\
\text { necessary treatment has been } \\
\text { performed }\end{array}$ & $\begin{array}{l}\text { Present no potential } \\
\text { major conflicts with the } \\
\text { missions or operations } \\
\text { of other existing or } \\
\text { planned INEL waste } \\
\text { management facilities } \\
\end{array}$ & $\begin{array}{l}\text { Present no } \\
\text { insurmountable } \\
\text { institutional problems }\end{array}$ \\
\hline MLLWDF - 1A & $\mathbf{P}$ & $\mathbf{P}$ & $\mathbf{P}$ & $\mathbf{P}$ & $\mathbf{P}$ \\
\hline MLLWDF - 1B & $\mathbf{P}$ & $\mathbf{P}$ & $\mathbf{P}$ & $\mathbf{P}$ & $\mathbf{P}$ \\
\hline MLLWDF - ID & $\mathbf{P}$ & $\mathbf{P}$ & $\mathbf{P}$ & $\mathbf{P}$ & $\mathbf{P}$ \\
\hline MLLWDF - 1E & $\mathbf{P}$ & $\mathbf{P}$ & $\mathbf{P}$ & $\mathbf{P}$ & $\mathbf{P}$ \\
\hline MLLWDF - 1F & $\mathbf{P}$ & $\mathbf{P}$ & $\mathbf{P}$ & $\mathbf{P}$ & $\mathbf{P}$ \\
\hline MLLWDF - 1G & $\mathbf{P}$ & $\mathbf{P}$ & $\mathbf{P}$ & $P$ & $\mathbf{P}$ \\
\hline MLLWDF - $1 \mathrm{H}$ & $\mathbf{P}$ & $\mathbf{P}$ & $\mathbf{P}$ & $P$ & $\mathbf{P}$ \\
\hline MLLWDF/RWMC & $\mathbf{P}$ & $\mathrm{P}$ & $\mathbf{P}^{\mathbf{b}}$ & $\mathbf{P}$ & $\mathbf{P}$ \\
\hline No action ${ }^{a}$ & F & $\mathbf{P}$ & F & $\mathbf{P}$ & $\mathrm{F}$ \\
\hline \multicolumn{6}{|c|}{$\mathbf{P}=$ Passed the "must" criterion. } \\
\hline \multicolumn{6}{|c|}{ a. Alternatives that are no longer considered viable. } \\
\hline b. RWMC and the & bination with $\mathrm{RWMC}$ will & pass the large-generation & imum-treatment scenario. & & \\
\hline
\end{tabular}


Table 15-2. Summary of performance of alternatives against "wants."

\begin{tabular}{|c|c|c|c|c|c|c|c|c|c|}
\hline \multirow[b]{2}{*}{ Alternative } & \multicolumn{8}{|c|}{ Wants } & \multirow[b]{2}{*}{ Sum } \\
\hline & $\begin{array}{l}\text { Minimize risk to } \\
\text { workers, the public, } \\
\text { and the environment } \\
\text { during the } \\
\text { construction and } \\
\text { operations phases } \\
\text { (5) }\end{array}$ & $\begin{array}{l}\text { Provide maximum } \\
\text { isolation of the } \\
\text { waste after the } \\
\text { operations period } \\
\text { (5) }\end{array}$ & $\begin{array}{l}\text { Minimize problems } \\
\text { in constructibility, } \\
\text { operability, } \\
\text { reliabiility, and } \\
\text { maintainability } \\
\text { (3) } \\
\end{array}$ & $\begin{array}{l}\text { Minimize the } \\
\text { development risk } \\
\text { associated with } \\
\text { the isolation } \\
\text { system } \\
\text { technologies (3) }\end{array}$ & $\begin{array}{l}\text { Minimize the } \\
\text { schedule for } \\
\text { beginning disposal } \\
\text { operations } \\
\text { (2) }\end{array}$ & $\begin{array}{l}\text { Minimize } \\
\text { life-cycle } \\
\text { cost } \\
\text { (2) } \\
\end{array}$ & $\begin{array}{l}\text { Minimize } \\
\text { potential } \\
\text { interface } \\
\text { problems with } \\
\text { other INEL } \\
\text { WM facilities } \\
\text { (1) } \\
\end{array}$ & $\begin{array}{l}\text { Minimize } \\
\text { potential } \\
\text { institutional } \\
\text { problems } \\
\text { (1) } \\
\end{array}$ & \\
\hline MLLWDF - 1A & 30 & 32.5 & 27 & 15 & 12 & 10 & 10 & 9 & 145.5 \\
\hline MLLWDF - 1B & 30 & 50 & 21 & 15 & 12 & 10 & 10 & 9 & 157 \\
\hline MLLWDF - 1C & 45 & 27.5 & 30 & 24 & 12 & 8 & 9 & 9 & 164.5 \\
\hline MLLWDF - 1D & 35 & 47.5 & 24 & 27 & 14 & 12 & 10 & 7 & 176.5 \\
\hline MLLWDF - IE & 40 & 42.5 & 27 & 27 & 14 & 12 & 10 & 5 & 177.5 \\
\hline MLLWDF - 1F & 35 & 30 & 24 & 21 & 12 & 12 & 10 & 7 & 151 \\
\hline MLLWDF - IG & 50 & 40 & 27 & 15 & 12 & 14 & 10 & 10 & 178 \\
\hline MLLWDF - 1H & 20 & 30 & 24 & 15 & 12 & 6 & 10 & 7 & 124 \\
\hline RWMC & 40 & 30 & 27 & 21 & 14 & 12 & 8 & 6 & 158 \\
\hline Offsite & 30 & 42.5 & 24 & 27 & 20 & 20 & 9 & 3 & 175.5 \\
\hline MLLWDF/Offsite & 30 & 35 & 21 & 18 & 16 & 8 & 9 & 5 & 142 \\
\hline RWMC/Offsite & 35 & 35 & 27 & 24 & 18 & 10 & 7 & 4 & 160 \\
\hline No action ${ }^{a}$ & 25 & 15 & 30 & 30 & 18 & 12 & 10 & $\mathbf{0}$ & 140 \\
\hline
\end{tabular}

()$=$ Importance weight.

a. Alternatives that failed a "must" and are not considered viable. 
The top row of Table 15-2 summarizes the "wants;" the importance weights appear in parentheses. Minimizing risk to the workers, the public, and the environment, and providing maximum isolation of the waste after the operations period are considered to be the most important "wants" and are assigned an importance weight of five each. Minimizing problems in constructibility, operability, reliability, and maintainability (including an assessment of the ability to monitor and retrieve waste), and minimizing the risk of technology development are each assigned a weight of three. Minimizing the schedule for beginning operations, and minimizing the life-cycle cost are each assigned a weight of two. Minimizing potential interface problems with other INEL waste management facilities and minimizing potential institutional problems are considered least important and are each assigned a weight of one.

The remainder of Table $15-2$ presents the weighted scores. The viable disposal alternatives are ranked as follows:

1. Subalternative 1E-Shallow land burial/boreholes

2. Subalternative 1D-Greater-depth burial/boreholes

3. Alternative 3-Ship waste offsite for disposal

4. Subalternative $1 \mathrm{C}-$ Covered modules

5. Alternative $4 \mathrm{~B}-\mathrm{RWMC}$ and ship offsite

6. Alternative 2-Continue Disposal at RWMC

7. Subalternative 1B-Belowgrade engineered structures

8. Subalternative 1F-Shallow land burial/abovegrade engineered structures

9. Subalternative $1 \mathrm{~A}-\mathrm{Abovegrade}$ engineered structures

10. Alternative 4A-MLLWDF and ship offsite

Subalternatives $1 \mathrm{G}$ and $1 \mathrm{H}$ are not included in the above rankings because they are scenarios not alternatives.

The degree of resolution of the ratings in Table $15-2$ has a limit. It is estimated that total scores within about $10 \%$ of one another, as scored against the maximum possible of 220 points, are within the uncertainty range. Thus, total scores within 22 points of one another are within the uncertainty range. The seven top-scoring viable alternatives are within that range of one another. The lower-scoring alternatives are probably not within the uncertainty range of the top-scoring seven alternatives. The major impacts on the total scores are the isolation-related and risk-related "wants," with a spread of 22.5 and 15 points, respectively, for the viable alternatives (not including scenarios). 


\subsection{Decisionmaking Considerations}

If the recommendation were based strictly on the "musts" and "wants," the preferred alternative for disposal of INEL mixed waste and LLW would be the onsite shallow land burial alternative. (The onsite greater depth disposal alternative scores within one point of the leader, and the offsite shallow land burial alternative scores within two points.) However, selecting an alternative is extremely complex and involves many factors; some do not readily lend themselves to lists of "musts" and "wants." The decision is not completely determined by any evaluation of numbers.

This subsection suggests several major factors that the decision maker needs to consider, in addition to the results of the "musts" and "wants" in Tables 15-1 and 15-2.

\subsubsection{Should a Decision on Enhanced Disposal Capability Be Pursued Now or Delayed?}

The first major consideration is whether a decision on a disposal alternative should be pursued now or delayed. (It is noted that nothing in the following discussion is intended to circumvent the NEPA proccss for decisionmaking. See the schedules in Section 12 for an indication of how technical and design studies would be integrated with the NEPA and other regulatory processes.)

If the decision were delayed for a year or two, would the key uncertainties identified in this study (see Section 14) be greatly reduced? A few uncertainties associated with selecting an alternative would be resolved by delaying the decision for a year or two. For example, revisions to some of the applicable regulations may be complete. The technical requirements of 10 CFR 61 were used as guidance for this study, based on the assumption that they would be incorporated into DOE Order 5820.2A. These requirements exceed what is required by the current DOE Order, so a selection based on these requirements is considered to be conservative. New location standards for TSD facilities are expected within the next year. These new standards would affect where on the INEL a new facility could be located. The selected location will impact the facility performance assessment and potentially the selected disposal technology. Improved projections of ER and D\&D waste may be available in 1 to 2 years. Finalization of the RWMC performance assessment within the next year would provide insights about isolation performance in the INEL environment. It should be noted, however, that the waste streams evaluated in the RWMC performance assessment are considerably different from those considered for disposal at the MLLWDF, so a direct comparison cannot be made.

On the other hand, there are several negative impacts of delay. For example, if the decision is delayed, the schedule for implementing an alternative is also delayed. Implementation of a new disposal facility requires approximately $7-12$ ycars, so a decision must be made 7-12 years prior to implementation. Meanwhile, there is no capacity for disposal of INEL mixed waste. The lack of a permitted mixed waste disposal facility places the burden on storage. Mixed waste storage capacity at the INEL is limited, and prolonged storage may require construction of new storage facilities. Prolonged storage is not a solution and also creates additional risk for workers due to inspection of the stored contact-handled waste. Also, a delay in the decision could eventually require that the operating life of the RWMC be correspondingly extended. The lifetime projections for the RWMC, as discussed in Section 4, indicate that under the minimum-treatment scenarios the RWMC in its current configuration would reach maximum capacity prior to beginning operations at a new disposal facility. Depending on the length of delay (more than 3 years), it is possible that even under the maximum-treatment scenario, the RWMC might not be able to accommodate the volume of waste requiring disposal. 
In conclusion, although there is some benefit to delaying a decision, the negative impacts of delay are more significant, favoring pursuing a decision now.

\subsubsection{The Effect of Waste Treatment on Disposal Requirements.}

Another consideration in making a decision is whether the INEL will implement the maximum or the minimum required treatment of LLW. At present, treatment of LLW is required to satisfy only DOE Orders and is not subject to State or Federal regulations. The key point of the DOE Orders is that LLW must be treated to satisfy the requirements of the performance assessment. In addition, DOE Orders require treatment to stabilize and reduce volume if it is cost effective. DOE Orders also require treatment if it is feasible.

The interpretation and implementation of these treatment requirements could impact the selection of a disposal alternative in two ways. The first way relates to the volume of waste requiring disposal. In the near term, implementation of minimum treatment would significantly impact the remaining life of the RWMC. The lifetime capacity of the RWMC would be reached several years earlier than planned. In the longer term, under minimum treatment and maximum generation, the modified RWMC (Alternative 2) and its combination (Alternative 4B) would not be capable of handling the large volume of waste requiring disposal within current facility boundaries.

A comparison of Subalternative $1 \mathrm{~A}$ and Subalternative $1 \mathrm{H}$ also illustrates the impacts that treatment has on disposal. Minimum treatment, represented by Subalternative $1 \mathrm{H}$, results in a larger volume of waste requiring disposal. The larger volume of waste increases the number of disposal units required, the total land area required for the disposal facility, and ultimately the cost involved in constructing and operating the disposal facility. Maximum treatment, represented by Subalternative $1 \mathrm{~A}$, requires fewer disposal units, less land area, and less funding for construction and operation of the disposal facility.

The second way in which treatment affects disposal relates to the isolation provided by the waste form. Maximum treatment would result in reduced mobility of the waste product and less dependence on the disposal facility to provide long-lerm isolation. Maximum treatment may result in the less expensive disposal technologies (e...., shallow land burial) providing sufficient isolation.

The conclusion is that the decision on disposal technology is linked with the decision on treatment of LLW.

\subsubsection{Waste Volume Projections}

Consideration should be given to the uncertainty associated with the waste volume projections used in this study.

The four sources of waste are listed in Table 15-3: waste from ongoing operations, waste from D\&D, waste from environmental remedial actions, and waste in storage at the INEL. Ideally, an analyst could obtain high estimates (upper bounds), low estimates (lower bounds), and best estimates of the waste volume expected from each source. Then, an informed judgment could be used in specifying the baseline assumption for waste volumes.

Reexamination of Section 2 reveals that the projection for waste from ongoing operations is probably a high estimate. The projection was based on current generation levels. Several INEL operations are ceasing, and waste generation reduction activities will also result in lower volumes in 
the future. Thus, an $\mathrm{X}$ is shown in Table 15-3 under high estimate for waste from ongoing operations.

Sections 2 and 3 reveal that (for all alternatives except 1G) the projection used for waste from $D \& D$ is also a high estimate. The projection assumed that the planned major expansion in D\&D activities will occur. The projection is also based on dismantlement of all facilities undergoing D\&D, the mode expected to result in the largest volume of waste. Thus, an $\mathrm{X}$ is shown in Table 15-3 under high estimate for waste from D\&D.

Sections 2 and 3 also reveal that the projection of waste from environmental remedial actions is a low estimate. Based on input from remedial action managers, nearly all the ER activities are scheduled to be completed and the waste dispositioned before a new waste disposal facility will be operating. These waste streams were, therefore, not considered for disposal as part of this study. Based on the milestones in the final INEL Action Plan for Implementation of The Federal Facility Agreement and Consent Order, it is, however, possible that some of the waste will not be dispositioned. Also, there is currently no disposal capacity at the INEL for mixed waste; therefore, such waste would likely remain in storage until a permitted disposal facility is on line. Thus, an $\mathrm{X}$ is shown in Table 15-3 under low estimate for waste from remedial actions.

The projection of waste currently in storage at the INEL MWSF and the RWMC TSA is relatively well documented. An $\mathrm{X}$ is shown in Table 15-3 under best estimate for waste in storage.

The available projections of the four sources of waste do not provide the desired complete set of bounding estimates. Projections for two of the waste sources are biased on the high side, and one projection is a lower bound.

To evaluate the impact that waste volume has on a disposal alternative, two cases of waste generation were considered in this study. The differences between Subalternative $1 \mathrm{~A}$ and Subalternative $1 G$ demonstrate the impact of the large generation scenario versus the small generation scenario. The volume of waste disposed of in Subalternative $1 \mathrm{~A}$ is three times greater than that disposed of in Subalternative 1G, resulting in more disposal units, space required, and funding for design and construction. These same impacts would be seen in the other alternatives if variations in waste volumes were evaluated.

If the actual waste volumes exceeded those used in the study, aside from the impacts discussed above, the disposal concept most likely to be affected would be the RWMC alternative and its combinations. In this case, the RWMC would be expected to reach lifetime capacity sooner than predicted, depending on the treatment philosophy adopted. As discussed in Section 15.4.2, the modified RWMC (Alternative 2) and its combination (Alternative 4B) would not be capable of handling the larger volume of waste, under a minimum-treatment scenario, within current boundaries.

Table 15-3. Projections used for waste volumes.

\begin{tabular}{lccc}
\hline \multicolumn{1}{c}{ Waste source } & $\begin{array}{c}\text { High } \\
\text { estimate }\end{array}$ & $\begin{array}{c}\text { Low } \\
\text { estimatc }\end{array}$ & $\begin{array}{c}\text { Best } \\
\text { estimate }\end{array}$ \\
\hline Ongoing operations & $\mathrm{X}$ & - & - \\
D\&D & $\mathrm{X}$ & - & - \\
Remedial actions & - & $X$ & - \\
Waste in storage & - & - & $X$ \\
\hline
\end{tabular}


If the actual waste volumes are less than those used in the study, the MLLWDF alternatives would be impacted as discussed above for Subalternative 1G. In this case, the RWMC would be expected to reach lifetime capacity later than predicted and would be capable of handling the volume of waste requiring disposal within current boundaries.

\subsubsection{Site Selection}

As discussed in Sections 4.4.2 and 5.3.3, the location of the MLLWDF affects the suitability and performance of various disposal technologies. The site selection process for the MLLWDF has not been completed yet. The current study addressed three potential candidate sites, with the baseline site being located in the central part of the site. Consideration should be given to which disposal technologies are best suited for a particular site. Table 15-4 indicates a preliminary judgment concerning the potential suitability of the evaluated disposal technologies at the three sites. It should be noted that this evaluation is based on a limited amount of data and is therefore subject to change.

The entries marked with a "Y" in Table 15-4 indicate the evaluated disposal technology is considered potentially suitable for a particular site. The entries marked with a " $\mathrm{N}$ " indicate the evaluated disposal technology is considered potentially not suitable for a particular site.

Table 15-4. Potential suitability of disposal technologies at candidate sites. ${ }^{a}$

\begin{tabular}{ccccc}
\hline Disposal alternative & Disposal concept & Site A & Site B & Site C \\
\hline MLLWDF - 1A & AGES & $\mathrm{Y}$ & $\mathrm{Y}$ & $\mathrm{Y}$ \\
MLLWDF - 1B & BGES & $\mathrm{Y}$ & $\mathrm{N}$ & $\mathrm{N}$ \\
MLLWDF - 1C & CM & $\mathrm{Y}$ & $\mathrm{Y}$ & $\mathrm{Y}$ \\
MLLWDF - 1D & GDB/GDBH & $\mathrm{Y}$ & $\mathrm{N}$ & $\mathrm{N}$ \\
MLLWDF - 1E & SLB/SBH & $\mathrm{Y}$ & $\mathrm{N}$ & $\mathrm{Y}^{\mathrm{b}}$ \\
MLLWDF - 1F & SLB/AGES & $\mathrm{Y}$ & $\mathrm{N}$ & $\mathrm{Y}^{\mathrm{b}}$ \\
MLLWDF - 1G & AGES & $\mathrm{Y}$ & $\mathrm{Y}$ & $\mathrm{Y}$ \\
MLLWDF - 1H & AGES & $\mathrm{Y}$ & $\mathrm{Y}$ & $\mathrm{Y}$
\end{tabular}

a. The acronyms and abbreviations used in this table are as follows:

$\begin{array}{llll}\text { AGES } & =\text { Abovegrade engineered structures } & \text { BGES } & =\text { Below-grade engineered structures } \\ \mathrm{CM} & =\text { Covered modules } & \mathrm{SLB} & =\text { Shallow land burial } \\ \mathrm{SBH} & =\text { Shallow boreholes } & \mathrm{GDB} & =\text { Greater-depth burial } \\ \text { GDBH } & =\text { Greater-depth boreholes } & \mathrm{Y} & =\text { suitable } \\ \text { Site } A=\text { Well Site } 14 & \mathrm{~N} & = \\ \text { Site } \mathrm{B}=\text { Tip of Lemhi Range } & & \\ \text { Site } \mathrm{C}=\text { East of RWMC } & \\ \text { b. Assuming limited blasting to necessary depth is feasible. }\end{array}$


Site $A$ is located in the central portion of the INEL and is otherwise known as well Site 14. The site characteristics appear to be favorable to all the proposed disposal concepts. Site B is located at the southern tip of the Lemhi Range, in the northwest corner of the INEL. Due to the shallow sediments, the potential for crosion, and surface water runon and runoff, the disposal concepts using BGES, shallow land burial, and greater depth burial are not considered suitable for this location. Site $C$ is located approximately 3 miles east of the existing RWMC. The sediments at this location are shallow and are not considered suitable for BGES or the greater depth burial concept.

\subsubsection{Technology Risk}

Another factor for consideration is the technological risk associated with the various disposal concepts. Because of the long tine periods over which isolation must be maintained, demonstration of these technologies is not possible. As discussed in Section 14.2.9, none of the disposal concepts has been demonstrated to provide successful confinement for any time period approaching 500 years.

Upon the tentative selection of a given alternative, a performance assessment will be prepared to project whether the disposal concept passes or fails the prescribed criteria. It is impossible to predict the performance of any alternative at this early stage, so a technology risk will exist.

\subsubsection{Will Other Facilities Be Available?}

Consideration must also be given to the question of whether treatment facilities and offsite waste disposal facilities will be available in the future as hoped. The disposal alternatives were based on the assumption that onsite or offsite treatment facilities will be available for treatment of INEL waste. Failure to treat INEL mixed waste would mean the waste could not be disposed of.

The offsite disposal alternative was based on the assumption that facilities will be available and able 10 accept INEL waste as planned. Selecting this alternative means accepting a risk that a disposal lacility will not be available in the fulure.

\subsubsection{Public Perception}

Another decisionmaking factor that needs consideration is public perception of disposal alternatives. Disposal of LLW and mixed waste may be controversial and may engender a high degree of public concern. Public perception of nonengineered onsite alternatives such as GDB and SLB may be tied to the pereeption of the current disposal methods at the INEL. The perception is expected to be negative, even if performance assessment criteria are met, due to existing wasteisolation problems at the RWMC. Even proposed disposal of commercial LLW using highly engineered disposal technologies has resulted in negative eneounters with the public and institutional groups.

Oftsite disposal alternatives may also be controversial in the eyes of the public and institutional groups (including those in the receiving state). Transportation of radioactive waste is often perceived as dangerous, due $t o$ a lack of accurate information and an abundance of misinformation available to the public.

\subsubsection{Offsite Waste}

Consideration should be given to the question of whether to accept other DOE waste for disposal at the INEL. Although this study addresses disposal of only INEL-generated or -stored 
waste, the INEL ER\&WM EIS is considering the possibility of the INEL's becoming a regional center for treatment, storage, and disposal of DOE waste.

Consideration should also be given to the question of accepting commercial mixed waste for storage, treatment, and disposal at the INEL. A preliminary evaluation of the legal and regulatory basis of DOE acceptance of commercial mixed waste for treatment, storage, and disposal was conducted by the National Low-Level Waste Program (NLLWP). The evaluation concluded that DOE disposal of commercial waste may he feasible. It was recommended that DOE proceed further to investigate the feasibility of accepting commercial mixed waste for disposal. 


\section{RECOMMENDATIONS}

Based on the analyses of results presented in Section 15, the recommendations from this preliminary study are the following:

1. It is recommended that a detailed building-by-building study be periormed as soon as possible to obtain the best estimate of future D\&D waste volumes anci characteristics.

As discussed in Sections 14 and 15, waste volume projections have a significant impact on selection of a disposal alternative. The performance assessment results depend on the curie inventory of the waste. The size or number of disposal units depends on the type and volume of waste. It is therefore important to obtain best-estimate projections. Arriving at best-estimate projections for the D\&D waste streams involves facility-by-facility walkthrough and evaluation for all significant INEL facilities. Projections would be conducted by D\&D personnel, INEL regulatory/waste treatment personnel, and past facility operators, if possible. No waste sampling would be performed. Perhaps the most significant 100 of the 300 to 400 INEL facilities would be addressed in this matter. The remaining facilities would be addressed by engineering judgment.

The time necessary to complete the study, including entry of the data into a database, is estimated to be 6 to 8 months. The cost is estimated to be $\$ 250 \mathrm{~K}$ to $\$ 350 \mathrm{~K}$. The quality of data to be obtained from the study would not match that developed in the usual detailed characterizations of facilities, which cost $\$ 50 \mathrm{~K}$ to $\$ 250 \mathrm{~K}$ each for nonreactor facilities. However, the data quality would far exceed that available today for waste projections in the INEL D\&D Program.

2. Based on the milestones in the final INEL Action Plan for Implementation of The Federal Facility Agreement and Consent Order, it is recommended that the environmental restoration personnel recvaluate periodically the projected long-term timing and volume of waste from remedial actions (including self-generated waste) and the need for treatment and disposal of such waste at the INEL.

Recommendations 1 and 2 reflect the requirements of the DOE-HQ Environmental Restoration Program EM-40 Management Plan, which states that EM-40 is responsible for "advising EM-30 of projected needs for waste treatment, storage, and disposal capability" (DOE 1992).

3. Because of the uncertaintics about isolation performance and the significance of the performance assessment, as discussed in Section 14.28, it is recommended that a preliminar: performance assessment be conducted in FY 1995 and FY 1996 when conceptual design is performed. It is rocommended that a detailed performance assessment be conducted in FY 1999 and FY 2000 when Title II design has been completed.

Although completion of a performance assessment has always been included in the plan for the MLLWDF, the near-term funding need and priority was not fully recognized. This need was identified at the beginning of this alternatives study. At the direction of the MLLWDF project manager, the performance assessment was included in the schedules and cost estimates. As shown in Section 12 and 13, a preliminary perlormance assessment should be performed during conceptual design and a detailed performance assessment 
should be performed after Title II design. Thus, early completion of a performance assessment has become a de facto recommendation.

4. Several performance-verification support studies are recommended in support of the selected disposal alternative. If the selected alternative is offsite disposal, these studies should be integrated with those performed by the host DOE site.

Feasibility studies should be performed on the multilayer cover that would be emplaced upon closure of any of the disposal facilities. Feasibility studies should entail literature reviews and planning during FY 1994, design and modeling studies during conceptual design and advanced conceptual design (FY 1995 and FY 1996), and field studies during Title I and Title II design (FY 1997 and FY 1998).

Feasibility studies should also be performed on the liners, leak detection systems, and the leachate collection and removal systems. Degradation studies should also be performed on the liners. The recommended timing is the same as discussed above under feasibility studies for the cover.

If a technology using concrete structures is selected, studies of the concrete composition, emplacement techniques, and CQA should be conducted to give a high assurance that these materials will last for at least 500 years. Studies should be conducted to determine the effect of weather on the concrete-pouring schedule. The recommended timing is the same as discussed above under feasibility studies for the cover.

If concrete modules are selected as the technology of choice, a material research and development program is recommended. Studies should be conducted to examine failure mechanisms and the effect of concrete thickness on performance.

5. It is recommended that detailed design tradeoff studies be conducted.

If an alternative using boreholes (i.e., Subalternative $1 \mathrm{D}$ or $1 \mathrm{E}$ or Alternative 2) is selected, studies should be performed to refine these concepts.

Studies are recommended to evaluate the optimum family of containers for disposal-space efficiency, ease of handling, minimum transport costs, etc. A tradeoff study of various packaging sizes and shapes is needed during conceptual design.

6. The offsite alternative, the onsite shallow land burial alternative, and the onsite greater depth burial alternative are the conditionally recommended alternatives for the following reasons:

- They satisfy all "must" criteria

- They rank highest against the "wants" criteria

- They are the least expensive, or among the least expensive, viable alternatives in lifecycle cost

The recommended alternatives are conditional on (a) the waste volume and characteristics projections, which would be confirmed in the studies discussed under Recommendations 
1 and 2, (b) the results of the performance assessment that would be performed as discussed under Recommendation 3, (c) the results of the performance-verification support studies of Recommendation 4, (d) the successful development and operation of a suitable offsite facility, and (e) the willingness of the offsite facility to accept INEL waste.

7. It is recommended that activities begin on the recommended alternative(s) as scheduled in the project documentation, while reevaluating the waste volume projections, so that additional time is not lost. This approach will help the INEL to develop the necessary disposal capacity for INEL LLW and mixed waste as soon as feasible. 


\subsection{References}

DOE (U.S. Department of Energy), 1992, Environmental Restoration Program EM-40 Management Plan, DOE/EM/RM/02, Rev. 1, February 1992. 


\section{APPENDIX A}

Detalls and Bases for Cost Estimates 


\section{CONTENTS}

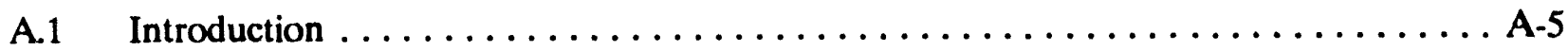

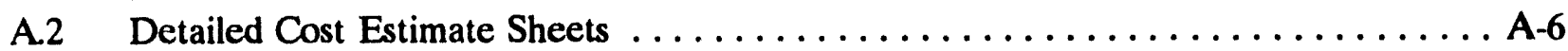

Overview of Evaluations of Disposal Alternatives for Mixed and Low-Level Waste . . A-8

Cost Estimate Support Data Recapitulation ..................... A-9

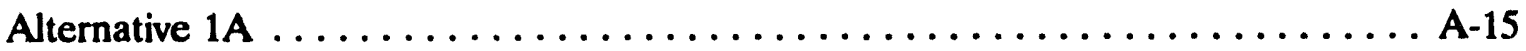

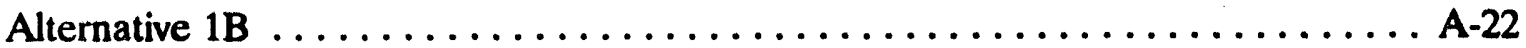

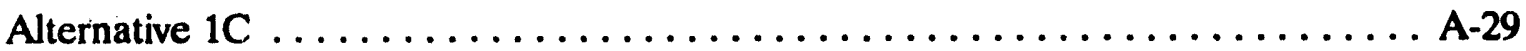

Alternative 1D . . . . . . . . . . . .

Alternative $1 \mathrm{E}, \ldots \ldots \ldots \ldots \ldots \ldots \ldots \ldots \ldots \ldots \ldots \ldots \ldots \ldots \ldots \ldots \ldots$

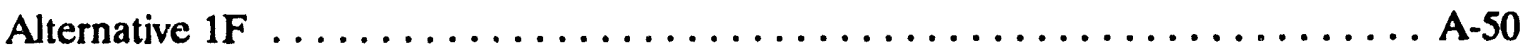

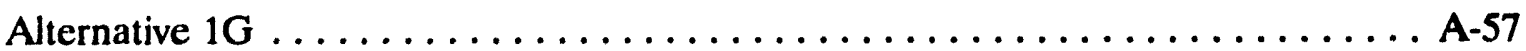

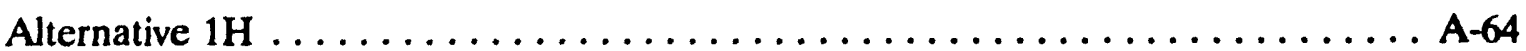

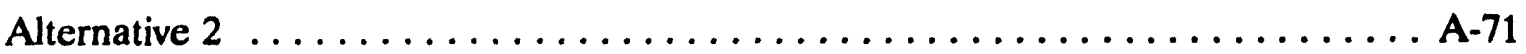

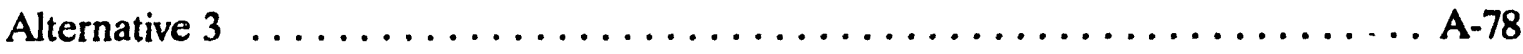

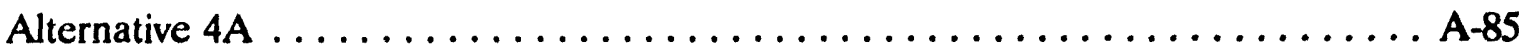

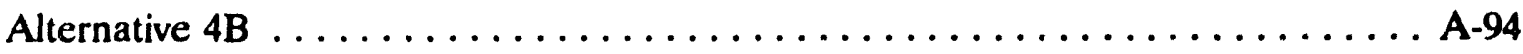

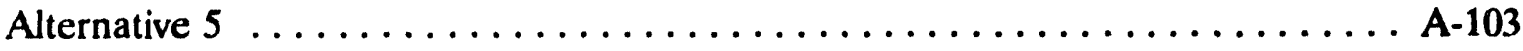




\section{A.1 INTRODUCTION}

This appendix provides the cost-estimate details that led to the estimates in Tables 13-1 and 13-2. The basis for the estimate of each cost element is also provided.

The remainder of the appendix (Section A.2) consists of a set of Detailed Cost Estimate Sheets for each disposal alternative. For each entry on an estimate sheet, the column headings call out the cost account number, activity description, type of source of information, estimated cost, unescalated total cost for that entry, escalated cost to year of expenditure, activity schedule for escalation, and the basis for the estimate. Subtotals are given for the seven categories of life cycle costs listed in Section 13.3.

Unescalated cost is generally rounded to the nearest thousand; Escalated cost is generally rounded to the nearest ten thousand, hundred thousand, or million. As a result, the totals are sometimes not exactly equal to the sum of the individual parts; however, the error involved in these round-offs is less than $1 \%$. 


\section{A.2 DETAILED COST ESTIMATE SHEETS}

Following are the Detailed Cost Estimate Sheets. The sheets for the various alternatives are ordered in the same sequence as that used throughout the main text of this document. 


\section{INTEROFFICE CORRESPONDENCE}

Date: $\quad$ July 7,1993

To: $\quad$ T. H. Smith, MS 3960

From: $\quad$ D. N. Stoddard, MS 3655

Subject: EVALUATIONS OF ALTERNATIVES FOR THE DISPOSAL OF MIXED AND LOW-LEVEL WASTE - DNS - 22-93

Cost Estimating has prepared the attached Cost Estimate Studies investigating thirteen (13) alternatives of the above referenced project. Each alternative investigated will be included in a report to be published by Mr. T. H. Smith on Alternative Methods for the Disposal of Mixed and Low-Level Waste at the Idaho National Engineering Laboratory (INEL). As instructed, the resulting data is based upon the information provided at the time the work package was received by Cost Estimating. Subsequent meetings were necessary to define the scope and parameters of each alternative. Based upon the above references and verbal communication, the resulting costs encompass those items as shown on the attached Overview, Data Recapitulation, Summary, Contingency Analysis and Detail Sheets. Rough Cost data has been adjusted to the specific application and escalated accordingly for the appropriate scheduled time frame of occurrence.

Should you have any questions, please feel free to contact either of us, T. E. Sivill at 526-9273 or D. N. Stoddard at 526-5484.

\section{References:}

(a) A Draft of Preliminary Evaluation of Alternatives for Disposal of INEL Low Level Waste and Low Level Mixed Waste

(b) Preliminary Evaluation of Alternatives for Treatment of INEL Low Level Waste and Low Level Mixed Waste, No. EGG-WMO-10321, June, 1992

(c) D.N. Stoddard letter to M. J. Sherick, DNS-1-93, Co-Location of Mixed and Low Level Waste, February 8, 1993

(d) D. N. Stoddard letter to S. R. Withers, DNS-8-91, INEL Contingency Landfill, October 31, 1991

(e) J. A. Logan letter to G. A. Beitel, JAL-29-90, Draft ID 5820.2A's Requirements for a 2 -meter Thick Cover Over Disposed LLW, September 21, 1990

(f) Decommissioning Handbook, DOE/EV/10128-1, November 1980

(g) Final Report/Guidance Manual: Cost Estimates for Closure and Post-Closure Plans (Subpart G and H) Volume III - Unit Costs, No. PB87-159018, EPA Contract No. 68-01-6861, November 1986

$\mathrm{mm}$

At tachments:

As Stated
CC: W. S. Roesener, MS 3505

M. J. Sherick, MS 3941

T. E. Sivill, MS 3655

M. Jorgenson-Waters, MS 3422
Central Files, MS 1651

Estimate File 5204 D. N. Stoddard File 
OVERVIEW OF EVALUATIONS OF DISPOSAL ALTERNATIVES

FOR MIXED AND LOW-LEVEL WASTE

\begin{tabular}{|c|c|c|}
\hline ALTERNATIVE & $\begin{array}{c}\text { UNESCALATED } \\
\text { TPC }\end{array}$ & $\begin{array}{c}\text { ESCALATED } \\
\text { TPC }\end{array}$ \\
\hline ALTERNATIVE 1A - Above-Grade Engineered Structures & $640,000,000$ & $3,400,000,000$ \\
\hline ALTERNATIVE 1B - Below - Grade Engineered Structures (BGES) & $650,000,000$ & $3,350,000,000$ \\
\hline ALTERNATIVE 1C - Covered Modules & $780,000,000$ & $4,000,000,000$ \\
\hline ALTERNATIVE 1D - Greater Depth Buria/Boreholes & $500,000,000$ & $3,000,000,000$ \\
\hline ALTERNATIVE 1E - Shallow Land Burial/Boreholes & $490,000,000$ & $3,000,000,000$ \\
\hline ALTERNATIVE 1F - Shallow Land Burial/AGES & $488,000,000$ & $2,950,000,000$ \\
\hline ALTERNATIVE 1G - Effect of Waste Volumes & $440,000,000$ & $2,750,000,000$ \\
\hline ALTERNATIVE 1H - Efiect of Waste Treatment & $970,000,000$ & $4,700,000,000$ \\
\hline ALTERNATIVE 2 - Continue Disposal at the RWMC & $510,000,000$ & $3,140,000,000$ \\
\hline ALTERNATIVE 3 - Ship Waste Oftsite for Disposal & $295,000,000$ & $994,100,000$ \\
\hline ALTERNATIVE 4A - MULWDF/Ship Offsite (Combination 1A \& 3) & $677,000,000$ & $3,400,000,000$ \\
\hline ALTERNATIVE 4B - RWMC/Ship Offsite (Combination $2 \& 3$ ) & $601,000,000$ & $3,090,000,000$ \\
\hline ALTERNATIVE 5 - No Action & $533,000,000$ & $10,299,000,000$ \\
\hline
\end{tabular}


$E G \& G$ Idaho, inc.

FORM EG\&G-1950

(Rev. 06-91)

\section{COST ESTIMATE SUPPORT DATA RECAPITULATION}

$\begin{array}{rrl}\text { Project Title: EVALUATIONS OF ALTERNATIVES FOR } & \text { Estimator: } & \text { T. E. Sivill } \\ \text { THE DISPOSAL OF MIXED AND LOW-LEVEL WASTE } & & \text { D. N. Stoddard }\end{array}$

Type of Estimate: Study

File No: 5204

Approved By:

$07 / 07 / 93$

I. SCOPE OF WORK: Brief description of the proposed project.

Perform a cost study based on thirteen (13) alternatives and subalternatives for the disposal of mixed and low-level waste at the Idaho National Engineering Laboratory (INEL). The site selected for the a)ternative studies $1 A$ through $1 H$ is referenced as Site $A$ and is located approximately 17 miles north of the Central Facilities Area (CFA) and one mile east of Lincoln Boulevard. Alternative 2 addresses continued operations at the Radioactive Waste Management Complex (RWMC), Alternative 3 addresses Offsite disposal, Alternative 4 addresses combinations of $1 A$, 3 and also 2, 3 . Alternative 5 addresses storage only (located at the Waste Experimental Reduction Facility, WERF) as a result of No Action.

The Cost Studies for each alternative are analyzed by comparison of Total Project costs (TPC). TPC includes the costs for Front End Documentation, i.e., Project Management, Studies, Permitting and Technology Development. Also included are Engineering/Design, Title III Inspection, Start-Up and Readiness, Operational Costs, Decontamination and Decommissioning (D\&D, Closure and Post Closure.

II. BASIS OF THE ESTIMATE: Drawings, Design Report, Engineers notes, and/or other documentation upon which the estimate is originated.

(a) A Draft of Preliminary Evaluation of Alternatives for Disposal of INEL Low Level Waste and Low Level Mixed Waste

(b) Preliminary Evaluation of Alternatives for Treatment of INEL Low Level Waste and Low Level Mixed Waste, No. EGG-WMO-10321, June, 1992

(c) D. N. Stoddard letter to M. J. Sherick, DNS-1-93, Co-Location of Mixed and Low Level Waste, February 8, 1993

(d) D.N. Stoddard letter to S. R. Withers, DNS-8-91, INEL Contingency Landfi11, October 31, 1991

(e) J. A. Logan letter to G. A. Beitel, JAL-29-90, Draft ID 5820.2A's Requirements for a 2-meter Thick Cover Over Disposed LLW, September 21,1990

(f) Decommissioning Handbook, DOE/EV/10128-1, November 1980

(g) Final Report/Guidance Manual: Cost Estimates for Closure and PostClosure Plans (Subpart $G$ and $H$ ) Volume III - Unit Costs, No. PB87159018, EPA Contract No, 68-01-6861, November 1986 
$E G \& G$ Idaho, inc.

\section{COST ESTIMATE SUPPORT DATA RECAPITULATION}

(CONTINUATION)

File No: $\quad 5204$

Page 2 of 6

III. ASSUMPTIONS: Condition statements accepted or supposed true without proof or demonstration. An assumption has a direct impact on total estimated cost.

Contingency is determined by the guidelines established by DOE/MA-0063 Vol 6., Cost Estimating Methods and Techniques, and as presented in the INEL Cost Estimating Guide. Contingency averages $20 \%$ to $21 \%$ based upon the risk associated with the changing climate in the area of regulatory requirements, process advancements, and code compliance.

Escalation is determined by the "Departmental Price Change Index, FY 1995 Guidance, Anticipated Economic Escalation Rates DOE Construction Projects. Escalation beyond 1998 is averaged at $3.8 \%$ for the outyears shown on the detail sheets.

\section{ALTERNATIVE 1A: ABOVE-GRADE ENGINEERED STRUCTURES (AGES)}

Alternative $1 \mathrm{~A}$ consists of 115 acres of site development to include the necessary facilities to accommodate waste disposal. Project scope includes fencing, support buildings (office/admin building, facility support building, materials storage, and heavy equipment storage and maintenance buildings), utility installation and roadways. A surface gallery drainage systein consists of catch basins and underground piping routed to a pumphouse and retention pond. A leachate drainage collection system includes two (2) sublayer single point drains from each disposal vault with underground piping to a collection sampling vault located within 200 feet of the facility. A total of twenty-five (25) concrete waste disposal vaults will be constructed throughout the project duration. Initial equipment required for day to day functions will be purchased and replaced throughout the forty (40) year operational life of the facility. D\&D Will encompass the support facilities and associated utilities. Closure will consist of a multi-layer engineered cover including revegetation. Post closure incudes monitoring and maintenance as required by regulations.

\section{ALTERNATIVE 1B: BELOW-GRADE ENGINEERED STRUCTURES (BGES)}

Alternative $1 B$ consists of 112 acres of site develorment to include the necessary facilities to accommodate waste disposal. Project scope differs from $1 A$ in that the concrete disposal vaults will be located below grade. The below grade concept requires modifications to the utilities, drainage and collection systems. 
$E G \& G$ Idaho, inc.

\section{COST ESTIMATE SUPPORT DATA RECAPITULATION}

(CONTINUATION)

File No: $\quad 5204$

Page 3 of 6

\section{ASSUMPTIONS: (CONTINUED)}

\section{ALTERNATIVE $1 C$ : COVERED MODULES}

Alternative $1 C$ consists of 132 acres of site development to include the necessary facilities to accommodate waste disposal. Project scope remains the same as $1 \mathrm{~A}$ for the support facilities. Waste will be placed on concrete pads in lieu of vaults.

\section{ALTERNATIVE 1D: GREATER DEPTH BURIAL/BOREHOLES}

Alternative 10 consists of 112 acres of site development to include the necessary facilities to accommodate waste disposal. Project scope remains the same as 1A for the support facilities. Waste will be placed on concrete pads in lieu of vaults. Disposal includes a combination of greater depth burial and greater depth boreholes.

\section{ALTERNATIVE 1E: SHALLOW LAND BURIAL/BOREHOLES}

Alternative $1 E$ consists of 108 acres of site development to include the necessary facilities to accommodate waste disposal. This concept is identical to alternative 10 except that the depth of the disposal is considerably less.

\section{ALTERNATIVE IF: SHALLOW LAND BURIAL/ABOVE GRADE ENGINEERED STRUCTURES}

Alternative IF consists of 122 acres of site development to include the necessary facilities to accommodate waste disposal. This alternative includes shallow land burial identical to IF with the bore hole concept replaced by above ground engineered structures similar to $1 A$.

\section{ALTERNATIVE 1G: EFFECT OF WASTE VOLUMES}

Alternative $1 G$ consists of 86 acres of site development to include the necessary facilities to accommodate waste disposal. This alternative is identical to Alternative $1 A$, in which all types of waste are disposed of in above ground engineered structures. The volume has been reduced assuming minimal ER and D\&D scenario. 
$E G \& G$ Idaho, ine.

COST ESTIMATE SUPPORT DATA RECAPITULATION

(CONTINUATION)

File No: $\quad 5204$

Page 4 of 6

\section{ASSUMPTIONS: (CONTINUED)}

\section{ALTERNATIVE 1H: EFFECT OF WASTE TREATMENT.}

Alternative $1 \mathrm{H}$ consists of 166 acres of site development to include the necessary facilities to accommodate waste disposal. This alternative is identical to alternative $1 \mathrm{~A}$, in which all types of waste received are disposed of in above ground engineered structures, except that the volume of waste is increased assuming minimal waste treatment scenario.

\section{ALTERNATIVE 2: CONTINUE DISPOSAL AT THE RWMC}

Alternative 2 assumes RWMC's present mission is completed by the year 2004. The existing support facilities will accommodate the functions for this facility through the year 2045. Three of the support facilities will be replaced during that time frame. Boundaries of the existing RWMC will not change their present configuration. Four existing areas within the Subsurface Disposal Area (SDA) denoted as Pits 18 through 20, area between Pits 8 and 9 , area west of Pit 3 , and area between Pits 3 and Pad $A$ will be utilized for the new disposal pads. Existing access roads, drainage areas, monitoring facilities, fencing, security gates, operational bulldings and parking and lighting will already be in place to accommodate this alternative requiring minimal upgrades or expansion.

\section{ALTERNATIVE 3: SHIP WASTE OFFSITE FOR DISPOSAL}

Alternative 3 assumes that a Mixed and Low-Level Waste Disposal Facility (MLLWDF) is not built, the RWMC is shut down permanently, the large-volume waste scenario and the maximum waste treatment scenario applies. This alternative assumes that general support will be housed through expansion of the existing waste treatment facility. A new transfer building and truck maintenance/decon facility will be built adjacent to the treatment complex. All waste will be shipped offsite approximately 1000 miles.

\section{ALTERNATIVE 4A: MLLWDF/SHIP OFFSITE (ALTERNATIVE IA AND 3 COMBINATION)}

Alternative $4 A$ assumes that disposal facility is built for low-level waste only. When the new disposal facility begins operation, RWMC is shut down permanently. All INEL mixed waste will be shipped offsite to a DOE disposal facility. The maximum generation and maximum treatment scenarios apply. 
EG\&G idaho, inc.

\section{COST ESTIMATE SUPPORT DATA RECAPITULATION} (CONTINUATION)

File No: $\quad 5204$

Page 5 of 6

III. ASSUMFTIONS: (CONTINUED)

ALTERNATIVE 4B: RWMC/SHIP OFFSITE (ALTERNATIVE 2 AND 3 COMBINATION)

Alternative $4 B$ assumes the disposal methods and current disposal space at the RWMC is modified to accommodate more low-level waste. A new disposal facility will not be required for this option. All INEL mixed waste will be shipped offsite to a DOE disposal facility. The maximum generation and maximum treatment scenarios apply.

\section{ALTERNATIVE 5: NO ACTION}

Alternative 5 consists of 47 acres of site development to include the necessary facilities to accommodate waste storage of mixed and low-level waste. This alternative assumes a disposal facility will not be built and no other disposal alternative will be implemented. Also, as descrtbed in Alternative 2, RWMC will no longer be accepting waste. The maximum generation and maximum treatment scenario apply to this alternative. This storage is assumed to be located near WERF. The support facilities required for this alternative are identical to that required in Alternative lí. 
$E G \& G$ Idaho, inc.

\section{COST ESTIMATE SUPPORT DATA RECAPITULATION} (CONTINUATION)

File No: $\quad 5204$

Page 6 of 6

\section{OTHER COMMENTS/CONCERNS SPECIFIC TO THE ESTIMATE: (CONTINUED)}

- FRONT END COSTS include Project Management, preliminary studies (project definition, design support, siting, etc.), documentation and permitting (NEPA, NESHAP, TSCA, RCRA, SAR, etc.) and Research and Development (technology development).

- ENGINEERING, DESIGN AND INSPECTION includes performance specifications, conceptual and Title I/II design and Title III inspection of the required facilities, disposal areas, utility runs and hookups to accommodate the proposed disposal functions at the RWMC.

- CONSTRUCTION COSTS include land improvements for the disposal pads, future replacement buildings, construction costs, utility installation and hookup. Construction Management for this phase has been include at the current rate of twenty-two percent $(22 \%)$ of construction costs.

- START-UP AND READINESS includes the costs associated with initial system testing, system operation and maintenance manuals, and training of personnel associated with the operations of the processes. The crew has been sized based upon existing operations presently functioning at the RWMC and adjusted to handle the requirements of this alternative.

- OPERATING COSTS include the staff, materials, equipment maintenance (improvements and replacement parts) and utilities necessary to the facility function over a period of forty (40) years. Again, the staff size and utility usage has been derived from the operations of existing facilities and adjusted for this specific alternative.

- DECONTAMINATION AND DECOMMISSIONING includes costs associated with the total dismantlement of the support facilities constructed for this alternative. Per Project Manager/Engineer direction, D\&D costs have been estimated at $10 \%$ of the original facility and process construction costs.

- Management Reserve has also been allowed for based upon the Construction costs. This is calculated in the overall percentage of Contingency 
EG\&G Idaho, Inc.

Type of Estimate: PLANNING

Date:

07/05/93

Project: EVALUATION OF ALTERNATIVES FOR DISPOSAL OF INEL MIXED AND LOWLEVEL WASTE

File No. $\quad 5204-$ Alt. $1 \mathrm{~A}$

Appr'd By:

sol

Prepared By: T.E. SIVIL

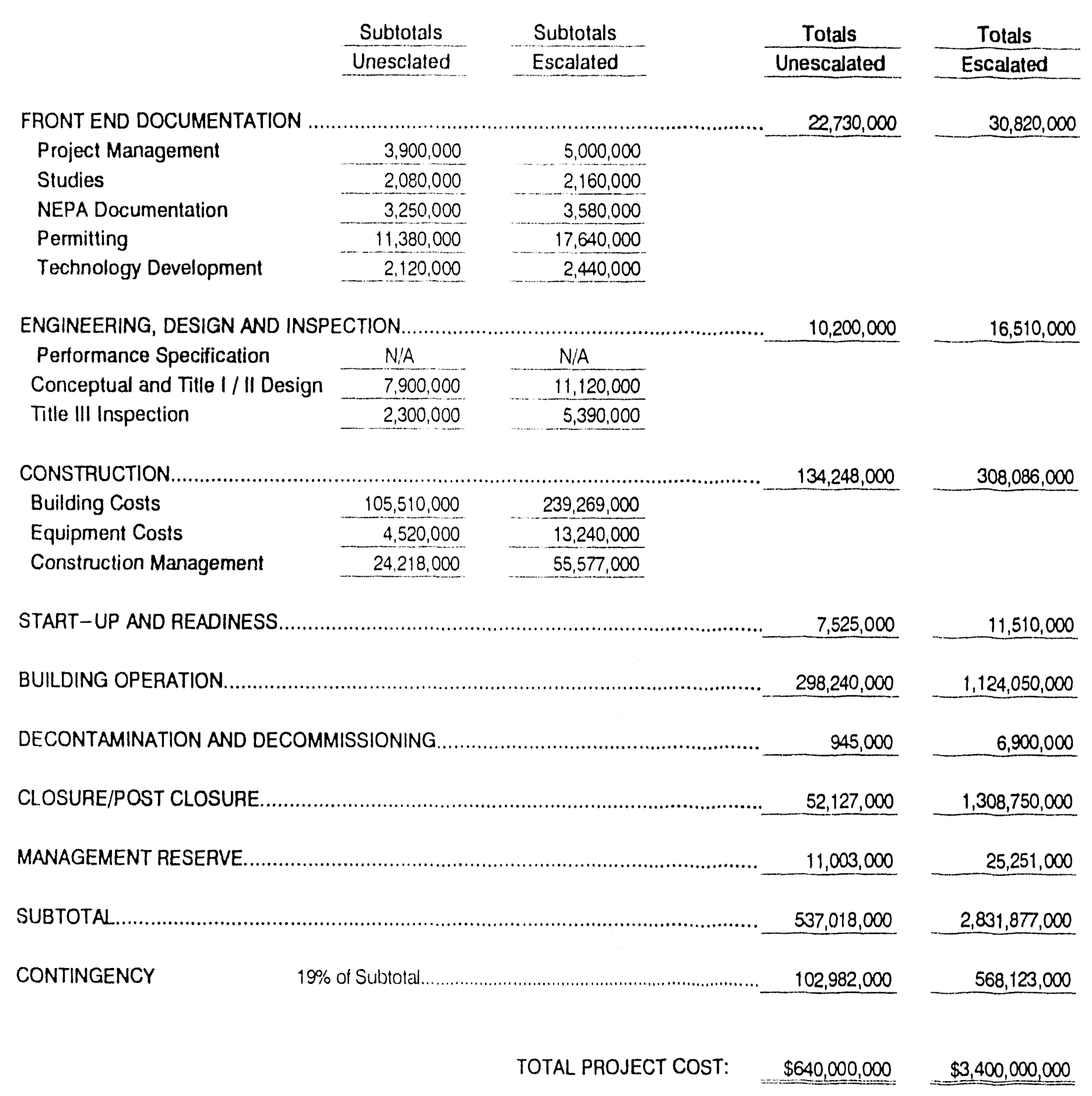

Comments: 
Rev 5-92

Project EVALUATION OF ALTERNATIVES For DISPOSAL OF INEL MIXED AND LOW LEVEL WASTE

Location INEL

Requester T.H.SMITH
Type of Estimate PLANNING

Source (E) Eng. Est

(V) Vendor

(P) Pur. Order

(H) Handbook Ret.
File No. 5204 - Alt. 1A

Date

Prep'd By:

Chk'd By:

Appr'd By: $\operatorname{sex}$

\begin{tabular}{|c|c|}
\hline $\begin{array}{c}\text { ACCT. } \\
\text { NO. }\end{array}$ & $\begin{array}{c}\text { ACTIVITY } \\
\text { DESCAIPTION }\end{array}$ \\
\hline & PROJECT COSTS \\
\hline & Project Management \\
\hline & Project Studies - 2 People. Full Time \\
\hline & Project Formulation (F\&OR's) \\
\hline & Siting Studies \\
\hline & Pre-Operational Monitoning \\
\hline & Safely Analysis Repon (SAR) \\
\hline & Performance Assessment \\
\hline & RCRA \\
\hline & NEPA (EIS) (CX'S) \\
\hline & Air Permits (PSD/PTC/NESHAP) \\
\hline & ESH \\
\hline & Oiner Regulatory llems (State Water Wull Approval! \\
\hline & Special O/A Program For Construction - 2 Peopte \\
\hline & \\
\hline & $\ldots$ \\
\hline & IECHNOLOGY DEVELOPMENT \\
\hline & Material Behavior (Conc. Clay. etc) \\
\hline & Cover Pertormance Control \\
\hline & Leachate Collection and Liner Pentormance \\
\hline$\ldots$ & Material Handling (Gantry Crane) \\
\hline & \\
\hline & \\
\hline & 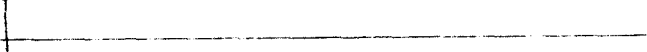 \\
\hline & \\
\hline & Subtutal tor Front End Costs \\
\hline
\end{tabular}

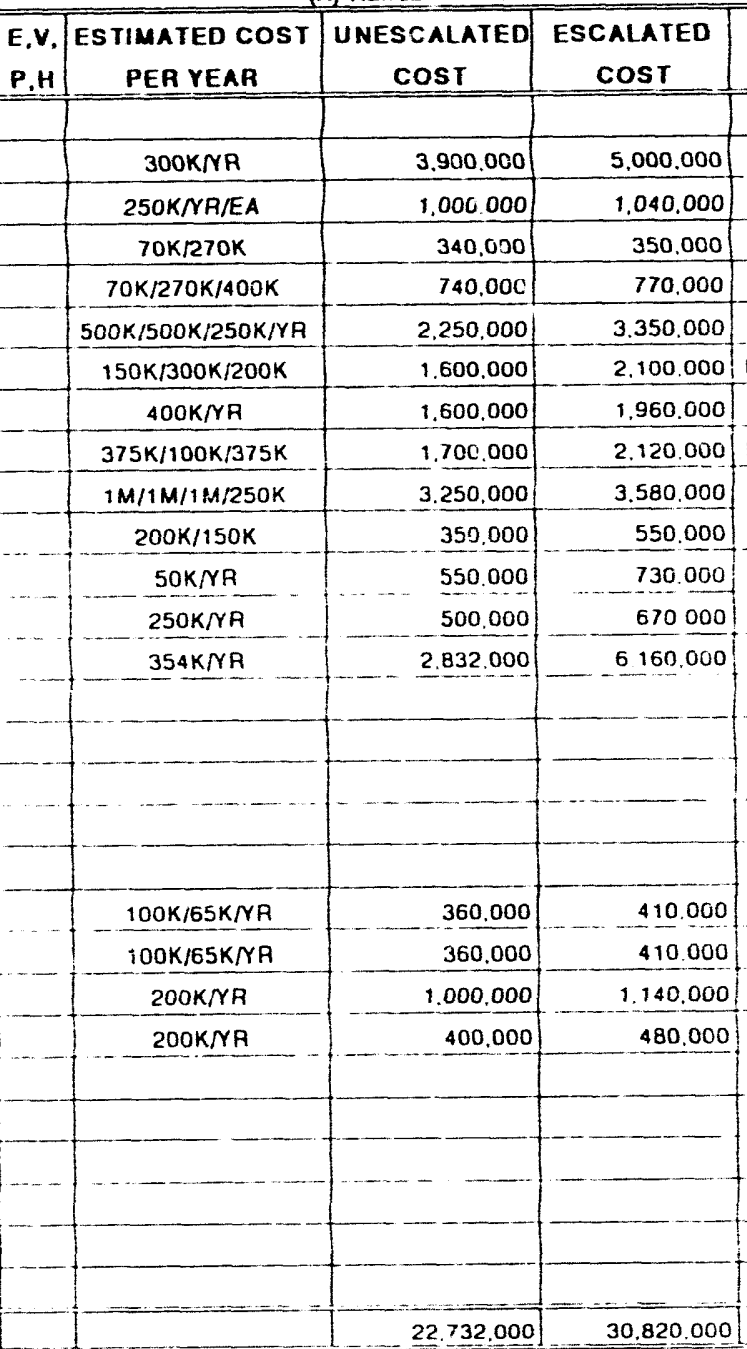

ACTIVITY SCHEDULE ESCALATION

\begin{tabular}{l|l} 
FY92 THRU FY2C05 & TOD Sheet. dated 4/28/93
\end{tabular}

\begin{tabular}{l|l}
\hline FY93 -94 & TDD Sneet, dated 4/28/93 \\
\hline FYg2 -93
\end{tabular}

FY92 - 93

FY92 - FY94

TDD Sheet, dated 4/28/93. Eng Estimate

TDD Sneet, dated 4/28/93, Eng Estimate

FY95.FY99,FY2000-2005 Engineer's Estimate

Y95-96.FY99-01.FYO2 - 03 Data from Similar SAR's

FY95-96.FY99-00 Engineer's Estimate

FY96-97.FY98-99.FY00-01 Engineer's Estimate (Includes Sampling)

\begin{tabular}{l|l} 
FY94-FYg7 & Engineer's Estimate
\end{tabular}

FV, 995 FY2000

Engineer's Estlmate

FY1995- 2005

Engineer's Estimate

FY2000-:301

Enyineer's Estimate

FY2001-04.13.23.24.33

Engineer's Estima

\begin{tabular}{|l|l|}
\hline & \\
\hline FY94.FY95-98 & Engineer's Estimate \\
\hline FY94. FY95-98 & Engineer's Estimate \\
\hline FY94-98 & Engineer's Estimate \\
\hline FY97-98 & Engineer's Estimate \\
\hline & \\
\hline & \\
\hline & \\
\hline
\end{tabular}


Rev 5-92

Page 2 of 5

Type of Estimate: PLANNING

File No. $5204-$ Alt. 1 A

Date:

$07 / 05 / 93$

Project EVALUATION OF ALTERNATIVES FOR DISPOSAL

OF INEL MIXED ANO LOW LEVEL WASTE

\begin{tabular}{|c|c|c|c|c|c|c|c|}
\hline $\begin{array}{c}\text { ACCT. } \\
\text { No. }\end{array}$ & $\begin{array}{c}\text { ACTIVITY } \\
\text { DESCAIPTION }\end{array}$ & $\begin{array}{l}\text { E.V. } \\
P . H\end{array}$ & $\begin{array}{c}\text { ESTIMATED COST } \\
\text { PER YEAR }\end{array}$ & $\begin{array}{c}\text { UNESCALATED } \\
\text { COST }\end{array}$ & $\begin{array}{c}\text { ESCALATED } \\
\text { COST }\end{array}$ & $\begin{array}{l}\text { ACTIVITY SCHEDULE } \\
\text { ESCALAIION }\end{array}$ & ESTIMATE BASIS \\
\hline & DESIGN & & & & & & \\
\hline & Conceptual & & $500 \mathrm{~K} / 500 \mathrm{~K}$ & $1.000,000$ & $1,110,000$ & FY95,FY96 & ToD Sheet \\
\hline & Tite I & & $3 M$ & 3.000 .000 & $3,530.000$ & FY97 & Engineers Estimate \\
\hline & Tive 11 & & $3 \mathrm{M} .300 \mathrm{~K} / \mathrm{NR}$ & 3.900 .000 & 6.480 .000 & FY98.2012.2022.2032 & Engineers Estimate \\
\hline$\ldots \ldots$ & Sublotal for Design (Based on $\%$ of Construction Costs) & $\ldots$ & 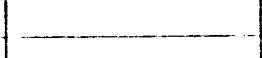 & $7.900,000$ & 11.120 .000 & & \\
\hline - - - - & Tive III Inspection (4 People for 30 Months) & & 575K/575K/288K EA & 2.300 .000 & 5.390 .000 & FY $2003,04,13,23,24,8,2033$ & Comparative Historical Data \\
\hline & & & & & & & \\
\hline & CONSTRUCIION & & & & & & \\
\hline 4000 & Improvements to Land & & & & & & \\
\hline & Surveying (115 Acres) & & 150,000 & 150.000 & 210,000 & FY2002 & Comparative Historical Data \\
\hline & Site Prep Clearing and Grubbing (115 Acres) & & 345.000 & 345.000 & 490.000 & FY2002 & Comparative Historical Data \\
\hline & Roadways and Parking & & $1.400,000$ & $1.400,000$ & 1.980 .000 & FY2002 & Comparative Historical Data \\
\hline & Fencing & & 400.000 & 400,000 & 570.000 & FY2002 & Comparative Historical Data \\
\hline & Retention Pond & & 40,000 & 40.000 & 57,000 & FY2002 & Comparative Historical Data \\
\hline & Drainage Gallery System & & 900,000 & 900,000 & $1,280,000$ & FY20ก2 & Comparative Historical Data \\
\hline & Drainage Pumphouse & & 100.000 & 100,000 & 142.000 & FY2002 & Comparative Historical Data \\
\hline & Collectlon /Sampling System Vault at Disposal Areas & & $1,000.000$ & $1.000,000$ & 1.420 .000 & FY2002 & Comparative Historical Data \\
\hline & Storage Vault Disposal Area Including: Engineered & & $1,800.000$ & 1.800 .000 & 2.550 .000 & FY2002 & Comparative Historical Data \\
\hline & Fill, and Pit Run & & & & & & \\
\hline & Drainage Ditches & & 100.000 & 100.000 & 140.000 & FY2002 & \\
\hline & TOTAL FOR 4000 ACCOUNT & & & $6,235,000$ & $8,839,000$ & & \\
\hline 6000 & Utiitles & & & & & & \\
\hline & Electrical Power and Distribution To Facillty & & $1,850,000$ & $1,850,000$ & $2,620.000$ & FY2002 & Engineers Estimate \\
\hline & Electrical L'ghting.Power,Alarms, \& Communication & & 1.240 .000 & $1.240,000$ & $1,750,000$ & FY2002 & Engineers Estimate \\
\hline & Distributicin System & & & & & & Engineers Estimate \\
\hline & Water Supply \& Fire Water Distribution to Facility & & $1.500,000$ & $1.500,000$ & $2,130,000$ & FY2002 & Engineers Estlmate \\
\hline & Water Supply and Fire Water Distribution Systems & & 550,000 & 550,000 & 780,000 & FY2002 & Engineers Estimate \\
\hline & Sanitary Sewage System & & 250.000 & 250,000 & 350.000 & FY2002 & Engineers Estimate \\
\hline & TOTAL FOR 6000 ACCOUNT & & & $5,390,000$ & $7.630,000$ & & \\
\hline
\end{tabular}


Project EVALUATION OF ALTERNATIVES For DISPOSAL OF INEL MIXED AND LOW LEVEL WASTE

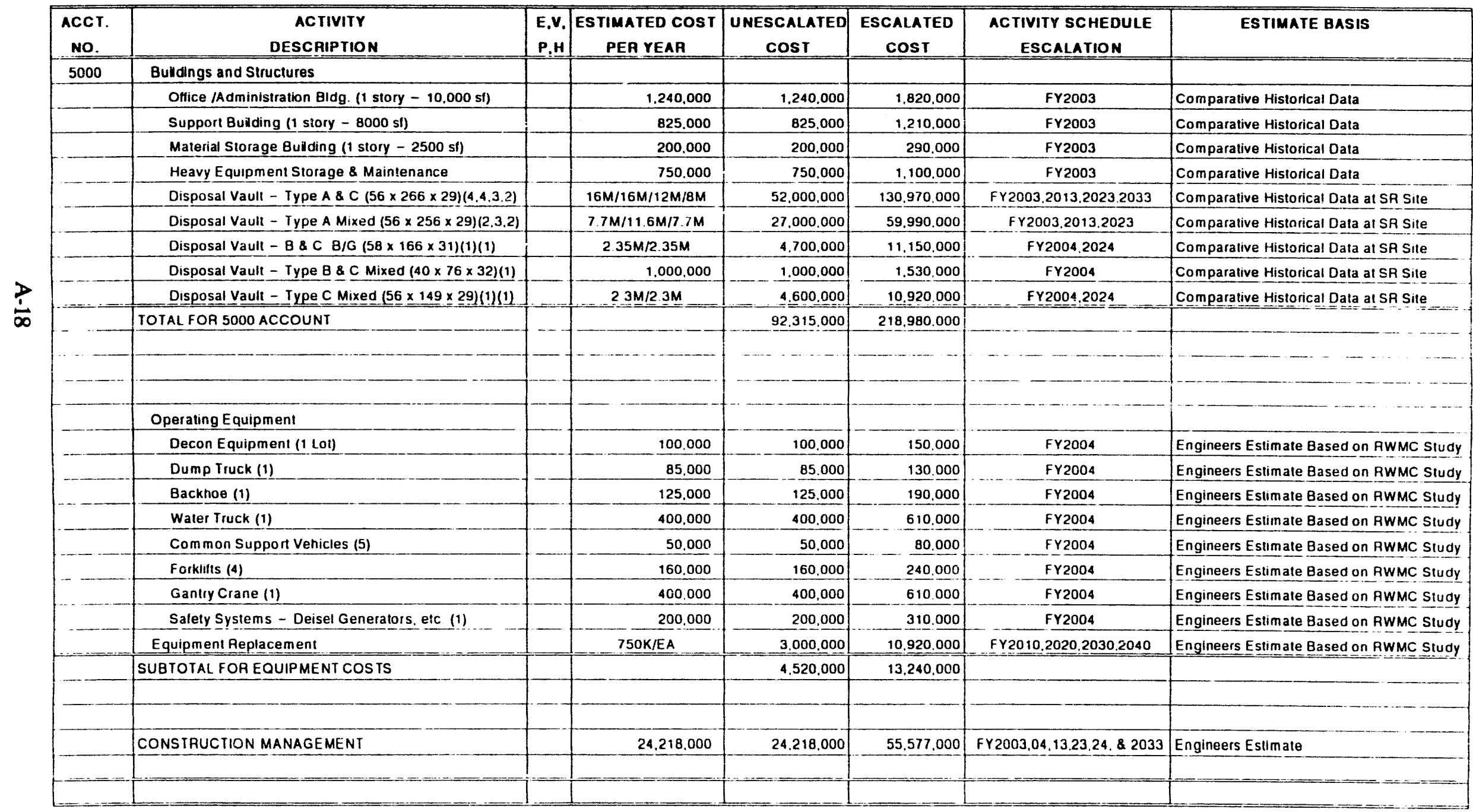


Page 4 of 5

Project evaluation of alternatives for disposal OF INEL MIXED AND LOW LEVEL WASTE

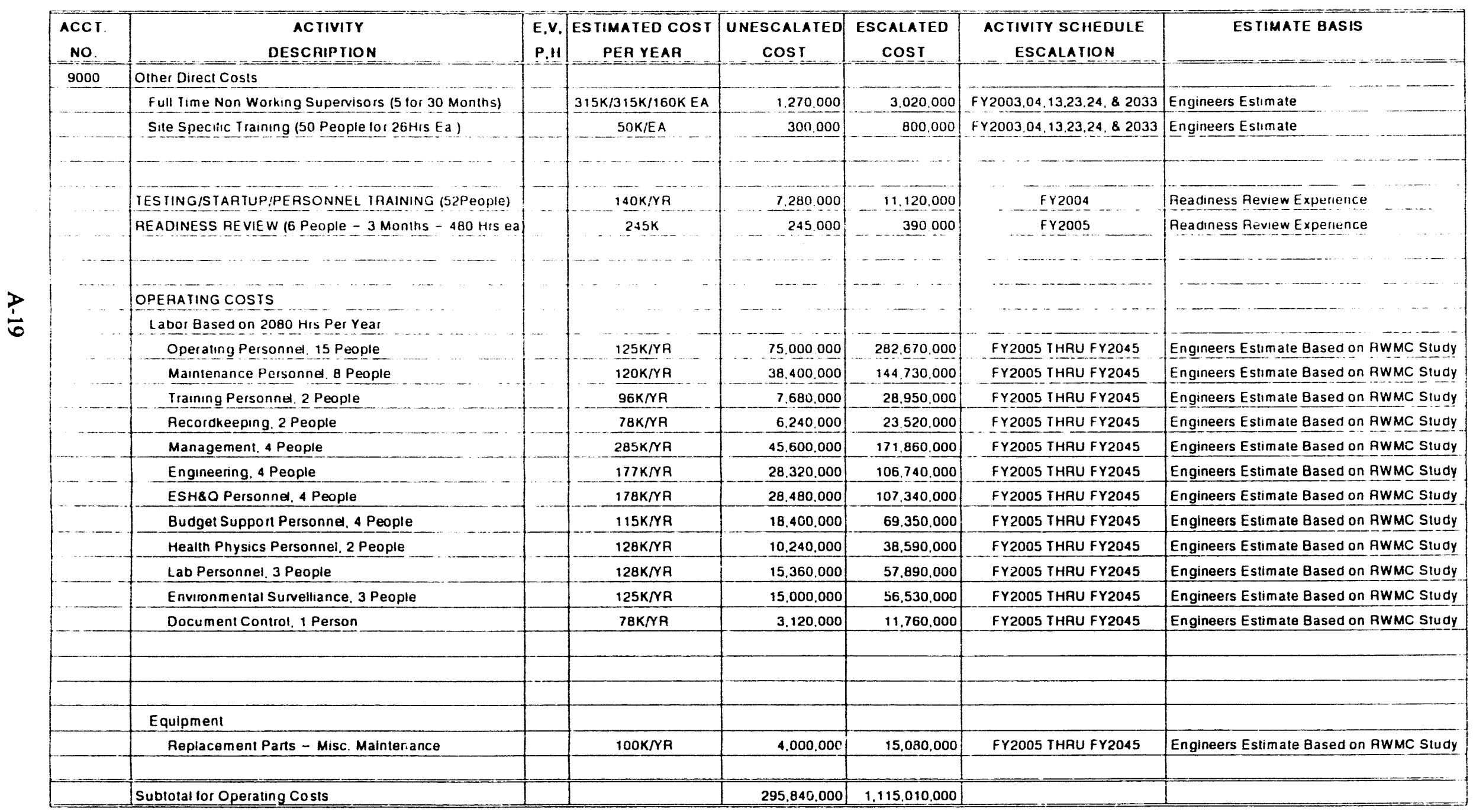


Prolect evaluation of alternatives for disposal

OF INEL MIXED AND LOW LEVEL WASTE

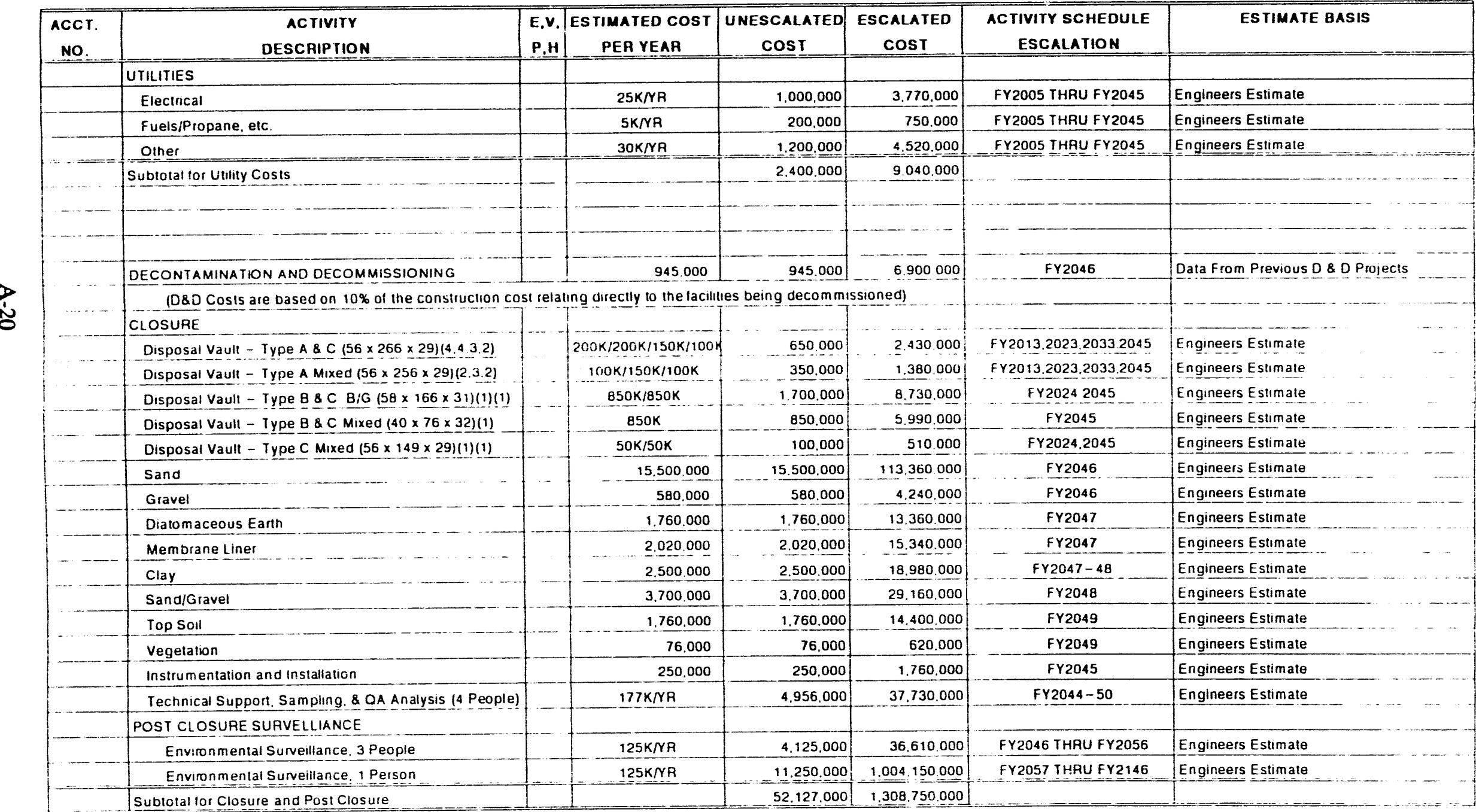


Rer 5-92

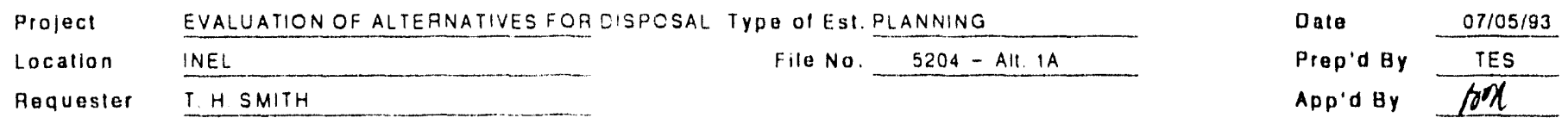

\begin{tabular}{|c|c|c|c|c|c|c|c|c|}
\hline \multirow{4}{*}{ ttem } & \multicolumn{4}{|c|}{ PROBABLE \% VARIAIION } & & & & \\
\hline & \multirow[t]{3}{*}{ Cosi } & \multirow{3}{*}{$\begin{array}{c}\% \\
\text { Total } \\
\text { Cosi }\end{array}$} & \multicolumn{2}{|l|}{ Est. Basis } & \multirow{2}{*}{\multicolumn{2}{|c|}{$\begin{array}{l}\text { Prob \% Var } \\
\text { From Est }\end{array}$}} & \multirow{2}{*}{\multicolumn{2}{|c|}{ Wi $\%$ of Prob }} \\
\hline & & & \multirow[t]{2}{*}{ Quote } & \multirow[t]{2}{*}{ Est } & & & & \\
\hline & & & & & - & + & - & + \\
\hline Prolect Management & 3.900 .000 & $1:$ & $25 !$ & $75 !$ & 5 & 20 & 0.04 & 0.15 \\
\hline Studies Permitting & 13.460 .000 & 3 & 25 & 75 & $10 !$ & 20 & 0.25 & 0.50 \\
\hline NEPA & 3.250 .000 & $1 \vdots$ & 5 & 95 & 201 & 25 & 0.12 & 0.15 \\
\hline Technology Developement & 2.120 .000 & 059 & 5 & 95 & 10 & 25 & 0.04 & 0.10 \\
\hline Tille 1 and Title $\|$ Design & 7.900 .000 & 1: & 25: & 75 & 101 & 15 & 0.15 & 0.22 \\
\hline Tille III Inspection & 2.300 .000 & 043 & 25 & 75 & 5 & 20 & 0.02 & 0.09 \\
\hline Construction Buildings \& Equipment & $110.030,000$ & 20 & 30 & 70 & 10 & 20 & 2.05 & 4.10 \\
\hline Construction Management & 24.218000 & 5 & 30 & 70 & 5 & 20 & 0.23 & 0.90 \\
\hline Startup and Readiness & 7.525 .000 & 1 & $25 !$ & 75 & $10 !$ & 15 & 0.14 & 0.21 \\
\hline Butlding Operation & 298.240 .000 & 56 & 5 & 95 & 20 & 25 & 11.11 & 13.88 \\
\hline Decontamination \& Decommissioning & 945.000 & 0 & 25 & 75 & 10 & 30 & 0.02 & 0.05 \\
\hline Closure/Post Closure & 52.127 .000 & 10 & 5 & $95 !$ & $10 !$ & 30 & 0.97 & 2.91 \\
\hline Management Reserve & 11.003000 & $=2$ & $=50$ & $50 !$ & 5 & 30 & 0.10 & 0.61 \\
\hline Tolal & 537018.000 & $100^{\prime}$ & & & & & 15.23 & 23.88 \\
\hline
\end{tabular}

Comments: Analysis results in an overall propeciconingency of

$20 \%$

In rounding. $\quad 19 \%$ was used. This is within the allowable $2 \%$ deviation $110 m$ the result of this analysis. 


\section{EG\&G Idaho, Inc.}

Type of Estimate: PLANNING

Project: EVALUATION OF ALTERNATIVES FOR DISPOSAL OF INEL MIXED AND LOW LEVEL WASTE

$\frac{\text { Subtotals }}{\text { Unescalated }}$

Sublotals
Escalated

Date:

File No.

Appr'd By:

Prepared By:

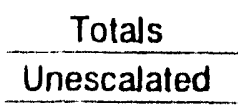

$22,730,000$

FRONT END DOCUMENTATION

Project Management

Studies

NEPA Documentation

Permitting

Technology Development

\begin{tabular}{rr}
$3,900,000$ & $5,000,000$ \\
\hline $2,080,000$ & $2,160,000$ \\
\hline $3,250,000$ & $3,580,000$ \\
\hline $11,380,000$ & $17,640,000$ \\
\hline $2,120,000$ & $2,440,000$ \\
\hline
\end{tabular}

$07 / 05 / 93$

$5204-$ Alt. $1 \mathrm{~B}$ Tan/

T.E. SIVILL

$\frac{\text { Totals }}{\text { Escalated }}$

$30,820,000$

$18,100,000$

ENGINEERING, DESIGN AND INSPECTION

$10,980,000$

Performance Specification

\begin{tabular}{rr}
$N / A$ & $N / A$ \\
\hline $8,100,000$ & $11,360,000$ \\
\hline $2,880,000$ & $6,740,000$ \\
\hline
\end{tabular}

Title III Inspection $2,880,000$

$144,459,000$

CONSTRUCTION $254,077,000$

$113,879,000$

$4,520,000$

$13,240,000$

$26,060,000$

$58,836,000$

Construction Management

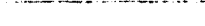

START-UP AND READINESS

$7,525,000$

$11,510,000$

BUILDING OPERATION.

$298,240,000$

$1,124,050,000$

DECONTAMINATION AND DECOMMISSIONING.

$1,000,000$

$7,310,000$

CLOSURE/POST CLOSURE

$43,126,000$

$1,241,420,000$

MANAGEMENT RESERVE

$11,840,000$

$26,732,000$

SUBTOTAL

$539,900,000$

$2,786,095,000$

CONTINGENCY

$20 \%$ of Subtotal.

$110,100,000$

$563,905,000$

TOTAL PROJECT COST:

$\$ 650,000,000$

$\$ 3,350,000,000$

Comments: 
Project evaluation of al teanatives for disposal OF INEL MIXED AND LOW LEVEL WASTE

Locatlon INEL

Requester T.H.SMITH

$+2$

\begin{tabular}{|c|c|}
\hline $\begin{array}{c}\text { ACCT. } \\
\text { No. }\end{array}$ & $\begin{array}{c}\text { ACTIVITY } \\
\text { DESCRIPIION }\end{array}$ \\
\hline
\end{tabular}

PROJECT COSTS

Project Management

Prolect Studies - 2 People. Full Time

Project Formulation (F $8 O R$ 's)

Siting Studies

Satety Analysis Report (SAR)

Pertormance Assessment

RCRA

NEPA (EIS)(CX's)

Air Permits (PSD/PTC/NESHAP)

ESHRO

Other Regulatory Items (State Water Well A pproval)

Special O/A Program for Construction - 2 People

-

-

\begin{tabular}{|l|l|}
\hline & TECHNOLOGY DEVELOPMENT \\
\hline & Material Behavior (Conc.. Clay, etc.) \\
\hline
\end{tabular}

Cover Pefformance Control

Leachate Collection and Liner Performance

\begin{tabular}{l|l}
- & Leachate Collection and Liner Pertormance \\
\hline & Material Handling (Gantry Crane) \\
\hline
\end{tabular}

\begin{tabular}{|l|l|}
\hline & \\
\hline & \\
\hline & \\
\hline & \\
\hline
\end{tabular}

Subtotal for Front End Costs

Type of Estimate PLANNING

Source (E) Eng. Est.

(V) Vendor

(P) Pur. Order

(H) Handbook ReA

\begin{tabular}{|l|l|l|}
\hline E.V. ESTIMATED COST & UNESCALATED & ESCALATED \\
\hline
\end{tabular}

P.H

$\left\{\begin{array}{l}1 \\ 500 x \\ \hdashline 1 \\ -1\end{array}\right.$

PER YEAR

30OKINA

250 KNR/EA

$70 K 1270 K$

$70 \mathrm{~K} / 270 \mathrm{~K} / 400 \mathrm{~K}$

$500 \mathrm{~K}: 500 \mathrm{~K}$ i250K/VA

$150 \mathrm{~K}$ !300K/200K

$400 \mathrm{~K} / \mathrm{YA}$

$375 K / 100 K / 375 K$

$1 \mathrm{M}: 1 \mathrm{M} / 1 \mathrm{M} / 250 \mathrm{~K}$

$200 K / 150 \mathrm{~K}$

5OK'YR

$250 K / N R$

$354 \mathrm{KNA}$

\begin{tabular}{r|r}
$250 K N R$ & 500.000 \\
\hline $354 K N R$ & 2.832 .000 \\
\hline
\end{tabular}

-

$100 \mathrm{~K} / 65 \mathrm{~K} / \mathrm{NA}$

$100 K / 65 K N R$

200KNA

$200 \mathrm{~K} / \mathrm{MR}$
File No.

\begin{tabular}{|c|c|}
\hline $\begin{array}{c}\text { UNESCALATED } \\
\text { COST }\end{array}$ & $\begin{array}{c}\text { ESCALATED } \\
\text { COST }\end{array}$ \\
\hline $3.900,000$ & $5,000.000$ \\
\hline $1.000,000$ & 1.040 .000 \\
\hline 340,000 & 350.000 \\
\hline 740,000 & 770.000 \\
\hline $2.250,000$ & 3.350 .000 \\
\hline $1,600,000$ & 2.100 .000 \\
\hline 1.600 .000 & 1.960 .000 \\
\hline $1.700,000$ & 2.120 .000 \\
\hline 3.250 .000 & 3.580 .000 \\
\hline 350.000 & 550.000 \\
\hline 550.000 & 730.000 \\
\hline 500.000 & 670.000 \\
\hline \multirow[t]{4}{*}{2.832 .000} & $6.160,000$ \\
\hline & \\
\hline & \\
\hline & \\
\hline 360.000 & 410,000 \\
\hline 360.000 & 410,000 \\
\hline $1,000,000$ & $1,140,000$ \\
\hline 400,000 & 480.000 \\
\hline & \\
\hline & \\
\hline & \\
\hline & \\
\hline & \\
\hline & \\
\hline & \\
\hline
\end{tabular}

30.820 .000

$5204-$ Alt. 18
Date:

Chk'd By:

Apprd By: $\overline{\text { LON }}$

ACTIVITY SCHEDULE ES TIMATE BASIS

ESCALATION

FY92 THRU FY2005

FY $93-94$

Frg2 - 93

FY92 - FY94

FY95.FY99.FY2000-2005 Engineer's Estimate

TDD Sneet, dated $4 / 28 / 93$

IDD Sheet, dated $4 / 28 / 93$

TDD Sheet. dated 4/28/93, Eng Estimale

TDD Sheel, dated 4/28/93 Eng Estimate

- 96.FY99 - 01 FY02 - 03 Data trom Similar SAR'S

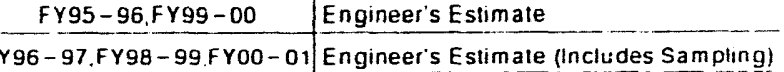

FY94 - FY97 Engineer's Estimate

FY1999. FY2000 Engineer's Estimate

FY1995-2005 Engineer's Estimate

FY2000-2001 Engineer's Estimate

Fr2001-04.13.23.24.33 Engineer's Estima

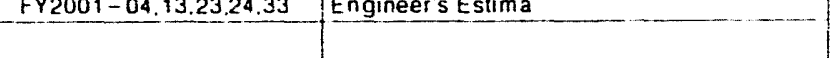

\begin{tabular}{|l|l|l|}
\hline & $200 \mathrm{~K} / \mathrm{F}$ \\
\hline & \\
\hline & \\
\hline & \\
\hline & \\
\hline
\end{tabular}

FY94, FY95-98

\begin{tabular}{|l}
\hline \\
\hline \\
\hline \\
\hline \\
\hline
\end{tabular}

Engineer's Estimate

Engineer's Estimate

Engineer's Estimate

ngineer's Estimate 
Project EVALUATION OF ALTERNATIVES FOR DISPOSAL OF INEL MIXED AND LOW LEVEL WASTE

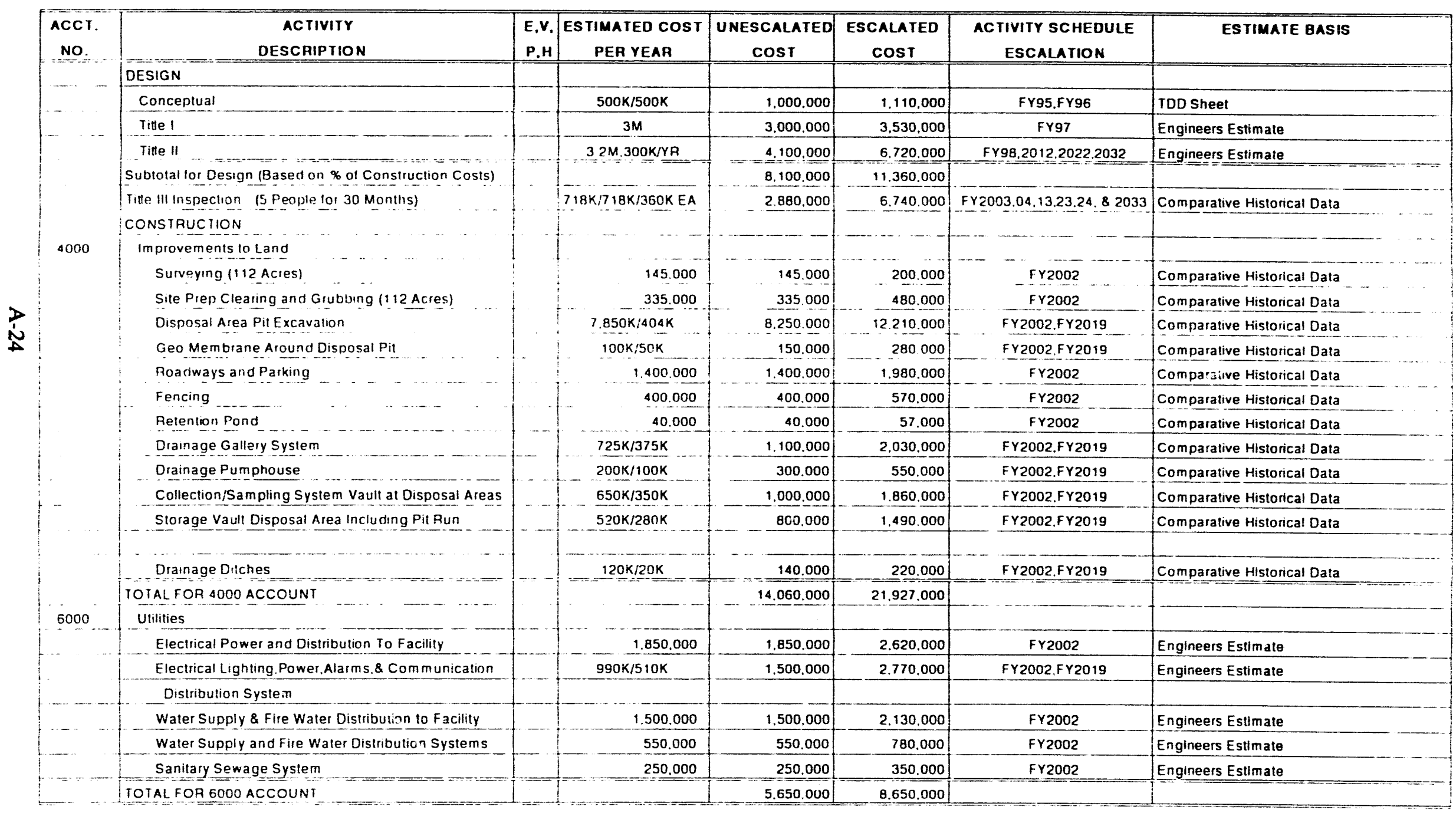


Page 3 o: 5

Project EVALUATION OF AL IERNATIVES FOA DISPOSAL

OF INEL MIXED AND LOW LEVEL WASTE

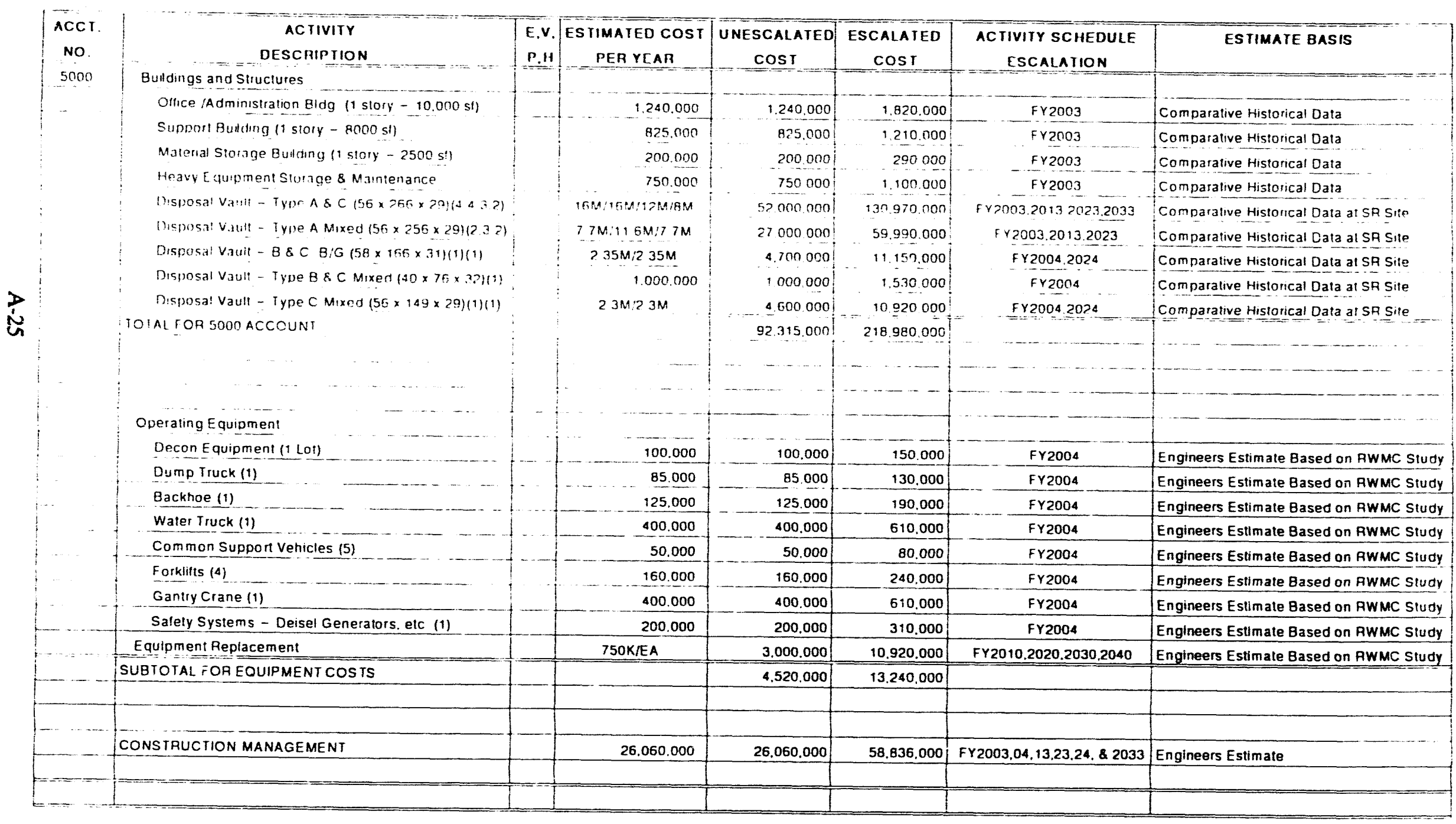


Page 4 of 5

Project EVALUATION OF ALTERNATIVES FOR DISPOSAL

OF INEL MIXED ANO LOW LEVEL WASTE

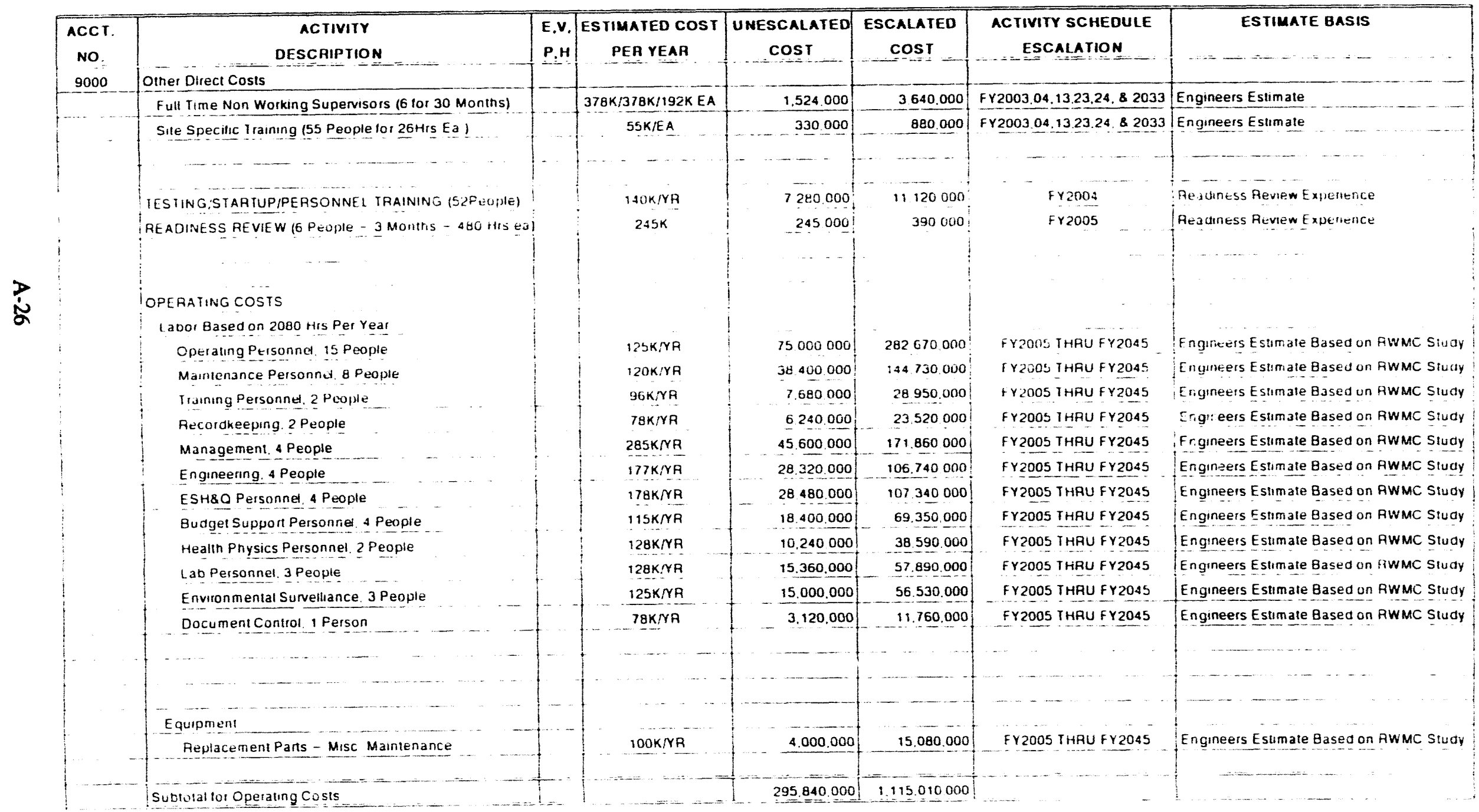


Project EVALUATION OF ALternatives For disPosal. OF INEL MIXED AND LOW LEVEL WASTE

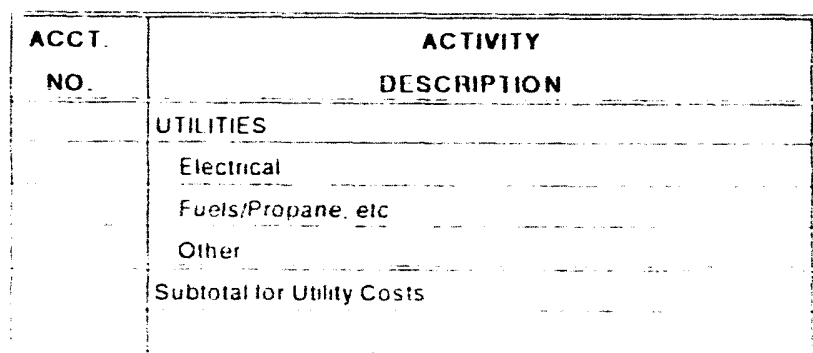

\begin{tabular}{|c|c|c|c|}
\hline $\begin{array}{l}\text { E.V. } \\
P .11\end{array}$ & $\begin{array}{c}\text { ESTIMATED COST } \\
\text { PER YEAR }\end{array}$ & $\begin{array}{c}\text { UNESCALATED } \\
\cos I\end{array}$ & $\begin{array}{c}\text { ESCALATED } \\
\text { COSI }\end{array}$ \\
\hline & & & \\
\hline & $25 \mathrm{~K} Y \mathrm{YA}$ & 1.000 .000 & 3.770 .000 \\
\hline & $5 K / Y R$ & 200.000 & 750000 \\
\hline & $30 K, Y F$ & 1.200 .000 & 4520000 \\
\hline & & 2.400000 & 9040000 \\
\hline & 1000000 & $1,000,000$ & 7310000 \\
\hline
\end{tabular}

DECONTAMINATION AND DECOMMISSIONINO

CSD COsts ate Dased on 10 \% of the construction cost felating threctly to the facilities berng decommissioned

\section{CLOSURE}

Disposal Vaull - Type A \& C $(56 \times 260 \times 29)(4432)$ Disposal Vault - Type A Mixed $(56 \times 256 \times 29)(232)$ Disposal Vault - Type B \& C B/G $(58 \times 166 \times 31)(1)(1)$ Disposal Vault - Type B 8 C Mixed $(40 \times 76 \times 32)(1)$ Disposal Vaull - Type C Mixed $(56 \times 149 \times 29)(1)(1)$ Backfill Around Vaults

Sand

Gravel

Diatomaceous Earin

Membrane Line

Clay Sand/Gravel

Top Soil Vegetation

Instrumentation and Installation

Technical Suppon. Sampling 8 OA Analysis (4 People) POST CLOSURE SURVELLIANCE Environmental Surveillance. 3 People Environmental Surveillance. 1 Person Subtotal for Closure and Post Closure

\section{$200 k: 200 k: 150 k / 100 k$ Bok:150K:100k \\ FY2013.2023.2033.2045}

850K:850K

$850 K$

$50 K 150 K$

6.810 .000

1.380 .000
1.680 .000

- 1.680 .000

$-\frac{1.680 .000}{2.460 .000}$

- $\frac{1,250.000}{50.000}$

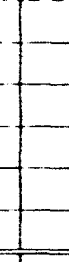

3.720000

385.000

1.160 .000

250.000
FY2013.2023 20332045 Fr2024.2045 FY2045 FY2024.2045

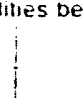

FY2005 THAU FY2045 FY2005 IHRU FY2045

FY2005 THRU FY2045

FY2046 
Rev 5-92

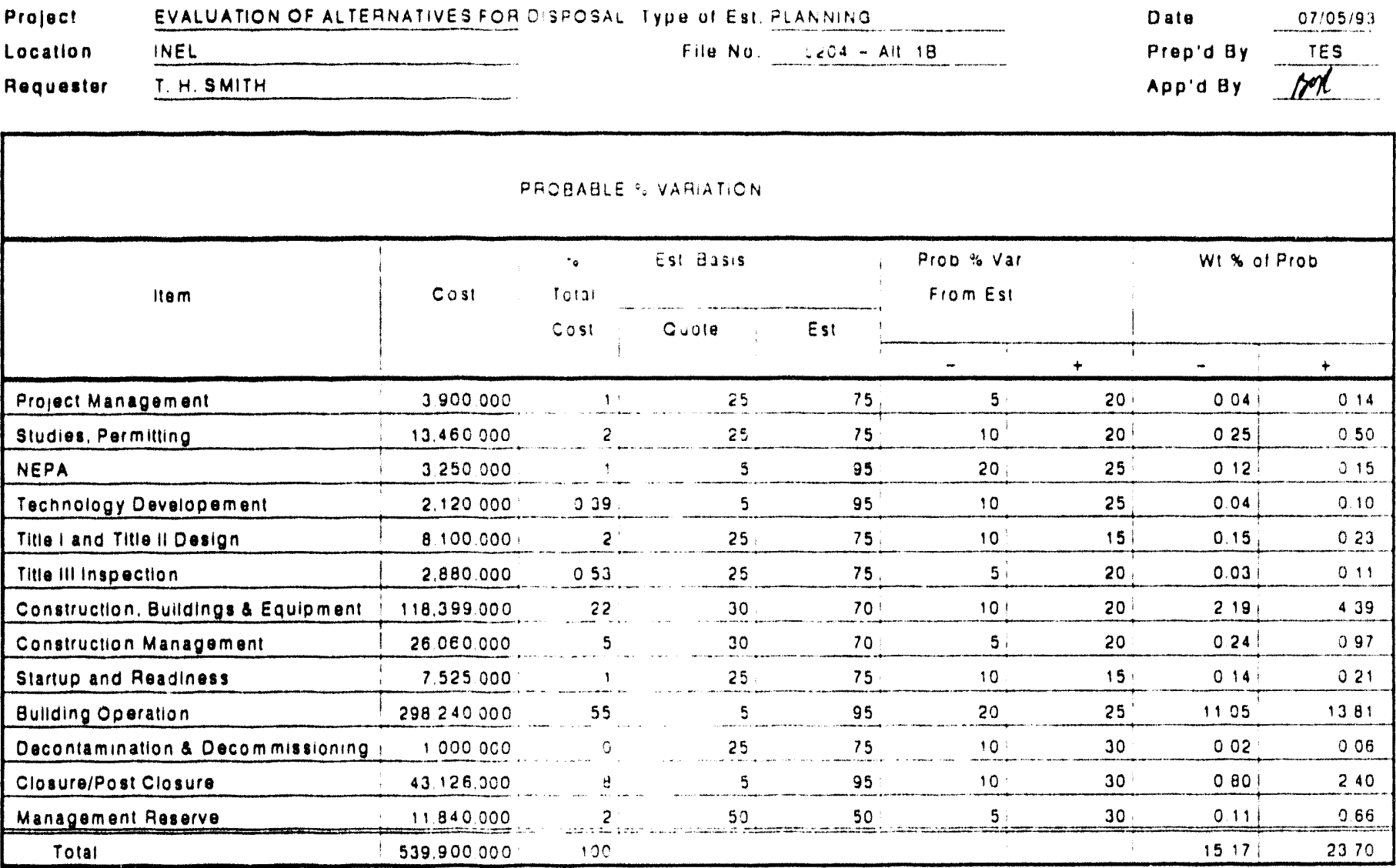


EG\&G Idaho, Inc.

Type of Estimate: PLANNING

Project: EVALUATION OF ALTERNATIVES FOR DISPOSAL OF INEL MIXED AND LOW LEVEL WASTE

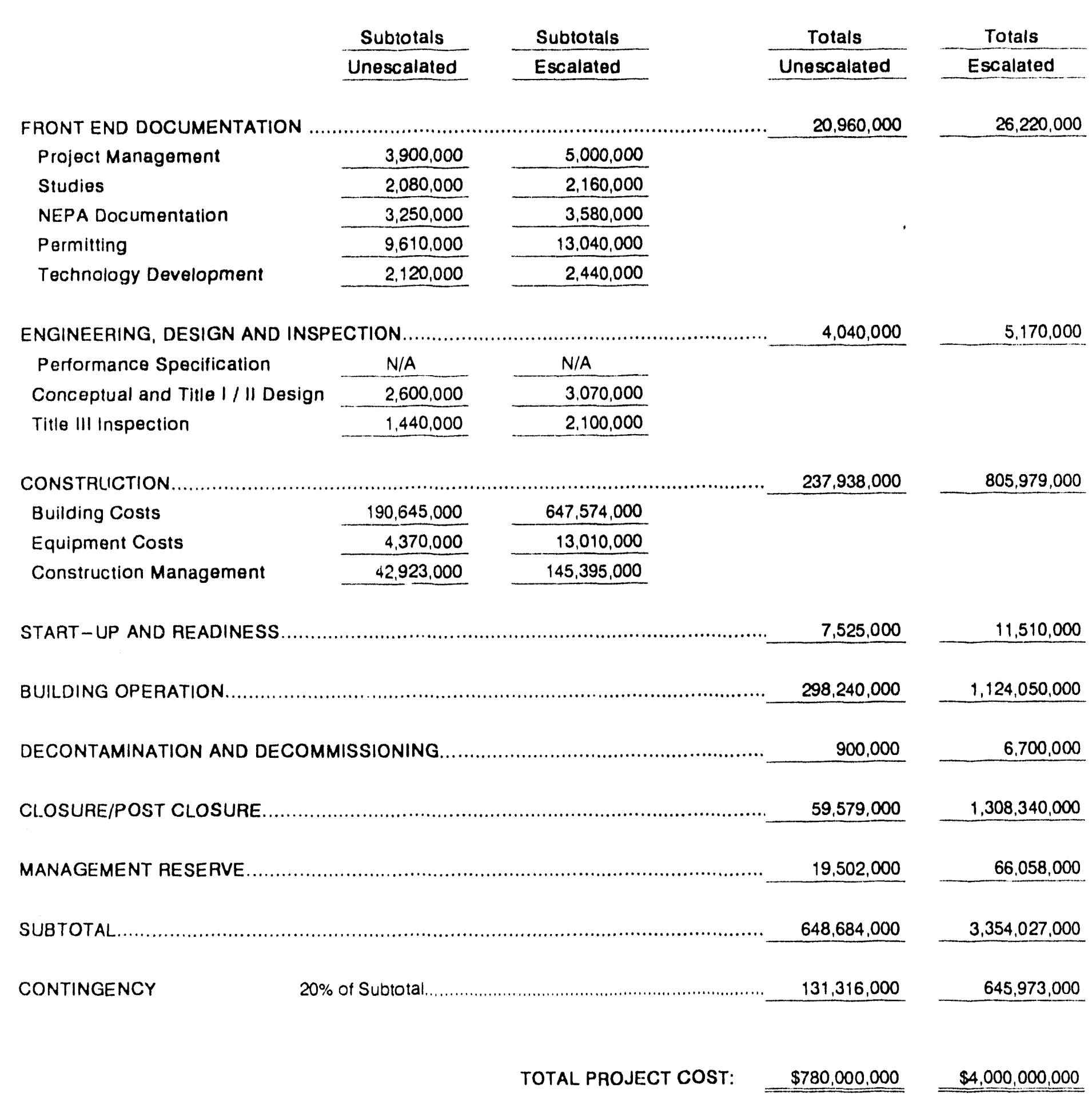

Date:

File No.

Appr'd By:

Prepared By: D. N. Stoddard

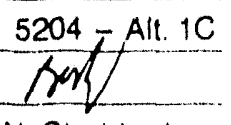


Project EVALUATION OF ALTERNATIVES FOR DISPOSAL OF INEL MIXED AND LOW LEVEL WASTE

Location INEL

Requester T.H SMITH

\begin{tabular}{|c|c|c|c|}
\hline \multirow[t]{2}{*}{$\begin{array}{l}\text { ACCT. } \\
\text { No. }\end{array}$} & $\begin{array}{c}\text { ACTIVITY } \\
\text { DESCPIPTION }\end{array}$ & \multirow[t]{2}{*}{$\begin{array}{l}\text { E.V. } \\
\text { P.H }\end{array}$} & \multirow[t]{2}{*}{$\begin{array}{c}\text { ESTIMATED COST } \\
\text { PER YEAR }\end{array}$} \\
\hline & PROJECT COSTS & & \\
\hline & Project Management & & $300 \mathrm{KYP}$ \\
\hline & Project Studies - 2 People, Full Time & & 250KVRVEA \\
\hline & Project Formulation (F\&OR's) & & $70 K / 270 K$ \\
\hline & Siting Studies & & $70 \mathrm{~K} / 270 \mathrm{~K} / 400 \mathrm{~K}$ \\
\hline \multirow{2}{*}{$\ldots$} & Pre-Operational Monitoring & & $500 \mathrm{~K} / 500 \mathrm{~K} / 250 \mathrm{KNR}$ \\
\hline & Salety Analysis Report (SAR) & & $150 \mathrm{~K} / 300 \mathrm{~K} / 200 \mathrm{~K}$ \\
\hline \multirow{7}{*}{ - } & Performance Assessment & & \$OOKNA \\
\hline & RCRA & & $375 \mathrm{~K} / 100 \mathrm{~K} / 375 \mathrm{~K}$ \\
\hline & NEPA (EIS)(CX's) & & $1 \mathrm{M} / 1 \mathrm{M} / 1 \mathrm{M} / 250 \mathrm{~K}$ \\
\hline & Air Permits (PSD/PTC/NESHAP) & & $200 \mathrm{~K} / 50 \mathrm{~K}$ \\
\hline & ESH\&O & & 50KMR \\
\hline & Other Regulatory Items (State Water Well Approval) & & 250KVR \\
\hline & Special Q/A Program For Construction - 2 People & & $354 K Y R$ \\
\hline \multicolumn{4}{|c|}{$-\ldots$} \\
\hline \multirow{2}{*}{\multicolumn{4}{|c|}{$\ldots$}} \\
\hline & & & \\
\hline \multirow{2}{*}{ 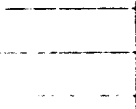 } & TECHNOLOGY DEVELOPMENT & & \\
\hline & Material Behavior (Conc. Clay. etc) & & 100K/65KYR \\
\hline \multirow{3}{*}{$\ldots$} & Cover Pertormance Control & & $100 \mathrm{~K} / 65 \mathrm{KNR}$ \\
\hline & Leachate Collection and Liner Performance & & 200KNA \\
\hline & Material Handting (Gantry Crane) & & $200 \mathrm{KNR}$ \\
\hline \multicolumn{4}{|c|}{ 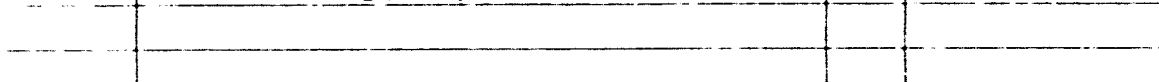 } \\
\hline$-\cdots$ & -- & & \\
\hline & $\ldots$ & & $-\cdots$ \\
\hline & 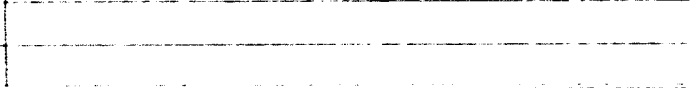 & & \\
\hline & Subtotal for Front End Costs & & \\
\hline
\end{tabular}

Type of Estimate PLANNING

Source (E) Eng. Est.

$M$ Vendor

(P) Pur. Order

(H) Handbook Ret

UNESCALATED ESCALATED ACTIVTY SCHEDULE

cost $\quad$ cost

ESCALATION

\begin{tabular}{|r|r|c|}
\hline 3.900 .000 & 5.000 .000 & FY92 THRU FY2005 \\
\hline 1.000 .000 & 1.040 .000 & FY93 -94 \\
\hline 340.000 & 350.000 & FY92 -93 \\
\hline 740.000 & 770.000 & FY92 FY94 \\
\hline
\end{tabular}

$740,000 \quad 770.000$

FY92 - FY94

\begin{tabular}{r|r|r|}
\hline 2.250 .000 & 3.350 .000 & FY95.FY99.FY2000-2005 \\
\hline
\end{tabular}

1.600 .000 1.600 .000

$1.700,000$

1.990.000 FY95-96.FY99-01.FY02-03

1.960 .000 FY95-96.FY99-00

2.120.000 FY96-97.FY98-99.FYOO-OI

\begin{tabular}{lll|l}
3.250 .000 & 3.580 .000 & FY94 - FY97
\end{tabular}

350.000

550.000

500.000

1.060 .000

$-1.060 .000$

…… 550.000

730.000

670.000

1.560 .000
-

$+\cdots$

FY1999. FY2000

FY2000-2001

FY2002.03.04

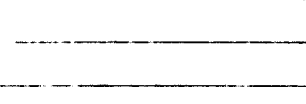

$\begin{array}{r} \\ -410.000 \\ 410.000 \\ \hline 1.140 .000 \\ \hline 480.000 \\ \hline \\ \hline \\ \hline \\ \hline \\ \hline\end{array}$

\begin{tabular}{l} 
FY94. FY95-98 \\
FY94. FY95-98 \\
FY94-98 \\
FY97-98 \\
\hline
\end{tabular}

Date:

Prep'd By:

Chin'd By:

Appr'd By: BCL

ESTMATE BASIS

\section{TDD Sheet, dated 4/28/93}

TDD Sheet. dated 4/28/93

TDD Sheet, dated 4/28/93. Eng Estimate TDD Sheet, dated 4/28/93. Eng Estimate

Engineer's Estimate

Data from Similar SAR's

Engineer's Estimate

Engineer's Estimate (includes Sampling)

Engineer's Estimale

Engineer's Estimate

Engineer's Estimate

Engineer's Estimate

Engineer's Estimate

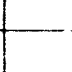

\section{Engineer's Estimate}

Engineer's Estrmate

Engineer's Estimate

Engineer's Estimate 
Project EVALUATION OF ALTERNATIVES FOR DISPOSAL OF INEL MIXED AND LOW LEVEL WASTE

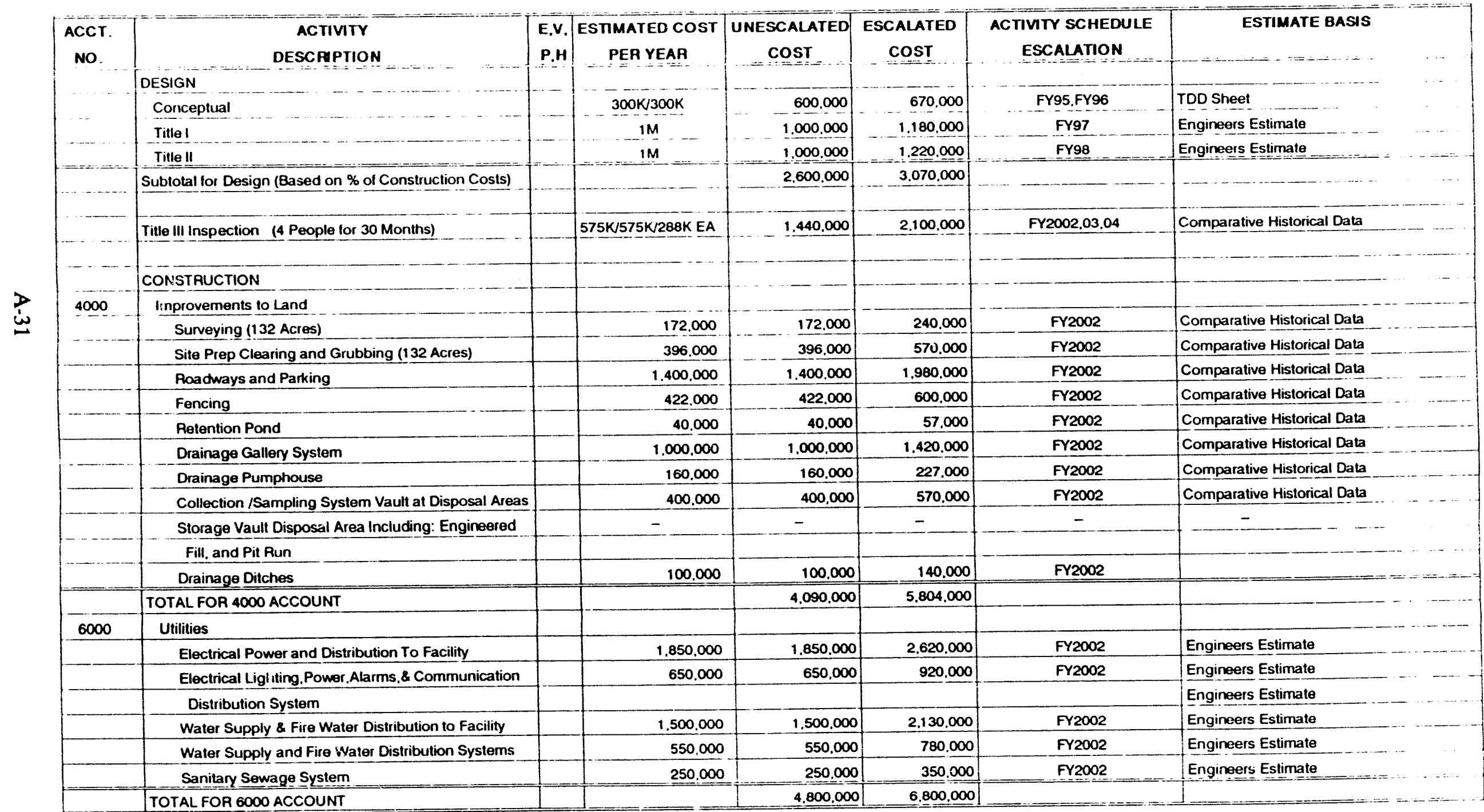


Rev $5-9]$

Page 3 of 5

Project EVALUATION OF ALTERNATVES FOR DISPOSAL OF INEL MIXED AND LOW LEVEL WASTE

\begin{tabular}{|c|c|c|c|c|c|c|c|}
\hline $\begin{array}{l}\text { ACCT. } \\
\text { No. }\end{array}$ & $\begin{array}{c}\text { ACTIVITY } \\
\text { DESCPAPTION } \\
\end{array}$ & $\begin{array}{l}\text { E.V. } \\
\text { P.H }\end{array}$ & $\begin{array}{c}\text { ESTMMATED COST } \\
\text { PER YEAR }\end{array}$ & $\begin{array}{c}\text { UNESCALATED } \\
\text { COST }\end{array}$ & $\begin{array}{l}\text { ESCALATED } \\
\text { COST } \\
\end{array}$ & $\begin{array}{l}\text { ACTIVITY SCHEDULE } \\
\text { ESCALATION } \\
\end{array}$ & ESTIMATE BASIS \\
\hline \multirow[t]{2}{*}{5000} & Buildings and Structures & & & & & & \\
\hline & Office /Administration Bidg (1 story - $10.000 \mathrm{~s}$ ) & & 1.240 .000 & $1,240,000$ & 1.820 .000 & FY2003 & Comparative Historical Data \\
\hline & Support Building (1 story - 8000 sf) & & 825.000 & 825.000 & 1.210 .000 & FY2004 & Comparative Historical Data \\
\hline & Material Storage Building (1 story - $2500 \mathrm{~s}$ ) & & 200.000 & 200,000 & 290,000 & FY2004 & Comparative Historical Data \\
\hline & Heavy Equipment Storage \& Maintenance & & 750,000 & 750,000 & $1.100,000$ & FY2004 & Comparative Historical Data \\
\hline \multirow{3}{*}{-1} & Disposal Pad - LLW/Mixed Waste $180 \times 815$ (3) & & 11.000 .000 & $11.000,000$ & 16.180 .000 & FY2003 & Comparative Historical Data \\
\hline & Disposal Pad - LLW/Mixed Waste $180 \times 486$ (3) & & 6.800 .000 & 6.800 .000 & 10.000 .000 & FY2003 & Comparative Historical Data \\
\hline & Concrele Disposal Containers LLW and Mixed Waste & & 4M/YR & 160.000 .000 & $603,000,000$ & FY2005 Thru 2045 & Comparative Historical Data \\
\hline 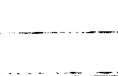 & & & & & & & $-\cdots$ \\
\hline \multirow{4}{*}{$\begin{array}{c}\ldots- \\
\cdots \\
\cdots\end{array}-$} & TOTAL FOR 5000 ACCOUNT & & & 180.815 .000 & 633.600 .000 & & \\
\hline & & - & & & & & $\ldots$ \\
\hline & -1 & & & & & & - \\
\hline & Operating Equipment & & & & & & $\ldots$ \\
\hline \multirow{2}{*}{ - - - . } & Docon Equipment (1 Lot) & & 100.000 & 100.000 & 150.000 & FY2004 & Engineers Estimale Based on RWMC Study \\
\hline & Dump Truck (1) & $\ldots$ & 85.000 & 85.000 & 130.000 & FY2004 & Engineers Estimate Based on RWMC Study \\
\hline \multirow{4}{*}{$\ldots$} & Backhoe (1) & & 125.000 & 125,000 & 190,000 & FY2004 & Engineers Estimale Based on RWMC Sludy \\
\hline & Water Truck (1) & & 400.000 & 400.000 & 610.000 & FY2004 & Engineers Estimate Based on RWMC Study \\
\hline & Common Support Vehicles (5) & & 50.000 & 50.000 & 80.000 & FY2004 & Engineers Estimate Based on RWMC Study \\
\hline & Forklifts (4) & & 160.000 & 160.000 & 240.000 & FY2004 & Engineers Estimate Based on RWMC Sludy \\
\hline \multirow[t]{2}{*}{$\ldots$} & Boom Crane (1) & & 250.000 & 250.000 & 380.000 & FY2004 & Engineers Estimate Based on RWMC Study \\
\hline & Salety Systerns - Deisel Generators, elc. (1) & & 200.000 & 200.000 & 310.000 & FY2004 & Engineers Estimate Based on RWMC Study \\
\hline \multirow{2}{*}{$\ldots$} & Equipment Replacement & & $750 \mathrm{~K} E \mathrm{EA}$ & $3.000,000$ & 10.920 .000 & $F Y 2010,2020,2030,2040$ & Engineers Estimate Based on RWMC Study \\
\hline & SUBTOTAL FOR EOUIPMENT COSTS & & & $4.370,000$ & 13.010 .000 & & \\
\hline \multirow[t]{2}{*}{$\ldots \ldots$} & - & & $\ldots$ & 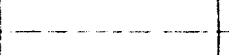 & $\ldots$ & -1 & - \\
\hline & CONSTRUCTION MANAGEMENT & & 42.923 .000 & 42.923 .000 & $145,395,000$ & FY2002,03.04 & Engineers Estimate \\
\hline
\end{tabular}


Page 4 of 5

Project EVALUATION OF ALTERNATIVES FOR IISPOSAL

OF INEL MIXED AND LOW LEVEL WA STE

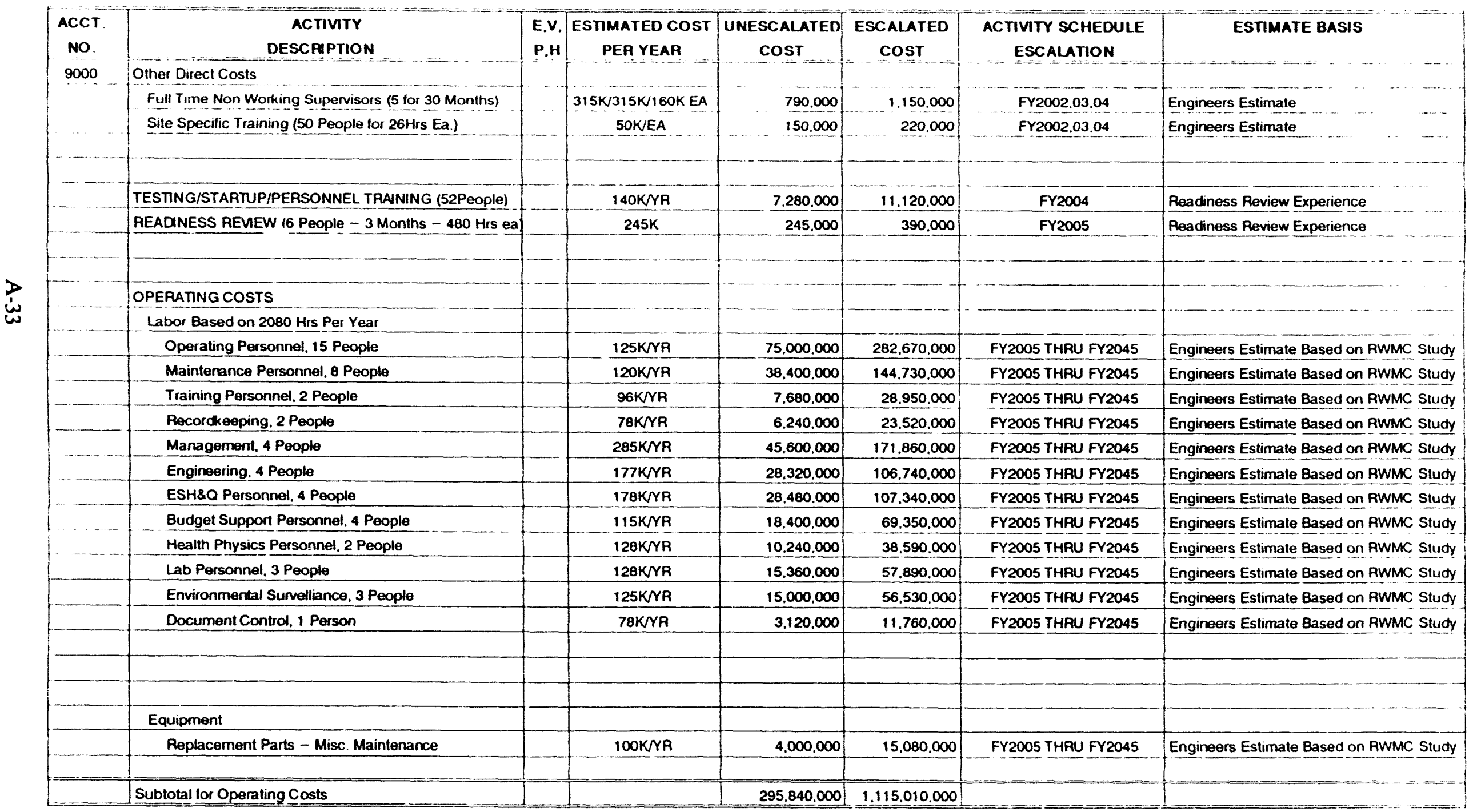


Project eVawation OF alternatives for Disposal OF INEL MIXED AND LOW LEVEL WASTE

\begin{tabular}{|c|c|c|c|c|c|c|c|}
\hline $\begin{array}{l}\text { ACCT. } \\
\text { No. }\end{array}$ & $\begin{array}{c}\text { ACTIVITY } \\
\text { DESCRIPTION }\end{array}$ & $\begin{array}{l}\text { E.V. } \\
\text { P.H } \\
\end{array}$ & $\begin{array}{c}\begin{array}{c}\text { ESTIMATED COST } \\
\text { PER YEAR }\end{array} \\
\end{array}$ & $\begin{array}{c}\text { UNESCALATED } \\
\text { COST }\end{array}$ & $\begin{array}{c}\text { ESCALATED } \\
\text { COST }\end{array}$ & $\begin{array}{l}\text { ACTIVTY SCHEDULE } \\
\text { ESCALATION } \\
\end{array}$ & ESTIMATE BASIS \\
\hline & UTIUITIES & & & & & & \\
\hline & Electrical & & 25KMR & 1.000 .000 & $3,770,000$ & FY2005 THRU FY2045 & Engineers Estimate \\
\hline & Fuels/Propane, etc. & & 5KNR & 200,000 & 750.000 & FY2005 THRU FY2045 & Engineers Estimate \\
\hline & Other & & 30KNA & 1.200 .000 & 4.520 .000 & FY2005 THRU FY2045 & Engineers Estimate \\
\hline & Sublotal for Utility Costs & & & 2.400 .000 & $9.040,000$ & & \\
\hline & & & & & & & \\
\hline$\ldots$ & & & & & & & \\
\hline & & & & & & & \\
\hline & DECONTAMINATION AND DECOMMISSIONING & & 900.000 & 900.000 & 6.700 .000 & FY2046 & Data From Previous D \& D Projects \\
\hline & (D8D Costs are based on $10 \%$ of the construction cos & st relati & ting directly to the facilit & ties being decommis & issioned! & & \\
\hline & IEMPORARY CLOSURE - (Pre-engineered metal bl'dg st & hell) & & & & & \\
\hline & Disposal Pad - LLW/Mixed Waste $180 \times 815$ (3) & & 275KNA & 11.002 .000 & 41.000 .000 & FY2005 THRU FY2045 & Engineers Estimate \\
\hline & Disposal Pad - LLW/Mixed Waste $180 \times 486$ (3) & & 164KNR & 6.560 .000 & 24.500 .000 & FY2005 THRU FY2045 & Engineers Estimate \\
\hline & & & & & & & $\ldots \ldots$ \\
\hline$\ldots$ & Sand & & 9.000 .000 & $9.000,000$ & 65.830 .000 & FY2046 & Engineers Estimate \\
\hline & Gravel & & 600,000 & 600.000 & 4.390 .000 & FY2046 & Engineers Estimate \\
\hline & Diatomaceous Earth & & $1,800.000$ & 1.800 .000 & 13.670 .000 & FY2047 & Engineers Estimate \\
\hline & Membrane Liner & & 2.000 .000 & 2.000 .000 & 15.180 .000 & FY2047 & Engineers Estimate \\
\hline & Clay & & $2,500,000$ & $2.500,000$ & 19.340 .000 & FY $2047-48$ & Engineers Estimate \\
\hline & Sand/Gravel & & 3.700 .000 & $3.700,000$ & 29.160 .000 & FY2048 & Engineers Estimate \\
\hline & Top Soil & & 1.760 .000 & 1.760 .000 & 14.400 .000 & FY2049 & Engineers Estimate \\
\hline & Vegetation & & 76,000 & 76.000 & 620.000 & FY2049 & Engineers Estimate \\
\hline I & Instrumentation and Installation & & 250.000 & 250.000 & 1.760 .000 & FY2045 & Engineers Estimate \\
\hline & Technical Support. Sampling. \& OA Analysis (4 People) & & 177KYYR & 4.956 .000 & 37.730 .000 & FY2044-50 & Engineers Estimate \\
\hline & POST CLOSURE SURVELUANCE & & & & & & \\
\hline & Environmental Surveillance. 3 People & & $125 \mathrm{~K} Y \mathrm{Y}$ & 4.125 .000 & 36.610 .000 & FY2046 THRU FY2056 & Engineers Estimate \\
\hline & Environmental Surveillance. 1 Person & & $125 \mathrm{KVR}$ & 11.250 .000 & $1.004 .150,000$ & FY2057 THRU FY21 46 & Engineers Estimate \\
\hline 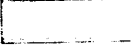 & Subtotal lor Closure and Post Closure & & & 59.579 .000 & 1.308 .340 .000 & & \\
\hline
\end{tabular}


Revi-92

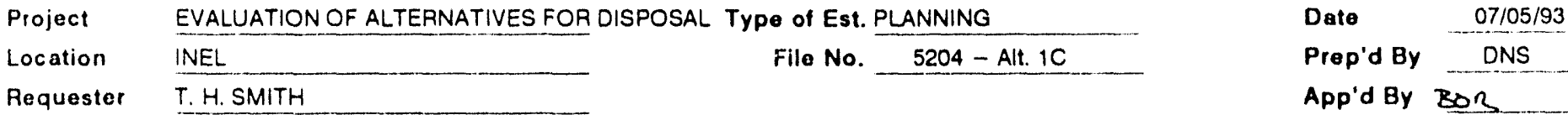

\begin{tabular}{|c|c|c|c|c|c|c|c|c|}
\hline \multicolumn{9}{|c|}{ PROBABLE \% VARIATION } \\
\hline \multirow{2}{*}{ Item } & \multirow{2}{*}{ Cost } & \multirow{2}{*}{$\begin{array}{c}\% \\
\text { Total } \\
\text { Cost }\end{array}$} & \multirow[t]{2}{*}{ Quote } & \multirow[t]{2}{*}{ Est } & \multicolumn{2}{|l|}{$\begin{array}{c}\text { Prob } \% \text { Var } \\
\text { From Est }\end{array}$} & \multicolumn{2}{|c|}{ Wt $\%$ of Prob } \\
\hline & & & & & - & + & - & + \\
\hline Studies, Permitting & $11,690,000$ & $2 !$ & 25 & 75 & 10 & 20 & 0.18 & 0.36 \\
\hline NEPA & $3.250,0001$ & 11 & 5 & 95 & 20 & 25 & 0.10 & 0.13 \\
\hline Technology Developement & $2.120,000$ & 0.33 & 5 & 95 & 10 & 25 & 0.03 & 0.08 \\
\hline Title I and Title $\|$ Design & $2,600,000$ & 01 & 25 & 75 & 10 & 15 & 0.04 & 0.06 \\
\hline Title III Inspection & $1,440,000$ & 0.22 & 25 & 75 & 5 & 20 & 0.01 & 0.04 \\
\hline Building Operation & $298,240,000$ & 46 & 5 & 95 & 20 & 25 & 9.20 & 11.49 \\
\hline Decontamination \& Decommissioning & 900,000 & 01 & 25 & 75 & 10 & 30 & 0.01 & 0.04 \\
\hline Closure/Post Closure & $59,579,000$ & 9 & 5 & 95 & 10 & 30 & 0.92 & 2.76 \\
\hline Management Reserve & 19.502 .000 & 31 & 50 & 50 & 5 & 30 & 0.15 & 0.90 \\
\hline Total & $648.684,000$ & 1001 & & & & & 14.13 & 23.50 \\
\hline
\end{tabular}


EG\&G Idaho, Inc.

Type of Estimate: PLANNING

Date:

07/05/93

File No.

5204 - Alt. 10

Project: EVALUATION OF ALTERNATIVES FOR DISPOSAL

OF INEL MIXED AND LOWLEVEL WASTE

Appr'd By:

Prepared By: T.E. SIVILL

$\begin{array}{ll}\text { Subtotals } & \text { Subtotals } \\ \text { Unescalated } & \text { Escalated }\end{array}$

$\frac{\text { Totals }}{\text { Unescalated }}$

Totals

Escalated

FRONT END DOCUMENTATION

$19,680,000$

$24,490,000$

Project Management

Studies

NEPA Documentation

Permitting

Technology Development

\begin{tabular}{rr}
\hline $3,900,000$ \\
\hline $2,080,000$ \\
\hline $3,250,000$ \\
\hline $8,900,000$ \\
\hline $1,550,000$ \\
\hline
\end{tabular}

ENGINEERING, DESIGN AND INSPECTION.

Performance Specification

$\begin{array}{r}\text { N/A } \\ \hline 3,100,000 \\ \hline 630,000\end{array}$

Title III Inspection

CONSTRUCTION

Building Costs

Equipment Costs

Construction Management

\begin{tabular}{rr}
$\frac{34,463,000}{5,070,000}$ & $\frac{63,602,000}{14,080,000}$ \\
\hline $8,701,000$ & $17,098,000$ \\
\hline
\end{tabular}

START-UP AND READINESS.

$7,204,000$

BUILDING OPERATION

DECONTAMINATION AND DECOMMISSIONING.

CLOSURE/POST CLOSURE.

$39,741,000$

MANAGEMENT RESERVE

SUBTOTAL.

CONTINGENCY
$21 \%$ of Subtotal.

$87,188,000$

$3,953,000$

$412,812,000$

$4,560,000$

$94,780,000$

$537,692,000$

$11,010,000$

$1,087,860,000$

$6,070,000$

$1,225,770,000$

$7,768,000$

$2,462,308,000$

TOTAL PROJECT COST: \$500,000,000 $\$ 3,000,000,000$

Comments: 
EGSislaho, Inc

Rev 5-92

Project

EVALUATION OF ALIERNATIVES FOR DISPOSAL OF INEL MIXED AND LOW LEVEL WASTE

INEL

Location

Requester T.H.SMITH

DE TAILED COST ESTIMATE SHEET

Iype of Estimate PLANNING

Source (E) Eng Est

(V) Vendor

(P) Pur. Order

(H) Handbook Ret
File No.

5204 - Alt. 10

Date:

Prep'd By: TESivill

Chk'd By:

Appr'd By: $\operatorname{sox}$

\begin{tabular}{|c|c|c|c|c|c|c|c|}
\hline $\begin{array}{l}\text { ACCT. } \\
\text { No. }\end{array}$ & $\begin{array}{c}\text { ACTIVITY } \\
\text { DESCRIPIION }\end{array}$ & $\begin{array}{l}E . V \\
P . H\end{array}$ & $\begin{array}{l}\text { ESTIMATED COST } \\
\text { PER YEAR }\end{array}$ & $\begin{array}{c}\text { UNESCALATED } \\
\text { COSI }\end{array}$ & $\begin{array}{c}\text { ESCALATED } \\
\text { COST }\end{array}$ & $\begin{array}{l}\text { ACTIVITY SCHEDULE } \\
\text { ESCALATION }\end{array}$ & ESTIMATE BASIS \\
\hline & PROJECT COSTS & & & & & & \\
\hline & Project Management & & $300 K N \mathrm{NA}$ & 3.900 .000 & $5,000.000$ & FY92 THAU FY2005 & IDD Sheet, dated $4 / 28 / 93$ \\
\hline & Project Siudies - 2 People. Full Time & & 25OK/YA/EA & $1,000.000$ & 1.040 .000 & Frg3-94 & TDD Sheet, dated $4 / 28 / 93$ \\
\hline & Prolect Formulation (F\&OR's) & & $70 \mathrm{~K} / 270 \mathrm{~K}$ & 340.000 & 350.000 & FY92 - 93 & TOD Sheet, dated 4/28/93. Eng Estimate \\
\hline & Siting Studies & & $70 \mathrm{~K} / 270 \mathrm{~K} / 400 \mathrm{~K}$ & 740,000 & 770,000 & FY92 - FY94 & IDD Sheet, dated 4/28/93. Eng Estimate \\
\hline & Pre-Operational Monitoring & & $500 \mathrm{~K} / 500 \mathrm{~K} / 250 \mathrm{~K} / \mathrm{KA}$ & $2,250,000$ & 3.350 .000 & FY95,FY99,FY2000-2005 & Engineer's Estimate \\
\hline & Safety Analysis Repori (SAR) & & $150 \mathrm{~K} / 300 \mathrm{~K} / 200 \mathrm{~K}$ & $1.600,000$ & $2,100.000$ & FY95-96.FY99-01.FY02-03 & Data from Similar SAR's \\
\hline & Pertormance Assessment & & $400 K N A$ & 1.600 .000 & $1.960,000$ & FY95-96.FY99-00 & Engineer's Estimate \\
\hline & RCAA & & $375 \mathrm{~K} / 100 \mathrm{~K} / 375 \mathrm{~K}$ & 1.700 .000 & 2.120 .000 & FY96-97.FY98-99.FYO0-01 & Engineer's Estimate (Includes Sampling) \\
\hline & NEPA (EIS)(CX's) & & $1 \mathrm{M} / 1 \mathrm{M} / 1 \mathrm{M} / 25 \mathrm{~K}$ & $3.250,000$ & 3.580 .000 & FY94 - FY97 & Engineer's Estimate \\
\hline & Air Permits (PSD/PTC/NESHAP) & & $200 K / 150 K$ & 350.000 & 550,000 & FY1999. FY2000 & Engineer's Estimate \\
\hline & ESHBO & & $50 K / Y R$ & 550.000 & 730.000 & FY1995-2005 & Engineer's Estimate \\
\hline & Other Reguiatory Items (State Water Well Approval) & & $250 K / \mathrm{NA}$ & 500,000 & 670,000 & FY2000-2001 & Engineer's Estimate \\
\hline & Special Q/A Program For Construction - 1 Person & & $177 \mathrm{~K} / \mathrm{NA}$ & 354.000 & 510.000 & FY2002.03 & Engineer's Estimate \\
\hline & & & & & & & \\
\hline & & & & & & & \\
\hline & & & & & & & \\
\hline & TECHNOLOGY DEVELOPMENT & & & & & & \\
\hline & Material Behavior (Clay, etc.) & & $50 \mathrm{~K} / 35 \mathrm{~K} / \mathrm{VR}$ & 190,000 & 210,000 & FY94, FY95-98 & Engineer's Estimate \\
\hline & Cover Performance Control & & $100 \mathrm{~K} / 65 \mathrm{~K} / \mathrm{RR}$ & 360.000 & 410,000 & FY94, FY95-98 & Engineer's Estimate \\
\hline & Leachate Collection and Liner Performance & & $200 \mathrm{~K} N \mathrm{R}$ & $1.000,000$ & $1,140,000$ & FY94-98 & Engineer's Estimate \\
\hline & & & & & & & \\
\hline & & & & & & & \\
\hline & & & & & & & \\
\hline & & & & & & & \\
\hline & & & & & & & \\
\hline & & & & & & & \\
\hline & & & & & & & \\
\hline & Subtotal for Front End Costs & & & 19.684 .000 & 24.490 .000 & & \\
\hline
\end{tabular}


Rev 5-92

Page 2 of 5

Type of Estimate: PLANNING

Flle No. $5204-$ Alt. 10

Date:

Prolect EVALUATION OF alteanatives for disposal OF INEL MIXED AND LOW LEVEL WASTE

\begin{tabular}{|c|c|c|c|c|c|c|c|}
\hline \multirow[t]{9}{*}{$\begin{array}{c}\text { ACCT. } \\
\text { No. }\end{array}$} & $\begin{array}{c}\text { ACTIVITY } \\
\text { DESCAIPIION }\end{array}$ & E.V. & $\begin{array}{c}\text { ES TIMATED COST } \\
\text { PER YEAR }\end{array}$ & $\begin{array}{c}\text { UNESCALATED } \\
\text { COST }\end{array}$ & $\begin{array}{c}\text { ESCALATED } \\
\text { Cost } \\
\end{array}$ & $\begin{array}{c}\text { ACTIVITY SCHEDULE } \\
\text { ESCALATION }\end{array}$ & ESTIMATE BASIS \\
\hline & DESIGN & & & & & & \\
\hline & Conceplual & & $350 \mathrm{~K} / 350 \mathrm{~K}$ & 700.000 & 780.000 & FY95,FY96 & TDD Sheet \\
\hline & Tive 1 & & $1.2 \mathrm{M}$ & 1.200 .000 & $1.420,000$ & FY97 & Engineers Estimate \\
\hline & Tive II & & $1.2 \mathrm{M}$ & $1,200,000$ & $1,460,000$ & Frg8 & Engineers Estimate \\
\hline & Subtotal for Design (Based on $\%$ of Construction Costs) & & & $3,100.000$ & 3.660 .000 & & \\
\hline & Tive III Inspection (3 People for 18 Months) & & $420 \mathrm{~K} / 210 \mathrm{~K}$ & 630,000 & 900,000 & FY2002. 03 & Comparative Historical Data \\
\hline & & & & & & & \\
\hline & CONSTRUCTION & & & & & & \\
\hline \multirow[t]{13}{*}{4000} & Improvements to Land & & & & & & \\
\hline & Surveying (112 Acres) & & 145.000 & 145.000 & 200.000 & FY2002 & Comparative Historical Data \\
\hline & Site Prep Clearing and Grubbing (112 Acres) & & 335.000 & 335.000 & 480,000 & FY2002 & Comparative Historical Data \\
\hline & Roadways and Parking & & 1.400 .000 & 1.400 .000 & 1.980 .000 & FY2002 & Comparative Historical Data \\
\hline & Fencing & & 400,000 & 400.000 & 570,000 & FY2002 & Comparative Historical Data \\
\hline & Retention Pond & & 40.000 & 40.000 & 57,000 & FY2002 & Comparattve Historical Data \\
\hline & Drainage Gallery System & & 430.000 & 430.000 & 610.000 & FY2002 & Comparative Historical Data \\
\hline & Drainage Pumphouse & & 420.000 & 420.000 & 595,000 & FY2002 & Comparative Historical Data \\
\hline & Collection /Sampling System Vault at Disposal Areas & & 1.000 .000 & 1.000 .000 & $1.420,000$ & FY2002 & Comparative Historical Data \\
\hline & Storage Vault Disposal Area Including: (Reter to & & - & - & - & - & - \\
\hline & Pad Constiuction Costs) & & $=$ & - & $=$ & - & - \\
\hline & Drainage Ditches & & 100,000 & 100.000 & 140.000 & FY2002 & \\
\hline & IOTAL FOR 4000 ACCOUNT & & & 4.270 .000 & 6.052 .000 & & \\
\hline \multirow[t]{8}{*}{6000.} & Utillties & & & & & & \\
\hline & Electrical Power and Distribution To Facillty & & $1.850,000$ & 1.850 .000 & 2.620 .000 & FY2002 & Engineers Estimate \\
\hline & Electrical Lighting.Power.Alarms.\& Communication & & 650.000 & 650.000 & 920,000 & FY2002 & Engineers Estimate \\
\hline & Distribution System & & & & & & Engineers Estimate \\
\hline & Water Supply \& Fire Water Distribution to Facility & & $1,500,000$ & 1.500 .000 & 2.130 .000 & FY2002 & Enginee:s Estimate \\
\hline & Water Supply and Fire Water Distribution Systems & & 150.000 & 150.000 & 210.000 & FY2002 & Engineers Estimate \\
\hline & Sanitary Sewage System & & 250.000 & 250.000 & 350,000 & FY2002 & Engineers Estimate \\
\hline & TOTAL FOR 6000 ACCOUNT & & & 4.400 .000 & 6.230 .000 & & \\
\hline
\end{tabular}


Page 3 of 5

Prolect EVALUATION OF ALTERNATIVES FOR DISPOSAL OF INEL MIXED AND LOW LEVEL WASTE

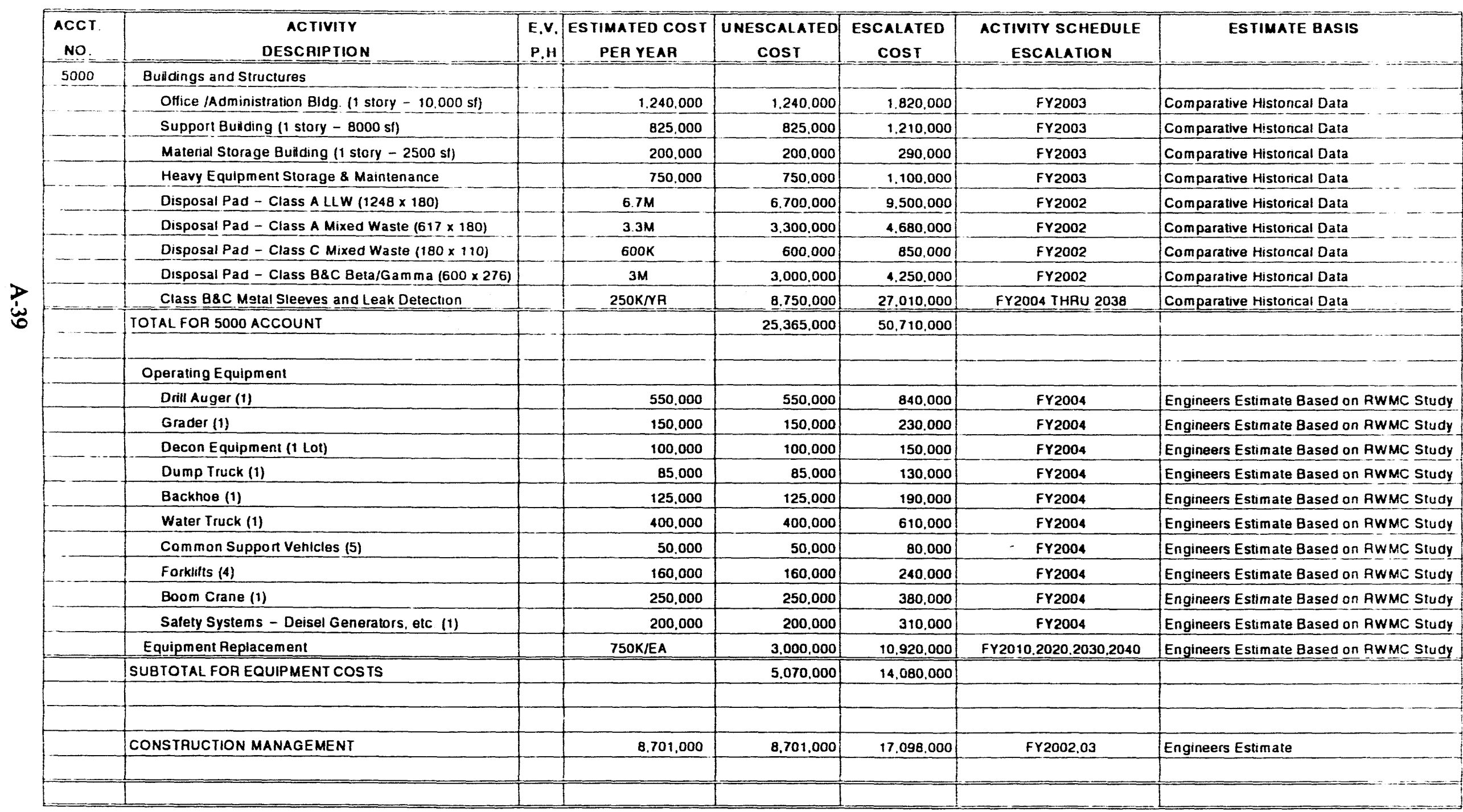


Propect evaluation of alternatives for disposal OF INEL MIXED AND LOW LEVEL WASTE

\begin{tabular}{|c|c|c|c|c|c|c|c|}
\hline $\begin{array}{l}\text { ACCT. } \\
\text { No. }\end{array}$ & $\begin{array}{c}\text { ACTIVITY } \\
\text { DESCRIPTION } \\
\end{array}$ & $\begin{array}{l}\text { E.V. } \\
\text { P.H. }\end{array}$ & $\begin{array}{c}\text { ESTIMATED COST } \\
\text { PEA YEAR }\end{array}$ & $\begin{array}{c}\text { UNESCALATED } \\
\text { COST }\end{array}$ & $\begin{array}{c}\text { ESCALATED } \\
\text { COST } \\
\end{array}$ & $\begin{array}{c}\text { ACTIVITY SCHEDULE } \\
\text { ESCALATION } \\
\end{array}$ & ESTIMATE BASIS \\
\hline \multirow[t]{3}{*}{9000} & Other Direct Costs & & & & & & \\
\hline & Full Time Non Working Supervisors (3 for 18 Months) & & $218 K / 110 K E A$ & 328.000 & 470.000 & FY2002. 03 & Engineers Estimate \\
\hline & Site Specific Training (50 People for $26 \mathrm{Hrs}$ Ea.) & & 5OK/EA & 100,000 & 140.000 & FY2002, 03 & Engineers Estimate \\
\hline & & & & & & & \\
\hline & TESTING/STARTUP/PERSONNEL TRAINING (5OPeople) & & $140 \mathrm{~K} / \mathrm{NR}$ & $7.000,000$ & 10.690 .000 & FY2004 & Readiness Review Expenence \\
\hline & AEADINESS REVIEW (5 Peopie -3 Months -480 Hrs ea) & & $204 K$ & 204.000 & 320.000 & FY2005 & Readiness Review Experience \\
\hline & & & & & & & \\
\hline & & & & & & & \\
\hline & OPERATING COSTS & & & & & & \\
\hline & Labor Based on 2080 Hrs Per Year & & & & & & \\
\hline & Operating Personnet. 15 People & & $125 \mathrm{~K} N \mathrm{NA}$ & 75.000 .000 & 282.670 .000 & FY2005 THAU FY2045 & Engineers Estimate Based on RWMC Study \\
\hline & Maintenance Personnel. 6 People & & $120 \mathrm{KNA}$ & 28.800 .000 & 108.540 .000 & FY2005 THRU FY2045 & Engineers Estimate Based on RWMC Study \\
\hline & Training Personne, 2 People & & 96K/NR & 7.680 .000 & 28.950 .000 & FY2005 THAU FY2045 & Engineers Estimate Based on RWMC Study \\
\hline & Recordkeeping. 2 People & & 78K/NR & 6.240 .000 & 23.520 .000 & FY2005 THRU FY2045 & Engineers Estimate Based on RWMC Sludy \\
\hline & Management. 4 People & & $285 \mathrm{~K} / \mathrm{NA}$ & 45.600 .000 & 171.860 .000 & FY2005 THAU FY2045 & Engineers Estimate Based on RWMC Study \\
\hline & Engineening 4 People & & $177 \mathrm{~K} / \mathrm{NR}$ & 28.320 .000 & 106.740 .000 & FY2005 THRU FY2045 & Engineers Estimate Based on RWMC Study \\
\hline & ESH8O Personned. 4 People & & $178 \mathrm{KNR}$ & $28,480.000$ & 107.340 .000 & FY2005 THRU FY2045 & Engineers Estimate Based on RWMC Study \\
\hline & Budget Suppon Personnel, 4 People & & $115 \mathrm{~K} / \mathrm{NA}$ & $18,400,000$ & 69.350 .000 & FY2005 THRU FY2045 & Engineers Estimate Based on RWMC Study \\
\hline & Health Physics Personnel. 2 People & & $128 \mathrm{~K} / \mathrm{NA}$ & $10,240.000$ & 38.590 .000 & FY2005 THRU FY2045 & Engineers Estimate Based on RWMC Study \\
\hline & Lab Personnel, 3 People & & $128 \mathrm{~K} / \mathrm{RR}$ & 15.360 .000 & $57,890,000$ & FY2005 THAU FY2045 & Engineers Estimate Based on RWMC Study \\
\hline & Environmental Surveltiance. 3 People & & $125 \mathrm{~K} N \mathrm{R}$ & $15,000,000$ & 56.530 .000 & FY2005 THAU FY2045 & Engineers Estimate Based on RWMC Study \\
\hline & Document Control, 1 Person & & $78 \mathrm{~K} / \mathrm{R}$ & 3.120 .000 & $11.760,000$ & FY2005 THRU FY2045 & Engineers Estimate Based on RWMC Study \\
\hline & (5) & & 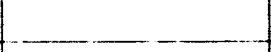 & & - & 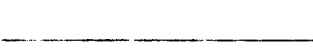 & 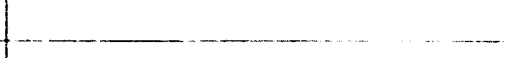 \\
\hline & & & & & & & $\ldots$ \\
\hline & Equipment & & & & & & 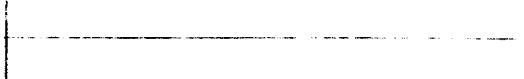 \\
\hline & Replacement Parts - Misc. Maintenance & & $120 \mathrm{~K} / \mathrm{KR}$ & $4.800,000$ & $15,080.000$ & FY2005 THRU FY2045 & Engineers Estimate Based on RWMC Study \\
\hline & & & & & & & \\
\hline & Subtotal for Operating Costs & & & 287.040 .000 & 1.078 .820 .000 & & \\
\hline
\end{tabular}


Project EVALUATION OF ALTERNATIVES FOR DISPOSAL OF INEL MIXED AND LOW LEVEL WASTE

\begin{tabular}{|c|c|c|c|c|c|c|c|}
\hline $\begin{array}{l}\text { ACCT. } \\
\text { No. }\end{array}$ & $\begin{array}{c}\text { ACTIVITY } \\
\text { DESCRIPIION } \\
\end{array}$ & E.V. & $\begin{array}{c}\text { ESTIMATED COST } \\
\text { PEA YEAR }\end{array}$ & $\begin{array}{c}\text { UNESCALATED } \\
\text { COST }\end{array}$ & $\begin{array}{c}\text { ESCALATED } \\
\text { COST }\end{array}$ & $\begin{array}{l}\text { ACTIVITY SCHEDULE } \\
\text { ESCALAIION }\end{array}$ & ESTIMATE BASIS \\
\hline & UTILITIES & & & & & & \\
\hline & Electrical & & $25 \mathrm{~K} / \mathrm{NA}$ & 1.000 .000 & 3.770 .000 & FY2005 THAU FY2045 & Engineers Estumate \\
\hline & Fuels/Propane, etc & & $5 K / N R$ & 200,000 & 750.000 & FY2005 THRU FY2045 & Engineers Estimate \\
\hline & Olner & & 30K/YR & 1.200 .000 & $4.520,000$ & FY2005 THRU FY2045 & Engineers Estimate \\
\hline & Subtotal for Utility Costs & & & $2,400,000$ & 9.040 .000 & & \\
\hline & & & & & & & \\
\hline & & & & & & & \\
\hline & & & & & & & \\
\hline & DECONTAMINATION AND DECOMMISSIONING & & 830.000 & 830.000 & 6.070 .000 & Fr2046 & Data From Previous D \& D Projects \\
\hline & (D8D Costs are based on $10 \%$ of the construction cos & st retati & ting directiy to the facilit & ties being decom mi & issioned) & & \\
\hline & & & & & & & \\
\hline & & & & & & & \\
\hline & & & & & & & \\
\hline & & & & & & & \\
\hline & & & & & & & \\
\hline & CLOSURE & & & & & & \\
\hline & Sand & & 7.720 .000 & 7.720 .000 & 56.460 .000 & FY2046 & Engineers Estimate \\
\hline & Gravel & & 533.000 & 533.000 & 3.900 .000 & FY2046 & Engineers Estimate \\
\hline & Diatomaceous Earn & & 1.610 .000 & $1.610,000$ & $12,220,000$ & FY2047 & Engineers Estimate \\
\hline & Membrane Liner & & $1.840,000$ & 1.840 .000 & 13.970 .000 & FY2047 & Engineers Estımate \\
\hline & Clay & & 2.280 .000 & 2.280 .000 & 17.640 .000 & $\mathrm{FY} 2047-48$ & Engineers Estimate \\
\hline & Sand/Gravel & & $3,390.000$ & $3,350,000$ & 26.710 .000 & FY2048 & Engineers Estimate \\
\hline & Top Soil & & 1.715 .000 & $1.715,000$ & $14.030,000$ & FY2049 & Engineers Estumate \\
\hline & Vegetation & & 72.000 & 72.000 & 590,000 & FY2049 & Engineers Estimate \\
\hline & Instrumentation and installation & & 250,000 & 250.000 & 1.760 .000 & FY2045 & Engineers Estımate \\
\hline & Technical Support, Sampling. \& OA Analysis (4 People) & & $177 \mathrm{~K} / \mathrm{VR}$ & 4.956 .000 & 37.730 .000 & FY2044-50 & Engineers Estumate \\
\hline & POST CLOSURE SURVELLIANCE & & & & & & \\
\hline & Environmental Sunveillance, 3 People & & $125 \mathrm{~K} / \mathrm{YR}$ & $4.125,000$ & 36.610 .000 & FY2046 THAU FY2056 & Engineers Estumate \\
\hline & Environmental Surveillance. 1 Person & & $125 \mathrm{~K} / \mathrm{NR}$ & 11.250 .000 & $1.004,150.000$ & FY2057 THRU FY2146 & Engineers Estimate \\
\hline & Subtotal for Closure and Post Closure & & & 39.741 .000 & 1.225 .770 .000 & & \\
\hline
\end{tabular}


Rev 5-92

Projact eValuation of alternatives for oISPOSAL Typo of Est. PLANNING

Location INEL

FllO NO. 5204 - Alt. 10

Date

Prep'd By

$07 / 05 / 93$

Aoquester

T. H. SMITH

App'd By

$3 x$

PROBABLE \% VARIATION

\begin{tabular}{|c|c|c|c|c|c|c|c|c|}
\hline \multirow[t]{2}{*}{ Item } & \multirow[t]{2}{*}{ Cost } & \multirow{2}{*}{$\begin{array}{c}\% \\
\text { Total } \\
\text { Cost }\end{array}$} & \multicolumn{2}{|l|}{ Est. Basis } & \multirow{2}{*}{\multicolumn{2}{|c|}{$\begin{array}{c}\text { Prod } \% \text { Var } \\
\text { From Est }\end{array}$}} & \multicolumn{2}{|c|}{ Wi $\%$ of Prob } \\
\hline & & & Quoto & Est & & & & \\
\hline Project Management & $3.900,000$ & 1 & 25 & 75 & 5 & 20 & 0.05 & 0.19 \\
\hline Studies, Permitting & $10,880,000$ & 3 & 25 & 75 & 10 & 20 & 027 & 053 \\
\hline Title I and Title \| Design & $3,100,000$ & 1. & 25 & 75 & 10 & 15 & 0.081 & 0.11 \\
\hline Title III Inspection & 830,000 & 0.15 & 25 & 75 & 5 & 20 & 0.011 & 0.03 \\
\hline Construction, Buildings \& Equipment & $39,533,000$ & 10 & $30 !$ & 70 & 10 & 20 & 0.96 & 192 \\
\hline Construction Management & $8.701,000$ & 2 & 301 & 70 & $5 !$ & 201 & 0.111 & 0.42 \\
\hline Slartup and Readiness & $7,204,000$ & 2 & 25 & 75 & 101 & 15 & $0.17^{i}$ & 0.26 \\
\hline Management Reserve & $3.953,000$ & 1 & 50 & 50 & 5 & 30 & 0.05 & 029 \\
\hline Total & 412.812 .000 & 100 & & & & & 16.88 & 24.52 \\
\hline
\end{tabular}


EG\&G Idaho, inc.

\section{COST ESTIMATE SUMMARY}

Type of Estimate: PLANNING

Project: EVALUATION OF AL TERNATIVES FOR DISPOSAL OF INEL MIXED AND LOW LEVEL WASTE

\begin{tabular}{|c|c|c|c|}
\hline & Subtotals & Subtotals & Totals \\
\hline & Unescalated & Escalated & Unescalated \\
\hline \multicolumn{3}{|l|}{ FRONT END DOCUMENTATION .. } & $19,680,00$ \\
\hline Project Management & $3,900,000$ & $5,000,000$ & \\
\hline Studies & $2,0 \longdiv { 0 8 0 , 0 0 0 }$ & $2,160,000$ & \\
\hline NEPA Documentation & $3,250,000$ & $3,580,000$ & \\
\hline Permitting & $8,900,000$ & $11,990,000$ & \\
\hline Technology Development & $1,550,000$ & $1,760,000$ & \\
\hline \multicolumn{3}{|c|}{ ENGINEERING, DESIGN AND INSPECTION... } & $3,330,000$ \\
\hline Performance Specification & N/A & N/A & \\
\hline Conceptual and Title I/ II Design & $2,700,000$ & $3,180,000$ & \\
\hline Title III Inspection & 630,000 & 900,000 & \\
\hline \multicolumn{3}{|l|}{ CONSTRUCTION... } & $40,383,000$ \\
\hline Building Costs & $28,028,000$ & $54,603,000$ & \\
\hline Equipment Costs & $5,070,000$ & $14,080,000$ & \\
\hline Construction Management & $7,285,000$ & $15,117,000$ & \\
\hline
\end{tabular}

START-UP AND READINESS.

BUILDING OPERATION. $289,440,000$

DECONTAMINATION AND DECOMMISSIONING.

CLOSURE/POST CLOSURE

MANAGEMENT RESERVE.

SUBTOTAL

CONTINGENCY

$21 \%$ of Subtotal. $7,204,000$

Date:

File No.

Appr'd By:

Prepared By: T.E. SIVILL

5204 - Alt. $1 E$
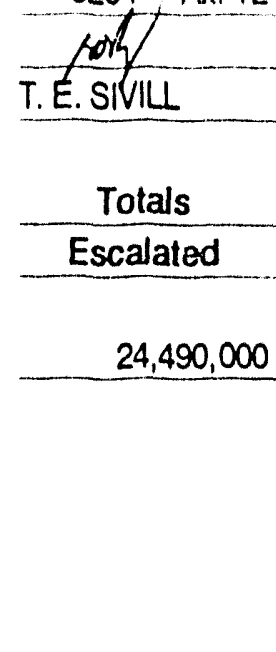

$4,080,000$

$83,800,000$

$11,010,000$

$1,087,860,000$

$5,920,000$

$1,225,770,000$

$6,868,000$

$2,449,798,000$

$550,202,000$

TOTAL PROJECT COST: \$490,000,000 \$3,000,000,000

Comments: 
Project EVALUATION OF ALTERnatives for disposal OF INEL MIXED AND LOW LEVEL WASTE

Location INEL

\section{Requester T.H.SMITH}

\begin{tabular}{|c|c|c|c|}
\hline $\begin{array}{l}\text { ACCT. } \\
\text { No. }\end{array}$ & $\begin{array}{c}\text { ACTIVITY } \\
\text { DESCRIPIION }\end{array}$ & $\begin{array}{l}\text { E.V. } \\
\text { P.H }\end{array}$ & $\begin{array}{c}\text { ESTIMATED COST } \\
\text { PER YEAR }\end{array}$ \\
\hline & PROJECT COSTS & & \\
\hline & Project Management & & 300KNA \\
\hline & Project Studies - 2 People, Full Time & & 250K/NR/EA \\
\hline & Project Formulation (F8OR's) & & $70 \mathrm{~K} / 270 \mathrm{~K}$ \\
\hline & Siting Studies & & $70 \mathrm{~K} / 270 \mathrm{~K} / 400 \mathrm{~K}$ \\
\hline & Pre-Operational Monitoring & & $500 \mathrm{~K} / 500 \mathrm{~K} / 250 \mathrm{~K} / \mathrm{KR}$ \\
\hline & Safety Analysis Repon (SAR) & & $150 \mathrm{~K} / 300 \mathrm{~K} / 200 \mathrm{~K}$ \\
\hline & Pertormance Assessment & & 400KNA \\
\hline & RCRA & & $375 \mathrm{~K} / 100 \mathrm{~K} / 375 \mathrm{~K}$ \\
\hline & NEPA (EIS)(CX's) & & $1 \mathrm{M} / 1 \mathrm{M} / 1 \mathrm{M} / 250 \mathrm{~K}$ \\
\hline & Air Permits (PSD/PTC/NESHAP) & & $200 K / 150 K$ \\
\hline & ESH8O & & 50K/NA \\
\hline & Other Regulatory Items (State Water Well Approval) & & 250K/NA \\
\hline & Special Q/A Program For Construction - 1 Person & & $177 \mathrm{~K} / \mathrm{NR}$ \\
\hline & & & \\
\hline & & & \\
\hline & & & \\
\hline & IECHNOLOGY DEVELCPMENT & & \\
\hline & Material Behavior (Clay, elc) & & $50 \mathrm{~K} / 35 \mathrm{~K} / \mathrm{NR}$ \\
\hline & Cover Performance Control & & $100 \mathrm{~K} / 65 \mathrm{~K} / \mathrm{YR}$ \\
\hline & Leachate Collection and Liner Peftormance & & $200 \mathrm{~K} / \mathrm{NR}$ \\
\hline & & & \\
\hline & & & \\
\hline & & & \\
\hline & & & \\
\hline & & & \\
\hline & & & \\
\hline & & & \\
\hline & Subtotal for Front End Costs & & \\
\hline
\end{tabular}

Type of Estimate PLANNING

Source (E) Eng. Est

(M) Vendor

(P) Pur. Order

(H) Handbook Rel.
FHe No. 5204 - Att. IE Dale

Prep'd 8y: T.E.Sivill

Chk'd By:

Apprd By

\begin{tabular}{|c|c|c|c|}
\hline $\begin{array}{c}\text { UNESCALATED } \\
\text { COST }\end{array}$ & $\begin{array}{c}\text { ESCALATED } \\
\text { COST } \\
\end{array}$ & $\begin{array}{l}\text { ACTIVITY SCHEDULE } \\
\text { ESCALATION }\end{array}$ & ESTIMATE BASIS \\
\hline 3.900 .000 & $5,000,000$ & FY92 THRU FY2005 & TDD Sheet, dated 4/28/93 \\
\hline $1,000,000$ & 1.040 .000 & FY93 - 94 & TDD Sheet, dated $4 / 28 / 93$ \\
\hline 340,000 & 350,000 & FY92 - 93 & TDD Sheet, dated 4/28/93. Eng. Estimate \\
\hline 740.000 & 770.000 & FY92 - FY94 & TOD Sheet, dated 4/28/93. Eng Estimate \\
\hline $2,250,000$ & $3,350,000$ & FY95,FY99,FY2000-2005 & Engineer's Estumate \\
\hline 1.600 .000 & $2.100,000$ & FY95-96,FY99-01,FY02-03 & Data from Similar SAR's \\
\hline 1.600 .000 & 1.960 .000 & FY95-96.FY99-00 & Engineer's Estimate \\
\hline $1.700,000$ & $2,120,000$ & FY96-97,FY98-99,FYO0-01 & Engineer's Estimate (includes Sampling) \\
\hline 3.250 .000 & 3.580 .000 & FY94 - FY97 & Engineer's Estimate \\
\hline 350.000 & 550.000 & FY1999, FY2000 & Engineer's Estimate \\
\hline 550.000 & 730,000 & FY1995-2005 & Engineer's Estimate \\
\hline 500.000 & 670.000 & FY $2000-2001$ & Engineer's Estimate \\
\hline 354.000 & 510,000 & FY2002.03 & Engineer's Estima \\
\hline & & & \\
\hline & & & \\
\hline & & & \\
\hline & & & \\
\hline 190.000 & 210,000 & FY94, FY95-98 & Engineer's Estimate \\
\hline 360.000 & 410.000 & FY94. FY95-98 & Engineer's Estimate \\
\hline $1,000,000$ & $1,140,000$ & FY94-98 & Engineer's Estimate \\
\hline & & & \\
\hline & & & 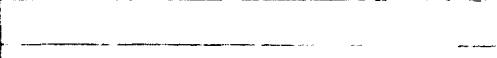 \\
\hline & & & $\cdots$ \\
\hline & & & $\cdots$ \\
\hline & & & $\ldots$ \\
\hline $19.684,000$ & 24.490 .000 & & \\
\hline
\end{tabular}


Project evaluation of alternatives for disposal

OF INEL :IXED AND LOW LEVEL WASTE

\begin{tabular}{|c|c|c|c|c|c|c|c|}
\hline \multirow[t]{6}{*}{ No } & $\begin{array}{c}\text { ACTIVITY } \\
\text { DESCRIPTION }\end{array}$ & E.V. & $\begin{array}{c}\text { ESTIMATED COST } \\
\text { PEA YEAR }\end{array}$ & $\begin{array}{c}\text { UNESCALATED } \\
\text { COST }\end{array}$ & $\begin{array}{c}\text { ESCALATED } \\
\text { COST } \\
\end{array}$ & $\begin{array}{l}\text { ACTIVITY SCHEDULE } \\
\text { ESCALATION }\end{array}$ & ESTIMATE BASIS \\
\hline & DESIGN & & & & & & \\
\hline & Conceptual & & $350 \mathrm{~K} / 350 \mathrm{~K}$ & 700,000 & 780,000 & FY95.FY96 & TDD Sheet \\
\hline & Iive 1 & & $1.0 \mathrm{M}$ & $1,000,000$ & $1,180,000$ & FY97 & Engineers Estimate \\
\hline & Tive II & & $1.0 \mathrm{M}$ & $1,000,000$ & 1.220 .000 & FY98 & Engineers Estimate \\
\hline & Subtotal for Design (Based on $\%$ of Construction Costs) & & & $2.700,000$ & $3.180,000$ & & \\
\hline \multirow{3}{*}{ 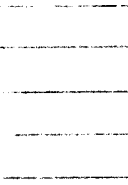 } & & & & & & & \\
\hline & Tive III Inspection (3 People lor 18 Months) & & $420 \mathrm{~K} / 210 \mathrm{~K}$ & 630.000 & 900,000 & FY2002.03 & Comparative Historical Data \\
\hline & & & & & & & \\
\hline \multirow[t]{13}{*}{4000} & $\begin{array}{l}\text { CONSTRUCTION } \\
\text { Improvements to Land }\end{array}$ & & & & & & \\
\hline & $\frac{\text { Improvements to Land }}{\text { Surveying (108 Acres) }}$ & & & & & & \\
\hline & $\begin{array}{l}\text { Surveying (108 Acres) } \\
\text { Site Prep Clearing and Grubbing (108 Acres) }\end{array}$ & & 141.000 & 141.000 & 200.000 & FY2002 & Comparative Historical Data \\
\hline & $\begin{array}{l}\text { Site Prep Clearing and Grubbing (108 Acres) } \\
\text { Roadways and Parking }\end{array}$ & & 324.000 & 324.000 & 460.000 & FY2002 & Comparative Historlcal Data \\
\hline & $\begin{array}{l}\text { Roadways and Parking } \\
\text { Fencing }\end{array}$ & & 1.380 .000 & 1.380 .000 & $1,960,000$ & FY2002 & Comparative Historical Data \\
\hline & $\begin{array}{l}\text { Fencing } \\
\text { Petention Pond }\end{array}$ & & 380,000 & 380.000 & 540,000 & FY2002 & Comparative Historical Data \\
\hline & $\begin{array}{l}\text { Petention Pond } \\
\text { Drainage Gallery System }\end{array}$ & & 40,000 & 40.000 & 57,000 & FY2002 & Comparative Historical Data \\
\hline & $\begin{array}{l}\text { Drainage Gallery System } \\
\text { Drainage Pumphouse }\end{array}$ & & 300.000 & 300.000 & 540.000 & FY2002 & Comparatlve HIstorical Data \\
\hline & $\begin{array}{l}\text { Dralnage Pumphouse } \\
\text { Collection /Sampling System Vault at Disposal Areas }\end{array}$ & & 350,000 & 350,000 & 496.000 & FY2002 & Comparatlve Historical Data \\
\hline & $\begin{array}{l}\text { Collection /Sampling System Vault at Disposal Areas } \\
\text { Storage Vault Disposal Area Including: (Refer to }\end{array}$ & & $1,000,000$ & 1.000 .000 & $1,420,000$ & FY2002 & Comparative Historlcal Data \\
\hline & $\begin{array}{l}\text { Storage Vault Disposal Area Including: (Refer to } \\
\text { - Pad Construction Costs) }\end{array}$ & & - & - & - & - & - \\
\hline & $\begin{array}{l}\text { Pad Construction Costs) } \\
\text { Drainage Ditches }\end{array}$ & & - & - & - & - & - \\
\hline & Drainage Ditches & & 100,000 & 100,000 & 140,000 & FY2002 & \\
\hline \multirow[t]{8}{*}{6000} & $\frac{\text { TOTAL FOR } 4000 \text { ACCOUNT }}{\text { Uillities }}$ & & & 4.015 .000 & $5,813,000$ & & \\
\hline & $\begin{array}{l}\text { Uillities } \\
\text { Electrical Power and Distribution To Facility }\end{array}$ & & & & & & \\
\hline & $\begin{array}{l}\text { Electrical Power and Distribution To Faclity } \\
\text { Electrical Lighting. Power,Alarm s, \& Communication }\end{array}$ & & $\begin{array}{r}1,850,000 \\
650,000 \\
\end{array}$ & $\begin{array}{r}1,850,000 \\
650,000 \\
\end{array}$ & $2.620,000$ & FY2002 & Engineers Estimate \\
\hline & $\begin{array}{l}\text { Electrical Lighting.Power,Alarms, \& Communication } \\
\text { Distribution System }\end{array}$ & & 650,000 & 650,000 & 920.000 & FY2002 & Engineers Estimate \\
\hline & $\begin{array}{l}\text { Distribution System } \\
\text { Water Supply } 8 \text { Fire Water Distribution to Facility }\end{array}$ & & & & & & Engineers Estimate \\
\hline & $\begin{array}{l}\text { Water Supply } 8 \text { Fire Water Distribution to Facility } \\
\text { Water Supply and Fire Water Distribution Systems }\end{array}$ & & $\begin{array}{r}1,500,000 \\
150,000 \\
\end{array}$ & $\begin{array}{r}1.500 .000 \\
150.000 \\
\end{array}$ & $\begin{array}{r}2,130,000 \\
210,000\end{array}$ & FY2002 & Engineers Estimate \\
\hline & $\begin{array}{l}\text { Water Supply and Fire Water Distribution Systems } \\
\text { Sanitary Sewage System }\end{array}$ & & $\begin{array}{l}150,000 \\
250,000 \\
\end{array}$ & $\begin{array}{l}150.000 \\
250,000 \\
\end{array}$ & $\begin{array}{l}210,000 \\
350,000\end{array}$ & FY2002 & Engineers Estimate \\
\hline & TOTAL FOR 6000 ACCOUNT & & 250,000 & $\begin{array}{r}250,000 \\
4.400,000 \\
\end{array}$ & $\frac{350,000}{6,230,000}$ & FY2002 & Engineers Estimate \\
\hline & & & & 4.400 .000 & $6.230,000$ & & \\
\hline
\end{tabular}


Project EVALUATION OF ALTERNaTIVES For DISPOSAL OF INEL MIXED AND LOW LEVEL WASTE

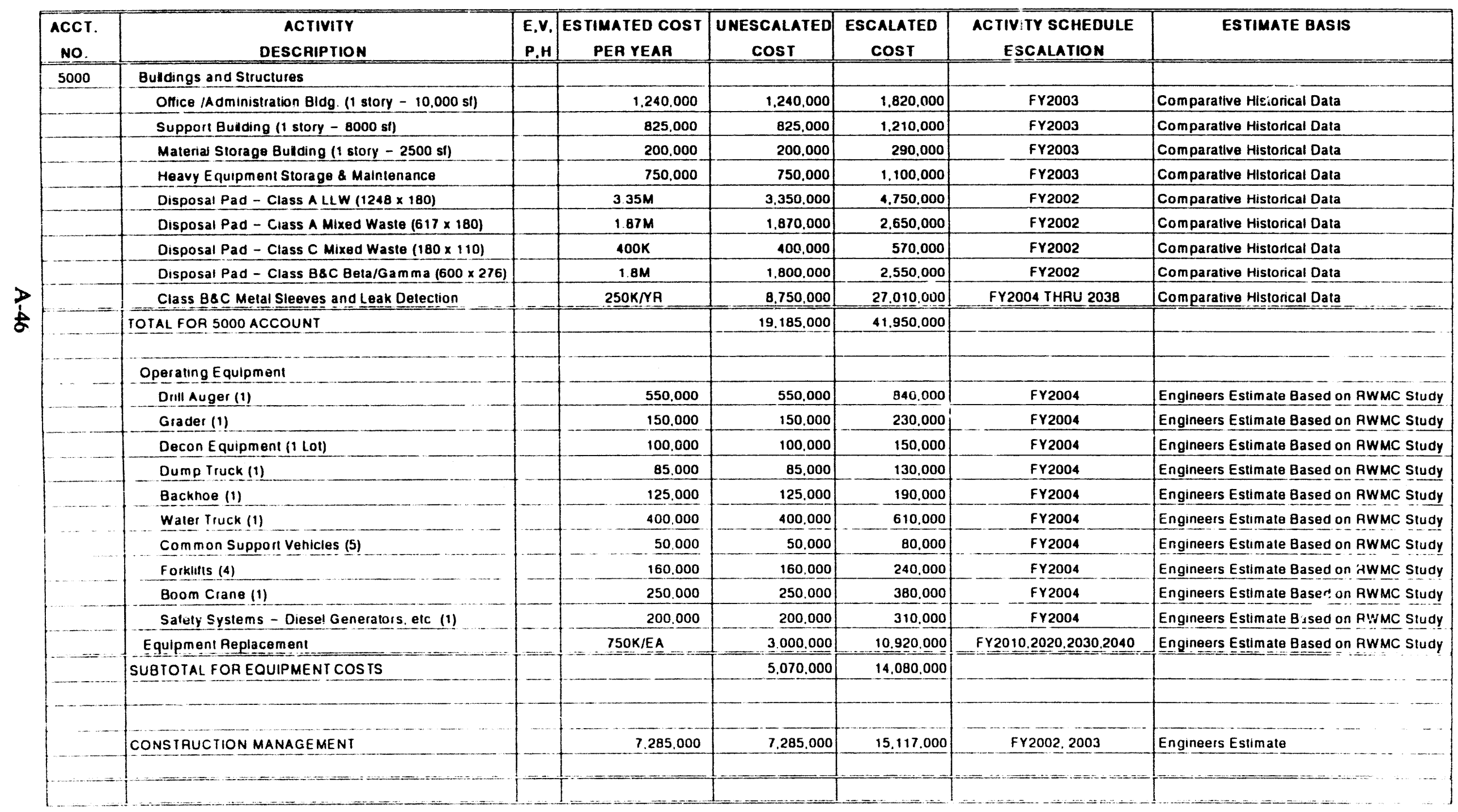


Page 4 of 5

Prolect evaluation of al ternatives for disposal

OF INEL MIXED AND LOW LEVEL WASTE

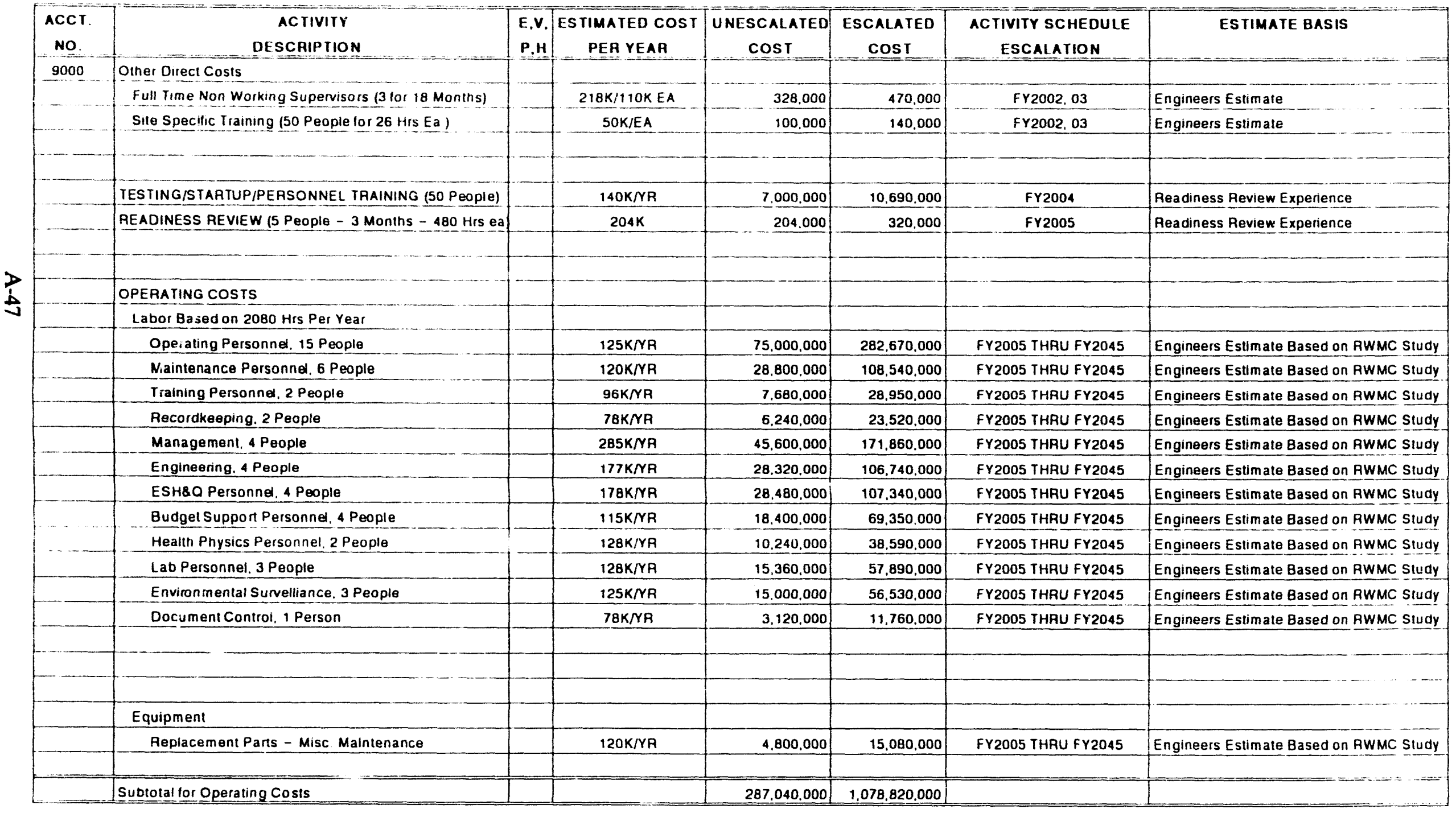


Project EVALUATION OF ALTERNATIVES For DisPosal OF INEL MIXED AND LOW LEVEL WASTE

\begin{tabular}{|c|c|c|c|c|c|c|c|}
\hline $\begin{array}{c}\text { ACCT. } \\
\text { No. }\end{array}$ & $\begin{array}{c}\text { ACTIVITY } \\
\text { DESCAIPIION } \\
\end{array}$ & $\begin{array}{l}\text { E.V. } \\
\text { P.H }\end{array}$ & $\begin{array}{c}\text { ESTIMATED COST } \\
\text { PEA YEAR }\end{array}$ & \begin{tabular}{|c|} 
UNESCALATED \\
COST
\end{tabular} & $\begin{array}{c}\text { ESCALATED } \\
\text { COST } \\
\end{array}$ & $\begin{array}{c}\text { ACTIVITY SCHEDULE } \\
\text { ESCALATION }\end{array}$ & ESTIMATE BASIS \\
\hline & UIILITIES & & & & & & \\
\hline & Electrical & & $25 \mathrm{~K} N \mathrm{R}$ & $1.000,000$ & $3,770,000$ & FY2005 THRU FY2045 & Engineers Estimate \\
\hline & Fuels/Propane. etc. & & 5K/NR & 200.000 & 750,000 & FY2005 THRU FY2045 & Engineers Estimate \\
\hline & Other & & 30K/YR & $1.200,000$ & 4.520 .000 & FY2005 IHRU FY2045 & Engineers Estimate \\
\hline & Subtolal for Utility Costs & & & 2.400 .000 & $9,040,000$ & & \\
\hline & & & & & & & \\
\hline & & & & & & & \\
\hline & & & & & & & \\
\hline & DECONIAMINATION ANO DECOMMISSIONING & & 810,000 & 810.000 & 5.920 .000 & FY2046 & Data From Previous D \& D Projects \\
\hline & (D\&D Costs are based on $10 \%$ of the construction co & it relat & ting directly to the facili & ies being decom mi & ssioned) & & \\
\hline & & & & & & & \\
\hline & & & & & & & \\
\hline & & & & & & & \\
\hline & & & & & & & \\
\hline & & & & & & & \\
\hline & CLOSUAE & & & & & & \\
\hline & Sand & & 7.720 .000 & $7.720,000$ & 56.460 .000 & FY2046 & Engineers Estimate \\
\hline & Gravel & & 533,000 & 533,000 & 3.900 .000 & FY2046 & Engineers Estimate \\
\hline & Diatomaceous Earth & & 1.610 .000 & 1.610 .000 & 12.220 .000 & FY2047 & Engineers Estimate \\
\hline & Membrane Liner & & $1,840.000$ & 1.840 .000 & 13.970 .000 & FY2047 & Engineers Estimate \\
\hline & Clay & & $2.280,000$ & $2.280,000$ & $17,640,000$ & FY2047-48 & Engineers Estimate \\
\hline & Sand/Gravel & & 3.390 .000 & 3.390 .000 & 26.710 .000 & FY2048 & Engineers Estimate \\
\hline & Top Soil & & 1.715 .000 & $1.715,000$ & $14,030.000$ & Fr2049 & Engineers Estimate \\
\hline & Vegetation & & 72.000 & 72.000 & 590.000 & FY2049 & Engineers Estimate \\
\hline & Instrumentarion and installation & & 250,000 & 250.000 & 1.760 .000 & FY2045 & Engineers Estlmate \\
\hline & Technical Support, Sampling. 8 OA Analysls (4 People) & & 177KNAR & $4,956,000$ & 37.730 .000 & Fr2044-50 & Engineers Estimate \\
\hline & POST CLOSURE SURVELLIANCE & & & & & & \\
\hline & Environmental Surveillance, 3 People & & $125 K / \mathrm{KA}$ & 4.125 .000 & $36,610,000$ & FY2046 THRU FY2056 & Engineers Estumate \\
\hline & Environmental Survellance. 1 Person & & $125 \mathrm{~K} / \mathrm{NR}$ & 11.250 .000 & 1.004 .150 .000 & FY2057 THRU FY2146 & Engineers Estimate \\
\hline & Subtotal for Closure and Post Closure & & & 39.741 .000 & 1.225 .770 .000 & & \\
\hline
\end{tabular}


Rev 5-92

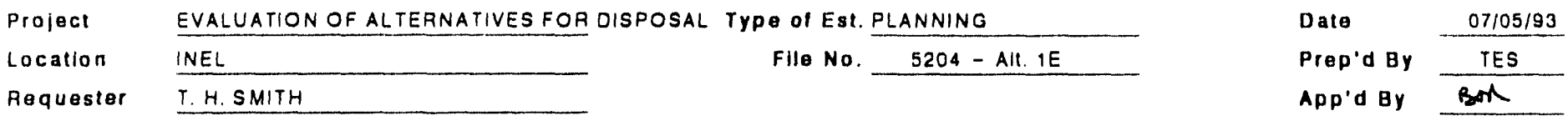

PROBABLE \% VARIATION

\begin{tabular}{|c|c|c|c|c|c|c|c|c|}
\hline \multirow[t]{3}{*}{ Item } & \multirow[t]{3}{*}{ Cosi } & \multirow{3}{*}{$\begin{array}{c}\% \\
\text { Total } \\
\text { Cost }\end{array}$} & \multicolumn{2}{|l|}{ Est. Basis } & \multirow{2}{*}{\multicolumn{2}{|c|}{$\begin{array}{l}\text { Prob \% Var } \\
\text { From Est }\end{array}$}} & \multirow{2}{*}{\multicolumn{2}{|c|}{ Wt $\%$ of Prob }} \\
\hline & & & Quote & Est & & & & \\
\hline & & & & & - & + & - & + \\
\hline Project Management & $3,900,000$ & 1 & 25 & 75 & 5 & 20 & 0.05 & 0.19 \\
\hline Studies. Permitting & $10,980,000 !$ & 3 & 25 & 7.5 & 10 & 20 & 0.27 & 0.54 \\
\hline NEPA & $3.250,000$ & 1 & 5 & 95 & 20 & 25 & 0.16 & 0.20 \\
\hline Tecnnology Developement & 1.550 .000 & 0.38 & 5 & 95 & 10 & 25 & 0.04 & 0.10 \\
\hline Title I and Title $1 /$ Design & 2.700 .000 & $1 !$ & 25 & 75 & 10 & 15 & 0.07 & 0.10 \\
\hline Title III Inspection & 630,000 & $0.16 !$ & 25 & 75 & 5 & 20 & 0.01 & 0.03 \\
\hline Construction. Bulldings \& Equipment & $33,098,000$ & 8 & 30 & 70 & 10 & 20 & 0.82 & 1.64 \\
\hline Construction Management & 7.285 .000 & 2 & 30 & 70 & 5 & 20 & 0.09 & 0.36 \\
\hline Startup and Readiness & 7204.000 & 2 & 25 & 75 & 10 & 15 & 0.18 & 0.27 \\
\hline Building Operation & $289,440.000$ & 72 & 5 & 95 & 20 & 25 & 14.33 & 17.92 \\
\hline Decontamination \& Cecommissioning & 810,000 & 0 & 25 & 75 & 10 & 30 & 0.02 & 0.06 \\
\hline Closure/Post Closure & $39.741 .000 i$ & 10 & 5 & 95 & 10 & 30 & 0.98 & 2.95 \\
\hline Management Reserve & 3.310 .000 & 11 & 50 & 50 & 5 & 30 & 0.04 & 025 \\
\hline Total & $403,898.000$ & 1001 & & & & & 17.08 & 24.61 \\
\hline
\end{tabular}

Comments: Analysis results in an overall project contingency of

In rounding. $\quad 21 \%$ was used. This is within the allowable $2 \%$ deviation from the result of this analysis. 
EG\&G Idaho, Inc.

COST ESTIMATE SUMMARY

Type of Estimate: PLANNING

Project: EVALUATION OF ALTERNATIVES FOR DISPOSAL OF INEL MIXED AND LOWLEVEL WASTE

Date:

File No.

Appr'd By:

Prepared By: T.E. SIVILL

Subtotals $\frac{\text { Subtotals }}{\text { Enescalated }}$

FRONT END DOCUMENTATION

Project Management

Studies

NEPA Documentation

Permitting

Technology Development

\begin{tabular}{rr}
\hline$, 3,900,000$ & $5,000,000$ \\
\hline $2,080,000$ & $2,160,000$ \\
\hline $3,250,000$ & $3,580,000$ \\
\hline $9,970,000$ & $14,180,000$ \\
\hline $2,120,000$ & $2,440,000$ \\
\hline
\end{tabular}

ENGINEERING, DESIGN AND INSPECTION.

$4,930,000$

\begin{tabular}{llll} 
Performance Specification & N/A & & N/A \\
Conceptual and Title I / II Design & $3,200,000$ & & $4,300,000$ \\
\cline { 2 - 3 } Title III Inspection & $-1,730,000$ & & $3,030,000$ \\
\hline
\end{tabular}

CONSTRUCTION.

Building Costs

Equipment Costs

Construction Management

$\quad 51,558,000$

$4,670,000-13,470,000$

$7,499,000$

$14,313,000$

START-UP AND READINESS.

$7,245,000$

$11,080,000$

BUILDING OPERATION.

$288,640,000$

$1,087,700,000$

DECONTAMINATION AND DECOMMISSIONING.

850,000

$6,330,000$

CLOSURE/POST CLOSURE

$37,701,000$

$1,205,270,000$

MANAGEMENT RESERVE

$3,407,000$

$6,503,000$

SUBTOTAL

$405,662,000$

$2,430,914,000$

CONTINGENCY

$20 \%$ of Subtotal.

$82,338,000$

$519,086,000$

TOTAL PROJECT COST: $\$ 488,000,000$

$\$ 2,950,000,000$

Comments: 
Rev 5-92

Project EVALUATION OF ALTERNaTIVES For DisPosal OF INEL MIXED AND LOW LEVEL WASTE

Location INEL

\section{Requester T.H.SMITH}

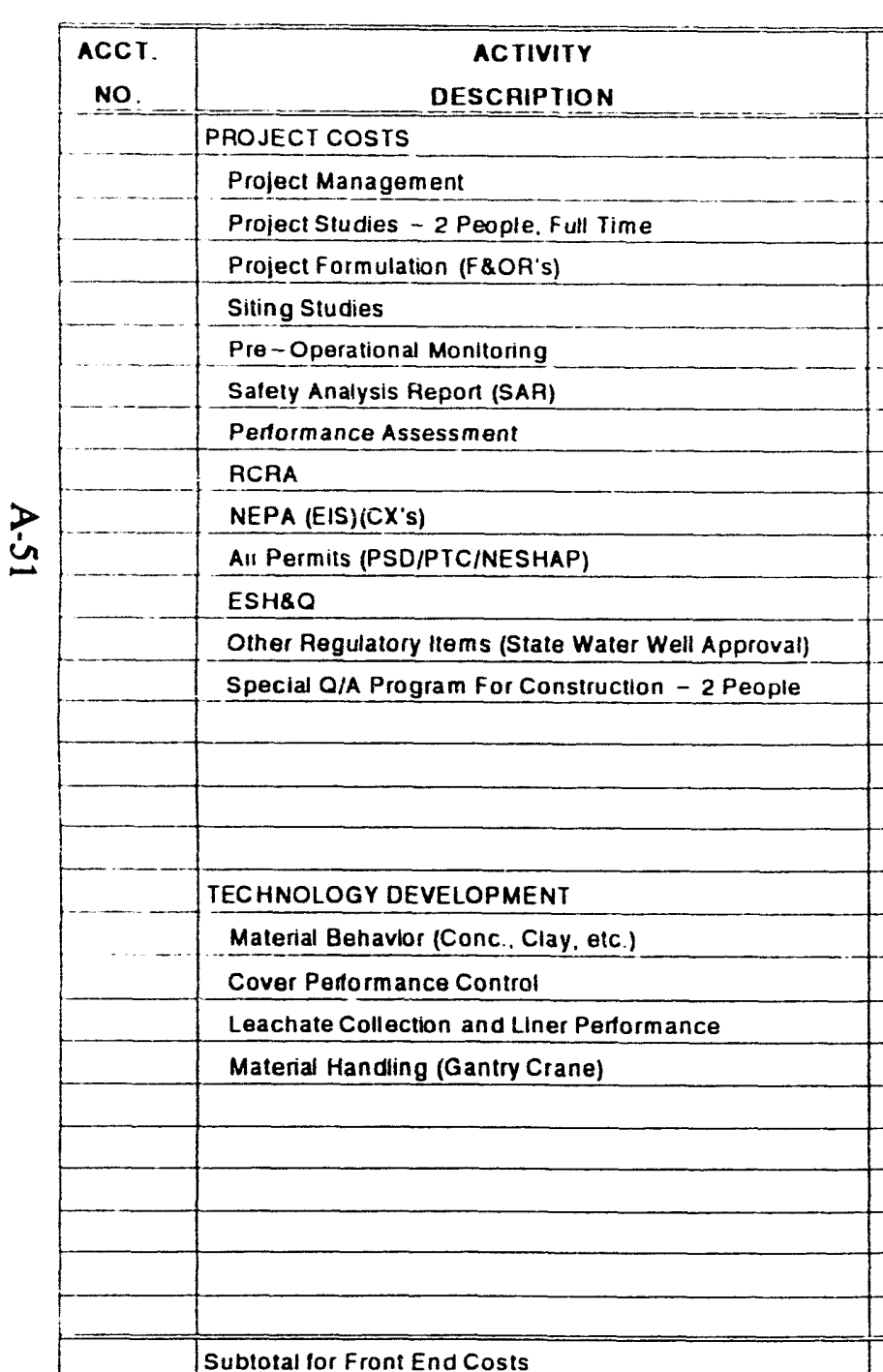

Type of Estimate PLANNING

Source (E) Eng. Est

(V) Vendor

(P) Pur. Order

(H) Handbook Ret.

\begin{tabular}{|c|c|c|c|c|c|}
\hline $\begin{array}{l}\text { E.V. } \\
\text { P.H }\end{array}$ & $\begin{array}{c}\text { ESTIMATED COST } \\
\text { PER YEAR }\end{array}$ & $\begin{array}{c}\text { UNESCALATED } \\
\text { COST }\end{array}$ & $\begin{array}{c}\text { ESCALATED } \\
\text { COST }\end{array}$ & $\begin{array}{l}\text { ACTIVITY SCHEDULE } \\
\text { ESCALATION }\end{array}$ & ESTIMATE BASIS \\
\hline & & & & & \\
\hline & $300 \mathrm{~K} / \mathrm{YR}$ & 3.900 .000 & 5.000 .000 & FY92 THRU FY2005 & IDD Sheet, dated $4 / 28 / 93$ \\
\hline & 250K/YR/EA & $1.000,000$ & $1,040,000$ & FYg3 -94 & IDD Sheet, dated $4 / 28 / 93$ \\
\hline & $70 \mathrm{~K} / 270 \mathrm{~K}$ & 340,000 & 350.000 & FY92 - 93 & TDO Sheet. dated 4/28/93. Eng. Estimate \\
\hline & $70 \mathrm{~K} / 270 \mathrm{~K} / 400 \mathrm{~K}$ & 740,000 & 770.000 & FY92 - FY94 & TDD Sheet, dated 4/28/93. Eng. Estimate \\
\hline & $500 \mathrm{~K} / 500 \mathrm{~K} / 250 \mathrm{~K} / \mathrm{NR}$ & 2.250 .000 & 3.350 .000 & FY95.FY99.FY2000-2005 & Engineer's Estimate \\
\hline & $150 \mathrm{~K} / 300 \mathrm{~K} / 200 \mathrm{~K}$ & 1.600 .000 & $2.100,000$ & FY95-96.FY99-01.FY02-03 & Data from Simllar SAR's \\
\hline & $400 \mathrm{~K} / \mathrm{NR}$ & $1,600.000$ & 1.960 .000 & FY95-96,FY99-00 & Engineer's Estimate \\
\hline & $375 K / 100 \mathrm{~K} / 375 \mathrm{~K}$ & 1.700 .000 & $2.120,000$ & FY96-97.FY98-99.FYO0-01 & Engineer's Estimate (inclu des Sampling) \\
\hline & $1 \mathrm{M} / 1 \mathrm{M} / 1 \mathrm{M} / 250 \mathrm{~K}$ & 3.250 .000 & 3.580 .000 & FY94-FY97 & Engineer's Estimate \\
\hline & $200 \mathrm{~K} / 150 \mathrm{~K}$ & 350.000 & 550,000 & FY1999, FY2000 & Engineer's Estimate \\
\hline & $50 K / \mathrm{R}$ & 550.000 & 730.000 & FY1995-2005 & Engineer's Estimate \\
\hline & $250 K / \mathrm{K}$ & 500.000 & 670.000 & FY $2000-2001$ & Engineer's Estimate \\
\hline & $354 \mathrm{~K} I \mathrm{R}$ & $1.420,000$ & $2,700,000$ & FY2002,03,04,24 & Engineer's Estimate \\
\hline & & & & & \\
\hline & & & & & \\
\hline & & & & & \\
\hline & & & & & \\
\hline & & & & & \\
\hline & $100 \mathrm{~K} / 65 \mathrm{KNR}$ & 360.000 & 410,000 & FY94, FY95-98 & Engineer's Estımate \\
\hline & $100 \mathrm{~K} / 65 \mathrm{~K} / \mathrm{YR}$ & 360.000 & 410.000 & FY94, FY95-98 & Engineer's Estimate \\
\hline & $200 \mathrm{~K} / \mathrm{YA}$ & $1,000,000$ & $1,140.000$ & FY94-98 & Engineer's Estimate \\
\hline & 200K/rR & 400.000 & 480,000 & FY97-98 & Engineer's Estimate \\
\hline & & & & & \\
\hline & & & & & \\
\hline & & & & & \\
\hline & & & & & \\
\hline & & & & & \\
\hline & & & & & \\
\hline & & 21.320 .000 & $27,360,000$ & & \\
\hline
\end{tabular}

Date

Chk'd By:

Approd By: T.E.Sivill

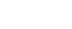
- 
Project evaluation of alternatives for disposal OF INEL MIXED AND LOW LEVEL WASTE

\begin{tabular}{|c|c|c|c|c|c|c|c|}
\hline $\begin{array}{c}\text { ACCT. } \\
\text { No. }\end{array}$ & $\begin{array}{c}\text { ACTIVITY } \\
\text { DESCAIPIION } \\
\end{array}$ & $\begin{array}{l}\text { E.V. } \\
P . H \\
\end{array}$ & $\begin{array}{c}\text { ESTIMATED COST } \\
\text { PEA YEAR } \\
\end{array}$ & $\begin{array}{c}\text { UNESCALATED } \\
\text { COST }\end{array}$ & $\begin{array}{c}\text { ESCALATED } \\
\text { COST } \\
\end{array}$ & $\begin{array}{c}\text { ACTIVITY SCHEDULE } \\
\text { ESCALATION }\end{array}$ & ESTIMATE BASIS \\
\hline & DESIGN & & & & & & \\
\hline & Conceptual & & $450 \mathrm{~K} / 45 \mathrm{CK}$ & 900,000 & $1.000,000$ & FY95.FY96 & TOD Sheet \\
\hline & Tive I & & $1 M$ & $1,000,000$ & $1.180,000$ & FY97 & Engineers Estimate \\
\hline & Tive II & & $1 \mathrm{M}, 300 \mathrm{~K}$ & 1.300 .000 & 2.120 .000 & FY98.2022 & Engineers Estimate \\
\hline & Subtotal for Design (Based on $x$ of Construction Costs) & & & $3,200,000$ & $4,300,000$ & & \\
\hline & & & & & & & \\
\hline & Tive III Inspection (4 People for 30 Months) & & $575 K / 575 K / 288 K E A$ & $1.730,000$ & $3.030,000$ & FY2002.03.04,24 & Comparative Historical Data \\
\hline & & & & & & & \\
\hline & CONSTRUCTION & & & & & & \\
\hline 4000 & Improvements to Land & & & & & & \\
\hline & Surveying (122 Acres) & & 159.000 & 159,000 & 230,000 & FY2002 & Comparative Historical Data \\
\hline & Site Prep Clearing and Grubbing (122 Acres) & & 366.000 & 366,000 & 520.000 & FY2002 & Comparative Historical Data \\
\hline & Roadways and Parking & & 1.400 .000 & $1,400,000$ & 1.980 .000 & FY2002 & Comparative Historical Data \\
\hline & Fencing & & 405,000 & 405.000 & 570.000 & FY2002 & Comparative Historical Data \\
\hline & Retention Pond & & 40.000 & 40,000 & 57,000 & FY2002 & Comparative Historical Data \\
\hline & Drainage Gallery System & & 460,000 & 460.000 & 650.000 & FY2002 & Comparative Historical Data \\
\hline & Drainage Pumphouse & & 375.000 & 375,000 & 531.000 & FY2002 & Comparative Historical Data \\
\hline & Collection /Sampling System Vault at Disposal Areas & & $1,100.000$ & 1.100 .000 & 1.560 .000 & FY2002 & Comparative Historical Data \\
\hline 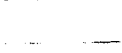 & Stolage Vault Disposal Area Including: Engineered & & 550.000 & 550.000 & 780.000 & FY2002 & Comparative Historical Data \\
\hline & Fili. and Pit Run & & & & & & \\
\hline & Drainage Ditches & & 120.000 & 120,000 & 170.000 & FY2002 & \\
\hline & IOTAL FOR 4000 ACCOUNT & & & 4.975 .000 & $7.048,000$ & & \\
\hline 6000 & Utilities & & & & & & \\
\hline & Electrical Power and Distribution To Facility & & $1.850,000$ & 1.850 .000 & 2.620 .000 & FY2002 & Engineers Estimate \\
\hline & Electrical Llghting. Power, Alarms, \& Communication & & 700.000 & 700.000 & 990.000 & FY2002 & Engineers Estimate \\
\hline & Distribution System & & & & & & Engineers Estimate \\
\hline & Water Supply \& Fire Water Distribution to Facility & & 1.500 .000 & 1.500 .000 & 2.130 .000 & FY2002 & Engineers Estimate \\
\hline & Water Supply and Fire Water Distribution Systems & & 260.000 & 260.000 & 370,000 & FY2002 & Engineers Estimate \\
\hline & Sanitary Sewage System & & 250,000 & 250.000 & 350.000 & FY2002 & Engineers Estimate \\
\hline & TOTAL FOR 6000 ACCOUNT & & & 4.560 .000 & 6.460 .000 & & \\
\hline
\end{tabular}


Project EVALUATION OF ALTERNATIVES For disPOSAL OF INEL MIXED AND LOW LEVEL WASTE

\begin{tabular}{|c|c|c|c|c|c|c|c|}
\hline $\begin{array}{l}\text { ACCT. } \\
\text { No. }\end{array}$ & $\begin{array}{c}\text { ACTIVITY } \\
\text { DESCAIPIION }\end{array}$ & $\begin{array}{l}\text { E.V. } \\
\text { P.H }\end{array}$ & $\begin{array}{c}\text { ES TIMATED COST } \\
\text { PER YEAR }\end{array}$ & $\begin{array}{c}\text { UNESCALATED } \\
\text { COST }\end{array}$ & $\begin{array}{c}\text { ESCALATED } \\
\text { COSI }\end{array}$ & $\begin{array}{l}\text { ACTIVITY SCHEDULE } \\
\text { ESCALATION }\end{array}$ & ESTIMATE BASIS \\
\hline \multirow[t]{8}{*}{5000} & Buid dings and Structures & & & & & & \\
\hline & Office /Administration Blag (1 story - 10.000 st) & & 1.240 .000 & $1,240.000$ & 1.820 .000 & FY2003 & Comparative Historical Data \\
\hline & Support Building (1 story - 8000 st) & & 825,000 & 825,000 & $1,210,000$ & FY2003 & Comparative Historical Data \\
\hline & Material Storage Buidding (1 story - $2500 \mathrm{sf}$ ) & & 200.000 & 200,000 & 290,000 & FY2003 & Comparative Historical Data \\
\hline & Heavy Equipment Storage 8 Maintenance & & 750.000 & 750,000 & 1.100 .000 & FY2003 & Comparative Historical Data \\
\hline & Disposal Pad - Class A LLW $(1248 \times 180)$ & & $3.35 \mathrm{M}$ & $3,350,000$ & $5,120,000$ & FY2004 & Comparative Historical Data at SR Site \\
\hline & Disposal Pad - Class A Mixed Waste $(617 \times 180)$ & & $187 \mathrm{M}$ & 1.870 .000 & 2.860 .000 & FY2004 & Comparative Historical Data at SR Site \\
\hline & Disposal Vault - $8 \& C$ B/G $(58 \times 166 \times 31)(1)(1)$ & & $2.35 \mathrm{M} / 2.35 \mathrm{M}$ & $4.700,000$ & 11.150 .000 & FY2004,2024 & Comparative Historical Data at SA Site \\
\hline & Disposal Vault - Type B \& C Mixed $(40 \times 76 \times 32)(1)$ & & $1 \mathrm{M}$ & $1.000,000$ & 1.530 .000 & FY2004 & Comparative Historical Data at SR Site \\
\hline & Disposal Vault - Type C Mixed $(56 \times 157 \times 29)$ (1)(1) & & $23 \mathrm{M} / 2.3 \mathrm{M}$ & $4.600,00,0$ & 10.920 .000 & FY2004,2024 & Comparative Historical Data at SR Site \\
\hline & TOTAL FOR 5000 ACCOUNT & & & $18.535,000$ & 36.000 .000 & & \\
\hline \multirow{4}{*}{ - } & & & & & & & \\
\hline & & & & & & & \\
\hline & Operating Equipment & & & & & & \\
\hline & Grader (1) & & 150.000 & 150.000 & 230.000 & FY2004 & Engineers Estimate Based on RWMC Study \\
\hline \multirow{6}{*}{\begin{tabular}{|l} 
\\
\\
\end{tabular}} & Decon Equipment (1 Lot) & & 100,000 & 100,000 & 150.000 & FY2004 & Engineers Estlmate Based on RWMC Study \\
\hline & Dump Truck (1) & & 85,000 & 85.000 & 130.000 & FY2004 & Engineers Estimate Based on FWMC Stuay \\
\hline & Backhoe (1) & & 125.000 & 125,000 & 190,000 & FY2004 & Engineers Estimate Based on RWMC study \\
\hline & Water Truck (1) & & 400.000 & 400.000 & 610,000 & FY2004 & Engineers Estimate Based on RWMC Stuíy \\
\hline & Common Support Venicles (5) & & 50,000 & 50,000 & 80,000 & FY2004 & Engineers Estimate Based on RWMC Study \\
\hline & Forklifts (4) & & 160,000 & 160.000 & 240,000 & FY2004 & Engineers Estumate Based on RWMC Study \\
\hline \multirow{2}{*}{$-\ldots$} & Gantry Crane (1) & & 400.000 & 400,000 & 610.000 & FY2004 & Engineers Estimate Based on RWMC Study \\
\hline & Safety Systems - Deisel Generators, etc. (1) & & 200,000 & 200,000 & 310.000 & FY2004 & Engineers Estimate Based on RWMC Study \\
\hline \multirow{2}{*}{$=$} & Equipment Replacement & & 750K/EA & $3.000,000$ & $10,920,000$ & FY2010.2020.2030.2040 & Engineers Estimate Based on RWMC Study \\
\hline & SUBTOTAL FOR EQUIPMENT COSTS & & & $4.670,000$ & 13.470 .000 & & \\
\hline \multirow[t]{2}{*}{ 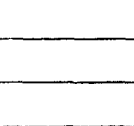 } & & & & & & & \\
\hline & CONSTAUCTION MANAGEMENT & & 7.499 .000 & $7.499,000$ & $14,313,000$ & FY2003,04, 13,23,24, \& 2033 & Engineers Estimate \\
\hline & & & & & & & \\
\hline & & & & & & & \\
\hline
\end{tabular}


Project EVALUATION OF ALTERNATIVES For DISPOSAL OF INEL MIXED AND LOW LEVEL WASTE

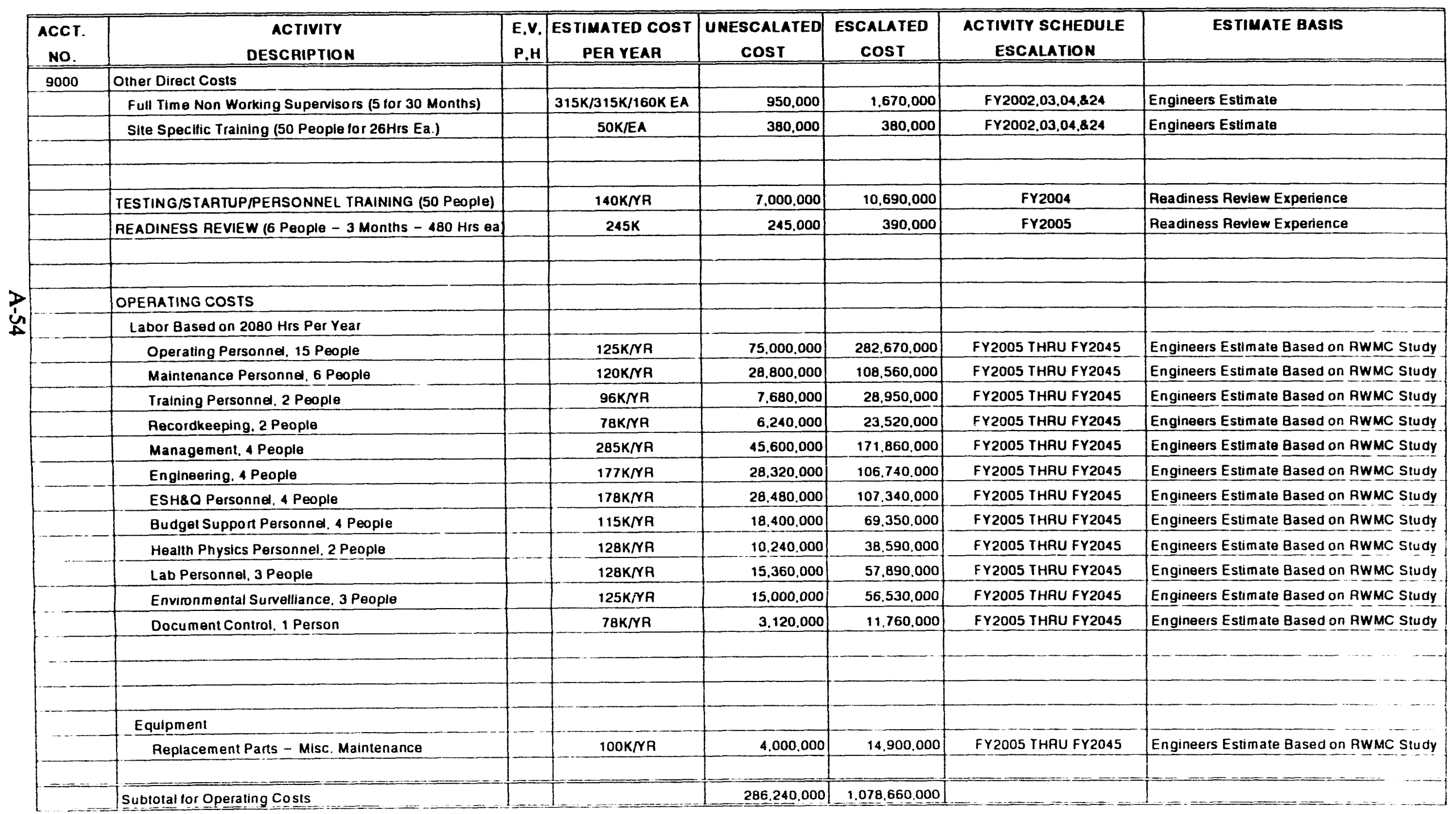


Rev 5-9?

Page 5 of 5

Project EVALUation of alternatives for Disposal OF INEL MIXED AND LOW LEVEL WASTE

\begin{tabular}{|c|c|c|c|c|c|c|c|}
\hline $\begin{array}{c}\text { ACCT. } \\
\text { No. }\end{array}$ & $\begin{array}{c}\text { ACTIVITY } \\
\text { DESCAIPIION } \\
\end{array}$ & $\begin{array}{l}\text { E.V. } \\
\text { P.H. }\end{array}$ & $\begin{array}{c}\text { ESTIMATED COST } \\
\text { PER YEAR }\end{array}$ & \begin{tabular}{|c|} 
UNESCALATED \\
COST
\end{tabular} & $\begin{array}{c}\text { ESCALATED } \\
\text { COST }\end{array}$ & $\begin{array}{c}\text { ACTIVITY SCHEDULE } \\
\text { ESCALATION }\end{array}$ & ESTIMATE BASIS \\
\hline & UTILITIES & & & & & & \\
\hline & Electrical & & $25 K / N R$ & $1,000.000$ & 3.770 .000 & FY2005 THRU FY2045 & Engineers Estimate \\
\hline & Fuels/Propane. otc. & & $5 \mathrm{~K} / \mathrm{KR}$ & 200.000 & 750.000 & FY2005 THAU FY2045 & Engineers Estimate \\
\hline & Other & & 30K/NR & 1.200 .000 & $4.520,000$ & FY2005 THAU FY2045 & Engineers Estimate \\
\hline & Subtotal for Utility Costs & & & $2.400,000$ & $9.040,000$ & & \\
\hline & & & & & & & \\
\hline & & & & & & & \\
\hline & & & & & & & \\
\hline & DECONIAMINATION AND DECOMMISSIONING & & 850.000 & 850.000 & 6.330 .000 & FY2046 & Data From Previous D \& D Projects \\
\hline & (L: Costs are based on $10 \%$ of the construction co & st relat & ing directly to the facilit & ies being decom mis & ssioned) & & \\
\hline & CLOSURE & & & & & & \\
\hline & Disposal P: - Class A LLW $(1248 \times 180)$ & & - & - & - & & \\
\hline & Disposal Pad - Ciass A Mixed Waste $(617 \times 180)$ & & - & - & - & & \\
\hline & Disposal Vault - B \& C B/G $(58 \times 166 \times 31)(1)(1)$ & & $850 \mathrm{~K} / 850 \mathrm{~K}$ & 1.700 .000 & $8.730,000$ & FY2024.2045 & Engineers Estimate \\
\hline & Disposal Vaull - Type B 8 C Mixed $(40 \times 76 \times 32)(1)$ & & $300 \mathrm{~K}$ & 300.000 & 2.110 .000 & FY2045 & Engineers Estimate \\
\hline & Disposal Vault - Type C Mixed $(56 \times 157 \times 29)(1)(1)$ & & $50 \mathrm{~K} / 50 \mathrm{~K}$ & 100.000 & 510,000 & FY2024.2045 & Engineers Estimate \\
\hline & Sand & & 6.300 .000 & 6.300 .000 & $46.080,000$ & FY2046 & Engineers Estimate \\
\hline & Gravel & & 440,000 & 440,000 & $3,220,000$ & FY2046 & Engineers Estimate \\
\hline & Diatomaceous Earth & & 1.330 .000 & 1.330 .000 & 10.100 .000 & FY2047 & Engineers Estimate \\
\hline & Membrane Liner & & 1.530 .000 & 1.530 .000 & $11,620,000$ & FY2047 & Engineers Estımate \\
\hline & Clay & & $1,900,000$ & $1.900,000$ & $14.700,000$ & FY2047-48 & Engineers Estimate \\
\hline & Sand/Gravel & & $2.800,000$ & 2.800 .000 & 22.060 .000 & FY2048 & Engineers Estimate \\
\hline & Top Soll & & 660,000 & 660.000 & $5.400,000$ & FY2049 & Engineers Estimate \\
\hline & Vegetation & & 60,000 & 60,000 & 490,000 & FY2049 & Engineers Estimate \\
\hline & Instrumentation and Installation & & 250,000 & 250,000 & $1,760,000$ & FY2045 & Engineers Estimate \\
\hline & Technical Support. Sampling. \& OA Analysis (4 Feople) & & $177 \mathrm{~K} / \mathrm{NR}$ & 4.956 .000 & $37,730,000$ & FY2044-50 & Engineers Estimate \\
\hline & POST CLOSUAE SUAVELLIANCE & & & & & & \\
\hline & Environmental Survellance, 3 People & & $125 \mathrm{~K} / \mathrm{RR}$ & $4,125,000$ & 36.610 .000 & FY2046 THRU FY2056 & Engineers Estimate \\
\hline & Environmental Survelliance, 1 Person & & $125 \mathrm{~K} / \mathrm{NA}$ & 11.250 .000 & $1.004 .150,000$ & FY2057 THRU FY2146 & Engineers Estimate \\
\hline & Subtotal for Closure and Post Closure & & & 37.701 .000 & 1.205 .270 .000 & & \\
\hline
\end{tabular}


Rev 5-92

\begin{tabular}{|c|c|c|c|c|}
\hline Project & EVALUATION OF ALTERNATIVES FOR DISPOSAL & Do of Est. PLANNING & Date & $07 / 05 / 92$ \\
\hline Location & INEL & Fllo No. 5204 - Alt. IF & Prop'd By & TES \\
\hline Pequester & T. H. SMITH & & App'd By & Bor \\
\hline
\end{tabular}

\begin{tabular}{|c|c|c|c|c|c|c|c|c|}
\hline \multirow{4}{*}{ Item } & \multicolumn{4}{|c|}{ PROBRBLE $\%$ VARIATION } & & & & \\
\hline & \multirow[t]{3}{*}{ Cost } & \multirow{3}{*}{$\begin{array}{c}\% \\
\text { Total } \\
\text { Cost }\end{array}$} & \multicolumn{2}{|l|}{ Est. Basis } & \multirow{2}{*}{\multicolumn{2}{|c|}{$\begin{array}{l}\text { Prob } \% \text { Var } \\
\text { From Est }\end{array}$}} & \multirow{2}{*}{\multicolumn{2}{|c|}{ Wt $\%$ of Prob }} \\
\hline & & & \multirow{2}{*}{ Quote } & \multirow[t]{2}{*}{ Est } & & & & \\
\hline & & & & & - & + & - & + \\
\hline Project Management & $3,900,000$ & 1 & 25 & 75 & 5 & 20 & $0.05 i$ & 0.19 \\
\hline Sludies, Permitting & $12,050,000$ & 3 & 25 & 75 & 10 & 20 & $0.30 i$ & 0.59 \\
\hline NEPA & $3,250,000$ & 1 & 5 & 95 & 20 & 25 & 0.16 & 0.20 \\
\hline Technology Developement & $2.120,000$ & 0.52 & 5 & 95 & 10 & 25 & 0.05 & 0.13 \\
\hline Tille I and Title \| Design & $3,200,000$ & 1 & 25 & 75 & 10 & 15 & 0.08 & 0.12 \\
\hline Title III Inspection & $1,730,000$ & 0.43 & 25 & 75 & 5 & 20 & 0.02 & 0.09 \\
\hline Construction, Buildings \& Equipment & $34.070 .0 n c$ & 8 & 30 & 70 & 10 & 20 & 0.84 & 1.68 \\
\hline Construction Management & $7,499,000$ & 2 & 30 & 70 & 5 & 20 & 0.09 & 0.37 \\
\hline Startup and Readinees & $7,245,000$ & 2 & 25 & 75 & 10 & 15 & 0.18 & 0.27 \\
\hline Bullding Operation & $288,640,000$ & 71 & 5 & 95 & 20 & 25 & 14.23 & 17.79 \\
\hline Decontamination \& Decommissioning & 850,000 & 0 & 25 & 75 & 10 & 30 & 0.02 & 0.06 \\
\hline Closure/Post Closure & $37,701,000$ & 9 & 5 & 85 & 10 & 30 & 0.93 & 2.79 \\
\hline Management Reserve & $3,407,000$ & 1 & 50 & 50 & 5 & 30 & 0.04 & 0.25 \\
\hline Total & $405,662,000$ & 100 & & & 1 & & 16.99 & 24.53 \\
\hline
\end{tabular}


Rev 5-92

Page 2 of 5

Project EVALUATION OF ALTERNATIVES FOR DISPOSAL

OF INEL MIXED AND LOW LEVEL WASTE

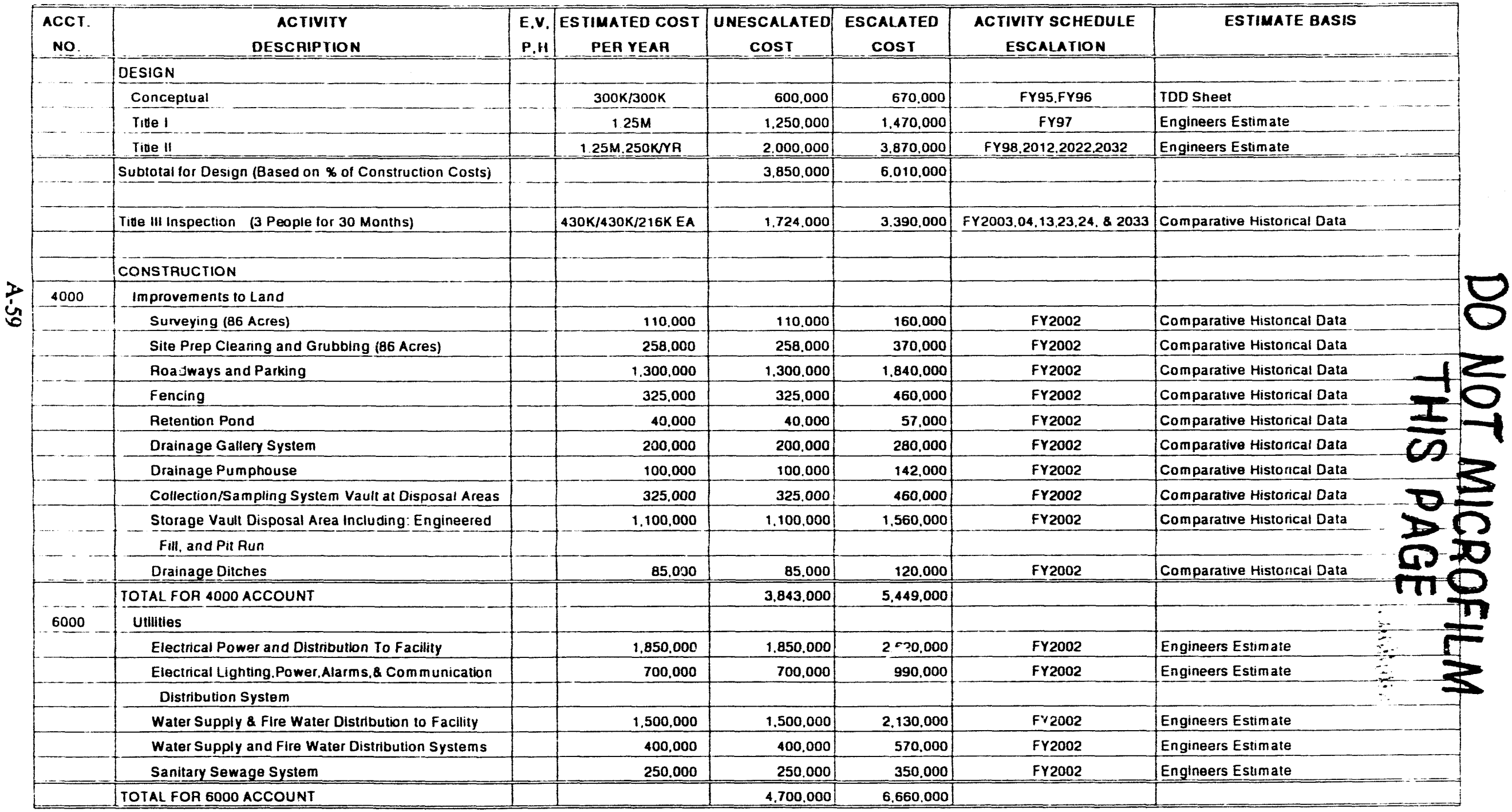


EG\&G Idaho. Inc. Rev 5-92

Page 3 of 5

\section{$\because 3$ PAGE} $\therefore$

DETAILED COST ESTIMATE (CONT.SHEET)

Type of Estimate: PLANNING

File No 5204 - Alt. 16

Date: 07/05/93

Project evaluation of alternatives for disposal OF INEL MIXED AND LOW LEVEL WASTE

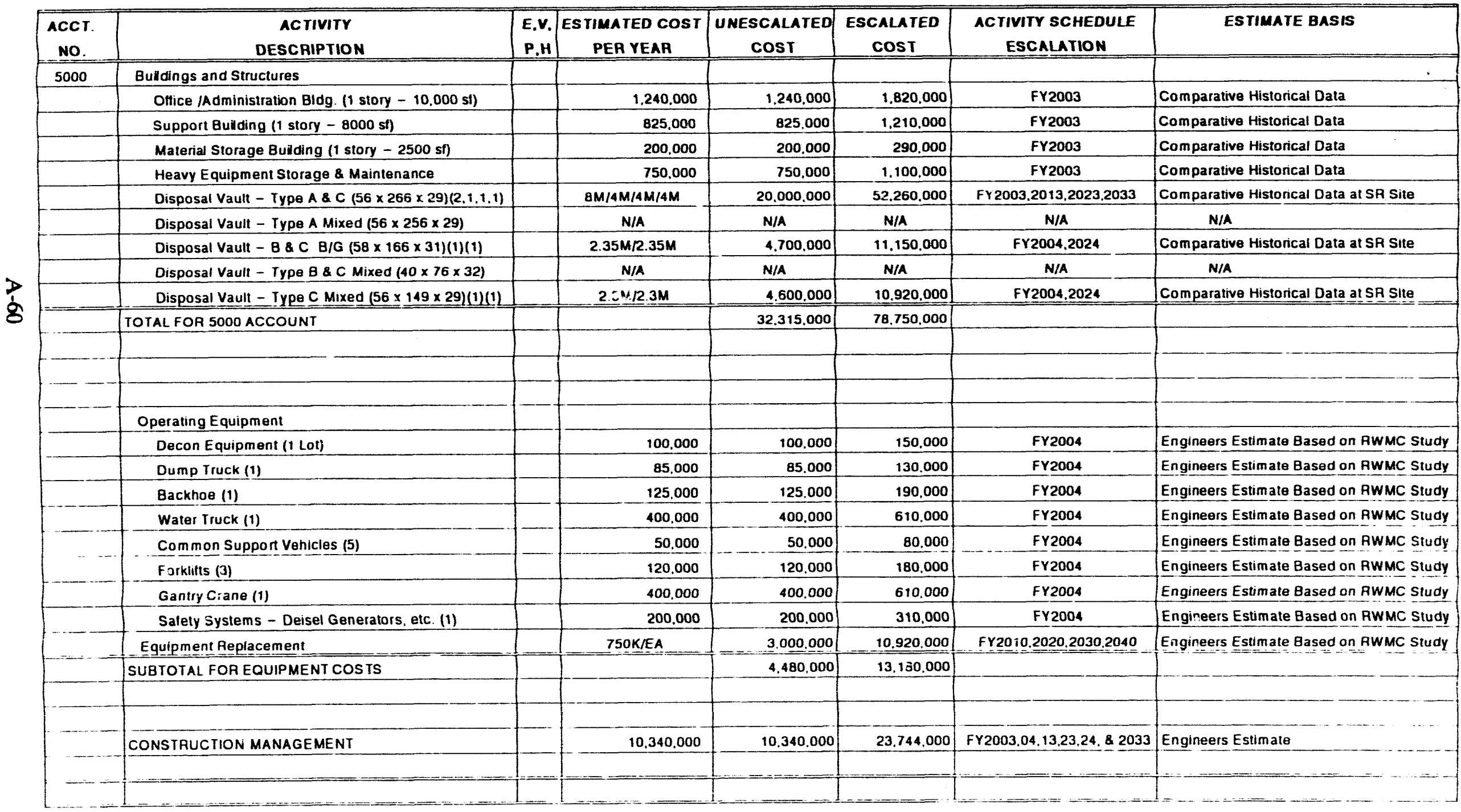




\section{COST ESTIMATE SUMMARY}

EG\&G Idaho, Inc.

Type of Estimate: PLANNING

Project: EVALUATION OF ALTERNATIVES FOR DISPOSAL OF INEL MIXED AND LOWLEVEL WASTE

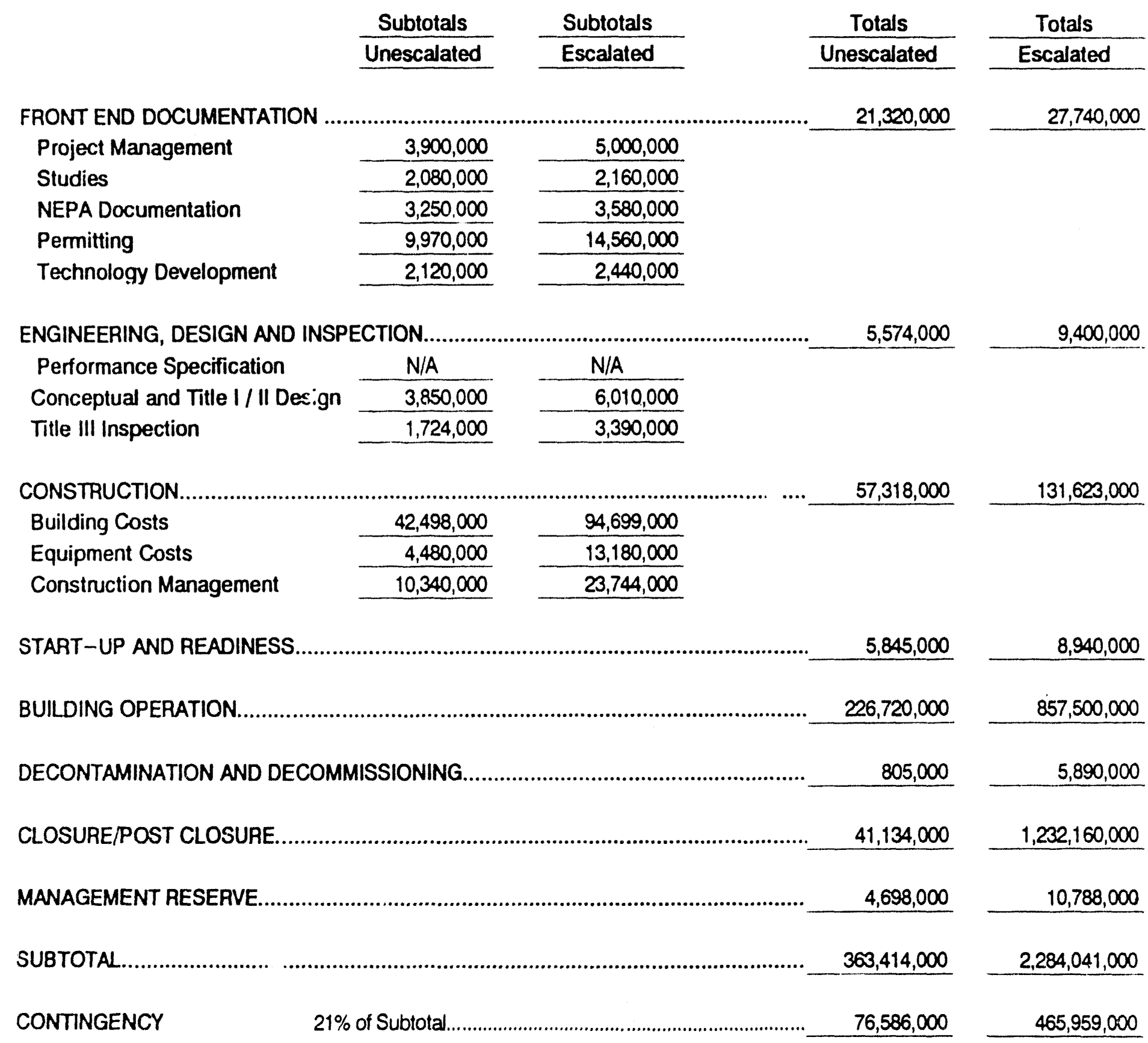

Date:

File No.

Appr'd By:

Prepared By:
07/05/93 5204 - Alt. 1G

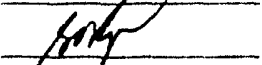

T.E. SIVILL

TOTAL PROJECT COST: 
Rev 5-92

Project

EVALUATION OF ALTERNATIVES FOR DISPOSAL OF INEL MIXED AND LOW LEVEL WASTE

Location INEL

Requesler T.H.SMITH

\begin{tabular}{|c|c|c|}
\hline $\begin{array}{l}\text { ACCT. } \\
\text { NO. }\end{array}$ & $\begin{array}{c}\text { ACTIVITY } \\
\text { DESCRIPTION } \\
\end{array}$ & $\begin{array}{l}\text { E.V. } \\
\text { P.H } \\
\end{array}$ \\
\hline & PROJECT COSTS & \\
\hline & Project Management & \\
\hline & Project Studies - 2 People. Full Time & \\
\hline & Project Formulation (F\&OR's) & \\
\hline & Siting Studies & \\
\hline & Pre-Operational Monitoring & \\
\hline & Satety Analysis Repon (SAR) & \\
\hline & Pertormance Assessment & \\
\hline & RCRA & \\
\hline & NEPA (EIS)(CX's) & \\
\hline & Air Permits (PSD/PTC/NESHAP) & \\
\hline & ESH8O & \\
\hline & Other Regulatory Items (State Water Well Approval) & \\
\hline & Speclat O/A Program For Construction - 1 Peison & \\
\hline & & \\
\hline & & \\
\hline ... & IECHNOLOGY DEVELOPMENT & \\
\hline & Material Behavior (Conc. Clay. etc.) & \\
\hline & Cover Performance Control & \\
\hline$-\ldots$ & Leachate Collection and Liner Performance & \\
\hline 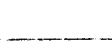 & Material Handing (Gantry Crane) & \\
\hline- & & \\
\hline & & \\
\hline & . & \\
\hline & $-\ldots-\ldots$ & \\
\hline & & \\
\hline & Subtotal for Front End Costs & \\
\hline
\end{tabular}

Type of Estimate PLANNING

Source (E) Eng Est.

(V) Vendor

(P) Pur. Order

(H) Handbook Ret

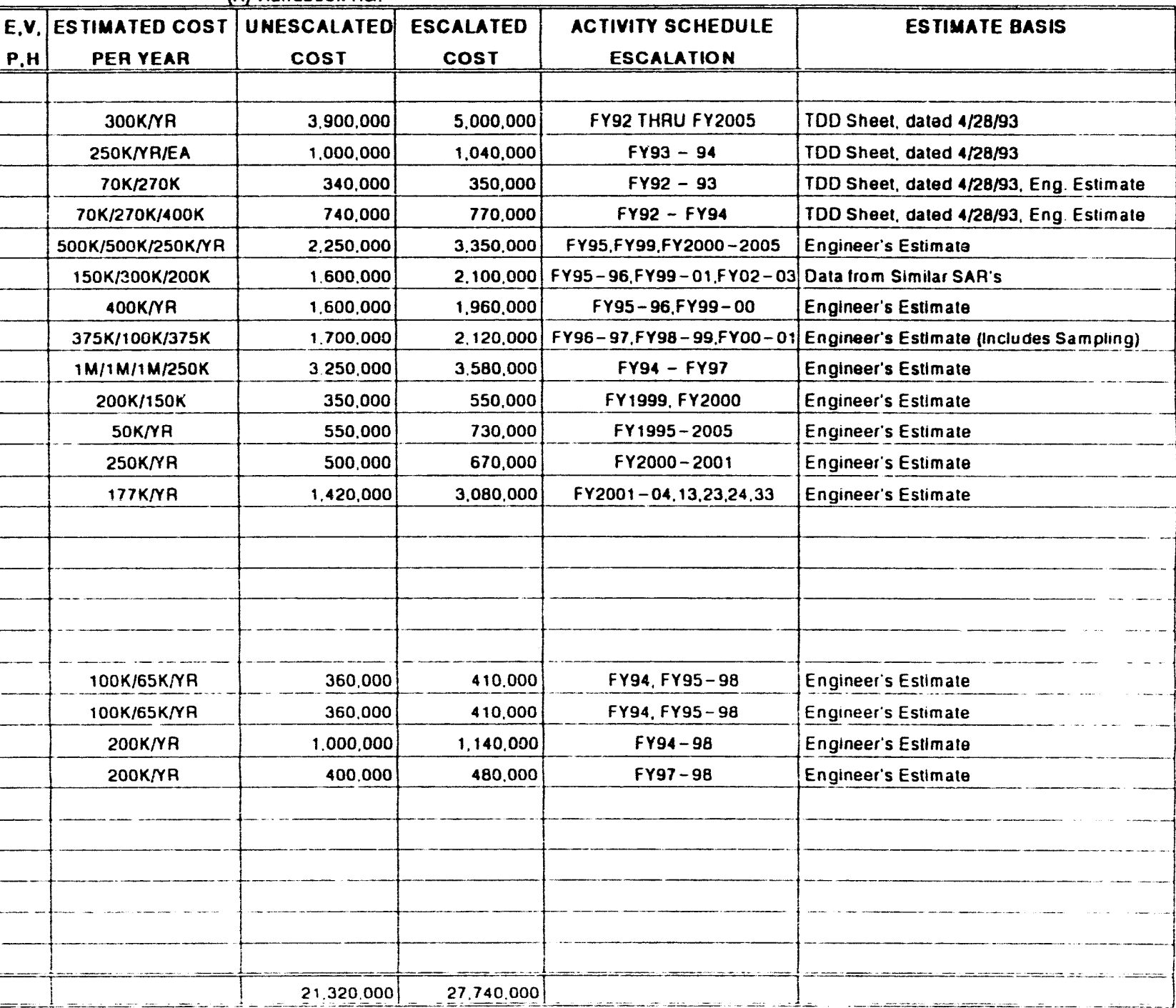

Date:

Prep'd By:

Chk'd By:

T.E.Sivill

$07 / 05 / 93$

Appr'd By:

4

(H) Handbook Ret. 
Proleci EVALUATION OF ALTERNATIVES FOR DISPOSAL

OF INEL MIXED AND LOW IEVEL WASTE

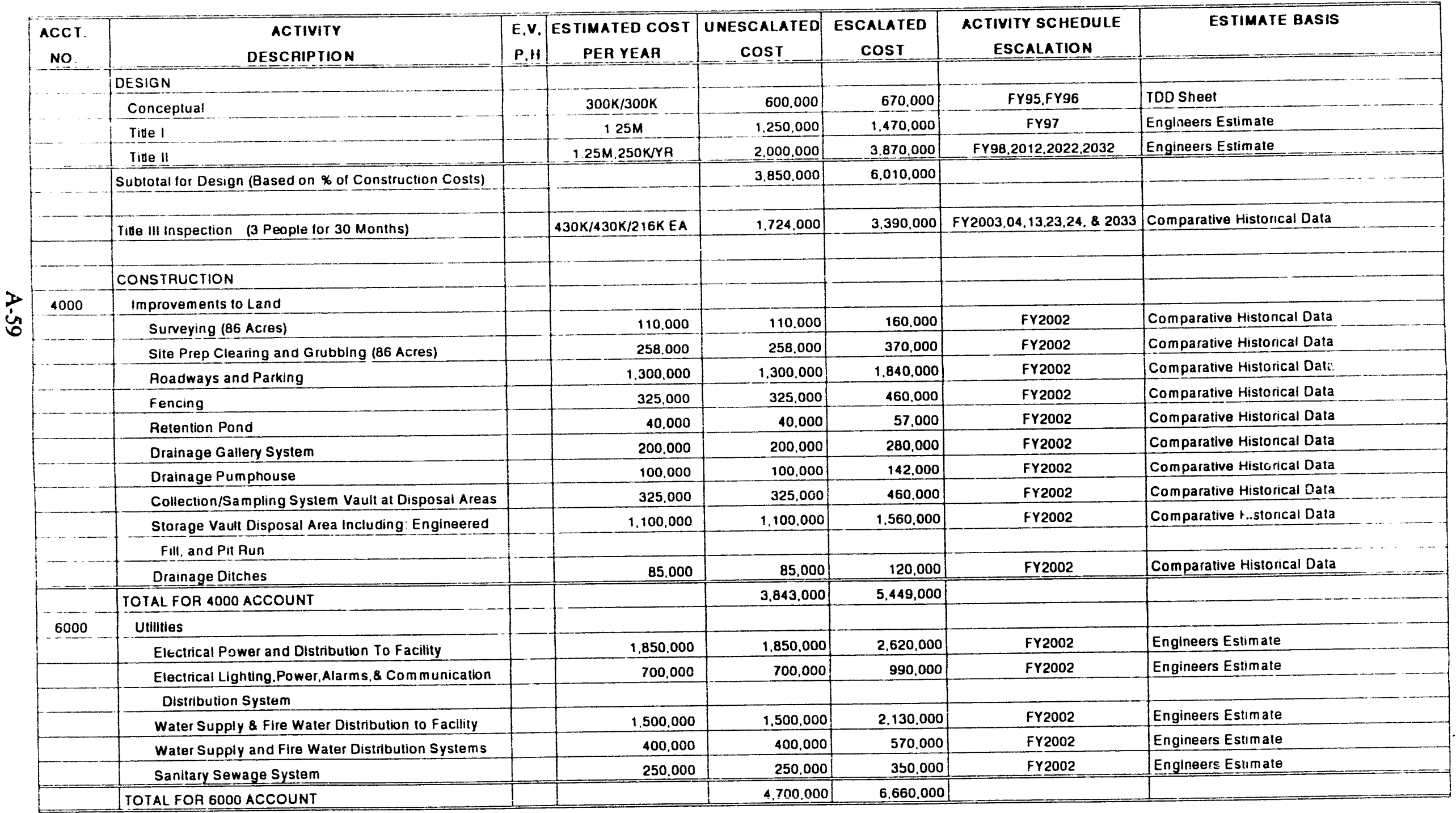


Project EVALUATION OF ALternatives for disposal OF INEL MIXED AND LOW LEVEL WASTE

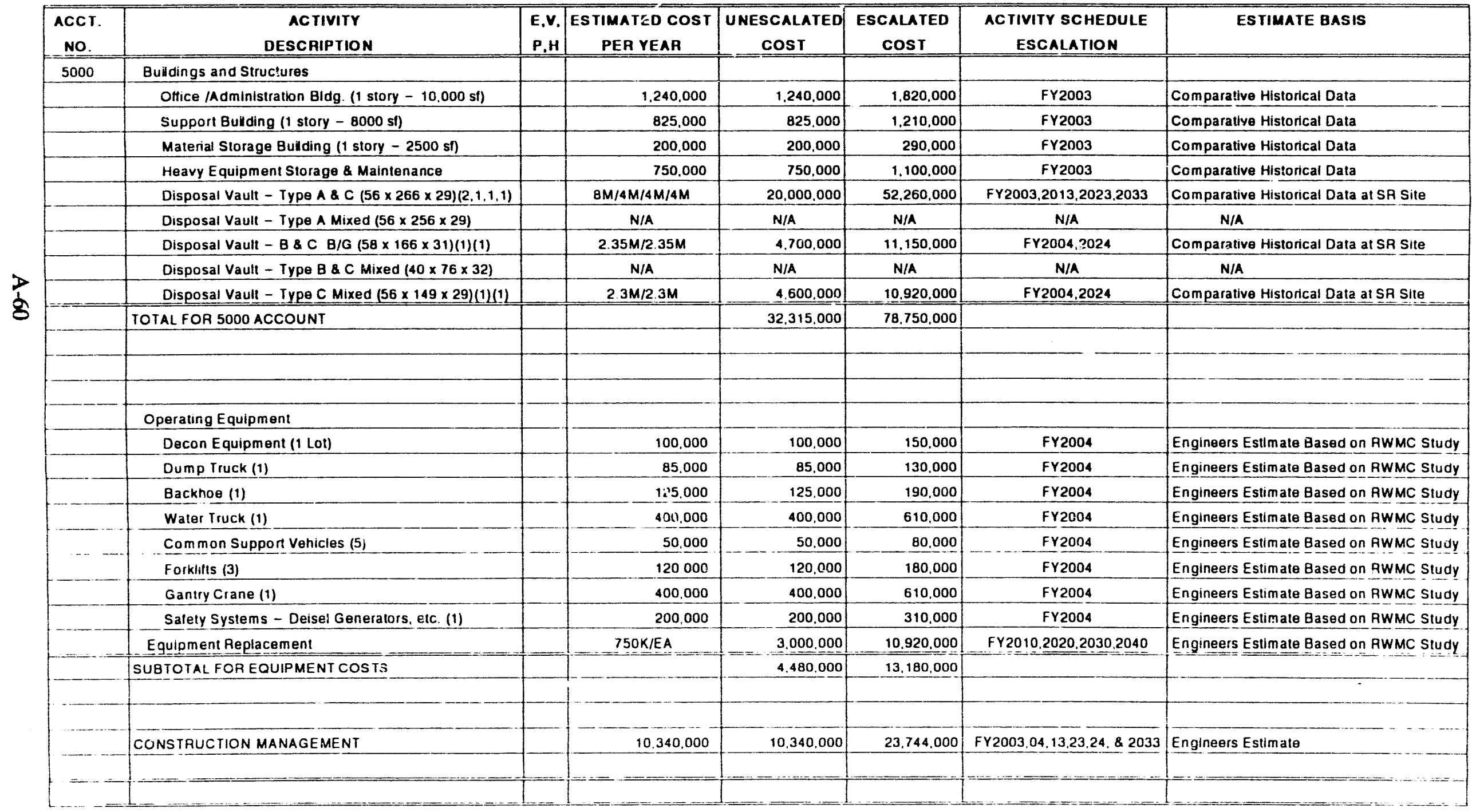


Rev 5-92

Page 4 of 5

Project EVALUATION OF ALTERNATIVES FOR DISPOSAL

OF INEL MIXED AND LOW LEVEL WASTE

\begin{tabular}{|c|c|c|c|c|c|c|c|}
\hline $\begin{array}{l}\text { ACCI. } \\
\text { NO. } \\
9000\end{array}$ & $\begin{array}{c}\text { ACTIVITY } \\
\text { DESCRIPIION } \\
\text { Oiner Direct Costs }\end{array}$ & $\begin{array}{l}\text { E.V. } \\
P .11\end{array}$ & $\begin{array}{c}\text { ESTIMATED COST } \\
\text { PER YEAR }\end{array}$ & $\begin{array}{c}\text { UNESCALATED } \\
\text { COST }\end{array}$ & $\begin{array}{c}\text { ESCALATED } \\
\text { COST }\end{array}$ & $\begin{array}{l}\text { AC TIVITY SCHEDULE } \\
\text { ESCALATION }\end{array}$ & ESTIMATE BASIS \\
\hline & Full Tıme Non Working Supervisors (5 tor 30 Months) & & $315 K / 315 K / 160 K E A$ & $1.430,000$ & $3.230,000$ & FY2002,03,04,13,23,24.33 & Engineers Estimate \\
\hline & Site Specific Training ( 35 People for $26 \mathrm{Hrs}$ Ea ) & & 35K/EA & 210.000 & 610.000 & $F Y 2002,03,04,13,23,24,33$ & Engineers Estimate \\
\hline$\ldots$ & 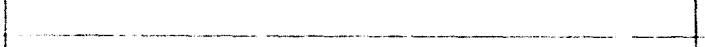 & & & & & & \\
\hline & TESTING/S IARTUP/PERSONNEL TRAINING (40PeOPIE) & & $140 \mathrm{~K} / \mathrm{YA}$ & 5.600 .000 & $8,550,000$ & FY2004 & Readiness Review Experience \\
\hline & READINESS REVIEW $(6$ People - 3 Months - 480 His ea & & $245 \mathrm{~K}$ & 245.000 & 390.000 & Fr2005 & Readiness Review Experience \\
\hline & & & & & & & \\
\hline & & & & & & & \\
\hline & OPERATING COSTS & & & & & & \\
\hline & Labor Based on $2080 \mathrm{Hrs}$ Per Year & & & & & & \\
\hline & Operating Personnel. 10 People & & $125 \mathrm{~K} / \mathrm{AP}$ & 50.000 .000 & $188.450,000$ & FY2005 THAU FY2045 & Engineers Estimate Based on RWMC Study \\
\hline & Mantenance Personnel, 6 People & & $120 \mathrm{~K} / \mathrm{YA}$ & $28.800,000$ & $108,540,000$ & FY2005 THAU FY2045 & Engineers Estimate Based on RWMC Siudy \\
\hline & Training Personnel. 2 People & & $96 \mathrm{~K} N \mathrm{R}$ & 7.680 .000 & $28,950,000$ & FY2005 THRU FY2045 & Engineers Estimate Based on PWMC Study \\
\hline & Recordkeeping. 2 People & & $78 \mathrm{KNA}$ & $6.240,000$ & $23,520,000$ & FY2005 THAU FY2045 & Engineers Estimate Based on RWMC Study \\
\hline & Management. 3 People & & $285 \mathrm{~K} / \mathrm{YA}$ & $34,200,000$ & $128,900,000$ & FY2005 THRU FY2045 & Engineers Estimate Based on RWMC Study \\
\hline & Engineering. 3 People & & $177 \mathrm{~K} / \mathrm{MA}$ & $21,240,000$ & $80,050,000$ & FY2005 THRU FY2045 & Englneers Estimate Based on RWMC Siudy \\
\hline & ESH\&O Personnet, 3 People & & $178 \mathrm{~K} / \mathrm{MA}$ & $21,360,000$ & 80.500 .000 & FY2005 THRU FY2045 & Engineers Estimale Based on RWMC Study \\
\hline & Budget Suppon Personnel. 3 People & & $115 \mathrm{~K} / \mathrm{NR}$ & 13.800 .000 & 52.010 .000 & FY2005 THRU FY2045 & Engineers Estimate Based on RWMC Study \\
\hline & Health Physics Personnel. 2 People & & $128 \mathrm{KNR}$ & 10.240 .000 & 38.590 .000 & FY2005 THRU FY2045 & Engineers Estimate Based on RWMC Sludy \\
\hline & Lab Personnel. 2 People & & $128 \mathrm{k} / \mathrm{NR}$ & $10,240,000$ & $38,590,000$ & FY2005 THRU FY2045 & Engineers Estimate Based on AWMC Study \\
\hline & Environmental Survelliance, 3 People & & $125 \mathrm{~K} / \mathrm{YR}$ & $15,000,000$ & $56,530,000$ & FY200S THRU FY2045 & Engineers Estimate Based on RWMC Study \\
\hline & Document Control, 1 Person & & $78 \mathrm{KNA}$ & $3,120,000$ & 11.760 .000 & FY2005 THRU FY2045 & Engineers Estimate Based on AWMC Study \\
\hline & & & & & & & \\
\hline & & & & & & & \\
\hline & & & & & & & \\
\hline & Equipment & & & & & & \\
\hline & Replacement Parts - Misc. Maintenance & & BOKNR & 3.200 .000 & $15,080,000$ & FY2005 THRU FY2045 & Engineers Estimate Based on AWMC Sludy \\
\hline & & & & & & & \\
\hline & Sublotal for Operating Costs & & & 225.120 .000 & 851.470 .000 & & \\
\hline
\end{tabular}


Rev 5-92

Page 5 of 5

Project EVALUation of alternatives for disposal OF INEL MIXED AND LOW LEVEL WASTE

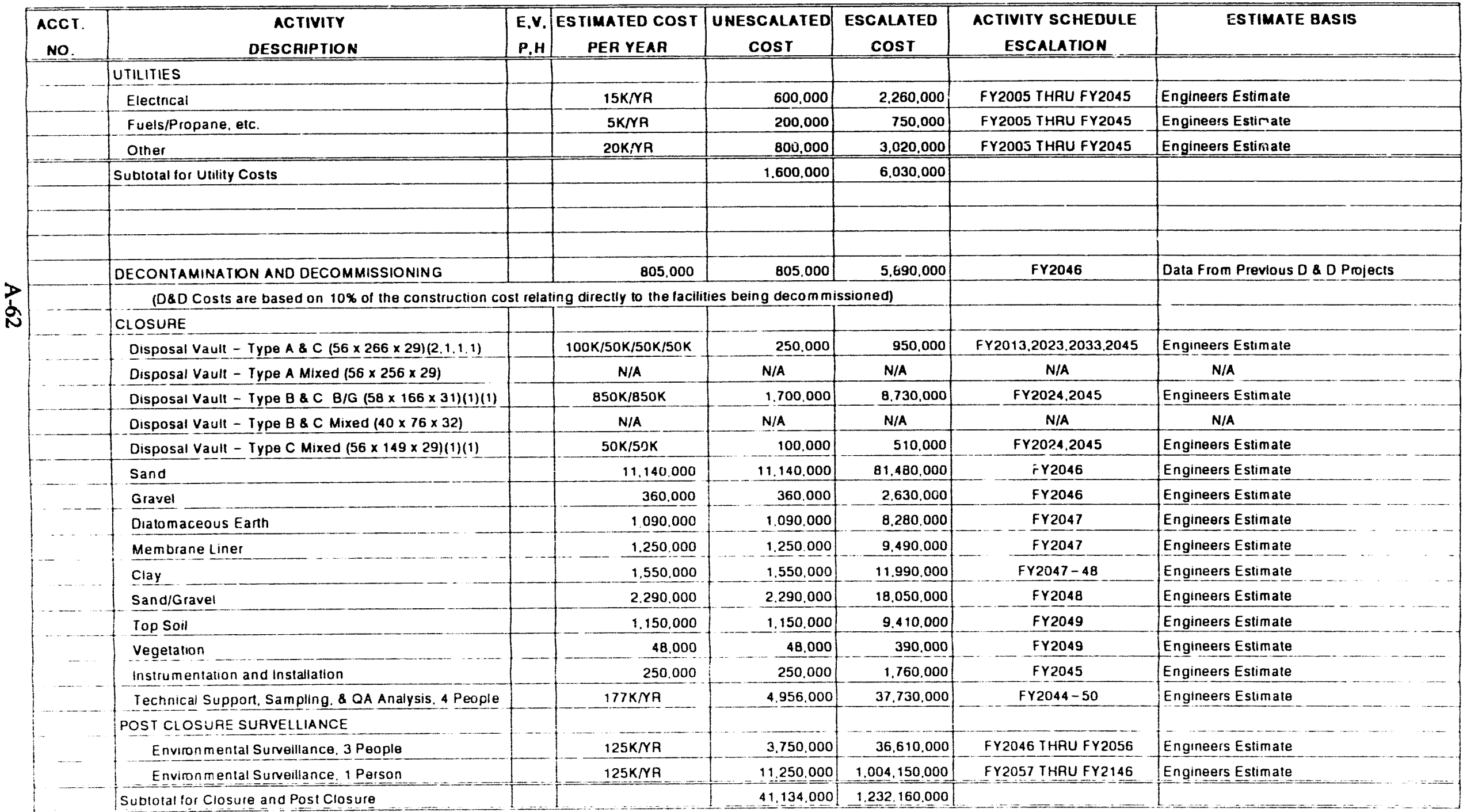


Projecl

Location

EVALUATION OF ALTERNATIVES FOA DISPOSAL

INEL

Requester
YPU of Est. PLANNING

Flle No. $5204-$ Alt. $1 G$
Date

Prep'd By

App'd By
$07 / 05 / 93$

TES

ferc

\begin{tabular}{|c|c|c|c|c|c|c|c|c|}
\hline \multicolumn{9}{|c|}{ PROBABLE $\%$ VARIATION } \\
\hline \multirow[t]{3}{*}{ Item } & \multirow[t]{3}{*}{ Cost } & \multirow{3}{*}{$\begin{array}{c}\% \\
\text { Total } \\
\text { Cosi }\end{array}$} & \multicolumn{2}{|l|}{ Est. Basis } & \multirow{2}{*}{\multicolumn{2}{|c|}{$\begin{array}{l}\text { Prob \% Var } \\
\text { From Est }\end{array}$}} & \multirow{2}{*}{\multicolumn{2}{|c|}{ Wt $\%$ of Prob }} \\
\hline & & & \multirow[t]{2}{*}{ Quote } & \multirow[t]{2}{*}{ Est } & & & & \\
\hline & & & & & - & + & - & + \\
\hline Project Management & $3,900,000$ & 1 & 25 & 75 & 5 & 20 & 0.05 & 0.21 \\
\hline Studies, Permitting & $12.050,000$ & 3 & 25 & 75 & 10 & 20 & 0.33 & 0.66 \\
\hline NEPA & 3.250 .000 & 11 & 5 & 95 & 20 & 25 & 0.18 & 0.22 \\
\hline Technology Developement & 2.120 .000 & 0.58 & 5) & 95 & 10 & 25 & 0.06 & 0.15 \\
\hline Tille I and Title II Design & $3,850.000$ & 1 & 25 & 75 & 10 & 15 & 0.11 & 0.16 \\
\hline Title III Inspection & 1.724 .000 & 0.47 & 25 & 75 & 5 & 20 & 0.02 & 0.09 \\
\hline Construction, Buildings \& Equipment & $46,978,000$ & 13 & 30 & 70 & 10 & 20 & 1.29 & 2.59 \\
\hline Construction Management & $10,340,000$ & 3 & 30 & 70 & 5 & 20 & 0.14 & 0.57 \\
\hline Slartup and Readiness & 5.845 .000 & 2 & 25 & 75 & 10 & 15 & 0.16 & 0.24 \\
\hline Bullding Operation & $226,720,000$ & 62 & 5 & 95 & 20 & 25 & 12.48 & 15.60 \\
\hline Decontamination \& Decommissioning & 805.000 & 0 & 25 & 75 & 10 & 30 & 0.02 & 0.07 \\
\hline CiosurelPosiclosure & $41,134,000$ & 11 & 5 & 95 & 10 & 30 & 1.13 & 3.40 \\
\hline Managemenl Reserve & 4.698 .000 & 11 & 50 & 50 & 5 & 30 & 0.06 & 0.39 \\
\hline Tolal & 363.414 .000 & 1001 & & & & & 16.04 & 24.34 \\
\hline
\end{tabular}


EG\&G Idaho, Inc.

COST ESTIMATE SUMNARY

Type of Estimate: PLANNING

Date:

File No.

Project: EVALUATION OF ALTERNATIVES FOR DISPOSAL

OF INEL MIXED AND LOWLEVEL WASTE

Appr'd By:

Prepared By: T.E. SIVRL

Subtotals
Unescalated

Totals

Unescalated

FRONT END DOCUMENTATION

Project Management

Studies

NEPA Documentation

Permitting

Technology Development

\begin{tabular}{|c|c|}
\hline $3,900,000$ & $5,000,000$ \\
\hline $2,080,000$ & $2,160,000$ \\
\hline $3,250,000$ & $3,580,000$ \\
\hline $11,380,000$ & $17,640,000$ \\
\hline $2,120,000$ & $2,440,000$ \\
\hline
\end{tabular}

ENGINEERING, DESIGN AND INSPECTION.

Performance Specification Conceptual and Title I / II Design

Title III Inspection

\begin{tabular}{rl}
$\frac{N}{11,500,000}$ & $\frac{N / A}{16,610,000}$ \\
\hline $4,600,000$ & $10,900,000$ \\
\hline
\end{tabular}

CONSTRUCTION

Building Costs

Equipment Costs

Construction Management

\begin{tabular}{rr}
$219,900,000$ & $567,589,000$ \\
\hline $4,600,000$ & $13,370,000$ \\
\hline $49,412,000$ & $127,869,000$ \\
\hline
\end{tabular}

$22,730,000$ $30,820,000$

START-UP AND READINESS.

BUILDING OPERATION.

$350,560,000$

$1,321,240,000$

DECONTAMINATION AND DECOMMISSIONING.

$1,200,000$

$8,780,000$

CLOSURE/POST CLOSURE.

$112,546,000$

$1,755,060,000$

MANAGEMENT RESERVE

$22,450,000$

$58,096,000$

SUBTOTAL

$808,423,000$

$3,923,974,000$

CONTINGENCY

$20 \%$ of Subtotal.

$161,577,000$

$776,026,000$

TOTAL PROJECT COST: $\$ 970,000,000$

Comments: 
Rev 5-92

Project EVALUATICN OF ALTERNATIVES FOR DISPOSAL OF INEL MIXED AND LOW LEVEL WASTE

Location INEL

Requester I.H.SMITH
Iype of Estimate PLANNING

Source (E) Eng. Est

(V) Vendor

(P) Pur. Order

(H) Handbook Re
Flle No. $5204-$ Alt. $1 \mathrm{H}$

Date:

Chk'd By:

Appr'd By:

\begin{tabular}{|c|c|}
\hline \multirow[t]{2}{*}{$\begin{array}{l}\text { ACCI. } \\
\text { No. }\end{array}$} & $\begin{array}{c}\text { AC TIVITY } \\
\text { DESCAIPTION }\end{array}$ \\
\hline & PROJECT COSTS \\
\hline & Project Management \\
\hline & Project Studies - 2 People. Full Time \\
\hline & Project Formulation (F\&OR's) \\
\hline & Sitting Sludies \\
\hline & Pre-Operational Monitoring \\
\hline & Safety Analysis Report (SAR) \\
\hline & Pertormance Assessment \\
\hline & RCRA \\
\hline & NEPA (EIS) (CX's) \\
\hline & Air Permits (P O/PTC/NESHAP) \\
\hline & ESHBO \\
\hline & Other Regulatory Items (State Water Well Approval) \\
\hline & Special O/A Program For Construction - 2 People \\
\hline & \\
\hline & \\
\hline & IECHNOLOGY DEVELOPMENT \\
\hline & Materlal Behavior (Conc. Clay, elc.) \\
\hline & Cover Performance Control \\
\hline & Leachate Collection and Liner Pefiormance \\
\hline & Material Handling (Gantry Crane) \\
\hline & \\
\hline & \\
\hline & \\
\hline & \\
\hline & \\
\hline & Subtotal for Front End Costs \\
\hline
\end{tabular}

E,V. ES TIMATED COST UNESCALATED P.V. PER YEAR
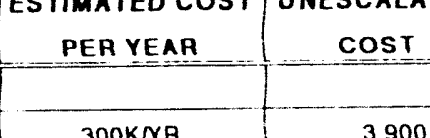

$300 K N A$

250K/NR/EA

$70 K 1270 K$

$70 \mathrm{~K} / 270 \mathrm{~K} / 400 \mathrm{~K}$

$500 \mathrm{~K} / 500 \mathrm{~K} / 250 \mathrm{~K} / \mathrm{NR}$

$150 \mathrm{~K} / 300 \mathrm{~K} / 200 \mathrm{~K}$

$$
400 \mathrm{~K} / \mathrm{VR}
$$

$375 K / 100 K / 375 K$

$1 \mathrm{M} / 1 \mathrm{M} / 1 \mathrm{M} / 250 \mathrm{~K}$

200K/150K

$50 K N \mathrm{NR}$

$250 K / Y R$

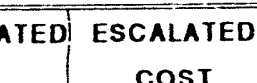

Cost

\begin{tabular}{r|r|}
\hline 3.900 .000 & 5.000 .000 \\
\hline
\end{tabular}

\begin{tabular}{r|r|r|}
\hline 1.000 .000 & 1.040 .000 & FY92 THRU FY2005 \\
\hline FY93 -94 & FY92 \\
\hline
\end{tabular}

\begin{tabular}{r|r|}
340.000 & 350.000 \\
\hline 740.000 & \\
\hline
\end{tabular}

740.000

770,600

\begin{tabular}{|r|r|c|l|}
\hline 2.250 .000 & 3.350 .000 & FY95.FY99,FY2000-2005 & Engineer's Estimate \\
\hline 1.600 .000 & 2.100 .000 & FY95-96.FY99-01.FY02-03 & Data from Similar SAR's
\end{tabular}

FY92 - FY94

ACTIVITY SCHEDULE

ESTIMATE BASIS

\begin{tabular}{ll|l|l}
1.600 .000 & 1.960 .000 & FY95-96.FY99-00 & Engineer's Estimate
\end{tabular}

\begin{tabular}{ll|l}
$1.700,000$ & $2.120,000$ & FY96-97,FY98-99.FY00-01 Engineer's Estimate (Includes Sa mpling)
\end{tabular}

\begin{tabular}{r|r|r|r|}
\hline 3.250 .000 & 3.580 .000 & FY94 - FY97 & Engineer's Estimate \\
\hline
\end{tabular}

\begin{tabular}{l|l|l}
550.000 & FY1999, FY2000 & Engineer's Estimate
\end{tabular}

\begin{tabular}{r|r|c|l}
\hline 350,000 & 550,000 & FY1999, FY2000 & Engineer's Estimate \\
\hline 550,000 & 730,000 & FY1995-2005 & Engineer's Estimate \\
\hline
\end{tabular}

\begin{tabular}{|l|l|l|l}
500.000 & 670.000 & FY2000-2001 & Engineer's Estimate
\end{tabular}

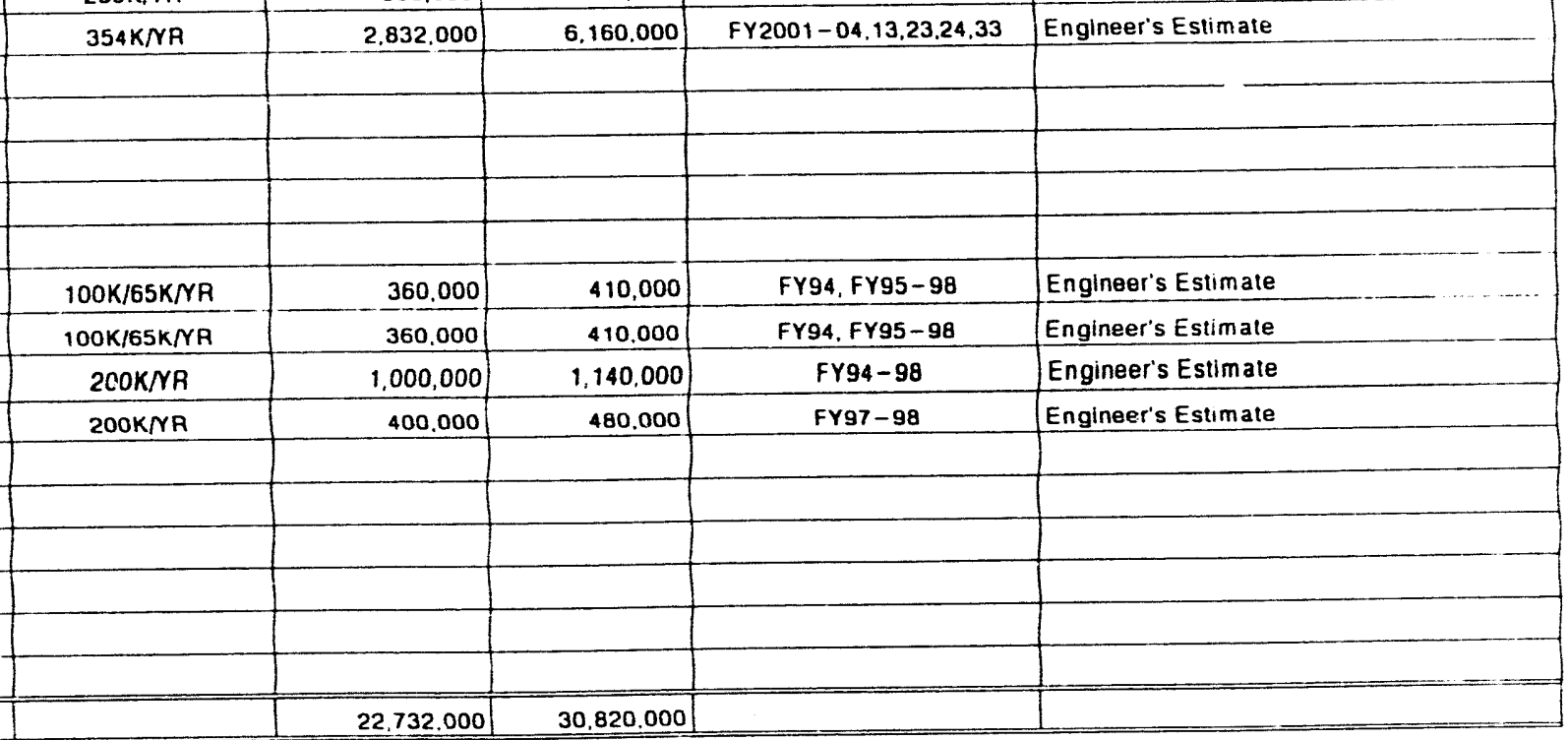


Rev 5-92

Page 2 of 5

Project eValuation of alternatives for disposal OF INEL MIXED AND LOW LEVEL WASTE

\begin{tabular}{|c|c|c|c|c|c|c|c|}
\hline $\begin{array}{l}\text { ACCI. } \\
\text { No. } \\
\end{array}$ & $\begin{array}{c}\text { ACTIVITY } \\
\text { DESCAIPIION } \\
\end{array}$ & $\begin{array}{l}E . V . \\
P . H \\
\end{array}$ & $\begin{array}{c}\text { ESTIMATED COST } \\
\text { PER YEAR }\end{array}$ & $\begin{array}{c}\text { UNESCALATED } \\
\text { COST }\end{array}$ & $\begin{array}{c}\text { ESCALATED } \\
\text { COST } \\
\end{array}$ & $\begin{array}{c}\text { ACTIVITY SCHEDULE } \\
\text { ESCALATION } \\
\end{array}$ & ESTIMATE BASIS \\
\hline & DESIGN & & & & & & \\
\hline & Conceptual & & $500 \mathrm{~K} / 500 \mathrm{~K}$ & $1,000,000$ & $1,110,000$ & FY95.FY96 & TDD Sneet \\
\hline & Tive 1 & & $4 M$ & $4,000,000$ & 4.700 .000 & FY97 & Engineers Estimate \\
\hline & Tive II & & 5M.500KMR & 6.500 .000 & 10.800 .000 & FY98.2012.2022.2032 & Engineers Estimate \\
\hline & Subtotal for Design (Based on $x$ of Construction Costs) & & & 11.500 .000 & 16.610 .000 & & \\
\hline & & & & & & & \\
\hline & Tive III Inspection (8 People for 30 Months) & & $1.15 \mathrm{M} / 1.15 \mathrm{M} / 575 \mathrm{KEA}$ & 4.600 .000 & $10,900.000$ & FY2003.04.13.23.24.8.2033 & Comparative Historical Data \\
\hline & CONSTAUCTION & & & & & & \\
\hline 4000 & Improvements to Land & & & & & & \\
\hline & Surveying (166 Acres) & & 215.000 & 215.000 & 300.000 & FY2002 & Comparative Historical Data \\
\hline & Site Prep Clearing and Grubbing (166 Acres) & & 500.000 & 500.000 & 710.000 & FY2002 & Comparative Histoncal Data \\
\hline & Roadways and Parking & & $1,600,000$ & 1.600 .000 & $2.340,000$ & FY2002 & Comparative Historical Data \\
\hline & Fencing & & 500.000 & 500,000 & 710.000 & FY2002 & Comparative Historical Data \\
\hline & Retention Pond & & 40.000 & 40.000 & 57.000 & FY2002 & Comparative Historical Data \\
\hline & Drainage Gallery System & & $1.000,000$ & 1.000 .000 & 1.420 .000 & FY2002 & Comparative Historical Data \\
\hline & Drainage Pumphouse & & 100.000 & 100,000 & 142.000 & FY2002 & Comparative Historical Data \\
\hline & Collection/Sampling System Vault @ Disposal Areas & & 1.800 .000 & 1.800 .000 & 2.550 .000 & FY2002 & Comparative Historical Data \\
\hline & Storage Vault Disposal Area Including: Engineered & & $3.000,000$ & 3.000 .000 & 4.250 .000 & FY2002 & Comparative Historical Data \\
\hline & Fill. and Pit Run & & & & & & \\
\hline & Drainage Ditches & & 135,000 & 135.000 & 190.000 & Fr2002 & Comparative Historical Data \\
\hline & IOIAL FOR 4000 ACCOUNT & & & $8.890,000$ & 12.669 .000 & & \\
\hline 6000 & Utilities & & & & & & \\
\hline & Electrical Power and Distribution To Facility & & 1.850 .000 & 1.850 .000 & $2,620,000$ & FY2002 & Engineers Estlmate \\
\hline & Electrical Lighting, Power Alarms, \& Communication & & 2.580 .000 & $2.580,000$ & 3.660 .000 & FY2002 & Engineers Estimate \\
\hline & Distribution System & & & & & & \\
\hline & Water Supply \& Fire Water Disiribution to Facility & & 1.500 .000 & $1,500,000$ & $2,130,000$ & FY2002 & Engineers Estimate \\
\hline & Water Supply and Fire Water Distribution Systems & & 650.000 & 650,000 & 920.000 & FY2002 & Engineers Estimate \\
\hline & Sanitary Sewage System & & 250.000 & 250.000 & 350,000 & FY2002 & Engineers Estimate \\
\hline & TOTAL FOP 6000 ACCOUNT & & & 6.830 .000 & 9.680 .000 & & \\
\hline
\end{tabular}


Page 3 of 5

Prolect EVALUATION OF AL IERNATIVES FOR DISPOSAL OF INEL MIXED AND LOW LEVEL WASTE

\begin{tabular}{|c|c|c|c|c|c|c|c|}
\hline $\begin{array}{l}\text { ACCT. } \\
\text { NO. }\end{array}$ & $\begin{array}{c}\text { ACTIVITY } \\
\text { DESCRIPTION }\end{array}$ & $\begin{array}{l}\text { E.V. } \\
\text { P.H }\end{array}$ & $\begin{array}{c}\text { ESTIMATED COST } \\
\text { PER YEAR }\end{array}$ & $\begin{array}{c}\text { UNESCALATED } \\
\text { COST }\end{array}$ & $\begin{array}{c}\text { ESCALATED } \\
\text { COST } \\
\end{array}$ & $\begin{array}{l}\text { ACTIVITY SCHEDULE } \\
\text { ESCALATION }\end{array}$ & ESTIMATE BASIS \\
\hline \multirow[t]{4}{*}{5000} & Buildings and Structures & & & & & & \\
\hline & Office /Administration B!dg (1 story - $12.500 \mathrm{st})$ & & 1.550 .000 & $1.550,000$ & 2.280 .000 & FY2CO3 & Comparative Historical Data \\
\hline & Supporl Building (1 story - 10.000 si) & & $1.000,000$ & 1.000 .000 & 1.470 .000 & FY2003 & Comparative Historical Data \\
\hline & Material Storage Building (1 story -3000 sf) & & 240.000 & 240,000 & 350.000 & FY2003 & Comparative Historical Data \\
\hline \multirow{3}{*}{$\ldots$} & Heavy Equip Storage 8 Maintenance $(10.000$ si) & & 950.000 & 950.000 & $1.400,000$ & FY2003 & Comparative Historical Data \\
\hline & Disposal Vauit - A \&C $(56 \times 266 \times 29)(10.10 .10,10)$ & & $40 \mathrm{M} / 40 \mathrm{M} / 40 \mathrm{M} / 40 \mathrm{M}$ & 160.000 .000 & 448.510 .000 & FY2003,2013,2023,2033 & Comparative Historical Data at SR Site \\
\hline & Disposal Vault - Type A Mixed $(56 \times 256 \times 29)(2,3.2)$ & & $77 \mathrm{M} / 11.6 \mathrm{M} / 7 \mathrm{M}$ & 27.000 .000 & 59.990 .000 & FY2003,2013,2023 & Comparative Historical Data at SR Site \\
\hline \multirow{2}{*}{$\ldots$} & Disposal Vault - B8CB/G $(58 \times 166 \times 31)(1)(1)$ & & $235 \mathrm{M} / 2.35 \mathrm{M}$ & 4.700 .000 & 11.150 .000 & FY2004.2024 & Comparative Historical Data at SR Site \\
\hline & Disposal Vault - Type B 8 C Mixed $(40 \times 76 \times 32)(1)$ & & 1.000 .000 & $1,000,000$ & 1.530 .000 & FY2004 & Comparative Historical Data at SA Site \\
\hline \multirow{3}{*}{$=-\cdots+\cdots$} & Disposal Vault - Type C Mixed (56 $\times 149 \times 29)(1)(1)$ & & $23 \mathrm{M} / 2.3 \mathrm{M}$ & 4.600 .000 & 10.920 .000 & Fr2004.2024 & Comparative Historical Data at SR Site \\
\hline & TOTAL FOR 5000 ACCOUNT & & & 201.040 .000 & $53 ? .600 .000$ & & \\
\hline & & & & & & & \\
\hline \multirow{3}{*}{ 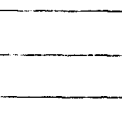 } & & & & & & & \\
\hline & & & & & & & \\
\hline & Operating Equipment & & & & & & \\
\hline \multirow{2}{*}{ 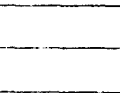 } & Decon Equipment (1 Lot) & & 100.000 & 100,000 & 150.000 & FY2004 & Engineers Estimate Based on RWMC Study \\
\hline & Dump Truck (1) & & 85.000 & 85.000 & 130,000 & FY2004 & Engineers Estimate Based on RWMC Study \\
\hline \multirow{3}{*}{$\ldots$} & Backhoe (1) & & 125.000 & 125,000 & 190.000 & FY2004 & Engineers Estlmate Based on AWMC Study \\
\hline & Water Truck (1) & & 400.000 & 400,000 & 610,000 & FY2004 & Engineers Estimate Based on AWMC Sludy \\
\hline & Common Support Vehicles (5) & & 50.000 & 50.000 & 80.000 & FY2004 & Engineers Estumate Based on RWMC Study \\
\hline \multirow[t]{2}{*}{$\ldots$} & Forklifts (6) & & 240.000 & 240.000 & 370,000 & FY2004 & Engineers Estimate Based on RWMC Study \\
\hline & Gantry Crane (1) & & 400.000 & 400.000 & 610.000 & FY2004 & Engineers Estimate Based on RWMC Study \\
\hline \multirow{2}{*}{$\ldots$} & Safety Systems - Deisel Generators, etc. (1) & & 200,000 & 200,000 & 310.000 & FY2004 & Engineers Estimate Based on RWMC Study \\
\hline & Equipment Replacement & & 750K/EA & 3.000 .000 & 10.920 .000 & FY2010.2020.2030.2040 & Engineers Estimate Based on RWMC Study \\
\hline 2 & SUBTOIAL FOR EQUIPMENT COSTS & & & $4.600,000$ & $13,370,000$ & & \\
\hline \multirow{3}{*}{$\ldots$} & & & & & & & \\
\hline & & & & & & & \\
\hline & CONSTRUCTION MANAGEMENT & & 49.412 .000 & 49.412 .000 & $127,869,000$ & FY2003,04,13,23,24,8,2033 & Engineers Estimate \\
\hline \multirow[t]{2}{*}{$=$} & & & & & & & \\
\hline & & & & & & & \\
\hline
\end{tabular}


Page 4 of 5

Project evaluation of alternatives for disposal OF INEL MIXED AND LOW LEVEL WASTE

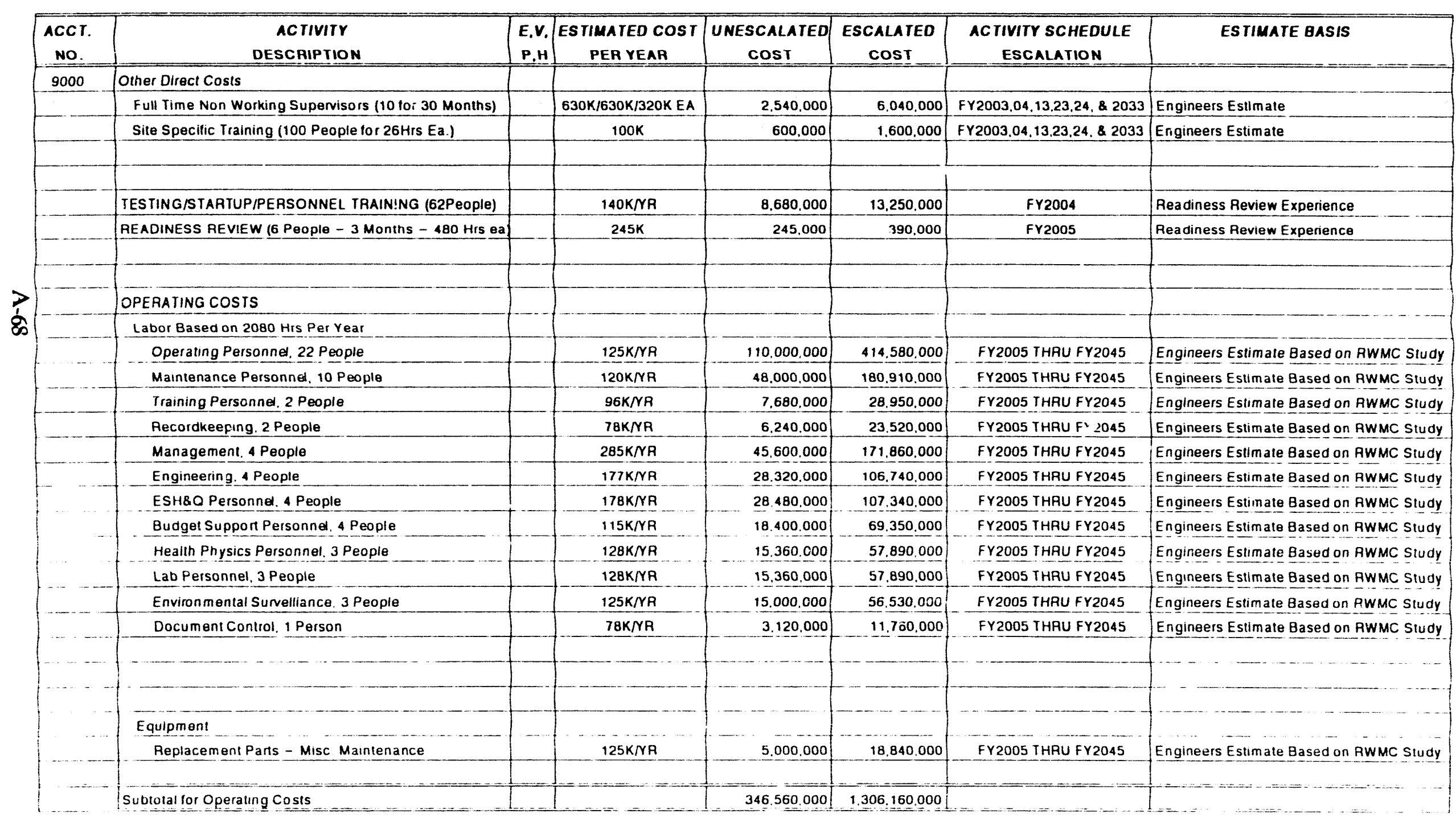


Prolect EVALUATION OF ALTERNATIVES FOR DISPOSAL OF INEL MIXEO AND LOW LEVEL WASTE

\begin{tabular}{|c|c|c|c|c|c|c|c|}
\hline $\begin{array}{l}\text { ACCI. } \\
\text { No. }\end{array}$ & $\begin{array}{c}\text { ACIIVITY } \\
\text { DESCAIPIION }\end{array}$ & $\begin{array}{l}E . V . \\
P . M\end{array}$ & $\begin{array}{c}\text { ESTIMATED COST } \\
\text { PEA YEAR }\end{array}$ & $\begin{array}{c}\text { UNESCALATED } \\
\text { COSI }\end{array}$ & $\begin{array}{c}\text { ESCALATED } \\
\text { COST }\end{array}$ & $\begin{array}{l}\text { ACTIVITY SCHEDULE } \\
\text { ESCALAIION }\end{array}$ & ESTIMATE BASIS \\
\hline & UTILITIES & & & & & & \\
\hline & Electrical & & 40K/VA & $1.600,000$ & $6,030,000$ & FY2005 THRU FY2045 & Engineers Estimate \\
\hline & Fuels/Propane etc & & 10KNR & 400.000 & 1.510 .000 & FY2005 THRU FY2045 & Engineers Estimate \\
\hline$\ldots=$ & Other & & SOKMR & 2.000 .000 & 7.540 .000 & FY2005 THAU FY2045 & Engineers Estimate \\
\hline & Subiotal for Utility Cosis & & & $4,000,000$ & $15,080.000$ & & \\
\hline & & & & & & & \\
\hline & & & & & & & \\
\hline & DECONTAMINATION AND DECOMMISSIONING & & & & & & \\
\hline & (D\&D Costs are based on $10 \%$ of the construction co & st relati & \begin{tabular}{|c|}
$1,200.000$ \\
ing directly to the faciliti
\end{tabular} & 1.200 .000 & 8.780 .000 & FY2046 & Data From Previous D \& D Projects \\
\hline & CLOSURE & & ing directly to the faciliti & ies being decommis & issioned) & & \\
\hline & Disposal Vault - A \&C $(56 \times 266 \times 29)(10,10,10.10)$ & & & & & & \\
\hline & Disposal Vault - Type A Mixed $(56 \times 256 \times 29)(2,3,2)$ & & $\begin{array}{c}500 \mathrm{~K} / 500 \mathrm{~K} / 500 \mathrm{~K} / 500 \mathrm{~K} \\
100 \mathrm{~K} / 150 \mathrm{~K} / 100 \mathrm{~K} \\
\end{array}$ & 2.000 .000 & $8,390,000$ & FY2013,2023.2033.2045 & Engineers Estimale \\
\hline & Disposal Vault - Type B \& C B/G $(58 \times 166 \times 31)(1)(1)$ & & $\frac{100 K / 150 K / 100 K}{850 K / 850 K}$ & 350.000 & $1,380,000$ & FY2013,2023,2033,2045 & Engineers Estimate \\
\hline & Disposal Vault - Type $88 \mathrm{C}$ Mixed $(40 \times 76 \times 32)(1)$ & & $\frac{850 K / 850 K}{850 K}$ & 1.700 .000 & $8,730,000$ & FY20 4.2045 & Engineers Estimale \\
\hline & Disposal Vaull - Type C Mixed $(56 \times 149 \times 29)(1)(1)$ & & $\begin{array}{c}850 \mathrm{~K} \\
50 \mathrm{~K} / 50 \mathrm{~K} \\
\end{array}$ & 850,000 & $5.990,000$ & FY'OU45 & Engineers Estimate \\
\hline & Sand & & $\frac{50 K / 50 K}{63.790 .000}$ & 100,000 & 510,000 & FY2024,2045 & Engineers Estimate \\
\hline & Gravel & & $\begin{array}{r}63.790 .000 \\
1.120 .000 \\
\end{array}$ & 63.790 .000 & $466,550,000$ & FY2046 & Engineers Estimate \\
\hline & Dlatomaceous Earth & & $\begin{array}{r}1,120,000 \\
3,370,000 \\
\end{array}$ & $1,120.000$ & $8,190,000$ & FY2046 & Engineers Estimate \\
\hline & Membrane Liner & & $\frac{3,370,000}{3,810,000}$ & 3.370 .000 & $25.580,000$ & FY2047 & Engineers Estimate \\
\hline & Ciay & & $\frac{3.810,000}{4.740,000}$ & 3.810 .000 & $28,920,000$ & FY2047 & Engineers Estimate \\
\hline & Sand/Gravel & & $\begin{array}{l}4,740,000 \\
6,900,000\end{array}$ & $4.740,000$ & $36,670,000$ & FY2047-48 & Engineers Estimate \\
\hline & Top Soil & & $\begin{array}{r}6,900,000 \\
3,470,000 \\
\end{array}$ & 6.900 .000 & $54,370,000$ & FY2048 & Engineers Estimate \\
\hline & Vegetation & & $\frac{3.470,000}{140,000}$ & $3.470,000$ & $28,380,000$ & FY2049 & Engineers Estimate \\
\hline & Instrumentation and Installation & & $\begin{array}{r}140.000 \\
250.000 \\
\end{array}$ & 140,000 & 1.150 .000 & FY2049 & Engineers Estimate \\
\hline & Technical Suppon, Sampling. 2 OA Analysis, 4 People & & $\frac{250.000}{177 \mathrm{~K} / \mathrm{KR}}$ & 250.000 & $1.760,000$ & FY2045 & Engineers Estimate \\
\hline & POST CLOSURE SURVELLIANCE & & $177 \mathrm{~K} / \mathrm{KR}$ & $4,956.000$ & 37.730 .000 & FY2044-50 & Engineers Estimate \\
\hline & Environmental Surveillance, 3 People & & & & & & \\
\hline & Environmental Surveillance, 1 Person & & $\begin{array}{l}125 \mathrm{~K} / \mathrm{NA} \\
125 \mathrm{~K} / \mathrm{KA} \\
\end{array}$ & 3.750 .000 & $36,610,000$ & FY2046 THRU FY2056 & Engineers Estimate \\
\hline & Subtotal for Closure and Post Closure & & $125 \mathrm{KMAR}$ & 11.250 .000 & $1.004,150,000$ & FY2057 THRU FY2146 & Engineers Estimate \\
\hline & & & & $112.546,000$ & 1.755 .060 .000 & & \\
\hline
\end{tabular}


EG\&G loano, Inc

CONTINGENCY ANALYSIS

Rev 5-92

Project EVALUATION OF ALTERNATIVES FOR DISPOSAL TyPe of Est. PLANNING

Location INEL

Flle No.

$5204-$ Alt. $1 \mathrm{H}$

Date

Prep'd By $07 / 05 / 93$

Aequester T. H. SMITH

App'd By

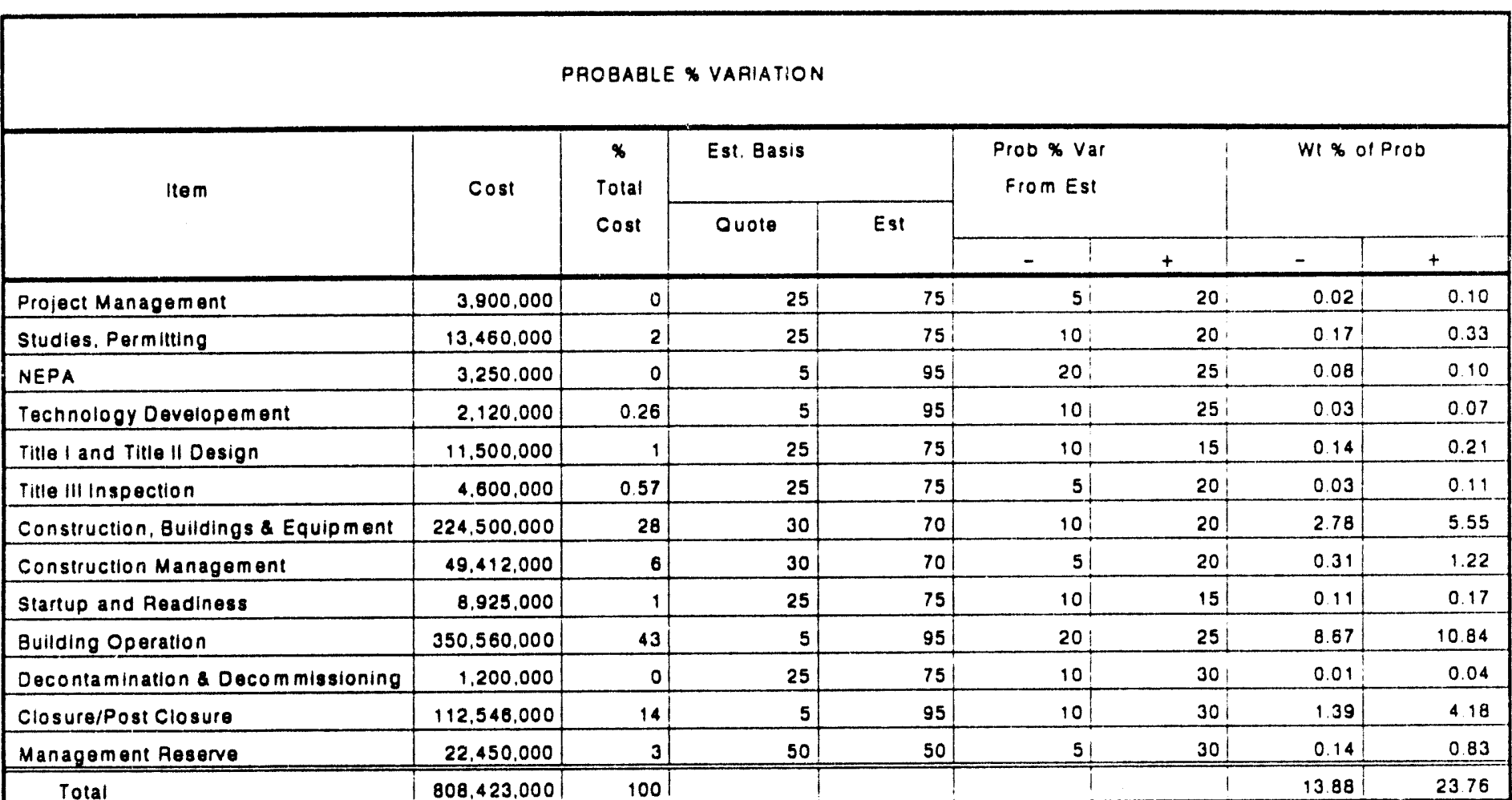




\section{COST ESTIMATE SUMMARY}

EG\&G Idaho, Inc.

Type of Estimate: PLANNING

Date:

07/05/93

Project: EVALUATION OF ALTERNATIVES FOR DISPOSAL OF INEL MIXED AND LOW LEVEL WASTE

File No. $5204-$ Alt. 2

Appr'd By:

Prepared By:

D. N. Stoddard

$\frac{\text { Totals }}{\text { Unescalated }}$

$12,900,000$

FRONT END DOCUMENTATION

Project Management

Studies

NEPA Documentation

Permitting

Technology Development
Subtotals

Escalated

\begin{tabular}{|c|c|}
\hline $3,300,000$ & $3,900,000$ \\
\hline $1,410.000$ & $1,464,000$ \\
\hline $1,750,000$ & $1,930,000$ \\
\hline $4,750,000$ & $6,170,000$ \\
\hline $1,690,000$ & $1,920,000$ \\
\hline
\end{tabular}

ENGINEERING, DESIGN AND INSPECTION

$3,860,000$

Performance Specification

\begin{tabular}{r} 
N/A \\
\hline $2,600,000$ \\
$\frac{N}{1.260,000}$
\end{tabular}

Title III Inspection $1.260,000$

$2,880,000$

CONSTRUCTION

Building Costs

Equipment Costs

Construction Management

$9,480,000$
$4,544,000$$\frac{70,955,000}{13,270,000}$

$7,489,000$

$18,538,000$

START-UP AND READINESS

$7,204,000$

BUILDING OPERATION.

$289,360,000$

DECONTAMINATION AND DECOMMISSIONING.

630,000

CLOSURE/POST CLOSURE

MANAGEMENT RESERVE.

$3,402,000$

$8,423,000$

SUBTOTAL.

$422,649,000$

$2,599,437,000$

CONTINGENCY

$21 \%$ of Subtotal

$87,351,000$

$540,563,000$

TOTAL PROJECT COST:

$=\$ 510,000,000$

$\$ 3,140,000,000$

Comments: 
EVALUATION OF ALTERNATIVES FOR DISPOSAL OF INEL MIXED AND LOW LEVEL WASTE

Projec INEL

H. SMITH
Type of Estimale PLANNING

Source (E) Eng. Est.

$M$ Vendor

(P) Pur. Order

(H) Handbook Ret

\begin{tabular}{|c|c|c|c|c|c|c|c|}
\hline $\begin{array}{l}\text { ACCT. } \\
\text { No. }\end{array}$ & $\begin{array}{c}\text { ACTIVTY } \\
\text { DESCPEPTION } \\
\end{array}$ & $\begin{array}{l}\text { E.V. } \\
\text { P.H }\end{array}$ & $\begin{array}{c}\text { ESTIMATED COST } \\
\text { PEA YEAR }\end{array}$ & $\begin{array}{c}\text { UNESCALATED } \\
\text { COST }\end{array}$ & $\begin{array}{c}\text { ESCALATED } \\
\text { COST } \\
\end{array}$ & $\begin{array}{l}\text { ACTIVITY SCHEDULE } \\
\text { ESCALATION }\end{array}$ & ESTMATE BASIS \\
\hline & PROJECT COSTS & & & & & & \\
\hline & Projecı Managemen & & 300KYYR & 3.300 .000 & 3.900 .000 & FY92 THRU FY2002 & TDO Sheet, dated 4/28/93 \\
\hline & Project Studies - 2 People. Full Time & & 250KYRIEA & 1.000 .000 & 1.040 .000 & FY93 - 94 & TDD Sheet, dated 4/28/93 \\
\hline & Project Formulation (F\&OR's) & & $70 \mathrm{~K} / 270 \mathrm{~K}$ & 340,000 & 350.000 & FY92 - 93 & TDD Shoet, dated 4/28/93, Eng. Estimate \\
\hline & Siting Studies (1/2 FTE) & & 70K & 70,000 & 74.000 & FY94 & Engineer's Estimate \\
\hline & Pre-Operational Monitoring & & - & - & - & & \\
\hline & Satety Analysis Repon (SAR) - Modifying existing & & $100 \mathrm{~K} / 200 \mathrm{~K} / 100 \mathrm{~K}$ & 1.000 .000 & 1.300 .000 & FY95-96.FY99-01.FY02-03 & Data from Similar SAR's \\
\hline & Pertorman - Assessment - Modify existing & & $200 \mathrm{~K} Y \mathrm{R}$ & 800.000 & 960.000 & FY95-96,FY99-00 & Engineer's Estimate \\
\hline & RCRA & & $375 \mathrm{~K} / 100 \mathrm{~K} / 375 \mathrm{~K}$ & 1.700 .000 & $2,120.000$ & FY96-97.FY98-99.FYOO-01 & Engineer's Estimate (Includes Sampling) \\
\hline & NEPA (EIS) (CX's) & & $500 \mathrm{~K} / 500 \mathrm{~K} / 50 \mathrm{~K} / 250 \mathrm{~K}$ & 1.750 .000 & 1.930 .000 & FY94 - FY97 & Engineer's Estimate \\
\hline & Air Permits (PSD/PTC/NESHAP) & & $200 \mathrm{~K} / 150 \mathrm{~K}$ & 350.000 & 550.000 & FY1999, FY2000 & Engineer's Estimate \\
\hline & ESH\&O & & SOKNR & 550,000 & 730,000 & FY1995-2005 & Engineer's Estimate \\
\hline & Other Regulatory ltems (State Water Well Approval) & & - & - & - & & \\
\hline & Special Q/A Program For Construction - 1 Person & & 177KYYR & 354.000 & 510.000 & FY2002, FY2003 & Engineer's Estimate \\
\hline & & & & & & & \\
\hline & & & & & & & \\
\hline & & & & & & & \\
\hline & & & & & & & \\
\hline & TECHNOLOGY DEVELOPMENT & & & & & & \\
\hline & Material Behavior (Clay, etc.) & & 65KYR & 330.000 & 370.000 & FY94-98 & Engineer's Estimate \\
\hline & Cover Performance Control & & $100 \mathrm{~K} / 65 \mathrm{~K} / \mathrm{YR}$ & 360.000 & 410.000 & FY94, FY95-98 & Engineer's Estimate \\
\hline & Leachate Collection and Liner Pertormance & & $200 \mathrm{KNA}$ & 1.000 .000 & 1.140 .000 & FY94-98 & Engineer's Estimate \\
\hline & Material Handling (Boom Crane) & & - & - & - & & \\
\hline & & & & & & & \\
\hline & & & & & & & \\
\hline & & & & & & & -.... \\
\hline & & & & & & & $\ldots$ \\
\hline & & & & & & & $\cdots$ \\
\hline$=$ & Sublotal for Front End Costs & & & 12.904 .000 & 15.384 .000 & & \\
\hline
\end{tabular}

Date: $\quad 07 / 05 / 93$

Prep'd By: D. N. Stoddard

Chicd By:

Appr'd By:

File No. 5204 - Alt. $2 \quad$ Date: $\frac{07 / 05 / 93}{\text { Prep'd By: D. N. Stoddard }}$

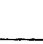


Project EVALUATION OF ALTERNATVES FOR DISPOSAL OF INEL MIXEO AND LOW LEVEL WASTE

\begin{tabular}{|c|c|c|c|c|c|c|c|}
\hline $\begin{array}{l}\text { ACCT. } \\
\text { NO. }\end{array}$ & $\begin{array}{c}\text { ACTIVITY } \\
\text { DESCRPTION }\end{array}$ & $\begin{array}{l}\text { E.V. } \\
\text { P.H }\end{array}$ & $\begin{array}{l}\text { ESTIMATED COST } \\
\text { PER YEAR }\end{array}$ & $\begin{array}{c}\text { UNESCALATED } \\
\text { COST }\end{array}$ & $\begin{array}{l}\text { ESCALATED } \\
\text { COST }\end{array}$ & $\begin{array}{l}\text { ACTIVITY SCHEDULE } \\
\text { ESCALATION }\end{array}$ & ESTIMATE BASIS \\
\hline & DESIGN & & & & & & \\
\hline & Conceptual & & $300 \mathrm{~K} / 300 \mathrm{~K}$ & 600.000 & 670.000 & FY95.FY96 & TDD Sheet \\
\hline- & Title 1 & & $1 M$ & 1.000 .000 & $1.180,000$ & FY97 & Engineers Estimate \\
\hline & Tille II & & $1 M$ & 1.000 .000 & 1.220 .000 & FY98 & Engineers Estimate \\
\hline & Subtotal for Design (Based on $\%$ of Construction Costs) & & & 2.600 .000 & 3.070 .000 & & \\
\hline & Title IIII Inspection (3 People for 18 Months) & & $420 \mathrm{~K} / 210 \mathrm{~K}$ & $1,260.000$ & $2,880.000$ & FY2002, 2003.23, 24 & Comparative Historical Data \\
\hline & & & & & & & \\
\hline & CONSTRUCTION & & & & & & \\
\hline 4000 & Improvements to Land & & & & & & \\
\hline & Surveying (20 acres) & & 26.000 & 26.000 & 37.000 & FY2002 & Comparative Historical Daia \\
\hline & Site Prep Clearing and Grubbing (20 Acres) & & 60.000 & 60.000 & 85.000 & FY2002 & Comparative Historical Data. \\
\hline & Roadways minimal service and Parking existing & & 750.000 & 750.000 & 1.063 .000 & FY2002 & Comparative Historical Data \\
\hline & Fencing. minimal & & 100.000 & 100.000 & 142.000 & FY2002 & Comparative Historical Data \\
\hline & Retention Pond & & - & - & - & & \\
\hline & Drainage Gallery Systern & & 1.000 .000 & 1.000 .000 & 1.420 .000 & FY2002 & Comparative Historical Data \\
\hline & Drainage Pumphouse & & 160.000 & 160.000 & 227,000 & FY2002 & Comparative Historical Data \\
\hline & Collection/Sampling System Vauh at Disposal Areas & & 400.000 & 400.000 & 567.000 & FY2002 & Comparative Historical Data \\
\hline & Storage Vaull Disposal Area Including: Engineered & & - & - & $=$ & & \\
\hline & Fill. Pit Run & & & & & & \\
\hline & Drainage Ditch/Berm Around Pads (10,500 if) & & 178.000 & 178.000 & 252.000 & FY2002 & Comparative Historical Data \\
\hline & TOTAL FOR 4000 ACCOUNT & & & 2.674 .000 & $3.793,000$ & & \\
\hline 6000 & Utilities & & & & & & \\
\hline & Electrical Power and Distribution to Site & & - & - & - & & \\
\hline & Electrical. Senvice to Disposal Area and Minimal & & 650.000 & 650.000 & 922.000 & FY2002 & Engineers Estimate \\
\hline & Upgrades Only & & & & & & \\
\hline & Water Supply \& Fire Water Distribution to Facility & & - & - & - & & \\
\hline & Water Supply and Fire Water Distribution Systems & & 150,000 & 150.000 & $2.130,000$ & FY2002 & Engineers Estimate \\
\hline & Sanitary Sewage System & & - & - & - & & \\
\hline & TOTAL FOR 6000 ACCOUNT & & & 800.000 & 3.052 .000 & & \\
\hline
\end{tabular}


Project EVALUATION OF ALTERnATVES FOR DISPOSAL OF INEL MIXED AND LOW LEVEL WASTE

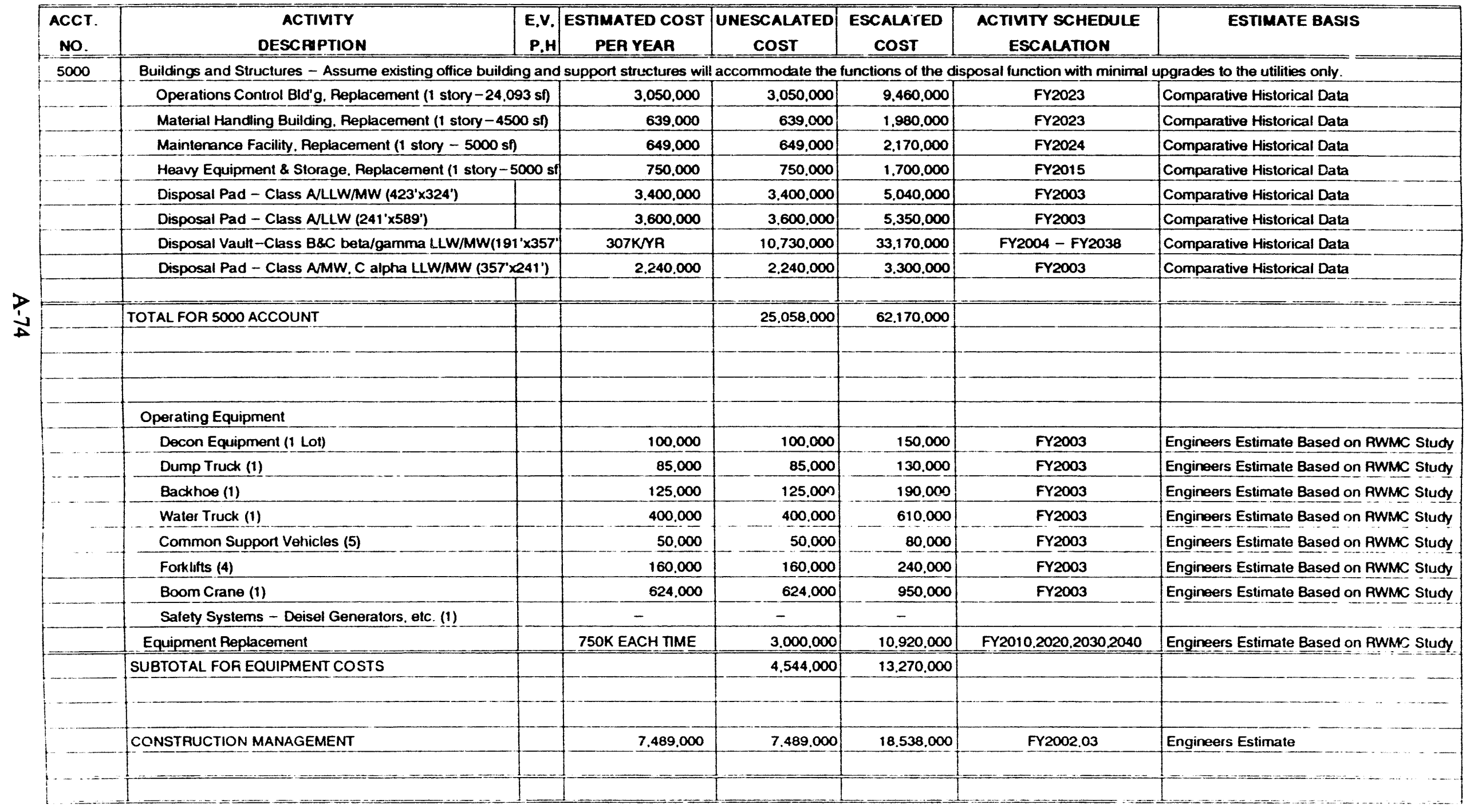


Project EVALUATION OF ALTEANATIVES FOR DISPOSAL OF INEL MIXED AND LOW LEVEL WASTE

\begin{tabular}{|c|c|c|c|c|c|c|c|}
\hline $\begin{array}{c}\text { ACCT. } \\
\text { NO. }\end{array}$ & $\begin{array}{c}\text { ACTIVITY } \\
\text { DESCPIPTION }\end{array}$ & E.V. & $\begin{array}{l}\text { ESTIMATED COST } \\
\text { PER YEAR }\end{array}$ & $\begin{array}{c}\text { UNESCALATED } \\
\text { COST }\end{array}$ & $\begin{array}{l}\text { ESCALATED } \\
\text { COST }\end{array}$ & $\begin{array}{l}\text { ACTIVITY SCHEDULE } \\
\text { ESCALATION }\end{array}$ & ESTIMATE BASIS \\
\hline \multirow[t]{6}{*}{9000} & Other Direct Costs & & & & & & \\
\hline & Full Time Non Working Supervisors (3 for 18 Months) & & $220 \mathrm{~K} / 110 \mathrm{~K} E \mathrm{~A}$ & 660.000 & 1.500 .000 & FY2002, 03, 23.24 & Engineers Estimate \\
\hline & Site Specific Training (48 People lor $26 \mathrm{Hrs}$ Sa.) & & 48KEA & 288.000 & 440.000 & $\mathrm{FY} 2002.03 .23 .24$ & Engineers Estimate \\
\hline & $-\ldots$ & & & & $\ldots$ & & $-\ldots \ldots$ \\
\hline & TESTING/STARTUP/PERSONNEL TRANING (50 People) & & $140 \mathrm{KNR}$ & 7.000 .000 & 9.900 .000 & FY2003 & Readiness Review Experience \\
\hline & READNESS REVEW ( 5 People -3 Months $-480 \mathrm{Hrs}$ ea) & & $204 \mathrm{~K}$ & 204.000 & 312.000 & FY2004 & Readiness Review Experience \\
\hline & & & & & & & \\
\hline \multirow{3}{*}{$\ldots$} & & & & & & & \\
\hline & OPERAMNG COSTS & & & & & & \\
\hline & Labor Based on 2080 Hrs Per Year & & & & & & \\
\hline & Operating Personnel. 15 People & & $125 \mathrm{~K} Y \mathrm{R}$ & 75.000 .000 & $282.670,000$ & FY2005 THRU FY2045 & Engineers Estimate Based on RWMC Study \\
\hline & Mainterance Personnel, 6 People & & 120KYR & 29.520 .000 & 108.545 .000 & FY2005 THRU FY2045 & Engineers Estimate Based on RWMC Study \\
\hline & Training Personnel, 2 People & & $96 \mathrm{KVR}$ & $7,680,000$ & 28.950 .000 & FY2005 THRU FY2045 & Engineers Estimate Based on RWMC Study \\
\hline & Recordkeeping. 2 People & & $78 \mathrm{KYR}$ & $6,240,000$ & $23,520.000$ & FY2005 THRU FY2045 & Engineers Estimate Based on RWMC Study \\
\hline & Management. 4 People & & 285KNR & 45.600 .000 & 171.860 .000 & FY2005 THRU FY2045 & Engineers Estimate Based on RWMC Study \\
\hline & Engineering. 4 People & & $177 \mathrm{KNR}$ & 28.320 .000 & 106.740 .000 & FY2005 THRU FY2045 & Engineers Estimate Based on RWMC Study \\
\hline & ESH\&O Personnel. 4 People & & 178KNR & 28.480 .000 & 107.340 .000 & FY2005 THRU FY2045 & Engineers Estimate Based on RWMC Study \\
\hline & Budget Support Personnel. 4 People & & $115 \mathrm{KNR}$ & 18.400 .000 & $69.350,000$ & FY2005 THRU FY2045 & Engineers Estimate Based on RWMC Stuc, \\
\hline & Health Physics Personnel, 2 People & & $128 \mathrm{kYR}$ & 10.240 .000 & 38.590 .000 & FY2005 THRU FY2045 & Engineers Estimate Based on RWMC Siudy \\
\hline & Lab Personnel, 3 People & & $128 \mathrm{~K} Y \mathrm{R}$ & $15.360,000$ & $57,890.000$ & FY2005 THAU FY2045 & Engineers Estimate Based on RWMC Study \\
\hline & Environmental Survelliance, 3 People & & $125 \mathrm{KNYR}$ & 15.000 .000 & 56.530 .000 & FY2005 THRU FY2045 & Engineers Estimate Based on RWMC Siudy \\
\hline & Document Control. 1 Person & & 78KYR & 3.120 .000 & 11.760 .000 & FY2005 THRU FY2045 & Engineers Estimate Based on RWMC Study \\
\hline & & & & & & & \\
\hline & & & & & & & \\
\hline & & & & & & & 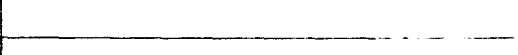 \\
\hline & Equipment & & & & & & \\
\hline - - - & Replacement Parts - Misc. Maintenance & & $100 \mathrm{KNR}$ & $4,000,000$ & 15.080 .000 & FY2005 THRU FY2045 & Engineers Estimate Based on RWMC Study \\
\hline & Subtotal for Operating Costs & & & 285060000 & $1070005 \mathrm{~mm}$ & & \\
\hline
\end{tabular}


Projoct EVALUATION OF ALTERNATIVES For DISPOSAL

OF INEL MIXED AND LOW LEVEL WASTE

\begin{tabular}{|c|c|c|c|c|c|c|c|}
\hline $\begin{array}{l}\text { ACCT. } \\
\text { No. }\end{array}$ & $\begin{array}{c}\text { ACTIVTY } \\
\text { DESCFIPTION }\end{array}$ & $\begin{array}{l}\text { E.V. } \\
\text { P.H }\end{array}$ & $\begin{array}{c}\text { ESTIMATED COST } \\
\text { PER YEAR }\end{array}$ & $\begin{array}{c}\text { UNESCALATED } \\
\text { COST }\end{array}$ & $\begin{array}{c}\text { ESCALATED } \\
\text { COST } \\
\end{array}$ & $\begin{array}{l}\text { ACTIVITY SCHEDULE } \\
\text { ESCALATION }\end{array}$ & ESTIMATE BASIS \\
\hline & UTILITIES & & & & & & \\
\hline & Electrical & & $25 \mathrm{KYN}$ & 1.000 .000 & 3.770 .000 & FY2005 THRU FY2045 & Engineers Estimate \\
\hline & Fuels/Propane. etc. & & SKYR & 200.000 & 750.000 & FY2005 THRU FY2045 & Engineers Estimate \\
\hline & Other & & 30KYR & 1.200 .000 & 4.520 .000 & FY2005 THRU FY2045 & Engineers Estimate \\
\hline \multirow{5}{*}{$-\cdots$} & Subtotal for Utility Costs & & & 2.400 .000 & 9.040 .000 & & \\
\hline & & & & & & & \\
\hline & & & & & & & \\
\hline & & & & & & & \\
\hline & DECONTAMINATON AND DECOMMISSIONING & & 630.000 & 630.000 & 4.610 .000 & FY2046 & \multirow{2}{*}{ Data From Previous D \& D Projects } \\
\hline \multicolumn{7}{|c|}{ (D\&D Costs are based on $10 \%$ of the construction cost relating directly to the lacilities being decornmissioned) } & \\
\hline & TEMPORARY CLOSURE - (Pre-engineered metal bl'dg SI & hell) & & & & & \\
\hline \multirow{4}{*}{$-\cdots$} & Disposal Pad - Class ALLW/MW $\left(423^{\prime} \times 324^{\prime}\right)$ & & 10OKYrR & 4.000 .000 & 14.900 .000 & FY2005 THRU FY2045 & Engineers Estimate \\
\hline & Disposal Pad-Class ALLWW (241'x589') & & $100 \mathrm{KNR}$ & 4.000 .000 & $14.900,000$ & FY2005 THRU FY2045 & Engineers Estimate \\
\hline & \multicolumn{2}{|l|}{ Disposal Vault-Class B8C beta/gamma LLW/MW(191' $\left.\times 357^{\prime}\right)$} & - & - & - & & Engineers Estimate \\
\hline & \multicolumn{2}{|l|}{ Disposal Pad-Class A\&C alpha LLW/MW(357'x241') } & 85KYR & 3.400 .000 & $12.700,000$ & FY2005 THRU FY2045 & Engineers Estimate \\
\hline \multirow{4}{*}{ 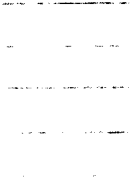 } & FINAL CLOSURE - (Built - up barrier) & & & & & & \\
\hline & Sand & & 16.567 .000 & $16.567,000$ & 121.200 .000 & FY2046 & Engineers Estimate \\
\hline & Gravel & & 562.000 & 562.000 & $4.100,000$ & FY2046 & Engineers Estimate \\
\hline & Diatomaceous Soil & & 1.640 .000 & 1.640 .000 & 12.500 .000 & FY2047 & Engineers Estimate \\
\hline \multirow{4}{*}{$\ldots \ldots$} & Membrane Liner & & 1.900 .000 & 1.900 .000 & 14.400 .000 & FY2047 & Engineers Estimate \\
\hline & Clay & & 2.600 .000 & 2.600 .000 & 20.100 .000 & FY $2047-48$ & Engineers Estimate \\
\hline & Sand/Gravel & & 6.780 .000 & 6.780 .000 & 53.400 .000 & FY2048 & Engineers Estimate \\
\hline & Top Soil & & 1.680 .000 & 1.680 .000 & 13.700 .000 & FY2049 & Engineers Estimate \\
\hline \multirow{7}{*}{, } & Vegetation. 35 Acres & & 70.000 & 70.000 & 570.000 & FY2049 & Engineers Estimate \\
\hline & Instrumentation and installation & & 250.000 & 250.000 & 1.760 .000 & FY2045 & Engineers Estimate \\
\hline & Technical Support. Sampling. \& OA Analysis, 4 People & & 177 KYR & 4.956 .000 & 38.000 .000 & FY2044-50 & Engineers Estimate \\
\hline & POST CLOSURE SURVEILLANCE & & & & & & \\
\hline & Environmental Surveillance. 3 People & & $125 \mathrm{KYr}$ & 4.125 .000 & 37.000 .000 & FY2046 THRU FY2056 & Engineers Estimate \\
\hline & Environmental Surveillance. 1 People & & $125 \mathrm{KYYR}$ & 11.250 .000 & 1.005 .000 .000 & FY2057 THRU FY2146 & Engineers Estimate \\
\hline & Sublotal for Closure and Post Closure & & & 63.780 .000 & 1.364 .230 .000 & & \\
\hline
\end{tabular}




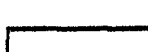

PROBABLE \% VARIATION

\begin{tabular}{|c|c|c|c|c|c|c|c|c|}
\hline \multirow[t]{2}{*}{ Item } & \multirow[t]{2}{*}{ Cost } & \multirow{2}{*}{$\begin{array}{c}\% \\
\text { Total } \\
\text { Cost }\end{array}$} & \multicolumn{2}{|l|}{ Est. Basis } & \multirow{2}{*}{\multicolumn{2}{|c|}{$\begin{array}{c}\text { Prob } \% \text { Var } \\
\text { From Est }\end{array}$}} & \multirow{2}{*}{\multicolumn{2}{|c|}{ Wt $\%$ of Prob }} \\
\hline & & & Quote & Est & & & & \\
\hline Project Management & $3,300,000$ & 11 & 25 & 75 & $5 i$ & 20 & 0.04 & 0.16 \\
\hline Studies, Permitting & 6.160 .000 & 1 & 25 & 75 & 10 & 20 & 0.15 & 0.29 \\
\hline NEPA & $1,750,000$ & 01 & $5 !$ & 95 & 20 & 25 & 0.08 & 0.10 \\
\hline Title / and Title ii Design & $2.600,000$ & $1 !$ & 25 & 75 & $10 !$ & 15 & 0.06 & 0.09 \\
\hline Title III Inspection & $1,260,000$ & 0.30 & 25 & 75 & 5 & 20 & 0.01 & 0.06 \\
\hline Construction. Buildings \& Equipment & 34.024 .000 & 8 & 30 & 70 & 10 & 20 & 0.81 & 1.61 \\
\hline Construction Management & $7,489,000$ & 2 & 30 & 70 & 5 & 20 & 0.09 & 0.35 \\
\hline Startup and Readiness & $7,204,000$ & 2 & 25 & 75 & 10 & 15 & 0.17 & 0.26 \\
\hline Management Reserve & $3,402,000$ & 1 & 50 & 50 & 51 & 30 & 0.04 & 0.24 \\
\hline Total & $422.649,000$ & 100 & & & & & 16.70 & 24.95 \\
\hline
\end{tabular}


EG\&G Idaho, Inc.

Type of Estimate: PLANNING

Date:

File No.

Project: EVALUATION OF ALTERNATIVES FOR DISPOSAL OF INEL MIXED AND LOW LEVEL WASTE

Appr'd By:

Prepared By:

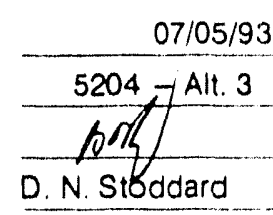

$\begin{array}{ll}\text { Subtotals } & \text { Subtotals } \\ \text { Unescalated } & \text { Escalated }\end{array}$

$\frac{\text { Totals }}{\text { Unescalated }}$

Totals
Escalated

FRONT END DOCUMENTATION

$8.390,000$

Project Management

Studies

NEPA Documentation

Permitting

Technology Development

\begin{tabular}{rr}
$\frac{2,700,000}{1,340,000}$ & $\frac{3,100,000}{1,390,000}$ \\
\hline $3,250,000$ & $\frac{3,580,000}{1,360,000}$ \\
\hline $1,100,000$ & - \\
\hline- & -
\end{tabular}

9.430 .000

ENGINEERING, DESIGN AND INSPECTION.

740,000

Performance Specification

Conceptual and Title I / II Design

Title III Inspection

\begin{tabular}{cc} 
N/A & N/A \\
\hline 600,000 & 698,000 \\
\hline 140,000 & 181,000 \\
\hline
\end{tabular}

CONSTRUCTION

$2,046,000$

Building Costs

$1,677,000$

$2,126,000$

Equipment Costs

Construction Management

$-$

369,000

468,000

TRANSPORT TO DISPOSAL SITE.

(n.............................................. $56,480,000$

$115,520,000$

WASTE DISPOSAL COSTS

100,000

879,000

START-UP AND READINESS

$39,740,000$

BUILDING OPERATION.

$\quad 100,000$

607,000

DECONTAMINATION AND DECOMMISSIONING

CLOSURE/POST CLOSURE.

$20,295,000$

$153,260,000$

MANAGEMENT RESERVE

168,000

213.000

SUBTOTAL

$243,579,000$

$823,891,000$

CONTINGENCY

$21 \%$ of Subtotal.

$51,253,000$

$170,209,000$

TOTAL PROJECT COST: $\quad \$ 295,000,000$

$\$ 994.100,000$

Comments: 
Project EVALUATION OF ALTERNATVES FOR DISPOSAL OF INEL MIXED AND LOW LEVEL WASTE

Location INEL

Requester

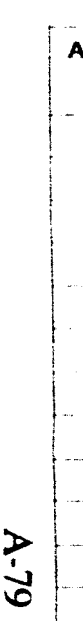

ACCT.

No.
T.H. SMITH ACTIVITY
DESCRPTION PAOJECT COSTS

Project Management

Prolect Studies - 2 People. Full Time

Project Formulation (F\&OR's)

Siting Studies

Pre-Operational Monitoring

Safety Analysis (minimal)

Modity OH site Pertormance Assessment

ACRA

NEPA (EIS)

Air Permits (PSD/PTC/NESHAP)

ESH\&O
Type of Estimate PLANNING

Source (E) Eng. Est.

$M$ Vendor

(P) Pur. Order

(H) Handbook Rel.

\begin{tabular}{|c|c|c|c|c|c|}
\hline E.V. & $\begin{array}{c}\text { ESTIMATED COST } \\
\text { PER YEAR }\end{array}$ & $\begin{array}{c}\text { UNESCALATED } \\
\text { COST }\end{array}$ & $\begin{array}{l}\text { ESCALATED } \\
\text { COST }\end{array}$ & $\begin{array}{l}\text { ACTIVITY SCHEDULE } \\
\text { ESCALATION }\end{array}$ & ESTMMATE BASIS \\
\hline & $300 \mathrm{KVR}$ & 2.700 .000 & 3.100 .000 & FY92 THRU FYZO0O & TDD Sheet. dated 4/28/93 \\
\hline & 250KYYR/EA & $1.000,000$ & 1.040 .000 & FY93-94 & TDD Sheet, dated 4/28/93 \\
\hline & $70 \mathrm{~K} / 270 \mathrm{~K}$ & 340.000 & 350.000 & FY92 - 93 & TDD Sheet. dated 4/28/93. Eng. Estumate \\
\hline & - & 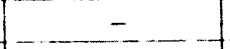 & - & & \\
\hline & - & - & - & & \\
\hline & $200 \mathrm{KYA}$ & 400.000 & 500.000 & FY98.FY99 & Data from Similar SAR's \\
\hline & $200 \mathrm{~K} Y \mathrm{R}$ & 400.000 & 500.000 & FY98.FY99 & Engineer's Estimate \\
\hline & - & - & - & & \\
\hline & $1 \mathrm{M} / 1 \mathrm{M} / 1 \mathrm{M} / 250 \mathrm{~K}$ & 3.250 .000 & 3.580 .000 & FY94 - FY97 & Engineer's Estimate \\
\hline & - & - & - & & \\
\hline & SOKNR & 300.000 & 360.000 & FY1995-2000 & Engineer's Estimate \\
\hline & - & - & - & & \\
\hline & - & - & - & & \\
\hline & & & & & \\
\hline & & & & & \\
\hline & 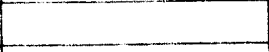 & & & & \\
\hline & - & - & - & & \\
\hline & - & - & - & & \\
\hline & - & - & - & & \\
\hline & & 8.390 .000 & 9.430 .000 & & \\
\hline & & & & & \\
\hline & & & & & \\
\hline & $200 \mathrm{~K}$ & 200.000 & 219.000 & FY95 & TOD Sheet \\
\hline & $200 \mathrm{~K}$ & 200.000 & 235.000 & FY97 & Engineer Estimate \\
\hline & $200 \mathrm{~K}$ & 200.000 & 244.000 & FY98 & Enginoer Estimate \\
\hline & & 600.000 & 698.000 & & \\
\hline & & & & & \\
\hline & $140 \mathrm{~K}$ & 140.000 & 181.000 & FY99 & Comparative Historical Data \\
\hline & & & & & \\
\hline
\end{tabular}

Date: $\quad 07 / 05 / 93$

Prep'd By: _ D. N. Stoddard

Chis'd By:

Other Regulabry Items (State Water Well Approval)

Special Q/A Program For Construction - 1 Person

TECHNOLOGY DEVELOPMENT

Material Behavior (Clay. etc.)

Cover Performance Control

Leachate Collection and Liner Performance

Subtotal for Front End Costs

\begin{tabular}{|l|l|l}
\hline & Subtotal for Front End Costs & \\
\hline & & \\
\hline & DESIGN & \\
\hline & Conceptual & \\
\hline & Tittel & \\
\hline & Titte II & \\
\hline & Subtotal for Design (Based on \% ol Construction Costs) \\
\hline & & \\
\hline & Title III Inspection (1 Person for 12 Months) & \\
\hline
\end{tabular}


Project EVAWATION OF ALTERNATIVES FOR DISPOSAL OF INEL MIXED AND LOW LEVEL WASTE

\begin{tabular}{|c|c|c|c|c|c|c|c|}
\hline $\begin{array}{l}\text { ACCT. } \\
\text { No. }\end{array}$ & $\begin{array}{c}\text { ACTIVTY } \\
\text { DESCPPPTION }\end{array}$ & E.V. & $\begin{array}{c}\text { ESTMAATED COST } \\
\text { PER YEAR }\end{array}$ & $\begin{array}{c}\text { UNESCALATED } \\
\text { COST }\end{array}$ & $\begin{array}{c}\text { ESCALATED } \\
\text { COST } \\
\end{array}$ & $\begin{array}{l}\text { ACTIVTY SCHEDULE } \\
\text { ESCALATK }\end{array}$ & ESTIMATE BASIS \\
\hline & CONSTRUCTION & & & & & & \\
\hline \multirow[t]{13}{*}{4000} & Improvements to Land & & & & & & \\
\hline & Surveying (2 Acres) & & 3,000 & 3.000 & 4,000 & FY99 & Comparative Historical Data \\
\hline & Site Prep Clearing and Grubbing (2 Acres) & & 6.000 & 6.000 & 8.000 & FY99 & Comparative Historical Data \\
\hline & Roadways and Parking & & 370.000 & 370,000 & 469,000 & FY99 & Comparative Historical Data \\
\hline & Fencing & & - & - & - & & \\
\hline & Retention Pond & & - & - & - & & \\
\hline & Drainage Gallery System & & - & - & - & & \\
\hline & Drainage Pumphouse & & - & - & 二. & & \\
\hline & Collection /Sampling System Vault at Disposal Areas & & - & - & - & & \\
\hline & Storage Vautt Disposal Area Including: (Refer to & & - & - & - & & \\
\hline & Pad Construction Costs) & & - & - & - & & \\
\hline & Drainage Ditches & & - & - & $=$ & & \\
\hline & TOTAL FOR 4000 ACCOUNT & & & 379.000 & 481.000 & & \\
\hline \multirow{2}{*}{$-\ldots$} & & & & & & & \\
\hline & & & & - - . & - & -1 & ... \\
\hline \multirow[t]{9}{*}{6000} & Ulitities (Assume 200' run to Treatment Facility) & & & & & & \\
\hline & Electrical Power and Distribution To Facility & & 100.000 & 100.000 & 128.000 & FY99 & Engineers Estimate \\
\hline & Electrical Lighting.Power,Alarms.\& Communication & & 75.000 & 75,000 & 95.000 & FY99 & Engineers Estimate \\
\hline & Distribution System & & $\ldots$ & 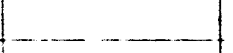 & $\ldots$ & & - ........ \\
\hline & Water Supply \& Fire Water Distribution to Facility & & 20.000 & 20,000 & 25,000 & FY99 & Engineers Estimale \\
\hline & Water Supply and Fire Water Distribution Systems & & - & - & - & & \\
\hline & Sanitary Sewage System & & 20.000 & 20.000 & 25.000 & FY99 & Engineers Estimate \\
\hline & TOTAL FOR 6000 ACCOUNT & & & 215.000 & 273.000 & & \\
\hline & -1 & - & $1 \ldots$ & $\ldots$ & $\ldots \ldots \ldots$ & 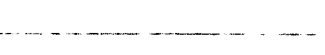 & - \\
\hline \multirow[t]{4}{*}{5000} & Buildings and Structures & & & & & ....... & 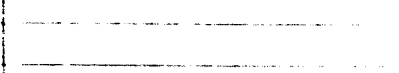 \\
\hline & Office Space Added to Treatment Facility (2500 st) & & 400.000 & 400.000 & 507.000 & FY99 & Comparative Historical Data \\
\hline & Truck Maintenance/Decon Facility ( $3500 \mathrm{sf}$ ) & & 450.000 & 450.000 & 570.000 & FY99 & Comparative Historical Data \\
\hline & TOTAL FOR 5000 ACCOUNT & & - & 850.000 & 1.077 .000 & 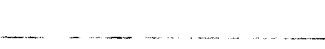 & 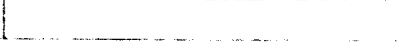 \\
\hline
\end{tabular}


Page 3 of 5

Project EVALUATION OF ALTERNATIVES FOR DISPOSAL OF INEL MIXED AND LOW LEVEL WASTE

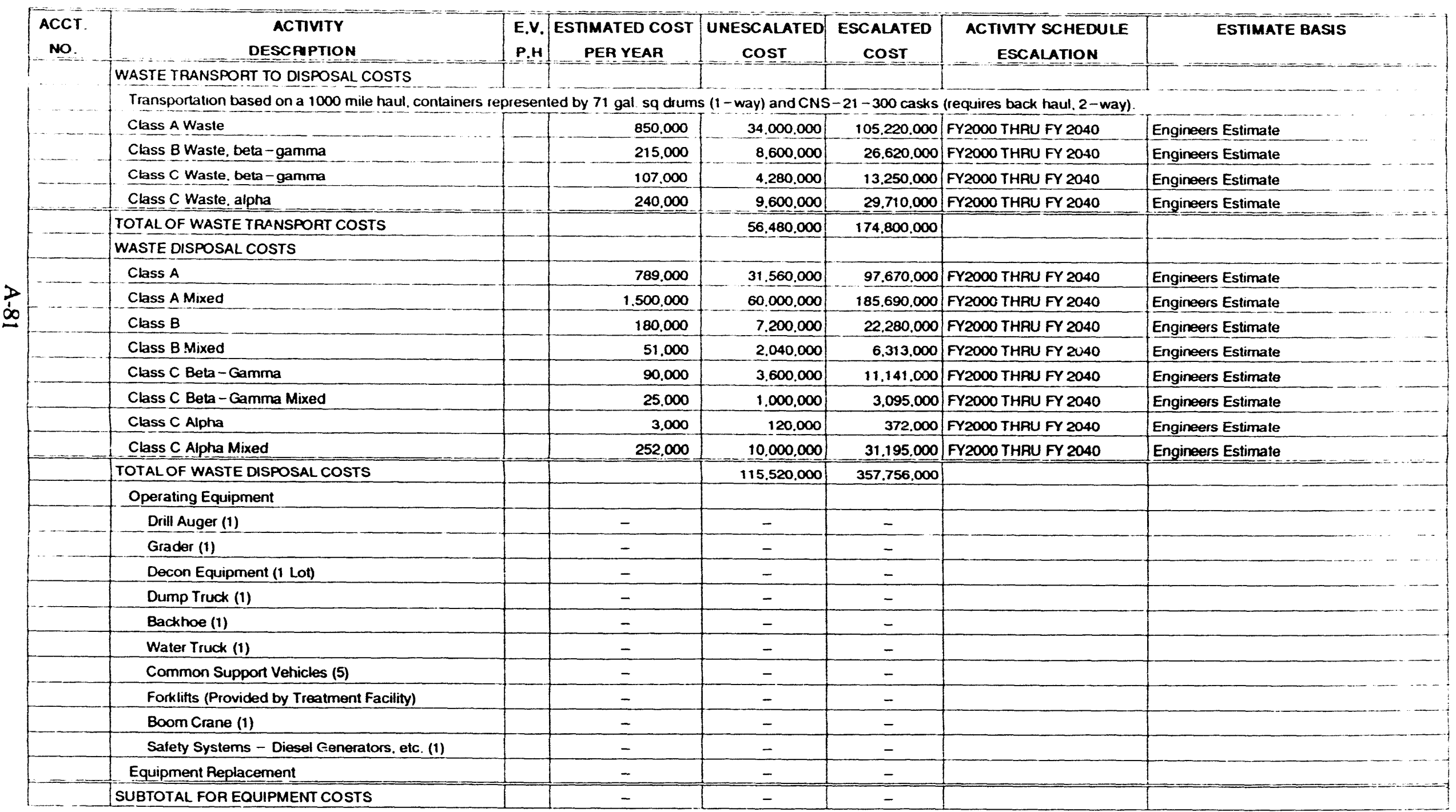


Project EVALUATION OF ALTERNATIVES For OISPOSAL OF INEL MIXED AND LOW LEVEL WASTE

\begin{tabular}{|c|c|c|c|c|c|c|c|}
\hline $\begin{array}{l}\text { ACCT. } \\
\text { №. }\end{array}$ & $\begin{array}{c}\text { ACTIVTY } \\
\text { DESCRPIION } \\
\end{array}$ & E.V. & $\begin{array}{c}\text { ESTMMATED COST } \\
\text { PER YEAR }\end{array}$ & $\begin{array}{c}\text { UNESCALATED } \\
\text { COST }\end{array}$ & $\begin{array}{c}\text { ESCALATED } \\
\text { COST } \\
\end{array}$ & $\begin{array}{l}\text { ACTIVTY SCHEDULE } \\
\text { ESCALATION }\end{array}$ & ESTIMATE BASAS \\
\hline & CONSTRUCTION MANAGEMENT & & 369.000 & 369.000 & 468,000 & FY99 & Engineers Estimate \\
\hline & & & & & & & \\
\hline & & & & & & & \\
\hline \multirow[t]{3}{*}{9000.} & Other Direct Costs & & & & & & \\
\hline & Full Time Non Working Supervisors ( 1 for 12 Months) & & $218 K$ & 218,000 & 276,000 & FY99 & Engineers Estimate \\
\hline & Site Specific Training (18 People lor 26 Hrs Ea.) & & $15 K$ & 15.000 & 19,000 & FY99 & Engineers Estimate \\
\hline \multirow{2}{*}{$\ldots$} & & & & & & & \\
\hline & \multicolumn{2}{|l|}{ IESTING/STARTUP/READINESS REV/PERSONNEL TRAN'G } & $100 K$ & 100.000 & 132.000 & FYzo00 & Readiness Review Experience \\
\hline & & & & & & & \\
\hline \multirow{4}{*}{$\ldots$} & OPERATING COSTS & & & & & & \\
\hline & Labor Based on 2080 His Per Year & & & & & & \\
\hline & Operating Personnei & & - & - & - & & \\
\hline & Repair/Decon Maintenance Personnel. 1 Person & & $120 \mathrm{KNA}$ & 4.800 .000 & 15.010 .000 & FY2000 THPU FY2040 & Engineers Estimate Based on RWMC Study \\
\hline & Training Personnel & & - & - & - & & \\
\hline & Recordkeeping. 2 People & & 78KNR & 6.240 .000 & 19.520 .000 & FY2000 THAU FY2040 & Engineers Estimale Based on RWMC Study \\
\hline & Management, 1 Person & & $285 K M A$ & 11.400 .000 & 35.660 .000 & FY2000 THAU FY2040 & Engineers Estimate Based on RWMC Study \\
\hline & Engineering. 1 Person & - & 177KNR & 7.100 .000 & 22.140 .000 & FY2000 THRU FY2040 & Engineers Estimate Based on RWMC Study \\
\hline & ESH8Q Personnel & & - & - & - & & \\
\hline & Budget Support Personnel. 1 Person & - & $115 \mathrm{KNR}$ & $4.600,000$ & 14.390 .000 & FY2000 THRU FY2040 & Engineers Estimate Based on RWMC Study \\
\hline & Health Physics Personnel & - & - & - & $=$ & & + \\
\hline & Lab Personnel & & - & - & - & & \\
\hline & Environmental Survelliance & & $=$ & - & $=$ & & - \\
\hline & Document Control & & - & - & - & & 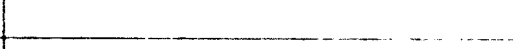 \\
\hline & Shipping Coordinator. 1 Person & & $130 \mathrm{KNR}$ & 5.200 .000 & 16.260 .000 & FY2000 THRU FY2040 & Engineers Estimate Based on RWMC Study \\
\hline & & & & & & & \\
\hline & Equipment & $\ldots$ & $\cdots$ & 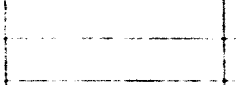 & $-\ldots-\ldots$ & $\ldots \ldots$ & $\ldots-\ldots$ \\
\hline & Replacement Parts - Misc. Maintenarxce & & - & - & - & & $-\cdots$ \\
\hline & & & & & & & \\
\hline & Subiotal lor Operating Costs & & & 39.340 .000 & 122.980 .000 & & \\
\hline
\end{tabular}


Rer $5-9$

Page 5 of 5

Type of Estimate: PLANNING

File No 5204 - Alt. 3

Date:

07/05/93

Pioject eVallation of alternatives for disposal OF INEL MIXED AND LOW LEVEL WASTE

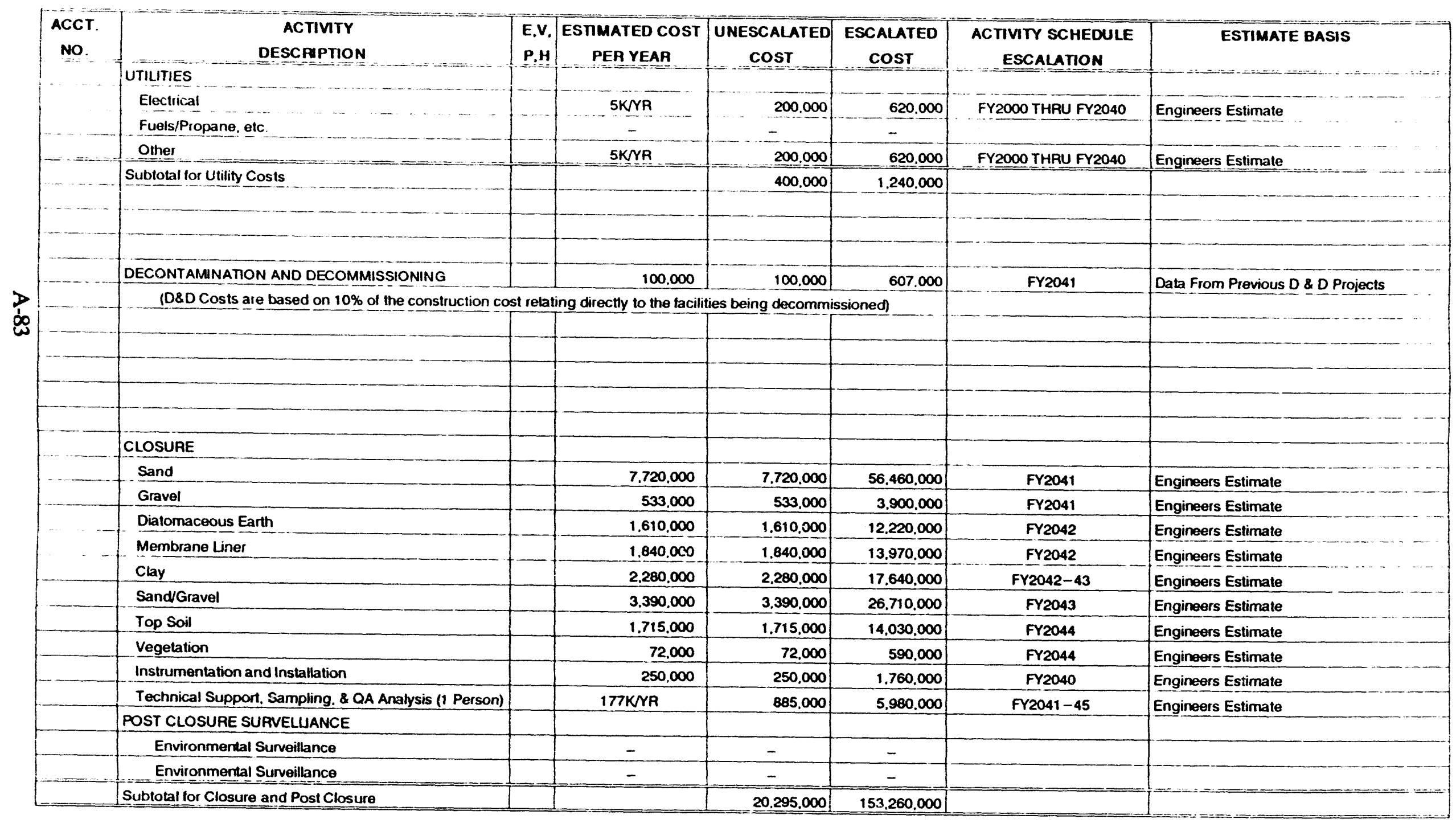


Project EVALUATION OF ALTERNATIVES FOR DISPOSAL TyPo of Est. PLANNING

Location INEL

File No. 5204 - Alt. 3

Date

Prep'd By 07/05/93

Requester

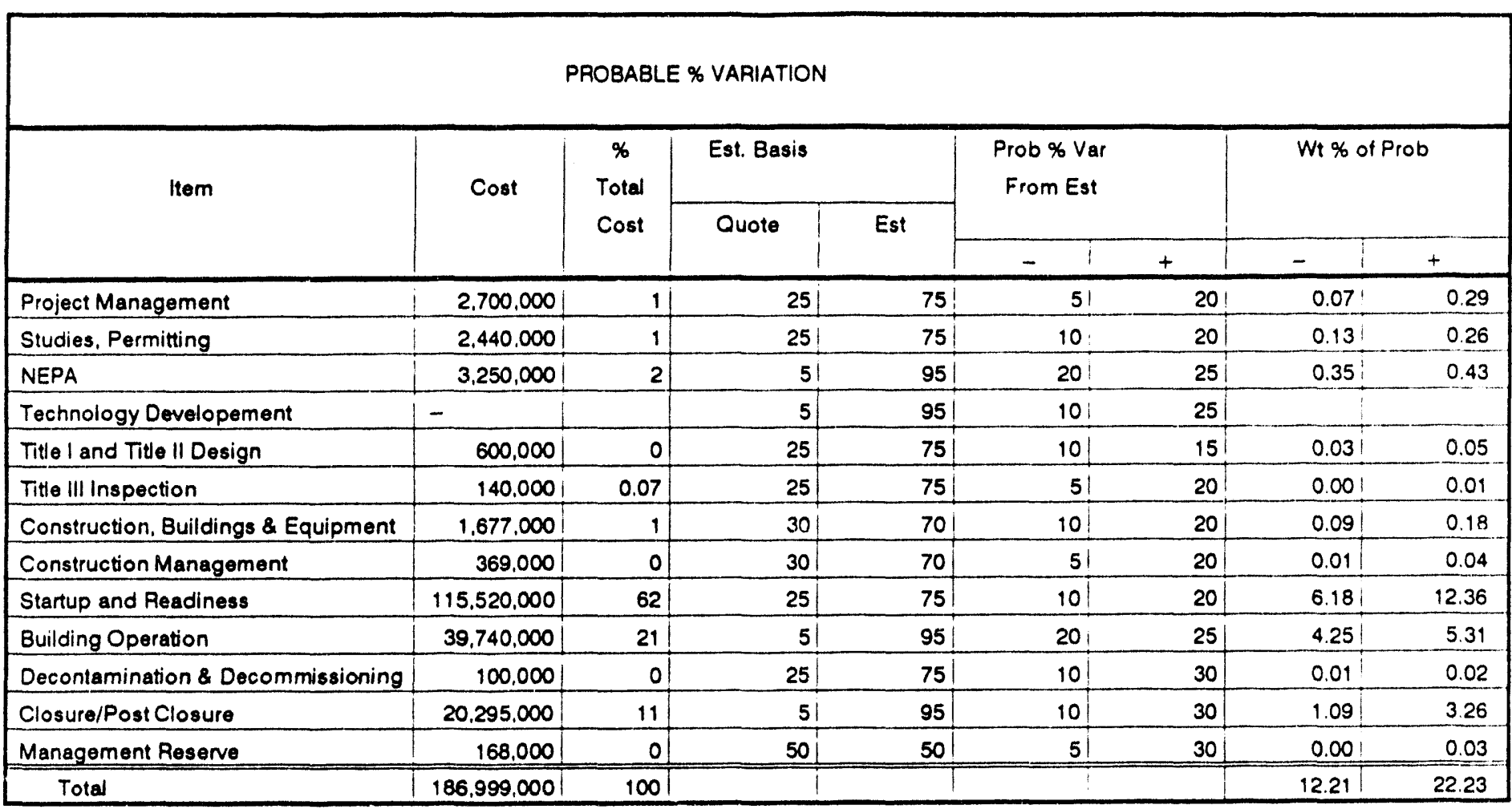


EG\&G Idaho, Inc.

Type of Estimate: PLANNING

Date:

File No.

Project: EVALUATION OF ALTERNATIVES FOR DISPOSAL OF INEL MIXED AND LOW LEVEL WASTE

$\frac{\text { Subtotals }}{\text { Unesclated }} \quad \frac{\text { Subtotals }}{\text { Escalated }}$

Appr'd By:

Prepared By:

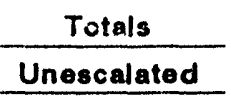

$18,660,000$

FRONT END DOCUMENTATION
Project Management
Studies
NEPA Documentation
Permitting
Technology Development

FRONT END DOCUMENTATION

\begin{tabular}{|c|c|}
\hline $3,300,000$ & $4,060,000$ \\
\hline $2,080,000$ & $2,160,000$ \\
\hline $3,250,000$ & $3,580,000$ \\
\hline $7,910,000$ & $10,320,000$ \\
\hline $2,120,000$ & $2,440,000$ \\
\hline
\end{tabular}

ENGINEERING, DESIGN AND INSPECTION

Performance Specification Conceptual and Title I/ II Design Title III Inspection

$\frac{N / A}{8,300,000}$

$\frac{N / A}{11,594,000}$

$10,455,000$

CONSTRUCTION. $93,931,000$ Building Costs Equipment Costs Construction Management \begin{tabular}{rr}
$\frac{72,506,000}{4,480,000}$ & $164,944,000$ \\
\hline $16,945,000$ & $13,050,000$ \\
\hline
\end{tabular}

TAANSPORT TO DISPOSAL SITE. $21,560,000$

WASTE DISPOSAL COSTS $73,120,000$

START-UP AND READINESS. $5,945,000$

BUILDING OPERATION $274,200,000$

DECONTAMINATION AND DECOMMISSIONING.

$1,045,000$

CLOSURE/POST CLOSURE.

$57,300,000$

MANAGEMENT RESERVE.

$7,699,000$

SUBTOTAL

$563,915,000$

CONTINGENCY

$20 \%$ of Subtotal.
$16,734,000$

07/02/93 5204 - Aly. 4 A hol

D. . S. Stdddard

$\frac{\text { Totals }}{\text { Escalatod }}$

$22,560,000$

$217,170,000$

$63,232,000$

$226,293,000$

$8,730,000$

$938,350,000$

$7,207,000$

$1,314,460,000$

$17,799,000$

$2,832,535,000$

$567,465,000$

TOTAL PROJECT COST: $\$ \$ 677,000,000 \quad \$ 3,400,000,000$ 
Project EVALUATION OF ALTERNATIVES FOR DISPOSAL OF INEL MIXED AND LOW LEVEL WASTE

Location INEL

Requester T.H. SMITH

\begin{tabular}{|c|c|}
\hline $\begin{array}{c}\text { ACCT. } \\
\text { NO. }\end{array}$ & $\begin{array}{c}\text { ACTIVTY } \\
\text { DESCRPTION }\end{array}$ \\
\hline & PAOJECT COSTS \\
\hline & Project Management \\
\hline & Project Studies - 2 People. Full Time \\
\hline & Project Formulation (F\&OR's) \\
\hline & Siting Studies \\
\hline & Pre-Operational Monitoring \\
\hline & Safety Analysis Report (SAR) \\
\hline & Perlormance Assessment \\
\hline & RCRA \\
\hline & NEPA (EIS)(CX's) \\
\hline & Air Permits (PSD/PTC/NESHAP) \\
\hline & ESH\&Q \\
\hline & Other Regulatory Items (State Water Well Approval) \\
\hline & Special O/A Program For Construction - 2 People \\
\hline & \\
\hline & \\
\hline & \\
\hline & \\
\hline & Cover Performance Control \\
\hline & Leachate Collection and Liner Performance \\
\hline & Material Handling (Gantry Crane) \\
\hline & \\
\hline & \\
\hline & \\
\hline & \\
\hline & Subtotal for Front End Costs \\
\hline
\end{tabular}

File No.

Type of Estimate PLANNING Source (E) Eng. Est.

M Vendor

(P) Pur. Order

(H) Handbook Ret

\begin{tabular}{|c|c|c|c|c|c|}
\hline $\begin{array}{l}\text { E.V. } \\
\text { P.H }\end{array}$ & $\begin{array}{c}\text { ESTMMATED COST } \\
\text { PER YEAR }\end{array}$ & $\begin{array}{c}\text { UNESCALATED } \\
\text { COST }\end{array}$ & $\begin{array}{c}\text { ESCALATED } \\
\text { COST }\end{array}$ & $\begin{array}{l}\text { ACTIVITY SCHEDULE } \\
\text { ESCALATION }\end{array}$ & ESTIMATE BASIS \\
\hline & 300KYR & $3,300,000$ & 4.060 .000 & FY92 THRU FY2003 & TDD Sheet, dated 4/28/93 \\
\hline & 250KYRUEA & 1.000 .000 & $1,040,000$ & FY93 - 94 & TDD Sheet, dated 4/28,93 \\
\hline & $70 \mathrm{~K} / 270 \mathrm{~K}$ & 340.000 & 350.000 & FY92 - 93 & TDD Sheet. dated 4/28/93. Eng. Estimate \\
\hline & $70 \mathrm{~K} / 270 \mathrm{~K} / 400 \mathrm{~K}$ & 740.000 & 770,000 & FY92 - FY94 & TDD Sheet, dated 4/28/93. Eng. Estimate \\
\hline & $500 \mathrm{~K} / 500 \mathrm{~K} 250 \mathrm{KVR}$ & $2.000,000$ & 2.570 .000 & FY95,FY99,FY2000-2003 & Engineer's Estimate \\
\hline & $150 \mathrm{~K} / 300 \mathrm{~K} 200 \mathrm{~K}$ & $2,000,000$ & $2.550,000$ & FY95-96.97-99.2000-03 & Data from Similar SAR's \\
\hline & 400KYR & $1.600,000$ & $1.960,000$ & FY95-96.FY99-00 & Engineer's Estimate \\
\hline & - & - & - & & \\
\hline & $1 \mathrm{M} / 1 \mathrm{M} / 1 \mathrm{M} / 25 \mathrm{KK}$ & 3.250 .000 & $3.580,000$ & FY94 - FY97 & Engineer's Estimate \\
\hline & $200 \mathrm{~K} / 150 \mathrm{~K}$ & 350,000 & 550,000 & FY1999, FY2000 & Engineer's Estimale \\
\hline & SOKVR & 400.000 & 570.000 & FY1995-2003 & Engineer's Estimate \\
\hline & 250KYA & 500.000 & 670.000 & FY98-FY99 & Enginoer's Estimate \\
\hline & 354KVYR & $1.062,000$ & $1,450.000$ & FY2000-01.02 & Engineer's Estima \\
\hline & & & & & \\
\hline & & & & & \\
\hline & & & & & \\
\hline & & & & & \\
\hline & 100KV65KNR & 360.000 & 410.000 & FY94, FY95-98 & Engineer's Estimate \\
\hline & $100 \mathrm{~K} / 65 \mathrm{KNYR}$ & 360.000 & 410,000 & FY94, FY95-98 & Engineer's Estimate \\
\hline & 200KNR & 1.000 .000 & $1,140,000$ & FY94-98 & Engineer's Estimate \\
\hline & 200KNR & 400.000 & 480.000 & FY97-98 & Engineer's Estimate \\
\hline & & & & & \\
\hline & & & & & \\
\hline & & & & & \\
\hline & & & & & \\
\hline & & & & & \\
\hline & & & & & \\
\hline & & 18.662 .000 & $22,560,000$ & & \\
\hline
\end{tabular}

$07 / 02 / 93$

Prep'd By: D. N. Stoddard

Cha'd By:

\section{-}

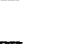


Page 2 of 7

Pioject evaluation of alternatives for disposal OF INEL MIXED AND LOW LEVEL WASTE

\begin{tabular}{|c|c|c|c|c|c|c|c|}
\hline $\begin{array}{l}\text { ACCT. } \\
\text { NO. }\end{array}$ & $\begin{array}{c}\text { ACTIVITY } \\
\text { DESCRIPTION }\end{array}$ & E.V. & $\begin{array}{c}\text { ESTMMATED COST } \\
\text { PEA YEAR }\end{array}$ & $\begin{array}{c}\text { UNESCALATED } \\
\text { COST }\end{array}$ & $\begin{array}{c}\text { ESCALATED } \\
\text { COST } \\
\end{array}$ & $\begin{array}{l}\text { ACTIVITY SCHEDULE } \\
\text { ESCALATION }\end{array}$ & ESTIMATE BASIS \\
\hline & DESIGN & & & & & & \\
\hline & Conceptual & & $600 \mathrm{~K} / 400 \mathrm{~K}$ & 1.000 .000 & 1.110 .000 & FY95.FY96 & TDD Sheet \\
\hline & Title I & & $3.2 \mathrm{M}$ & 3.200 .000 & 3.764 .000 & FY97 & Engineers Estimate \\
\hline & Title 11 & & $3.2 \mathrm{M}, 300 \mathrm{KNR}$ & 4.100 .000 & 6.720 .000 & FY98.2012.2022.2032 & Engineers Estimate \\
\hline \multirow[t]{4}{*}{$\ldots$} & Subtotal for Design (Based on $\%$ of Construction Costs) & & & 8.300 .000 & 11.594 .000 & & \\
\hline & & & & & & & \\
\hline & Title III Inspection (1 Person/12 Mths, 4 People/30 Mths) & & $140 \mathrm{~K} / 575 \mathrm{~K} / 288 \mathrm{KNR}$ & $2,155,000$ & 5.140 .000 & $F Y 99,00-02,04,13,23,24, \& 3$ & Comparative Historical Data \\
\hline & CONSTRUCTION & & & & & & \\
\hline \multirow[t]{13}{*}{4000} & Improvements to Land & & & & & & \\
\hline & Surveying (89 Acres \& 2 Acres) & & $110 \mathrm{~K} \& 3 \mathrm{~K}$ & 113.000 & 149,000 & FY2000 & Comparative Historical Data \\
\hline & Site Prep Clearing and Grubbing (89 Acres \& 2 Acres) & & $260 K \& 6 K$ & 345.000 & 454.000 & FY2000 & Comparative Historical Data \\
\hline & Roadways and Parking & & $1.3 \mathrm{M} \& 370 \mathrm{~K}$ & 1.400 .000 & $1,842,000$ & FY2000 & Comparative Historical Data \\
\hline & Fencing & & 325,000 & 325,000 & 428,000 & FY2000 & Comparative Historica! Data \\
\hline & Retention Pond & & 40.000 & 40,000 & 53,000 & FY2000 & Comparative Historical Data \\
\hline & Drainage Gallery System & & 600.000 & 600.000 & 790.000 & FY2000 & Comparative Historical Data \\
\hline & Drainage Pumphouse & & 100.000 & 100,000 & 132.000 & FY2000 & Comparative Historical Data \\
\hline & Collection / Sampling System Vault at Disposal Areas & & 600,000 & 600.000 & 790.000 & FY2000 & Comparative Historical Data \\
\hline & Storage Vault Disposal Area Including: Engineered & & $1.200,000$ & $1,200,000$ & $1.580,000$ & FY2000 & Comparative Historical Data \\
\hline & Fill, and Pit Pun & & & & & & \\
\hline & Drainage Ditches & & 100.000 & 100,000 & 132,000 & FY2000 & \\
\hline & TOTAL FOR 4000 ACCOUNT & & & 4.823 .000 & $6.350,000$ & & \\
\hline \multirow[t]{8}{*}{6000} & Utilities & & & & & & \\
\hline & Electrical Power and Distribution To Facility & & $1.950,000$ & 1.950 .000 & 2.565 .000 & FY2000 & Engineers Estimate \\
\hline & Electrical Lighting.Power,Alarms, \& Communication & & $1,075,000$ & 1.075 .000 & $1,414,000$ & FY2000 & Engineers Estimate \\
\hline & Distribution System & & & & & & Engineers Estimate \\
\hline & Water Supply \& Fire Water Distribution to Facility & & $1.570,000$ & 1.570 .000 & $2,065.000$ & FY2000 & Engineers Estimate \\
\hline & Water Supply and Fire Water Distribution Systems & & 450,000 & 450,000 & 592.000 & FY2000 & Engineers Estimate \\
\hline & Sanitary Sowage System & & 270,000 & 270,000 & 356.000 & FY2000 & Engineers Estimate \\
\hline & IOTAL FOR 6000 ACCOUNT & & & 5.315 .000 & 6.922 .000 & & \\
\hline
\end{tabular}


Project EVALUATION OF ALTERNATIVES FOR DISPOSAL OF INEL MIXED AND LOW LEVEL WASTE

\begin{tabular}{|c|c|c|c|c|c|c|c|}
\hline $\begin{array}{l}\text { ACCT. } \\
\text { No. }\end{array}$ & $\begin{array}{c}\text { ACTIVTY } \\
\text { DESCFPIION }\end{array}$ & $\begin{array}{l}\text { E.V. } \\
\text { P.H } \\
\end{array}$ & $\begin{array}{c}\begin{array}{c}\text { ESTIMATED COST } \\
\text { PEA YEAR }\end{array} \\
\end{array}$ & \begin{tabular}{|c|} 
UNESCALATED \\
COST
\end{tabular} & $\begin{array}{c}\text { ESCALATED } \\
\text { COST } \\
\end{array}$ & $\begin{array}{c}\text { ACTIVTY SCHEDULE } \\
\text { ESCALATION } \\
\end{array}$ & ESTIMATE BASIS \\
\hline \multirow[t]{7}{*}{5000} & Buildings and Structures - Low Level Waste Disposal & & & & & & \\
\hline & Office /Administration Bldg. (1 story $-10,000 \mathrm{~s}$ ) & & $1,240,000$ & $1,240.000$ & $1,700,000$ & FY2001 & Comparative Historical Data \\
\hline & Support Building (1 story - $8000 \mathrm{~s}$ ) & & 825.000 & 825,000 & $1,200,000$ & FY2002 & Comparative Historical Data \\
\hline & Material Storage Building (1 story - $2500 \mathrm{sf}$ ) & & 200,000 & 200,000 & 290.000 & FY2002 & Comparative Historical Data \\
\hline & Heavy Equipment Storage \& Maintenance & & 750.000 & 750,000 & $1,100,000$ & FY2002 & Comparative Historical Data \\
\hline & Disposal Vauh - Type A \& C $(56 \times 266 \times 29)(4.4 .3 .2)$ & & 16M/16M/12M/BM & $52,000.000$ & $130,970,000$ & FY2003,2013,2023,2033 & Comparative Historical Data at SR Site \\
\hline & Disposal Vautt - B \& C B/G $(58 \times 166 \times 31)(1)(1)$ & & $2.35 \mathrm{M} / 2.35 \mathrm{M}$ & 4.700 .000 & 11.150 .000 & FY2004.2024 & Comparative Historical Data at SR Site \\
\hline & Buildings and Structures - Mixed Waste Disposal & & & & & & \\
\hline & Office Space Added to Treatment Facility (2500 st) & & 400.000 & 400,000 & 507,000 & FY99 & Comparative Historical Data \\
\hline & Truck Maintenance/Decon Facility ( $3500 \mathrm{sf}$ ) & & 450.000 & 450.000 & 570,000 & FY99 & Comparative Historical Data \\
\hline & & & & & & & \\
\hline & TOTAL FOR 5000 ACCOUNT & & & $60,565,000$ & 147.487 .000 & & \\
\hline & & & & & & & \\
\hline & WASTE TRANSPORT TO DISPOSAL COSTS & & & & & & \\
\hline & \multicolumn{7}{|c|}{ Transportation based on a 1000 mile haul. containers represented by 71 gallon sq drums (1 - way) and CNS-21-300 casks (requires back - haul, 2-way) } \\
\hline & Class A Mixed & & 273.000 & $10.920,000$ & 33.796 .000 & FY2000 THRU FY 2040 & Comparative Historical Data \\
\hline & Class B Mixed & & 30,000 & 1.200 .000 & 176.000 & FY2000 THRU FY 2040 & Comparative Historical Data \\
\hline & Class C Beta-Gamma Mixed & & 15.000 & 600.000 & $1.860,000$ & FY2000 THRU FY 2040 & Comparative Historical Data \\
\hline & Class C Alpha Mixed & & 221.000 & $8,840,000$ & 27.400 .000 & FY2000 THRU FY 2040 & Comparative Historical Data \\
\hline & & & & & & & \\
\hline & TOTALOF WASTE TRANSPORT COSTS & & & $21,560,000$ & 63.232 .000 & & \\
\hline & & & & & & & \\
\hline & WASTE DISPOSAL COSTS & & & & & & \\
\hline & Class A Mixed & & 1.500 .000 & 60.000 .000 & 185.690 .000 & FY2000 THRU FY 2040 & Estimate from NTS \\
\hline & Class B Mixed & & 51.000 & $2,040,000$ & $6,313.000$ & FY2000 THRU FY 2040 & Estimate from NTS \\
\hline & Class C Beta-Gamma Mixed & & 25.000 & 1.000 .000 & 3.095 .000 & FY2000 THRU FY 2040 & Estimate from NTS \\
\hline & Class C Alpha Mixed & & 252,000 & $10.080,000$ & 31.195 .000 & FY2000 THRU FY 2040 & Estimate from NTS \\
\hline & TOTAL OF WASTE DISPOSAL COSTS & & & $73.120,000$ & 226.293 .000 & & \\
\hline
\end{tabular}


Page 4 of 7

Project eValuation of alternatives for disposal

OF INEL MIXED AND LOW LEVEL WASTE

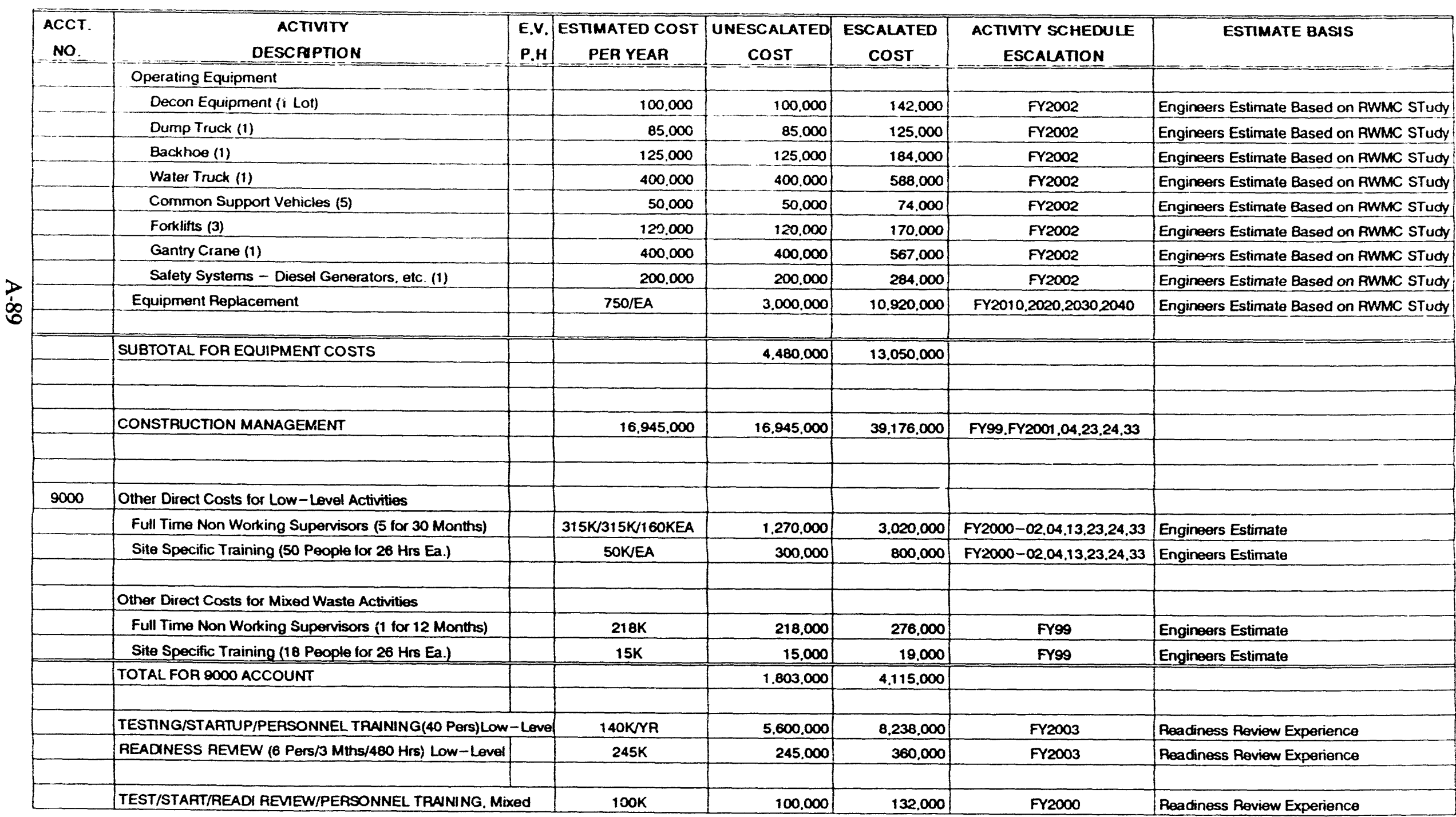


Project EVAUATION OF ALTERNATIVES FOR DISPOSAL OF INEL MIXED AND LOW LEVEL WASTE

\begin{tabular}{|c|c|c|c|c|c|c|c|}
\hline $\begin{array}{l}\text { ACCT. } \\
\text { No. }\end{array}$ & $\begin{array}{c}\text { ACTIVITY } \\
\text { DESCRIPTION }\end{array}$ & $\begin{array}{l}\text { E.V. } \\
\text { P.H } \\
\end{array}$ & $\begin{array}{c}\text { ESTIMATED COST } \\
\text { PEA YEAR }\end{array}$ & $\begin{array}{c}\text { UNESCALATED } \\
\text { COST }\end{array}$ & $\begin{array}{c}\text { ESCALATED } \\
\text { COST }\end{array}$ & $\begin{array}{c}\text { ACTIVITY SCHEDULE } \\
\text { ESCALATION }\end{array}$ & ESTIMATE BASIS \\
\hline & OPERATING COSTS (Low-Level Waste Operations) & & & & & & \\
\hline & Operating Personnel, 12 People & & $125 \mathrm{KNR}$ & 60.000 .000 & 208.000 .000 & FY2003 THRU FY 2043 & Engineers Estimate Based on RWMC Study \\
\hline & Maintenance Personnel. 7 People & & $120 \mathrm{KNR}$ & $33,600,000$ & 116.300 .000 & FY2003 THRU FY 2043 & Engineers Estimate Based on RWMC Study \\
\hline & Training Personnel, 2 People & & $96 \mathrm{KNR}$ & 7.680 .000 & 27.000 .000 & FY2003 THRU FY 2043 & Engineers Estimate Based on RWMC Study \\
\hline & Recordkeeping. 2 People & & 78KNA & 6.240 .000 & $21,940.000$ & FY2003 THRU FY 2043 & Engineers Estimate Based on RWMMC Study \\
\hline & Management. 3 People & & 285KYR & $34.200,000$ & $118.400,000$ & FY2003 THRU FY 2043 & Engineers Estimate Based on RWMC Study \\
\hline & Engineering. 3 People & & 177KNR & $21,240,000$ & $73.500,000$ & FY2003 THRU FY 2043 & Engineers Estimate Based on RWMC Study \\
\hline & ESH\&Q Personnel. 3 People & & 178KYRR & $21.360,000$ & $74,000,000$ & FY2003 THRU FY 2043 & Engineers Estimate Based on RWMC Study \\
\hline & Budget Support Personnel. 3 People & & 115KYR & $13.800,000$ & 47.800 .000 & FY2003 THRU FY 2043 & Engineers Estimate Based on RWMC Study \\
\hline & Health Physics Personnet, 2 People & & 128KYYR & $10,240,000$ & $35.400,000$ & FY2003 THRU FY 2043 & Engineers Estimate Based on RWMC Study \\
\hline & Lab Personnel, 1 Person & & $128 \mathrm{KNA}$ & 5.120 .000 & 17.700 .000 & FY2003 THRU FY 2043 & Engineers Estimate Based on RWMC Study \\
\hline & Environmertal Surveillance, 3 People & & 125KNR & 15.000 .000 & $52.000,000$ & FY2003 THRU FY 2043 & Engineers Estimate Based on RWMC Study \\
\hline & Document Control. 1 Person & & 78KYR & 3.120 .000 & 10.800 .000 & FY2003 THRU FY 2043 & Engineers Estimate Based on RWMC Study \\
\hline & OPERATING COSTS (Mixed Waste Operations @ INEL) & & & & & & \\
\hline & Labor Based on 2080 Hrs Per Year & & & & & & \\
\hline & Operating Personnet, 0 & & - & - & - & & \\
\hline & Repair/Decon Maintenance Personnel. 1 Person & & $120 \mathrm{KNR}$ & 4.800 .000 & 14.900 .000 & FY2000 THRU FY2040 & EngineersEstimate Based on RWMC Study \\
\hline & Training Personnel, 0 & & - & - & - & & \\
\hline & Recordkeeping. 1 Person & & 7OKYR & $3,120,000$ & 9.700 .000 & FY2000 THRU FY2040 & EngineersEstimate Based on RWMC Study \\
\hline & Management, 1 Person & & 285KNR & 11.400 .000 & $35,300,000$ & FY2000 THRU FY2040 & Engineers Estimate Based on RWMC Sludy \\
\hline & Engineering. 1 Person & & 177KYR & $7.080,000$ & 22.000 .000 & FY2000 THRU FY2040 & EngineersEstimate Based on RWMC Sludy \\
\hline & ESH\&O Personnel, 0 & & - & - & - & & \\
\hline & Budget Support Personnel. 1 Person & & $115 \mathrm{~K} Y \mathrm{R}$ & 4.600 .000 & 14.200 .000 & FY2000 THRU FY2040 & EngineersEstimate Based on RWMC Study \\
\hline & Health Physics Personnel, 0 & & - & - & - & & \\
\hline & Lab Personnel, 0 & & - & - & - & & \\
\hline & Environmental Surveillance, 0 & & - & - & - & & \\
\hline & Document Control, 0 & & - & - & - & & \\
\hline & Shipping Coordinator, 1 Person & & $130 \mathrm{KNR}$ & 5.200 .000 & 16.100 .000 & FY2000 THRU FY2040 & EngineersEstimate Based on RWMC Study \\
\hline & Subtotal for Operating Costs & & & $267.800,000$ & 915.040 .000 & & \\
\hline
\end{tabular}


Page 6 of 7

Project EVALUATION OF ALTERNATVES FOR DISPOSAL OF INEL MIXED AND LOW LEVEL WASTE

\begin{tabular}{|c|c|c|c|c|c|c|c|}
\hline $\begin{array}{c}\text { ACCT. } \\
\text { NO. }\end{array}$ & $\begin{array}{c}\text { ACTIVITY } \\
\text { DESCRAPTION }\end{array}$ & $\begin{array}{l}\text { E.V. } \\
\text { P.H }\end{array}$ & $\begin{array}{c}\text { ESTIMATED COST } \\
\text { PER YEAR }\end{array}$ & $\begin{array}{c}\text { UNESCALATED } \\
\text { COST }\end{array}$ & $\begin{array}{l}\text { ESCALATED } \\
\text { COST } \\
\end{array}$ & $\begin{array}{l}\text { ACTIVITY SCHEDULE } \\
\text { ESCALATION }\end{array}$ & ESTIMATE BASIS \\
\hline & OPERATNG COSTS - Equipment & & & & & & \\
\hline & Equipment & & & & & & \\
\hline & Reptacement Parts - Misc Maintenance & & $100 \mathrm{KN}$ R & 4.000 .000 & $15.080,000$ & FY2003 THRU FY2043 & Engineers Estimate Based on RWMC Sludy \\
\hline & & & & & & & \\
\hline & & & & & & & \\
\hline & UTILITIES - Low-Levei Waste Operations & & & & & & \\
\hline & Electrical & & 2OKNR & 800,000 & 2.800 .000 & FY2003 THRU FY2043 & Engineers Estimate Based on RWMC Study \\
\hline & Fuels/Propane, etc. & & 5KYYR & 200.000 & 692.000 & FY2003 THRU FY2043 & Engineers Estimate Based on RWMC Study \\
\hline & Other & & $25 \mathrm{KYA}$ & 1.000 .000 & 3.500 .000 & FY2003 THRU FY2043 & Enginoers Estimate Based on RWMC Sludy \\
\hline & & & & & & & \\
\hline & UTILITIES - Mixed Waste Operations & & & & & & \\
\hline & Electrical & & 5KYR & 200.000 & 619.000 & FY2000 THRU FY2040 & Engineers Estimate Based on RWMC Study \\
\hline & Fuels/Propane. etc. & & - & - & - & & \\
\hline & Other & & $5 K M Y R$ & 200.000 & 619.000 & FY2000 THRU FY2040 & Engineers Estirnate Based on RWMMC Study \\
\hline & & & & & & & \\
\hline & fuototar uperaung Costs & & & $6,400,000$ & $23.310,000$ & & \\
\hline & TOTAL OPERATING COSTS & & & 274.200 .000 & 938.350 .000 & & \\
\hline & & & & & & & \\
\hline & & & & & & & \\
\hline & DECONTAMINATION AND DECOMMISSIONING & & & & & & \\
\hline & (D\&D Costs are based on $10 \%$ of the constructi & relatin & g directly to the tacilitie & s being decommiss & ioned) & & \\
\hline & Low-Level Waste Facilities & & 945.000 & 945.000 & 6.600 .000 & FY2044 & Data From Provious D \& D Projects \\
\hline & Mixed Waste Facilities & & 100.000 & 100.000 & 607.000 & FY2042 & Data From Provious D \& D Projects \\
\hline & & & & & & & \\
\hline & ITOTAL DER ROTC & & & & & & \\
\hline & TOTAL D O D COSTS & & & 1.045 .000 & 7.207 .000 & & \\
\hline & & & & & & & \\
\hline & & & & & & & \\
\hline
\end{tabular}


Project EVAWATION OF ALTERNATVES FOR DISPOSAL OF INEL MIXED AND LOW LEVEL WASTE

\begin{tabular}{|c|c|c|c|c|c|c|c|}
\hline $\begin{array}{l}\text { ACCT. } \\
\text { NO. }\end{array}$ & $\begin{array}{c}\text { ACTIVITY } \\
\text { DESCFIPTION }\end{array}$ & $\begin{array}{l}\text { E.V. } \\
\text { P.H }\end{array}$ & $\begin{array}{c}\text { ESTMAATED COST } \\
\text { PER YEAR }\end{array}$ & $\begin{array}{c}\text { UNESCALATED } \\
\text { COST }\end{array}$ & $\begin{array}{c}\text { ESCALATED } \\
\text { COST } \\
\end{array}$ & $\begin{array}{c}\text { ACTIVTY SCHEDULE } \\
\text { ESCALATION } \\
\end{array}$ & ESTIMATE BASIS \\
\hline & CLOSURE - Low-Level Waste & & & & & & \\
\hline & 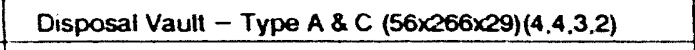 & & $200 \mathrm{~K} / 200 \mathrm{~K} / 150 \mathrm{~K} / 100 \mathrm{~K}$ & 650.000 & 2.430 .000 & FY2013.23.33,43 & Engineers Estimate \\
\hline & Disposal Vault - Type B \& C B/G $(58 \times 166 \times 31)$ (1) (1) & & 850K/850K & 1.700 .000 & 8.730 .000 & FY2024.2043 & Engineers Estimale \\
\hline & Sand & & $10.168,000$ & 10.168 .000 & 69.000 .000 & FY2044 & Engineers Estimate \\
\hline & Gravel & & 380,500 & 380.500 & $2,600,000$ & FY2044 & Engineers Estimate \\
\hline & Diatomaceous Earth & & 1.155 .000 & 1.155 .000 & 8.000 .000 & FY2045 & Engineers Estimate \\
\hline & Membrane Liner & & $1.326,000$ & $1,326,000$ & $9.300,000$ & FY2045 & Engineers Estimate \\
\hline & Clay & & 4.640 .000 & 4.640 .000 & 34.000 .000 & FY2045-46 & Engineers Estimate \\
\hline & Sand/Gravel & & $2.428,000$ & 2.428 .000 & 17.800 .000 & FY2046 & Engineers Estimate \\
\hline & Top Soil & & 1.155 .000 & 1.155 .000 & 8.800 .000 & FY2047 & Engineers Estimate \\
\hline & Vegetation & & 50.000 & 50.000 & 380.000 & FY2047 & Engineers Estimate \\
\hline & Instrumentation and Installation & & 150.000 & 150.000 & 1.000 .000 & FY2043 & Engineers Estimate \\
\hline & Technical Support. Sampling. \& OA Analysis (4 People) & & $177 \mathrm{~K} Y \mathrm{R}$ & 4.950 .000 & $35.000,000$ & FY2042-48 & Engineers Estimate \\
\hline & POST CLOSURE SURVEILLANCE & & & & & & \\
\hline & Environmental Surveillance. 3 People & & 125KYA & 4.125 .000 & 34.000 .000 & FY2044 THRU FY2054 & Engineers Estimate \\
\hline & Environmental Surveillance. 1 Person & & 125KNA & $11.250,000$ & 1.000 .000 .000 & FY2055 THRU FY 2144 & Engineers Estimate \\
\hline & CLOSURE - Mixed Waste Offsite & & & & & & \\
\hline & Sand & & 4.883 .000 & 4.883 .000 & 29.600 .000 & FY2041 & Engineers Estimate \\
\hline & Gravel & & 340.000 & 340.000 & 2.100 .000 & FY2041 & Engineers Estimate \\
\hline & Diatomaceous Earth & & 1.020 .000 & 1.020 .000 & 6.400 .000 & FY2042 & Engineers Estimate \\
\hline & Membrane Liner & & 1.164 .000 & $1.164,000$ & $7.300,000$ & FY2042 & Engineers Estimate \\
\hline & Clay & & 1.442 .000 & 1.442 .000 & 9.400 .000 & FY2042-43 & Engineers Estimate \\
\hline & Sand/Gravel & & 2.145 .000 & $2,145.000$ & $14,000,000$ & FY2043 & Engineers Estimate \\
\hline & Top Soil & & 1.085 .000 & $1,085,000$ & 7.400 .000 & FY2044 & Engineers Estimate \\
\hline & Vegetation & & 50.000 & 50.000 & 340.000 & FY2044 & Engineers Estimato \\
\hline & Instrumentation and Installation & & 150.000 & 150,000 & 900.000 & FY2040 & Engineers Estimate \\
\hline & Technical Suprort, Sampling. \& OA Analysis (1 People) & & 177/MA & 885.000 & 5.980 .000 & FY2041-45 & Engineers Estimate \\
\hline & POST CLOSURE SURVEILLANCE & & - & - & - & & \\
\hline & Subtotal for Closure and Post Closure & & & 57.300 .000 & $1,314.460,000$ & & \\
\hline
\end{tabular}


PROBABLE \% VARIATION

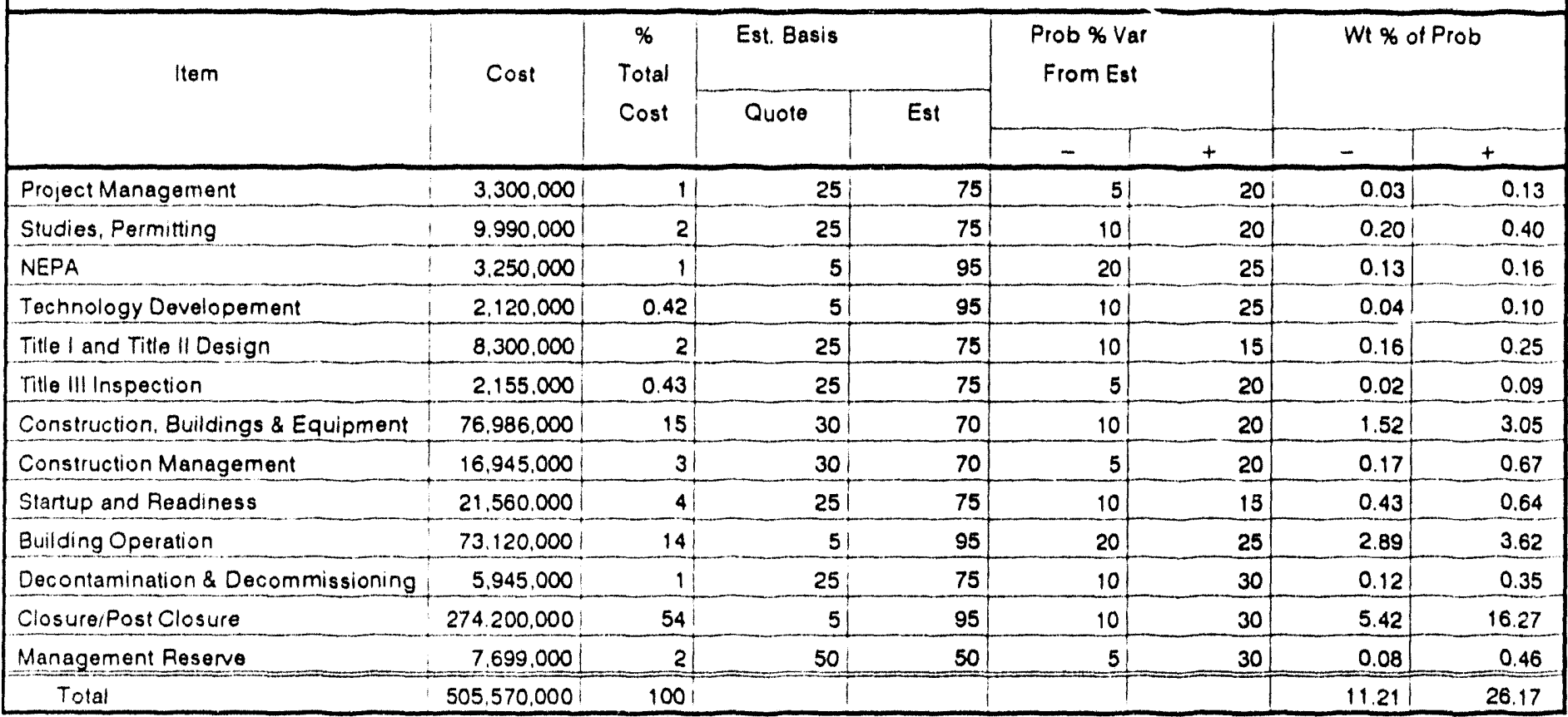




\section{EG\&G Idaho, Inc.}

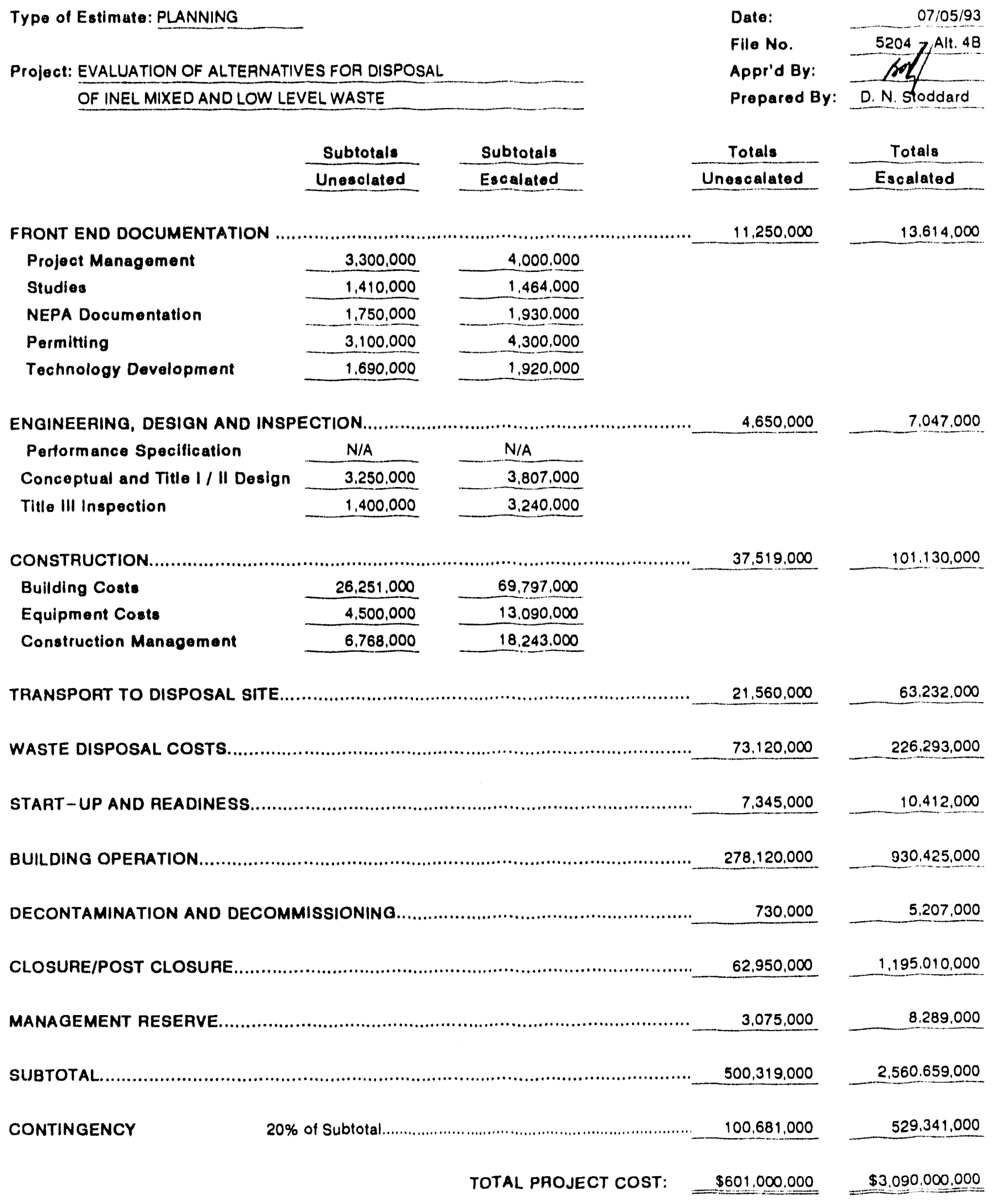


Piojecl EVALUATION OF ALTERNATIVES FOR DISPOSAL OF INEL MIXED AND LOW LEVEL WASTE INEL

Location

T. H. SMITH

Requester T.H.SMITH

\begin{tabular}{|c|c|}
\hline $\begin{array}{c}\text { ACCT. } \\
\text { NO. }\end{array}$ & $\begin{array}{c}\text { ACTIVITY } \\
\text { DESCPIPTION }\end{array}$ \\
\hline & PROJECT COSTS \\
\hline & Project Management \\
\hline & Project Studies - 2 People. Full Time \\
\hline & Project Formulation (F3OR's) \\
\hline & Siting Studies \\
\hline & Pre-Operational Monitoring \\
\hline & Safety Analysis Report (SAR) \\
\hline & Performance Assessment \\
\hline & RCRA \\
\hline & NEPA (EIS) (CX's) \\
\hline & Air Permits (PSD/PTC/NESHAP) \\
\hline & ESH\&O \\
\hline & Other Regulatory Items (State Water Well Approval) \\
\hline & Special O/A Program For Construction - 1 Person \\
\hline & \\
\hline & \\
\hline & \\
\hline & ( \\
\hline & Cover Perlormance Control \\
\hline & Leachate Collection and Liner Performance \\
\hline & Material Handling (Gantry Crane) \\
\hline & \\
\hline & \\
\hline & \\
\hline & \\
\hline & Subtotal for Front End Costs \\
\hline
\end{tabular}

Type of Estimate PLANNING

Source (E) Eng. Est.

(M) Vendor

(P) Pur. Order

(H) Handbook Ref

\begin{tabular}{|c|c|c|c|c|c|}
\hline $\begin{array}{l}\text { E.V. } \\
\text { P.H }\end{array}$ & $\begin{array}{c}\text { ESTMATED COST } \\
\text { PER YEAR }\end{array}$ & $\begin{array}{c}\text { UNESCALATED } \\
\text { COST }\end{array}$ & $\begin{array}{c}\text { ESCALATED } \\
\text { COST }\end{array}$ & $\begin{array}{l}\text { ACTIVIY SCHEDULE } \\
\text { ESCALATION }\end{array}$ & ESTIMATE BASIS \\
\hline & & & & & \\
\hline & $300 \mathrm{KYYR}$ & 3.300 .000 & 4.000 .000 & FY92 THRU FY2002 & TDD Sheet. dated $4 / 28 / 93$ \\
\hline & 25OKVRVEA & 1.000 .000 & 1.040 .000 & FY $93-94$ & TDD Sheet. dated 4/28/93 \\
\hline & $70 \mathrm{~K} / 270 \mathrm{~K}$ & 340.000 & 350.000 & FY92 - 93 & IDD Sheet. dated 4/28/93. Eng Estimate \\
\hline & $70 \mathrm{~K}$ & 70.000 & 74.000 & FY94 & TOD Sheet. dated 4/28/93. Eng Estimate \\
\hline & - & - & - & & \\
\hline & $100 \mathrm{~K} / 200 \mathrm{~K} / 400 \mathrm{~K} / 100 \mathrm{~K}$ & 1.200 .000 & 1.720 .000 & $95-96.97 .98-99.00-01$ & Engireer's Estimate \\
\hline & $200 k$ & 800,000 & 960.000 & FY95-96.FY99-00 & Eng noer's Estimate \\
\hline & - & - & - & & \\
\hline & $500 \mathrm{~K} 500 \mathrm{~K} / 500 \mathrm{~K} / 250 \mathrm{~K}$ & 1.750 .000 & 1.930 .000 & FY94-FY97 & Engineer's Estimate \\
\hline & $200 \mathrm{~K} / 150 \mathrm{~K}$ & 350.000 & 550.000 & FY1998. FY1999 & Engineer's Estimate \\
\hline & 5OKNR & 400,000 & 570.000 & FY1995-2002 & Engineer's Estimate \\
\hline & - & - & - & & \\
\hline & $177 \mathrm{~K} N \mathrm{R}$ & 354.000 & 500.000 & FY2000-01 & Engineer's Estimate \\
\hline & & & & & \\
\hline & & & & & \\
\hline & & & & & \\
\hline & & & & & \\
\hline & & & & & \\
\hline & $65 \mathrm{KNR}$ & 330.000 & 370.000 & FY94, FY95-98 & Engineer's Estimate \\
\hline & $100 \mathrm{~K} / 65 \mathrm{KNR}$ & 360.000 & 410.000 & FY94, FY95-98 & Engineer's Estimate \\
\hline & $200 \mathrm{KNA}$ & $1.000,000$ & $1.140,000$ & FY94-98 & Engineer's Estimate \\
\hline & - & - & $=$ & & \\
\hline & & & & & \\
\hline & & & & & \\
\hline & & & & & \\
\hline & & & & & \\
\hline & & & & & \\
\hline & & 11.254 .000 & 13.614 .000 & & \\
\hline
\end{tabular}

Date:

Chk'd By:

Appr'd By: fogf

ESTIMATE BASIS 
Project EVALUATION OF ALTERNATIVES FOR DISPOSAL OF INEL MIXED AND LOW LEVEL WASTE

\begin{tabular}{|c|c|c|c|c|c|c|c|}
\hline \multirow[t]{9}{*}{ No. } & $\begin{array}{c}\text { ACTIVIT } \\
\text { DESCFIPTION }\end{array}$ & E.V. & $\begin{array}{c}\text { ESTIMATED COST } \\
\text { PEA YEAA }\end{array}$ & $\begin{array}{c}\text { UNESCALATED } \\
\text { COST }\end{array}$ & $\begin{array}{c}\text { ESCALATED } \\
\text { COST } \\
\end{array}$ & $\begin{array}{l}\text { ACTIVTY SCHEDULE } \\
\text { ESCALATION }\end{array}$ & ESTIMATE BASIS \\
\hline & DESIGN & & & & & & \\
\hline & Conceptual & & $500 \mathrm{~K} / 300 \mathrm{~K}$ & 800.000 & 887.000 & FY95.FY96 & TDD Sheet \\
\hline & Title 1 & & $1.2 \mathrm{M}$ & 1.200 .000 & 1.410 .000 & FY97 & Engineers Estimate \\
\hline & Titie II & & $1 \mathrm{M} / 250 \mathrm{~K}$ & 1.200 .000 & 1.510 .000 & FY98.FY2022 & Engineers Estimate \\
\hline & Subtotal for Design (Based on $\%$ ol Construction Costs) & & & 3.250 .000 & $3.807,000$ & & \\
\hline & & & & & & & \\
\hline & Tille III Inspection (1 Person/12 Miths. 4 People/30 Miths) & & $140 \mathrm{~K} / 420 \mathrm{~K} / 420 \mathrm{~K} / 210 \mathrm{~K}$ & 1.400 .000 & 3.240 .000 & FY99.FY2000,01.02,03.23.24 & Comparative Historical Data \\
\hline & CONSTRUCTION & & & & & & \\
\hline \multirow[t]{13}{*}{4000} & Improvements to Land & & & & & & \\
\hline & Surveying (15 Acres \& 2 Acres) & & $20 K \& 3 K$ & 23.000 & 30.000 & FY1999.FY2002 & Comparative Historical Data \\
\hline & Site Prep Clearing and Grubbing (15 Acres \& 2 Acres) & & $45 K \& 3 K$ & 48.000 & 61.000 & FY1999,FY2002 & Comparative Hislorical Data \\
\hline & Roadways and Parking & & $370 K \& 750 K$ & 1.120 .000 & 1.530 .000 & FY1999,FY2002 & Comparative Historical Data \\
\hline & Fencing & & 100.000 & 325.000 & 428.000 & FY2002 & Comparative Historical Data \\
\hline & Retention Pond & & - & - & - & & \\
\hline & Drainage Gallery System & & 600.000 & 600.000 & 1.130 .000 & FY2002 & Comparative Historical Data \\
\hline & Drainage Pumphouse & & 100.000 & 100.000 & 142.000 & FY2002 & Comparative Historical Data \\
\hline & Collection /Sampling System Vault at Disposal Areas & & 600.000 & 600.000 & 454.000 & FY2002 & Comparative Historical Data \\
\hline & Storage Vault Disposal Area Including: Engineered & & - & - & - & & \\
\hline & Fill. and Pit Run & & & & & & \\
\hline & Drainage Ditch/Berm Around Pads @RWMC (8,000 In) & & 60.000 & 60.000 & 85.000 & FY2002 & Comparative Historical Data \\
\hline & TOTAL FOR 4000 ACCOUNT & & & 2.876 .000 & 3.860 .000 & & \\
\hline \multirow[t]{8}{*}{6000} & Utilities (@WERF. Assume 200' Run To Treatment Facility & & & & & & \\
\hline & Electrical Power and Distribution To Facility & & 100.000 & 100.000 & 128.000 & FY99 & Engineers Estimate \\
\hline & Electrical Lighting.Power.Alarms.\& Communication & & 700.000 & 700.000 & 1.710 .000 & FY1999. FY 2002 & Engineers Estimate \\
\hline & Distribution System. RWMC Upgrades Onty & & & & & & \\
\hline & Water Supply \& Fire Water Distribution to Facility & & 20,000 & 20.000 & 25.000 & FY1999 & Engineers Estimate \\
\hline & Water Supply and Fire Water Distribution Systems & & 150.000 & 150.000 & 2.130 .000 & FY2000 & Engineers Estumate \\
\hline & Sanitary Sewage System & & 20.000 & 20.000 & 25.000 & FY1999 & Engineers Estimate \\
\hline & TOTAL FOR $600 \mathrm{U}$ ACCOUNT & & & 990.000 & 4.018 .000 & & \\
\hline
\end{tabular}


Page 3 of 7

Project EVALUATION OF ALTERNATIVES FOR DISPOSAL OF INEL MIXED AND LOW LEVEL WASTE

\begin{tabular}{|c|c|c|c|c|c|c|c|}
\hline $\begin{array}{l}\text { ACCT. } \\
\text { NO. }\end{array}$ & $\begin{array}{c}\text { ACTIVITY } \\
\text { DESCPAPTION }\end{array}$ & E.V. & $\begin{array}{c}\text { ESTIMATED COST } \\
\text { PER YEAR }\end{array}$ & $\begin{array}{c}\text { UNESCALATED } \\
\text { COST }\end{array}$ & $\begin{array}{c}\text { ESCALATED } \\
\text { COST }\end{array}$ & $\begin{array}{l}\text { ACTIVIT SCHEDULE } \\
\text { ESCALATION }\end{array}$ & ESTIMATE BASIS \\
\hline \multirow[t]{30}{*}{5000} & Buildings and Structures - Low Level Waste Disposal & & & & & & \\
\hline & Operations Control BId' $\mathrm{g}$. Replacement (1 story-24.0 & $93 \mathrm{sl}$ & 3.050 .000 & $3.050,000$ & 9.460 .000 & FY2023 & Comparative Historical Data \\
\hline & Material Storage Building. Replacement (1 story - 450 & $\underline{0}$ sf & 639.000 & 639.000 & 1.980 .000 & FY2023 & Comparative Historical Data \\
\hline & Mainterance Facility. Replacement (1 story - 3500 sn) & & 649,000 & 649.000 & 2.117 .000 & FY2024 & Comparative Historical Data \\
\hline & Disposal Pad - Class A. L w-Level Waste (423' $\times 324^{\prime}$ & & 3.400 .000 & 3.400 .000 & 4.150 .000 & FY2001 & Comparative Historical Data \\
\hline & Disposal Pad - Class A. Low - Level Waste (241'x589' & & $3.600,000$ & 3.600 .000 & 4.400 .000 & FY2001 & Comparative Historical Data \\
\hline & Disposal Vaults - Class BaC Beta/Gamma & & $260 \mathrm{KYR}$ & $9.100,000$ & $28,100,000$ & FY2004-FY2038 & Comparative Historical Data \\
\hline & & & & & & & \\
\hline & Buildings and Structures - Mixed Waste Disposal & & & & & & \\
\hline & Office Space Added to Treatment Faciip (2500 sf) & & 400.000 & 400.000 & 507.000 & FY99 & Comparative Historical Data \\
\hline & Truck Maintenance/Decon Facility ( $3500 \mathrm{sf}$ ) & & 450.000 & 450.000 & 570.000 & FYg9 & Comparative Historical Data \\
\hline & & & & & & & \\
\hline & TOTAL FOR 5000 ACCOUNT & & & 21.288 .000 & 51.284 .000 & & \\
\hline & & & & & & & \\
\hline & & & & & & & \\
\hline & WASTE TRANSPORT TO DISPOSAL COSTS (Mixed Waste & Only) & & & & & \\
\hline & Transportation based on a 1000 mile haul. containers re & resente & ted by 71 gallon sq drur & $\mathrm{ms}(1-$ way $)$ and $\mathrm{CN}$ & VS $-21-300$ cask & is (requires back - haul, 2-wa & \\
\hline & Class A Mixed & & 273.000 & 10.920 .000 & 33.796 .000 & FY2000 THRU FY 2040 & Comparative Historical Data \\
\hline & Class B Mixed & & 30.000 & $1,200.000$ & 176.000 & FY2000 THRU FY 2040 & Comparative Historical Data \\
\hline & Class C Beta-Gamma Mixed & & 15.000 & 600.000 & $1.860,000$ & FY2000 THRU FY 2040 & Comparative Historical Data \\
\hline & Class C Alpha Mixed & & 221.000 & $8.840,000$ & $27,400,000$ & FY2000 THRU FY 2040 & Comparative Historical Data \\
\hline & & & & & & & \\
\hline & TOTAL OF WASTE TRANSPORT COSTS & & & $21,560,000$ & $63,232,000$ & & \\
\hline & & & & & & & \\
\hline & WASTE DISPOSAL COSTS (Offsite Disposal of Mixed Was & Only) & & & & & \\
\hline & Class A Mixed & & 1.500 .000 & 60.000 .000 & 185.690 .000 & FY2000 THRU FY 2040 & Estimate From NTS \\
\hline & Class B Mixed & & 51.000 & $2,040.000$ & $6,313,000$ & FY2000 THRU FY 2040 & Estimate From NTS \\
\hline & Class C Beta-Gamma Mixed & & 25,000 & 1.000 .000 & $3,095,000$ & FY2000 THRU FY 2040 & Estimate From NTS \\
\hline & Class C Alpha Mixed & & 252.000 & 10.080 .000 & 31.195 .000 & FY2000 THRU FY 2040 & Estimate From NTS \\
\hline & TOTAL OF WASTE DISPOSAL COSTS & & & 73.120 .000 & 226.293 .000 & & \\
\hline
\end{tabular}


Rev $5-y$ -

Page 4 of 7

Project

EVALUATION OF ALTERNATIVES FOR DISPOSAL OF INEL MIXED AND LOW LEVEL WASTE

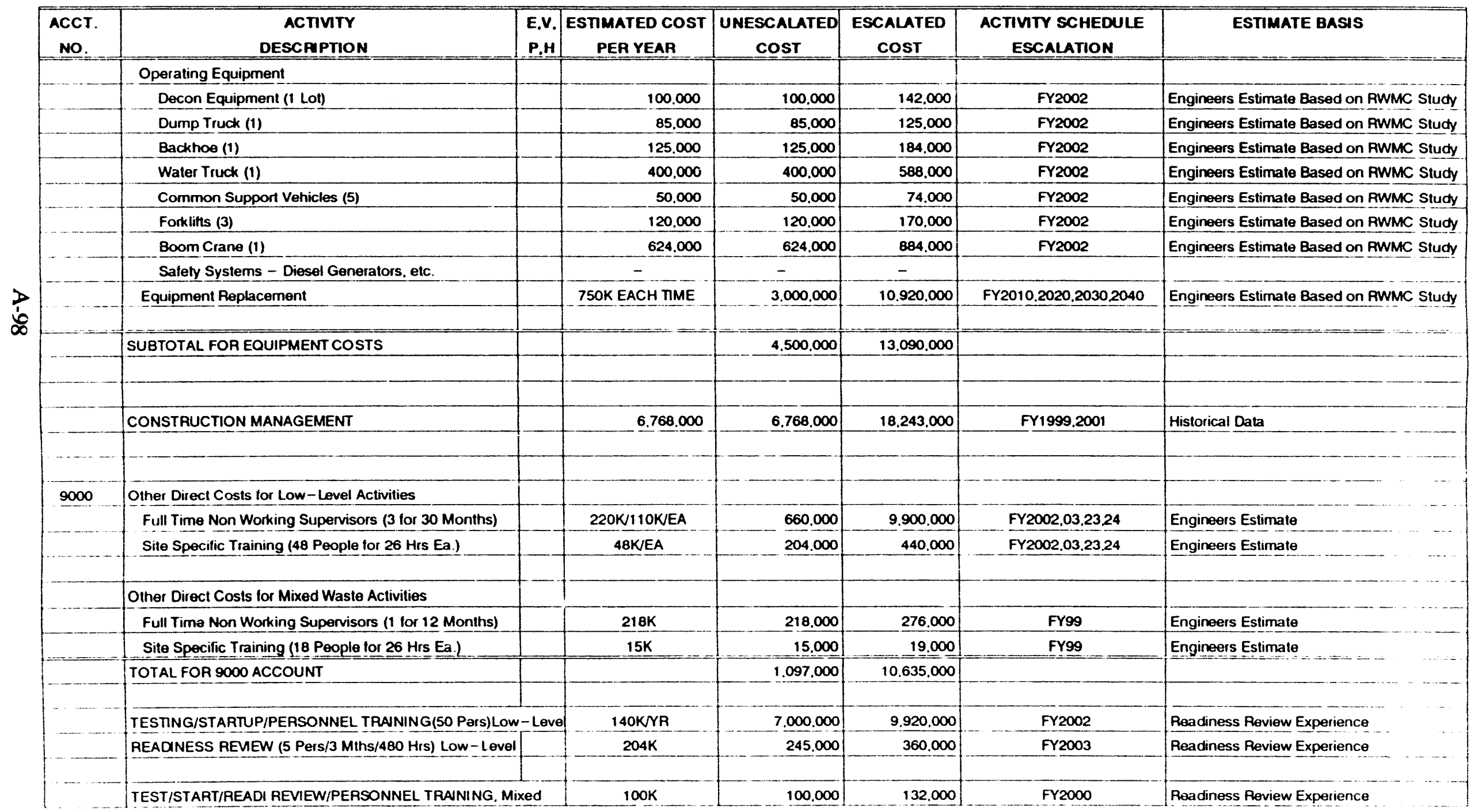


Project EVALUATION OF ALTERNATIES FOR DISPOSAL OF INEL MIXED AND LOW LEVEL WASTE

\begin{tabular}{|c|c|c|c|c|c|c|c|}
\hline $\begin{array}{l}\text { ACCT. } \\
\text { NO. }\end{array}$ & $\begin{array}{c}\text { ACTIVITY } \\
\text { DESCPIPTION }\end{array}$ & E.V. & $\begin{array}{l}\text { ESTMATED COST } \\
\text { PER YEAR }\end{array}$ & $\begin{array}{l}\text { UNESCALATED } \\
\text { COST }\end{array}$ & $\begin{array}{l}\text { ESCALATED } \\
\text { COST }\end{array}$ & $\begin{array}{l}\text { ACTIVTY SCHEDULE } \\
\text { ESCALATION }\end{array}$ & ESTIMATE BASIS \\
\hline$\ldots$ & OPERATING COSTS (Low - Level Waste Operations) & & & & & & \\
\hline & Operaling Personnel. 13 People & & $125 \mathrm{KNR}$ & 65.000 .000 & $219.050,000$ & FY2002 THRU FY 2042 & Engineers Estimate Based on RWMC Sludy \\
\hline & Maintenance Personnet. 6 People & & $120 \mathrm{KNR}$ & 29.520 .000 & 108.545 .000 & FY2002 THRU FY 2042 & Engineers Estimate Based on RWMC Study \\
\hline & Training Personnel. 2 People & & 96KYR & 7.680 .000 & 27.000 .000 & FY2002 THRU FY 2042 & Engineers Estimate Based on RWMC Study \\
\hline & Recorckeeping. 2 People & & $78 \mathrm{KNR}$ & 6.240 .000 & 10.800 .000 & FY2002 THRU FY 2042 & Engineers Estimate Based on RWMC Study \\
\hline & Management, 3 People & & $285 K N R$ & $34.200,000$ & 118.400 .000 & FY2002 THRU FY 2042 & Engineers Estimate Based on RWMC Study \\
\hline & Engineering. 3 People & & 177KNR & 21.240 .000 & 73.500 .000 & FY2002 THRU FY 2042 & Engineers Estimate Based on RWMC Study \\
\hline & ESH\&O Personnel, 3 People & & 178KNA & 24.360 .000 & $74,000,000$ & FY2002 THAU FY 2042 & Engineers Estimate Based on RWMC Study \\
\hline & Budgel Suppont Personnel. 3 People & & $115 \mathrm{KNA}$ & 13.800 .000 & 47.800 .000 & FY2002 THRU FY 2042 & Engineers Estimate Based on RWMC Study \\
\hline & Health Physics Personnel. 2 People & & $128 \mathrm{~K} Y \mathrm{P}$ & 10.240 .000 & 35.400 .000 & FY2002 THRU FY 2042 & Engineers Estimate Based on RWMC Study \\
\hline & Lab Personnel. 1 Person & & $128 \mathrm{~K} Y \mathrm{R}$ & 5.120 .000 & 17.700 .000 & FY2002 THRU FY 2042 & Engineers Estimate Based on RWMC Study \\
\hline & Environmental Surveillance. 3 People & & $125 \mathrm{KMR}$ & 15.000 .000 & 52.000 .000 & FY2002 THRU FY 2042 & Engineers Estimate Based on RWMC Study \\
\hline & Document Control. 1 Person & & 78KYYR & $3,120,000$ & 10.800 .000 & FY2002 THRU FY 2042 & Engineers Estimate Based on RWMC Study \\
\hline & & & & & & & \\
\hline & OPERATING COSTS (Mixed Waste Operations) & & & & & & \\
\hline & Labor Based on 2080 Hrs Per Year & & & & & & \\
\hline & Operating Personnel, 0 & & - & - & - & & \\
\hline & Repair/Decon Maintenance Personnel. 1 Person & & 120KVY & $4.800,000$ & $14.900,000$ & FY2000 THRU FY2040 & EngineersEstimale Based on RWMC Study \\
\hline & Training Personnel. 0 & & - & - & $=$ & & \\
\hline & Recordkeeping. 1 Person & & 78KNR & 3.120 .000 & 9.700 .000 & FY2000 THAU FY2040 & EngineersEstimate Based on RWMC Study \\
\hline & Management. 1 Person & & $285 \mathrm{KNR}$ & $11.400,000$ & $35.300,000$ & FY2000 THRU FY2040 & Engineers Estimate Based on RWMC Study \\
\hline & Engineering. 1 Person & & 177KNR & 7.080 .000 & $22,000,000$ & FY2000 THRU FY2O40 & EngineersEstimate Based on RWMC Study \\
\hline & ESH\&O Personnel. 0 & & - & - & - & & \\
\hline & Budget Support Personnel. 1 Person & & $115 \mathrm{KNR}$ & 4.600 .000 & $14.200,000$ & FY2000 THRU FY2040 & EngineersEstimate Based on RWMC Study \\
\hline & Health Physics Personnel. 0 & & - & - & - & & \\
\hline & Lab Personnel. 0 & & - & - & - & & \\
\hline & Environmental Surveillance. 0 & & - & - & - & & \\
\hline & Document Control. 0 & & - & - & - & & \\
\hline & Shipping Coordinator, 1 Person & & $130 \mathrm{KYYR}$ & 5.200 .000 & 16.100 .000 & FY2000 THRU FY2040 & Engineers Estimate Based on RWMC Study \\
\hline & Subtotal for Operating Costs & & & 271.720 .000 & 907.195 .000 & & \\
\hline
\end{tabular}


Project EVALATION OF ALTERNATIVES FOR DISPOSAL OF INEL MIXED AND LOW LEVEL WASTE

\begin{tabular}{|c|c|c|c|c|c|c|c|}
\hline $\begin{array}{l}\text { ACCT. } \\
\text { NO. }\end{array}$ & $\begin{array}{c}\text { ACTIVIY } \\
\text { DESCPIPTION }\end{array}$ & $\begin{array}{l}\text { E.V. } \\
\text { P.H }\end{array}$ & $\begin{array}{c}\text { ESTIMATED COST } \\
\text { PER YEAR }\end{array}$ & $\begin{array}{c}\text { UNESCALATED } \\
\text { COST }\end{array}$ & $\begin{array}{c}\text { ESCALATED } \\
\text { COST }\end{array}$ & $\begin{array}{l}\text { ACTIVITY SCHEDULE } \\
\text { ESCALATION }\end{array}$ & ESTIMATE BASIS \\
\hline & OPERATING COSTS - Equipment & & & & & & \\
\hline & Equipment & & & & & & \\
\hline & Replacement Parts - Misc. Maintenance & & 100KNR & $4.000,000$ & 15.000 .000 & FY2002 THRU FY 2042 & Engineers Estimate Based on RWMC Study \\
\hline & & & & & & & \\
\hline & & & & & & & \\
\hline & UTIUTIES - Low - Lovel Waste Operations & & & & & & \\
\hline & Electrical & & 20KYR & 800.000 & 2.800 .000 & FY2002 THRU FY 2042 & Engineers Estimate Based on RWMC Study \\
\hline & Fuels/Propane, etc. & & 5KYYR & 200,000 & 692.000 & FY2002 THRU FY 2042 & Engineers Estimate Based on RWMC Study \\
\hline & Other & & $25 \mathrm{KNR}$ & 1.000 .000 & 3.500 .000 & FY2002 THRU FY 2042 & Engineers Estimate Based on RWMC Study \\
\hline & & & & & & & \\
\hline & UTIUTIES - Mixed Waste Operations & & & & & & \\
\hline & Electrical & & 5KYYA & 200.000 & 619.000 & FY2000 THRU FY2040 & Engineers Estimate Based on RWMC Study \\
\hline & Fuels/Propane. etc. & & $=$ & - & - & & \\
\hline & Other & & 5K/YA & 200.000 & 619.000 & FY2000 THRU FY2040 & Engineers Estimate Based on RWMC Study \\
\hline & Subtotal Operating Costs & & & 6.400 .000 & $23,230,000$ & & \\
\hline & & & & & & & \\
\hline & TOTAL OPERATING COSTS & & & 278.120 .000 & 930.425 .000 & & \\
\hline & & & & & & & $\cdots$ \\
\hline & & & & & & & - \\
\hline & DECONTAMINATION AND DECOMMISSIONING & & & & & & \\
\hline & (D\&D Costs are based on $10 \%$ of the construction costs & relating & g directly to the facilities & s being decommissi & ioned) & & \\
\hline & Low-Level Waste Facilities & & 630.000 & 630,000 & 4.600 .000 & FY2043 & Data From Previous D \& D Projects \\
\hline & Mixed Waste Facilities & & 100.000 & 100.000 & 607.000 & FY2041 & Data From Previous D\& D Projects \\
\hline & & & & & & & - \\
\hline & 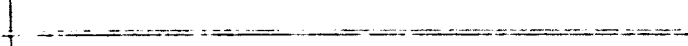 & $=$ & 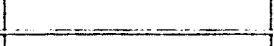 & $\ldots=$ & $==-=-=$ & 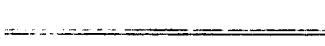 & $\ldots \ldots$ \\
\hline & TOTALD \& D COSTS & & & 730.000 & 5.207 .000 & & $\ldots$ \\
\hline & & & & & & & $\cdots$ \\
\hline-7 & $\ldots$ & & & & & & $\ldots-\ldots-$ \\
\hline
\end{tabular}


Page 7 of 7

Project EVALUATION OF ALTERNATIVES FOR DISPOSAL

OF INEL MIXED AND LOW LEVEL WASTE

\begin{tabular}{|c|c|c|c|c|c|c|c|}
\hline $\begin{array}{c}\text { ACCT. } \\
\text { №. }\end{array}$ & $\begin{array}{c}\text { ACTIVITY } \\
\text { DESCPAPIION }\end{array}$ & $\begin{array}{l}\text { E.V. } \\
\text { P.H }\end{array}$ & $\begin{array}{c}\text { ESTMMATED COST } \\
\text { PER YEAR }\end{array}$ & $\begin{array}{c}\text { UNESCALATED } \\
\text { COST }\end{array}$ & $\begin{array}{c}\text { ESCALATED } \\
\text { COST } \\
\end{array}$ & $\begin{array}{l}\text { ACTIVTY SCHEDULE } \\
\text { ESCALATION }\end{array}$ & ESTIMATE BASIS \\
\hline & \multicolumn{7}{|c|}{ TEMPORARY CLOSURE - LOW - Level Waste (Pre-engineered metal building shell) } \\
\hline & Disposal Pad = Class AVLLW (423'x324') & & 10OKYR & 4.000 .000 & 13.340 .000 & FY2002 THRU FY 2042 & Engineers Estimate \\
\hline & Disposal Pad - Class AVLLW $\left(241^{\prime} \times 589^{\prime}\right)$ & & 10OKYR & 4.000 .000 & $13.340,000$ & FY2002 THRU FY 2042 & Engineers Estimate \\
\hline & FINAL CLOSURE - Low - Level Waste & & & & & & \\
\hline & Sand & & 10.168 .000 & 10.168 .000 & 69.000 .000 & FY2043 & Engineers Estimate \\
\hline & Gravel & & 380.500 & 380.500 & 2.600 .000 & FY2043 & Engineers Estimate \\
\hline & Diatomaceous Earth & & 1.155 .000 & 1.155 .000 & 8.000 .000 & FY2044 & Engineers Estimate \\
\hline & Membrane Liner & & 1.326 .000 & 1.326 .000 & 9.300 .000 & FY2044 & Engineers Estimate \\
\hline & Clay & & $4.640,000$ & $4,640,000$ & 34.000 .000 & FY2044-45 & Engineers Estimate \\
\hline & Sand/Gravel & & 2.428 .000 & 2.428 .000 & 17.800 .000 & FY2045 & Engineers Estimate \\
\hline & Top Soil & & 1.155 .000 & 1.155 .000 & $8.800,000$ & FY2046 & Engineers Estimate \\
\hline & Vegetation, 27 Acres & & 50.000 & 50,000 & 380,000 & FY2046 & Engineers Estimate \\
\hline & Instrumentation and Installation & & 150.000 & 150,000 & $1,000.000$ & FY2042 & Engineers Estimate \\
\hline & Technical Support, Sampling. \& QA Analysis (4 People) & & $177 \mathrm{KYYR}$ & 4.956 .000 & 35.000 .000 & FY2041-47 & Engineers Estimate \\
\hline & POST CLOSURE SURVEILLANCE & & & & & & \\
\hline & Environmental Surveillance, 3 People & & $125 \mathrm{KMR}$ & 4.125 .000 & 34.000 .000 & FY2042 THRU FY2052 & Engineers Estimate \\
\hline & Environmental Surveillance. 1 Person & & $125 \mathrm{KYR}$ & 11.250 .000 & 864.990 .000 & FY2053 THRU FY21 42 & Engineers Estimate \\
\hline & CLOSURE - Mixed Waste & & & & & & \\
\hline & Sand & & $4.883,000$ & 4.883 .000 & 29.600 .000 & FY2041 & Engineers Estimate \\
\hline & Gravel & & 340.000 & 340,000 & 2.100 .000 & FY2041 & Engineers Estimate \\
\hline & Diatomaceous Earth & & $1.020,000$ & 1.020 .000 & 6.400 .000 & FY2042 & Engineers Estimate \\
\hline & Membrane Liner & & 1.164 .000 & $1,164.000$ & $7,300,000$ & FY2042 & Engineers Estimate \\
\hline & Clay & & $1.442,000$ & $1.442,000$ & $9.400,000$ & FY2042-43 & Engineers Estimate \\
\hline & Sand/Gravel & & 2.145 .000 & 2.145 .000 & $14.000,000$ & $\mathrm{FY} 2043$ & Engineers Estimate \\
\hline & Top Soil & & 1.085 .000 & 1.085 .000 & 7.400 .000 & FY2044 & Enginoers Estimate \\
\hline & Vegetation & & 54.000 & 54.000 & 380.000 & FY2044 & Engineers Estimate \\
\hline & Instrumentation and Installation & & 150,000 & 150.000 & 900.000 & FY2040 & Engineers Estimate \\
\hline & Technical Support. Sampling. \& OA Analysis (1 People) & & 177/YR & 885,000 & 5.980 .000 & FY2041-45 & Engineers Estimate \\
\hline & POST CLOSURE SURVEILLANCE & & - & - & - & & \\
\hline & Subtotal for Closure and Post Closure & & & 62.950 .000 & 1.195 .010 .000 & & \\
\hline
\end{tabular}


Project EVALUATION OF ALTERNATIVES FOR DISPOSAL Type of Est. PLANNING

Location INEL

File No.

$5204-$ Alt. $4 B$

Date

Prep'd By

Requester T. H. SMITH

App'd By $07 / 05 / 93$ DNS

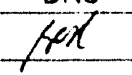

\section{PROBABLE $\%$ VARIATION}

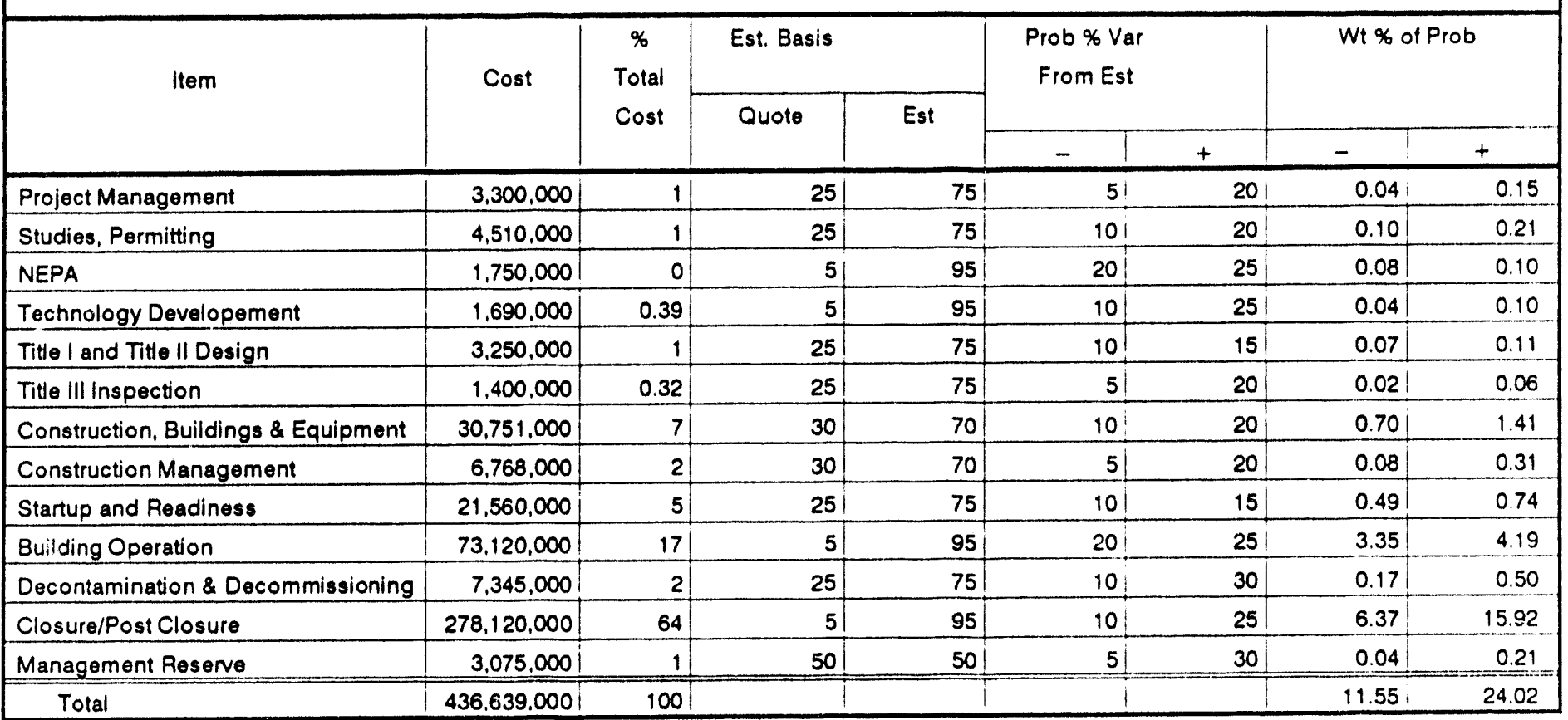


EG\&G Idaho, Inc.

Type of Estimate: PLANNING

Date:

File No.

Project: EVALUATION OF ALTERNATIVES FOR DISPOSAL OF INEL MIXED AND LOW LEVEL WASTE

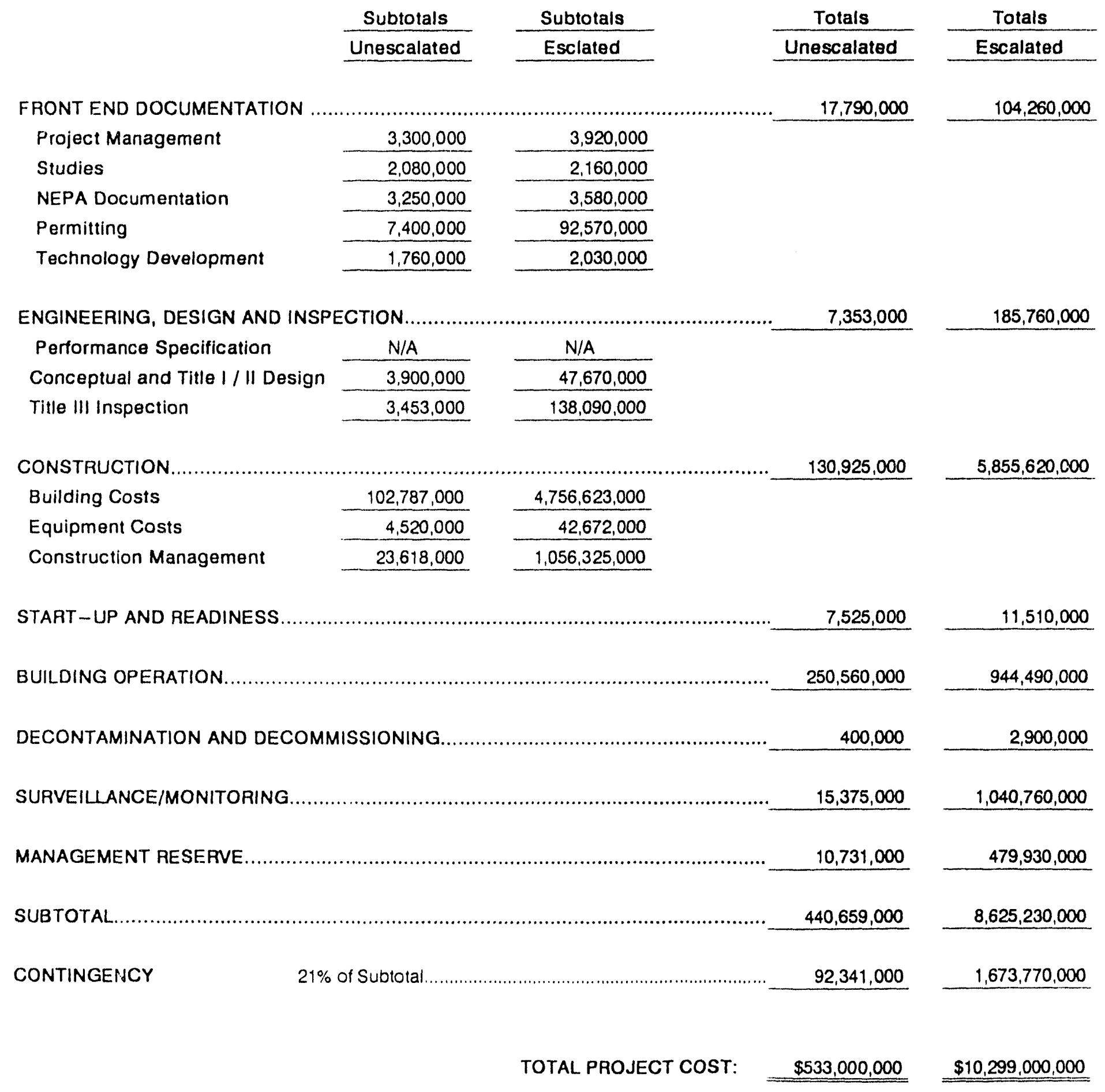

Comments: 
Kevs-92

Project EVAWATION OF ALTERNATIVES FOR DISPOSAL OF INEL MIXED AND LOW LEVEL WASTE

Location INEL

Pequester T.H.SMITH

\begin{tabular}{|c|c|}
\hline $\begin{array}{l}\text { ACCT. } \\
\text { No. }\end{array}$ & $\begin{array}{c}\text { ACTIVITY } \\
\text { DESCPIPTION }\end{array}$ \\
\hline & PROJECT COSTS \\
\hline & Project Management \\
\hline & Project Studies - 2 People. Full Time \\
\hline & Project Formulation (F\&OR's) \\
\hline & Siting Studies \\
\hline & Pre-Operational Monitoring \\
\hline & Safety Analysis Report (SAR) \\
\hline & Performarice Assessment \\
\hline & RCRA \\
\hline & NEPA (ESS)(CX's) \\
\hline & Air Permits (PSD/PTC/NESHAP) \\
\hline & ESH\&O \\
\hline & Other Regulatory Items (State Water Well Approval) \\
\hline & Special Q/A Program For Construction - 1 People \\
\hline & \\
\hline & \\
\hline & TECHNOLOGY DEVELOPMENT \\
\hline & Material Behavior (Conc.. Clay. etc.) \\
\hline & Cover Performance Control \\
\hline & Leachate Collection and Liner Performance \\
\hline & Material Handing (Gantry Crane) \\
\hline & \\
\hline & \\
\hline & \\
\hline & \\
\hline & \\
\hline & Subtotal for Front End Costs \\
\hline
\end{tabular}

Type of Estimate PLANNING

Source (E) Eng. Est.

$M$ Vendor

(P) Pur. Order

(H) Handbook Rol

\begin{tabular}{|c|c|c|c|c|c|}
\hline $\begin{array}{l}\text { E.V. } \\
\text { P.H }\end{array}$ & $\begin{array}{c}\text { ESTIMATED COST } \\
\text { PER YEAR }\end{array}$ & $\begin{array}{c}\text { UNESCALATED } \\
\text { COST }\end{array}$ & $\begin{array}{c}\text { ESCALATED } \\
\text { COST }\end{array}$ & $\begin{array}{l}\text { ACTIVITY SCHEDULE } \\
\text { ESCALATION }\end{array}$ & ESTIMATE BASIS \\
\hline & $300 \mathrm{KYR}$ & 3.300 .000 & $3.920,000$ & FY92 THRU FY2002 & TDD Sheet, dated 4/28/93 \\
\hline & 250KNRVEA & 1.000 .000 & 1.040 .000 & FY93 - 94 & TDD Sheet, dated 4/28/93 \\
\hline & $70 \mathrm{~K} / 270 \mathrm{~K}$ & 340.000 & 350,000 & FY92 - 93 & TDD Sheet, dated 4/28/93. Eng. Estimate \\
\hline & $70 \mathrm{~K} / 270 \mathrm{~K} / 400 \mathrm{~K}$ & 740.000 & 770.000 & FY92 - FY94 & TDD Sheet, dated 4/28/93. Eng. Estimate \\
\hline & $500 \mathrm{~K} / 500 \mathrm{~K} 250 \mathrm{KNA}$ & 1.750 .000 & 2.200 .000 & FY95,FY99.FY2000-2002 & Engineer's Estimate \\
\hline & $150 \mathrm{~K} 400 \mathrm{~K} / 200 \mathrm{~K}$ & $1,300,000$ & 1.640 .000 & FY95-96.FY99-2000.FY2001 & Data from Similar SAR's \\
\hline & N/A & N/A & N/A & & \\
\hline & $375 \mathrm{~K} / 100 \mathrm{~K} / 200 \mathrm{~K}$ & 1.150 .000 & $1,380,000$ & FY96-97.FY98-99.FY2000 & Engineer's Estimate (Includes Sampling) \\
\hline & $1 \mathrm{M} / 1 \mathrm{M} / 1 \mathrm{M} / 25 \mathrm{~K}$ & 3.250 .000 & 3.580 .000 & FY94 - FY97 & Engineer's Estimate \\
\hline & $200 \mathrm{~K} / 150 \mathrm{~K}$ & 350.000 & 430.000 & FY1998-FY1999 & Engineer's Estimate \\
\hline & SOKNA & 400,000 & 500.000 & FY1995-2002 & Engineer's Estimate \\
\hline & $250 \mathrm{KNR}$ & 500,000 & 650.000 & FY1999-2000 & Engineer's Estimate \\
\hline & 177KNY & 1.947 .000 & 85.770 .000 & FY2000.01.11.21.31.41,61.81.2 & Engineer's Estumate \\
\hline & & & & & \\
\hline & & & & & \\
\hline & & & & & \\
\hline & $100 K / 65 K N D$ & am & $410 \mathrm{mot}$ & FYo4 EYa5-09 & \\
\hline & rownosivin & 300,000 & 410.000 & FY94. PY9s-98 & Engineer's Esumate \\
\hline & $200 \mathrm{KYA}$ & & $1.140,000$ & & \\
\hline & $200 \mathrm{KNR}$ & & 480,000 & FY97-98 & \\
\hline & & & & & \\
\hline & & & & & \\
\hline & & & & & \\
\hline & & & & & \\
\hline & & & & & \\
\hline & & & & & \\
\hline & & 17.787 .000 & 104.260 .000 & & \\
\hline
\end{tabular}

Date:

Crk'd By:

D. N Stoddard

File No. $5204-$ Alt. 5

93

Appr'd By: $A$ PCl

STMATE BASIS 
Page 2 of 5

Project EVALUATION OF ALTERNATIVES FOR DISPOSAL

OF INEL MIXED AND LOW LEVEL WASTE

\begin{tabular}{|c|c|c|c|c|c|c|c|}
\hline $\begin{array}{l}\text { ACCT. } \\
\text { NO. }\end{array}$ & $\begin{array}{c}\text { ACTIVITY } \\
\text { DESCrAPIION }\end{array}$ & $\begin{array}{l}\text { E.V. } \\
\text { P.H }\end{array}$ & $\begin{array}{l}\text { ESTMATED COST } \\
\text { PER YEAR }\end{array}$ & $\begin{array}{l}\text { UNESCALATED } \\
\text { COST }\end{array}$ & $\begin{array}{c}\text { ESCALATED } \\
\text { COST }\end{array}$ & $\begin{array}{l}\text { ACTIVITY SCHEDULE } \\
\text { ESCALATION }\end{array}$ & ESTIMATE BASIS \\
\hline & DESIGN & & & & & & \\
\hline & Conceptual & & $500 \mathrm{~K} 1500 \mathrm{~K}$ & 1.000 .000 & $1.030,000$ & FY95.FY96 & TDD Sheet \\
\hline$\ldots$ & $\ldots \ldots$ & & $1 M$ & 1.000 .000 & 1.180 .000 & FY97 & Engineers Estimate \\
\hline$\ldots$ & Title II & & $1 \mathrm{M} / 100 K$ EA YRAFT & 1.900 .000 & $45.460,000$ & FY $98,1010.20 .30 .40 .60,80.21$ & Engineers Estimate \\
\hline & Sublotal for Design (Based on $\%$ or Construction Costs) & & & 3.900 .000 & 47.670 .000 & & \\
\hline & & & & & & & \\
\hline & Title III Inspection (4 People for 18 Months initial const) & & $575 \mathrm{~K} / 288 \mathrm{~K} \mathrm{EA}$ & 863.000 & $1.150,000$ & FY2000,20001 & Comparative Historical Data \\
\hline & Title III Inspection (2 People for 12 Months storage bld' gs & & $288 \mathrm{KNR}$ & 2.590 .000 & 136.940 .000 & FY2011.21.31.41.61.81.2101.2 & Comparative Hist. Data \\
\hline 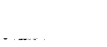 & CONSTRUCTION & & & & & & \\
\hline 4000 & Improvements to Land & & & & & & \\
\hline & Surveying (47 Acres) & & 61.000 & 51.000 & 80.000 & FY2000 & Comparative Historical Data \\
\hline & Site Prep Clearing and Grubbing ( 47 Acres) & & 141.000 & 141.000 & 185.000 & FY2000 & Comparative Historical Data \\
\hline & Roadways and Parking & & 810.000 & 810.000 & 1.065 .000 & FY20W & Comparative Historical Data \\
\hline & Fencing & & 160.000 & 160.000 & 210,000 & FY2000 & Comparative Historical Data \\
\hline & Retention Pond & & 40.000 & 40.000 & 53.000 & FY2000 & Comparative Historical Data \\
\hline & Drainage Gallery System & & 1.260 .000 & 1.260 .000 & 1.657 .000 & FY2000 & Comparative Historical Data \\
\hline & Drainage Pumphouse & & 100.000 & 100,000 & 132.000 & FY2000 & Comparative Historical Data \\
\hline & Collection / Sampling System Vault at Disposal Areas & & 1.170 .000 & 1.170 .000 & 1.539 .000 & FY2000 & Comparative Historical Data \\
\hline & Storage Vault Disposal Area Including: Engineered & & N/A & N/A & N/A & & \\
\hline & Fill, and Pit Run & & & & & & \\
\hline & Drainage Ditches & & 90,000 & 90,000 & 118.000 & FY2000 & Comparative Historical Data \\
\hline & TOTAL FOR 4000 ACCOUNT & & & $3,832,000$ & 5.039 .000 & FY2000 & \\
\hline 6000 & Utiitities & & & & & & \\
\hline & Electrical Power and Distribution To Facility & & 175.000 & 175,000 & 230.000 & FY2000 & Engineers Estimate \\
\hline & Electrical Lighting, Power, Alarms, \& Cormmunication & & $1.080,000$ & $1,080,000$ & 1.421 .000 & FY2000 & Engineers Estimate \\
\hline & Distribution System & & & & & & Engineers Estimate \\
\hline & Water Supply \& Fire Water Distribution to Facility & & 320,000 & 320,000 & 421.000 & FY2000 & Engineers Estimate \\
\hline & Water Supply and Fire Water Distribution Systerns & & 770.000 & 770.000 & $1.013,000$ & FY2000 & Engineers Estimate \\
\hline & Sanitary Sewage System & & 250.000 & 250,000 & 329.000 & FY2000 & Engineers Estimate \\
\hline & TOTAL FOR 6000 ACCOUNT & & & 2.595 .000 & 3.414 .000 & & \\
\hline
\end{tabular}


Project EVALUATION OF ALTERNATIVES FOR DISPOSAL OF INEL MIXED AND LOW LEVEL WASTE

\begin{tabular}{|c|c|c|c|c|c|c|c|}
\hline $\begin{array}{l}\text { ACCT. } \\
\text { No. }\end{array}$ & $\begin{array}{c}\text { ACTIVITY } \\
\text { DESCAIPTION }\end{array}$ & $\begin{array}{l}\text { E.V. } \\
\text { P.H. } \\
\end{array}$ & $\begin{array}{c}\text { ESTMATED COST } \\
\text { PER YEAR }\end{array}$ & $\begin{array}{c}\text { UNESCALATED } \\
\text { COST }\end{array}$ & $\begin{array}{c}\text { ESCALATED } \\
\text { COST }\end{array}$ & $\begin{array}{c}\text { ACTIVITY SCHEDULE } \\
\text { ESCALATION }\end{array}$ & ESTIMATE BASIS \\
\hline \multirow[t]{2}{*}{5000} & Buildings and Struclures & & & & & & \\
\hline & Otfice /Administration Bldg. (1 story - $10.000 \mathrm{~s}$ ) & & $1.240,000$ & 1.240 .000 & 1.690 .000 & FY2001 & Comparative Historical Data \\
\hline & Support Building (1 story - 8000 s) & & 825.000 & 825.000 & 1.130 .000 & Fr2001 & Comparative Historical Data \\
\hline & Material Storage Building ( 1 story -2500 sf) & & 200.000 & 200,000 & 273.000 & FY2001 & Comparative Historical Data \\
\hline & Heavy Equipment Storage \& Maintenance & & 750,000 & 750,000 & 1.020 .000 & FY2001 & Comparative Historical Data \\
\hline & \multicolumn{2}{|l|}{ a- Metal Storage Bldg-Type A Mixed $(104 \times 224 \times 18)(5.5,3.3)$} & $6.4 \mathrm{M} / 6.4 \mathrm{M} / 3.8 \mathrm{M} / 3.8 \mathrm{M}$ & 20.400 .000 & $47.930,000$ & FY2000,11.21,31 & Comparative Historical Data at SR Site \\
\hline & \multicolumn{2}{|l|}{ b- Metal Storage Bldg-Type A LLW $(100 \times 224 \times 26)(2.1 .1 .1)$} & $2.6 \mathrm{M} / 1.3 \mathrm{M} / 2.3 \mathrm{M} / 1.3 \mathrm{M}$ & 6.500 .000 & 15.170 .000 & FY2000.11.21.31 & Comparative Historical Data at SR Site \\
\hline & \multicolumn{2}{|l|}{ 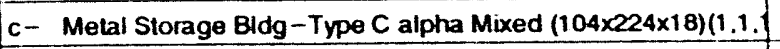 } & $1.3 \mathrm{M} / 1.3 \mathrm{M} / 1.3 \mathrm{M}$ & $3,900,000$ & 8.030 .000 & FY2000.11.21.31 & Comparative Historical Data at SR Site \\
\hline & \multicolumn{2}{|l|}{ d- Conc Storage Unit-Type B\&C beta/gamma $(58 \times 166 \times 31)(1.1$} & $2.35 M / 235 M$ & 4.700 .000 & 9.900 .000 & FY2000.2121 & Comparative Historical Data at SR $S: \subseteq$ \\
\hline & \multicolumn{2}{|l|}{ 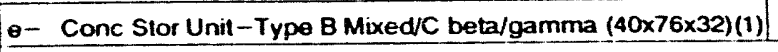 } & 1.000 .000 & 1.000 .000 & 1.300 .000 & FY2000 & Comparative Historical Data at SR Site \\
\hline & \multicolumn{3}{|c|}{ a- Metal Stor Bldg 40 Yr D\&D/Replace'l $(5.5 .3 .3 .5 .5)-7.5 \mathrm{M} / 7.5 \mathrm{M} / 46 \mathrm{M} / 4.6 \mathrm{M} / 7.5 \mathrm{M} / 7.5 \mathrm{~N}$} & 39.200 .000 & 3.323 .000 .000 & FY2041.61.81,FY2101.21.41 & Comparative Historical Data at SR Site \\
\hline & \multicolumn{3}{|c|}{ b- Metal Stor Bldg 40 Yr D8D/Replace't $(2.1 .1 .1 .2 .1)-3.1 \mathrm{M} / 1.6 \mathrm{M} / 1.6 \mathrm{M} / 1.6 \mathrm{M} / 3.1 \mathrm{M} / 1$. $6 \mathrm{f}$} & 10.400 .000 & $495.000,000$ & FY2041.61.81.FY2101.21.41 & Comparative Historical Data at SR Site \\
\hline & \multicolumn{3}{|l|}{ c- Metal Stor Bldg 40 Yr Replacement (1,1.1.1)-1.6M/EA YA } & 6.400 .000 & 670.000 .000 & FY2041,61,81.FY2121.41 & Comparative Historical Data at SR Site \\
\hline & TOTAL FOR 5000 ACCOUNT & & & 92.500 .000 & $4.570,330.000$ & & \\
\hline & & & & & & & \\
\hline & & & & & & & \\
\hline & Operating Equipment & & & & & & \\
\hline & Decon Equipment (1 Lot) & & 100.000 & 100.000 & 142.000 & FY2002 & Engineers Estimate Based on RWMC Study \\
\hline & Dump Truck (1) & & 85.000 & 85.000 & 120.000 & FY2002 & Engineers Estimale Based on RWMC Study \\
\hline & Backhoe (1) & & 125.000 & 125.000 & 177,000 & FY2002 & Engineers Estimate Based on RWMC Study \\
\hline & Water Truck (1) & & 400.000 & 400.000 & 570,000 & FY2002 & Engineers Estimate Based on RWMMC Study \\
\hline & Common Support Vehicles (5) & & 50.000 & 50.000 & 70.000 & FY2002 & Engineers Estimate Based on RWMK Study \\
\hline & Forklifts (4) & & 160.000 & 160.000 & 230.000 & FY2002 & Engineers Estimate Based on RWMC Study \\
\hline & Gantry Crane (1) & & 400.000 & 400.000 & 570,000 & FY2002 & Engineers Estimate Based on RWMC Study \\
\hline & Safety Systems - Deisel Generators, etc. (1) & & 200.000 & 200.000 & 280.000 & Fr2002 & Engineers Estimate Based on RWMC Study \\
\hline & Equipment Replacement & & 750KVEA & 3.000 .000 & 40.513 .000 & $F Y 2008.2018 .2028 .2038$ & Engineers Estimate Based on RWMC Study \\
\hline & SUBTOTAL FOR EOUIPMENT COSTS & & & 4.520 .000 & 42.672 .000 & & \\
\hline & & & & & & & \\
\hline & CONSTRUCTION MANAGEMENT & & & 23.618 .000 & 1.056 .325 .000 & FY2000.01.13.23.24 \& 2033 & Engineers Estumate \\
\hline
\end{tabular}


Rer 5-9:

Page 4 of 5

Project EVALUATION OF ALTERNATIVES FOR DISPOSAL OF INEL MIXED AND LOW LEVEL WASTE

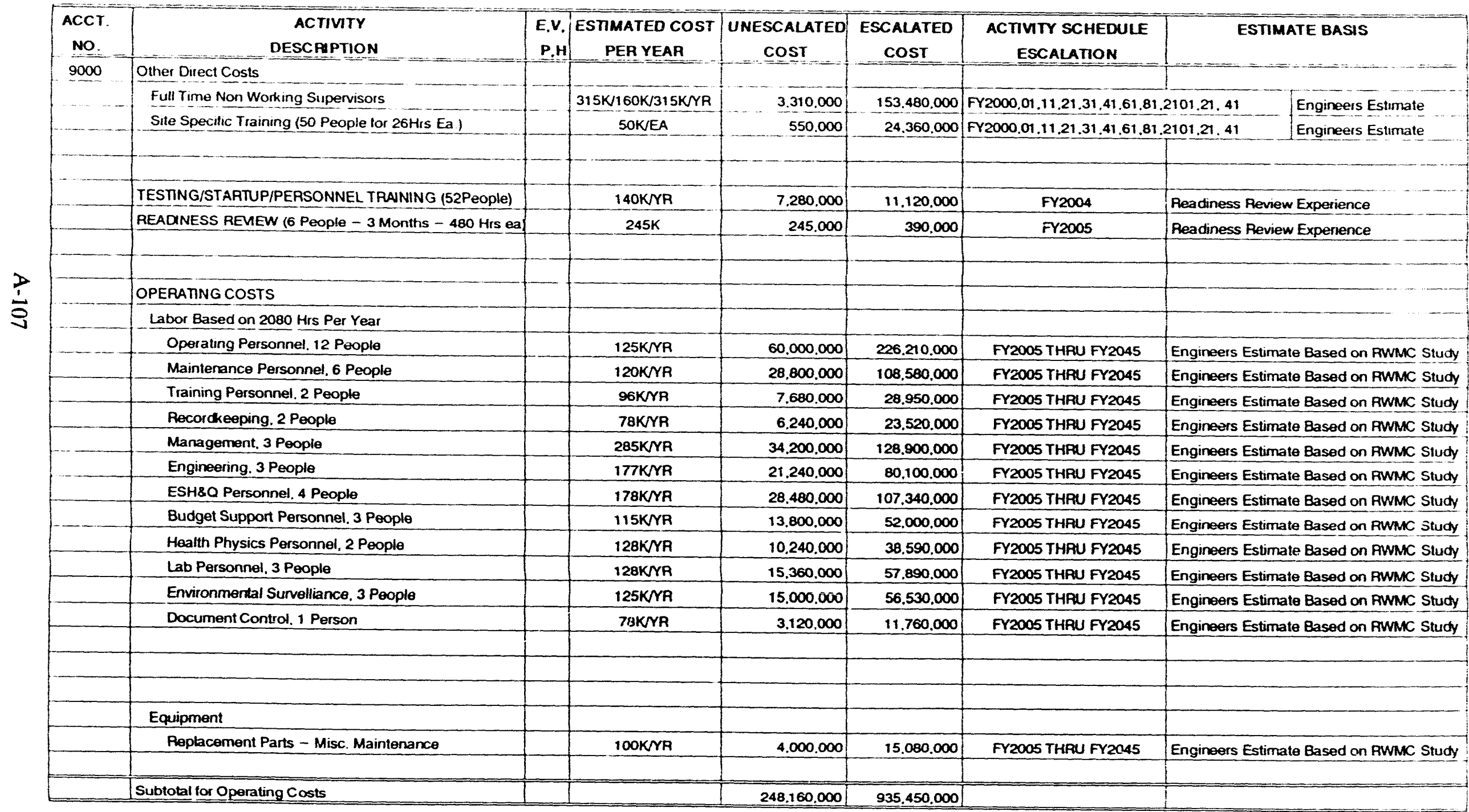


Rev 5-92

Page 5 of 5

EVALUATION OF ALTERNATVES FOR DISPOSAL OF INEL MIXED AND LOW LEVEL WASTE

\begin{tabular}{|c|c|c|c|c|c|c|c|}
\hline $\begin{array}{l}\text { ACCT. } \\
\text { No. }\end{array}$ & $\begin{array}{c}\text { ACTIVITY } \\
\text { DESCRIPTION } \\
\end{array}$ & $\begin{array}{l}\text { E.V. } \\
\text { P.H }\end{array}$ & $\begin{array}{c}\text { ESTMATED COST } \\
\text { PEA YEAA }\end{array}$ & $\begin{array}{c}\text { UNESCALATED } \\
\text { COST }\end{array}$ & $\begin{array}{c}\text { ESCALATED } \\
\text { COST } \\
\end{array}$ & $\begin{array}{c}\text { ACTIVITY SCHEDULE } \\
\text { ESCALATION }\end{array}$ & ESTIMATE BASIS \\
\hline & UTIUTIES & & & & & & \\
\hline & Electrical & & $25 \mathrm{KNR}$ & 1.000 .000 & 3.770 .000 & FY2005 THAU FY2045 & Engineers Estimate \\
\hline & Fuels/Propane, etc. & & 5KVR & 200,000 & 750,000 & FY2005 THRU FY2045 & Engineers Estimate \\
\hline & Other & & 30KVR & 1.200 .000 & 4.520 .000 & FY2005 THRU FY2045 & Engineers Estimate \\
\hline & Subtotal for Utility Costs & & & $2.400,000$ & $9.040,000$ & & \\
\hline & & & & & & & \\
\hline & & & & & & & \\
\hline & & & & & & & \\
\hline & DECONTAMINATION AND DECOMMISSIONING & & 400.000 & 400.000 & 2.900 .000 & FY2046 & Data From Previous D \& D Projects \\
\hline & (D\&D Costs are based on $10 \%$ of the construction co & st relatir & ing directly to the lacilit & ties being decommis & issioned) & & \\
\hline & & & & & & & \\
\hline & & & & & & & \\
\hline & SURVELLANCEMONTORING & & & & & & \\
\hline & Environmental Surveillance. 3 People & & $125 \mathrm{KVYR}$ & 4.125 .000 & 36.610 .000 & FY2046 THRU FY2056 & Engineers Estimate \\
\hline & Environmental Surveillance. 1 Person & & 125KNR & 11.250 .000 & $1,004.150 .000$ & FY2057 THRU FY2146 & Engineers Estimate \\
\hline & Subtotal Surveillance/Monitoring & & & 15.375 .000 & 1.040 .760 .000 & & \\
\hline & & & & & & & \\
\hline & & & & & & & \\
\hline & & & & & & & \\
\hline & & & & & & & \\
\hline & & & & & & & \\
\hline & & & & & & & \\
\hline & & & & & & & \\
\hline & & & & & & & \\
\hline & & & & & & & \\
\hline & & & & & & & \\
\hline & & & & & & & \\
\hline & & & & & & & \\
\hline & & & & & & & \\
\hline & & & & & & & \\
\hline
\end{tabular}


Filo No. 5204 - Alt. 5

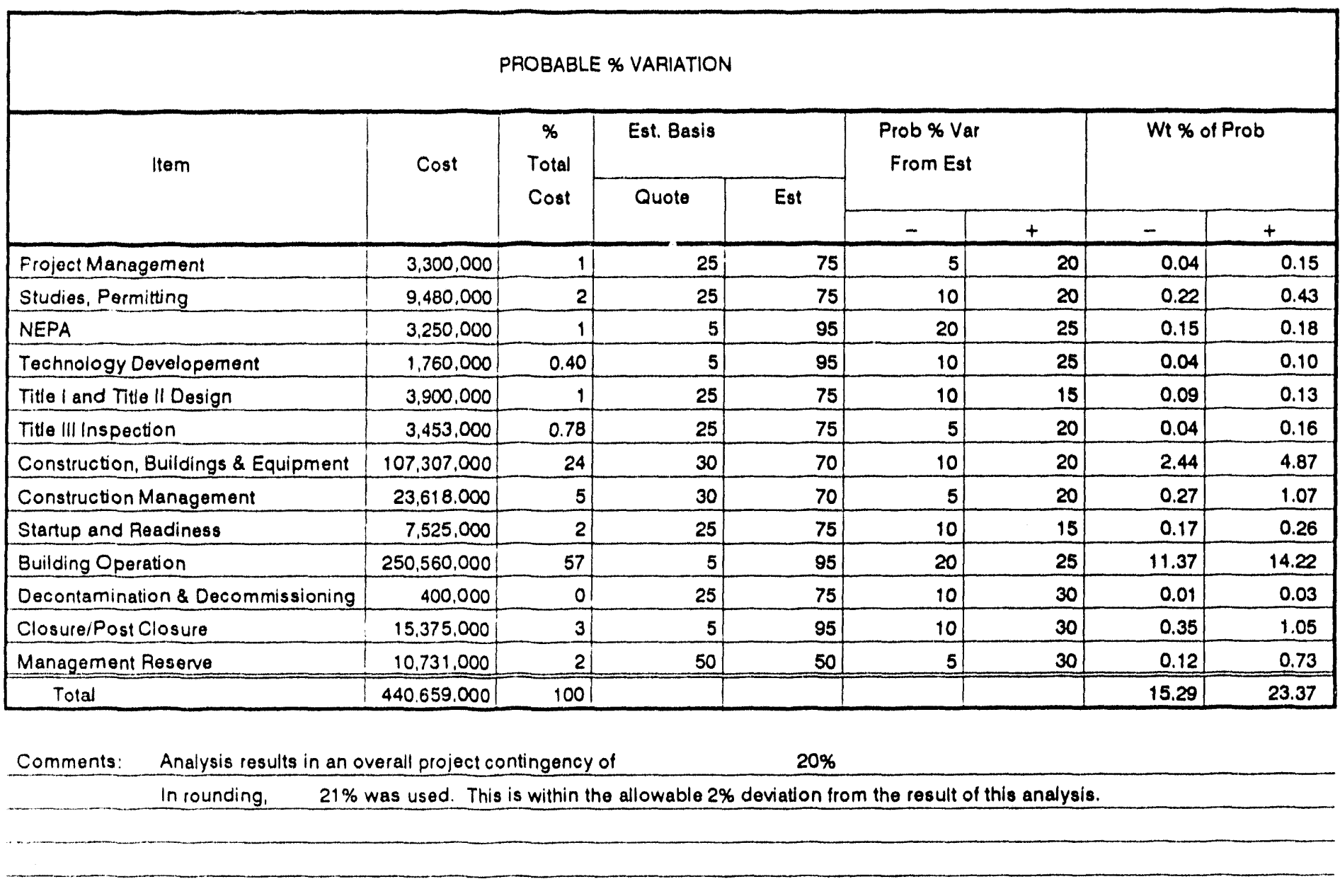




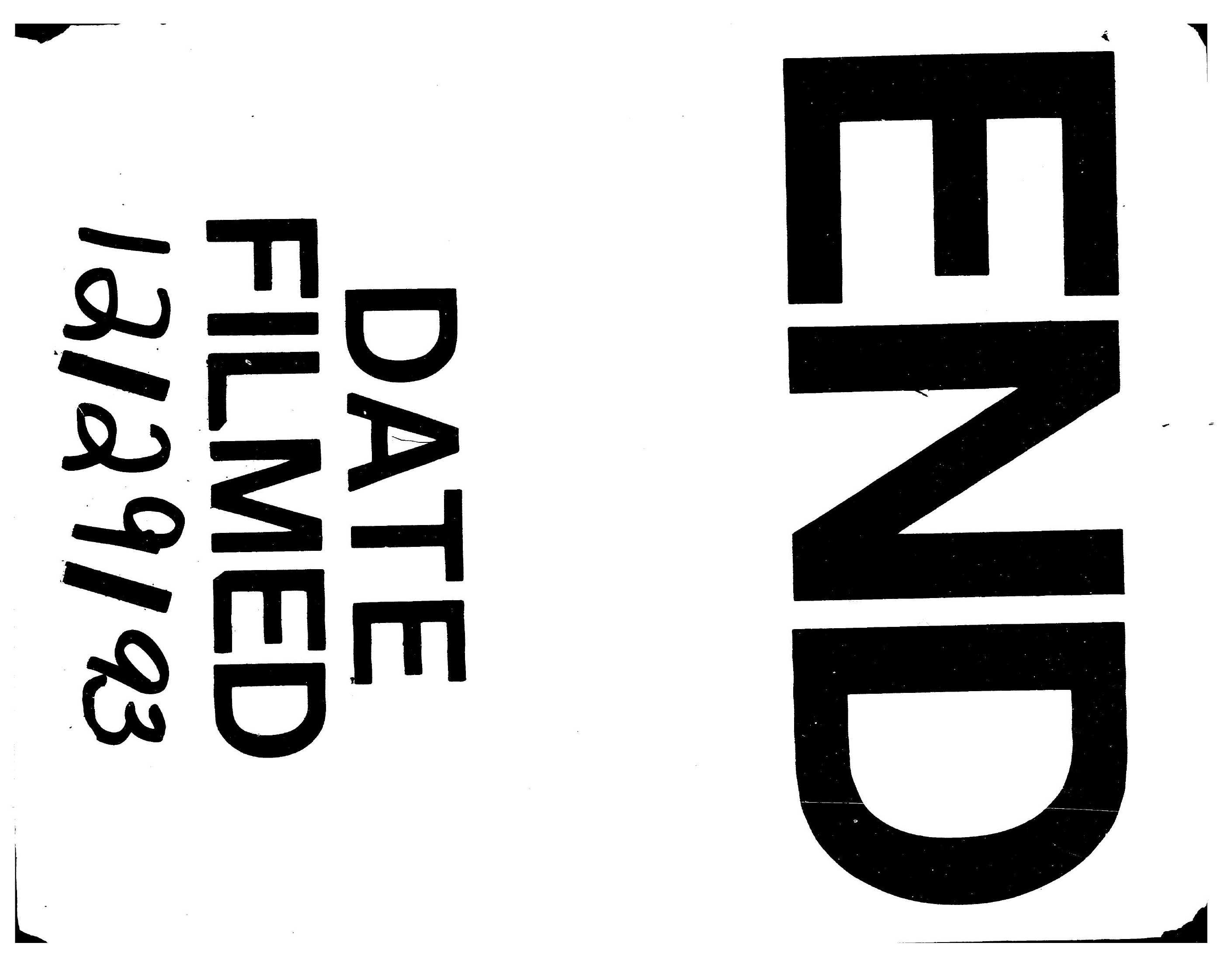

